\title{
ARBEITSTEILUNG DER ELTERN
}

Die Aufteilung der Erwerbsarbeit, der Hausarbeit und der Kinderbetreuung in Abhängigkeit vom Alter des Kindes

\section{DISSERTATION}

zur Erlangung des Doktorgrades Dr. disc. pol. an der Sozialwissenschaftlichen Fakultät der Georg-August-Universität Göttingen

\author{
vorgelegt von \\ Magdalena Gerum \\ geboren in Rosenheim
}

Göttingen, 2021 


\section{Betreuungsausschuss}

Erstgutachterin: Prof. Dr. Karin Kurz

Zweitgutachterin: Prof. Dr. Daniela Grunow

Weitere Mitglieder der Prüfungskommission:

Dr. Claudia Zerle-Elsäßer

Tag der mündlichen Prüfung: $\quad$..09.06.2021 


\section{Inhaltsverzeichnis}

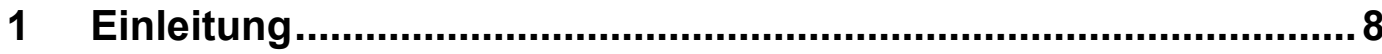

2 Konzeptionelle Rahmung: Das Familienzyklus-Modell und die Lebensverlaufsperspektive...................................................14

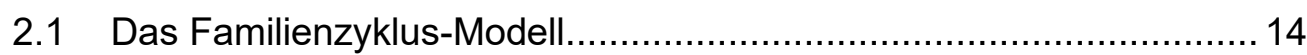

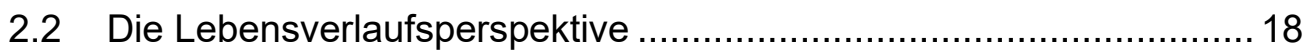

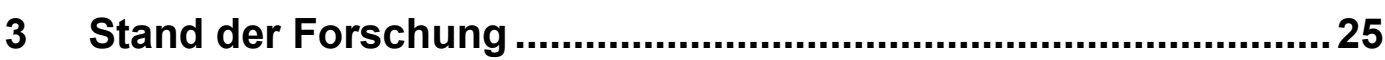

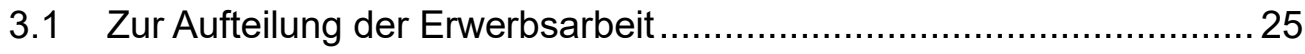

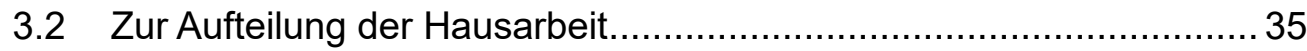

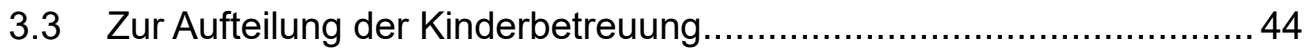

3.4 Forschungslücke und Forschungsfragen.......................................... 49

4 Theoretische Ansätze zur Aufteilung von Erwerbsarbeit,

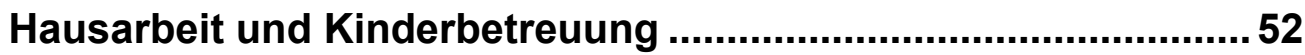

4.1 Ressourcenbasierte Erklärungsansätze ………………………….... 54

4.1.1 Familienökonomische Theorie ..............................................54

4.1.2 Ökonomische Verhandlungstheorie........................................59

4.2 Geschlechterrollen-, normen- und identitätsbasierte Ansätze .............66

4.2.1 (Geschlechter-)Rollentheorien, Normen und Einstellungen .... 66

4.2.2 „Doing Gender“ und Kompensationshypothese ........................ 68

4.2.3 Identitätsformationsmodell ...................................................... 72

4.2.4 Stabilität oder Wandelbarkeit von Einstellungen ...................... 74

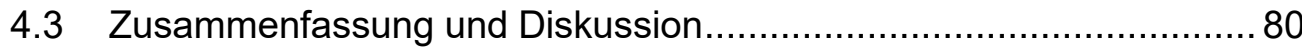

5 Institutionelle und kulturelle Rahmenbedingungen der Arbeitsteilung der Eltern ......................................................... 84

5.1 Deutschland aus wohlfahrtsstaatlicher Perspektive ......................... 84

5.2 Geschlechterarrangements und kulturelle Leitbilder in Ost- und

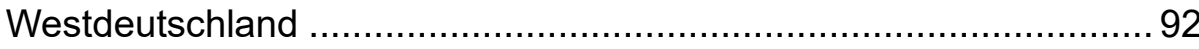

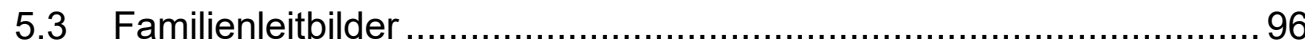


5.4 Zeit, Geld und Infrastruktur: Zur Entwicklung der ,Trias der Familienpolitik' in Deutschland. 104

5.5 Verknüpfung der theoretischen Ansätze mit den institutionellen Rahmenbedingungen

6 Hypothesengenerierung ........................................................... 121

$7 \quad$ Daten und Methoden ............................................................... 129

7.1 Datengrundlage: DJI-Survey 2013/14 „AID:A“............................. 129

7.2 Operationalisierung der Variablen ............................................ 131

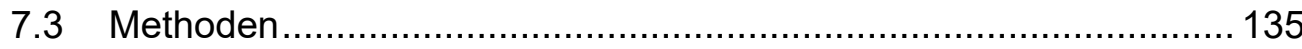

7.4 Verteilungen von zentralen Variablen ......................................... 139

7.5 Exkurs: Latente Klassenanalyse zur multidimensionalen Abbildung von Geschlechterrolleneinstellungen ........................... 144

8 Aufteilung der Erwerbsarbeit .................................................... 157

8.1 Deskriptive Befunde ............................................................ 157

8.2 Multivariate Analysen ............................................................. 165

$9 \quad$ Aufteilung der Hausarbeit ........................................................ 190

9.1 Deskriptive Befunde .................................................................. 190

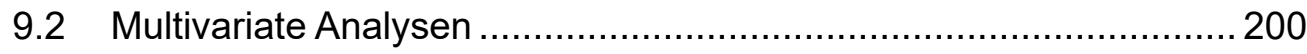

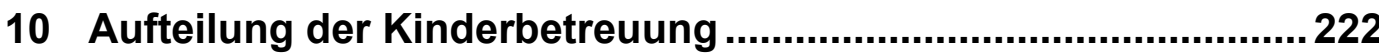

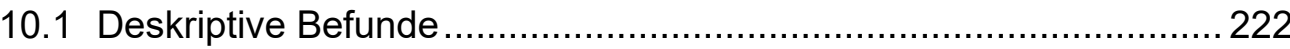

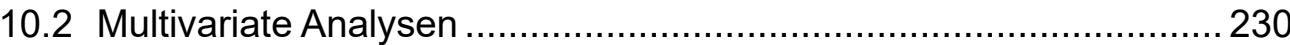

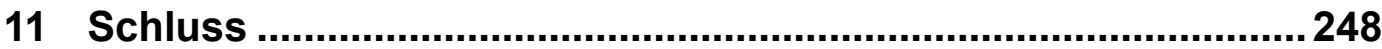

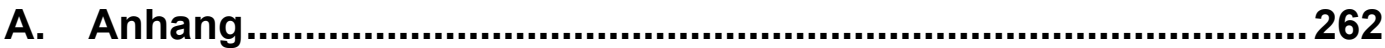

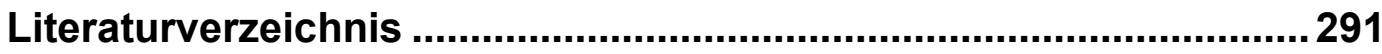

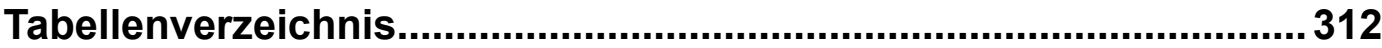

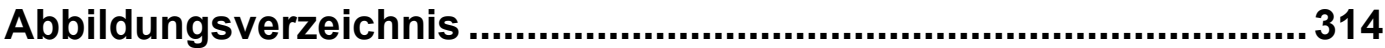




\section{Danksagung}

Viele Personen haben dazu beigetragen, dass diese Dissertation entstehen konnte und an dieser Stelle möchte ich mich gerne ausdrücklich bei diesen Personen bedanken.

Mein besonderer Dank gilt meiner Doktormutter Frau Prof. Dr. Karin Kurz. Ich danke Ihnen für die hervorragende Betreuung, die zahlreichen Gespräche und die enorme Unterstützung bei der Umsetzung der gesamten Arbeit. Vielen, herzlichen Dank!

Ebenfalls besonders bedanken möchte ich mich bei meiner Zweibetreuerin Frau Prof. Dr. Daniela Grunow. Ich danke Ihnen für Ihre intensive Auseinandersetzung mit meiner Arbeit und für ihre umfangreichen Hilfestellungen!

Die Idee zur Dissertation ist aus einem Forschungsprojekt am Deutschen Jugendinstitut e.V. München (DJI) entstanden. Ich danke dem DJI für die Bereitstellung der Datengrundlage „Aufwachsen in Deutschland: Alltagswelten“ (AID:A) und für ein Stipendium, dank dem ich meine Dissertation abschließen konnte.

Mein Dank gilt auch vielen Kolleginnen und Kollegen des DJls: Besonders danke ich meiner Fachgruppenleitung Frau Dr. Claudia Zerle-Elsäßer, die mich von Anfang an begleitet und unterstützt hat. Ebenso danke ich den Kolleginnen und Kollegen dieser Fachgruppe sowie dem „Kompetenzteam Familie“ unter der Leitung von Frau Dr. Claudia Hoffmann-Recksiedler. Ich danke der Abteilungsleiterin Frau Dr. Christina Boll sowie der ehemaligen Abteilungsleiterin Frau Dr. Karin Jurczyk.

Des Weiteren danke ich der Abteilung „Zentrum für Dauerbeobachtung und Methoden“ für die methodische Unterstützung im Umgang mit den „AID:A“-Daten. Ich danke der Forschungsdirektorin Frau Prof. Dr. Sabine Walper sowie den Teilnehmenden ihres DJIKolloquiums für die inhaltliche und methodische Auseinandersetzung mit meiner Arbeit. Herzlich bedanken möchte ich mich auch bei Leonie Kleinschrot für die vielen, hilfreichen Gespräche und für die intensive Auseinandersetzung mit meiner Arbeit!

Darüber hinaus möchte ich mich bei den Doktorandinnen und Doktoranden von Frau Prof. Dr. Karin Kurz bedanken, die mir in den Kolloquien wertvolle Tipps und Feedback gegeben haben. Namentlich erwähnt seien hier Dr. Jörg Hartmann, Katharina Kilburger, Judith Lehmann, Henrik Schwarze, Barbara Franke und Qiuren Wang. Ich danke euch für den großartigen Austausch!

Besonderer Dank gilt auch meinem Partner Jürgen Schaller, der mich während der ganzen Promotionsphase unterstützt hat und hinter mir stand. Ich danke dir für deine Geduld und deine Ermutigungen!

Ganz herzlich möchte ich mich auch bei meinen Eltern Maria Gerum und Dr. Eduard Gerum bedanken, die mich während des gesamten Studiums unterstützt haben. Meinen Eltern möchte ich diese Dissertation daher widmen. 


\section{Zusammenfassung}

Familiensoziologische Forschung vernachlässigt bislang die Bedeutung des Kindesalters als Erklärung dafür, wie sich Eltern die Erwerbsarbeit, die Hausarbeit und die Kinderbetreuung aufteilen. Dabei ist es offenkundig, dass sich mit einem jugendlichen Kind andere Ausgestaltungsspielräume für die Eltern ergeben als mit einem Säugling.

Im Fokus dieser Dissertation stehen daher Forschungsfragen, die sich darauf beziehen, wie Eltern sich die Erwerbsarbeit, die Hausarbeit und die Kinderbetreuung in Abhängigkeit vom Alter des Kindes aufteilen. Des Weiteren interessiert die Frage danach, welche Rolle Einflussfaktoren wie z.B. relative Ressourcen oder Geschlechterrolleneinstellungen für die Arbeitsteilung spielen und ob sich deren Bedeutung in Abhängigkeit vom Alter des Kindes verändert. Einen Theoriestrang stellen dabei ökonomische Theorien wie die familienökonomische Theorie und die Verhandlungstheorie dar; einen weiteren Theoriestrang bilden die geschlechterrollen-, normen- und identitätsbasierten Ansätze. Bei beiden Theoriesträngen wird in dieser Dissertation herausgearbeitet, welche Bedeutung dem Alter des Kindes jeweils zugeschrieben werden kann.

Doch zusätzlich spielen auch in Deutschland gegebene institutionelle und kulturelle Rahmenbedingungen wie etwa familienpolitische Maßnahmen (wie z.B. Geldpolitiken, Zeitpolitiken und infrastrukturelle Regelungen) und Familienleitbilder eine wichtige Rolle. Daher werden Hypothesen abgeleitet, die sich aus der Verknüpfung der theoretischen Ansätze mit den institutionellen und kulturellen Rahmenbedingungen ergeben und die zusätzlich zwischen den Altersstufen des Kindes differenzieren. Diese altersspezifischen Hypothesen machen Annahmen darüber, welche Rolle die Einflussfaktoren in verschiedenen Altersstufen des Kindes und unter den jeweiligen Rahmenbedingungen (wie z.B. Familienleitbilder, Geldpolitiken, Zeitpolitiken und infrastrukturelle Regelungen) spielen.

Als Datengrundlage zur Beantwortung der Forschungsfragen dient der DJI-Survey „Aufwachsen in Deutschland: Alltagswelten“ (kurz: „AID:A“) von 2013/14. Anhand von diversen, multivariaten Analysen wird herausgearbeitet, welche Rolle das Alter des Kindes für die Aufteilung der Erwerbsarbeit, der Hausarbeit und der Kinderbetreuung spielt und inwiefern sich die Bedeutung weiterer Einflussfaktoren in Abhängigkeit vom Alter des Kindes verändert. Die Ergebnisse zeigen, dass das Kindesalter von zentraler Bedeutung für die Aufteilung der Erwerbsarbeit ist, während es für die Aufteilung der Hausarbeit und der Kinderbetreuung nur eine geringere Rolle spielt. Während die Aufteilung der Erwerbsarbeit mit zunehmendem Alter des Kindes deutlich egalitärer wird, sind die Aufteilung der Hausarbeit und der Kinderbetreuung über das Kindesalter hinweg relativ traditionell. In der Tendenz zeigt sich jedoch, dass die Aufteilung der Hausarbeit bei älteren Kindern etwas traditioneller ausfällt als bei kleineren Kindern, wohingegen die Aufteilung der Kinderbetreuung bei älteren Kindern dagegen etwas egalitärer ist. Insgesamt kann daraus abgeleitet werden, dass Mütter mit dem Älterwerden des Kindes größere Erwerbsoptionen realisieren können, aber offenbar nicht, weil die Väter stärker in die Kinderbetreuung einsteigen, sondern eventuell, weil die Kinder weniger betreuungsintensiv 
sind oder die institutionellen und kulturellen Rahmenbedingungen dies besser ermöglichen.

Aus den Ergebnissen geht hervor, dass niedrigere Ressourcen der Mütter (im Vergleich zu den Ressourcen der Väter) einen negativen Effekt auf den Erwerbsanteil der Mütter haben, aber höhere Ressourcen der Mütter zeigen dagegen keinen positiven Effekt auf den Erwerbsanteil der Mütter. Die relativen Ressourcen haben somit keinen symmetrischen, geschlechtsunabhängigen Effekt. Gleiches trifft auch für die Aufteilung der unbezahlten Arbeit zu: Hier zeigt sich, dass Mütter mehr Hausarbeit und mehr Kinderbetreuung übernehmen, wenn sie niedrigere Ressourcen haben, aber sie übernehmen umgekehrt nicht weniger Hausarbeit und nicht weniger Kinderbetreuung, wenn sie höhere Ressourcen als die Väter haben. Die altersspezifischen Hypothesen hierzu, d.h. dass die Bedeutung der relativen Ressourcen bei kleineren Kindern geringer ist als bei älteren Kindern, können in den meisten Fällen nicht bestätigt werden. Insgesamt kommt den relativen Ressourcen nur eine geringe Bedeutung zu.

Bei den Geschlechterrolleneinstellungen zeigt sich, dass egalitäre Einstellungen mit einer egalitäreren Aufteilung der Erwerbsarbeit, der Hausarbeit und der Kinderbetreuung einhergehen. Die altersspezifischen Annahmen, dass die Geschlechterrolleneinstellungen bei kleineren Kindern (aufgrund institutioneller Rahmenbedingungen) einen geringeren Effekt und bei älteren Kindern einen stärkeren Effekt haben, können für alle drei Dimensionen der Arbeitsteilung dagegen größtenteils nicht bestätigt werden. In vertiefenden Analysen werden die Geschlechterrolleneinstellungen darüber hinaus anhand einer latenten Klassenanalyse multidimensional abgebildet: Hieraus gehen die drei latenten Klassen „intensive mothering/parenting“, „egalitärer Essentialismus“ und „egalitär“ hervor. Wenn diese latenten Klassen als Einflussfaktoren für die Arbeitsteilung verwendet werden, so zeigt sich, dass die Aufteilung der Erwerbsarbeit, der Hausarbeit und der Kinderbetreuung in der Klasse des "intensive mothering/parenting" am traditionellsten aufgeteilt ist und in der „egalitären“ Klasse am egalitärsten, während sich die Klasse des "egalitären Essentialismus“ zwischen diesen beiden Extremen befindet. Allerdings gibt es auch bei dieser multidimensionalen Abbildung der Einstellungen in der Regel keine signifikante Veränderung in Abhängigkeit vom Alter des Kindes, d.h. die Einstellungen spielen für die Arbeitsteilung der Eltern in den meisten Altersstufen des Kindes eine gleich große Rolle.

Darüber hinaus untersucht die Dissertation die Bedeutung weiterer Einflussfaktoren wie etwa die Kinderanzahl, die absoluten Ressourcen der Mütter oder die Unterschiede zwischen Ost- und Westdeutschland für die Arbeitsteilung der Eltern. Die vorliegende Dissertation liefert neue und detaillierte Einblicke darüber, wie sich Eltern die Erwerbsarbeit und die Familienarbeit in Abhängigkeit vom Alter des Kindes aufteilen und bildet dabei alle Altersstufen des Kindes ab - angefangen vom Kleinkind- und Kindergartenalter bis hin zum Grundschul- und Jugendalter. 


\section{$1 \quad$ Einleitung}

Warum teilen sich manche Eltern die Arbeit egalitärer auf als andere und welche Unterschiede gibt es dabei zwischen Eltern mit kleineren Kindern und Eltern mit größeren Kindern? Dieser Forschungsfrage möchte die vorliegende Arbeit nachgehen. Die Frage nach der Arbeitsteilung der Eltern ist nach wie vor aktuell, wie sich insbesondere im Kontext jüngster Familienpolitik zeigt: Seit 2007 setzt sich die deutsche Familienpolitik verstärkt für eine egalitäre Arbeitsteilung zwischen Müttern und Vätern ein. Dieser neue Kurs der deutschen Familienpolitik wird auch als „Paradigmenwechsel“ bezeichnet (Schutter und Zerle-Elsäßer 2012, S. 219; Bujard 2013, S. 133; Menke und Klammer 2017, S. 213). „Moderne Familienpolitik setzt auf Partnerschaftlichkeit“ (BMFSFJ 2014b) - so wirbt das Bundesministerium für Familie, Senioren, Frauen und Jugend. Beispiele für neuere familienpolitische Maßnahmen, durch die eine egalitäre Arbeitsteilung gefördert wird, sind etwa die Verabschiedung des Elterngeldes mit den Optionen des ElterngeldPlus und des Partnerschaftsbonus, ebenso wie der verstärkte Ausbau von Betreuungsstätten für unter 3-jährige Kinder. Diese neuere Familienpolitik scheint daher eine egalitärere Arbeitsteilung der Eltern eher zu ermöglichen als die frühere Familienpolitik. Ist deshalb davon auszugehen, dass Eltern - sofern sie sich dies wünschen - eine egalitäre Arbeitsteilung auch realisieren können?

Bevor auf die aktuelle Arbeitsteilung zwischen Müttern und Vätern eingegangen wird, wird zunächst ein Blick in die Historie geworfen. Hierbei zeigt sich, dass sich die Arbeitsteilung zwischen Frauen und Männern seit Mitte der 1960er Jahre deutlich verändert hat: So ist in den letzten Jahrzehnten die Erwerbspartizipation der Frauen deutlich gestiegen und Väter beteiligen sich mehr an der Familienarbeit. Dennoch sind sowohl die Erwerbsarbeit als auch die Familienarbeit nach wie vor sehr ungleich zwischen den Geschlechtern verteilt. So zeigt sich etwa bei der Erwerbsarbeit, dass diese in Deutschland insbesondere nach der Familiengründung sehr geschlechtsasymmetrisch verteilt ist: Zwar ist die Erwerbsquote der Mütter in den letzten Jahrzehnten stark angestiegen ist, aber es gibt weiterhin große Unterschiede im Erwerbsumfang, da der Großteil der erwerbstätigen Mütter in Deutschland lediglich in Teilzeit erwerbstätig ist (BMFSFJ 2014a, S. 38; OECD 2016, S. 145-146), während die meisten Väter in Vollzeit beschäftigt sind (BMFSFJ 2018b, S. 31). Obwohl viele kinderlose Paare in gleichem Umfang erwerbstätig sind, so ist es weiterhin ein seltenes Phänomen, wenn Paare auch nach der Familiengründung noch in gleich hohem Umfang erwerbstätig sind, insbesondere dann, wenn die Kinder noch klein sind (Buschner et al. 2018).

Auch bei der Hausarbeit zeigt sich weiterhin eine geschlechtsspezifische Arbeitsteilung: Obwohl sich die Hausarbeitszeiten der Frauen seit den 1960er Jahren stark reduziert haben und die Hausarbeitszeiten der Männer leicht gestiegen sind (Bianchi et al. 2000; Coltrane 2000; Gershuny 2000; Hook 2010; Sayer 2010; Bianchi et al. 2012; Grunow 2014; Hook 2017), übernehmen Frauen weiterhin den Löwenanteil der Hausarbeit (Geist 2005; Gershuny et al. 2005; Lachance-Grzela und Bouchard 2010; Nitsche und Grunow 
2016; Grunow 2019). Neben der Aufteilung der Hausarbeit ist auch die Aufteilung der Kinderbetreuung in den letzten Jahrzehnten egalitärer geworden: Die „neuen“, „aktiven“ Väter beteiligen sich mehr an der Kinderbetreuung und verbringen im Vergleich zu früher mehr Zeit mit ihren Kindern (Sayer et al. 2004a; Wall und Arnold 2007; Craig et al. 2014; McGill 2014). Betrachtet man die Kinderbetreuungszeit der Väter jedoch differenziert nach Werktagen und Wochenendtagen, so zeigt sich, dass die Väter v.a. an den Wochenenden viel Zeit mit den Kindern verbringen, während ihre Kinderbetreuungszeit an den Werktagen immer noch gering ausfällt (Walper und Lien 2018). Des Weiteren gibt es geschlechtsspezifische Unterschiede bzgl. der Art der Kinderbetreuung: So übernehmen Mütter häufiger die Routine-Betreuung (worunter beispielsweise die Körperpflege und die allgemeine Beaufsichtigung fällt), während Väter überwiegend an der angenehmeren, interaktiven „quality-time“ (wie etwa Gespräche, Spiele und Sport) beteiligt sind (Walper und Lien 2018).

Insgesamt lässt sich konstatieren, dass eine geschlechtsspezifische Arbeitsteilung noch immer präsent ist, obwohl sich in den letzten Jahrzehnten sowohl auf internationaler als auch auf nationaler Ebene ein Einstellungswandel vollzogen hat: Traditionelle Geschlechterrolleneinstellungen, die auf eine geschlechtsspezifische Arbeitsteilung abzielen, haben abgenommen, und moderne Geschlechterrolleneinstellungen, die eine egalitäre Arbeitsteilung befürworten, haben zugenommen (Bolzendahl und Myers 2004; Brooks und Bolzendahl 2004; Lee et al. 2007; Davis und Greenstein 2009; Bühlmann et al. 2010; Cotter et al. 2011; Knight und Brinton 2017; Grunow et al. 2018; Inglehart 2018).

Wie jedoch aus vielen Studien hervorgeht, wird die Arbeitsteilung mit dem Übergang zur Elternschaft weiterhin traditioneller, da sich Mütter verstärkt auf die Sphäre der Familienarbeit und Väter auf die Sphäre der Erwerbarbeit spezialisieren (Evertsson und Nermo 2007; Grunow et al. 2007; Bühlmann et al. 2010; Schulz 2010; Grunow und Müller 2012; Kühhirt 2012; Schober 2013a; Grunow und Baur 2014; Baxter et al. 2015; Hipp und Leuze 2015). Diese traditionelle Arbeitsteilung steht somit im Widerspruch zu den egalitärer gewordenen Geschlechterrolleneinstellungen.

Dieser Widerspruch konnte in Deutschland lange Zeit anhand von ungünstigen, institutionellen Rahmenbedingungen erklärt werden, die das männliche Ernährermodell unterstützen, wie beispielsweise dem Ehegattensplitting, dem Erziehungsgeld und der Verbreitung von Halbtagsschulen und Halbtagskindergärten, die allesamt für eine egalitäre Arbeitsteilung hinderlich sind (Grunow et al. 2011, S. 398; Bertram und Bujard 2012, S. 6; Müller et al. 2013, S. 3-4; Menke und Klammer 2017, S. 226). Auch wenn einige dieser Maßnahmen (wie etwa das Ehegattensplitting) immer noch in Kraft sind, so setzt sich die deutsche Familienpolitik, wie eingangs erwähnt, seit 2007 verstärkt für eine egalitäre Arbeitsteilung ein. Doch wenn sowohl die individuellen Einstellungen egalitärer geworden sind und sich auch die institutionellen Rahmenbedingungen dahingehend entwickelt haben, dass sie eine egalitäre Arbeitsteilung zunehmend fördern, warum sind dann nicht mehr Mütter in gleichem Umfang erwerbstätig wie die Väter? Warum übernehmen Mütter 
weiterhin den Großteil der Hausarbeit und der Kinderbetreuung? Möglicherweise sind die institutionellen Rahmenbedingungen immer noch nicht egalitätsfördernd genug oder es sind kulturelle Rahmenbedingungen, wie etwa Familienleitbilder, die einer egalitären Arbeitsteilung im Wege stehen.

Diesen Fragen bzgl. der Arbeitsteilung der Eltern möchte die vorliegende Arbeit nachgehen und stellt dabei die Bedeutung des Alters des Kindes in den Mittelpunkt, da dieses eine Schlüsselrolle spielt. So geht aus bisheriger Forschung hervor, dass das Alter des Kindes eine wichtige Determinante für die Arbeitsteilung der Eltern ist, aber es noch unzureichend erforscht ist, wie die Arbeitsteilung in verschiedenen Altersstufen des Kindes (inkl. des Jugendalters) ausfällt und welche Rolle andere Einflussfaktoren in den verschiedenen Altersstufen des Kindes spielen. Des Weiteren verändern sich die institutionellen und kulturellen Rahmenbedingungen mit dem Alter des Kindes: So beziehen sich sämtliche familienpolitische Regelungen wie etwa das Elterngeld oder die Elternzeit oder der Ausbau von Kinderbetreuungsstätten auf ein bestimmtes Kindesalter; ebenso variieren z.B. die Erwartungen an die Mutterrolle mit dem Alter des Kindes. In der vorliegenden Arbeit werden theoretische Ansätze zur Erklärung der Arbeitsteilung mit den jeweiligen, altersspezifischen institutionellen Rahmenbedingungen verknüpft, um damit die folgenden Forschungsfragen beantworten zu können:

- Welchen Einfluss hat das Alter des Kindes auf die Aufteilung der Erwerbsarbeit, der Hausarbeit und der Kinderbetreuung?

- Wie unterscheiden sich die Effekte von Einflussfaktoren (wie z.B. von relativen Ressourcen und Geschlechterrolleneinstellungen) in Abhängigkeit vom Alter des jüngsten Kindes?

- Sind bei Eltern von kleinen Kindern bestimmte Einflussfaktoren für die Arbeitsteilung relevanter (oder weniger relevant) als bei Eltern von größeren Kindern?

- Wie unterscheiden sich die Dimensionen der Aufteilung der Erwerbsarbeit, der Hausarbeit und der Kinderbetreuung im Hinblick auf das Alter des Kindes voneinander? Kann etwa davon ausgegangen werden, dass bei Eltern mit größeren Kindern alle drei Dimensionen egalitärer (resp. traditioneller) aufgeteilt werden oder ist anzunehmen, dass manche Dimensionen egalitärer und andere traditioneller aufgeteilt werden?

\section{Datengrundlage der Arbeit}

Als Datengrundlage dient der DJI-Survey „Aufwachsen in Deutschland: Alltagswelten“ (AID:A) von 2013/14, der das Aufwachsen von Kindern und Jugendlichen bis ins Erwachsenenalter abbildet. Der Survey „AID:A“ eignet sich besonders gut zur Beantwortung der Forschungsfragen, da er neben seiner Vielzahl an sozialstrukturellen und demographischen Grunddaten auch dezidiert nach der Arbeitsteilung der Erwerbsarbeit, der Haus- 
arbeit und Kinderbetreuung sowie nach Verschränkungen und Entgrenzungen der einzelnen Bereiche fragt. Des Weiteren liegen vielfältige Angaben zum Familienalltag und zu Geschlechterrolleneinstellungen vor. Da der Survey „AID:A“ ein sehr breites Themenfeld abdeckt und detaillierte Einblicke in die Lebenslagen und den Prozess des Aufwachsens von Kindern, Jugendlichen und jungen Erwachsenen liefert, gilt er aktuell als die umfangreichste Erhebung zu den Lebensverhältnissen junger Menschen in Deutschland (Rauschenbach 2015, S. 4). Mit seinen rund 22.000 Zielpersonen ist der Survey in der Lage, alle Altersstufen - und insbesondere die ersten Lebensjahre sowie die frühe und mittlere Kindheit - mit besonders hohen Fallzahlen abzubilden. Der Survey liegt im MultiActor-Design vor, da neben den Zielkindern auch deren Mütter und Väter befragt wurden.

\section{Aufbau der Arbeit}

In Kapitel 2 wird als konzeptionelle Rahmung auf das Familienzyklus-Modell (Glick 1947; Duvall und Hill 1945; Rodgers 1973; Steffens 1988; Aldous 1990; Brady 2011) und die Lebensverlaufsperspektive (Clausen 1972; Riley et al. 1972; Elder 1974, 1998) eingegangen. Aus dem Familienzyklus-Modell wird gut ersichtlich, welche zentrale Rolle das Alter des Kindes für Familien spielt: So befinden sich Familien in Abhängigkeit vom Alter des Kindes in unterschiedlichen Phasen, die unterschiedliche Entwicklungsaufgaben und Herausforderungen mit sich bringen. Das Familienzyklus-Modell ist als eine heuristische Rahmung zu verstehen, die einen idealtypischen Ablauf von Familienphasen skizziert. Eine weitere heuristische Rahmung stellt die Lebensverlaufsperspektive dar, deren Grundannahme ist, dass das Leben durch Phasen und Übergänge beeinflusst wird und dass die Lebensläufe von Familienmitgliedern stark miteinander verflochten sind. Wieviel Erwerbsarbeit, Hausarbeit oder Kinderbetreuung ein Elternteil übernimmt, wird beispielsweise durch das Alter des Kindes beeinflusst, aber auch dadurch, wieviel der andere Elternteil übernimmt bzw. nicht übernimmt.

Nach dieser konzeptionellen Rahmung wird in Kapitel 3 der aktuelle Forschungsstand zur Aufteilung der Erwerbsarbeit, der Hausarbeit und der Kinderbetreuung aufgearbeitet. Dabei werden Forschungslücken aufgedeckt und es werden die damit einhergehenden Forschungsfragen formuliert. So geht aus dem Forschungsstand hervor, dass die Bedeutung des Alters des Kindes für die Aufteilung der Erwerbsarbeit, der Hausarbeit und der Kinderbetreuung noch unzureichend erforscht ist. Die vorliegende Arbeit leistet einen wichtigen Beitrag zur Schließung dieser Forschungslücke, indem sie der Frage nachgeht, welche Rolle das Alter des Kindes für die Arbeitsteilung spielt und inwiefern sich die Bedeutung von Einflussfaktoren wie etwa den relativen Ressourcen oder den Geschlechterrolleneinstellungen in Abhängigkeit vom Alter des Kindes verändert. Indem diese Arbeit alle Altersstufen des Kindes bis zur Volljährigkeit betrachtet, liefert sie einen breiteren Einblick als Studien, die nur die frühe oder mittlere Kindheit beleuchten. Da die Analysen dieser Arbeit auf sehr hohen Fallzahlen beruhen, können zudem robuste Ergebnisse erwartet werden. 
Kapitel 4 beschreibt die theoretischen Ansätze zur Aufteilung der Erwerbsarbeit, der Hausarbeit und der Kinderbetreuung und welche Bedeutung die theoretischen Ansätze dem Alter des Kindes zuschreiben. Im Mittelpunkt stehen dabei zwei Theoriestränge: Den ersten Theoriestrang stellen die ökonomischen Theorien dar, die durch die familienökonomische Theorie von Becker (Becker 1993) und durch die Verhandlungstheorie nach Ott (Ott 1992) vertreten werden. Die ökonomischen Theorien basieren auf einer Kosten-Nutzen-Logik und bei innen stehen relative Ressourcen als Prädiktor für Einkommenspotentiale und innerpartnerschaftliche Machtverhältnisse im Fokus. Der zweite Theoriestrang beinhaltet geschlechterrollen-, identitäts- und normenbasierte Ansätze (z.B. West und Zimmerman 1987; Bielby und Bielby 1989; Wetterer 1992a; Brines 1994). Hier ist der Fokus auf die Bedeutung von Sozialisationsprozessen, Rollenerwartungen, Identitäten, Präferenzen und Einstellungen gerichtet. Bei beiden Theoriesträngen wird erarbeitet, welche Bedeutung der Rolle des Alters des Kindes für die Arbeitsteilung der Eltern zugeschrieben wird.

In Kapitel 5 stehen die institutionellen und kulturellen Rahmenbedingungen im Vordergrund. Hierbei wird Deutschland zunächst kurz im Lichte der Typologie der Wohlfahrtsstaaten (Esping-Andersen 1990, 1999) beschrieben und anschließend auf die Geschlechterarrangements (Pfau-Effinger 1997, 2000, 2005) sowie die unterschiedlichen Leitbilder in Ost- und Westdeutschland eingegangen. Eine besondere Bedeutung haben auch die Familienleitbilder (Diabaté und Lück 2014), da die Arbeitsteilung der Eltern von Leitbildern wie dem „intensive mothering“ (Hays 1996) oder dem „intensive parenting“ (Wall 2010; Liss et al. 2013; Craig et al. 2014; Faircloth 2014) bzw. der "verantworteten Elternschaft" (Ruckdeschel 2015) mitbeeinflusst wird. Ein weiterer wichtiger Bestandteil der institutionellen Rahmenbedingungen ist die sog. „Trias der Familienpolitik“ (BMFSFJ 2006b), die familienpolitische Maßnahmen wie die Geldpolitiken, die Zeitpolitiken und die Infrastruktur beinhaltet. Anhand der Entwicklung dieser Trias der Familienpolitik werden etwa Mutterschaftsleitbilder ersichtlich (Gangl und Ziefle 2015). Darüber hinaus können diese (familien-)politischen Rahmenbedingungen auch wiederum die individuellen Einstellungen und Präferenzen prägen („policy feedback-theory“) (Campbell 2012; Gangl und Ziefle 2015). Nachdem die theoretischen Ansätze und die institutionellen Rahmenbedingungen bislang getrennt voneinander betrachtet wurden, werden sie in 5.5 explizit miteinander verknüpft.

Diese Verknüpfung der theoretischen Ansätze mit den institutionellen Rahmenbedingungen ist die Grundlage der Hypothesengenerierung in Kapitel 6. Hierbei werden in einem ersten Schritt zunächst allgemeine Hypothesen formuliert, die auf einer ausschließlich theoretischen Perspektive basieren. Danach werden altersspezifische Hypothesen formuliert, die sowohl das Alter des Kindes als auch die Verknüpfung der theoretischen Ansätze mit den jeweiligen, institutionellen Rahmenbedingungen explizit mitberücksichtigen. 
Ab Kapitel 7 beginnt der empirische Teil dieser Arbeit. In Kapitel 7 wird zunächst der DJISurvey „Aufwachsen in Deutschland: Alltagswelten“ beschrieben, der die Datengrundlage zur Beantwortung der Forschungsfragen darstellt. Anschließend wird die Operationalisierung der abhängigen Variablen und der wichtigsten, unabhängigen Variablen geschildert. Nachdem die verwendete Methodik dargelegt wurde, wird ein Blick auf die univariaten Verteilungen der unabhängigen Variablen geworfen. Zentrale unabhängige Variablen sind in der vorliegenden Arbeit auch die Geschlechterrolleneinstellungen. Hierbei wird jedoch nicht nur auf einzelne Einstellungsitems zurückgegriffen, sondern es wird ein Versuch unternommen, diese anhand einer latenten Klassenanalyse multidimensional darzustellen. Diesem Vorhaben zur multidimensionalen Abbildung der Geschlechterrolleneinstellungen ist ein eigener Exkurs am Ende des Kapitels gewidmet (Kapitel 7.5), der die genaue Operationalisierung sowie eine Beschreibung der Klassen beinhaltet. Damit wird an neuere Forschung angeknüpft, aus der hervorgeht, dass sich Einstellungen nicht nur eindimensional zwischen den Polen „traditionell“ und „egalitär“ bewegen, sondern dass Einstellungen komplexer sind (Knight und Brinton 2017; Grunow et al. 2018).

In den Kapiteln 8, 9 und 10 wird auf die empirischen Ergebnisse eingegangen. Hierbei werden zunächst die deskriptiven und anschließend die multivariaten Ergebnisse dargestellt. Die Unterkapitel zu den multivariaten Ergebnissen (Kapitel 8.2, 9.2 und 10.2) sind jeweils so aufgebaut, dass zunächst auf die Bedeutung des Alters des Kindes für die Arbeitsaufteilung eingegangen wird. Dann werden jeweils stufenweise zunächst die relativen Ressourcen (abgeleitet aus den ökonomischen Theorien), dann die Items zu den Geschlechterrolleneinstellungen (abgeleitet aus den geschlechterrollen-, identitäts- und normenbasierte Ansätze) und schließlich weitere wichtige Variablen mitaufgenommen. Anschließend werden diese Modelle (sowohl bei der Aufteilung der Erwerbsarbeit als auch bei der Aufteilung der Hausarbeit und der Kinderbetreuung) getrennt nach dem Alter des Kindes berechnet. Auf diese Weise wird der zentralen Forschungsfrage nachgegangen, da so herausgefunden werden kann, ob sich die Effektstärken von Einflussfaktoren wie etwa den relativen Ressourcen oder den Geschlechterrolleneinstellungen in Abhängigkeit vom Alter des Kindes verändern. So könnte es beispielsweise sein, dass die Effekte in Familien mit kleinen Kindern geringer ausfallen als in Familien mit älteren Kindern. Abschließend werden vertiefende Analysen zu den Geschlechterrolleneinstellungen vorgenommen, indem diese nicht mehr als einzelne Items vertreten sind, sondern - wie bei Kapitel 7.5 angesprochen - multidimensional abgebildet sind.

Abschließend werden im Schlusskapitel (Kapitel 11) die Ergebnisse zusammengefasst und kritisch diskutiert, gefolgt von einem kurzen Ausblick. 


\section{Konzeptionelle Rahmung: Das Familienzyklus- Modell und die Lebensverlaufsperspektive}

Wie stabil ist die Arbeitsteilung von Müttern und Vätern, welchen Schwankungen unterliegt sie und welche Rolle spielt dabei das Alter des Kindes? Die Konzepte des Familienzyklus und des Lebenslaufs nehmen Bezug darauf, welche Veränderungen im Familien- bzw. im Lebensverlauf stattfinden und dienen daher als konzeptionelle Rahmung. An späterer Stelle (siehe Kapitel 5) wird zudem darauf eingegangen, dass für die Entwicklung des Lebenslaufs Faktoren wie institutionelle und kulturelle Rahmenbedingungen eine wichtige Rolle spielen.

\subsection{Das Familienzyklus-Modell}

Das Modell des Familienzyklus bzw. der Familienentwicklung (Glick 1947; Duvall und Hill 1945; Rodgers 1973; Brady 2011; Aldous 1990) geht davon aus, dass Familien unterschiedliche Phasen durchlaufen. Es unterstellt, dass es einen idealtypischen „Normalverlauf" der Familienentwicklung gibt und somit ein zeitlich klar normierter Lebenslauf existiert (Huinink und Konietzka 2007, S. 40). Nach Glick (1947) ist der Familienverlauf durch sieben Phasen gekennzeichnet: Die erste Phase beginnt typischerweise mit der Eheschließung eines Paares und der gemeinsamen Haushaltsgründung, gefolgt von den Phasen des Übergangs zur Elternschaft mit der Geburt des ersten Kindes und der Erweiterung der Familiengröße bis zur Geburt des letzten Kindes. Die weiteren Phasen werden mit dem Auszug des ältesten Kindes und schließlich auch der weiteren Kinder aus dem gemeinsamen elterlichen Haushalt eingeleitet. Die Familiengröße verkleinert sich dabei wieder, bis sie nur noch aus den beiden Elternteilen besteht. Die letzte Phase setzt ein, wenn ein Elternteil und später auch das andere Elternteil verstirbt (Glick 1947, S. 164-165). Der Familienzyklus beginnt somit mit dem Eingehen einer (ehelichen) Partnerschaft und endet mit der Auflösung der Partnerschaft durch das Versterben der Partner. Ein neuer Familienzyklus in der Kindergeneration beginnt dann, wenn das Kind aus dem Elternhaus auszieht bzw. mit der Eheschließung selbst eine Partnerschaft eingeht.

Glick $(1947,1977)$ beschreibt, in welchem durchschnittlichen Alter Familien sich in welcher Phase befinden: So lag beispielsweise das durchschnittliche Heiratsalter 1940 bei Männern bei 24,3 Jahren und bei Frauen bei 21,6 Jahren. Wenn das erste Kind geboren wurde, waren 1940 Männer im Durchschnitt 25,3 Jahre und Frauen 22,6 Jahre alt (Glick 1947, S. 165). Innerhalb der Familie interessiert sich Glick besonders für die Veränderung der Haushaltszusammensetzung. So greift Glick für die Einteilung der unterschiedlichen Phasen insbesondere auf die Anzahl der Kinder zurück, etwa indem die Geburt des ersten Kindes eine eigene Phase markiert, ebenso wie die Geburt des letzten Kindes; auch die Auszüge des ersten sowie des letzten Kindes aus dem Elternhaushalt stellen je eine eigene Phase dar. Die Kinderanzahl hält Glick somit für einen wichtigen 
Bestandteil zur Beschreibung der Zusammensetzung des Haushalts. Was bei inm jedoch unberücksichtigt bleibt, ist das Alter des Kindes: Er definiert die einzelnen Phasen nicht etwa danach, ob das Kind im Kleinkind- oder Grundschulalter ist.

Im Unterschied zu Glick definieren Weiterentwicklungen des Familienzyklusmodells diese einzelnen Phasen schon unter der Einbeziehung des Alters des Kindes. Denn es gibt gewisse Knotenpunkte im Familienzyklus, an denen eine „Integration der verschiedenen Rollen und ein adäquater Rollenwechsel stattfinden“ (Steffens 1988, S. 33) müssen und diese Knotenpunkte werden unter anderem auch durch das Alter der Kinder bestimmt. Steffens (1988) beschreibt (modifiziert nach Duvall 1971) die folgenden acht Phasen, die sich wesentlich am Alter des ältesten Kindes orientieren (Steffens 1988, S. 33-34):

\section{Abbildung 2.1: Das Familienzyklus-Modell}

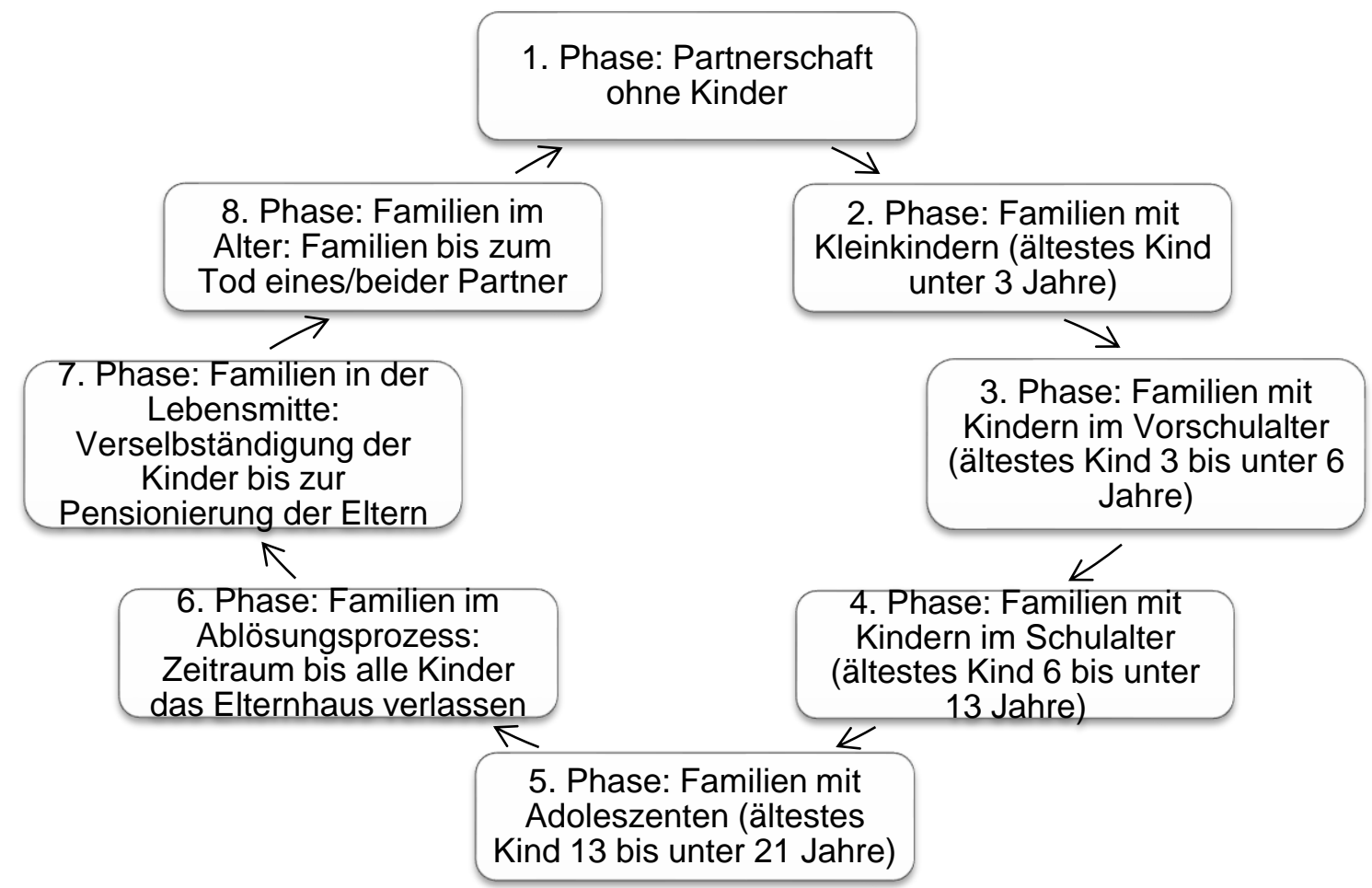

Quelle: Steffens 1988, S. 33-34, eigene Darstellung.

Je nach Phase ergeben sich für die Familien neue Entwicklungsaufgaben, da sie einen Rollenwechsel bzw. eine Integration neuer Rollen bewältigen müssen: Ein Beispiel wäre der Übergang von der Kindheit ins Erwachsenenalter, gefolgt vom Eingehen einer Partnerschaft und schließlich der Übernahme der Elternrolle (Steffens 1988, S. 34). Oder anders formuliert: Familien, die sich in denselben Lebensphasen befinden, erleben ähnliche Ereignisse und ähnliche Krisen, die sie bewältigen müssen, sodass sie ähnliche Entwicklungsaufgaben haben. Aufgrund der Verschiedenheit zwischen den einzelnen Phasen sind Familien deshalb nicht als homogene Gruppe zu betrachten, denn je nach Phase unterscheiden sie sich z.B. nach der Anzahl der beteiligten Personen oder nach 
dem Alter des Kindes. Umgekehrt können die Familien, die sich in derselben Phase befinden, eher als homogene Gruppe angesehen werden, da sie etwa die Altershomogenität der Kinder und die damit einhergehenden Erlebnisse und Anforderungen vereinen (Mattessich und Hill 1987; zit. n. Erickson et al. 2010, S. 957-958).

Es gibt mehrere Punkte, die am Familienzyklus-Modell kritisiert werden können. Ein Kritikpunkt bezieht sich darauf, dass ein „Normalverlauf“ der Familienentwicklung bzw. eine "Normalbiographie“ unterstellt wird. Nicht bei allen Paaren kommt es jedoch zu einer Familiengründung, da ein größer werdender Anteil an Paaren kinderlos bleibt: 2018 betrug in Deutschland die sog. endgültige Kinderlosenquote, die den Anteil der kinderlosen Frauen an allen Frauen zwischen 45 und 49 Jahren beschreibt, 21\% (Destatis 2019, S. 17). Ebenso kann kritisiert werden, dass ausschließlich heterosexuelle Paare betrachten werden und homosexuelle Paare somit unberücksichtigt bleiben. Des Weiteren kommt es nach dem Familienzyklus-Modell erst dann zur Auflösung, wenn die Kinder bereits erwachsen und ausgezogen sind und ein Partner verstirbt, d.h. es wird an der lebenslangen Stabilität einer Partnerschaft festgehalten. Der Anstieg der Scheidungen verweist jedoch darauf, dass Partnerschaften instabiler geworden sind. Wenn nach der Scheidung neue Partnerschaften eingegangen werden und einer oder beide Partner die Kinder aus der vorherigen Beziehung mitbringen, finden sich die Beteiligten in Stieffamilien wieder. Diese oder anderweitige Formen, die auf eine Pluralisierung der Lebensformen hinweisen, werden im Familienzyklus-Modell außer Acht gelassen. Am FamilienzyklusModell wird daher allgemein kritisiert, dass es zu eng gefasst und zu normativ ausgerichtet ist (Huinink und Konietzka 2007, S. 41). Ebenso kann die Annahme, dass es einen „richtigen“ Zeitpunkt für die einzelnen Phasen oder Entwicklungsschritte gibt, kritisiert werden, da der „richtige“ Zeitpunkt stark von gesellschaftlichen Normenvorstellungen abhängig (Rosow 1978). Ein weiterer Kritikpunkt bezieht sich darauf, dass die Einteilung der Phasen zu unscharf und willkürlich sei (Nock 1979; Spanier et al. 1979). Da sich das Familienzyklus-Modell nur auf die Kernfamilie bezieht, ist es auf die heutige Zeit nur eingeschränkt übertragbar, da die in modernen Gesellschaften stattfindende Pluralisierung familialer Lebensformen nicht adäquat abgebildet werden kann. Diese Kritikpunkte lassen vermuten, dass der Nutzen des Familienzyklus-Modells gering ist und die Kenntnisse der Familienphasen nicht über den Nutzen hinausgehen, den man auch durch Variablen wie die Ehedauer oder das Alter erhält (Nock 1979; Spanier et al. 1979).

Es gibt jedoch auch Studien, die den Nutzen des Familienzyklus-Modells verteidigen: Kapinus und Johnson (2003) zeigen, dass die Vorhersagekraft des Familienzyklus-Modells für viele abhängige Variablen ziemlich unabhängig vom Alter oder der Dauer der Ehe ist. Sie argumentieren, dass das Familienzyklus-Modell insbesondere bei abhängigen Variablen, bei denen man einen Zusammenhang mit dem Alter des Kindes erwarten wird, ein gutes Hilfsmittel ist (Kapinus und Johnson 2003). Ein Beispiel für solch eine abhängige Variable könnte etwa die Zeit sein, die Eltern mit ihren Kindern verbringen, da anzunehmen ist, dass dies in starken Zusammenhang mit dem Alter des Kindes steht. $\mathrm{Da}$ in der vorliegenden Arbeit die Aufteilung der Erwerbsarbeit, der Hausarbeit und der 
Kinderbetreuung in Abhängigkeit vom Alter des Kindes im Vordergrund steht, wird explizit auf den Zusammenhang mit dem Kindesalter und damit auch auf den Familienzyklus verwiesen. Es wird deshalb angenommen, dass der Familienzyklus (in dieser Arbeit operationalisiert durch die Altersstufen des jüngsten Kindes) hilfreich ist, um die Arbeitsteilung in Familien besser verstehen zu können.

Auch eine Studie von Bühlmann et al. (2010), die im europäischen Vergleich die Kohärenz von Einstellungen und Praktiken in verschiedenen Stadien des Lebenslaufs untersucht, verwendet das Familienzyklus-Modell als heuristische Rahmung. Ihre Ergebnisse zeigen, dass die biographischen Phasen in allen untersuchten, europäischen Ländern ein wichtiger Faktor für die Aufteilung der Erwerbsarbeit in Paaren sind und dass sie sogar noch wichtiger als sämtliche, anderen Faktoren sind:

"[T]he biographical stage of a couple is a forceful factor affecting the distribution of occupational work between spouses. It is more important than socio-occupational status, residential context, marital status, or cohort" (Bühlmann et al. 2010, S. 62).

Insbesondere die Geburt des Kindes stellt einen Wendepunkt der Arbeitsteilung dar und führt zu einer Inkohärenz zwischen den Werten und der Arbeitsteilung: Während viele kinderlose Paare ihre egalitären Werte in eine egalitäre Arbeitsteilung umsetzen können, wird die Arbeitsteilung mit dem Übergang zur Elternschaft bei den meisten Paaren traditioneller, sodass dann ein Widerspruch zwischen den egalitären Werten und der traditionellen Arbeitsteilung besteht (Bühlmann et al. 2010). Die Studie von Bühlmann et al. (2010) gewinnt somit Erkenntnisse darüber, inwiefern Werte und Arbeitsteilungsmuster einander entsprechen, wenn Paare kinderlos sind, ein Kind erwarten oder ein Kind in den Altersstufen null bis unter zwei Jahre, zwei bis unter fünf Jahre und über fünf Jahre haben.

Das Familienzyklus-Modell wird nicht nur in Studien herangezogen, die sich unmittelbar mit der Arbeitsteilung befassen, sondern auch in Studien, die im weiteren Sinne die Vereinbarkeit von Beruf und Familie erforschen. So analysierten Erickson et al. (2010) mit den Daten aus 79 Ländern („IBM 2004 Global Work and Life Issues Survey“), welche Erfahrungen Eltern mit der Schnittstelle von Beruf und Familie in sechs verschiedenen Phasen des Familienzyklus machen. Im Vordergrund stand die Frage danach, inwiefern sich Faktoren wie Arbeitszeiten, Arbeitsverantwortung, Arbeitsflexibilität, Hausarbeitszeit und Familienstand je nach Familienphase unterschiedlich auf die abhängigen Variablen „work-family-conflict“, „family-work-conflict“ und die allgemeine Vereinbarkeit von Beruf und Familie auswirken. Die Ergebnisse zeigen, dass die Zusammenhänge je nach Familienphase unterschiedlich stark ausgeprägt sind: Der Übergang zur Elternschaft ist mit hohen Arbeits- und Betreuungsanforderungen verbunden, sodass „work-family-conflicts“ und „family-work-conflicts“ zunehmen. Das Ausmaß dieser beiden Konflikte steigert sich bis zu den Familienphasen, in denen das Kind im Vorschul- und im Schulalter ist, anschließend reduzieren sich die Konflikte wieder (Erickson et al. 2010). 
Eine ähnliche Forschungsfrage hatte die Studie von Allen und Finkelstein (2014), die mit amerikanischen Daten untersuchte, wie in „dual-career-couples“ je nach Phase des Familienzyklus der „work-family-conflict“ ist. Am stärksten fällt der Konflikt zwischen Arbeit und Familie dann aus, wenn das jüngste Kind unter fünf Jahre alt ist. In den nachfolgenden Phasen verringert sich der Konflikt und ist schließlich in der sog. „empty nest“Phase, d.h. wenn alle Kinder bereits aus dem Elternhaushalt ausgezogen sind, am geringsten. Die Studie kontrolliert auch das Alter der Eltern und zeigt, dass es sich bei den gefundenen Zusammenhängen zwischen den Familienphasen und dem „work-familyconflict" nicht einfach nur um Alterseffekte handelt, sondern den Familienphasen eine darüber hinausgehende Bedeutung zukommt (Allen und Finkelstein 2014).

Diese Studien zeigen, dass das Familienzyklus-Modell trotz der vielen Kritikpunkte ein gutes Hilfsinstrument sein kann und es angebracht ist zwischen verschiedenen Familienphasen zu differenzieren. Auch wenn das Familienzyklus-Modell nicht auf die Pluralisierung der Lebensformen und auch nicht auf die De-Standardisierung der Lebensverläufe eingeht, so dient es vielmehr als heuristische Rahmung, die einen idealtypischen Ablauf von Familienphasen nachzeichnet und dazu beiträgt, die einzelnen Familienphasen besser zu verstehen sowie die Gemeinsamkeiten und Unterschiede mit anderen Familienphasen zu identifizieren.

\subsection{Die Lebensverlaufsperspektive}

Ein weiteres Konzept ist die Perspektive des Lebenslaufs, die sich über Jahrzehnte entwickelt hat und in ihren Anfängen auf Clausen (1972), Elder (1974) und Riley et al. (1972) zurückgeht (Bernardi et al. 2019). Eine Grundannahme der Lebenslaufperspektive ist, dass der Lebenslauf durch Phasen bzw. Übergange beeinflusst wird. Dabei wird eine sog. Pfadabhängigkeit unterstellt, d.h. es wird davon ausgegangen, dass

„zeitlich vorangehende Entscheidungen - etwa zur Partnerschaft oder zur Elternschaft - spätere Entscheidungen - etwa zum Erwerb von Wohneigentum oder über Bildungsinvestitionen in die Kinder - mehr oder weniger stark beeinflussen. Aktuelle Entscheidungen haben eine Vorgeschichte mit prägendem Einfluss; ein einmal beschrittener Weg wird tendenziell fortgesetzt, es besteht eine Pfadabhängigkeit“ (Hill und Kopp 2013, S. 56-57).

Im Folgenden wird auf die vier Grundprinzipien der Lebenslaufperspektive nach Elder (1998) eingegangen, anschließend wird in aller Kürze die „Institutionalisierung des Lebenslaufs" nach Kohli (1985) vorgestellt, danach werden Kritiken genannt und eine Weiterentwicklung der Lebenslaufperspektive - der sog. „Lebenslaufwürfel“ (Bernardi et al. 2019) - vorgestellt; abschließend werden Studien skizziert, die die Veränderung der Arbeitsteilung im Lebenslauf behandeln.

Die Lebenslaufperspektive nach Elder (1998) basiert auf vier Grundprinzipien, die im Folgenden kurz skizziert werden: 
1) „the principle of historical time and place“,

2) „the principle of timing“,

3) „the principle of linked lives“ und

4) „the principle of human agency“ (Elder 1998, S. 3-4).

Das erste Prinzip der Lebens(ver-)laufperspektive (,the principle of historical time and place“) verweist darauf, dass die Lebensverläufe von Individuen in unterschiedliche, historische Zeiten und Orte eingebettet sind und von diesen Erfahrungen geprägt sind (Elder 1994, 1998). Eine wichtige Bedeutung spielt nach Prinzip zwei („the principle of timing") auch das Timing, denn die Bedeutung, die Lebensübergängen oder Ereignissen zukommt, hängt auch davon ab, wann die Veränderungen im Leben einer Person auftreten. Beispiele hierfür sind etwa der Zeitpunkt der Eheschließung oder der Familiengründung, deren langfristige Konsequenzen sich danach unterscheiden, ob sie früher oder später im Lebenslauf geschehen:

„Early marriage tended to produce a cumulation of disadvantages, from socioeconomic hardships to the loss of education. Early childbearing had similar consequences" (Elder 1998, S. 3).

Des Weiteren betont die Lebenslaufperspektive, dass Menschen in sozialer Beziehung zueinander stehen und ihre Lebensläufe miteinander verwoben sind (Elder 1994, 1998). Die Einbettung eines Menschenlebens in seine sozialen Beziehungen und die gegenseitigen Interdependenzen werden in diesem Kontext auch als „linked lives" beschrieben (Elder 1994, S. 6) und sind das dritte Prinzip der Lebenslaufperspektive. Besonders deutlich zeigen sich „linked lives“ innerhalb der Familie: Die Lebensläufe der Familienmitglieder sind aufs Engste miteinander verflochten und die Entscheidungen eines Familienmitgliedes stehen mit den Entscheidungen anderer Familienmitglieder in Wechselwirkung. Schließlich nimmt das vierte Prinzip (,the principle of human agency“) an, dass Individuen ihren eigenen Lebenslauf durch die Entscheidungen und Handlungen konstruieren, die sie innerhalb der gegebenen historischen und sozialen Umstände ergreifen. Dabei wirken sich vorhergegangene Entscheidungen auf spätere Entscheidungen aus. Entscheidungen legen somit einen Pfad fest und ein einmal eingeschlagener Pfad wird tendenziell fortgesetzt, woraus sich pfadabhängige, typische Lebensverläufe ergeben (Hill und Kopp 2013, S. 56-57).

Die Soziologie des Lebenslaufs erforscht Lebensläufe sowohl auf der Makro- als auch auf der Mikroebene:

„Sie [die Lebensläufe] dienen der Charakterisierung ganzer Gesellschaften und sie dienen der Charakterisierung individueller Lebensläufe. (...) Auf der Makroebene wird der Lebenslauf als eine soziale Institution betrachtet. (...) Auf der Mikroebene wird der Lebenslauf als Abfolge von Rollen und Positionen betrachtet, die Menschen in ihrem Leben einnehmen und verlassen“ (Sørensen 1990, S. 304, Hervorhebung im Original).

Bezugnehmend auf die Makroebene des Lebenslaufs wird auch von einer „Institutionalisierung des Lebenslaufs“ (Kohli 1985) gesprochen. Kohli argumentiert, dass das Leben 
als eine zusätzliche Dimension die zeitliche Dimension aufweist und dass „Lebenslauf und Lebensalter als eine eigenständige gesellschaftliche Strukturdimension aufzufassen sind“ (Kohli 2017, S. 495, Hervorhebung im Original). Nach Kohli (1985) lassen sich drei Phasen des Lebenslaufs unterscheiden, die alle in Zusammenhang mit der Erwerbsarbeit stehen: „die Phase der Vorbereitung auf die Erwerbsarbeit, die Phase der Erwerbsarbeit und die Phase des Rückzugs von der Erwerbsarbeit" (Sørensen 1990, S. 305). Diese starke Ausrichtung der Lebensphasen an der Erwerbsarbeit ist in Deutschland stark ausgeprägt. In anderen Ländern, wie beispielsweise den USA, spielt die Erwerbsarbeit eine geringere Rolle und dafür die Familie eine gravierendere Rolle (Krüger 2010).

Sørensen hält diese Ausrichtung der Lebensphasen an der Erwerbsarbeit für zu einseitig und vor allem nur auf das Leben der Männer ausgerichtet, da nicht berücksichtigt wird, dass das Leben von Frauen nicht ohne Bezug auf ihre Familie verstanden werden kann (Sørensen 1990, S. 305-306). Für eine geschlechterübergreifende Klassifizierung von Lebensphasen ist es deshalb notwendig, den familiären Bereich miteinzubeziehen. Anstelle von den Phasen der Vorbereitung auf die Erwerbsarbeit, der Erwerbsarbeit und des Rückzugs aus der Erwerbsarbeit, könnte allgemeiner von der „Vorbereitung auf Arbeit“, „Arbeit“ und „Rückzug von der Arbeit“ gesprochen werden:

„,Arbeit' umfaßt dann Tätigkeiten, die außerhalb der Familie im Arbeitsmarkt geleistet werden; ,Arbeit' umfaßt aber auch Tätigkeiten, die in der Familie und für die Familie geleistet werden“" (Sørensen 1990, S. 306).

Auch Krüger und Levy (2000) kritisieren, dass es nicht ausreichend ist, die Institutionenstrukturierung des Lebenslaufs anhand der Erwerbsarbeit auszurichten und dabei die Familie unberücksichtigt zu lassen (Krüger und Levy 2000, S. 382). Stattdessen sei es notwendig, die Lebenslaufforschung mit der Familiensoziologie zu verknüpfen. Krüger und Levy (2000) nehmen an, dass das Geschlecht eine Brückenfunktion zwischen der Familie und anderen, außerfamiliären Institutionen hat und entwickeln ihr theoretisches Konzept, das dem Geschlecht einen „Masterstatus“ zuschreibt (Krüger und Levy 2000): Der „Masterstatus“ bezeichnet ein

„institutionelles doing gender, das die strukturelle Umwelt sich bildender Paare und Familien kennzeichnet und rational ihre Organisationsform wählenden Paaren traditional ausgerichtete Entscheide zumindest stark nahe legt, bzw. Paarstrategien, die gegen die strukturell induzierten Regeln verlaufen, mit hohen Penalitäten belegt“ (Levy und Ernst 2002, S. 128, Hervorhebung im Original).

Wenn Krüger und Levy (2000) das Geschlecht als einen „Masterstatus“ bezeichnen, so verstehen sie darunter „ein soziale Positionen und Interaktionen überlagerndes Prinzip“ (Krüger und Levy 2000, S. 384). Biographische Ereignisse wie etwa der Übergang zur Elternschaft prägen den Masterstatus und führen dazu, dass Frauen familienorientiertere Präferenzen und Männer erwerbsorientiertere Präferenzen entwickeln. Indem bei der Institutionalisierung des Lebenslaufs die Familie mitberücksichtigt wird, können Lebensläufe und insbesondere die Reproduktion von geschlechtsspezifischen Ungleichheiten besser nachgezeichnet werden. 
Eine neuere Konzeptualisierung des Lebenslaufs ist der sog. „life course cube“ (Bernardi et al. 2019). Dieser Lebenslaufwürfel (siehe Abbildung 2.2) zeichnet sich durch seine dreidimensionale Struktur aus, die aus den Dimensionen a) Zeit, b) Lebensbereiche und c) Ebenen besteht. Bei diesen drei Dimensionen gibt es Interdependenzen innerhalb dieser einzelnen Dimensionen (Interdependenzen erster Ordnung) sowie auch Interdependenzen zwischen den einzelnen Dimensionen (Interdependenzen zweiter und dritter Ordnung). Im Folgenden wird auf die Interdependenzen erster Ordnung näher eingegangen.

\section{Abbildung 2.2: $\quad$ Der Lebenslaufwürfel}

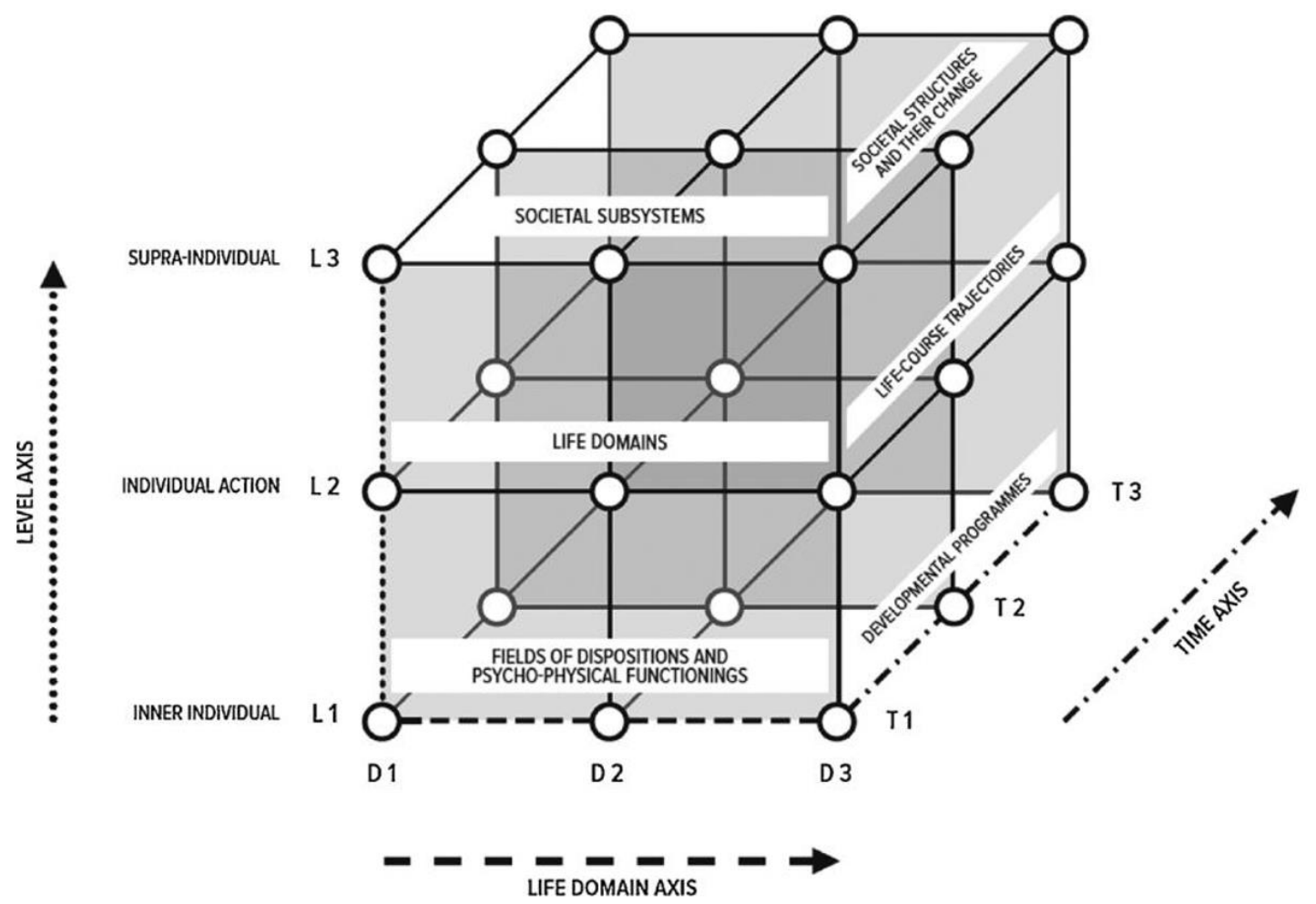

Quelle: Bernardi et al. 2019, S. 4.

Ad a): Interdependenzen auf der zeitlichen Dimension umfassen Pfadabhängigkeiten, Antizipationen und Wendepunkte (Bernardi et al. 2019, S. 3). Pfadabhängigkeiten bedeuten, dass Ereignisse aus der Vergangenheit die Gegenwart beeinflussen; bei Antizipationen ist der Fokus dagegen darauf gerichtet, dass die Erwartung von bestimmten, zukünftigen Ergebnissen die Gegenwart beeinflusst (Bernardi et al. 2019, S. 3-4). Während Pfadabhängigkeiten somit als „Schatten der Vergangenheit“ bezeichnet werden können, können Antizipationen als „Schatten der Zukunft" begriffen werden (Bernardi et al. 2019, S. 4). Bei Wendepunkten handelt es sich um radikale Abweichungen vom bisherigen Lebenslauf eines Individuums bzw. von den individuellen oder gesellschaftlichen Erwartungen, d.h. bestimmte individuelle Verhaltensweisen sind weder aufgrund der Vergangenheit (Pfadabhängigkeit) noch aufgrund der erwarteten Zukunft (Antizipation) als wahrscheinlich anzunehmen und stellen deshalb eine „Wendung“ dar. Wendepunkte 
sind so gesehen mit Pfadabhängigkeiten und Antizipationen verknüpft, denn „[t]urning points cannot be understood without reference to the history or the anticipated future of the life course" (Bernardi et al. 2019, S. 4).

Ad b): Bei den Interdependenzen der Lebensbereiche geht es darum, dass verschiedene Lebensbereiche wie beispielsweise die Erwerbsarbeit, die Familie und die Freizeit miteinander verflochten sind und sich etwa in Form von Opportunitätskosten gegenseitig beeinflussen können. Je mehr Zeit oder Geld in einen bestimmten Bereich investiert werden, umso weniger Zeit oder Geld kann in andere Bereiche investiert werden. Gleichzeitig können die Ergebnisse eines Lebensbereichs auch die anderen Lebensbereiche unterstützen: Beispielsweise kann das durch die Erwerbsarbeit verdiente Geld in bestimmte Freizeitaktivitäten investiert werden und die aus den Freizeitaktivitäten hervorgehende Erholung dient umgekehrt wiederum der Leistungsfähigkeit im Erwerbsleben (Bernardi et al. 2019, S. 5).

Ad c): Die Interdependenzen der Ebenen beziehen sich darauf, dass es neben der „individuellen Handlungsebene“ auch noch eine Ebene darunter, die „innerindividuelle Ebene“, und eine Ebene darüber, die „supra-individuelle Ebene“, gibt und dass diese Ebenen in gegenseitiger Wechselwirkung zueinanderstehen (Bernardi et al. 2019, S. 5). Die „supra-individuelle Ebene“ umfasst das, was in der Soziologie als „Meso-“ und „Makroebene" bezeichnet wird, während die „innerindividuelle Ebene" auf physiologische und psychologische Entwicklungsveränderungen ausgerichtet ist und deshalb eher im Bereich der Psychologie angesiedelt ist (Bernardi et al. 2019, S. 5).

Auf Ausführungen zu den Interdependenzen höherer Ordnung wird aus Komplexitätsgründen an dieser Stelle verzichtet. Anhand des Lebenslaufwürfels sollte vielmehr deutlich gemacht werden, welche Dimensionen zum Verständnis von Lebensläufen relevant sind (die zeitliche Dimension, die Dimension der Lebensbereiche und die Dimension der Ebenen) und dass diese in wechselseitiger Abhängigkeit zueinanderstehen. Beim Lebenslaufwürfel handelt es sich um eine Konzeptualisierung des Lebenslaufs, die sich darum bemüht, möglichst sparsam zu sein und disziplinübergreifend genutzt werden zu können (Bernardi et al. 2019).

Abschließend wird noch auf Studien, die die Arbeitsteilung aus einer Lebenslaufperspektive heraus betrachten, Bezug genommen. So wurde in der bisherigen Forschung der Übergang zur Elternschaft als ein wichtiger Wendepunkt identifiziert, wobei dies für Frauen stärker zutrifft als für Männer: Bei Frauen ist der Übergang zur Elternschaft mit einer (zumindest kurzzeitigen) Erwerbsunterbrechung verbunden und mit zunehmendem Alter des Kindes steigt die Wahrscheinlichkeit, dass die Erwerbsarbeit wieder aufgenommen bzw. der Erwerbsumfang wieder erhöht wird (Gjerdingen und Center 2005; Misra et al. 2011; Kühhirt 2012; Berghammer 2014; BMFSFJ 2012, 2014a; Boeckmann et al. 2015; Hipp und Leuze 2015; OECD 2016; Steiber et al. 2016; Buschner et al. 2018). Im Gegensatz dazu führt der Übergang zur Elternschaft bei den Vätern zu keiner relevanten Verringerung ihrer Erwerbsumfänge (Gjerdingen und Center 2005; Kühhirt 2012; 
Berghammer 2014; OECD 2016). Stattdessen wird bei ihnen häufig sogar eine Zunahme ihrer Erwerbsumfänge festgestellt. Da der Anteil der Mütter am Erwerbsvolumen der Paare mit zunehmendem Alter des Kindes ansteigt, wird die Aufteilung der Erwerbsarbeit dadurch egalitärer.

Mit der Familiengründung wird auch die unbezahlte Familienarbeit ungleicher aufgeteilt: Mütter übernehmen mehr Hausarbeit als zuvor (Nomaguchi und Milkie 2003; Baxter et al. 2008; Grunow et al. 2012; Kühhirt 2012; Schober 2013a; Dechant et al. 2014; Nitsche und Grunow 2016) und sie übernehmen auch bei der neu hinzukommenden Kinderbetreuung deutlich mehr als die Väter - obwohl sich der Anteil der Väter an der Kinderbetreuung in den letzten Jahrzehnten bereits erhöht hat (Sayer et al. 2004a; Craig 2006a; Bonke und Esping-Andersen 2011; Raley et al. 2012; Craig et al. 2014). Während die Aufteilung der Erwerbsarbeit mit zunehmendem Alter des Kindes weniger geschlechtsspezifisch wird, lässt sich diese Annahme nicht ohne Weiteres auf die Aufteilung der unbezahlten Arbeit übertragen: Studien deuten darauf hin, dass die traditionelle Aufteilung der Hausarbeit, die mit dem Übergang zur Elternschaft einhergeht, auch in den nachfolgenden Jahren bzw. Familienphasen beibehalten wird (Gershuny et al. 2005; Grunow et al. 2012; Kühhirt 2012; Nitsche und Grunow 2016). Bei der innerpartnerschaftlichen Aufteilung der Kinderbetreuung zeigt sich, dass diese mit zunehmendem Alter des Kindes egalitärer wird (Nitsche und Grunow 2018). Dies liegt insbesondere daran, dass sich die Betreuungsintensität mit zunehmendem Alter des Kindes verringert und Mütter deswegen ihre absolute Kinderbetreuungszeit reduzieren (Craig und Sawrikar 2009; Kühhirt 2012). Eine andere Erklärung könnte sein, dass Väter mit älteren Kindern mehr Zeit verbringen als mit jüngeren Kindern (Dermott 2008; Craig und Sawrikar 2009; Norman et al. 2014). Die Studienlage ist hierzu jedoch nicht eindeutig, da einige Studien darauf hindeuten, dass Väter mit jüngeren Kindern mehr Zeit verbringen (Mühling 2007; Hook und Wolfe 2012; Walper und Lien 2018) oder aber, dass das Alter des jüngsten Kindes keinen signifikanten Effekt darauf hat, ob Väter eine „aktive Vaterschaft" praktizieren oder nicht (Zerle-Elsäßer und Li 2017). Es besteht somit noch Forschungsbedarf dazu, wie sich die Aufteilung der Kinderbetreuung in den einzelnen Altersstufen des Kindes verhält.

Sowohl beim Familienzyklus-Modell als auch bei der Lebenslaufperspektive handelt es sich weniger um Theorien, aus denen sich explizite Vorhersagen ableiten lassen, sondern vielmehr sind sie als eine Art „Forschungsprogramm" zu verstehen und dienen als konzeptionelle Rahmung (Aldous 1990). In einem späteren Kapitel werden daher theoretische Ansätze vorgestellt, um besser erklären zu können, warum sich manche Eltern die Erwerbsarbeit, Hausarbeit und Kinderbetreuung egalitärer aufteilen, während andere sie sich traditioneller aufteilen. Im Zuge dessen wird darauf eingegangen, welche Bedeutung Einflussfaktoren wie den relativen Ressourcen oder den Geschlechterrolleneinstellungen zukommt. Darüber hinaus wird aus den Theorien abgeleitet, welche Rolle jeweils dem Alter des Kindes für die Arbeitsteilung der Eltern beigemessen wird, denn wie 
bereits aus den Ausführungen zum Familienzyklus und zur Lebenslaufperspektive hervorgegangen ist, werden Arbeitsteilungsmuster durch Familienphasen bzw. durch wichtige Übergänge im Lebenslauf wesentlich beeinflusst. Zunächst wird jedoch auf den aktuellen Forschungsstand zur Aufteilung der Erwerbsarbeit, Hausarbeit und Kinderbetreuung eingegangen sowie auf die sich daraus ergebenden Forschungslücken und Forschungsfragen. 


\section{Stand der Forschung}

Im Folgenden wird ein Überblick gegeben, welche Studien sich bereits mit der Aufteilung der Erwerbsarbeit, der Hausarbeit und der Kinderbetreuung auseinandergesetzt haben und welche Einflussfaktoren dabei identifiziert werden konnten. Dabei gibt es viele Faktoren, die sowohl die Aufteilung der Erwerbsarbeit als auch die Aufteilung der Hausarbeit und Kinderbetreuung beeinflussen. Zudem beeinflussen sich die verschiedenen Bereiche gegenseitig: So wird beispielsweise die Aufteilung der Hausarbeit und der Kinderbetreuung stark durch die Erwerbskonstellation der Eltern beeinflusst; umgekehrt können auch die Hausarbeit und die Kinderbetreuung die Aufteilung der Erwerbsarbeit beeinflussen. Dies ist insbesondere dann der Fall, wenn die Bedarfe an Familienarbeit etwa aufgrund von mehreren Kindern oder von noch besonders kleinen Kindern sehr hoch sind und sich ein Elternteil (i.d.R. die Mütter) deswegen aus der Erwerbstätigkeit zurückzieht. Auch wenn diese gegenseitigen Verschränkungen von Erwerbsarbeit, Hausarbeit und Kinderbetreuung immer mitgedacht werden müssen, wird der Forschungsstand für eine bessere Übersichtlichkeit dennoch getrennt nach diesen Bereichen aufgearbeitet.

\subsection{Zur Aufteilung der Erwerbsarbeit}

Obwohl die Erwerbspartizipation von Frauen in den letzten Jahrzehnten extrem gestiegen ist, sind die Erwerbsumfänge der Frauen häufig immer noch deutlich niedriger als die der Männer (Konietzka und Kreyenfeld 2010; Goldscheider et al. 2015; OECD 2016). Diese Unterschiede bestehen bereits vor dem Übergang zur Elternschaft, spitzen sich aber mit der Elternschaft extrem zu (van der Lippe und van Dijk 2002; Kühhirt 2012; Nieuwenhuis et al. 2012; OECD 2016; Buschner et al. 2018).

Diese geschlechtsspezifischen Unterschiede in der Erwerbspartizipation und den Erwerbsumfängen bestehen in den meisten Ländern, jedoch in unterschiedlichem Ausmaß, sodass sich das Erwerbsmuster von Frauen in vier Gruppen einteilen lässt (vgl. für den folgenden Abschnitt OECD 2016, S. 139):

(1) Frauen in langfristiger Teilzeitbeschäftigung (in Ländern wie Deutschland, Österreich oder Niederlande);

(2) eine polarisierte Frauenerwerbstätigkeit, d.h. Frauen sind entweder in Vollzeit erwerbstätig oder nicht-erwerbstätig (in Ländern wie z.B. Italien, Griechenland oder Spanien);

(3) Frauen in kurzfristiger Nichterwerbstätigkeit, hier kehren Frauen nach einer kurzen Erwerbsunterbrechung wieder in die Vollzeiterwerbstätigkeit zurück (dies trifft auf Länder wie z.B. Estland, Finnland und Lettland zu) und

(4) geringe geschlechtsspezifische Arbeitszeitunterschiede, d.h. Frauen arbeiten auch hier etwas weniger als Männer, aber die Stundendifferenzen sind vergleichsweise gering (dies ist in Ländern wie Dänemark, Norwegen oder Schweden der Fall). 
Deutschland zählt damit zu den Ländern mit den größten geschlechtsspezifischen Unterschieden. Während sich bei den Frauen im internationalen Vergleich große Unterschiede in der Erwerbstätigkeit zeigen, ist das Bild der Männer dagegen einheitlicher: Die meisten Männer arbeiten sowohl vor als auch nach der Familiengründung in Vollzeit - unabhängig davon, in welchem Land sie leben (OECD 2016). Wird neben der Erwerbstätigkeit von Frauen und Männern auch noch die Zuständigkeit für die Familienarbeit berücksichtigt, so lassen sich nach Lewis (2001) daraus mindestens die folgenden Idealtypen an Arbeitsteilungsmustern ableiten (Lewis 2001, S. 157; vgl. auch Leitner et al. 2004, S.13):

Tabelle 3.1: $\quad$ Typen der Aufteilung von Erwerbs- und Familienarbeit nach Lewis

\begin{tabular}{|c|c|}
\hline Typen der Arbeitsteilung & Bedeutung/ konkrete Aufteilung \\
\hline - „male breadwinner model“ & $\begin{array}{l}\text { Mann VZ (=Vollzeit), Frau nicht erwerbstätig und al- } \\
\text { lein für Familienarbeit zuständig; }\end{array}$ \\
\hline - „dual career model“ & beide VZ, Familienarbeit überwiegend externalisiert; \\
\hline - „single earner model“ & $\begin{array}{l}\text { Ein-Elternteil-Familie: ein Elternteil arbeitet in VZ } \\
\text { oder TZ (=Teilzeit), Familienarbeit wird entweder } \\
\text { komplett allein übernommen oder externalisiert; }\end{array}$ \\
\hline - „dual breadwinner model“ & $\begin{array}{l}\text { Variante 1: Mann VZ, Frau kurze TZ, Familienarbeit } \\
\text { wird von Frau (oder Verwandten) übernommen; } \\
\text { Variante 2: Mann VZ, Frau lange TZ, Familienarbeit } \\
\text { externalisiert; } \\
\text { Variante 3: beide TZ, beide teilen sich Familienarbeit }\end{array}$ \\
\hline
\end{tabular}

Quelle: Lewis 2001, S. 157, eigene Darstellung.

Beim traditionellsten Modell, dem „male breadwinner model“, ist ausschließlich der Mann erwerbstätig, während beim „dual career model“ beide in Vollzeit erwerbstätig sind und die Familienarbeit größtenteils externalisiert wird, sodass bei letzterem die bezahlte Arbeit egalitär aufgeteilt ist und die unbezahlte Arbeit ausgelagert ist (siehe Tabelle 3.1). Zwischen diesen Extremen gibt es verschiedene Abstufungen in Form von „dual breadwinner models“: Die erste Variante des „dual breadwinner models“, in der die Frau in Teilzeit arbeitet, kann als semi-traditionell bezeichnet werden kann. Die zweite Variante des „dual breadwinner models" kann als annähernd egalitär betrachtet werden, da die Erwerbsumfänge ähnlich sind und keine Ungleichheit bei der Familienarbeit gegeben ist. Eine egalitäre Arbeitsteilung im strengeren Sinne liegt neben dem „dual career model“ auch in der dritten Variante des „dual breadwinner models“ vor, in der beide in Teilzeit erwerbstätig sind und sich die Familienarbeit gleich aufteilen. Bei diesen Typen handelt es sich um Idealtypen, die nicht erschöpfend sind. So bilden diese Typen beispielsweise nicht ab, dass Frauen oftmals trotz gleich hoher Erwerbsumfänge wie Männer mehr Familienarbeit übernehmen. 
Nach diesen Idealtypen stellt sich die Frage, wie sich Familien in Deutschland die Erwerbsarbeit tatsächlich aufteilen. In Deutschland ist nach dem Übergang zur Elternschaft ein Vollzeit-/Teilzeit-Modell üblich, wobei die Erwerbstätigkeit der Mütter stark vom Kindesalter abhängig ist, nicht jedoch die Erwerbstätigkeit der Väter. Wie aus der folgenden Abbildung 3.1 hervorgeht, betrug 2018 die Erwerbsquote von Vätern in Abhängigkeit vom Kindesalter zwischen $81 \%$ und 85\% (Demografie Portal 2019). Der Großteil der Väter ist in Vollzeit beschäftigt und lediglich $4 \%$ bis $6 \%$ der Väter arbeiten in Teilzeit (Demografie Portal 2019). Sowohl die Erwerbstätigenquote der Väter als auch ihre Erwerbsumfänge sind damit relativ robust gegenüber dem Kindesalter. Bei Müttern mit einem Kind im Alter von unter drei Jahren sind nur 33\% der Mütter erwerbstätig: $23 \%$ in Teilzeit und $10 \%$ in Vollzeit. Wenn das Kind im Kindergartenalter ist, steigt die Erwerbsquote der Mütter rapide auf insgesamt $65 \%$ an, wobei $18 \%$ in Teilzeit und $47 \%$ in Vollzeit beschäftigt sind. Mit dem Älterwerden des Kindes steigt die Erwerbsquote der Mütter nur noch geringfügig an, jedoch erhöht sich der Anteil der Mütter, die in Vollzeit arbeiten auf $25 \%$ bzw. 30\%, wenn das Kind im Jugendalter ist (Demografie Portal 2019).

\section{Abbildung 3.1 Erwerbstätigenquote nach Alter des jüngsten Kindes und Beschäfti- gungsumfang, 2018}

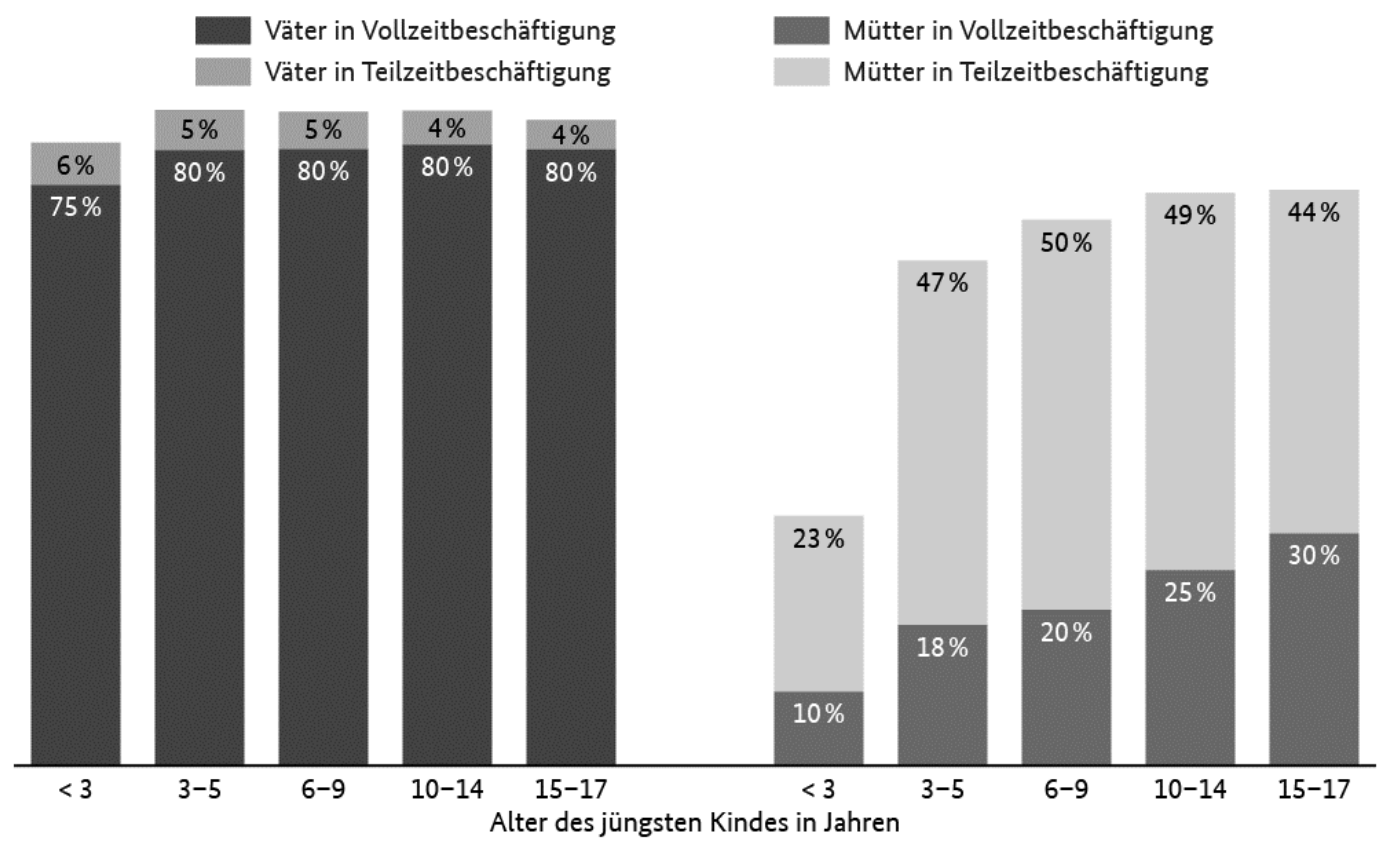

Quelle: Demografie Portal 2019, Daten: Statistisches Bundesamt 2018, Berechnungen: Bundesinstitut für Bevölkerungsforschung.

Neben der Altersabhängigkeit des Kindes wird die Aufteilung der Erwerbsarbeit auch stark dadurch geprägt, ob Familien in Ost- oder Westdeutschland leben. Während ostdeutsche Mütter relativ schnell nach der Geburt des Kindes wieder erwerbstätig sind, sind westdeutsche Mütter für einen längeren Zeitraum nicht erwerbstätig und kehren mit einem geringerem Umfang als ostdeutsche Mütter wieder in die Erwerbstätigkeit zurück (BMFSFJ 2014a, S. 42). Mit dem Alter des Kindes und den Unterschieden zwischen Ost- 
und Westdeutschland wurden bereits zwei wesentliche Einflussfaktoren für die Aufteilung der Erwerbstätigkeit kurz angeschnitten. Im Folgenden wird auf diese sowie auf weitere Einflussfaktoren für die innerpartnerschaftliche Aufteilung der Erwerbstätigkeit näher eingegangen. Dabei wird zunächst auf die Bedeutung von Ressourcen eingegangen und anschließend werden familiale Einflussfaktoren (wie der Übergang zur Elternschaft sowie das Alter und die Anzahl der Kinder) aufgezeigt. Danach wird der Forschungsstand zum Zusammenhang von Geschlechterrolleneinstellungen bzw. -ideologien und der Aufteilung der Erwerbsarbeit vorgestellt. Abschließend werden weitere Einflussfaktoren wie etwa die Bedeutung der öffentlichen Kinderbetreuung für die Aufteilung der Erwerbsarbeit behandelt.

\section{Zentrale Einflussfaktoren für die Aufteilung der Erwerbsarbeit}

\section{Bedeutung der Ressourcen der Mütter und Väter}

Viele Studien belegen, dass höher gebildete Frauen stärker in den Arbeitsmarkt integriert sind (Kurz 1998; Evertsson et al. 2007; England et al. 2012; Nieuwenhuis et al. 2012; Steiber et al. 2016; Buschner et al. 2018) und Mütter nach einer kindbezogenen Erwerbsunterbrechung wieder schneller in den Arbeitsmarkt zurückkehren (Kurz 1998; GutiérrezDomènech 2005; Grunow et al. 2011). Ein hohes Bildungsniveau der Frauen wirkt sich somit positiv auf ihre Erwerbstätigkeit, ihren Erwerbsumfang und ihren Wiedereinstieg aus. So konnte beispielsweise eine Studie von England et al. (2012) im internationalen Vergleich zeigen, dass Frauen mit hoher Bildung länderübergreifend eine höhere Erwerbsquote haben und in höherem Umfang erwerbstätig sind als Frauen mit niedriger Bildung. Auch eine Studie von Konietzka und Kreyenfeld (2010) belegt, dass Frauen mit hoher Bildung eine größere Wahrscheinlichkeit haben in Vollzeit oder in Teilzeit beschäftigt zu sein als Frauen mit niedriger Bildung.

Ein Großteil der Studien, die den Zusammenhang zwischen Bildung und Erwerbstätigkeit analysieren, verwenden für die Bildung die individuelle Bildung von einem oder von beiden Partnern und für die Erwerbstätigkeit den Erwerbsstatus (erwerbstätig/nicht-erwerbstätig) oder den Erwerbsumfang in Form der absoluten Stundenanzahl (Bernasco et al. 1998; Steiber und Haas 2009; Verbakel und Graaf 2009; Konietzka und Kreyenfeld 2010; Verbakel 2010; van der Lippe et al. 2011; England et al. 2012; BMFSFJ 2014a) (vgl. auch Buschner et al. 2018, S. 97-98). Zur Vorhersage der Aufteilung der Erwerbsarbeit zwischen den Partnern, stellt sich jedoch auch die Frage, wie sich die relative Bildung auf den relativen Erwerbsanteil eines Partners auswirkt (Buschner et al. 2018, S. 98). Im Folgenden wird daher näher auf die Bedeutung der relativen Bildung eingegangen.

Buschner et al. (2018) konnten in ihrer Studie zeigen, dass Frauen mit höherer, relativer Bildung einen höheren Anteil der Erwerbsarbeit übernehmen als Frauen mit niedrigerer oder gleicher Bildung. Dennoch ist bei den höhergebildeten Frauen der Anteil an der Erwerbsarbeit immer noch unter 50\%, d.h. die Erwerbsarbeit wird zwar egalitärer, aber nicht egalitär aufgeteilt. In Ostdeutschland rücken Frauen mit hoher, relativer Bildung 
deutlich näher an die 50\%-Marke heran als in Westdeutschland, unterschreiten die 50\%Marke aber dennoch, d.h. selbst hier sind Männer, deren Bildung niedriger ist als die ihrer Partnerinnen, in höherem Umfang erwerbstätig (Buschner et al. 2018). Aus einer Studie von Kreyenfeld et al. (2007) zur Bildungsungleichheit des Erwerbsverhaltens von Müttern geht hervor, dass Mütter mit größerer Wahrscheinlichkeit in eine Vollzeiterwerbstätigkeit zurückkehren, wenn sie höher qualifiziert sind als die Väter und umgekehrt mit niedrigerer Wahrscheinlich, wenn sie geringer qualifiziert sind als die Väter. Dass sich eine höhere, relative Bildung der Mütter positiv auf die Erwerbstätigkeit auswirkt, geht auch aus einer Studie von Berghammer (2014) für Österreich hervor: Die Wahrscheinlichkeit für ein „modernized male breadwinner“-Modell und für ein „dual breadwinner“Modell nimmt im Vergleich zur Referenzkategorie „male breadwinner“-Modell deutlich zu, wenn Mütter eine höhere Bildung als die Väter haben (Berghammer 2014, S. 621).

Die Verwendung der Bildung als Einflussfaktor für die Arbeitsteilung bringt jedoch das Problem mit sich, dass die Bildung nicht ausschließlich ein Indikator für das Humankapital ist, sondern auch ein Indikator für Werte und Einstellungen sein kann (van Berkel und Graaf 1999; Bonke und Esping-Andersen 2011; Steiber et al. 2016). Aufgrund dieser doppeldeutigen Bedeutung der Bildung, ist es daher von Vorteil, einen anderen Indikator zu verwenden, der nicht sowohl für arbeitsmarktrelevante Ressourcen als auch für Einstellungen steht, sondern eindeutiger ist, sodass dadurch mehr analytische Klarheit gewonnen werden kann (Nitsche und Grunow 2018). Ein Beispiel für einen alternativen Indikator ist der Berufsstatus. Der Berufsstatus wird häufig anhand des ISEI's (International Socio-Economic Index of Occupational Status) nach Ganzeboom et al. (1992) operationalisiert. Eine Studie von Hipp und Leuze (2015), in der die relativen Ressourcen durch den relativen Berufsstatus (nach ISEI) operationalisiert wurden, findet einen ähnlichen Zusammenhang wie bei der relativen Bildung: Die Erwerbsarbeit wird dann partnerschaftlicher aufgeteilt, wenn Frauen einen höheren oder ähnlichen Berufsstatus wie die Männer haben.

Warum selbst bei Frauen mit höheren Ressourcen lediglich eine egalitärere Arbeitsteilung und kein Rollentausch zu erwarten ist, kann u.a. dadurch erklärt werden, dass Frauen - und insbesondere Mütter - auf dem Arbeitsmarkt benachteiligt werden. So verdienen Frauen, wie der Gender Pay Gap verdeutlicht, immer noch weniger als Männer: 2017 betrug der unbereinigte Verdienstunterschied zwischen Männern und Frauen 22,3\% (Glassdoor Economic Research 2019, S. 50). Wenn Alter, Bildung und Berufserfahrung bereinigt werden, reduziert sich die Lohnlücke auf 15,3\%; werden des Weiteren auch firmenspezifische und berufsspezifische Faktoren bereinigt, so reduziert sie sich zwar auf 6,4\% (Glassdoor Economic Research 2019, S. 50). Aber diese bestehenbleibende Lohnlücke zeigt, dass Frauen selbst mit gleicher Bildung oder gleichem Berufsstatus wie der Partner nicht die gleichen Verdienstmöglichkeiten haben. Deshalb kann angenommen werden, dass die Arbeitsteilung bei Frauen mit gleichen oder höheren 
Ressourcen zwar egalitärer ist, aber Männer weiterhin - selbst dann, wenn sie mit schlechteren Ressourcen ausgestattet sind - die Hauptverdiener sind. ${ }^{1}$

Bedeutung des Übergangs zur Elternschaft und des Haushaltskontextes

Wie bereits von zahlreichen Studien erforscht ist, geht mit der Familiengründung eine geschlechtsspezifischere Arbeitsteilung einher (Sanchez und Thomson 1997; Gjerdingen und Center 2005; Dribe und Stanfors 2009; Bühlmann et al. 2010; Craig und Mullan 2010; Kühhirt 2012; Grunow 2014; Buschner et al. 2018). Das Vorhandensein von Kindern gilt als ein sehr großer - wenn nicht sogar als der größte - Einflussfaktor für die Erwerbstätigkeit von Frauen (van der Lippe und van Dijk 2002; Bühlmann et al. 2010; Kühhirt 2012). Die Wahrscheinlichkeit, erwerbstätig zu sein, ist für Mütter deutlich geringer als für kinderlose Frauen (Nieuwenhuis et al. 2012; Boeckmann et al. 2015). Nach der Familiengründung ist insbesondere das Alter des jüngsten Kindes für die Arbeitsteilung ausschlaggebend.

Aus einer Studie von Kühhirt (2012) geht hervor, dass Frauen vor der Geburt des Kindes etwa sieben Stunden pro Tag erwerbstätig sind, mit der Geburt des Kindes ihre Erwerbsstunden rapide abnehmen und im Durchschnitt nahe bei null Erwerbsstunden pro Tag liegen. Mit zunehmendem Alter des Kindes steigt ihr Erwerbsumfang zwar wieder auf durchschnittlich ca. drei Erwerbsstunden pro Tag an, aber sie bleiben damit noch weit unterhalb des Erwerbsumfangs vor der Geburt des Kindes zurück (Kühhirt 2012, 2012, S. 571-572). Eine Studie von Buschner et al. (2018) zeigt, dass der Erwerbsanteil von Frauen am gesamten Erwerbsvolumen des Paares vor der Geburt des Kindes bei etwa $43 \%$ liegt und im ersten Lebensjahr des Kindes nur noch bei 5\%; mit dem Älterwerden des Kindes steigt ihr Erwerbsanteil wieder auf bis zu 32\% an, ist damit aber immer noch niedriger als ihr Erwerbsanteil vor der Geburt des Kindes (Buschner et al. 2018, S. 117). Auch Hipp und Leuze (2015) konnten in ihrer Studie bestätigen, dass sich kleine Kinder negativ auf den Erwerbsanteil der Frauen auswirken. Berghammer (2014) findet in ihrer Studie heraus, dass sich die Wahrscheinlichkeit für eine Vollzeit- oder Teilzeitbeschäftigung für Mütter (im Vergleich zu einer Erwerbslosigkeit) deutlich erhöht, wenn sich das Kind im Kindergartenalter und nicht mehr im Kleinkindalter befindet.

Diese sowie weitere Studienergebnisse stimmen dahingehend überein, dass sich mit dem Übergang zur Elternschaft die Erwerbstätigkeit der Frauen enorm reduziert oder sie ganz aus der Erwerbstätigkeit aussteigen und Frauen mit zunehmendem Alter des Kindes wieder in die Erwerbstätigkeit einsteigen bzw. ihren Erwerbsumfang wieder erhöhen (Konietzka und Kreyenfeld 2010; Verbakel 2010; Berghammer 2014; Buschner et al. 2018). Neben dem Alter des Kindes wird die Aufteilung der Erwerbsarbeit entscheidend

\footnotetext{
${ }^{1}$ Auf kulturelle Gründe, die gegen einen Rollentausch sprechen oder inn zumindest unwahrscheinlich machen, wird an späterer Stelle bei den Ausführungen zur Bedeutung der Geschlechterrolleneinstellungen eingegangen.
} 
durch die Anzahl der Kinder mitbeeinflusst, wobei eine höhere Kinderanzahl mit einer geringeren Erwerbsbeteiligung der Mütter assoziiert ist. Dieser negative Effekt der Kinderanzahl auf die Erwerbstätigkeit der Mütter konnte von vielen Studien bestätigt werden (Konietzka und Kreyenfeld 2010; Berghammer 2014; BMFSFJ 2014a; Hipp und Leuze 2015; OECD 2016).

\section{Bedeutung von Geschlechterrolleneinstellungen}

Nach der Präferenztheorie von Hakim (2002) besteht ein enger Zusammenhang zwischen den Präferenzen und der Erwerbstätigkeit: Frauen mit erwerbsorientierten Einstellungen setzen ihren Fokus auf die Erwerbsarbeit, Frauen mit familienorientierten Einstellungen auf die Familie und die sog. adaptiven Frauen stehen zwischen diesen beiden Extremen. Viele Studien belegen, dass Frauen mit egalitären bzw. erwerbsorientierten Einstellungen häufiger und in höherem Umfang erwerbstätig sind als Frauen mit traditionellen Einstellungen (Fortin 2005; Corrigall und Konrad 2007; Cunningham 2008; Davis und Greenstein 2009; Diener und Berngruber 2018). Khoudja und Fleischmann (2018) untersuchten in ihrer Studie, wie sich die Geschlechterrolleneinstellungen von Frauen und Männer auf die Arbeitsmarkteintritte und -austritte von Frauen sowie auf die Erwerbsumfänge von Frauen auswirken. Die Ergebnisse zeigen, dass traditionelle Einstellungen der Frauen ihren Arbeitsmarkteintritt unwahrscheinlicher und ihren -austritt wahrscheinlicher machen. Ihr Arbeitsmarktaustritt wird auch dann wahrscheinlicher, wenn der Mann traditionelle Einstellungen vertritt. Zwischen den Einstellungen der Frauen und Männer und dem Erwerbsumfang der Frauen konnten dagegen keine signifikanten Zusammenhänge gefunden werden. Aus einer Studie von Diener und Berngruber (2018) geht hervor, dass sich der Erwerbsumfang der Mütter dann erhöht, wenn sie eine positive Einstellung zur außerhäuslichen Kinderbetreuung von Kindern im Alter von ein bis zwei Jahren haben. Cunningham (2008) konnte zeigen, dass Frauen mit egalitären Einstellungen eher erwerbstätig und auch in höherem Umfang erwerbstätig sind im Vergleich zu Frauen mit traditionellen Einstellungen und dass ihre Erwerbstätigkeit gefördert wird, wenn der Mann sie bei der Hausarbeit unterstützt. Während der Zusammenhang zwischen den Einstellungen und der Erwerbstätigkeit bei Frauen vielfach bestätigt wurde, konnten Corrigall und Konrad (2007) diesen Zusammenhang nicht auf Männer übertragen, da sie bei ihnen keinen signifikanten Zusammenhang zwischen den Einstellungen und der Erwerbstätigkeit der Männer feststellen konnten.

Bislang wurden die Einstellungen als eindimensionales Kontinuum von „traditionell“ bis "egalitär" betrachtet. Einstellungsitems, die verschiedene Bereiche abfragen, wurden in der bisherigen Forschung oftmals zu einem Index zusammengefasst. Da dies zunehmend kritisiert wird und neuere Forschung für eine multidimensionale Betrachtungsweise plädiert, wird zunächst ein kurzer Exkurs zur Operationalisierung der Geschlechterrolleneinstellungen eingeschoben. Im Anschluss an diesen Exkurs wird wieder auf weitere Einflussfaktoren eingegangen. 
Exkurs zur Operationalisierung der Geschlechterrolleneinstellungen: Von unidimensionalen zu multidimensionalen Konzepten

Geschlechterrolleneinstellungen beschreiben geschlechtsspezifische Rollenvorstellungen von Männern und Frauen. Synonym zum Begriff der Geschlechterrolleneinstellungen werden in der Literatur auch Begriffe wie "gender ideologies“, „gender roles“, „gender role attitudes“ oder "gender-related attitudes“ verwendet um Einstellungen zu geschlechtsspezifischer Arbeitsteilung abzubilden (Davis und Greenstein 2009, S. 88-89). Sämtliche Studien, die die Entwicklung der Geschlechterrolleneinstellungen in den letzten Jahrzehnten erforschen, kommen zu dem Ergebnis, dass traditionelle Einstellungen abgenommen und egalitärere Einstellungen zugenommen haben (Bolzendahl und Myers 2004; Lee et al. 2007; Davis und Greenstein 2009; Cotter et al. 2011; Knight und Brinton 2017).

Zur Operationalisierung der Einstellungen wurden in bisherigen Studien viele unterschiedliche Items verwendet. Davis und Greenstein (2009) vergleichen die in früheren Studien verwendeten Items und kategorisieren sie nach den folgenden, inhaltlichen Dimensionen:

(1) „primacy of the breadwinner role”,

(2) "belief in gendered separate spheres",

(3) "working women and relationship quality",

(4) "motherhood and the feminine self",

(5) "household utility" und

(6) "acceptance of male privilege" (Davis und Greenstein 2009, S. 90-91).

Items der ersten Dimension, „primacy of the breadwinner role”, fragen zum Beispiel danach, ob der Mann mehr verdienen soll als die Frau oder ob der Mann bei einem Mangel an Arbeitsplätzen bevorzugt werden soll. In der zweiten Dimension, "belief in gendered separate spheres", wird abgefragt, ob man der Ansicht ist, dass an der Aufteilung in weibliche, innerhäusliche und männliche, außerhäusliche Zuständigkeitsbereiche festgehalten werden soll. Dimension drei, „working women and relationship quality”, fragt nach der Beziehungsqualität erwerbstätiger Frauen bzw. Mütter und inwiefern Kinder unter dieser Situation leiden. Unter der vierten Dimension, „motherhood and the feminine self", werden Items zusammengefasst, die nach dem weiblichen Naturell fragen und welche Rolle hierfür die Familie und die Erwerbstätigkeit spielen, d.h. ob die Familie und die Mutterrolle essentiell für Frauen sind, oder ob Frauen auch in der Rolle der Erwerbstätigkeit aufgehen können. Die fünfte Dimension, "household utility", bezieht sich auf die Aufteilung der Hausarbeit. Fällt die Hausarbeit weiterhin in den Zuständigkeitsbereich der Frau, in dem sie nur gelegentlich vom Mann Unterstützung erfährt, oder sollte im Falle eines gleichen Erwerbsumfangs auch die Hausarbeit zwischen beiden Geschlechtern gleich verteilt werden? Die letzte Dimension, "acceptance of male privilege", bezieht sich auf die Frage, ob beide Geschlechter den gleichen Stellenwert haben und gleichbe- 
rechtigt sind oder ob eine Geschlechterhierarchie vorliegt, in der das männliche Geschlecht eine höhere Position einnimmt und bevorzugt behandelt wird (Davis und Greenstein 2009, S. 90-91).

Während sich Davis und Greenstein (2009) auf den Vergleich amerikanischer Daten beschränken, vergleicht Walter (2018b) die in internationalen und nationalen Surveys verwendeten Gender-Items miteinander. Sie kritisiert, dass die Items oftmals veraltet sind, da sie in den 1970er und 1980er Jahren entwickelt und vielfach unverändert übernommen wurden (Walter 2018b). Dabei beziehen sich viele Items insbesondere auf die Dimension „male breadwinner model“ und lassen andere Dimensionen unberücksichtigt.

In früheren Studien wurden Einstellungen bislang als ein unidimensionales Kontinuum mit einer Skala von traditionell bis egalitär begriffen (Davis und Greenstein 2009). Traditionelle Einstellungen gehen von "gendered separate spheres” aus, nehmen also an, dass es getrennte, geschlechtstypische Zuständigkeiten für Lebensbereiche wie die Erwerbs- und die Familienarbeit gibt, wobei Männer für die Erwerbs- und Frauen für die Familienarbeit zuständig sind (Davis und Greenstein 2009; Grunow et al. 2018). Egalitäre Einstellungen vertreten dagegen die Ansicht, dass beide Geschlechter gemeinsam für die Erwerbs- und Familienarbeit verantwortlich und zuständig sind (Davis und Greenstein 2009; Grunow et al. 2018). Für die Konstruktion eines unidimensionalen Kontinuums mit den Skalenenden "traditionell“ und „egalitär" werden sämtliche Items zu einem Index zusammengefasst. Dabei handelt es sich jedoch um Items, die - wie weiter oben ausgeführt - verschiedene Dimensionen abbilden (wie z.B. „primacy of the breadwinner role”, „belief in gendered separate spheres”, „working women and relationship quality” usw.). Wenn diese verschiedenen Items dann zu einem einzigen Index zusammengefasst werden, so gehen dadurch wertvolle Informationen verloren. Außerdem sind Geschlechterrolleneinstellungen komplexer, als es ein unidimensionales Kontinuum von traditionell bis egalitär abbilden könnte, da die Einstellungen auch je nach Lebensbereich variieren können: So können bei einer Person zum Beispiel hinsichtlich der Erwerbsarbeit egalitäre Rollenvorstellungen vertreten werden, aber bezüglich der Familienarbeit traditionellere Einstellungen vorliegen. Um diese Einstellungen differenzierter erfassen zu können, ist es deshalb angebracht, eine multidimensionale Herangehensweise zu wählen (Grunow et al. 2018, S. 43).

Neuere Studien wenden diese multidimensionale Auffassung bereits an und kommen damit zu differenzierteren Gender-Ideologien (Knight und Brinton 2017; Grunow et al. 2018). Es kann beispielsweise danach differenziert werden, ob von einer gemeinsamen oder getrennten Zuständigkeit der Geschlechter sowohl für die Erwerbs- als auch für die Sorgearbeit ausgegangen wird und ob diese Zuständigkeiten als durch Entscheidungen bedingt oder als durch die Natur der Geschlechter bedingt angesehen werden (Grunow 
et al. 2018). ${ }^{2}$ Mittels einer latenten Klassenanalyse identifizieren Grunow et al. (2018) insgesamt fünf Klassen an Gender-Ideologien: 1) „traditional“, 2) „moderate traditional“, 3) „intensive parenting“, 4) „egalitarian essentialism“ und 5) „egalitarian“ (Grunow et al. 2018, S. 52). Neben den beiden unidimensionalen Klassen, die zwischen einer getrennten Zuständigkeit („traditional“) und einer gemeinsamen Zuständigkeit („egalitarian“) der Geschlechter unterscheiden, gibt es somit auch noch Mischformen, bei denen je nach Lebensbereich traditionellere oder egalitärere Einstellungen vorliegen („moderate traditional“, ,intensive parenting“, „egalitarian essentialism“) (Grunow et al. 2018, S. 51-52). ${ }^{3}$

Auch Knight und Brinton (2017) finden anhand einer latenten Klassenanalyse mehrere Klassen von Gender-Ideologien: Neben einer traditionellen Gender-Ideologie finden sie noch drei Varianten von egalitären Gender-Ideologien, die sie als „liberal egalitarianism“, „egalitarian familism" und "flexible egalitarianism“ benennen (Knight und Brinton 2017). Der „liberale Egalitarismus“ stellt dabei die strengste Form des Egalitarismus dar, in der das höchste Ausmaß an Geschlechtergleichheit angenommen wird. Die eigenständige Erwerbstätigkeit der Frau wird als wichtig angesehen und die Annahme vertreten, dass Frauen auch trotz Erwerbstätigkeit eine gute Mutter sein können. Der „egalitäre Familismus“ ist dem „liberalen Egalitarismus“ ähnlich, unterscheidet sich von ihm aber in der Annahme, dass Frauen nur mit Kindern ein erfülltes Leben haben können und die Familie Vorrang hat (Knight und Brinton 2017, S. 1502-1503). Der "flexible Egalitarismus" kombiniert traditionelle Einstellungen zur Rolle der Frau in der Familie mit egalitären Einstellungen zur Rolle der Frau auf dem Arbeitsmarkt (Knight und Brinton 2017, S. 1503). Knight und Brinton (2017) vergleichen insgesamt 17 verschiedene, europäische Länder und kommen zu dem Ergebnis, dass die traditionellen Einstellungen im Laufe der letzten zwei Jahrzehnten zwar in allen 17 Ländern abgenommen haben, die Länder sich aber weiterhin darin unterscheiden, welche der drei Varianten an egalitären Einstellungen in den jeweiligen Ländern vorherrschend sind (Knight und Brinton 2017).

Nach diesem kurzen Abriss zur Entwicklung von der eindimensionalen zur multidimensionalen Operationalisierung der Geschlechterrolleneinstellungen, wird im Folgenden auf weitere Einflussfaktoren für die Aufteilung der Erwerbsarbeit eingegangen.

\section{Bedeutung der öffentlichen Kinderbetreuung}

Die Aufteilung der Erwerbsarbeit in Familien wird des Weiteren dadurch beeinflusst, welche Möglichkeiten zur öffentlichen Kinderbetreuung vorhanden sind. Die bisherige Forschung zeigt, dass sich eine gut ausgebaute, öffentliche Kinderbetreuung sowie eine gut finanzierbare Kinderbetreuung förderlich auf die Erwerbstätigkeit von Müttern auswirken (Uunk et al. 2005; Del Boca et al. 2009; Steiber und Haas 2009; Bauernschuster und Schlotter 2015; Hipp und Leuze 2015; Diener und Berngruber 2018). Eine Studie von

\footnotetext{
2 Im Kapitel zur latenten Klassenanalyse wird hierauf tiefer eingegangen.

${ }^{3}$ Auch hierauf wird im Kapitel zur latenten Klassenanalyse noch näher eingegangen.
} 
Schober und Spiess (2015) konnte Zusammenhänge zwischen der Qualität der Kinderbetreuung und der Erwerbstätigkeit der Mütter zeigen: So waren Mütter in Ostdeutschland dann häufiger erwerbstätig und tendierten dazu, ihren Erwerbsumfang zu erhöhen, wenn sie in Bezirken mit kleinen Betreuungsgruppen wohnten; für westdeutsche Mütter konnte kein signifikanter Zusammenhang zwischen der Größe der Betreuungsgruppen und der Erwerbsneigung der Mütter gefunden werden (Schober und Spiess 2015). Diener und Berngruber (2018) fanden heraus, dass Mütter in höherem Umfang erwerbstätig sind, wenn sie positive Einstellungen zur außerhäuslichen Kinderbetreuung für Kinder im Alter von ein bis zwei Jahren haben. Nach dieser Studie sind Mütter auch dann in höherem Umfang erwerbstätig, wenn eine hohe Betreuungsquote auf Kreisebene vorliegt und wenn sie während der Elternzeit eine Kita oder eine Tagespflege in Anspruch nahmen (Diener und Berngruber 2018). Hipp und Leuze (2015) verwendeten zur Erklärung des Erwerbsanteil der Frauen am gesamten Erwerbsvolumen des Paares als unabhängige Variable einen Interaktionsterm aus der Kinderbetreuung für unter dreijährige Kinder und dem Vorhandensein von kleinen Kinder. Dahinter steckt die Annahme, dass öffentliche Kinderbetreuungsmöglichkeiten vor allem für Mütter von kleinen Kindern relevant sind. Ihre Ergebnisse bestätigen dies, da sie zeigen, dass eine höhere Kinderbetreuungsquote mit einem höheren Erwerbsanteil von Müttern mit kleinen Kindern einhergeht (Hipp und Leuze 2015, S. 676).

\section{Weitere Einflussfaktoren}

Ein weiterer Einflussfaktor ist der Familienstand, da sämtliche Studien belegen, dass die Erwerbsarbeit bei verheirateten Paaren traditioneller aufgeteilt wird als bei unverheirateten Paaren (van der Lippe et al. 2011; Kühhirt 2012; Hipp und Leuze 2015; OECD 2016; Buschner et al. 2018). Darüber hinaus gibt es in Deutschland auch Jahrzehnte nach der Wiedervereinigung noch deutliche Unterschiede zwischen den alten und den neuen Bundesländern: Ostdeutsche Frauen sind in höheren Umfang erwerbstätig als westdeutsche Frauen und kehren nach der Geburt des Kindes schneller wieder in die Erwerbstätigkeit zurück (Pfau-Effinger und Smidt 2011; Grunow und Müller 2012; BMFSFJ 2014a; Buschner et al. 2018). Mehrere Studien kontrollieren darüber hinaus das Alter der Frauen bzw. das Alter der Männer mit. In einigen Studien hat das Alter keinen signifikanten Effekt (OECD 2016; Buschner et al. 2018), in anderen Studien wurde herausgefunden, dass bei älteren Frauen der Erwerbsanteil signifikant höher ist als bei jüngeren Frauen (Berghammer 2014; Hipp und Leuze 2015).

\subsection{Zur Aufteilung der Hausarbeit}

Die Aufteilung der Hausarbeit wird bereits seit Jahrzehnten intensiv erforscht, so dass hierzu zahlreiche Studien vorliegen. Einen guten Überblick über bisherige Studien geben u.a. Shelton und John (1996), Coltrane (2000), Künzler und Walter (2001), Bianchi und 
Milkie (2010), Lachance-Grzela und Bouchard (2010), Treas und Drobnič (2010), Sullivan (2011), Grunow (2019), Esping-Andersen und Schmitt (2020) und Perry-Jenkins und Gerstel (2020).

\section{Gender Revolution?}

In den letzten Jahrzehnten kam es zu einer Veränderung der Hausarbeitszeiten von Frauen und Männern: Die Hausarbeitszeiten der Frauen haben sich seit 1965 stark reduziert, während die Hausarbeitszeiten der Männer leicht angestiegen sind (Bianchi et al. 2000; Coltrane 2000; Gershuny 2000; Hook 2006; Sayer 2010; Hook 2010; Grunow 2014). Diesen Befund interpretieren Sullivan und Coltrane (2008) als Beleg dafür, dass von einer „stalled revolution“ (Hochschild und Machung 1989), d.h. von einer blockierten oder verzögerten Revolution der Geschlechterrollen, nicht die Rede sein kann, sondern der Wandel der Geschlechterrollen stetig voranschreitet und Frauenforscher und -forscherinnen diesen Wandel lediglich in zu hohem Tempo erwarten (Grunow 2009, S. 158). Diese tendenzielle Angleichung der Hausarbeitszeiten von Männer und Frauen darf jedoch nicht darüber hinwegtäuschen, dass eine Gleichheit der Hausarbeitszeiten noch meilenweit entfernt ist, da Frauen bei der Hausarbeit immer noch den Löwenanteil übernehmen (Geist 2005; Gershuny et al. 2005; Lachance-Grzela und Bouchard 2010). Bittman et al. (2003) konnten mit Daten für Australien und den USA nachweisen, dass sich die stärkere Beteiligung der Frauen an der Hausarbeit nicht allein durch ihren reduzierten Erwerbsumfang erklären lässt, sondern dies selbst dann noch der Fall ist, wenn Frauen in Vollzeit erwerbstätig sind und sämtliche Faktoren wie etwa der Familienkontext oder das Einkommen kontrolliert werden. Dieser Sachverhalt wird von den Autoren pointiert mit "gender trumps money“ zusammengefasst (Bittman et al. 2003, S. 186). England (2010) hält es deshalb für angemessen schon von einer „stalled revolution“ oder zumindest von einer „uneven revolution“ beim Geschlechterrollenwandel zu sprechen, da sich beim Geschlechterrollenwandel deutliche Asymmetrien zeigen: Während sich die Lebensführung von Frauen deutlich geändert hat (etwa indem die Erwerbspartizipation von Frauen angestiegen ist und Frauen häufiger männer-typische Berufe oder Aktivitäten ausüben), hat sich die Lebensführung der Männer weit weniger verändert und sie sind kaum in frauen-typische Bereiche vorgedrungen (England 2010; Yavorsky et al. 2015).

\section{Kategorisierung und Messung der Hausarbeit}

Zwischen den Studien, die die Aufteilung der Hausarbeit untersuchen, gibt es große Unterschiede in der Kategorisierung und Messung der Hausarbeit. So gibt es einerseits Studien, die nach der allgemeinen Hausarbeit fragen und andererseits Studien, die nach einzelnen Tätigkeiten der Hausarbeit fragen. Diese einzelnen Tätigkeiten lassen sich danach kategorisieren, ob sie regelmäßig anfallen, wie etwa Kochen, Einkaufen, Wäsche waschen und Putzen, oder ob es sich dabei um außeralltägliche Angelegenheiten wie Reparaturen, Gartenpflege oder Autopflege handelt (Blair und Lichter 1991; Presser 1994). Die regelmäßig anfallenden Tätigkeiten („routine housework“) können nach tradi- 
tionellen Geschlechterstereotypen als „weiblich“ konnotiert und die nur gelegentlich anfallenden Tätigkeiten („occasional housework“) als „männlich“ konnotiert betrachtet werden (Blair und Lichter 1991; Lachance-Grzela und Bouchard 2010). Darüber hinaus können einige Tätigkeiten, wie beispielsweise das Bezahlen von Rechnungen oder Fahrdienstleistungen für Familienmitglieder, als geschlechtsneutral bezeichnet werden (Presser 1994). Anstelle einer Kategorisierung in „routine housework“ und „occasional housework", kann auch danach unterschieden werden, ob es sich um innerhäusliche oder außerhäusliche Tätigkeiten handelt (Baxter 1997).

Es empfiehlt sich, in Fragebögen die einzelnen Tätigkeiten der Hausarbeit getrennt abzufragen (anstatt nach der gesamten Hausarbeit zu fragen), da die Erinnerung an einzelne Tätigkeiten weniger fehleranfällig ist als die Erinnerung der gesamten Hausarbeitszeit und weil damit das Problem umgegangen wird, dass Befragte unter der Hausarbeit oft unterschiedliche Tätigkeiten subsumieren (Schulz und Grunow 2011; Grunow und Baur 2014). Zudem bringt die Analyse der einzelnen Tätigkeiten den Vorteil mit sich, dass nicht mehr nur betrachtet wird, wer im Allgemeinen mehr Hausarbeit übernimmt, sondern detailliertere Aussagen möglich sind, inwiefern sich bei Frauen und Männern der Anteil an geschlechtstypischen, -neutralen sowie -untypischen Tätigkeiten verändert hat (Kroska 2004).

Bei der Operationalisierung der Hausarbeit kann zudem danach unterschieden werden, ob nach dem Zeitaufwand gefragt wird, d.h. wie viel Zeit Männer und Frauen mit der Hausarbeit verbringen, oder ob ein Beteiligungsindex verwendet wird und somit danach gefragt wird, wer welche Hausarbeit überwiegend übernimmt. Erhebungsdaten unterscheiden sich des Weiteren danach, ob die Hausarbeit retrospektiv abgefragt wird oder ob sie auf Tagebucheinträgen zur Zeitverwendung basieren. Zeitverwendungsdaten auf Basis von Tagebüchern gelten allgemein als zuverlässiger als Umfragedaten, haben aber den Nachteil, dass sie aufwendig und teuer sind. Schulz und Grunow (2011) haben die Ähnlichkeiten und Unterschiede zwischen Tagebuchdaten und Umfragedaten untersucht und fanden heraus, dass beide Messgrößen ziemlich konsistente Ergebnisse liefern.

Eine weitere Unterscheidung bei Studien zur Aufteilung der Hausarbeit besteht darin, wie weit oder eng der Begriff der Hausarbeit aufgefasst wird. Beispielsweise sind die Grenzziehungen zwischen der Hausarbeit und der Kinderbetreuung teilweise unscharf. In einer Studie von Hook (2006) werden die Zeiten für die Hausarbeit und die Zeiten für die Kinderbetreuung zu einer gemeinsamen abhängigen Variable zusammengefasst. Damit ist Hook jedoch eine Ausnahme, denn die meisten Studien analysieren die Hausarbeit unter explizitem Ausschluss der Kinderbetreuung (Schulz und Blossfeld 2006; Fuwa und Cohen 2007; Davis et al. 2007; Grunow et al. 2007; Aassve et al. 2014) oder die Hausarbeit und die Kinderbetreuung werden jeweils als separate, abhängige Variablen betrachtet (Kühhirt 2012). Da sich die beiden Bereiche jedoch in ihrer Art und in ihren Einflussfaktoren zum Teil voneinander unterscheiden, erscheint eine getrennte Analyse 
von Hausarbeit und Kinderbetreuung angebracht (Lachance-Grzela und Bouchard 2010; Sullivan 2013).

\section{Zentrale Einflussfaktoren für die Aufteilung der Hausarbeit}

\section{Bedeutung der Ressourcen der Mütter und Väter}

Die Hausarbeit wird allgemein als unangenehm wahrgenommen und will deshalb möglichst vermieden werden (Blood und Wolfe 1960; Gershuny 2013; Sullivan 2013). Ressourcentheoretische Ansätze nehmen an, dass derjenige Partner mit den höheren Ressourcen weniger oder gar keine Hausarbeit übernehmen muss, da er sich aus ihr herausverhandeln bzw. von ihr freikaufen kann. Im Folgenden wird daher auf die Bedeutung von Ressourcen wie dem Einkommen und der Bildung eingegangen.

Ein Großteil der Studien hat die Bedeutung des relativen Verdienstes für die Aufteilung der Hausarbeit untersucht, jedoch sind die Befunde nicht einheitlich. Das „economic dependency model“ (Brines 1994) geht davon aus, dass ein Zusammenhang zwischen der ökonomischen Abhängigkeit vom Partner und dem eigenen Anteil an der Hausarbeit besteht. Diese Annahme konnte Brines (1994) für Frauen auf Basis der amerikanischen Daten „Panel Study of Income Dynamics“ von 1985 bestätigen, da Frauen mit zunehmender finanzieller Unabhängigkeit weniger Hausarbeit verrichteten. Für Männer zeigte sich jedoch ein kurvilinearer Zusammenhang: Entgegen der Erwartungen ist die Hausarbeitsbeteiligung von Männern dann am geringsten, wenn sie von den Frauen ökonomisch stark abhängig sind. Den Verlauf der Hausarbeitsbeteiligung der Frauen erklärt Brines anhand des Modells der ökonomischen Abhängigkeit, den Verlauf der Männer erklärt sie dagegen mit "gender display“ und entwickelt daraus ihre bekannte Kompensationshypothese.

Greenstein (2000) replizierte die Studie von Brines mit dem amerikanischen Datensatz „National Survey of Families and Households“ von 1987 und konnte ihre Ergebnisse bestätigen, solange er wie Brines als abhängige Variable die absolute Hausarbeitszeit verwendete. Sobald er jedoch als abhängige Variable den Anteil der Frauen bzw. der Männer an der gesamten Hausarbeitszeit relativierte, kam er zu dem Ergebnis, dass sowohl bei den Frauen als auch bei den Männern ein kurvilinearer Zusammenhang vorliegt, d.h. es kompensieren beide Geschlechter eine geschlechtsuntypische, ökonomische Abhängigkeit. Greenstein interpretiert seine Ergebnisse daher nicht als „gender display“, sondern allgemeiner als „deviance neutralization“.

Bittman et al. (2003) replizierten Greensteins Analysen mit denselben amerikanischen Daten (NSFH) und fanden heraus, dass der Hausarbeitsumfang von Frauen mit steigendem relativen Einkommen abnimmt bis zu dem Punkt, wo beide gleich viel verdienen. Dies entspricht den Erwartungen der Ressourcen- und Verhandlungstheorie. Sobald Frauen jedoch ein höheres Einkommen als die Partner haben, nehmen ihre Hausarbeitsumfänge wieder zu, d.h. zwischen der Hausarbeitszeit der Frauen und ihrem relativen Einkommen liegt ein u-förmiger Zusammenhang vor. Die Zunahme der Hausarbeitszeit 
der Frau bei höherem, relativem Einkommen widerspricht den ressourcentheoretischen Erwartungen. Stattdessen spielt das Geschlecht ab dem Moment, wo Frauen ein höheres Einkommen haben als ihre Partner, die größere Rolle, indem Frauen dieses geschlechtsuntypische Merkmal durch eine verstärkte Übernahme der Hausarbeit neutralisieren. Während Brines (1994) von „gender display“ und Greenstein (2000) von „deviance neutralization" spricht, interpretieren Bittman et al. (2003) ihre eigenen Ergebnisse deshalb als „gender deviance neutralization“.

Weitere Studien haben ebenfalls den Zusammenhang zwischen dem relativen Einkommen und der Aufteilung der Hausarbeit untersucht: So verglichen Evertsson und Nermo (2007), wie sich in Schweden zwischen 1991 und 2000 eine Veränderung der relativen Ressourcen auf eine veränderte Aufteilung der Hausarbeit auswirkt. Ihre Ergebnisse zeigen, dass der Anteil der Frauen an der Hausarbeit abnimmt, wenn sich das relative Einkommen der Frauen erhöht, wenn sich ihre relative Bildung erhöht und wenn sich ihre ökonomische Abhängigkeit vom Mann reduziert. Obwohl die Effekte der relativen Ressourcen signifikant sind, spielen sie insgesamt nur eine kleine Rolle für die Aufteilung der Hausarbeit (Evertsson und Nermo 2007).

Eine Studie von Grunow et al. (2012) erforschte, wie sich die Aufteilung der Hausarbeit im Eheverlauf verändert. Zu Beginn der Ehe liegt bei fast der Hälfte der Paare eine egalitäre Aufteilung der Hausarbeit vor, die sich jedoch im Eheverlauf - weitgehend unabhängig von Einkommen - traditionalisiert. Wenn beide Partner ein gleich hohes Einkommen haben, dann ist es unwahrscheinlicher, dass der Mann seinen Anteil an der Hausarbeit reduziert und begünstigt somit eine egalitärere Aufteilung der Hausarbeit. Andererseits führt ein gleich hohes Einkommen oder ein höheres Einkommen der Frau nicht dazu, dass der Mann seinen Anteil erhöht. Und sobald Kinder vorhanden sind, sind ehemals signifikante Effekte des relativen Einkommens nicht mehr signifikant. Insgesamt zeigt auch diese Studie, dass die relativen Ressourcen nur eine sehr geringe Rolle spielen und traditionelle Geschlechterrollen bedeutender sind (Grunow et al. 2012).

Zusammenfassend lässt sich festhalten, dass sowohl die oben erwähnten Studien als auch viele weitere Studien (Cunningham 2007; Kan 2008; Aassve et al. 2014; Hook 2017) signifikante Effekte zwischen dem relativen Einkommen und der Aufteilung der Hausarbeit finden, wobei die Effekte in einigen Studien trotz ihrer Signifikanz gering ausfallen.

Studien von Gupta kritisieren, dass in bisheriger Forschung der Fokus auf dem relativen Einkommen lag und dabei die Bedeutung des absoluten Einkommens vernachlässigt wurde (Gupta 2006, 2007; Gupta und Ash 2008; Gupta et al. 2015). In Guptas Studien konnte nachgewiesen werden, dass das absolute Einkommen der Frauen eine größere Erklärungskraft als das relative Einkommen hat. Dies führt Gupta darauf zurück, dass Frauen mit hohem, absolutem Einkommen unabhängiger als Frauen mit niedrigem, absolutem Einkommen sind - ungeachtet der Tatsache, wieviel sie im Vergleich zum Partner verdienen. Aufgrund dieser Erkenntnisse wurde in nachfolgenden Studien neben 
dem relativen Einkommen häufig auch noch das absolute Einkommen mitberücksichtigt. Sobald das absolute Einkommen der Frauen in die Analysen mitaufgenommen wurde, konnte die „gender deviance neutralization“-Hypothese nicht mehr bestätigt werden und es finden sich stattdessen Belege für die „autonomy theory“ (Gupta 2006, 2007; Gupta und Ash 2008; Sullivan 2011; Gupta et al. 2015; Carlson und Lynch 2017).

Die Verwendung des relativen Einkommens für die Aufteilung der unbezahlten Arbeit ist in Querschnittsstudien kritisch zu betrachten, da auch eine entgegengesetzte Kausalitätsrichtung möglich ist, d.h. dass die unbezahlte Arbeit das Einkommen beeinflusst (Carlson und Lynch 2017; Nitsche und Grunow 2018). Hier liegt somit ein Endogenitätsproblem vor, welches sich jedoch durch die Verwendung von Längsschnittdaten mit speziellen Verfahren umgehen lässt (Carlson und Lynch 2017; Nitsche und Grunow 2018).

Neben dem relativen Einkommen wird auch häufig die relative Bildung als Indikator für die Aufteilung der Hausarbeit verwendet. So finden Aassve et al. (2014) für einige Länder empirische Evidenz, dass bei Frauen mit höherer, relativer Bildung eine egalitärere Aufteilung der Hausarbeit vorliegt. Auch Evertsson und Nermo (2007) finden Belege für den Zusammenhang zwischen der relativen Bildung und der Aufteilung der Hausarbeit: Ihre Ergebnisse zeigen, dass sich der Anteil der Frauen an der Hausarbeit reduziert, wenn sich ihre relative Bildung zwischen den Jahren 1991 und 2000 erhöht hat. Sullivan und Gershuny (2016) kommen zu einem ähnlichen Ergebnis, wenn sie das relative Humankapital betrachten, da ihre Ergebnisse zeigen, dass sich bei Frauen mit höherem Humankapital die Hausarbeit reduziert. Für die Operationalisierung des Humankapitals verwenden Sullivan und Gershuny (2016) jedoch nicht nur die Bildung, sondern generieren einen Indikator der neben der Bildung auch weitere arbeitsmarktrelevante Informationen wie die Beschäftigungshistorie enthalten.

Dass eine höhere Bildung mit einer egalitäreren Arbeitsteilung assoziiert wird, liegt jedoch nicht nur daran, dass Bildung ein Indikator für Humankapital ist, sondern darüber hinaus auch mit Werten und Einstellungen in Verbindung gebracht wird. So nimmt das "Egalitarian-Values-Modell" (van Berkel und Graaf 1999) an, dass mit einem höherem Bildungsniveau egalitärere Werte und Geschlechterrolleneinstellungen einhergehen und diese sich in einer egalitäreren Aufteilung der Hausarbeit manifestieren. Entscheidend ist jedoch, dass nicht nur ein Partner, sondern beide Partner ein hohes Bildungsniveau innehaben (van Berkel und Graaf 1999). Die positiven Effekte von Bildungshomogamie auf hohem Niveau für eine partnerschaftliche Aufteilung der Hausarbeit können neben der Studie von van Berkel und Graaf (1999) auch von Schulz (2010) und von Dechant et al. (2014) bestätigt werden. Einen Beleg für den Zusammenhang zwischen der Bildung und der Aufteilung der Hausarbeit liefert auch eine Studie von Davis und Greenstein (2004), aus der hervorgeht, dass die Aufteilung dann egalitärer ist, wenn Frauen eine gleich hohe oder eine höhere Bildung als die Männer haben (Davis und Greenstein 2004). Eine Studie von Nitsche und Grunow (2016), die die Aufteilung der Hausarbeit im 
Lebenslauf untersucht, konnte dagegen keinen Zusammenhang zwischen der relativen Bildung und der Hausarbeit finden - weder in dem Subsample derjenigen Paare, die im beobachteten Zeitfenster kinderlos bleiben, noch in dem anderen Subsample mit denjenigen Paaren, die den Übergang zur Elternschaft erleben.

\section{Bedeutung der Erwerbstätigkeit}

Neben dem Übergang zur Elternschaft (der Forschungsstand hierzu wird weiter unten vorgestellt) zählt die Aufteilung der Erwerbsarbeit zu den wichtigsten Einflussfaktoren für die Aufteilung der Hausarbeit. In Haushalten mit erwerbstätigen Frauen ist die Hausarbeit egalitärer aufgeteilt als in Haushalten, in denen die Frauen nicht erwerbstätig sind; wenn Frauen in Vollzeit erwerbstätig sind, ist sie wiederum egalitärer als wenn Frauen in Teilzeit erwerbstätig sind (Gershuny et al. 2005; Cunningham 2007; Fuwa und Cohen 2007; Baxter et al. 2008; Hook 2010; Grunow et al. 2012; Zabel und Heintz-Martin 2013; Aassve et al. 2014; Dechant et al. 2014). Greenstein (1996) konnte mit amerikanischen Daten zeigen, dass sich Männer stärker an der alltäglichen Routine-Hausarbeit beteiligen, wenn ihre Frauen erwerbstätig sind, jedoch ist dieser Unterschied nur sehr gering. Auch Cunningham (2007) kann nachweisen, dass sich Männer von Frauen, die mehrere Jahre vollzeitbeschäftigt waren, stärker an der Routine-Hausarbeit beteiligen.

Eine weitere Ursache für die egalitärere Hausarbeitsaufteilung bei erwerbstätigen Frauen kann ein Einstellungswandel sein, der durch die Erwerbstätigkeit der Frauen bedingt ist: Bolzendahl und Myers (2004) sprechen in diesem Zusammenhang von erfahrungsbasierten Faktoren (wie etwa die eigene Erwerbstätigkeit), die zu egalitäreren Einstellungen führen können. Denn durch die Erwerbstätigkeit werden Frauen einerseits ökonomisch unabhängiger und andererseits treffen sie im Beruf auf gleichgesinnte, egalitär eingestellte Frauen, mit denen sie soziale Netzwerke schließen können (Bolzendahl und Myers 2004). Mehrere Studien belegen diese Annahme, wonach erwerbstätige Frauen egalitärere Einstellungen innehaben (Coverdill et al. 1996; Fan und Marini 2000; Cunningham 2007). Aus Studien, die den Erwerbsumfang verwenden, geht zudem hervor: Je höher die Erwerbsumfänge der Frauen sind, umso egalitärer ist die Aufteilung der Hausarbeit (Cunningham 2007; Lachance-Grzela und Bouchard 2010; Aassve et al. 2014; OECD 2016). Dabei ist die egalitärere Aufteilung stärker dadurch bedingt, dass Frauen aufgrund der hohen zeitlichen Belastung durch den Beruf ihren eigenen Anteil an der Hausarbeit reduzieren und weniger dadurch, dass Männer ihren eigenen Anteil an der Hausarbeit erhöhen (OECD 2016, S. 180).

Zur Erklärung des Einflusses der Erwerbstätigkeit auf die Aufteilung der Hausarbeit verweisen viele Studien auf den Time-Availability-Ansatz von Coverman (1985): Hierbei wird argumentiert, dass jeder Person nur ein begrenztes Zeitbudget zur Verfügung steht und wenn mehr Zeit in eine bestimmte Tätigkeit investiert wird, kann weniger Zeit für andere Tätigkeiten aufgewendet werden. Bezogen auf die 
Arbeitsteilung von Eltern bedeutet dies, dass derjenige Elternteil, der erwerbstätig ist bzw. der in höherem Umfang erwerbstätig ist, weniger von der Hausarbeit übernehmen kann als der Elternteil, der nicht erwerbstätig ist bzw. nur in geringerem Umfang erwerbstätig ist. Am Time-Availability-Ansatz ist jedoch zu kritisieren, dass von der Logik her eine umgekehrte Kausalitätsrichtung ebenso denkbar ist: Übernehmen Frauen mehr Hausarbeit, weil sie weniger Erwerbsarbeit übernehmen oder übernehmen sie weniger Erwerbsarbeit, weil sie mehr Hausarbeit übernehmen? Der Time-Availability-Ansatz nimmt keine theoretische Stellungnahme dazu, warum eine Kausalitätsrichtung schlüssiger sein sollte als die andere (Geist und Ruppanner 2018, S. 243-244). Wenn beide Kausalitätsrichtungen möglich sind, hat man jedoch ein Endogenitätsproblem. Um dieses Endogenitätsproblem umgehen zu können, ist man auf die Verwendung von Längsschnittdaten angewiesen (Nitsche und Grunow 2018).

\section{Bedeutung von Geschlechterrolleneinstellungen}

Der Einfluss von Geschlechterrolleneinstellungen auf die Aufteilung der Hausarbeit ist bereits vielfach erforscht und bestätigt worden (Davis und Greenstein 2009; LachanceGrzela und Bouchard 2010): Sämtliche Studien konnten zeigen, dass Frauen mit egalitären Einstellungen weniger Hausarbeit übernehmen als Frauen mit traditionellen Einstellungen und dass Männer mit egalitären Einstellungen mehr Hausarbeit übernehmen als Männer mit traditionellen Einstellungen (Arrighi und Mauma 2000; Fuwa 2004; Cunningham 2005; Lewin-Epstein et al. 2006; Davis et al. 2007; Davis und Greenstein 2009; Treas und Drobnič 2010; Aassve et al. 2014; Evertsson 2014; Nitsche und Grunow 2016). Greenstein (1996) kontrollierte erstmalig einen Interaktionseffekt zwischen den Geschlechterrolleneinstellungen von Frauen und Männern. Dieser war signifikant und deutete darauf hin, dass die Geschlechterrolleneinstellungen des Mannes keinen Einfluss auf seinen Hausarbeitsanteil hatten, wenn die Frau traditionelle Einstellungen hatte und dass die Hausarbeitsteilung dann am egalitärsten war, wenn beide Partner egalitäre Einstellungen hatten (Greenstein 1996, S. 593). Eine Studie von Evertsson (2014) untersuchte mit schwedischen Daten sowohl den Einfluss der Einstellungen der Männer als auch der Einstellungen der Frauen auf die Aufteilung der Hausarbeit. Die Studie fand heraus, dass Frauen weniger Hausarbeit übernehmen, wenn sie selbst egalitäre Einstellungen haben und wenn die Männer egalitäre Einstellungen haben. Auch eine Studie von Nitsche und Grunow (2016) analysierte die Einstellungen von beiden Geschlechtern und deren Effekte auf die Hausarbeitsaufteilung. Sie fanden mit deutschen Daten (pairfam) heraus, dass sowohl durch egalitärere Einstellungen der Frauen als auch durch egalitärere Einstellungen des Mannes die Aufteilung der Hausarbeit egalitärer wird.

Bedeutung des Übergangs zur Elternschaft und des Haushaltskontextes

Die bisherige Forschung zeigt, dass die Aufteilung der Hausarbeit bei kinderlosen Paaren am egalitärsten ist und nach der Familiengründung deutlich ungleicher aufgeteilt wird 
(Evertsson und Nermo 2007; Baxter et al. 2008; Grunow et al. 2012; Kühhirt 2012; Schober 2013b; Dechant et al. 2014; Baxter et al. 2015). Während sich die Hausarbeitszeit für Frauen nach der Geburt des Kindes rapide erhöht, bleibt die Hausarbeitszeit der Männer davon relativ unberührt und die geringe Veränderung, die bei Männern stattfindet, geht in die entgegengesetzte Richtung, d.h. ihre Hausarbeitszeit wird nicht höher, sondern etwas geringer (Grunow et al. 2012; Kühhirt 2012).

Während die Aufteilung der Hausarbeit mit dem Übergang zur Elternschaft besonders ungleich aufgeteilt ist, könnte erwartet werden, dass sie mit zunehmendem Alter des Kindes wieder etwas egalitärer aufgeteilt wird. Diese Schlussfolgerung wäre naheliegend, da die Aufteilung der Erwerbsarbeit nach der Geburt eines Kindes sehr ungleich verteilt ist, aber mit zunehmendem Alter des Kindes egalitärer wird. Studien deuten jedoch darauf hin, dass die Hausarbeit mit zunehmendem Alter des Kindes nicht egalitärer, sondern noch traditioneller wird (Zabel und Heintz-Martin 2013). So untersuchte eine Studie von Zabel und Heintz-Martin (2013) die Aufteilung der Hausarbeit in Frankreich, in Ost- und in Westdeutschland in Abhängigkeit vom Alter des jüngsten Kindes. Sie fanden heraus, dass in Westdeutschland die Aufteilung der Hausarbeit bis zu einem Kindesalter von 13 Jahren traditioneller wurde und erst dann, wenn das Kind über 13 Jahre alt war, wieder etwas egalitärer wurde. Für Frankreich und Ostdeutschland wurde eine kontinuierliche Traditionalisierung der Hausarbeit festgestellt, d.h. die Aufteilung wurde mit zunehmendem Kindesalter traditioneller und flachte somit auch nicht im Jugendalter des Kindes ab (Zabel und Heintz-Martin 2013). Diese zunehmende Traditionalisierung steht in Einklang mit Studien, die die Beziehungsdauer berücksichtigen und feststellen, dass die Aufteilung der Hausarbeit mit zunehmender Beziehungsdauer traditioneller wird (Schulz und Blossfeld 2006; Grunow et al. 2007, 2012).

Neben dem Alter des Kindes spielt auch die Anzahl der Kinder eine Rolle für die Aufteilung der Hausarbeit, denn einige Studien kommen zu dem Ergebnis, dass die Aufteilung mit zunehmender Kinderanzahl traditioneller wird (Baxter et al. 2008; Kühhirt 2012; Fahlén 2016). Eine andere Studie von Schulz und Blossfeld (2006) findet dagegen nur beim Übergang zur Geburt des ersten Kindes einen signifikanten, traditionalisierenden Effekt auf die Aufteilung der Hausarbeit, aber nicht mehr bei der Geburt des zweiten oder dritten Kindes.

\section{Weitere Einflussfaktoren}

Ein weiterer Einflussfaktor ist der Institutionalisierungsgrad, denn verheiratete Paare haben eine traditionellere Aufteilung der Hausarbeit als unverheiratete, zusammenlebende Paare (South und Spitze 1994; Baxter 2005; Baxter et al. 2008; Domínguez-Folgueras 2012). Dies ist darauf zurückzuführen, dass in ehelichen und nicht-ehelichen Lebensgemeinschaften unterschiedliche Kohäsionsprinzipien wirken: Während sich verheiratete Paare mehr an dem kulturellen Leitbild einer geschlechtsspezifischen Arbeitsteilung orientieren, stehen bei unverheirateten, zusammenlebenden Paaren Gleichheitsprinzipien 
und egalitärere Geschlechterrollenbilder im Vordergrund (Brines und Joyner 1999). Darüber hinaus spielt das Bundesgebiet eine Rolle für die Aufteilung der Hausarbeit, da die Hausarbeit in Ostdeutschland egalitärer aufgeteilt wird als in Westdeutschland (Cooke 2006; Wengler et al. 2008; Grunow 2014).

\subsection{Zur Aufteilung der Kinderbetreuung}

Während die Hausarbeitszeit von Paaren in den letzten Jahrzehnten abgenommen hat, ist die Zeit, die Eltern mit ihren Kindern verbringen, gestiegen (Sayer 2010). Ähnlich wie bei der Aufteilung der Hausarbeit haben sich auch bei der Aufteilung der Kinderbetreuung die Geschlechterunterschiede zwischen Müttern und Vätern ab Mitte der 1960er verringert (Sayer 2005). Diese Angleichung der Aufteilung der Kinderbetreuung ist darauf zurückzuführen, dass Väter mehr Zeit mit ihren Kindern verbringen (Bianchi et al. 2006). Die Zeit, die Mütter mit ihren Kindern verbringen, hat sich jedoch durch ihre zunehmende Erwerbstätigkeit nicht reduziert, sondern bleibt auf einen hohem Niveau (Sayer et al. 2004a; Bianchi et al. 2006). Insgesamt übernehmen Mütter weiterhin - trotz der gestiegenen Zeit, die Väter mit ihren Kindern verbringen - den größeren Anteil der Kinderbetreuung und verbringen insgesamt etwa doppelt bis drei Mal so viel Zeit mit ihren Kindern als Väter (Craig und Bittman 2005, S. 16).

Die Entwicklung, dass sich Väter verstärkt in die Kinderbetreuung einbringen und Mütter trotz ihrer Erwerbstätigkeit weiterhin viel Kinderbetreuung übernehmen, wird auch mit den Begriffen „involved fathering“, „intensive mothering“ oder allgemeiner für beide Elternteile mit "intensive parenting“ umschrieben (Hays 1996; Wall 2010; Walls et al. 2016). Die Begriffe gehen jedoch noch darüber hinaus: Sie betonen, dass Elternschaft nicht nur zeitintensiver geworden ist, sondern dass Eltern auch für immer mehr Aspekte der kindlichen Entwicklung und des kindlichen Wohlbefindens verantwortlich sind (Hays 1996; Wall 2010). Die gesteigerten Erwartungen an Elternschaft erhöhen den Druck und den Stress, der auf Eltern lastet (Wall 2010). Im Folgenden wird zunächst näher auf die Kategorisierung und Messung der Kinderbetreuung eingegangen, anschließend werden Einflussfaktoren wie etwa die Erwerbstätigkeit, die Ressourcenverteilung, die Geschlechterrolleneinstellungen oder der Familienkontext beleuchtet.

\section{Kategorisierung und Messung der Kinderbetreuung}

Analog zur Aufteilung der Hausarbeit kann auch bei der Aufteilung der Kinderbetreuung danach unterschieden werden, ob es sich um einen Zeitindex handelt, bei dem die Kinderbetreuungszeit abgefragt wird (z.B. Kühhirt 2012) oder ob es sich um einen Beteiligungsindex handelt, bei dem danach gefragt wird, wer die Kinderbetreuung überwiegend übernimmt (z.B. Nitsche und Grunow 2018). Bei den einzelnen Tätigkeiten kann des Weiteren - unabhängig davon, ob dabei zeitliche Angaben oder die Beteiligung daran abgefragt werden - nach verschiedenen Dimensionen differenziert werden: So differenziert Craig (2006a) zwischen der körperlichen und der interaktiven Kinderbetreuung: Die 
Ergebnisse auf Basis von australischen Daten zeigen, dass Mütter öfter Routine-Betreuung (,physical and emotional care“) übernehmen, während Väter am ehesten interaktive Betreuung wie das Sprechen und Spielen mit den Kindern übernehmen (,interactive child care") (Craig 2006a). Ein weiterer Unterschied zwischen Müttern und Vätern besteht darin, dass Mütter häufiger mehrere Aufgaben parallel erledigen, wohingegen dies auf Väter seltener zutrifft. Insgesamt verbringen Müttern in allen Formen der Kinderbetreuung mehr Zeit mit ihren Kindern als die Väter: So wenden Mütter im Vergleich zu Vätern etwa drei Mal mehr Zeit für „physical care“ auf, doppelt so viel Zeit für „interactive care“ und viermal so viel Zeit für „transporting children“ (Craig 2006a, S. 274).

Eine andere Art der Operationalisierung wählen Raley et al. (2012), indem sie auf Basis von Zeittagebuchdaten danach differenzieren, wie viel Zeit insgesamt mit der Kinderbetreuung verbracht wird („total time with children“), wie viel Zeit davon alleine (ohne Anwesenheit des Partners) mit der Betreuung verbracht wird („solo time with children“) und wie viel Zeit dabei für Betreuungsaktivitäten im engeren Sinn aufgewendet wird (,primary child care time“) (Raley et al. 2012, S. 1433). Sie fanden anhand von amerikanischen Daten heraus, dass Väter dann mehr Zeit mit den Kindern allein verbringen („fathers“ solo time with children“), wenn Mütter berufstätig sind und dass Väter mit steigendem Einkommen der Mütter mehr Zeit mit Kinderbetreuung im engeren Sinne („fathers‘ primary child care time") verbringen.

\section{Zentrale Einflussfaktoren für die Aufteilung der Kinderbetreuung}

\section{Bedeutung der Erwerbstätigkeit}

Die Aufteilung der Kinderbetreuung wird stark durch die Erwerbstätigkeit der Eltern mitbestimmt. ${ }^{4}$ Im Folgenden wird daher näher auf Studien eingegangen, die die Erwerbstätigkeit bzw. die Erwerbskonstellation der Eltern in den Blick nehmen. Gracia (2014) konnte anhand von spanischen Daten zeigen, dass sich die Erwerbstätigkeit der Mütter positiv auf die physische Kinderbetreuungszeit der Väter auswirkte, jedoch bestand kein signifikanter Effekt zwischen der Erwerbstätigkeit der Mütter und der interaktiven Kinderbetreuungszeit der Väter. Die physische Kinderbetreuung, worunter Aufgaben wie Füttern, Baden, Beaufsichtigen und ins Bett bringen fallen, gilt als aufwendig, zeitintensiv und unflexibel (Gracia 2014); zur interaktiven Kinderbetreuung zählen dagegen andere Aufgaben wie z.B. Spielen oder Vorlesen (Gracia 2014). Eine daran anschließende Studie von Gracia und Esping-Andersen (2015) untersuchte diesen Zusammenhang zwischen der Erwerbstätigkeit der Mütter und den beiden Arten der Kinderbetreuung neben Spanien auch für Dänemark und England. Auch in diesen Ländern konnte ein signifikanter Effekt der mütterlichen Erwerbstätigkeit auf die physische Kinderbetreuungszeit der

\footnotetext{
${ }^{4}$ Die Kausalitätsrichtung ist hier unklar: Es ist genauso denkbar, dass der Umfang der Kinderbetreuung die Erwerbstätigkeit beeinflusst. Hier liegt daher ein Endogenitätsproblem vor. Um dieses Problem zu umgehen, sollte man bei empirischen Analysen Längsschnittdaten verwenden (Carlson und Lynch 2017; Nitsche und Grunow 2018).
} 
Väter gefunden werden und analog zur vorherigen Studie kein signifikanter Effekt auf die interaktive Kinderbetreuungszeit der Väter.

Eine Studie von Raley et al. (2012) bestätigte mit amerikanischen Daten, dass Väter mit zunehmendem Erwerbsumfang der Mütter mehr Kinderbetreuung übernehmen. Umgekehrt besteht zwischen einem höherem Erwerbsumfang der Väter und der Kinderbetreuungszeit der Väter ein negativer Zusammenhang (Roeters et al. 2009; Evertsson 2014; Gracia und Esping-Andersen 2015).

Obwohl viele Studien signifikante Effekte zwischen der Erwerbstätigkeit der Eltern und der Aufteilung der Kinderbetreuung finden, gibt es auch Studien, die keine oder nur schwache Effekte finden. Beispielsweise können Hook und Wolfe (2012) für Deutschland und für Norwegen keinen Zusammenhang zwischen der Erwerbstätigkeit der Mütter und der Kinderbetreuungszeit der Väter entdecken. Bünning (2020) untersuchte mit deutschen Daten, wie viel Kinderbetreuung (und Hausarbeit) Väter übernehmen, wenn sie nicht mehr in Vollzeit, sondern nur noch in Teilzeit arbeiten und wie viel sie übernehmen, nachdem sie wieder in die Vollzeit zurückgekehrt sind. Sie fand heraus, dass Väter, die in Teilzeit arbeiten, mehr Kinderbetreuung (und auch mehr Hausarbeit) übernehmen als Väter, die in Vollzeit arbeiten. Sobald sie jedoch wieder in die Vollzeit zurückgekehrt sind, reduziert sich ihr Anteil wieder - außer die Mutter ist in Vollzeit erwerbstätig (Bünning 2020).

\section{Bedeutung der Ressourcen}

Führt eine hohe Bildung der Eltern dazu, dass sie mehr Zeit oder weniger Zeit mit der Kinderbetreuung verbringen, ist Bildung also „a pull to work or a pull to care" (Craig 2006b, S. 554)? Die theoretischen Ansätze unterscheiden sich darin, welchen Effekt sie der Bildung der Eltern im Hinblick auf die Kinderbetreuung zuschreiben. Ökonomische Theorien gehen davon aus, dass sich mit höherer Bildung ein höheres Einkommenspotential erzielen lässt und eine Ausrichtung auf die Erwerbarbeit damit attraktiver erscheint, wohingegen eine Fokussierung auf die Kinderbetreuung mit hohen Opportunitätskosten verbunden ist (Becker 1975, 1993). Gleichzeitig erhöht eine hohe Bildung die Macht in den innerpartnerschaftlichen Austausch- und Verhandlungsprozessen (Lundberg und Pollak 1993, 1996; Ott 1992). Für Frauen mit höherer Bildung kann es daher finanziell attraktiver sein, ihre unbezahlte Zeit für Hausarbeit und Kinderbetreuung zu reduzieren und dafür mehr Erwerbsarbeit zu übernehmen. Im Falle einer Bildungshomogamie ist davon auszugehen, dass eine egalitäre Aufteilung der Kinderbetreuung (und auch der Hausarbeit) deutlich wahrscheinlicher wird. Im Gegensatz dazu wird Bildung jedoch auch mit Werten und Einstellungen assoziiert (Bolzendahl und Myers 2004). Auch muss berücksichtigt werden, dass die Kinderbetreuung nicht wie die Hausarbeit vermieden werden will, denn im Gegensatz zur Hausarbeit ist die Kinderbetreuung (zumindest meistens) bereichernd und angenehm (Sullivan 2013, S. 74). Im Folgenden wird näher auf Studien eingegangen, die die absoluten und/oder relativen Ressourcen zur Erklärung der Aufteilung der Kinderbetreuung verwenden. 
Viele Studien belegen, dass Eltern mit höherem Bildungsniveau mehr Zeit mit ihren Kindern verbringen als Eltern mit niedrigerem Bildungsniveau (Sayer et al. 2004b; Bianchi et al. 2006; Craig 2006b; England und Srivastava 2013; Gracia 2014; Sani und Treas 2016). Nicht alle Studien finden jedoch für beide Geschlechter starke Effekte der Bildung auf die Kinderbetreuung: So zeigt eine Studie von Sayer et al. (2004b) mit internationalen Daten, dass hoch gebildete Mütter zwar in allen untersuchten Ländern (Kanada, Deutschland, Italien und Norwegen) mehr Zeit mit der Kinderbetreuung verbringen als Mütter mit niedriger Bildung; aber bei den Vätern konnten nicht für alle Länder signifikante Unterschiede zwischen ihrem Bildungsniveau und ihrer Kinderbetreuungszeit gefunden werden (Sayer et al. 2004b). Dagegen fand Craig (2006b) auch für Väter starke Unterschiede je nach Bildungsniveau: Craig (2006b) konnte anhand von australischen Daten nicht nur zeigen, dass hochgebildete Eltern mehr Zeit mit Kinderbetreuung verbringen, sondern auch, dass bei hochgebildete Eltern die Aufteilung der Kinderbetreuung egalitärer war als in anderen Bildungskonstellationen, da Väter mit hoher Bildung mehr Zeit mit Kinderbetreuung verbrachten als Väter mit niedriger Bildung. Diese uneindeutigen Ergebnisse zur Bildung der Väter und ihrer Beteiligung an der Kinderbetreuung spiegeln sich auch in der Studie von England und Srivastava (2013) wider: Während die eigene Bildung der Väter nur teilweise Auswirkungen auf ihre Kinderbetreuungszeit hat, scheint die Bildung der Partnerinnen mehr Einfluss auf die Kinderbetreuungszeit der Väter zu haben (England und Srivastava 2013). Bonke und Esping-Andersen (2011) untersuchten den Einfluss von Bildungshomogamie und der Kinderbetreuungszeit der Eltern. In Einklang mit den Ergebnissen von Craig (2006b) fanden sie heraus, dass Eltern, in denen beide hoch gebildet sind, mehr Zeit mit Kinderbetreuung verbringen. Aus ihren Analysen geht darüber hinaus hervor, dass dieser Effekt größer ist als die Summe der Effekte der individuellen Bildung beider Elternteile. Paradoxerweise fanden viele Studien heraus, dass höher gebildete Mütter und Väter nicht nur mehr Zeit mit ihren Kindern verbringen, sondern darüber hinaus auch öfter erwerbstätig sind im Vergleich zu niedriger gebildeten Müttern und Vätern (Sayer et al. 2004b; Craig 2006b; England und Srivastava 2013), so dass sie einem besonders hohen Stress ausgesetzt sind.

Neben der Bildung untersuchen viele Studien den Einfluss des Einkommens auf die Aufteilung der Kinderbetreuung (Bonke und Esping-Andersen 2011; Kühhirt 2012; Raley et al. 2012; England und Srivastava 2013; Nitsche und Grunow 2018). So konnten etwa Raley et al. (2012) zeigen, dass sich mit steigendem Einkommensanteil der Mütter die Kinderbetreuungszeit der Mütter reduziert - sowohl die Zeit, die sie insgesamt mit der Kinderbetreuung verbringen („mothers' total time“) als auch die Zeit, die sie alleine mit den Kindern verbringen („mothers' solo time“). Umgekehrt führt ein steigender Einkommensanteil der Mütter dazu, dass Väter mehr von der routinemäßig, anfallenden Kinderbetreuung übernehmen („fathers“ primary child care time“) (Raley et al. 2012, S. 14401441). Nitsche und Grunow (2018) betrachteten in ihrer Studie die wirtschaftlichen Ressourcen nicht mehr nur isoliert, sondern untersuchten eine Kombination der wirtschaftlichen Ressourcen und der Einstellungen. Dabei bezogen sie sich auf die Einstellungen 
beider Elternteile und gewannen dadurch Erkenntnisse darüber, ob Eltern in ihren Einstellungen übereinstimmen oder nicht. Ihre Ergebnisse zeigen, dass die Kombination der Einstellungen und des Einkommensanteils der Mutter positive Effekte auf den Kinderbetreuungsanteil des Vaters hat, wobei die Effekte dann am stärksten sind, wenn Eltern in den Einstellungen übereinstimmen und gemeinsam egalitäre Einstellungen vertreten (Nitsche und Grunow 2018).

\section{Bedeutung der Geschlechterrolleneinstellungen}

Einige Studien verwenden auch Geschlechterrolleneinstellungen zur Vorhersage der Aufteilung der Kinderbetreuung (Bulanda 2004; Evertsson 2014; Nitsche und Grunow 2018). Bulanda (2004) untersuchte mit amerikanischen Daten, wie sich die Einstellungen von Müttern und Vätern auf das väterliche Engagement bei der Kinderbetreuung auswirkten. Er stellt fest, dass egalitärer eingestellte Väter sich gemäß den Erwartungen mehr an der Kinderbetreuung beteiligen als Väter mit traditionelleren Einstellungen; aber entgegen der Erwartungen spielten die Einstellungen der Mütter keine Rolle für die väterliche Beteiligung an der Kinderbetreuung (Bulanda 2004). Evertsson (2014) untersuchte diese Fragestellung mit schwedischen Daten und bestätige, dass der Betreuungsumfang der Väter steigt, wenn die Väter egalitäre Einstellungen vertreten. Im Gegensatz zur Studie von Bulanda (2004) konnte Evertsson (2014) jedoch auch einen positiven, signifikanten Effekt zwischen den egalitären Einstellungen der Mütter und der Betreuungsintensität der Väter feststellen. Goldscheider et al. (2011) konnten mit schwedischen Daten aufzeigen, dass traditionelle Einstellungen im Jahr 1999 zu einer weniger egalitären Aufteilung der Kinderbetreuung im Jahr 2003 führten. Nitsche und Grunow (2018) untersuchten mit deutschen Daten die Effekte der Einstellungen auf die Aufteilung der Kinderbetreuung. Ihre Ergebnisse zeigen, dass bei Paaren, in denen beide traditionelle Einstellungen vertreten, die Aufteilung der Kinderbetreuung erwartungsgemäß am traditionellsten ausfällt und bei Paaren, in denen beide egalitäre Einstellungen vertreten am egalitärsten. Paare, bei denen beide moderate Einstellungen vertreten, befinden sich zwischen diesen beiden Extremen.

\section{Bedeutung des Kindesalters und der Kinderanzahl}

Das Alter und die Anzahl der Kinder legen den Umfang der zu verrichtenden Kinderbetreuung fest. Kleinere Kinder sind betreuungsbedürftiger als ältere Kinder und mehrere Kinder führen zu einem größeren Betreuungsaufwand als ein Kind. Bisherige Studien zeigen, dass in Familien mit kleineren Kindern und/oder mehreren Kindern die Arbeitsteilung besonders häufig geschlechtsspezifisch arrangiert ist (Craig und Bittman 2005; Kühhirt 2012; Nitsche und Grunow 2018). Eine egalitäre Arbeitsteilung ist demzufolge dann am wahrscheinlichsten, wenn das jüngste Kind die betreuungsintensivsten ersten Lebensjahre überschritten hat und wenn in Familien nur ein Kind vorhanden ist. Craig und Bittman (2005) zeigen, dass die Stunden, die Eltern für die Kinderbetreuung aufnehmen, mit dem Übergang des jüngsten Kindes in das Grundschulalter rapide abnehmen: Ist das jüngste Kind 0-2 Jahre alt, werden in Ein-Kind-Familien täglich etwa 4,5 Stunden 
für die Kinderbetreuung aufgebracht (in Zwei- und Mehr-Kind-Familien sind 5 bis 5,5 Stunden); ist das jüngste Kind dagegen 5-11 Jahre alt, sind es in Ein-Kind-Familien täglich nur noch etwa 1,2 Stunden und in Zwei- und Mehr-Kind-Familien 1,5 bis 2 Stunden (Craig und Bittman 2005, S. 7).

\section{Bedeutung der Dauer der Elternzeit}

Ein weiterer Faktor, der die Aufteilung der Kinderbetreuung (und auch der Hausarbeit) beeinflussen kann, ist die Dauer der Elternzeit (Almqvist und Duvander 2014; Bünning 2015; Evertsson et al. 2018; Schober und Zoch 2019; Bünning 2020). Almqvist und Duvander (2014) konnten mit schwedischen Daten zeigen, dass Väter, die lange Zeit in Elternzeit sind, sich danach mehr an der Kinderbetreuung und auch an der Hausarbeit beteiligen. Auch Evertsson et al. (2018) kommen mit schwedischen Daten zu dem Befund, dass sich die Dauer der Elternzeit nachhaltig darauf auswirkt, wie sehr sich Väter an der Kinderbetreuung beteiligen. Einen positiven Effekt zwischen der Elternzeitdauer der Väter und ihrer Beteiligung an der Kinderbetreuung und an der Hausarbeit konnte Bünning $(2015,2020)$ auch mit deutschen Daten belegen.

\subsection{Forschungslücke und Forschungsfragen}

Welche Forschungslücken ergeben sich auf Basis des aufgezeigten Forschungsstandes und welche darauf aufbauenden Forschungsfragen will die vorliegende Arbeit beantworten? Auf diese Fragen und welchen Beitrag hierzu diese Arbeit leisten will, wird im Folgenden näher eingegangen.

Während die Veränderung der Arbeitsteilung mit dem Übergang zur Elternschaft bereits vielfach erforscht ist (Künzler und Walter 2001; Baxter et al. 2008; Schulz 2010; Grunow et al. 2012; Schober 2013a; Dechant und Schulz 2014), besteht noch Unklarheit darüber, wie sich die Arbeitsteilung der Eltern mit dem Älterwerden des Kindes entwickelt. Es mangelt noch an Studien, die die Arbeitsteilung differenziert nach dem Alter des Kindes betrachten und Beiträge dazu liefern können, wie sich Wirkmechanismen, auf denen die Arbeitsteilung basiert, in unterschiedlichen Phasen des Familienzyklus unterscheiden können. Auf der Mikroebene werden zur Erklärung der Aufteilung von Erwerbsarbeit, Hausarbeit und Kinderbetreuung insbesondere relative Ressourcen (wie Einkommen, Bildung und Berufsstatus) und Geschlechterrolleneinstellungen herangezogen (Coltrane 2000; Lachance-Grzela und Bouchard 2010; Bianchi und Milkie 2010; Grunow 2014; Hipp und Leuze 2015; Buschner et al. 2018). Es hat sich jedoch gezeigt, dass es für die Arbeitsteilung in Familien besonders ausschlaggebend ist, in welchen Phasen des Familienzyklus (Glick 1947) sich eine Familie befindet (Bühlmann et al. 2010). Die Frage danach, welche Rolle relative Ressourcen und Geschlechterrolleneinstellungen innerhalb des Familienzyklus spielen, ist jedoch noch unerforscht.

Neue Erkenntnisse hierzu helfen dabei, besser begreifen zu können, wie sich die Arbeitsteilung im Familienzyklus verändert und welche Faktoren in den unterschiedlichen 
Phasen des Familienzyklus eine egalitäre oder traditionelle Arbeitsteilung begünstigen. Geschlechter(un-)gleichheiten können so besser nachvollzogen werden und etwa durch familienpolitische Maßnahmen gezielter kompensiert werden. Gleichzeitig erhält man detailliertere Einblicke in die Machtverhältnisse von Eltern als dies bislang der Fall war. Ebenso werden Einsichten darüber möglich, welche Familienleitbilder in welchen Phasen des Familienzyklus dominant sind und wann Diskrepanzen zwischen geäußerten Einstellungen und praktizierter Arbeitsteilung besonders ausgeprägt sind.

Betrachtet man diese Thematik aus einer Lebensverlaufsperspektive heraus, so lässt dies Aufschlüsse darüber zu, ob anfängliche Arbeitsteilungsmuster im Sinne einer Pfadabhängigkeit weiter beibehalten werden (und sich ggf. noch weiter zuspitzen) oder ob diese einer gewissen Dynamik und Flexibilität unterliegen und somit deutliche Wandlungen im Familienzyklus möglich sind. Zwei verschiedene Entwicklungen sind denkbar: Es könnte einerseits sein, dass mit der Geburt des Kindes bzw. mit der Arbeitsteilung unmittelbar nach der Geburt Weichen gestellt werden, durch die die in den nächsten Jahren (und Jahrzehnten) stattfindende Arbeitsteilung weitgehend vorbestimmt ist. Wenn beispielsweise im Säuglings- und Kleinkindalter eine traditionelle Arbeitsteilung praktiziert wird, in der der Vater in Vollzeit erwerbstätig ist und die Mutter nicht oder nur in geringem Umfang erwerbstätig ist, kann angenommen werden, dass diese Arbeitsteilung auch mit dem Älterwerden des Kindes beibehalten wird. Die zu Beginn gestellten Weichen lassen demzufolge nur wenig Spielraum zu, sodass entweder gar keine Veränderung der Arbeitsteilung stattfindet und wenn dann nur dahingehend, dass sich die eingeschlagene, traditionelle Arbeitsteilung noch weiter zuspitzt und noch traditioneller wird.

Andererseits wäre es auch denkbar, dass die Arbeitsteilung mit zunehmendem Alter des Kindes egalitärer wird. Diese Überlegung fußt auf der Annahme, dass die Betreuungsintensität mit zunehmendem Alter des Kindes abnimmt: Zum einen, weil Kinder von ihrer Natur her mit zunehmendem Alter selbstständiger werden und weniger Betreuung bedürfen; zum anderen, weil externe Betreuungseinrichtungen (etwa der Kindergartenbesuch oder die Schulzeit) für Kinder, die sich nicht mehr im Kleinkindalter befinden, besser ausgebaut sind. Indem die Betreuungsnotwendigkeit mit zunehmendem Alter des Kindes abnimmt, eröffnen sich für Mütter auch trotz einer anfänglich traditionellen Arbeitsteilung neue Möglichkeiten und lassen die Wiederaufnahme der Erwerbstätigkeit bzw. eine Erhöhung des Erwerbsumfangs zu.

Einen weiteren Beitrag leistet die vorliegende Arbeit bzgl. der Relevanz der Geschlechterrolleneinstellungen. Wie aus dem Exkurs zur Operationalisierung der Geschlechterrolleneinstellungen hervorgegangen ist, ist eine eindimensionale Betrachtungsweise der Einstellungen mit den Polen „traditionell“ und „egalitär“ unterkomplex und bildet die Einstellungen nicht adäquat ab. Indem die vorliegende Arbeit die Geschlechterrolleneinstellungen multidimensional abbildet, leistet sie einen wertvollen Beitrag für ein tiefergehendes Verständnis der Zusammenhänge von Einstellungen und Arbeitsteilungsmustern: 
Anhand einer latenten Klassenanalyse werden Einstellungsprofile erstellt, die als unabhängige Variablen für die Aufteilung der Erwerbsarbeit, Hausarbeit und Kinderbetreuung dienen. Dadurch können Aussagen darüber gemacht werden, welche Einstellungsprofile zu welcher Arbeitsteilung führen und inwiefern sich die Effekte der Einstellungsprofile je nach Alter des Kindes unterscheiden.

Im Fokus stehen daher die folgenden Forschungsfragen:

- Wie egalitär oder traditionell ist die Aufteilung der Erwerbsarbeit, Hausarbeit und Kinderbetreuung und wie verändert sich diese Arbeitsteilung im Laufe des Familienzyklus?

- Unterscheiden sich die Arbeitsteilungsmuster nach den Stadien des Familienzyklus und somit in Abhängigkeit vom Alter des Kindes?

- Inwiefern lassen sich bei den unterschiedlichen Dimensionen der Arbeitsteilung (Erwerbsarbeit, Hausarbeit und Kinderbetreuung) verschiedene Entwicklungen im Laufe des Familienzyklus beobachten? Unterscheiden sie sich in ihrer Starrheit bzw. Flexibilität?

- Welche Rolle spielen relative Ressourcen und Geschlechterrolleneinstellungen bei den unterschiedlichen Dimensionen der Arbeitsteilung und wie unterscheidet sich deren Bedeutung in den verschiedenen Phasen des Familienzyklus? Spielen relative Ressourcen und Geschlechterrolleneinstellungen etwa nur in bestimmten Phasen des Familienzyklus eine wichtige Rolle und evtl. nur bei bestimmten Dimensionen der Arbeitsteilung?

Auch wenn für die Beantwortung dieser Fragestellungen idealerweise Längsschnittdaten verwendet werden, können sie annäherungsweise anhand eines „synthetischen Querschnittslebenslaufs" (Panova et al. 2017, S. 46) untersucht werden, indem Familien mit Kindern in unterschiedlichen Altersstufen miteinander verglichen werden. Hierfür wurde in Kapitel 2 der Familienzyklus einer Familie nachgezeichnet, deren Kind sich im Säuglingsalter, Kleinkindalter, Kindergartenalter, Grundschulalter und Jugendalter befindet. Im folgenden Kapitel 4 wird aufgezeigt, welche Annahmen zur Arbeitsteilung der Eltern in Abhängigkeit vom Alter des Kindes aus den einzelnen Theorien hierzu hervorgehen und inwiefern diese durch bisherige Forschung bestätigt oder widerlegt wurden. In dem darauffolgenden Kapitel 5 wird auf die in Deutschland vorliegenden, institutionellen Rahmenbedingungen eingegangen, wobei in einem nächsten Schritt die theoretischen Ansätze mit den institutionellen Rahmenbedingungen verknüpft werden (Kapitel 5.5) und daraus dann schlussendlich die Hypothesen abgeleitet werden (Kapitel 6). 


\section{Theoretische Ansätze zur Aufteilung von Erwerbs- arbeit, Hausarbeit und Kinderbetreuung}

Welche Theorien zur Erklärung der Arbeitsteilung im Allgemeinen verwendet werden, ist in der nachfolgenden Abbildung 4.1 von Boll (2016) schematisch dargestellt. Dabei handelt es sich zum einen um ökonomische Theorien wie beispielsweise der Neuen Haushaltsökonomik, der kooperativen Verhandlungstheorie und dem Time-Availability-Ansatz (siehe unterste Zeile in Abbildung 4.1). Diese ökonomischen Theorien sind (prinzipiell) geschlechtsneutral und machen daher für beide Geschlechter symmetrische Vorhersagen. Zum anderen gibt es noch weitere Theorien wie etwa den Gender-Display-Ansatz oder das Identitätsformationsmodell, bei denen es sich um geschlechtsspezifische Theorien handelt, die für die Geschlechter asymmetrische Vorhersagen machen.

In der darüber liegenden Zeile (zweite Zeile von unten) sind jene Einflussfaktoren abgetragen, die für die entsprechenden Theorien von besonderer Relevanz sind. So sind etwa für die neue Haushaltsökonomik und die kooperative Verhandlungstheorie Faktoren wie das Bildungsniveau, der Institutionalisierungsgrad, die Ehedauer und die Einkommensanteile am gemeinsamen Einkommensvolumen entscheidend, wohingegen beim Gender-Display-Ansatz sowie beim Identitätsformationsmodell die Geschlechterrollen für die Art der Arbeitsteilung ausschlaggebend sind.

Aus der Graphik geht weiter hervor, dass die Theorien auf unterschiedlichen Optimierungskalkülen der Partner bzw. der Paare basieren: So ist bei der Neuen Haushaltsökonomik das oberste Ziel die Maximierung des Haushaltsnutzens, während bei der kooperativen Verhandlungstheorie das oberste Ziel die individuelle Nutzenmaximierung ist und individuelle Präferenzen oberste Priorität haben. Beim Gender-Display-Ansatz wird die Arbeitsteilung dahingehend bewertet, ob sie der Konstruktion der individuellen geschlechtlichen Identität dienlich ist.

Im Folgenden wird zunächst näher auf die ökonomischen, ressourcenbasierten Erklärungsansätze wie die familienökonomische Theorie und die ökonomische Verhandlungstheorie eingegangen; daran anschließend werden geschlechterrollen-, normen- und identitätsbasierte Ansätze wie der Geschlechterrollenansatz, der Doing-Gender-Ansatz, die Kompensationshypothese, das Identitätsformationsmodell und Familienleitbilder behandelt. Neben einer allgemeinen Darstellung der Theorien wird insbesondere darauf eingegangen, welche Annahmen sich daraus für die Arbeitsteilung der Eltern in unterschiedlichen Phasen des Familienzyklus ableiten lassen: D.h., ob die Theorien eher davon ausgehen, dass mit der Familiengründung Weichen gestellt werden und Arbeitsteilung im Sinne einer Pfadabhängigkeit für die folgenden Jahre weitestgehend vorbestimmt ist - oder ob die Theorien im Gegenteil davon ausgehen, dass sich mit zunehmendem Alter des Kindes die Handlungsspielräume vergrößern und so etwa eine egalitärere Arbeitsteilung wieder eher möglich ist. 


\section{Abbildung 4.1: Wirkungsschema zur Arbeitsteilung im Paar}

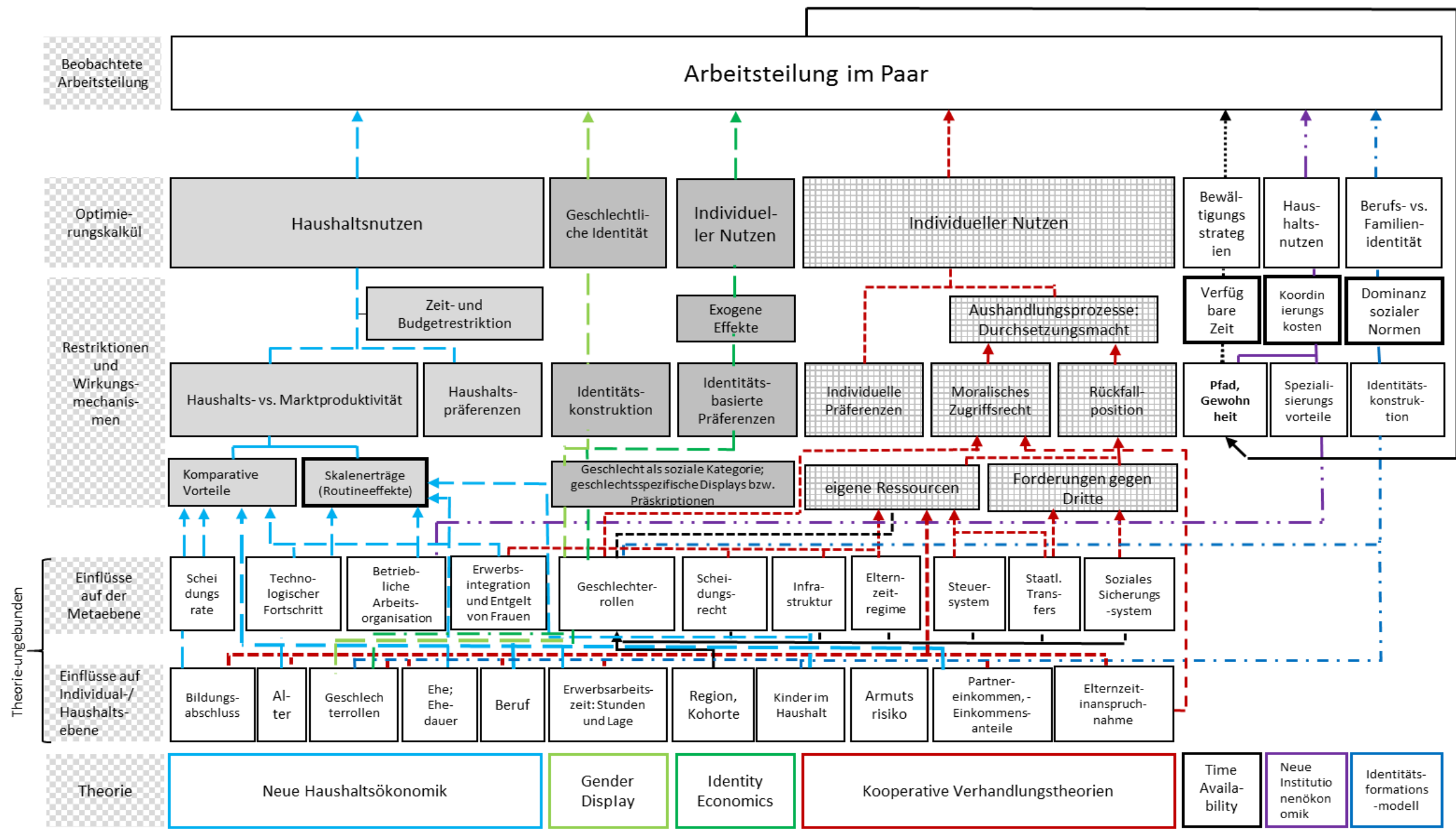

Quelle: Boll 2016, S. 17. 


\subsection{Ressourcenbasierte Erklärungsansätze}

\subsubsection{Familienökonomische Theorie}

Als bekanntester Vertreter der familienökonomischen Theorien gilt Gary S. Becker, der gemeinsam mit Jacob Mincer die „New Home Economics“ (Becker 1993) begründet hat. Die „New Home Economics“ erforschen insbesondere die Fertilitätsentscheidungen (Becker 1960), die Erwerbsbeteiligung verheirateter Frauen (Mincer 1962), die Bedeutung des Humankapitals (Becker 1975, 1985) sowie die Zeitallokation im Haushalt (Becker 1965) und liefern somit Erklärungsansätze für die Aufteilung von Erwerbs-, Haus- und Familienarbeit (vgl. auch Boll 2018).

Die New Home Economics gehen davon aus, dass Personen rational handelnde Personen sind, die bestimmte Präferenzen haben und im Rahmen ihres monetär und zeitlich begrenzten Budgets auf eine Bedürfnisbefriedigung bzw. eine Nutzenmaximierung ausgerichtet sind. Dabei können sich die Präferenzen zwischen den Individuen unterscheiden, aber die Präferenzen innerhalb eines Individuums sind zeitkonstant (Becker 1993, S. IX). Bei den Bedürfnissen kann es sich sowohl um materielle als auch um immaterielle Bedürfnisse (wie etwa Freizeit oder gemeinsame Zeit mit den Kindern oder dem Partner) handeln. Becker stellt konkrete Annahmen darüber auf, welche Arbeitsteilung des Paares die maximale Wohlfahrt und den maximalen Haushaltsnutzen generiert und greift hierfür insbesondere auf die Theorie der Zeitallokation (Becker 1965) und die Humankapitaltheorie (Becker 1975, 1985) zurück.

Der Theorie der Zeitallokation liegt der Gedanke zu Grunde, dass Zeit eine knappe Ressource (zeitliche Budgetrestriktion) ist und pro Tag nicht mehr als 24 Stunden zur Verrichtung der Hausarbeit und Erwerbsarbeit zur Verfügung stehen. Der Haushalt gilt in der Neuen Haushaltsökonomie nicht mehr nur als Konsument, sondern auch als Produzent von Gütern (Becker 1965). Deshalb ist der Haushalt mit einer kleinen Fabrik vergleichbar, die Zeit und erworbene Marktgüter investiert, um selbst wiederum bestimmte nutzenstiftende „commodities“ (Güter oder Haushaltsendprodukte) zu produzieren (Becker 1965, S. 495). Die Herstellung dieser „commodities“ dient dazu, die materiellen und immateriellen Bedürfnisse der Familienmitglieder zu stillen. Konkret handelt es sich bei den „commodities“ beispielsweise um gemeinsame Kinder, Ehestabilität oder die Gesundheit und Sicherheit der Haushaltsmitglieder (Becker 1965).

Die Zeit und die erworbenen Güter, die der Haushalt zunächst investieren muss, haben jedoch ihren Preis. Wird Zeit in die Hausarbeit investiert, kann in dieser Zeit kein Einkommen erzielt werden. Die Entscheidung für eine bestimmte Zeitverwendung implementiert gleichzeitig auch die Entscheidung gegen eine alternative Zeitverwendung. Der entgangene Nutzen, den man mit einer anderen Zeitverwendung hätte erzielen können, wird auch als Opportunitäts- oder Alternativkosten bezeichnet. Wie hoch die Opportuni- 
tätskosten sind, hängt von den individuellen Ressourcen ab. Verzichtet eine hoch gebildete Frau mit hohem Stundenlohn auf ihre Erwerbstätigkeit zugunsten der Hausarbeit, sind ihre Opportunitätskosten wesentlich höher als bei einer niedriger gebildeten Frau mit geringem Stundenlohn. Die Annahme, dass ein hohes Bildungsniveau zu einer höheren Produktivität und mehr finanziellen Ertrag führt, ist der Grundgedanke der Humankapitaltheorie (Becker 1975; Mincer 1958).

Für die Haushaltsnutzenmaximierung ist eine Spezialisierung der Arbeitsteilung innerhalb des Paares erforderlich (Becker 1993). Da der Betreuungsaufwand bei Familien mit Kleinkindern und in Mehr-Kind-Familien besonders hoch ist, sind die Spezialisierungsvorteile in diesen Familien entsprechend groß (Becker 1976). Wer sich auf die Erwerbsund wer sich auf die Hausarbeit spezialisiert, hängt von den individuellen Ressourcen $\mathrm{ab}$, da diese den zu erwartenden Nutzen und die Opportunitätskosten festlegen. Wenn die Haushaltsmitglieder unterschiedliche Fähigkeiten haben und sich jeder auf denjenigen Bereich spezialisiert, in dem er höhere Fähigkeiten besitzt, also entweder auf die Erwerbs- oder die Hausarbeit, ergeben sich daraus komparative Vorteile und kommen so der Nutzenmaximierung des Haushalts zugute:

„If all members of an efficient household have different comparative advantages, no more than one member would allocate time to both the market and household sectors. Everyone with a greater comparative advantage in the market than this member's would specialize completely in the market, and everyone with a greater comparative advantage in the household would specialize completely there" (Becker 1993, S. 33).

Die Logik der familienökonomischen Theorie ist zunächst geschlechtsneutral, da sie ressourcenbasiert argumentiert, d.h. wenn Frauen auf den Arbeitsmarkt ein höheres Einkommen als ihre Partner erzielen können, ist es für die Haushaltsnutzenmaximierung am besten, wenn sie sich auf die Erwerbs- und die Männer auf die Hausarbeit spezialisieren.

„[A]t the beginning everyone is identical; differences in efficiency are not determined by biological or other intrinsic differences. Variations in skill result from different experiences and other investments in human capital" (Becker 1993, S. 32).

Becker argumentiert somit, dass eine geschlechtstypische Spezialisierung von Männern auf die Erwerbs- und von Frauen auf die Hausarbeit v.a. durch unterschiedliche Investitionen in deren Humankapital bedingt ist. Wenn eine Frau mehr in ihr Humankapital investiert hat als ihr Mann, dann würde die Arbeitsteilung umgekehrt ausfallen, sodass sie sich auf die Erwerbs- und der Mann auf die Hausarbeit spezialisiert. Dennoch kommt noch eine geschlechtsspezifische Komponente hinzu, da Becker Frauen aufgrund biologischer Faktoren eher für die Hausarbeit und Kinderbetreuung geeignet hält, da nur Frauen das Gebären und Stillen der Kinder vorbehalten ist. Zudem investieren Frauen viel Zeit und Energie in die Fürsorge der Kinder, wodurch sie in diesem Bereich mehr Kompetenzen akkumulieren als Männer. 
„Women not only have a heavy biological commitment to the production and feeding of children, but they also are biologically committed to the care of children in other, more subtle ways. Moreover, women have been willing to spend much time and energy caring for their children because they want their heavy biological investment in production to be worthwhile. In addition, a mother can more readily feed and watch her older children while she produces additional children than while she engages in most other activities" (Becker 1993, S. 37-38).

Der Nutzenvorteil bei einer Spezialisierung der Arbeitsteilung ist einleuchtend, wenn die Kompetenzen beider Partner komplementär zueinander sind, d.h. wenn ein Partner ein höheres Bildungsniveau oder Einkommen hat und der andere Partner höhere Kompetenzen in Bereichen wie der Hausarbeit und Kinderbetreuung mitbringt. In diesem Fall wird sich ein Partner ausschließlich auf die Erwerbs- und der andere Partner ausschließlich auf die Hausarbeit und Kinderbetreuung spezialisieren.

„If all members of a household have different comparative advantages, no more than one member would invest in both market and household capital. Members specializing in the market sector would invest only in market capital, and members specializing in the household sector would invest only in household capital“ (Becker 1993, S. 34).

Weniger eindeutig ist der Nutzenvorteil hingegen, wenn beide Partner mit ähnlichen oder sogar identischen Ressourcen ausgestattet sind. Dann würde die Überlegung naheliegen, dass sich beide Partner auf denselben Bereich spezialisieren. Während eine beiderseitige Spezialisierung auf die Erwerbsarbeit möglich ist, gilt gleiches nicht für die Hausarbeit, da in diesem Falle kein Einkommen erwirtschaftet werden kann. Ein Partner muss sich also gänzlich auf die Erwerbsarbeit spezialisieren, der andere Partner kann sich dann entweder ganz auf die Hausarbeit spezialisieren oder sowohl der Hausarbeit als auch der Erwerbsarbeit nachgehen:

"[C]ould several members have the same comparative advantage and invest in both market and household capital and allocate time to both sectors? The answer, which can be stated as follows, is clearly no. [...] At most one member of an efficient household would invest in both market and household capital and would allocate time to both sectors" (Becker 1993, S. 34).

Bei Paaren mit gleichen Ressourcen kommt es somit nicht zu einer egalitären Arbeitsteilung, da diese nicht zu einer Haushaltsnutzenmaximierung führen würde, sondern wie bei Paaren mit unterschiedlichen Ressourcen ebenfalls zu einer Spezialisierung. Anfangs haben Paare mit gleichen Ressourcen in dem jeweiligen Arbeitsfeld noch keinen Kompetenzvorsprung gegenüber dem Partner. Diesen können sie sich jedoch mit der Zeit erarbeiten, da sie in ihrem Bereich durch Routineeffekte immer besser werden.

Paare mit unterschiedlichen Ressourcen haben von Anfang an komparative Vorteile, die sich im Laufe der Zeit weiter verschärfen. Ökonomen und Ökonominnen sprechen hier auch von Skalenerträge und meinen damit Routineeffekte in der Produktion, d.h. bei „positiven Skalenerträgen ist für eine gegebene Einheit Output immer weniger Ressourcen-Input nötig, da der Mensch in der Ausführung der Tätigkeit durch die Spezialisierung 
auf dieselbe immer produktiver wird“ (Boll 2018, S. 325). Aufgrund dieser Routineeffekte bzw. Skalenerträge wird die einmal eingeschlagene Spezialisierungsrichtung beibehalten und nicht mehr umgeändert.

Auch wenn die familienökonomische Theorie einen großen Beitrag zur Erklärung der innerpartnerschaftlichen Arbeitsteilung geleistet hat, enthält sie auch mehrere Schwachstellen. Ein erster Kritikpunkt ist, dass sie ein statisches Modell ist, das keinerlei Änderungen berücksichtigt und davon ausgeht, dass die einmal eingeschlagene Spezialisationsrichtung beibehalten wird. Lebensbiographien zeigen jedoch, dass heutzutage Erwerbsverläufe häufig durch Unterbrechungen gezeichnet sind. Zwar war das sogenannte „Normalarbeitsverhältnis“ mit einer Vollzeitbeschäftigung, einem unbefristeten Vertrag und einer hohen Arbeitsplatzsicherheit in den 1960ern und Anfang der 1970ern zumindest bei den Männern noch stark vertreten, aber ab Mitte der 1970er erodierte es, woraufhin die Erwerbsverläufe instabiler und flexibler wurden (Mückenberger 1985a, 1985b, 2010; Buchholz und Blossfeld 2009; Kurz et al. 2006).

Darüber hinaus haben sich die Positionen der Frauen verbessert, da ihre Erwerbspartizipation gestiegen ist und sie im Bildungsniveau aufgeholt haben, so dass sich der Anteil bildungshomogamer Paare in den letzten Jahrzehnten kontinuierlich erhöht hat (Timm 2004; Blossfeld und Timm 2003). Als Folge davon werden die von der Theorie prognostizierten Spezialisierungsvorteile abgeschwächt, da es mit hohen Opportunitätskosten verbunden wäre, wenn sich in Paaren mit ähnlich hohen Ressourcen ein Partner auf die Hausarbeit spezialisiert.

Als unitäres Modell betrachtet die familienökonomische Theorie die Haushaltsgemeinschaft als eine Einheit und ignoriert, dass es Interessenkonflikte zwischen den einzelnen Haushaltsmitgliedern geben kann (Berk und Berk 1983) und diese ausgehandelt werden müssen (Ott 1989) - ein Kritikpunkt, der von der nachfolgend skizzierten, ökonomischen Verhandlungstheorie aufgegriffen wird. Ein weiterer Kritikpunkt bezieht sich auf Beckers Annahme, dass Ehen stabil bleiben und es zu keiner Trennung kommt. Die Tatsache, dass die Scheidungsrate seit den 1970ern stark angestiegen ist (Destatis 2018), steht in Widerspruch zu Beckers Annahme.

Ott (1999) führt noch einen weiteren Kritikpunkt an den neoklassischen Theorien an, den sie als „Teufelskreis ökonomischer Rationalität“ beschreibt (Ott 1999, S. 172). Dieser Teufelskreis komme dadurch zustande, dass die "geschlechtsspezifische innerfamiliäre Arbeitsteilung aufgrund unternehmerischen Rentabilitätskalküls zu Benachteiligung von Frauen am Arbeitsmarkt - zu geringeren Löhnen und schlechteren Arbeitsbedingungen“" führt (Ott 1999, S. 172). Sobald diese Benachteiligung von Frauen einmal eingetreten ist, verfestigt sie sich und „rationales Verhalten [führt] sowohl auf der Nachfrage - wie der Angebotsseite zu einem Teufelskreis, über den die Verhaltensmuster reproduziert werden“ (Ott 1999, S. 174). Dieser Teufelskreis ist in Abbildung 4.2 abgetragen. 
Abbildung 4.2: Teufelskreis ökonomischer Rationalität

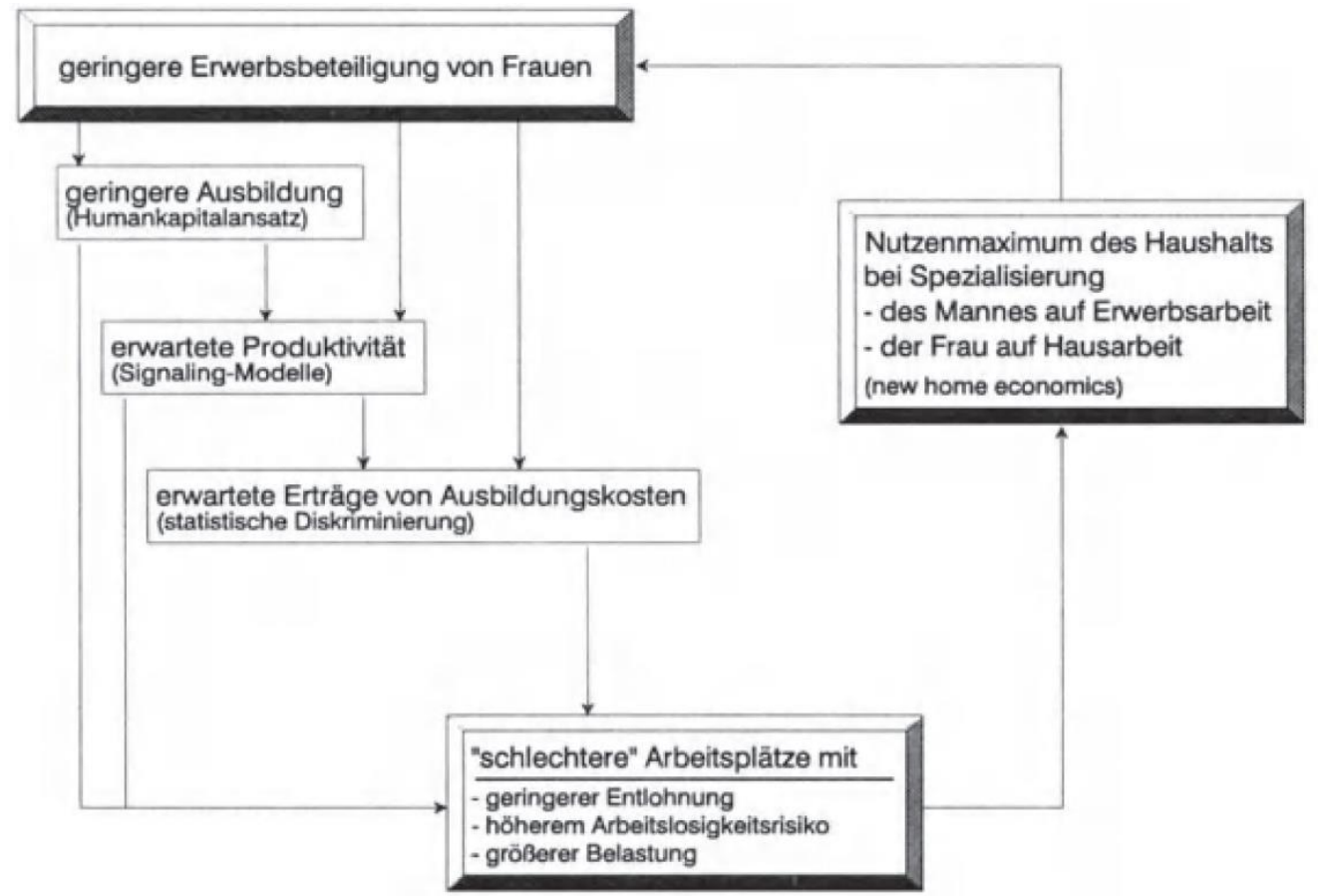

Quelle: Ott 1999, S. 173.

Der Hauptkritikpunkt von Ott bezieht sich nun darauf, dass diese Zirkularität nirgends explizit dargestellt wird und

„die daraus folgenden Entscheidungen den Anschein der ,Normalität', der ,Natürlichkeit' [haben]: im Rahmen der Arbeitsmarkttheorien ist es ,normal', daß [sic!] Frauen Erwerbsunterbrechungen planen, sie akzeptieren somit aus ,freier Entscheidung' geringere Löhne; in Zeitallokationstheorien ist es dagegen ,normal', daß [sic!] Frauen geringere Löhne vorfinden, sie entscheiden sich daher ,freiwillig' für die traditionelle Rollenverteilung“ (Ott 1999, S. 173).

Eine Möglichkeit, diesen „Teufelskreis ökonomischer Rationalität“ (Ott 1999, S. 172) zu durchbrechen und Frauen in ihrer benachteiligten Situation abzusichern, sieht Ott in langfristigen und verbindlichen Verträgen (Ott 1999, S. 182). Aufgrund der asymmetrischen Machtverteilung besteht jedoch für den mächtigeren Partner (i.d.R. der Mann) ein großer Anreiz zum Vertragsbruch. Daher ist es für den unterlegenen Partner (i.d.R. die Frau) umso wichtiger, dass die Verbindlichkeit der Verträge entsprechend hoch ist - etwa indem die Kosten, die bei Vertragsbruch entstehen, derart hoch ausfallen, dass ein Vertragsbruch seitens des mächtigeren Partners immens an Attraktivität verliert.

Angewandt auf die Forschungsfrage der vorliegenden Arbeit stellt sich folgende Frage: Welche Rolle spielt laut der familienökonomischen Theorie das Alter des Kindes für die Arbeitsteilung der Eltern? Kleine Kinder sind betreuungs- und zeitintensiver als ältere Kinder, weshalb die Spezialisierungsvorteile in Familien mit kleinen Kindern besonders groß sind (Becker 1976, S. 207-214). Indem sich ein Partner auf die Hausarbeit und 
Kinderbetreuung spezialisiert, leistet er zwar einen wichtigen Beitrag zur Haushaltsnutzenmaximierung und zur Familienwohlfahrt, allerdings verzichtet er damit zugleich auf Investitionen in das Humankapital, die er nicht mehr aufholen kann (Boll 2018, S. 330). Die Spezialisierungsrichtung, die Familien mit kleinen Kindern wählen, wird sich mit dem Älterwerden des Kindes nicht verändern. Stattdessen verschärfen sich die Spezialisierungsvorteile aufgrund von Routineeffekten und Skalenerträge und aufgrund des Verzichts eines Partners, in sein Humankapital zu investieren. Die Theorie nimmt also nicht an, dass Paare auf sich verändernde Situationen, wie beispielsweise das Aufwachsen des Kindes, flexibel reagieren, sondern die Vorteile ergeben sich gerade aus der langfristigen Beibehaltung der Spezialisierungsrichtung. Dem Alter des Kindes kommt in den New Home Economics somit keine wichtige Bedeutung zu.

Im Folgenden wird näher auf die Ressourcen- und Verhandlungstheorien eingegangen und deren Grundprinzipien beispielhaft anhand der ökonomischen Verhandlungstheorie nach Ott skizziert.

\subsection{2 Ökonomische Verhandlungstheorie}

Die Ressourcen- und Verhandlungstheorie (Blood und Wolfe 1960; Lundberg und Pollak 1993, 1996; Ott 1992, 1993) stellen eine Erweiterung der familienökonomischen Theorie dar. Im Fokus stehen hierbei zwar ebenso ökonomische Ressourcen wie das Einkommen bzw. Indikatoren für das Einkommenspotential wie etwa die Bildung oder die berufliche Position. Aber im Gegensatz zur familienökonomischen Theorie wird nicht unterstellt, dass die Haushaltsmitglieder ihre individuellen Präferenzen den Präferenzen des Haushalts unterordnen, d.h. im Mittelpunkt steht nicht länger die Haushaltsnutzenmaximierung, sondern die individuelle Nutzenmaximierung. Die individuellen Präferenzen können in Konkurrenz zueinanderstehen und dadurch zu Konflikten führen. Diese Konflikte werden dann unter Bezugnahme auf die individuellen Ressourcen (wie z.B. Einkommen, Bildung und berufliche Position) ausgehandelt. Die individuellen Ressourcen geben dabei Aufschluss über die Machtverhältnisse innerhalb der Beziehung und verbessern die Verhandlungsposition derjenigen Person mit den höheren Ressourcen und vice versa. Bei den Aushandlungsprozessen handelt es sich somit um machtgesteuerte Prozesse.

Dies sei am Beispiel der Hausarbeit erläutert: Da die Hausarbeit als unangenehm empfunden wird, wollen sie beide Partner möglichst vermeiden. Schließlich ist die Verrichtung der Hausarbeit mit Opportunitätskosten verbunden, weil in derselben Zeit auch Einkommen verdient werden könnte (oder z.B. auch die Freizeit genossen werden könnte). Da beide Partner auf eine individuelle Nutzenmaximierung ausgerichtet sind und beide die unangenehme Hausarbeit nicht übernehmen wollen, wird die Aufteilung auf Basis der Ressourcenverteilung getroffen. Derjenige mit den geringeren Ressourcen hat eine geringere Verhandlungsmacht und muss deswegen die Last der Hausarbeit auf sich 
nehmen, derjenige mit den höheren Ressourcen kann sich davon befreien. Die wichtigste relative Ressource ist dabei das Einkommen, sodass angenommen werden kann, dass diejenige Person mit dem höheren Einkommen weniger Hausarbeit übernimmt.

Weil bei der Verhandlungstheorie die relativen Ressourcen im Vordergrund stehen und nicht das biologische Geschlecht, ist sie in ihren Grundannahmen (ebenso wie familienökonomischen Theorie) geschlechtsneutral. Wenn es zu einer traditionellen Arbeitsteilung kommt, dann ist dies dadurch bedingt, dass Frauen geringere Ressourcen - insbesondere ein geringeres Einkommen - haben und ihre Verhandlungsposition dadurch unterlegen ist. Die ausgehandelte Arbeitsteilung ist jedoch reversibel, denn anders als bei der familienökonomischen Theorie, die annimmt, dass die einmal eingeschlagene Spezialisierungsrichtung beibehalten wird, handelt es sich bei der Verhandlungstheorie um ein dynamisches Modell: Die gewählte Arbeitsteilung ist nicht zeitkonstant, sondern wird immer wieder neu ausgehandelt (Ott 1992).

Bei den spieltheoretischen Modellen kann zwischen kooperativen und nicht-kooperativen Verhandlungsmodellen unterschieden werden. Bei den kooperativen Verhandlungsmodellen können beide Partner miteinander kommunizieren und ebenso ist es innen erlaubt, verbindliche Verträge abzuschließen (Schulz 2010, S. 79). Da beide Bedingungen in Partnerschaften gegeben sind, verfolgt Ott zur Erklärung der Arbeitsteilung in Partnerschaften die Argumentationslinie der kooperativen Verhandlungsmodelle (Ott 1992, S. 27), auf die im Folgenden beispielhaft näher eingegangen wird.

Die Spezialisierung auf die Hausarbeit hat für den ressourcenschwächeren Partner sowohl nach der Auflösung der Partnerschaft als auch bereits innerhalb der Partnerschaft negative Folgen: Nach der Auflösung der Partnerschaft hat er aufgrund des Humankapitalverlustes schlechtere Chancen auf dem Arbeitsmarkt und wird ein geringeres Einkommen erzielen. Deshalb ist für inn eine Trennung schmerzhafter und er ist gewissermaßen vom Partner abhängig. Aber auch bereits innerhalb der Partnerschaft zeigen sich negative Konsequenzen, da derjenige Partner mit den geringeren Ressourcen aufgrund eines geringeren „Drohpunktes“ ein schwächeres Durchsetzungsvermögen hat.

„Der auf Hausarbeit spezialisierte Partner geht nicht nur das Risiko ein, im Konfliktfall auf die eigene verminderte Einkommenskapazität angewiesen zu sein, sondern schwächt durch diese Verschlechterung der Alternativmöglichkeiten auch seine interne Verhandlungsposition“ (Ott 1989, S. 101).

Damit beide Partner in eine Kooperation einwilligen, muss der zu erwartende Nutzen mit Partner größer bleiben als der individuell erreichbare Nutzen ohne den Partner. Bei dem Partner mit geringeren Ressourcen übersteigt der Haushaltsnutzen schneller den individuell erreichbaren Nutzen. Daraus folgt, dass für inn ein Fortbestehen der Partnerschaft attraktiver ist. Da der Partner mit den höheren Ressourcen dies weiß, ist es schwerer inn in der Partnerschaft zu halten und sein Drohpotential ist deshalb größer (Ott 1992, 1993; Boll 2018; Seel 2001). 
Da es bei einer Spezialisierung auf die Erwerbsarbeit zu einer Akkumulation von Humankapital kommt und dieses auch außerhalb der Beziehung verwendbar ist, wohingegen es bei einer Spezialisierung auf die Hausarbeit zu einem Verlust an Humankapital kommt, welches nicht außerhalb der Beziehung von Nutzen ist, sind beide Partner darum bemüht, die eigene Erwerbstätigkeit nicht vollkommen aufzugeben. Da eine vollständige Spezialisierung zur Haushaltsnutzenmaximierung beiträgt, nicht aber zur individuellen Nutzenmaximierung, treten Interessenskonflikte zwischen den Partnern auf, die immer wieder ausgehandelt werden müssen. Gleichzeitig sind beide Partner auch darum bemüht, miteinander zu kooperieren, da durch eine langfristige Kooperation Zusatzgewinne entstehen, die die individuellen Nutzengewinne übersteigen (Ott 1999).

Aus den bisherigen Erläuterungen geht hervor, dass eine ausschließliche Spezialisierung auf die Hausarbeit äußerst unattraktiv ist und mit langfristigen Nachteilen verbunden ist. Beide Partner streben es deshalb an, ihre Erwerbsarbeit zumindest nicht vollständig aufzugeben. Im Falle einer Ressourcengleichheit und somit eines ausgeglichenen Machtverhältnisses kann deshalb - im Gegensatz zur familienökonomischen Theorie - eine egalitäre Arbeitsteilung angenommen werden.

An der Verhandlungstheorie kann kritisiert werden, dass sie ebenso wie die familienökonomische Theorie nur die ökonomischen Ressourcen berücksichtigt. Aufgrund ihrer geschlechtsneutralen Thesen ist anzunehmen, dass sich Paare mit identischem Humankapital die Erwerbs- und die Hausarbeit egalitär aufteilen und dass sich in Paaren mit unterschiedlichen Humankapital diejenige Person mit den höheren Ressourcen aus der unangenehmen Hausarbeit herausverhandeln kann. Aufgrund der Geschlechtsneutralität dieser Annahmen bedeutet dies somit auch, dass sich Frauen von der Hausarbeit befreien können, wenn sie ein höheres Humankapital haben, weil dann die Männer den Großteil der Hausarbeit übernehmen (Auspurg et al. 2017, S. 120). Dies steht jedoch im Widerspruch zu empirischen Befunden, aus denen hervorgeht, dass die Arbeitsteilung geschlechtsspezifisch ist und in der Regel die Frauen den Großteil der Hausarbeit übernehmen - selbst dann, wenn sie in selben Umfang oder sogar in höherem Umfang als die Männer erwerbstätig sind (z.B. Brines 1994; Greenstein 2000; Bittman et al. 2003; Gershuny et al. 2005; Lachance-Grzela und Bouchard 2010).

Des Weiteren wird kritisiert, dass das Einkommen als Schlüsselindikator für die Machtverteilung angesehen wird und andere Faktoren, die ebenfalls das Machtgefüge beeinflussen können, unberücksichtigt bleiben. Beispielsweise stellen feministische Wissenschaftlerinnen die rationale Wahlfreiheit der Verhandlungsmodelle in Frage und sehen Frauen durch das Patriarchat und durch hegemoniale Normen in einer schlechteren Verhandlungsposition als Männer (Geist und Ruppanner 2018, S. 245). Eine weitere Schwäche der Verhandlungstheorien besteht darin, dass das Machtkonzept den Fokus sehr stark auf das Paar richtet und dadurch komplexere Familienmodelle, wie etwa Mehrgenerationenfamilien oder das Vorhandensein von erwachsenen Kindern im Haushalt, nicht vollständig berücksichtigt (Geist und Ruppanner 2018, S. 245). Ebenso wird nicht 
darauf eingegangen, dass sich andere Dimensionen wie etwa eine rassen- oder klassenbedingte Diskriminierungen auf das innerpartnerschaftliche Machtverhältnis auswirken können (Geist und Ruppanner 2018, S. 245). Schließlich können auch die Arbeitsplatzbedingungen wie die Arbeitsplatzsicherheit oder die Einkommenskonstanz in unterschiedlicher Macht resultieren: So kann ein Partner zwar im Moment das gleiche Einkommen haben wie der andere Partner, aber dennoch eine geringe Macht haben, wenn sein Arbeitsvertrag befristet ist oder sein Einkommen sehr starken Schwankungen unterworfen ist (Geist und Ruppanner 2018, S. 246).

Im Folgenden interessiert die Fragestellung, wie wahrscheinlich es nach der Verhandlungstheorie ist, dass es zu einer Traditionalisierung der Arbeitsteilung im Familienzyklus kommt. Gegen eine Traditionalisierung spricht zum einen die Tatsache, dass die Verhandlungstheorie geschlechtsneutral ist, da sie sich auf Ressourcen bezieht und nicht auf das biologische Geschlecht; zum anderen macht die Tatsache, dass es sich dabei um ein dynamisches Modell handelt und die Arbeitsteilung immer wieder neu ausgehandelt wird, eine Traditionalisierung unwahrscheinlicher. Trotzdem sprechen viele Faktoren für eine Traditionalisierung der Arbeitsteilung, auf die im Folgenden näher eingegangen wird.

Die entscheidendste Ressource für die Arbeitsteilung ist das Einkommen. Obwohl Frauen in den letzten Jahrzehnten bzgl. ihres Bildungsniveaus und ihrer Erwerbspartizipation deutlich aufgeholt haben, verdienen Frauen selbst bei gleicher Qualifikation immer noch weniger als die Männer, wie aus dem Gender Pay Gap hervorgeht (Glassdoor Economic Research 2019). Aufgrund dieser Lohnlücke haben selbst Frauen mit gleicher Bildung oder gleichem Berufsstatus wie der Partner nicht die gleichen Verdienstmöglichkeiten und somit eine schlechtere Verhandlungsposition. Wenn sich Frauen mit geringeren Verdienstmöglichkeiten nun auf die Hausarbeit spezialisieren, können sie nicht gleichzeitig in ihr arbeitsmarktrelevantes Humankapital investieren, sodass dies mit einem Humankapitalverlust verbunden ist. Die Unterschiede zwischen den beiden Partnern verschärfen sich somit, weil die ressourcenschwächere Frau durch ihre Spezialisierung auf die Hausarbeit weiter an Humankapital verliert, während der ressourcenstärkere Mann durch seine Spezialisierung auf die Erwerbsarbeit weiter Humankapital akkumuliert.

Zu einer Traditionalisierung kommt es, wenn sich die Ressourcen der Frau im Vergleich zu den Ressourcen des Mannes verschlechtern. Ein Beispiel hierfür ist der Übergang zur Elternschaft, weil dieser in der Regel mit einer Erwerbsunterbrechung der Frauen, nicht jedoch der Männer, verbunden ist und sich eine Erwerbsunterbrechung negativ auf die Lohnentwicklung auswirkt. Eine Studie von Budig und England (2001) mit amerikanischen Daten zeigt, dass Mutterschaft mit einer Lohnstrafe einhergeht, weil Mütter durch die kindbedingte Erwerbsunterbrechung weniger Berufserfahrung haben, am Arbeitsplatz weniger produktiv sind, sie ihre höheren Löhne für mutterfreundlichere Arbeitsplätze eintauschen oder sie von Arbeitgebern diskriminiert werden (Budig und England 
2001). Studien, die sich auf Deutschland beziehen, bestätigen, dass es ein Lohngefälle zwischen Müttern und kinderlosen Frauen gibt und dass sich mit zunehmender Dauer der kindbedingten Erwerbsunterbrechung die Karrierechancen der Mütter weiter verschlechtern (Ziefle 2004; Gangl und Ziefle 2009). Eine Studie von Schmelzer et al. (2015) kommt zu dem Ergebnis, dass eine Erwerbsunterbrechung, die die gesetzlich vorgesehene Dauer der Erwerbsunterbrechung nicht überschreitet, noch zu keiner dauerhaften „motherhood wage penalty“ führt; sobald die Erwerbsunterbrechung jedoch für diese Zeitspanne hinaus geht, kommt es schon zu Lohneinbußen der Mütter.

Durch den Übergang zur Elternschaft haben Mütter somit eine nachhaltige Verschlechterung inrer Verhandlungsposition als vor der Mutterschaft. Besonders schlecht ist die Situation von Müttern mit kleinen Kindern, da Mütter dann häufig nicht erwerbstätig sind oder aufgrund der kindbedingten Erwerbsunterbrechung nur geringere Löhnen erzielen können oder zu einem familienfreundlicheren Arbeitgeber unter Inkaufnahme eines Lohnverlustes gewechselt sind (Budig und England 2001). Mit zunehmendem Alter des Kindes kann sich die Situation der Mütter zwar insofern wieder verbessern, weil sie wieder verstärkt erwerbstätig sind und in ihr Humankapital investieren können, allerdings haben Väter währenddessen durch ihre kontinuierliche Erwerbstätigkeit auch in ihr Humankapital investiert. Diesen Vorsprung können Mütter nur schwer wieder aufholen, so dass es naheliegend ist, dass Mütter aufgrund ihres schlechteren Einkommenspotentials und ihrer schlechteren Verhandlungsposition auch dann mehr Hausarbeit übernehmen, wenn die Kinder älter sind.

Wenn Mütter ein gleich hohes Einkommen haben wie ihre Partner, dann ist somit eine egalitäre Arbeitsteilung sehr wahrscheinlich und wenn Mütter ein höheres Einkommen haben, dann sogar eine umgekehrte Arbeitsteilung in Form eines Rollentausches. Aufgrund der zuvor genannten Gründe (wie etwa dem allgemeinen Gender Pay Gap, den geburtsbedingten Erwerbsunterbrechungen und der Lohnstrafe für Mutterschaft) ist es jedoch unwahrscheinlicher, dass Mütter ein gleiches oder höheres Einkommen haben als die Väter.

Weitere ressourcenbasierte Theorien: Theorie des sozialen Tauschs und Zeitbudget-Ansatz

a) Theorie des sozialen Tauschs

Eine weitere Austauschtheorie ist die Theorie des sozialen Tauschs (Blau 1964), die sich im Gegensatz zur Verhandlungstheorie nicht auf ökonomische Ressourcen beschränkt, sondern ihre Annahmen auf soziale Austauschprozesse überträgt. Als Beispiel für soziale Austauschprozesse nennt Blau etwa die gegenseitige Hilfe unter Nachbarn, Kindern, Freunden und Kollegen (Blau 2005: 127). Sowohl bei ökonomischen als auch bei sozialen Austauschprozessen liegen Reziprozitätserwartungen vor: Man erbringt für ein Gegenüber eine Leistung in irgendeiner Form und erwartet eine Gegenleistung. Entschei- 
dend ist hierbei, dass die zu erwartende Gegenleistung sowohl bezüglich ihres Zeitpunktes als auch bezüglich ihrer Art unspezifisch ist (Blau 1964, S. 93, 2005, S. 130). Die Gegenleistung kann nicht nur, sondern sie muss unspezifisch bleiben, denn

„[w]enn man eine Hilfeleistung für jemanden erbringt und gleichzeitig benennt, welche Gegenleistung man dafür erwartet, offenbart man, dass man diese Hilfe nicht als Gefallen betrachtet, sondern als Teil eines Geschäfts“" (Blau 2005, S. 130).

Stattdessen vertraut derjenige, der in Vorleistung gegangen ist, darauf, dass er irgendwann in irgendeiner Form eine Gegenleistung bekommt. Er leistet somit gewissermaßen einen Vertrauensvorschuss: „Since there is no way to assure an appropriate return for a favor, social exchange requires trusting others to discharge their obligations" (Blau 1964, S. 94).

Besondern dann, wenn ein Partner von den belohnenden Reaktionen des anderen abhängig ist, besteht eine ungleiche Machtverteilung zwischen den Partnern. Wenn sich ein Partner etwa auf die Hausarbeit und die Kinderbetreuung spezialisiert und der andere Partner auf die Erwerbsarbeit, so ist ersterer von letzterem finanziell abhängig. Da sich (wie bereits bei den Erläuterungen zur Verhandlungstheorie dargestellt) das auf dem Arbeitsmarkt erworbene Wissen akkumulieren lässt und auch außerhalb der Beziehung verwendet werden kann, selbiges jedoch nicht bei einer Spezialisierung auf die Hausarbeit erfolgt, verschärfen sich die Machtunterschiede weiter: Der auf die Erwerbsarbeit fokussierte Partner gewinnt weiter an Macht, während der auf die Hausarbeit oder die Kinderbetreuung ausgerichtete Partner weiter an Macht verliert. Das Vertrauen, dass der Machtunterlegene aufzubringen hat, ist dementsprechend groß und die Vertrauenswürdigkeit des Partners muss erprobt sein. Ehen wie auch langjährige Partnerschaften können daher „als eine Art ,implizite Verträge“ angesehen werden, die aus vielen unausgesprochenen und daher weitgehend unverbindlichen Verpflichtungen bestehen" (Schulz 2010, S. 85), bei denen so gehandelt wird, als ob formale Verträge vorliegen würden, obwohl dies de facto nicht der Fall ist (England und Farkas 1986, S. 47; Schulz 2010, S. 85).

In Bezug auf die Arbeitsteilung gehen aus der Theorie des sozialen Tauschs dieselben Annahmen wie aus der ökonomischen Verhandlungstheorie hervor (Schulz 2010, S. 86). Denn auch hier gilt, dass Ressourcen wie das Einkommen oder die Erwerbstätigkeit das Machtpotential eines Partners erhöhen, weil diese Ressourcen auch außerhalb der Beziehung genutzt werden können. Dies bringt wiederum den ressourcenschwächeren Partner dazu, noch mehr unangenehme Hausarbeit zu verrichten (Schulz 2010, S. 86). In welchem Ausmaß dieser dazu bereit ist, eher die Hausarbeit zu übernehmen, hängt darüber hinaus vom Vertrauensniveau und der Stabilität der Partnerschaft ab. 


\section{b) Zeitbudget-Ansatz}

Ein weiterer ressourcenbasierter Ansatz ist der Zeitbudget-Ansatz (Coverman 1985), der sich auf die Knappheit der zeitlichen Ressourcen bezieht. Im Einklang mit anderen ressourcenbasierten Ansätzen geht er zunächst davon aus, dass derjenige Partner mit dem höheren Humankapital und den besseren Chancen auf dem Arbeitsmarkt in stärkeren Umfang erwerbstätig ist. Wie viel Zeit Müttern und Vätern nun für die Hausarbeit und die Kinderbetreuung zur Verfügung steht, hängt in erster Linie von ihren Erwerbsumfängen ab: Je höher der Erwerbsumfang ausfällt, umso weniger Zeit bleibt für Hausarbeit und Kinderbetreuung. Der Demand/Response-Capability-Ansatz (Coverman 1985) bezieht die Überlegung mit ein, dass die Nachfrage an Hausarbeit und Kinderbetreuung von Faktoren wie der Anzahl der Kinder und dem Alter der Kinder abhängen, da bei mehr Kindern mehr Pflege als bei einem Kind und bei jüngeren Kindern mehr Pflege als bei älteren Kindern anfällt. Neben der Anzahl und dem Alter der Kinder spielen darüber hinaus weitere Faktoren wie die Wohnungsgröße oder die Ausstattung des Haushalts mit technischen Geräten eine Rolle für die Nachfrage an zu verrichtender Hausarbeit. In welchem Ausmaß Eltern dieser Nachfrage gerecht werden können, hängt wiederum von ihren Erwerbsumfängen ab. Der Zeitbudget-Ansatz ist damit ebenfalls geschlechtsneutral, da geschlechtsspezifische Unterschiede in der Hausarbeitsbeteiligung auf die unterschiedlichen Erwerbsumfänge der Partner zurückzuführen sind (Künzler und Walter 2001, S. 193).

Ein besonders gravierender Kritikpunkt an Covermans Zeitbudget-Ansatz bezieht sich darauf, dass die Kausalitätsrichtung nicht angemessen theoretisiert wird. Es bleibt offen, ob Frauen wegen ihrem geringem Erwerbsumfang mehr Hausarbeit übernehmen oder ob sie wegen ihrem hohem Hausarbeitsumfang weniger Erwerbsarbeit übernehmen (Geist und Ruppanner 2018, S. 243-244). Beide Kausalitätsrichtungen sind möglich und es fehlt eine theoretische Stellungnahme hierzu. Des Weiteren berücksichtigt der TimeAvailability-Ansatz nicht die Tatsache, dass wohlhabendere Familien eher in der Lage sind, sich etwa durch das Einstellen einer Haushaltshilfe von der Hausarbeit freizukaufen (Geist und Ruppanner 2018). Feministische Frauenforschung kritisiert am Time-Availability-Ansatz darüber hinaus, dass es sich bei der Hausarbeitsaufteilung um weit mehr handelt als um Kosten-Nutzen-Kalküle und zeitliche Verfügbarkeit (Bianchi et al. 2000, S. 194). Die Genderforschung sieht die Hausarbeitsaufteilung als Bühne der Inszenierung des eigenen, sozialen Geschlechts an. Aufgrund dieser Herstellungsleistung von geschlechtlicher Identität können die Zeiten, die von Frauen und Männern in der Erwerbs- und der Hausarbeit verbracht werden, nicht einfach ausgetauscht werden (West und Zimmerman 1987; Greenstein 1996; Bittman et al. 2003).

Die familienökonomische Theorie und die ökonomische Verhandlungstheorie (ebenso wie der Zeitbudget-Ansatz und die Theorie des sozialen Tausches) gehen davon aus, dass die Arbeitsteilung auf Basis von Ressourcen entschieden wird. Da sie in ihren Annahmen geschlechtsneutral sind, schreiben sie weder dem biologischen Geschlecht 
noch den Einstellungen, Normen und Leitbildern eine wichtige Rolle zu. Dies steht jedoch im Widerspruch zu einer Vielzahl von empirischen Befunden, die belegen, dass die Arbeitsteilung entscheidend durch Werte, Normen, (Familien-)Leitbilder, Geschlechterrolleneinstellungen und Geschlechterideologien mit beeinflusst wird (Cunningham 2008; Kan 2008; Davis und Greenstein 2009; Grunow et al. 2012; Evertsson 2014; Nitsche und Grunow 2016; Khoudja und Fleischmann 2018).

Im Folgenden wird daher auf den zweiten großen Theoriestrang eingegangen, der den ökonomischen, geschlechtsneutralen Theorien entgegengestellt ist: den geschlechtsspezifischen Ansätzen, die die Bedeutung von Normen, Leitbildern und Rollenerwartungen für die Arbeitsteilung mitberücksichtigen.

\subsection{Geschlechterrollen-, normen- und identitätsbasierte An- sätze}

Ökonomische Theorien können zwar gut erklären, warum es zu einer traditionellen Arbeitsteilung kommt, wenn Männer die höheren, relativen Ressourcen haben. Sie können jedoch nicht erklären, warum dies für Frauen mit höheren, relativen Ressourcen nicht im gleichen Ausmaß gilt und es nur in Ausnahmefällen zu einem Rollentausch kommt, in dem die Frau die Hauptverdienerin ist und der Mann den Großteil der Hausarbeit und der Kinderbetreuung übernimmt. Dies zeigt, dass hier noch andere Mechanismen vorhanden sind, die die Arbeitsteilung beeinflussen und die von den ökonomischen Theorien unberücksichtigt bleiben. Konkret handelt es sich dabei um Sozialisationsprozesse, Rollenerwartungen, Präferenzen und familiale Leitbilder, die die Arbeitsteilung entscheidend mitbeeinflussen.

\subsection{1 (Geschlechter-)Rollentheorien, Normen und Einstellungen}

Soziologische Rollentheorien gehen in ihren Anfängen auf Linton (1936), Parsons (1951) und Komarovsky (1946) zurück (West und Zimmerman 1987, S. 128). Rollentheorien nehmen an, dass Menschen durch Sozialisationsprozesse Normen, Werte und Einstellungen aufnehmen und verinnerlichen. Des Weiteren gehen Rollentheorien davon aus, dass sich Personen einstellungskonform verhalten. Bei Geschlechterrollen handelt es sich um sozial geteilte Verhaltenserwartungen, die sich auf das sozial zugeschriebene Geschlecht beziehen (Eckes 2010). Geschlechterrolleneinstellungen nehmen dabei Bezug auf „die Angemessenheit von Geschlechtsrollenerwartungen, wobei es vornehmlich um die Frage der geschlechtstypischen Arbeitsteilung und der Regeln des sozialen Umgangs von Männern und Frauen geht“ (Alfermann 1996, S. 47; Hervorhebung im Original).

Geschlechterrollenerwartungen orientieren sich dabei an Geschlechterstereotypen. Dem liegt die Annahme der „Polarisierung der Geschlechtscharaktere“ (Hausen 1976) 
zugrunde, wonach sich sämtliche Lebensbereiche, Tätigkeiten, Eigenschaften oder Verhaltensweisen als „männlich“ oder „weiblich“ einordnen lassen. Als männlich gilt etwa das außerhäusliche, öffentliche Leben, Aktivität (z.B. Energie, Kraft und Tapferkeit), Tun (z.B. Selbstständigkeit und Durchsetzungsvermögen) und Rationalität (z.B. Vernunft, Denken und Wissen); konträr dazu gilt das innerhäusliche, private Leben, Sein (z.B. Abhängigkeit und Anpassung) und Emotionalität (z.B. Gefühle, Empfindungen und Religiosität) als weiblich (Hausen 1976, S. 368). Wenn der Mann mehr Hausarbeit übernimmt, so steht dies nicht im Einklang mit traditionellen Geschlechterrolleneinstellungen.

Die normativen Überzeugungen und Einstellungen zu Geschlechterrollen werden als eindimensional und bipolar mit den Extremen „Traditionalität“ und „Modernität“ betrachtet (Künzler und Walter 2001, S. 194). Traditionelle Geschlechterrolleneinstellungen befürworten eine stark geschlechtsspezifische Arbeitsteilung, während moderne Geschlechterrolleneinstellungen diese geschlechtsspezifische Arbeitsteilung ablehnen (Künzler und Walter 2001, S. 194). Männer mit modernen Einstellungen übernehmen somit mehr Hausarbeit und Frauen mit modernen Einstellungen weniger Hausarbeit (Künzler und Walter 2001, S. 194-195).

Gemäß der Annahme, dass sich Einstellungen auf das Verhalten auswirken, müssten egalitäre Einstellungen zu einer egalitären Arbeitsteilung führen. Eine Studie von Greenstein (1996) ergab, dass es für eine egalitärere Aufteilung der Hausarbeit jedoch nicht ausreichend ist, wenn lediglich der Mann oder die Frau moderne Geschlechterrolleneinstellungen haben: Indem er erstmalig Interaktionseffekte zwischen den Geschlechterrolleneinstellungen von Männern und Frauen rechnete, konnte er aufzeigen, dass eine egalitärere Aufteilung der Hausarbeit erst dann eintritt, wenn sowohl beim Mann als auch bei der Frau moderne Geschlechterrolleneinstellungen vorliegen (Greenstein 1996).

Welche Vorhersagen lassen sich auf Basis des Geschlechterrollenansatzes für die Arbeitsteilung der Eltern in Abhängigkeit vom Alter des Kindes machen? Da angenommen wird, dass Einstellungen und Verhalten im Einklang stehen, kann vorneweg erwartet werden, dass Individuen mit traditionellen Einstellungen eine traditionellere Arbeitsteilung und Individuen mit egalitären Einstellungen eine egalitärere Arbeitsteilung praktizieren. Im Familienzyklus und somit auch über die Altersstufen des Kindes hinweg sind keine großen Veränderungen der Arbeitsteilung zu erwarten. Dies liegt darin begründet, dass der Geschlechterrollen-Ansatz auf Sozialisationstheorien basiert und davon ausgeht, dass sich die Einstellungen in der Kindheit und im Jugendalter entwickeln und im Erwachsenenalter stabil bleiben (Künzler und Walter 2001, S. 195). Wenn sich die Einstellungen im Erwachsenenalter nicht mehr ändern, dürfte sich somit bei einstellungskonformen Verhalten auch die Arbeitsteilung im Familienzyklus nicht ändern.

Diese Stabilitätsannahme wird jedoch heftig kritisiert. So konnten beispielsweise Baxter et al. (2015) nachweisen, dass Geschlechterrolleneinstellungen mit dem Übergang zur Elternschaft traditioneller werden. Dies ist jedoch ein Beleg dafür, dass Einstellungen instabil sind und sich durchaus im Erwachsenenalter noch verändern können. Auch eine 
Längsschnittstudie von Corrigall und Konrad (2007) konnte zeigen, dass sich Geschlechterrolleneinstellungen von Frauen nicht nur auf ihre Erwerbsumfänge auswirken, sondern ihre Erwerbsumfänge auch auf ihre späteren Einstellungen Einfluss nahmen. Weitere Längsschnittstudien belegen ebenfalls, dass sich die Einstellungen einer Person durch Lebensereignisse wie etwa durch eine Heirat, eine Familiengründung oder einen Arbeitsplatzwechsel ändern können und somit nicht stabil sind (Schober und Scott 2012; Evertsson 2013)

Eine weitere Schwäche des Geschlechterrollen-Ansatzes ist, dass er aufgrund seiner Annahme des einstellungskonformen Handelns nicht die Diskrepanz zwischen Normen und Handlungen erklären kann. Hinsichtlich der Arbeitsteilung konnten viele Studien nachweisen, dass insbesondere Männer häufig egalitäre Einstellungen äußern, aber nur selten eine egalitäre Arbeitsteilung praktizieren (Zulehner und Volz 1998; Oberndorfer und Rost 2005; Mühling und Rost 2006; Grunow und Baur 2014).

Ein weiterer Kritikpunkt bezieht sich darauf, dass sich Geschlechterrolleneinstellungen eindimensional zwischen Traditionalität und Modernität bewegen. Neuste Studien von Knight und Brinton (2017) und Grunow et al. (2018) kritisieren diese eindimensionale Betrachtungsweise als unterkomplex und plädieren für ein multidimensionales Konzept (in Kapitel 7.5 wird darauf näher eingegangen). In ihren Studien finden sie empirische Evidenz dafür, dass es mehrere Dimensionen von Geschlechterrolleneinstellungen gibt (Knight und Brinton 2017; Grunow et al. 2018). Die einzelnen Dimensionen unterscheiden sich danach, ob beide Geschlechter gemeinsam für die Erwerbstätigkeit und für die Kinderbetreuung zuständig sind oder nicht, und ob über diese Zuständigkeit frei entscheiden werden kann oder ob diese durch geschlechtsspezifische Merkmale vorbestimmt ist (Grunow et al. 2018).

\subsection{2 „Doing Gender" und Kompensationshypothese}

Die geschlechtsspezifische Arbeitsteilung von Frauen und Männern lässt sich nach dem „Doing Gender“-Ansatz von West und Zimmerman (1987) als Herstellungsleistung der geschlechtlichen Identität erklären. Dabei knüpfen sie an Überlegung von Garfinkel (1967) und Kessler und McKenna (1978) an, die reflektieren, anhand welcher Kriterien im Alltag darüber entschieden wird, ob eine Person für weiblich oder männlich gehalten wird. Weiter fragen sie, welche Eigenschaften oder Verhaltensweisen als männlich oder weiblich gelten, ob es überhaupt die männlichen und die weiblichen Eigenschaften gibt und wie das soziale Umfeld darauf reagiert, wenn das antizipierte Geschlecht und das im Verhalten gezeigte Geschlecht einander nicht entsprechen.

„If we ask by what criteria a person might classify someone as being either male or female, the answers appear to be so self-evident as to make the question trivial. But consider a list of items that differentiate females from males. There are none that always and without exception are true of only one gender. No behavioral charcteristic (e.g., crying or physical aggression) is always present or never present for one 
gender. Neither can physical characteristics - either visible (e.g., beards), unexposed (e.g., genitals), or normally unexamined (e.g., gonads) - always differentiate the genders" (Kessler und McKenna 1978, S. 1-2; Hervorhebung im Original).

Kessler und McKenna führen hierbei eine Unterscheidung zwischen der körperlichen Geschlechtszugehörigkeit „sex“ und der sozialen Geschlechtszugehörigkeit „gender“ ein. Zudem differenzieren sie zwischen der Geschlechtszuweisung (,gender assignment") und Geschlechtszuschreibung („gender attribution“) (Kessler und McKenna 1978). Die Geschlechtszuweisung erfolgt „als ein einmaliger Vorgang bei der Geburt (...), z.B. wenn der Arzt oder die Hebamme nach der Geburt durch einen Blick auf die Vagina oder den Penis die Geschlechtsbestimmung vornimmt" (Riegraf 2010, S. 67). Die Geschlechtszuschreibung („gender attribution“) verweist dagegen darauf, dass das Geschlecht mehr ist als nur eine einmalige Etikettierung bei der Geburt, sondern dass das eigene Geschlecht lebenslang immer wieder produziert und reproduziert wird. Ist eine Geschlechtszuweisung und -zuschreibung bei einer Person erfolgt, so hat dies nachreichende Folgen:

"[O]nce people decide what you are, they interpret everything you do in light of that" (Kessler und McKenna 1978, S. 6).

Das Geschlecht und die Geschlechtszugehörigkeit werden hierbei nicht als etwas Natürliches begriffen. Vielmehr sind sie das Ergebnis von komplexen, sozialen Prozessen. Zum Ausdruck kommen sie durch „alltägliche Darstellungsarbeit, d.h. es sind Gesten, Mimiken, Kleidung, Berufswahl“, die die geschlechtliche Identität tagtäglich konstruieren (Riegraf 2010, S. 67).

Die Unterscheidung zwischen körperlichem und sozialem Geschlecht hat sich bis heute in der Geschlechterforschung etabliert und sämtliche späteren Geschlechterforscher und -forscherinnen knüpfen daran an. So differenzieren West und Zimmerman etwa zwischen „sex“, „sex category“ und „gender“. In die Kategorie „sex“ fällt das biologische Geschlecht, das sich z.B. anhand der Genitalien bei der Geburt oder anhand des Chromosomensatzes bestimmen lassen (West und Zimmerman 1987, S. 127). Daneben gibt es aber auch noch die Zuschreibung zu einem Geschlecht (,sex category“) und die geschlechtliche Identität als ein soziales Merkmal („gender“). Letzteres wird durch performative Tätigkeiten und soziale Interaktionen ständig produziert und reproduziert (West und Zimmerman 1987, S. 127).

Die drei Kategorien müssen einander nicht entsprechen, d.h. eine Person kann die biologischen Merkmale eines bestimmten Geschlechtes besitzen, sich aber dem anderen Geschlecht zugehörig fühlen und sich entsprechend dieses anderen Geschlechts verhalten (wie etwa im Falle der Transsexualität). West und Zimmerman gehen sogar so weit, dass sie diese drei Kategorien als voneinander unabhängig begreifen, so dass "gender“ gerade eben nicht durch „sex“ und „sex category“ vorbestimmt sein muss:

"Gender depictions are less a consequence of our ,essential sexual natures' than interactional portrayals of what we would like to convey about sexual natures, using 
conventionalized gestures. Our human nature gives us the ability to learn to produce and recognize masculine and feminine gender displays" (West und Zimmerman 1987, S. 131; Hervorhebung im Original).

Gesellschaftlich relevant ist somit insbesondere "gender", genauer gesagt das „doing gender“, also die alltägliche Herstellungsleistung und Inszenierung der geschlechtlichen Identität.

„[G]ender is not a set of traits, nor a variable, nor a role, but the product of social doings of some sort" (West und Zimmerman 1987, S. 129).

Dabei wird auf internalisierte soziale Normen und Geschlechterrollenerwartungen zurückgegriffen, die darüber bestimmen, welches Verhalten und welche Tätigkeiten als weiblich konnotiert und welche als männlich konnotiert gelten. Frauen können ihre geschlechtliche Identität etwa durch die Ausrichtung auf die Haus- und Familienarbeit unterstreichen und Männer durch die Fokussierung auf das Erwerbsleben. Darüber hinaus gibt es auch innerhalb der Sphären Tätigkeiten, die als männlich oder weiblich gelten: Bei der Hausarbeit sind beispielsweise Tätigkeiten wie Reparieren und Handwerken männlich konnotiert, wohingegen Tätigkeiten wie Kochen oder Putzen weiblich konnotiert sind. Innerhalb der Berufstätigkeit gelten Berufe, die mit Vorsorge oder Dienstleistungen assoziiert sind als weiblich und Berufe, die etwa mit Technik oder Mathematik in Zusammenhang stehen, als männlich.

Aus diesen Überlegungen heraus hat sich der „doing gender while doing work“-Ansatz entwickelt (Wetterer 1992a, 1992b; Gottschall 1998). Hierbei wird die „Polarisierung der Geschlechtscharaktere" (Hausen 1976) auf die Aufteilung des Arbeitsmarktes in Frauenberufe und Männerberufe übertragen und gleichzeitig in Zusammenhang mit der Reproduktion von Hierarchien hergestellt. Verändern sich die typischen Geschlechterverhältnisse in einem Beruf, so geht dies auch mit einer Veränderung des Prestiges einher: Kommt es bei Berufen, die ursprünglich männlich waren, zu einer Erhöhung des Frauenanteils, so ist dies mit einem Prestigeverlust verbunden, kommt es umgekehrt bei einem ursprünglich weiblichen Beruf zu einer Erhöhung des Männeranteils, so geht dies mit einer Prestigeerhöhung einher. Als Beispiel für eine Feminisierung und damit einer Abwertung des Berufs, wählt Wetterer den Wandel „vom Sekretär zur Sekretärin“; die Vermännlichung und damit die Aufwertung eines Berufsbildes illustriert Wetterer mit dem Übergang „von der Putzfrau zum Lehrberuf des Gebäudereinigers“ (Wetterer 1992b, S. 23). Indem Wetterer die Bedeutung von Organisationen, Institutionen und Arbeitsplätzen für die Geschlechterkonstruktion herausarbeitet, zeigt sie auf, dass Geschlechterkonstruktionen nicht nur auf der Mikroebene, sondern auch auf der Mesoebene geschehen (Wetterer 1992a, 1992b).

Welche Konsequenzen eine geschlechtsuntypische Ausrichtung bzw. Ressourcenausstattung haben kann, geht aus Brines (1994) Kompensationshypothese hervor: Sie fand heraus, dass Männer, deren Frauen ein höheres Einkommen oder einen höheren Erwerbsumfang haben als sie selbst und von denen sie deshalb ökonomisch abhängig 
sind, mit einer reduzierten Übernahme der Hausarbeit reagieren. Diese geschlechtsuntypische Einkommensungleichheit bzw. Erwerbskonstellation widerspricht der männlichen Geschlechtsidentität des Hauptverdieners und wird kompensiert, indem Männer weniger von der weiblich konnotierten Hausarbeit verrichten (,gender-deviance-neutralization“) (Brines 1994). Brines konnte erstmalig mit einer groß angelegten, quantitativen Studie die Konstruktion von Männlichkeit anhand des „Doing Genders“ nachweisen und damit die Theorie der ökonomischen Abhängigkeit, die annimmt, dass ökonomisch abhängige Männer mehr Hausarbeit übernehmen, zurückweisen. Darüber hinaus deuten ihre Ergebnisse darauf hin, dass Frauen die geschlechtsuntypische Rolle als Familienernährerin kompensieren, indem sie mehr Hausarbeit übernehmen, allerdings erlangen diese Ergebnisse keine statistische Signifikanz (Brines 1994; Sullivan 2011).

Greenstein (2000) setzte an Brines Studie an und erweiterte sie, indem er Geschlechterrolleneinstellungen kontrollierte und die Aufteilung der Hausarbeit nicht in absoluter Stundenanzahl maß, sondern die Anteile einer Person am gesamten Hausarbeitsvolumen. Mit dieser anteiligen Messung der Hausarbeitsbeteiligung konnte Greenstein eine „gender-deviance-neutralization“ auch für Frauen bestätigen: Frauen, die ein höheres Einkommen als ihre Männer haben, übernehmen zur Kompensation einen höheren Anteil an der Hausarbeit (Greenstein 2000). Studien von Gupta $(2006,2007)$ und Gupta und Ash (2008) finden jedoch keine Bestätigung für die Annahme der "gender-devianceneutralization“, sondern gehen davon aus, dass der Befund dadurch zustande gekommen ist, dass nur das relationale Einkommen ins Modell mit aufgenommen wurde ohne dem absoluten Einkommen als Kontrollvariable (Gupta 2006, 2007; Gupta und Ash 2008). Das absolute Einkommen ist jedoch relevant, da Frauen, die ein höheres Einkommen haben als Männer, sich oftmals in einem Haushalt mit niedrigem Haushaltseinkommen befinden, in dem traditionellere Gender-Ideologien vorliegen (Sullivan 2011). Gupta $(2006,2007)$ und Gupta und Ash (2008) konnten nachweisen, dass der Effekt, der die "gender-deviance-neutralization" stützt, verschwindet, wenn anstelle des relativen Einkommens das absolute Einkommen beider Partner, insbesondere jedoch das absolute Einkommen der Frauen, kontrolliert wird.

Am „Doing Gender“-Ansatz kann kritisiert werden, dass in Interaktionen „gender" nie allein, sondern stets gleichzeitig mit Klassenunterschieden und ethnischen Unterschieden generiert wird (Gildemeister 2008, S. 192). West und Fenstermaker (1995) identifizieren diesen sogenannten „white middle-class bias“ im feministischen Denken und reagieren darauf mit "doing difference“, einer theoretischen Erweiterung, die neben "gender" auch "class" und „race" als wichtige Komponenten von sozialer Ungleichheit berücksichtigen (West und Fenstermaker 1995). Hierauf verweist auch Hirschauer, indem er auf die rhetorische Frage „[c]an we ever not do gender?" (West und Zimmerman 1987, S. 137; Hervorhebung im Original) mit der Gegenfrage "can we ever not do age/ethnicity/class?“ (Hirschauer 1994, S. 676) reagiert. Damit kritisiert Hirschauer bereits einen ersten Aspekt an der sog. Omnirelevanzannahme, die davon ausgeht, dass Geschlecht immer eine Rolle spielt und es sich dabei um einen kontinuierlichen Prozess handelt, der nie in 
den Hintergrund rückt oder ganz ausbleibt. Als zweiten Aspekt kritisiert Hirschauer die Unterstellung der Kontinuität: So müsse neben einer Konstruktion von Geschlecht durch Tun („doing gender“ und „doing difference“) auch eine Dekonstruktion durch Nicht-Tun („undoing gender“ und „undoing difference“) möglich sein, indem das Geschlecht in sozialen Situationen oder Interaktionen lediglich registriert wird, aber die Relevanz variieren oder ganz in den Hintergrund treten kann. Nach Hirschauer ist stattdessen „von einer Diskontinuität der Geschlechtskonstruktion auszugehen: der Prozess der Geschlechtskonstruktion besteht aus Episoden, in denen Geschlecht in sozialen Situationen auftaucht und verschwindet" (Hirschauer 1994, S. 677; Hervorhebungen im Original).

Welche Annahmen lassen sich aus dem „Doing Gender“-Ansatz für die Arbeitsteilung der Eltern in unterschiedlichen Phasen des Familienzyklus ableiten? Zunächst lässt sich festhalten, dass dem „Doing Gender“-Ansatz traditionelle Rollenbilder zugrunde liegen: Männer stellen ihr Geschlecht her, indem sie sich gemäß männlichen Geschlechterstereotypen verhalten und Frauen, indem sie sich gemäß weiblichen Geschlechterstereotypen verhalten. Deshalb ist zu erwarten, dass die Arbeitsteilung bereits am Anfang traditionell ist und auch über die verschiedenen Phasen des Familienzyklus bzw. über die verschiedenen Altersstufen des Kindes relativ stabil bleibt (Schulz 2010, S. 93).

Dies ist darauf zurückzuführen, dass sich der „Doing Gender“-Ansatz an denjenigen Geschlechterrollen orientiert, die dominant sind. Zwar gibt es neue Geschlechterrollen wie etwa die "neuen Väter“, die sich verstärkt an der Kinderbetreuung beteiligen, aber diese sind noch nicht so präsent, dass es sich dabei um die größte Gruppe unter den Vätern handelt. Der „Doing Gender“-Ansatz orientiert sich somit an den vorherrschenden Geschlechterstereotypen, ohne dabei die Vielfältigkeit von Geschlechterrollen zu berücksichtigen (Schulz 2010, S. 93). Sollte es bei den Geschlechterrollen zu einer starken Veränderung kommen und etwa egalitäre Arbeitsteilungsmuster zur neuen Norm werden, wird sich der „Doing Gender“-Ansatz an diesen neuen Rollen orientieren und könnte dann eine traditionelle Arbeitsteilung nicht mehr erklären (Schulz 2010, S. 93). Obwohl sich in den letzten Jahrzehnten die Erwerbsbeteiligung der Frauen stark erhöht hat und die Aufteilung der Hausarbeit etwas egalitärer geworden ist, ist das Leitbild des männlichen Hauptverdieners und der Mutter als primären Betreuungsperson nach wie vor sehr dominant.

\subsubsection{Identitätsformationsmodell}

Ein weiterer Ansatz, der auf sozialen Normen und Erwartungen basiert, ist das Identitätsformationsmodell von Bielby und Bielby (1989). Dieses Modell nimmt an, dass sich bei Frauen und Männern im Laufe des Lebens Bindungen an bestimmte Lebensbereiche herausbilden. Im Fokus stehen dabei Berufs- und Familienidentitäten, die sich bei Frauen und Männer in unterschiedlichem Ausmaß herausbilden und mit der Zeit verfestigen. 
„We find that for both men and women, engagement in work and family roles leads to identification with those roles. However, the process of identity formation differs for men and women in ways that correspond to gender-based differentiation in household and workplace activities“ (Bielby und Bielby 1989, S. 776).

Für die Herausbildung von Identitäten sind gesellschaftliche Normen und Leitbilder von entscheidender Bedeutung. Indem Männer und Frauen ihre „Zeit und Energie darauf verwenden, gesellschaftlich vorgegebene Rollen im Erwerbsleben und der Familie zu spielen, identifizieren sie sich mit diesen" (Schulz 2010, S. 96).

„Work and family are more than just complications, they are sources of meaning and identity to which men and women balance commitment" (Bielby 1992: 283).

Bielby und Bielby (1989) gehen davon aus, dass in modernen Gesellschaften weiterhin traditionelle Geschlechterrollen dominant sind und sich Frauen deshalb verstärkt mit der Familie und Männer verstärkt mit dem Beruf identifizieren (Bielby und Bielby 1989, S. 785). Wenn Frauen eine starke Familienidentität entwickeln, heißt das nicht, dass sie nicht gleichzeitig auch eine starke Berufsidentität entwickeln können, aber diese gleichzeitige Herausbildung einer Berufs- und Familienidentität bei Frauen würde mit Geschlechterrollenkonflikte einhergehen (Grunow et al. 2007, S. 165). Während Brines (1994) eher die Männer in Geschlechterrollenkonflikte verwickelt sieht, trifft dies laut Bielby und Bielby (1989) somit vielmehr auf die Frauen zu (Grunow et al. 2007, S. 165; Boll 2016, S. 31):

„We expect that wives employed outside the home balance dual role identities by trading one off against the other. In contrast, for men, contemporary- normative expectations for the ,husband' and ,father' roles still do not include fully shared responsibility and involvement in household and child-care activities. Furthermore, a husband's role in the workplace is consistent with his family obligations as ,provider" (Bielby \& Bielby 1989: 777).

Frauen mit starker Berufsidentität sind somit konkurrierenden Erwartungen ausgesetzt, denn bei Frauen ist

„die Stärkung der Berufsidentität nur auf Kosten einer Schwächung ihrer Aktivitäten in Familie und Haushalt möglich, da von innen, zusätzlich zu ihrem beruflichen Engagement, der volle Einsatz als ,Ehefrau' und ,Mutter' erwartet wird“" (Grunow et al. 2007, S. 165).

Bei Frauen kommt es daher zwar leichter zu Interrollenkonflikten zwischen ihrer Berufsund Familienidentität, dafür spielt die Erwerbstätigkeit für ihre geschlechtliche Identität keine derart große Rolle wie für die Identität der Männer: Wenn Frauen nicht erwerbstätig sind, so ist zwar ihre Berufsidentität geschwächt, nicht jedoch ihre Familienidentität. Wenn dagegen Männer ihren Arbeitsplatz verlieren, ist nicht nur ihre Berufsidentität gefährdet, sondern darüber hinaus auch noch ihre Familienidentität, da die Rolle des Familienernährers dann nicht mehr erfüllt werden kann: 
„Labor force interruptions eroded family identity for males but not for females, while having an employed spouse increased family identity for females and decreased work identity for males“ (Bielby \& Bielby 1989: 786).

Es stellt sich die Frage, wie sich die Arbeitsteilung nach dem Identitätsformationsmodell im Laufe des Familienzyklus verändert. Allgemein ist eine traditionelle Arbeitsteilung zu erwarten, da die Ablösung von traditionellen Rollenbildern nach Bielby und Bielby (1989) nur sehr langsam geschieht und Frauen auch heute noch die primäre Verantwortung für die Hausarbeit und die Kinderbetreuung zugeschrieben wird - selbst dann wenn sie über ein hohes Humankapital und ein hohes Einkommen verfügen (Schulz und Blossfeld 2006, S. 30). Deshalb ist bereits zu Beginn einer Beziehung eine traditionelle Arbeitsteilung zu erwarten. Mit der Familiengründung wird die „Identitätsformation als Mutter bedeutsam und eine Fokussierung auf die Familienidentität wahrscheinlicher werden“ (Grunow et al. 2007, S. 165). Indem die Mutter viel Zeit für die Erziehung des Kindes aufbringt, verfestigt sie ihre Familienidentität noch weiter. Es kann deshalb davon ausgegangen werden, dass die Aufteilung der Hausarbeit und Kinderbetreuung nach der Familiengründung traditionell ist und auch traditionell bleibt (Grunow et al. 2007, S. 165). Mütter können zwar weiterhin erwerbstätig sein, aber es kann davon ausgegangen werden, dass Familienidentität die Berufsidentität übertrumpft und Rollenkonflikte zwischen den beiden Identitäten zugunsten der Familienidentität ausgetragen werden. Aus dem Identitätsformationsmodell kann somit abgeleitet werden, dass sich die Arbeitsteilung im Familienzyklus traditionalisiert.

\subsubsection{Stabilität oder Wandelbarkeit von Einstellungen}

Die vorgestellten geschlechtsspezifischen Ansätze gehen davon aus, dass die Geschlechterrollen, Einstellungen und Identitäten über den Lebenslauf hinweg weitgehend stabil bleiben und dass sie traditionell ausgerichtet sind. Aus empirischen Befunden geht jedoch hervor, dass die Geschlechterrolleneinstellungen weder traditionell sind, noch, dass sie stabil bleiben müssen: So sind die Geschlechterrolleneinstellungen zum einen in den letzten Jahrzehnten egalitärer geworden sind (Kurz 1998; Bolzendahl und Myers 2004; Brooks und Bolzendahl 2004; Lee et al. 2007; Davis und Greenstein 2009; Cotter et al. 2011; Knight und Brinton 2017; Grunow et al. 2018); und zum anderen können sich die Einstellungen auch innerhalb eines Lebenslaufs verändern (Fan und Marini 2000; Cunningham 2005; Corrigall und Konrad 2007; Berrington et al. 2008; Schober und Scott 2012; Evertsson 2013; Baxter et al. 2015; Buchler et al. 2017).

Im Folgenden wird daher zunächst auf den Einstellungswandel hin zu egalitäreren Einstellungen eingegangen. Dies ist zugleich als Kritik gegen die Annahme der Geschlechterrollenansätzen, dass Geschlechterrollen und Einstellungen traditionell und stabil seien, zu verstehen. Anschließend wird die Einstellungsentwicklung innerhalb eines Lebenslaufs näher beleuchtet, da es für die vorliegende Arbeit von besonderem Interesse ist, wie sich Einstellungen innerhalb eines Lebenslaufs - und speziell in Abhängigkeit vom Alter des Kindes - verändern können. 
Eine Erklärung für den Trend zu egalitäreren Einstellungen liefert die „cohort replacement theory" (Brewster und Padavic 2000; Brooks und Bolzendahl 2004). Sie nimmt an, dass die Veränderung der Einstellungen darin begründet liegt, dass Einstellungen von älteren Kohorten durch Einstellungen von jüngeren Kohorten ersetzt werden. Weil jüngere Kohorten in ihrer Kindheit andere Erfahrungen gemacht haben als ältere Kohorten, generieren sich daraus unterschiedliche Einstellungen (Brooks und Bolzendahl 2004, S. 110). Zu einem Einstellungswandel kommt es also aufgrund eines Wechsels zwischen den Kohorten. Innerhalb des Lebenslaufs eines Individuums wird jedoch kein Einstellungswechsel prognostiziert, was auf der Annahme fußt, dass Einstellungen wesentlich durch die Kindheitserfahrungen geprägt sind und die Einstellungen im Erwachsenenalter stabil bleiben (Inglehart 1977; Alwin und Korsnick 1991).

Doch warum sollten jüngere Kohorten egalitärere Einstellungen haben als ältere Kohorten? Ein wichtiger Aspekt liegt hier in dem Zusammenhang von Bildung und Einstellungen. Nach dem „Egalitarian Values Model“ von van Berkel und Graaf (1999) kommt der Bildung eine doppelte theoretische Bedeutung zu, da sie einerseits für das Humankapital und andererseits für die Einstellungen relevant ist. Nach der ersten theoretischen Bedeutung fungiert Bildung als Indikator für Humankapital. Bzgl. der Arbeitsteilung beziehen sich van Berkel und Graaf (1999) in ihrer Studie auf die Beteiligung der Männer an der Hausarbeit. Betrachtet man die Bildung in ihrer Funktion als Humankapital, so ist ein negativer Zusammenhang zu erwarten, also dass Männer mit höherer Bildung weniger Hausarbeit verrichten. Da die Bedeutung von Humankapital und Arbeitsteilung bei den ökonomischen Theorien bereits eingehend dargestellt wurde, wird hierauf an dieser Stelle nicht weiter eingegangen. Die zweite theoretische Bedeutung nimmt an, dass Männer mit höherer Bildung mehr Hausarbeit übernehmen, weil Männer mit höherer Bildung egalitärere Einstellungen haben. Dabei ist nicht nur die eigene Bildung entscheidend, sondern auch die Bildung der Frau und die damit einhergehenden Bildungskonstellationen. Der Effekt der Bildung ist dabei wie folgt:

„Whereas for the lower educated inequality between the sexes is given, the higher educated are aiming at gender equality in public and private spheres as much as possible. Since this equalizing property of higher education applies irrespectively of sex, we may hypothesise: the higher the level of education of the couple, the less skewed the division of labour" (van Berkel und Graaf 1999, S. 790).

Es wird angenommen, dass Höhergebildete eher demokratische Werte befürworten und dies auch mit einer Befürwortung von Geschlechtergleichheit einhergeht (van Berkel und Graaf 1999, S. 790). Wenn Personen mit hoher Bildung egalitärere Werte vertreten, ist anzunehmen, dass folglich auch die Arbeitsteilung egalitärer aufgeteilt ist. Zwar beziehen sich van Berkel und Graaf (1999) in ihrer Studie lediglich auf die Aufteilung der Hausarbeit, aber die Annahmen lassen sich auch auf die Aufteilung der Erwerbsarbeit und der Kinderbetreuung übertragen. Ihre empirischen Befunde zeigen, dass die Hausarbeit dann am egalitären aufgeteilt ist, wenn Frauen und Männer eine hohe Bildung 
haben. Die Ergebnisse zeigen, dass die Bildung in ihrer Bedeutung als Humankapital weniger Erklärungskraft besitzt als in ihrer Bedeutung für egalitärere Werte:

„The effects of education are such that an explanation based on egalitarian values fares better than one based on human capital“ (van Berkel und Graaf 1999, S. 785).

Eine weitere Theorie zur Erklärung der egalitäreren Werte liefern Bolzendahl und Myers (2004). Dass Frauen in der Regel egalitärere Einstellungen aufweisen als Männer, lässt sich anhand von interessen- und erfahrungsbasierten Faktoren erklären (Bolzendahl und Myers 2004; Lee et al. 2007; Davis und Greenstein 2009; Mays 2012). Gemäß der interessenbasierten Perspektive haben Frauen egalitärere Werte als Männer, weil sie von einer zunehmenden Geschlechtergleichheit stärker profitieren als Männer (Bolzendahl und Myers 2004, S. 761). Die erfahrungsbasierten Faktoren gehen insbesondere aus der Sozialisation, der Arbeitsmarkterfahrung und der Bildung hervor (Bolzendahl und Myers 2004, S. 762). Beim Sozialisationshintergrund wird beispielsweise die Erwerbstätigkeit der Mutter sowie das Bildungsniveau der Mutter als wichtige Einflussgröße für die eigenen Geschlechterrolleneinstellungen angesehen. Auch die eigene Arbeitsmarkterfahrung spielt eine wichtige Rolle für egalitärere Werte, was darauf zurückzuführen ist, dass berufstätige Frauen von ihren Männern finanziell unabhängiger sind und sie Kontakte zu gleichgesinnten, berufstätigen Frauen knüpfen können (Bolzendahl und Myers 2004, S. 762).

Des Weiteren stellt die Bildung einen relevanten Faktor dar, weil höher gebildete Frauen eher mit feministischen Gedankengut in Berührung kommen und dadurch traditionelle Geschlechterstereotype eher kritisch hinterfragen und alternative Lebenswege für Frauen kennenlernen (Bolzendahl und Myers 2004, S. 762). Wie beim „Egalitarian Values Model“" von van Berkel und Graaf (1999) stellen somit auch Bolzendahl und Myers (2004) einen Zusammenhang zwischen der Bildung und der Ausrichtung der Werte her. Darüber hinaus haben Frauen mit hoher Bildung ein größeres Interesse Karriere zu machen und sind damit einhergehend danach bestrebt Diskriminierungen auf dem Arbeitsmarkt abzuschaffen (Bolzendahl und Myers 2004, S. 762).

Indem Bolzendahl und Myers (2004) auf erfahrungsbasierte Faktoren verweisen und diese Erfahrungen nicht ausschließlich in der Kindheit und Jugend stattgefunden haben müssen, sondern auch Erfahrungen im Erwachsenenalter inkludieren, lässt sich schlussfolgern, dass sich Einstellungen auch im Erwachsenenalter noch ändern können. Bolzendahl und Myers (2004) beziehen sich dabei v.a. auf Erfahrungen, die Frauen zu feministischeren Einstellungen bringen, wie die Erfahrung der Erwerbstätigkeit und der damit einhergehenden finanziellen Unabhängigkeit und der Austausch zu Kolleginnen. Umgekehrt sind jedoch auch Erfahrungen möglich, die dazu führen, dass die Einstellungen von Frauen im Erwachsenenalter wieder traditioneller werden. Ein Beispiel hierfür ist der Übergang zur Elternschaft, der bei Frauen traditionellere Einstellungen bewirken kann: 
„Women who experience higher demands from household activities and childrearing will have less time for outside activities and therefore receive less exposure to feminist ideas" (Bolzendahl und Myers 2004, S. 765).

Die empirischen Ergebnisse von Bolzendahl und Myers (2004) zeigen, dass Mütter mit mehreren Kindern bei einigen, bereichsspezifischen Einstellungen (hinsichtlich Abtreibung und vorehelicher Geschlechtsverkehr) traditionellere Einstellungen haben. Dies bestätigt die Annahme, dass durch Erfahrungen wie einer erhöhten Kinderanzahl die Einstellungen der Mütter traditioneller werden. Gleichzeitig zeigen die Ergebnisse auch, dass bei Müttern mit mehreren Kindern bereichsspezifische Einstellungen zu Familienverantwortlichkeiten nicht traditioneller, sondern sogar liberaler ausfallen. Bolzendahl und Meyers erklären dies mit den Interessen der Mütter: Weil Mütter (insbesondere mit mehreren Kindern) sich eine verstärkte Unterstützung der Väter wünschen, sie also ein Interesse an einer egalitäreren Arbeitsteilung haben, sind ihre Einstellungen diesbezüglich egalitärer (Bolzendahl und Myers 2004, S. 775).

Viele Studien, die die Einstellungen untersuchen, basieren auf Querschnittsdaten. Da für die vorliegende Arbeit von Interesse ist, wie sich die Arbeitsteilung von Eltern je nach Alter des Kindes verändert und damit einhergehend auch, wie sich die Einstellungen von Eltern je nach Alter des Kindes verändern, sind Erkenntnisse aus bisherigen Längsschnittstudien besonders gewinnbringend. Der Einstellungswandel innerhalb des Lebenslaufs ist mit Längsschnittdaten noch unzureichend erforscht. Im Folgenden wird auf die Längsschnittstudien, die es hierzu bereits gibt, näher eingegangen (Fan und Marini 2000; Cunningham 2005; Corrigall und Konrad 2007; Berrington et al. 2008; Schober und Scott 2012; Evertsson 2013; Baxter et al. 2015; Buchler et al. 2017).

Fan und Marini (2000) untersuchten in ihrer Studie an amerikanischen Längsschnittdaten den Übergang von Jugendlichen ins Erwachsenenalter und konnte zeigen, dass sich die Einstellungen durch Übergänge im Lebenslauf verändern können. Faktoren wie die Fortsetzung der Ausbildung oder der Einstieg ins Erwerbsleben führten sowohl bei Frauen als auch bei Männern zu egalitäreren Einstellungen. Das Heiraten bewirkte nur bei Frauen einen Einstellungswandel hin zu traditionelleren Einstellungen, während der Übergang zur Elternschaft bei beiden Geschlechtern mit traditionelleren Einstellungen verbunden ist. Damit zeigt die Studie, dass Einstellungen nicht als stabil betrachtet werden sollten, sondern Änderungen auch noch im Erwachsenenalter stattfinden.

Eine Längsschnittstudie von Cunningham et al. (2005) mit amerikanischen Daten erforschte über den Zeitraum von 1962 bis 1993 die Einstellungen von jungen Erwachsenen im Alter von 18 bis 31 Jahren. Sie konnten in ihren Analysen nur wenig Unterstützung dafür finden, dass sich die Einstellungen mit dem Übergang zur Elternschaft verändern. Auch der Übergang in die Ehe bewirkte keine Einstellungsänderung. Eine Einstellungsänderung fanden sie stattdessen bei in Vollzeit erwerbstätigen Frauen, da diese in ihrem späteren, jungen Erwachsenenalter egalitärere Einstellungen zu Familienrollen hatten (Cunningham et al. 2005, S. 887). Somit bestätigen auch die Ergebnisse von 
Cunningham et al. (2005), dass sich die Einstellungen im Erwachsenenalter noch verändern können, auch wenn sie speziell beim Übergang zur Elternschaft keine empirische Evidenz für einen Einstellungswandel gefunden haben.

Auch Corrigall und Konrad (2007) haben mit amerikanischen Längsschnittdaten von 1976 bis 1990 die Veränderung der Einstellungen von High-School-Absolventen mit dem Übergang ins Erwachsenenalter analysiert. Während der Übergang zur Ehe keine signifikante Veränderung der Einstellungen bewirkte, war dies beim Übergang zur Elternschaft schon der Fall: Sowohl bei den Frauen als auch bei den Männern waren die Einstellungen nach dem Übergang zur Elternschaft traditioneller.

Berrington et al. (2008) betrachteten mit britischen Längsschnittdaten den Zusammenhang zwischen dem Übergang zur Elternschaft und der Veränderung der Einstellungen. Besonderes Augenmerk richteten sie hierbei darauf, wie sich die Erwerbsbeteiligung der Frauen nach dem Übergang zur Elternschaft verändert. Sie stellten fest, dass die mit der Elternschaft einhergehende Einstellungsänderung wesentlich durch die Erwerbsbeteiligung der Frauen bestimmt ist. Sie kommen daher zu dem Resümee, dass nicht die Elternschaft per se entscheidend ist, sondern die Veränderung des Erwerbsstatus der Frauen.

Evertsson (2013) untersuchte mit schwedischen Längsschnittdaten, wie sich die Einstellungen von Frauen mit dem Übergang zur Mutterschaft verändern. Die Einstellungen bezogen sich auf die Erwerbsorientierung der Frauen und wurde operationalisiert, indem danach gefragt wurde, wie wichtig die Erwerbsarbeit für die Befragten ist. Das betrachtete Zeitfenster bezog sich auf die Jahre 1999 bis 2003. Zu Beginn im Jahr 1999 gab es keine Unterschiede zwischen den Frauen, die in den nachfolgenden Jahren den Übergang zur Mutterschaft erleben und denen, die diesen Übergang nicht erleben. Im Jahr 2003 gab es jedoch schon Unterschiede zwischen den neuen Müttern und den NichtMüttern: Bei den Müttern hat sich die Erwerbsorientierung verringert, bei den kinderlosen Frauen ist sie unverändert geblieben. Die Ergebnisse von Evertsson (2013) zeigen jedoch auch, dass die reduzierte Erwerbsorientierung der Mütter nicht dauerhaft ist, sondern sich auf die ersten Lebensjahre des Kindes beschränkt. Ab einem Kindesalter von vier Jahren ist die Erwerbsorientierung von Müttern nicht mehr signifikant niedriger als bei den kinderlosen Frauen. Eine Erklärung dafür, dass die reduzierte Erwerbsorientierung nur auf Kinder von unter vier Jahre beschränkt ist, liegt am schwedischen Wohlfahrtssystem, das den Müttern eine Rückkehr in die Erwerbsarbeit erleichtert.

Schober und Scott (2012) untersuchten mit britischen Längsschnittdaten, wie sich die Einstellungen mit Frauen und Männern mit dem Übergang zur Elternschaft verändern. Ihre Ergebnisse zeigen, dass die Einstellungen mit dem Übergang zur Elternschaft dann relativ stabil bleiben, wenn ihre nach der Geburt praktizierten Arbeits- und Betreuungsarrangements nicht im Widerspruch zu ihren Einstellungen vor der Geburt stehen. Bei einem Viertel der Eltern kommt es jedoch zu einem Einstellungswechsel und dies ist besonders dann wahrscheinlich, wenn die Arbeits- und Betreuungsarrangements nach 
der Geburt mit den pränatalen Einstellungen konfligieren (Schober und Scott 2012, S. 526). Weil die früheren Einstellungen dann nicht mehr mit dem aktuellen Verhalten kongruent sind, werden die Einstellungen angepasst.

Auch Baxter et al. (2015) konnten mit australischen Längsschnittdaten nachweisen, dass die Einstellungen im Lebenslauf nicht stabil sind und sich bei wichtigen Lebensereignissen wie dem Übergang zur Elternschaft verändern. Die Einstellungen werden anhand von sieben Items erfasst und jeweils als eigene abhängige Variable verwendet. Die Ergebnisse zeigen, dass sowohl bei Frauen als auch bei Männern nach dem Übergang zur Elternschaft ein Einstellungswechsel stattgefunden hat. Sowohl bei den Frauen als auch bei den Männern sind die Einstellungen zur Betreuung der Kinder traditioneller geworden: Die Mutterrolle wird als wichtigste Rolle der Frau angesehen und Frauen sollen zu Hause bleiben, um die Kinder zu betreuen. Bei den Frauen sind jedoch nicht alle Einstellungen mit dem Übergang zur Elternschaft traditioneller geworden, was sich z.B. daran zeigt, dass Frauen weiterhin egalitäre Einstellungen zur Berufstätigkeit der Frauen vertreten. Aber indem Frauen sowohl an der Wichtigkeit der Mutterrolle als auch an der Bedeutung der Berufstätigkeit festhalten, vertreten sie widersprüchliche Einstellungen. Ähnlich wie bei der Studie von Schober und Scott (2012) ist der Übergang zur Elternschaft für Frauen dabei mit einer kognitiven Dissonanz verbunden (Baxter et al. 2015, S. 1006).

Buchler et al. (2017) haben mit Längsschnittdaten aus Großbritannien und Australien untersucht, wie sich die Einstellungen zur Vaterschaft mit dem Übergang zur Elternschaft verändern. Ihre Ergebnisse zeigen, dass Männer mit dem Übergang zur Elternschaft sowohl in Großbritannien als auch in Australien enthusiastischer werden, sich an der Kinderbetreuung zu beteiligen, d.h. die Einstellungen der Männer werden egalitärer. Eine andere Entwicklung zeigt sich dagegen bei den Frauen: Im Gegensatz zu den Männern werden die Einstellungen der Frauen in Australien mit dem Übergang zur Elternschaft traditioneller; bei den Frauen in Großbritannien findet keine Veränderung der Einstellungen mit dem Übergang zur Elternschaft statt.

Aus den erwähnten Längsschnittstudien wird ersichtlich, dass Einstellungen im Erwachsenenalter nicht stabil bleiben müssen, sondern sich auch noch im Erwachsenenalter verändern können. Sie zeigen, dass sich die Einstellungen durch Übergänge im Lebenslauf wie etwa durch die Heirat, durch den Übergang zur Elternschaft oder durch den Einstieg in die Erwerbstätigkeit verändern können.

Besonders interessant für die vorliegende Arbeit ist die Frage danach, wie sich die Einstellungen mit dem Übergang zur Elternschaft und anschließend mit dem Älterwerden des Kindes verändern. Die Studien zeigen, dass die Einstellungen mit dem Übergang zur Elternschaft in der Regel traditioneller werden (Corrigall und Konrad 2007; Evertsson 2013; Baxter et al. 2015), mit Ausnahme der Studie von Buchler et al. (2017), aus der hervorgeht, dass die Einstellungen zur Vaterschaft bei den Männern mit dem Übergang zur Elternschaft egalitärer werden. 
Während diese Studien zwar Bezug auf den Übergang zur Elternschaft nehmen, ist noch wenig darüber bekannt, wie sich die Einstellungen in Abhängigkeit vom Kindesalter verändern. Dies mag damit zusammenhängen, dass es aufgrund von Kollinearität problematisch ist, wenn in Längsschnittmodellen neben dem Befragungsjahr gleichzeitig auch Altersangaben enthalten sind (Baxter et al. 2015, S. 1009, Fußnote 8). In einigen Studien werden jedoch die Jahre seit der Geburt als Kontrollvariable verwendet (und Variablen, die damit in Kollinearität stehen, entsprechend weggelassen).

In der Studie von Buchler et al. (2017) zeigt sich, dass die Einstellungen zur Vaterschaft in Großbritannien mit zunehmendem Alter des Kindes traditioneller werden, für Australien wurde keine signifikante Veränderung mit dem Kindesalter festgestellt (Buchler et al. 2017, S. 670-671). Auch bei den Einstellungen zur Mutterschaft konnte bei Baxter et al. (2015) mit australischen Daten keine signifikante Änderung in Abhängigkeit vom Kindesalter gefunden werden. Dies zeigt jedoch nur, dass kein linearer Zusammenhang vorliegt. Separate Analysen der Autoren, auf die nicht näher eingegangen wird, deuten auf eine quadratische Beziehung zwischen dem Kindesalter und den Einstellungen zur Mutterschaft hin (Baxter et al. 2015, S. 1005). Aus der Studie von Evertsson (2013) mit schwedischen Daten geht hervor, dass die Einstellungen nur in den ersten vier Jahren nach der Geburt traditioneller sind und der Effekt danach verblasst.

Für den deutschen Kontext kann angenommen werden, dass die Einstellungen bei kleinen Kindern besonders traditionell sind. Dies geht aus institutionellen Rahmenbedingungen hervor, etwa daraus, dass externe Betreuungsmöglichkeiten für Kleinkinder schlecht ausgebaut sind und es den Eltern durch das Elterngeld bzw. die Elternzeit ermöglicht wird, sich selbst um das Kind zu kümmern. Mit zunehmendem Alter des Kindes werden mehr Fürsorgeleistungen externalisiert und mehr Mütter kehren wieder in die Erwerbstätigkeit zurück. Damit einhergehend kann angenommen werden, dass die Einstellungen mit zunehmendem Alter des Kindes egalitärer werden.

\subsection{Zusammenfassung und Diskussion}

Die nachfolgende Tabelle 4.1 liefert eine Zusammenfassung der bisherigen, theoretischen Erklärungsansätze und stellt die ökonomischen bzw. ressourcenbasierten Ansätze den normenbasierten Ansätzen gegenüber. Die ökonomische Familientheorie geht von einer geschlechtsspezifischen Arbeitsteilung aus, da diese aufgrund von komparativen Vorteilen zur Haushaltsnutzenmaximierung beiträgt. Derjenige Elternteil mit den höheren Ressourcen spzialisiert sich auf die Erwerbsarbeit und der andere Elternteil auf die Hausarbeit und die Kinderbetreuung. Die einmal eingeschlagene Spezialisierungsrichtung wird unabhängig vom Alter des Kindes beibehalten, sodass mit zunehmendem Alter des Kindes kein Wechsel der Spezialisierungsrichtung zu erwarten ist. Ein Wechsel der Spezialisierungsrichtung wäre nicht im Sinne der Haushaltsnutzenmaximierung, weil beide Elternteile in ihrem Spezialisierungsbereich, also im Bereich der Erwerbsarbeit 
oder im Bereich der Familienarbeit, Wissen akkumuliert haben, sodass sich die komparativen Vorteile im Laufe der Zeit noch verschärfen.

Die ökonomische Verhandlungstheorie unterscheidet sich von der ökonomischen Familientheorie darin, dass sie auf eine individuelle Nutzenmaximierung ausgerichtet ist. Sie nimmt des Weiteren an, dass die Arbeitsteilung dynamisch ist und sie bei einer Veränderung der Ressourcenverteilung erneut verhandelt wird. Beide Partner wollen die Hausarbeit möglichst vermeiden, da sie einerseits als unangenehm empfunden wird und da andererseits das bei der Hausarbeit erworbene Wissen nicht außerhalb der Beziehung nutzbar ist. Derjenige Partner mit den höheren Ressourcen besitzt mehr Macht und verfügt über eine bessere Verhandlungsposition, sodass er seine eigenen Präferenzen besser durchsetzen kann. Der ressourcenstärkere Partner kann sich somit aus der Hausarbeit herausverhandeln. Im Gegensatz zur familienökonomischen Theorie ist bei der Verhandlungstheorie bei einer Ressourcengleichheit der Partner auch eine egalitäre Aufteilung der Hausarbeit denkbar. Auch bei der Verhandlungstheorie handelt es sich in ihren Grundannahmen um eine geschlechtsneutrale, symmetrische Theorie. Doch durch die mit der Familiengründung einhergehende Erwerbsunterbrechung der Frauen verschlechtert sich die Verhandlungsposition der Frauen nachhaltig. Bei Müttern mit kleinen Kindern ist die Hausarbeit deswegen besonders traditionell aufgeteilt. Sobald Mütter wieder in die Erwerbstätigkeit zurückkehren, erhöhen sich ihre Ressourcen und es kann eine egalitärere Arbeitsteilung ausgehandelt werden. Allerdings haben sich die Väter durch die ununterbrochene Erwerbstätigkeit einen Karrierevorsprung erschaffen, den Mütter kaum aufholen können. Deshalb kann angenommen werden, dass Mütter selbst dann den Großteil der Hausarbeit übernehmen, wenn die Kinder älter sind und die Mütter wieder erwerbstätig sind.

Die genannten, ökonomischen Theorien gehen davon aus, dass es sich bei Individuen um rational handelnde Akteure handelt, die auf eine Haushalts- bzw. individuelle Nutzenmaximierung ausgerichtet sind. Sie berücksichtigen nicht, dass Handlungen auch durch Sozialisationsprozesse, Rollenerwartungen oder Präferenzen geprägt sind. Im Gegensatz dazu richten normenbasierte Ansätze ihren Fokus auf diese kulturellen Einflüsse.

So nimmt die Rollentheorie an, dass Menschen durch Sozialisationsprozesse Normen, Werte und Einstellungen aufnehmen und verinnerlichen. Geschlechterrollenerwartungen orientieren sich dabei an Geschlechterstereotypen. Die normativen Überzeugungen und Einstellungen zu Geschlechterrollen bewegen sich dabei zwischen den Extremen „traditionell“ und „modern“. Die Rollentheorie nimmt weiter an, dass sich Individuen einstellungskonform verhalten, d.h. Individuen mit traditionellen Einstellungen haben demnach eine traditionelle Arbeitsteilung und Individuen mit egalitären Einstellungen eine egalitäre Arbeitsteilung. Rollentheorien können damit nicht die Kluft zwischen Einstellungen und tatsächlichem Verhalten erklären. Da die Rollentheorie annimmt, dass sich Einstellungen in der Kindheit und im Jugendalter entwickeln und im Erwachsenenalter stabil bleiben, dürfte sich bei einstellungskonformen Verhalten die Arbeitsteilung im Familienzyklus 
nicht ändern. Die Stabilitätsannahme der Einstellungen ist kritisch zu hinterfragen, da viele Studien belegen, dass sich die Einstellungen auch im Erwachsenenalter noch ändern können.

Der „Doing Gender“-Ansatz führt die Unterscheidung zwischen dem körperlichen Geschlecht (,sex“) und dem sozialen Geschlecht („gender“) ein und betrachtet die geschlechtliche Identität als Herstellungsleistung. Zur Herstellung der geschlechtlichen Identität wird auf internalisierte soziale Normen und Geschlechterrollenerwartungen zurückgegriffen, die darüber bestimmen, welches Verhalten und welche Tätigkeiten als weiblich oder als männlich gelten. Frauen können ihre geschlechtliche Identität durch die Ausrichtung auf die Haus- und Familienarbeit betonen und Männer durch die Fokussierung auf das Erwerbsleben. Die Kompensationshypothese besagt, dass die Bedrohung der eigenen geschlechtlichen Identität durch geschlechtsuntypisches Verhalten in einem bestimmten Bereich durch geschlechtstypisches Verhalten in einem anderen Bereich kompensiert wird. Dem „Doing Gender“-Ansatz und der Kompensationshypothese liegen traditionelle Geschlechterrollenbilder zugrunde, weshalb anzunehmen ist, dass die Arbeitsteilung bereits zu Beginn der Beziehung traditionell ist und auch mit zunehmendem Alter des Kindes traditionell bleibt.

Auch aus dem Identitätsformationsansatz lässt sich eine traditionelle Arbeitsteilung ableiten, denn es wird angenommen, dass die Ablösung von traditionellen Rollenbildern nur sehr langsam geschieht. Der Identitätsformationsansatz geht davon aus, dass sich bei Männern und Frauen im Laufe des Lebens Berufs- und Familienidentitäten herausbilden, wobei bei den Männern die Berufs- und bei den Frauen die Familienidentität von besonderer Bedeutung ist. Prinzipiell können bei einer Person auch beide Identitäten stark entwickelt sein, allerdings führt dies aufgrund von konkurrierenden Erwartungen zu Geschlechterrollenkonflikten. Da sich bei Frauen durch die Familiengründung und die intensive Betreuung des Kindes ihre Familienidentität verstärkt, ist davon auszugehen, dass die traditionelle Arbeitsteilung auch mit dem Älterwerden des Kindes traditionell bleibt.

Diese theoretischen Annahmen finden jedoch nicht ohne Kontext statt, sondern sind eingebettet in institutionelle und kulturelle Rahmenbedingungen, die mitberücksichtigt werden müssen. Auf diese Rahmenbedingungen wird im Folgenden näher eingegangen. 
Tabelle 4.1: Zusammenfassung der theoretischen Ansätze

\begin{tabular}{l} 
Ansatz \\
\hline Ökonomische Theo-
\end{tabular} rie der Familie

„New Home Economics"

Ressourcenabhängiger Verhandlungsansatz

"Relative Resource and Bargaining" (auch Theorie des sozialen Tauschs)

\section{Normenbasierte An-} sätze

Rollentheorie, DoingGender-Ansatz, Identitätsformationsansatz

Ausgangslage

Spezifika

Ungleichheit bei

Paaren (ressourcen-

Spezialisierung nach Resabhängig) sourcen beider Partner auf die Erwerbsarbeit oder auf die Hausarbeit und Kinderbetreuung;

Maximaler Nutzen für das Paar;

Steigende Gleichheit Aufgabenverteilung wird je bei Paaren (ressourcenabhängig) delt;

Maximaler Nutzen für das Individuum;

traditionelle vs. egalitäre Geschlechterrollenvorstellungen

Arbeitsverteilung richtet sich nach der Rolleneinstellung, Identitätsbildung und gesellschaftlichen Normen.

\section{Implikation}

Meist übernimmt die Frau aufgrund komparativer Vorteile den deutlich größeren Anteil an der Hausarbeit und an der Kinderbetreuung.

Der Partner mit den höheren Ressourcen (z.B. Bildung, Berufsstatus, Einkommen) übernimmt weniger Hausarbeit.

Die Verteilung ist egalitärer je progressiver die Rollenorientierung ist; aber da traditionelle Einstellungen auch in modernen Gesellschaften unterstellt werden wird Arbeitsteilung eher traditionell ausfallen.

\section{Bedeutung des Alters des Kindes}

Beibehaltung der geschlechtsspezifischen Arbeitsteilung unabhängig vom Alter des Kindes :

aufgrund von komparativen Vorteilen wird sich die eingeschlagene Spezialisierungsrichtung verschärfen und die Arbeitsteilung wird noch rentabler;

Verhandlungsposition von Frauen mit kleinen Kindern hat sich durch die Geburt nachhaltig verschlechtert, weshalb sie mehr Hausarbeit übernehmen;

Verhandlungsposition von Frauen kann sich bei älteren Kindern wieder erhöhen, aber Karrierevorsprung des Mannes kann kaum aufgeholt werden

die Arbeitsteilung ist nach der Rollentheorie und dem Doing-Gender-Ansatz bereits zu Beginn einer Partnerschaft traditionell und bleibt unabhängig vom Alter des Kindes traditionell;

nach dem Identitätsformationsansatz verstärkt sich die Familienidentität der Frauen nach der Familiengründung, sodass die Arbeitsteilung traditioneller wird

Quelle: in abgewandelter und erweiterter Form angelehnt an Cesinger et al. 2012, S. 5. 


\section{Institutionelle und kulturelle Rahmenbedingungen der Arbeitsteilung der Eltern}

Welche institutionellen Rahmenbedingungen in Deutschland vorliegen, lässt sich leichter erkennen, wenn man Deutschland mit anderen Ländern vergleicht. Daher werden die Unterschiede zwischen Deutschland und anderen Ländern zunächst aus der Perspektive der Wohlfahrtsstaatentypologie nach Esping-Andersen $(1990,1999)$ betrachtet. Anschließend wird auf sog. Geschlechterarrangements eingegangen, die sich aus einer Geschlechterkultur und einer Geschlechterordnung zusammensetzen (Pfau-Effinger 2000 , 2005). In einem nächsten Schritt werden Familienleitbilder, die in Deutschland besonders präsent sind, vorgestellt. Danach wird auf die familienpolitischen Rahmenbedingungen in Deutschland eingegangen und dabei insbesondere auf Zeitpolitiken, Geldpolitiken und infrastrukturelle Rahmenbedingungen Bezug genommen. Im nächsten Unterkapitel werden diese institutionellen und kulturellen Rahmenbedingungen als Vorbereitung für die Hypothesengenerierung mit den theoretischen Ansätzen aus dem Theoriekapitel verknüpft, denn schließlich können die theoretischen Ansätze nicht losgelöst von diesen Rahmenbedingungen betrachtet werden.

\subsection{Deutschland aus wohlfahrtsstaatlicher Perspektive}

Zur Einordnung des deutschen, institutionellen Kontextes wird Deutschland aus der Perspektive der Wohlfahrtsstaatentypologie nach Esping-Andersen $(1990,1999)$ betrachtet, denn im internationalen Vergleich zeigen sich Länderunterschiede im Hinblick auf vorherrschende Geschlechterrollen, Geschlechterpolitiken und typische Arbeitsteilungsmuster besonders deutlich. Hierfür wird zunächst in aller Kürze die Wohlfahrtsstaatentypologie von Esping-Andersens skizziert. Da diese Typologie jedoch auch stark kritisiert und weiterentwickelt wurde, werden im Anschluss diese Kritikpunkte und Weiterentwicklungen diskutiert.

Die Typologie von Wohlfahrtsregimen nach Esping-Andersen $(1990,1999)$ unterscheidet zwischen liberalen, sozialdemokratischen und konservativen Wohlfahrtsregimen. Dabei handelt es sich um Idealtypen von Wohlfahrtsstaaten, die sich im Verhältnis von Staat, Familie und Markt sowie im Dekommodifizierungsgrad und der Art der Stratifizierung unterscheiden.

Der Grad der Dekommodifizierung gibt an, in welchem Umfang die Wohlfahrt der Individuen vom Markt abhängig ist, d.h. inwiefern es auch Möglichkeiten der Wohlfahrtsproduktion und der sozialen Sicherung gibt, die außerhalb des Marktes liegen (Esping-Andersen 1998, S. 36). Gemeint sind damit sozialpolitische Maßnahmen, anhand derer die Individuen - etwa im Fall einer Erkrankung - soziale Absicherung erfahren und zu denen sie unabhängig von ihrer Marktposition Zugang haben (Esping-Andersen 1990, 21-23, 37). Beispiele einer Form der Dekommodifizierung sind Lohnersatzzahlungen (z.B. bei 
Krankheit, Elternschaft oder im Alter) sowie Dienstleistungen (z.B. für die Kinderbetreuung oder Altenbetreuung). Entscheidend ist hierbei, dass der Verteilungsmechanismus von der Arbeitsmarktposition der Individuen entkoppelt ist, denn

„die bloße Existenz von Sozialfürsorge und Sozialversicherung [bringt] nicht notwendigerweise auch eine spürbare De-Kommodifizierung mit sich (...), solange sie die Individuen nicht substantiell von ihrer Marktabhängigkeit befreit" (Esping-Andersen 1998, S. 36).

Ein weiteres Distinktionsmerkmal ist die soziale Form der Stratifizierung, d.h. die Art und Weise wie eine Schichtung innerhalb der Gesellschaft hergestellt wird, denn der

„Wohlfahrtsstaat ist nicht allein ein Instrument zur Beeinflussung und gegebenenfalls Korrektur der gesellschaftlichen Ungleichheitsstruktur. Er stellt vielmehr ein eigenständiges System der Stratifizierung dar, indem er in aktiver und direkter Weise soziale Beziehungsmuster ordnet“" (Esping-Andersen 1998, S. 39).

Die Art der Stratifizierung beschreibt, inwiefern Wohlfahrtsstaaten die Produktion und Reproduktion von sozialer Schichtung hemmen oder fördern und inwiefern sie es den Individuen ermöglichen zwischen den Schichten zu wechseln. Die Stratifizierung ist in Tabelle 5.1 anhand des Kriteriums der dominanten Form sowie des dominanten Ortes der Solidarität abgebildet. Als Form der Solidarität kann zwischen bedarfsgeprüften Fürsorgeleistungen, Sozialversicherungsmodellen und universalistischen Systemen unterschieden werden (Esping-Andersen 1998, S. 41). Während die bedarfsgeprüften Fürsorgeleistungen ebenso wie die Sozialversicherungsmodelle eine Stigmatisierung und Statusdifferenzierung begünstigen, sind universalistische Systeme auf Statusgleichheit ausgerichtet:

„Alle Bürger werden [im universalistischen System], unabhängig von ihrer Klassenzugehörigkeit oder Marktstellung, mit ähnlichen Rechten ausgestattet. In diesem Sinne zielt das System darauf ab, Solidarität zwischen den Klassen hervorzurufen, eine Solidarität der Nation“" (Esping-Andersen 1998, S. 41).

Im Folgenden wird skizziert, welches Verhältnis von Staat, Familie und Markt, welcher Dekommodifizierungsgrad und welche Art der Stratifizierung für die jeweiligen Wohlfahrtsstaaten typisch sind. Die Idealtypen sind mit ihren typischen Ausprägungen zusammenfassend in Tabelle 5.1 dargestellt. 
Tabelle 5.1: Zusammenfassung der Regimetypen nach Esping-Andersen

\begin{tabular}{|c|c|c|c|}
\hline & Liberal & $\begin{array}{l}\text { Sozialdemo- } \\
\text { kratisch }\end{array}$ & Konservativ \\
\hline \multicolumn{4}{|l|}{ Rolle von: } \\
\hline Familie & Marginal & Marginal & Zentral \\
\hline Arbeitsmarkt & Zentral & Marginal & Marginal \\
\hline Staat & Marginal & Zentral & Subsidiär \\
\hline \multicolumn{4}{|l|}{ Wohlfahrtsstaaten: } \\
\hline $\begin{array}{l}\text { Dominante Form } \\
\text { der Solidarität }\end{array}$ & Individuell & Universell & $\begin{array}{l}\text { Verwandtschaft } \\
\text { Korporatismus } \\
\text { Etatismus }\end{array}$ \\
\hline $\begin{array}{l}\text { Dominanter Ort } \\
\text { der Solidarität }\end{array}$ & Arbeitsmarkt & Staat & Familie \\
\hline $\begin{array}{l}\text { Grad der Dekom- } \\
\text { modifizierung }\end{array}$ & Minimal & Maximal & $\begin{array}{l}\text { Hoch (für Haupt- } \\
\text { verdiener) }\end{array}$ \\
\hline Beispiele & USA & Schweden & $\begin{array}{l}\text { Deutschland } \\
\text { Italien }\end{array}$ \\
\hline
\end{tabular}

Quelle: Esping-Andersen 1999, S. 85, ins Deutsche übersetzt.

Für den liberalen Wohlfahrtsstaat ist charakteristisch, dass der Arbeitsmarkt die zentrale Instanz darstellt. D.h. er nimmt im Hinblick auf das Wohlergehen der Bürger die wichtigste Rolle ein und gilt als Hauptgarant für soziale Sicherung. Der Familie und dem Staat kommt für die Wohlfahrtsproduktion dagegen nur eine nebensächliche Rolle zu. Im liberalen Wohlfahrtsstaat gibt es eine „bedarfsgeprüfte Sozialfürsorge, niedrige universelle Transferleistungen und ebenso bescheidene Sozialversicherungsprogramme“, die sich in erster Linie an die Arbeiterschicht richten (Esping-Andersen 1998, S. 43). Dabei sind die Fürsorgeleistungen „residual, d.h. sie sollen erst dann gewährt werden, wenn die Kapazität zur Selbsthilfe erschöpft ist" (Kulawik 2005, S. 6; Hervorhebung im Original). Die Inanspruchnahme solcher Fürsorgeleistungen ist oftmals mit einer Stigmatisierung verbunden, sodass Individuen bestrebt sind, eine Inanspruchnahme zu verhindern. Beim liberalen Typ wird der Markt in mehrerlei Hinsicht gefördert, etwa „in passiver Form, indem er nur minimale Leistungen bietet, oder aber in aktiver Weise durch die Subventionierung privater Sicherungsformen“ (Esping-Andersen 1998, S. 43). Dementsprechend ist der Grad der Dekommodifizierung hier nur minimal ausgeprägt, so dass die Wohlfahrtsproduktion der Individuen weiterhin sehr stark von ihrer Arbeitsmarktposition abhängig ist. Beispiele für einen liberalen Wohlfahrtsstaat sind die USA, Kanada und Australien.

Der sozialdemokratische Wohlfahrtsstaat zeichnet sich dadurch aus, dass hier der Staat die zentrale Instanz darstellt, wohingegen die Familie und der Arbeitsmarkt eine geringere Bedeutung haben. Das oberste Ziel in sozialdemokratischen Wohlfahrtsstaaten ist es, Gleichheit auf höchstem Niveau zu bieten (Esping-Andersen 1998, S. 45). 
„Alle Klassen und Schichten sind in ein einziges, universelles Versicherungssystem einbezogen; gleichwohl orientieren sich die Leistungen am vorherigen Einkommen. Dieses Modell drängt den Markt zurück und sorgt so für eine ihrem Wesen nach universale Solidarität (...). Jeder profitiert, jeder ist abhängig - und jeder wird sich voraussichtlich zum Zahlen verpflichtet fühlen“ (Esping-Andersen 1998, S. 45).

Folglich ist der Dekommodifizierungsgrad hier sehr hoch ausgeprägt. Die sozialen Fürsorgeleistungen sind im Vergleich zum liberalen Wohlfahrtsstaat viel besser ausgebaut und der Zugang zu denselben Rechten ist schichtunabhängig garantiert. Als Beispiel für einen sozialdemokratischen Wohlfahrtsstaat seien hier Finnland, Norwegen und Schweden genannt.

Beim konservativen Wohlfahrtsstaat ist die Familie von zentraler Bedeutung für die Wohlfahrtsproduktion der Individuen. Der Staat wirkt lediglich subsidiär, d.h. er springt nur dann stellvertretend ein, wenn die Mechanismen der Familie versagen und auf diesem Wege keine Selbsthilfe mehr möglich ist (Esping-Andersen 1998, S. 44). Der starke Familienbezug und die Persistenz von traditionellen Normen in konservative Wohlfahrtsstaaten ist u.a. darauf zurückzuführen, dass die Kirche hier noch einen größeren Einfluss als in anderen Typen an Wohlfahrtsstaaten hat. Der konservative Wohlfahrtsstaat ist auf eine Aufrechterhaltung von Statusunterschieden ausgerichtet. Soziale Rechte sind im Gegensatz zu sozialdemokratischen Wohlfahrtsstaaten nicht universell, sondern statusund klassengebunden (Esping-Andersen 1998, S. 44). Der Grad der Dekommodifizierung ist nur moderat ausgeprägt, was sich beispielsweise darin zeigt, dass „[n]ichterwerbstätige Frauen aus der Sozialversicherung ausgeschlossen [sind], familienpolitische Leistungen zur Mutterschaft [ermutigen]“ und „Kindertageseinrichtungen und ähnliche familienbezogene Dienste (...) deutlich unterentwickelt" sind (Esping-Andersen 1998, S. 44). Deutschland, Österreich und Italien stellen Beispiele für einen konservativen Wohlfahrtsstaat dar.

Es gilt zu berücksichtigen, dass die Typologie der Wohlfahrtsstaaten aus den 1990ern stammt, sodass neuere familienpolitische Maßnahmen von Esping-Andersen zum damaligen Zeitpunkt nicht berücksichtigt werden konnten. So ähneln in Deutschland neuere familienpolitische Maßnahme zusehends einem sozialdemokratischen und weniger einem konservativen Wohlfahrtsstaat. Da jedoch andere konservativen Politiken (wie etwa das Ehegattensplitting) weiterhin Bestand haben, ergeben die Politiken in Deutschland zum Teil ein uneindeutiges Bild. Hinzu kommt, dass es in Deutschland große Unterschiede zwischen West- und Ostdeutschland gibt: Während Esping-Andersen Deutschland als konservativen Wohlfahrtsstaat einordnet, erscheint eine differenzierte Betrachtung von West- und Ostdeutschland angebracht, da lediglich Westdeutschland dem konservativen Wohlfahrtsstaat entspricht, wohingegen in Ostdeutschland ein sozialdemokratischer Wohlfahrtsstaat vorliegt (Pfau-Effinger 2005). Auf diese Unterschiede zwischen Ost- und Westdeutschland wird im nachfolgenden Kapitel näher eingegangen.

Die Typologie der Wohlfahrtsstaaten von Esping-Andersen bietet ein hilfreiches Instrument zur Verortung der für Deutschland typischen, institutionellen Rahmenbedingungen 
und Arbeitsteilungsmuster im internationalen Vergleich. Die Wohlfahrtsstaatentypologie wurde jedoch auch in vielerlei Hinsicht kritisiert und weiterentwickelt. Ein wesentlicher Kritikpunkt bezieht sich auf die Geschlechterblindheit: So lautet der Vorwurf von Feministinnen, dass das Geschlecht in Esping-Andersens Theorie vernachlässigt wird. Lewis, Ostner und Orloff gehörten zu den ersten, die die Dimension Geschlecht in die Typologie integriert haben (Lewis 1992; Orloff 1993; Lewis und Ostner 1994; Ostner 1995). So unterscheidet Lewis zwischen starken, moderaten und schwachen „männlichen Ernährermodellen“. Als Unterscheidungskriterien dienen

„,neben dem Ausmaß der Erwerbsbeteiligung von Müttern das Ausmaß der Abhängigkeit der Frau von einer vom Mann abgeleiteten sozialen Sicherung und die Folgen der jeweils institutionalisierten Norm für Frauen“ (Ostner 2004, S. 53).

Ein starkes „männliches Ernährermodell“, für das eine niedrige Erwerbsbeteiligung von Frauen und Müttern charakteristisch ist und in dem Frauen häufig über ihre Männer mitversichert sind, liegt etwa in Deutschland und Großbritannien vor; ein moderates „männliches Ernährermodell“ mit einer verhältnismäßig hohen Frauen- und Müttererwerbsbeteiligung ist für Frankreich und Belgien typisch; und ein schwaches „männliches Ernährermodell" mit einer sehr hohen Erwerbsbeteiligung von Frauen und Mütter ist schließlich für Länder wie Schweden, Finnland und Dänemark kennzeichnend (Lewis 1992).

Aber nicht nur Frauen, sondern auch Familien - so die Kritik von Feministinnen - seien bei Esping-Andersen nicht adäquat berücksichtigt. Stattdessen stehe bei ihm „das Potential wohlfahrtsstaatlicher Aktivität" im Vordergrund (Ostner 1998, S. 228; Hervorhebung im Original). Dies wird auch daran ersichtlich, dass Esping-Andersens

„Begriff vom Wohlfahrtsstaat (...) der Familie und inrem Versorgungspotential tatsächlich kein Eigengewicht zu[gesteht], das dem von Markt und Staat vergleichbar wäre. Nicht die Familie, sondern das Ausmaß ihrer Markt- oder Staatsintegration interessiert - sei es in Gestalt der Frauenerwerbsarbeit oder der öffentlicher Dienstleistungsproduktion“ (Ostner 1998, S. 228).

Ein weiterer Kritikpunkt bezieht sich auf Esping-Andersens Annahme der kontinuierlichen Vollzeiterwerbstätigkeit und den damit einhergehenden sozialen Rechten. Frauen tauchen in dieser Typologie nur auf, sofern sie erwerbstätig sind: „Bürgerrechte werden so verkürzt auf Rechte von Erwerbsbürgern“ (Ostner 1998, S. 229; Hervorhebung im Original). Die unbezahlte Sorgearbeit von Frauen bleibt hierbei jedoch unreflektiert.

Ebenso kritisch sieht die feministische Forschung die Kategorie der De-Kommodifizierung, d.h. die Abfederung des Ausstiegs aus dem Arbeitsmarkt in Form von Lohnersatzleistungen. Denn um de-kommodifizieren zu können muss zunächst eine Kommodifizierung, also ein Einstieg in den Arbeitsmarkt, stattgefunden haben. Bei Frauen ist die Kommodifizierung jedoch erschwert, da sie mit unbezahlter Familienarbeit belastet sind. Prozesse der Kommodifizierung und De-Kommodifizierung stammen deshalb vor allem aus einer androzentrischen Sichtweise (Orloff 1993, S. 307; Ostner 1998, S. 229). 
Wie sehr Frauen in die Familienarbeit eingebunden und von den Familienverhältnissen abhängig bzw. davon losgelöst sind, wurde durch die Einführung der Begriffe „Familialismus“ bzw. „De-Familialisierung“ greifbar gemacht. Zwischen der (De-)Kommodifizierung und der (De-)Familialisierung besteht dabei folgender Zusammenhang: Damit Frauen in gleicher Weise wie Männer in den Arbeitsmarkt integriert werden können, müssen sie zunächst „kommodifiziert“ werden, d.h. beschäftigungsfähig gemacht werden (Leitner et al. 2004, S. 16). Hierfür müssen Frauen jedoch „von der Pflicht, zuhause für Kinder und ältere Familienangehörige zu sorgen, befreit, also ,de-familialisiert" werden“ (Leitner et al. 2004, S. 16). Eine „(Re-)Kommodifizierung“ von Frauen bedarf also einer „De-Familialisierung“, wobei ein „Mehr an De-Familialisierung [gleichzeitig] ein Weniger an Familialisierung“ bedeutet (Leitner et al. 2004, S. 17).

Esping-Andersen nahm sich dieser Kritik an und übernahm 1999 die Konzepte der Familialismus und der De-Familialisierung als weitere Distinktionsmerkmale für die Typologie der Wohlfahrtsstaaten. Um die Bedeutung der Familie hervorzuheben, spricht er nun auch nicht mehr von Wohlfahrtsstaaten, sondern von Wohlfahrtsregimen (Esping-Andersen 1999, S. 34-35).

„A welfare regime can be defined as the combined, interdependent way in which welfare is produced and allocated between state, market and family" (Esping-Andersen 1999, S. 34-35).

In konservativen Wohlfahrtsregimen ist der Grad der Dekommodifizierung gering und die Betreuung und Pflege von Familienangehörigen wird als Aufgabe der Frauen und Mütter aufgefasst. Folglich ist hier die De-Familialisierung äußerst gering ausgeprägt. In liberalen Wohlfahrtsregimen erfolgt die Defamilialisierung über marktabhängige Dienstleistungen. Mit Abstand am stärksten ausgeprägt ist die De-Familialisierung in sozialdemokratischen Wohlfahrtsstaaten. Leitner (2003) hat die Idee der (De-)Familialisierung weiterentwickelt, indem sie Varianten des Familialismus aufgedeckt hat. Sie differenziert zwischen familialisierenden und de-familialisierenden Maßnahmen. Je nach Ausprägung beider Maßnahmen ergeben sich daraus die Varianten des optionalen Familialismus, des expliziten Familialismus, des De-Familialismus sowie des impliziten Familialismus (siehe Tabelle 5.2).

Tabelle 5.2: $\quad$ Varianten von Familialismus nach Leitner

\begin{tabular}{llc}
\hline \multirow{2}{*}{$\begin{array}{l}\text { Familialisierende Maßnah- } \\
\text { men }\end{array}$} & \multicolumn{2}{c}{ De-familialisierende Maßnahmen } \\
& \multicolumn{1}{c}{ stark } & \multicolumn{1}{c}{ schwach } \\
\cline { 2 - 3 } stark & Optionaler Familialismus & Expliziter Familialismus \\
schwach & De-Familialismus & Impliziter Familialismus \\
\hline
\end{tabular}

Quelle: Leitner 2003, S. 358, ins Deutsche übersetzt.

Der explizite Familialismus unterstützt die Familie darin, die Betreuung und Pflege von Familienangehörigen selbst zu übernehmen. Da de-familialisierende Maßnahmen, also 
Maßnahmen, die die Familien davon befreien würden, fehlen, sind die Familien gewissermaßen dazu gezwungen, diese Aufgaben selbst zu übernehmen (Leitner 2003, S. 358-359).

Bei der zweiten Varianten, dem optionalen Familialismus, sind sowohl familialisierende wie auch de-familialisierende Maßnahmen stark ausgeprägt, d.h. Familien haben die Möglichkeit zu wählen: Sie können die Betreuungs- und Pflegearbeit selbst übernehmen oder aber sie externalisieren, sodass sie - im Gegensatz zum expliziten Familialismus - nicht zur Fürsorgearbeit verpflichtet sind (Leitner 2003, S. 359).

Beim impliziten Familialismus sind weder die familialisierenden noch die de-familialisierenden Maßnahmen stark ausgeprägt. Die Übernahme der Betreuung und Pflege ist hier primär in der Familie angesiedelt, was auf einen Mangel an Alternativen zurückzuführen ist (Leitner 2003, S. 359).

Der vierte und letzte Typus von Leitner, der De-Familialismus, ist durch starke de-familialisierende Maßnahmen gekennzeichnet. Aufgrund der fehlenden oder zumindest nur schwach ausgeprägten familialisierenden Maßnahmen sind Familien einerseits vom Betreuungs- und Pflegeaufwand befreit; andererseits sind Familien damit auch zugleich von einem Recht auf Fürsorgearbeit in der Familie ausgeschlossen (Leitner 2003, S. 359).

In Tabelle 5.3 sind unterschiedliche familienpolitische Cluster abgetragen sowie Länderbeispiele, in denen die entsprechenden familienpolitischen Cluster präsent sind. So lässt sich Deutschland etwa als ein Land mit einem starken Ernährermodell beschreiben und mit einem expliziten Familialismus (vgl. Tabelle 5.3).

Tabelle 5.3: $\quad$ Familienpolitische Cluster

\begin{tabular}{lllll}
\hline & I & II & III & V \\
\hline Lewis & Schwaches & Moderates Er- & Starkes Er- & \\
(1992), & Ernährermo- & nährermodell: & nährer- \\
Ostner & dell: & Belgien & modell: & \\
(1995) & Dänemark & Frankreich & Deutschland & \\
& Finnland & & Irland & \\
& Schweden & & Niederlande & \\
& & & Großbritan- & \\
& & & nien & \\
Esping- & Sozialdemo- & Liberal: & Konservativ: & Südeuropa: \\
(1999) & kratisch: & Australien & Kontinental- & Italien \\
& Dänemark & Kanada & europa & Portugal \\
& Finnland & Irland & Österreich & Spanien \\
& Norwegen & Großbritan- & Belgien & \\
& Schweden & nien & Frankreich & \\
& & USA & Deutschland & \\
& & & Niederlande &
\end{tabular}




\begin{tabular}{|c|c|c|c|c|c|}
\hline & I & II & III & IV & $\mathbf{V}$ \\
\hline $\begin{array}{l}\text { Leitner } \\
\text { (2003) }\end{array}$ & $\begin{array}{l}\text { Optionaler } \\
\text { Familialis- } \\
\text { mus: } \\
\text { Belgien } \\
\text { Dänemark } \\
\text { Finnland } \\
\text { Frankreich } \\
\text { Schweden }\end{array}$ & $\begin{array}{l}\text { Expliziter } \\
\text { Familialis- } \\
\text { mus: } \\
\text { Österreich } \\
\text { Deutschland } \\
\text { Italien } \\
\text { Luxemburg } \\
\text { Niederlande }\end{array}$ & $\begin{array}{l}\text { De-Familialis- } \\
\text { mus: } \\
\text { Irland } \\
\text { Großbritan- } \\
\text { nien }\end{array}$ & $\begin{array}{l}\text { Impliziter } \\
\text { Familialis- } \\
\text { mus: } \\
\text { Griechenland } \\
\text { Portugal } \\
\text { Spanien }\end{array}$ & \\
\hline $\begin{array}{l}\text { Pfau- } \\
\text { Effinger } \\
(2005)\end{array}$ & $\begin{array}{l}\text { Dual bread- } \\
\text { winner family } \\
\text { model: } \\
\text { Frankreich } \\
\text { Dänemark } \\
\text { Schweden } \\
\text { Finnland }\end{array}$ & $\begin{array}{l}\text { Modernisa- } \\
\text { tion of the } \\
\text { male bread- } \\
\text { winner } \\
\text { model: } \\
\text { Großbritan- } \\
\text { nien } \\
\text { Norwegen } \\
\text { Niederlande } \\
\text { Westdeutsch- } \\
\text { land }\end{array}$ & & & \\
\hline $\begin{array}{l}\text { Thévenon } \\
\text { (2011) }\end{array}$ & $\begin{array}{l}\text { Fortlaufende } \\
\text { starke Unter- } \\
\text { stützung be- } \\
\text { rufstätiger El- } \\
\text { tern mit Kin- } \\
\text { dern unter } 3 \\
\text { Jahren: } \\
\text { Dänemark } \\
\text { Finnland } \\
\text { Island } \\
\text { Norwegen } \\
\text { Schweden }\end{array}$ & $\begin{array}{l}\text { Starke finan- } \\
\text { zielle Unter- } \\
\text { stützung, je- } \\
\text { doch einge- } \\
\text { schränkte Un- } \\
\text { terstützung } \\
\text { von Doppel- } \\
\text { verdienern } \\
\text { mit Kindern } \\
\text { unter } 3 \text { Jah- } \\
\text { ren: } \\
\text { Österreich } \\
\text { Belgien } \\
\text { Frankreich } \\
\text { Deutschland } \\
\text { Luxemburg } \\
\text { Niederland }\end{array}$ & $\begin{array}{l}\text { Kurze Eltern- } \\
\text { zeit, gezielte } \\
\text { Unterstützung } \\
\text { geringverdie- } \\
\text { nender Allein- } \\
\text { verdienender } \\
\text { u. von Fami- } \\
\text { lien mit Kin- } \\
\text { dern im Vor- } \\
\text { schulalter: } \\
\text { Australien } \\
\text { Kanada } \\
\text { Irland } \\
\text { Neuseeland } \\
\text { Schweiz } \\
\text { Großbritan- } \\
\text { nien } \\
\text { USA }\end{array}$ & $\begin{array}{l}\text { Einge- } \\
\text { schränkte Un- } \\
\text { terstützung } \\
\text { von Familien: } \\
\text { Griechenland } \\
\text { Italien } \\
\text { Korea } \\
\text { Japan } \\
\text { Portugal } \\
\text { Spanien }\end{array}$ & $\begin{array}{l}\text { Lange Eltern- } \\
\text { zeit, aber ge- } \\
\text { ringe finanzi- } \\
\text { elle Unterstüt- } \\
\text { zung u. we- } \\
\text { nige Betreu- } \\
\text { ungseinrich- } \\
\text { tungen für } \\
\text { Kinder unter } \\
3 \text { Jahren: } \\
\text { Tschechische } \\
\text { Republik } \\
\text { Ungarn } \\
\text { Polen } \\
\text { Slowakische } \\
\text { Republik }\end{array}$ \\
\hline
\end{tabular}

Quelle: Schleutker 2014, S. 163.

Es gilt jedoch zu berücksichtigen, dass sich diese Einordnungen v.a. auf die 1990er und 2000er Jahre beziehen. Die deutsche Familienpolitik hat sich in den letzten Jahren jedoch stark verändert und einen sog. „Paradigmenwechsel“ (Schutter und Zerle-Elsäßer 2012, S. 219; Bujard 2013, S. 133; Menke und Klammer 2017, S. 213) eingeschlagen. Deutschland hat sich dadurch in verschiedenen, familienpolitischen Aspekten mehr in Richtung eines sozialdemokratischen Wohlfahrtsstaats entwickelt. Auf die neuere Familienpolitik wird in Kapitel 5.4 vertiefend eingegangen. Zuvor wird jedoch im folgenden Kapitel noch näher auf das Konzept des „Geschlechterarrangements“ von Pfau-Effinger 
eingegangen, aus dem gut hervorgeht, dass neben den wohlfahrtsstaatlichen Aspekten auch Normen, Werte und kulturelle Einstellungen eine wichtige Rolle spielen.

\subsection{Geschlechterarrangements und kulturelle Leitbilder in Ost- und Westdeutschland}

Bei Pfau-Effingers Konzept der „Geschlechterarrangements“ stehen sowohl Institutionen (wie der Arbeitsmarkt, die Familie und der Wohlfahrtsstaat) als auch kulturelle Leitbilder im Mittelpunkt. Hierbei differenziert sie zwischen den „Geschlechterarrangements“, der "Geschlechterkultur" und der "Geschlechterordnung" und beleuchtet, wie diese zueinander im Verhältnis stehen (Pfau-Effinger 1997, 2000, 2005).

Unter "Geschlechterkultur" sind "dominierende Werte und Leitbilder in Bezug auf Geschlechterbeziehungen" zu verstehen (Pfau-Effinger 2005, S. 3). Dies inkludiert auch Vorstellungen darüber, wie die Arbeitsteilung innerhalb der Familie von statten gehen soll oder welches Geschlecht die primäre Verantwortung für die Betreuung und Pflege sowohl der jüngeren als auch der älteren Generation übernehmen soll. Diese kulturellen Leitbilder sind anhand „von Normen im institutionellen System verankert und deshalb relativ stabil“ (Pfau-Effinger 2005, S. 3). Die vorherrschenden, kulturellen Leitbilder können sich von Land zu Land unterscheiden. Internationale Unterschiede in der Erwerbsbeteiligung von Frauen und Müttern führt Pfau-Effinger demzufolge auf unterschiedliche Geschlechterkulturen zurück (Pfau-Effinger 2000). Die „Geschlechterordnung" bezieht sich auf die Ebene der Institutionen und lässt sich wie folgt beschreiben:

„Die Geschlechterordnung umfaßt [sic!] die real vorfindlichen Strukturen des Geschlechterverhältnisses und die Beziehungen zwischen den verschiedenen gesellschaftlichen Institutionen im Hinblick auf die geschlechtsspezifische Arbeitsteilung“ (Pfau-Effinger 1997, S. 517-518).

Als besonders relevante Institutionen nennt Pfau-Effinger den Arbeitsmarkt, die Familie (bzw. den Haushalt) und den Wohlfahrtsstaat (Pfau-Effinger 1997, S. 518). Das Geschlechterarrangement bildet nun gewissermaßen eine Klammer um die Geschlechterkultur und die Geschlechterordnung und verknüpft beides miteinander (Pfau-Effinger 1997, 2000, 2005). Oder anders formuliert: Das Geschlechterarrangement basiert auf der Geschlechterkultur und der Geschlechterordnung und wenn sich die Geschlechterkultur und die Geschlechterordnung verändern, so verändert sich dementsprechend auch das Geschlechterarrangement (siehe Abbildung 5.1). 
Abbildung 5.1: Das Geschlechterarrangement nach Pfau-Effinger

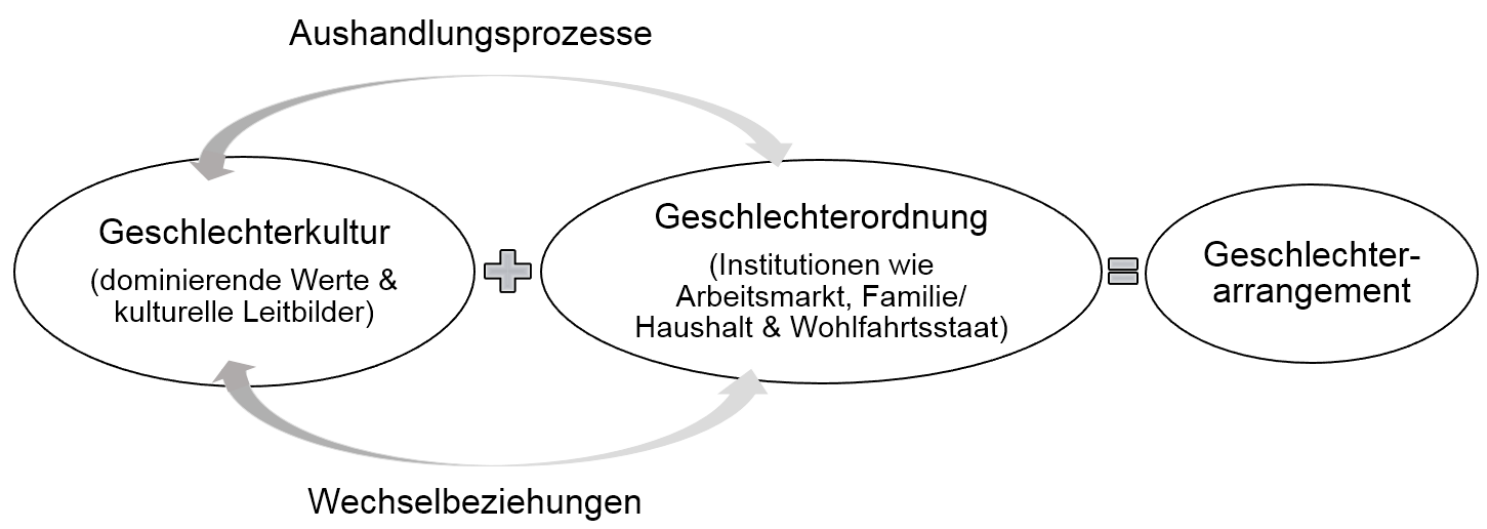

Quelle: eigene Darstellung.

Welche kulturellen Leitbilder dominieren und wie sich Institutionen hierzu verhalten ist das Ergebnis von Aushandlungs- und Kompromissprozessen zwischen sozialen Akteuren. Beides steht zudem in Wechselbeziehung zueinander, d.h. die Geschlechterkultur beeinflusst die Geschlechterordnung und umgekehrt beeinflusst auch die Geschlechterordnung die Geschlechterkultur. Geschlechterkultur und -ordnung werden von sozialen Akteuren vermittelt und stimmen deshalb in der Regel weitestgehend überein. Dass dies aber nicht zwangsläufig so sein muss, wird anhand von sog. „institutional lags“ und „cultural lags“ ersichtlich (Pfau-Effinger 1998). Bei „institutional lags“ hinken die Institutionen zeitlich hinterher und können sich einer wandelnden Geschlechterkultur nicht im gleichen Tempo anpassen. Als Beispiel sei hier eine Geschlechterkultur genannt, in der Frauen eine hohe Erwerbsorientierung haben, während die Familienpolitik für ein traditionelles Familienmodell mit einem männlichen Alleinversorger plädiert und deshalb Institutionen wie etwa Kinderbetreuungseinrichtungen nur unzureichend ausgebaut sind. Umgekehrt verhält es sich bei den „cultural lags“: Hier sind die Institutionen schon weiterentwickelt und auf kulturelle Leitbilder ausgerichtet, die noch nicht in der Mitte der Gesellschaft angekommen sind (Pfau-Effinger 1998).

Ein Kritikpunkt, den Pfau-Effinger an der Wohlfahrtsstaatentypologie äußert, ist die Eindimensionalität, d.h. dass ein Wohlfahrtsstaat komplett einem Typ zugeordnet wird. Stattdessen schlägt sie eine zweidimensionale Klassifizierung vor, deren erste Dimension das Wohlfahrtsregime abbildet und deren zweite Dimension die Geschlechterpolitik wiedergibt (Pfau-Effinger 2005, S. 2). Das Wohlfahrtsregime beinhaltet die Politik zur Erwerbsarbeit und der Dekommodifizierung im Alter oder bei Krankheit und Arbeitslosigkeit (Pfau-Effinger 2005, S. 3), während es sich bei der zweiten Dimension, der Geschlechterpolitik, um Politiken zu Geschlechterbeziehungen, Familien und Fürsorgeleistungen handelt. Indem man beide Dimensionen gemeinsam betrachtet, erhält man differenziertere Einblicke als bei einer eindimensionalen Betrachtung: So ist Deutschland insgesamt als konservatives Wohlfahrtsregime zu klassifizieren, aber insbesondere bei den Geschlechterpolitiken bewegt sich Deutschland weg vom konservativen Wohlfahrtsregime (Pfau-Effinger 2005, S. 7). An späterer Stelle in Kapitel 5.4 wird noch genauer 
auf die neueren Familienpolitiken in Deutschland eingegangen. Hierbei wird ersichtlich, dass die Familienpolitik in Deutschland seit 2007 einen deutlich egalitäreren Kurs eingeschlagen hat. Zunächst wird jedoch noch ein Blick auf die Unterschiede zwischen Ostund Westdeutschland geworfen und anschließend werden familiale Leitbilder vorgestellt.

Kulturelle Leitbilder in Ost- und Westdeutschland

Auch mehrere Jahrzehnte nach der Wiedervereinigung von Ost- und Westdeutschland gibt es in der Aufteilung von Erwerbsarbeit, Hausarbeit und Kinderbetreuung noch eklatante Unterschiede zwischen den beiden Bundesgebieten. Zwar unterscheiden sich kinderlose Frauen in Ost- und Westdeutschland in ihren Erwerbsumfängen kaum voneinander - nach dem Übergang zur Elternschaft ist der durchschnittlich Erwerbsumfang von westdeutschen Müttern mit nur 15,7 Wochenstunden jedoch deutlich geringer als der von ostdeutschen Müttern mit 22,4 Wochenstunden (BMFSFJ 2014a, S. 41). Während in Ostdeutschland die Externalisierung der Kinderbetreuung weit verbreitet ist, überwiegt in Westdeutschland in den ersten Lebensjahren des Kindes die innerfamiliale Kinderbetreuung durch eine nicht-erwerbstätige oder nur in sehr geringem Umfang erwerbstätige Mutter. Betrachtet man schließlich die Aufteilung der Hausarbeit, so zählt Ostdeutschland im internationalen Vergleich zu den Ländern mit der egalitärsten Aufteilung, Westdeutschland dagegen zu denjenigen mit der traditionellsten Aufteilung (Grunow 2014, S. 237).

Zur Skizzierung dieser Unterschiede zwischen Ost- und Westdeutschland wird im Folgenden sowohl auf die Typologie der Wohlfahrtsregime als auch auf die darauf aufbauenden theoretischen Weiterentwicklungen, wie etwa dem Geschlechterarrangement, zurückgegriffen.

Das (west-)deutsche Bundesgebiet gilt - zumindest nach der ursprünglichen, inzwischen etwas veralteten Einordnung nach Esping-Andersen - als ein konservativer Wohlfahrtsstaat. ${ }^{5}$ Es ist durch ein traditionelles Familienbild geprägt, in dem die Betreuung und Pflege von Familienangehörigen primär innerhalb der Familie verortet ist (Esping-Andersen 1999; Gerhards und Rössel 2000; Pfau-Effinger 2005). Männer übernehmen die Funktion des Familienernährers: Sie sind in Vollzeit erwerbstätig und es sind keine Unterbrechungen der Erwerbstätigkeit im Lebenslaufzyklus vorgesehen; Frauen sind dagegen - insbesondere nach der Familiengründung - häufig nur in reduziertem Umfang erwerbstätig und unterbrechen ihre Erwerbstätigkeit, wenn es die familialen Umstände fordern (Rosenfeld et al. 2004, S. 111; Pfau-Effinger und Smidt 2011, S. 222). Der Staat

\footnotetext{
${ }^{5}$ Auf die neuere, deutsche Familienpolitik wird in Kapitel 5.4 näher eingegangen. An dieser Stelle sei nur angemerkt, dass es einige, neuere Regelungen gibt (wie etwa das ElterngeldPlus oder der intensive Ausbau von U3-Kinderbetreuungseinrichtungen), die zeigen, dass sich Deutschland von einem konservativen Wohlfahrtsstaat wegentwickelt und in einigen Aspekten einem sozialdemokratischen Wohlfahrtsstaat ähnlicher wird.
} 
unterstützt dieses traditionelle Familienmodell durch „familialisierende Maßnahmen“ (Esping-Andersen 1999) wie dem Ehegattensplittung, einer langen Elternzeit und schlecht ausgebauten, öffentlichen Kinderbetreuungsplätzen für Kinder unter drei Jahren (Kreyenfeld und Geisler 2006, S. 335; Hummelsheim 2009, S. 71; Grunow und Müller 2012, S. 56). Eine geschlechtsspezifische Arbeitsteilung, in der sich Männer auf die Erwerbsarbeit und Frauen auf die Hausarbeit und Kinderbetreuung fokussieren, wird somit explizit unterstützt und alternative Modelle wie eine egalitäre Arbeitsteilung gleichzeitig erschwert.

Im Gegensatz dazu sind in Ostdeutschland noch die Folgen der ehemaligen DDR zu spüren, die als sozialdemokratischer Wohlfahrtsstaat galt. In der DDR zählte die Gleichberechtigung der Frauen zu den obersten, politischen Zielen. Dementsprechend stellte eine hohe Frauenerwerbstätigkeit die Norm dar - selbst nach dem Übergang zur Elternschaft (Kreyenfeld und Geisler 2006, S. 333; Grunow und Müller 2012, S. 56). Dies wurde anhand von gut ausgebauten und ganztägig geöffneten Kinderbetreuungseinrichtungen ermöglicht. Da die erwerbstätige Mutter in der DDR dem Familienleitbild entsprach, sah sie sich deswegen auch nicht wie in Westdeutschland mit dem Stigma einer schlechten Mutter konfrontiert.

Kreyenfeld und Geisler (2006) haben in ihrer Studie untersucht, inwiefern sich die Müttererwerbstätigkeit und die kulturellen Leitbilder in Ost- und Westdeutschland auch Jahrzehnte nach der Wiedervereinigung noch unterscheiden. Als Ausgangsbasis verwenden sie die Frage danach, „ob ein Kleinkind darunter leidet, wenn seine Mutter erwerbstätig ist" (Kreyenfeld und Geisler 2006, S. 338). Die Ergebnisse zeigen, dass im Zeitraum von 1992 bis 2004 die Einstellungen zu einer erwerbstätigen Mutter zwar in beiden Bundesgebieten liberaler geworden sind. Dennoch gibt es weiterhin starke Differenzen zwischen Ost und West: Während im Jahr 2004 nur 8\% der Ostdeutschen der Meinung sind, dass das Kleinkind einer erwerbstätigen Mutter leidet, sind 31\% der Westdeutschen dieser Meinung (Kreyenfeld und Geisler 2006, S. 339). Dass die Einstellungen in Ost- und Westdeutschland nicht konvergieren, sondern sogar noch größer werden, hat eine Studie von Lee et al. (2007) herausgefunden.

Die Situation der Wiedervereinigung, in der Ostdeutschland die westdeutsche Familienpolitik wie auch sämtliche anderen westdeutschen Regelungen übergestülpt bekam, wird als ideales, quasi-natürliches Experiment betrachtet, anhand dessen die Einflüsse von kulturellen und institutionellen Strukturen untersucht werden können (Rosenfeld et al. 2004). Grunow und Müller (2012) kritisieren jedoch, dass bei diesen Ost-West-Vergleichen das Potential der Vergleichbarkeit eingeschränkt ist, da sich Ost- und Westdeutschland weiterhin in strukturellen Rahmenbedingungen wie beispielsweise der Verfügbarkeit von Kinderbetreuungsplätzen unterscheiden, so dass unterschiedliche Verhaltensweisen nicht automatisch anhand kultureller Unterschiede erklärt werden können (Grunow und Müller 2012, S. 56-57). Um den Einfluss von kulturellen und strukturellen Faktoren bei der Frage nach der Rückkehr von Müttern in die Erwerbstätigkeit besser 
voneinander trennen zu können, analysieren Grunow und Müller (2012) ostdeutsche, westdeutsche und ost-west-mobile Mütter. Auf diese Weise kann analytisch zwischen kulturellen, im Sozialisationsprozess erworbenen Einstellungen und den strukturellen Rahmenbedingungen differenziert werden. Sie kommen zu dem Ergebnis, dass ostwest-mobile Mütter wieder schneller in die Erwerbsarbeit zurückkehren als westdeutsche Mütter, aber langsamer zurückkehren als ostdeutsche Mütter. Damit wird ersichtlich, wie sich sowohl strukturelle Rahmenbedingungen als auch kulturelle Leitbilder auf die Dauer der Erwerbsunterbrechung beim Übergang zur Elternschaft auswirken (Grunow und Müller 2012).

Dass sich die Unterschiede zwischen Ost- und Westdeutschland nach wie vor so hartnäckig halten, wird somit auch auf unterschiedliche kulturelle Leitbilder zurückgeführt. In Westdeutschland mit der „männlichen Versorgerehe“ (Pfau-Effinger 2005, S. 4) hält sich das Ideal einer „mutterzentrierten Erziehung“ (Gerhards und Rössel 2000, S. 250). Kindheit wird als „private Kindheit“ inszeniert, die zu Hause im Rahmen der Familie stattfindet und durch viel gemeinsame Zeit mit der Familie gekennzeichnet ist (Pfau-Effinger 2005, S. 4). In Ostdeutschland herrscht dagegen das „Doppelversorgermodell mit externer Kinderbetreuung" (Pfau-Effinger 2005, S. 5). Es hat sich zu einem Leitbild entwickelt, da dieses Modell zu DDR-Zeiten vom Staat massiv propagiert wurde und es keine Alternativen gab, sodass die Bevölkerung gezwungen war, dieses Modell anzunehmen. Dass dieses Modell zum Leitbild werden konnte, liegt somit zum einen in der Monopolstellung dieses Modells begründet, zum anderen aber auch darin, dass mit diesem Modell schlussendlich auch positive Erfahrungen gemacht wurden (Pfau-Effinger 2005, S. 5). Im Folgenden wird näher darauf eingegangen, wie (Familien-)Leitbilder definiert werden können, welche Familienleitbilder in Deutschland präsent sind und welche Rolle sie für die Arbeitsteilung der Eltern spielen.

\subsection{Familienleitbilder}

Leitbilder basieren auf Normen, Werten und Geschlechterrolleneinstellungen und knüpfen dadurch an die bisherigen, normenbasierten Ansätze an. Unter einem Leitbild ist im Allgemeinen

„ein Bündel aus kollektiv geteilten bildhaften Vorstellungen des ,Normalen', das heißt von etwas Erstrebenswertem, sozial Erwünschtem und/oder mutmaßlich weit Verbreitetem, also Selbstverständlichem“ (Diabaté und Lück 2014, S. 56)

zu verstehen. Indem es sich bei Leitbildern um ein Bündel von Normen, Werten und Vorstellungen handelt, weisen sie eine größere Vielfalt auf. Weiter handelt es sich bei Leitbildern um gesellschaftliche Konstrukte, die im Laufe der Sozialisation erworben und internalisiert werden (Mühling et al. 2006). Familienleitbilder sind Leitbilder, die sich auf die Familie beziehen und eine Vorstellung davon sind, 
„wie bestimmte Aspekte von Familie - immer oder ,normalerweise - sind und wie sie dementsprechend auch sein müssten oder ,richtigerweise' sein sollten“ (Diabaté und Lück 2014, S. 56).

Familienleitbilder sind multidimensional, d.h. es gibt nicht nur das eine Leitbild der Familie, sondern vielfältige Leitbilder, die sich auf verschiedene Dimensionen des Familienlebens beziehen (Mühling et al. 2006). Auch können mehrere familiale Leitbilder gleichzeitig aktiv sein. Diese müssen dabei nicht im Einklang zueinanderstehen, sondern können sich auch widersprechen. Ein Beispiel wäre das gleichzeitige Vorhandensein des Leitbilds der guten Mutter (wonach das Kind oberste Priorität hat) und des Leitbilds der berufstätigen, unabhängigen Mutter, die finanziell selbst für sich sorgen kann (Diabaté und Lück 2014). Wenn sich eine Person nach mehreren, widersprüchlichen Familienleitbildern ausrichtet, führt dies zu inneren Konflikten. Eine Möglichkeit, diese „kognitive Dissonanz" (Festinger 1957) abzumildern, besteht darin, die entsprechenden Familienleitbilder anzupassen bzw. die inneren Einstellungen neu auszurichten (Johnston und Swanson 2006). Im Folgenden wird näher auf die Leitbilder zur Elternschaft und insbesondere zur Mutterschaft eingegangen.

\section{Leitbilder zur Mutterschaft}

Ebenso wie die allgemeinen Familienleitbilder sind auch die Leitbilder zur Mutterschaft facettenreicher geworden. Welche Mutterleitbilder in Deutschland aktuell vorherrschend sind, ist noch ziemlich unerforscht. Aus einer Studie von Diabaté (2015) geht hervor, dass die Mutterschaftsleitbilder besonders durch die folgenden, drei Prinzipien geprägt sind (Diabaté 2015, S. 209): durch das Prinzip der Gleichberechtigung innerhalb der Partnerschaft, durch das Prinzip der „verantworteten Elternschaft" (Kaufmann 1990) und durch den „Mythos Mutterliebe“ (Schütze 1986). Letzteres, das Leitbild der Mutterliebe, ist in Deutschland besonders präsent (Diabaté 2015, S. 209). Das Konzept der Mutterliebe nimmt an, dass Mütter eine stärkere Bindung zum Kind haben als Väter, was durch körperliche Vorgänge wie die Geburt oder das Stillen bedingt ist. Es wird angenommen, dass Mütter von Natur aus wissen, was das Kind gerade benötigt (Diabaté 2014).

Ein Leitbild, das die Elemente des Konzepts der Mutterliebe in sich vereint, ist das Leitbild des „intensive mothering“. Der Begriff des „intensive mothering“ geht auf Hays (1996) zurück. Nach Hays (1996) ist Mutterschaft eine natürliche, weibliche Eigenschaft und Mütter sind die primäre Betreuungsperson des Kindes. Mütter tragen die Hauptverantwortung für die Erziehung und die kindliche Entwicklung auf sämtlichen Ebenen (z.B. die emotionale, soziale und intellektuelle Entwicklung). Der „intensive mothering“-Ansatz ist kindzentriert und die Bedürfnisse des Kindes haben Priorität gegenüber den Bedürfnissen der Mutter (Hays 1996). Darüber hinaus zeichnet sich das „intensive mothering“ dadurch aus, dass es arbeitsintensiv, zeitintensiv und finanziell teuer ist (Hays 1996). Aus diesen hohen Anforderungen geht hervor, dass diese kaum neben einer Erwerbstätigkeit in Vollzeit machbar sind, da die ständige, zeitliche Anwesenheit der Mutter erwartet wird. Gleichzeitig sind jedoch auch viele Mütter in Vollzeit erwerbstätig und somit den 
Anforderungen des Arbeitsmarkts ausgesetzt. Erwerbstätige Mütter versuchen trotz der zeitlichen Restriktionen, die mit einer Erwerbstätigkeit verbunden sind, ihr bestmögliches für ihre Kinder zu geben. Diese gleichzeitigen, widersprüchlichen Anforderungen, denen Mütter ausgesetzt sind, bezeichnet Hays als „cultural contradictions“ (Hays 1996, S. 149).

Etliche Studien können aufzeigen, wie verbreitet die Ideologie des „intensive mothering“ bereits ist: Garey (1995) untersuchte in einer qualitativen Studie Krankenschwestern und fand heraus, dass erwerbstätige Mütter häufig die Nachtschicht übernehmen, um tagsüber zu Hause bei den Kindern sein zu können. Trotz ihrer Erwerbstätigkeit versuchen die Mütter somit, das Bild einer „stay-at-home-mom“ aufrechtzuerhalten (Garey 1995, S. 421). Dadurch wird ihre eigene Erwerbstätigkeit öffentlich weniger sichtbar und das Bild der guten Mutter bzw. das „intensive mothering“ kann eher gewahrt werden. Eine qualitative Studie von Johnston und Swanson (2006) belegt, wie sich Erwerbsarbeit und Vorstellungen von „intensive mothering“ gegenseitig beeinflussen, d.h. Mütter passen nicht nur ihre Erwerbsarbeit an das Ideal des „intensive mothering“ an, sondern gleichzeitig verändern sie auch ihre Vorstellungen von „intensive mothering“, um beides besser miteinander in Einklang zu bringen. Aus einer Studie von Papastefanou und Thies (2018) mit deutschen Daten geht hervor, dass die tägliche Zeit, die Mütter für die Kinderbetreuung aufwenden, seit 1991 zugenommen hat. Insgesamt geht aus einem Literaturüberblick zum Thema Mutterschaft hervor, dass „intensive mothering“ den vorherrschenden Diskurs über Mutterschaft darstellt (Liss et al. 2013, S. 624).

In engem Zusammenhang dazu steht auch das "maternal gatekeeping“ (Allen und Hawkins 1999), denn gewissermaßen kann das „Konzept des, intensive mothering ( ...) auch als Nährboden des ,maternal gatekeeping' (...) eingeordnet werden“ (Diabaté und Beringer 2018, S. 298). Das „maternal gatekeeping“ bezeichnet den Umstand, dass Mütter das väterliche Engagement begrenzen wollen, um selbst die primäre Betreuungsperson zu sein. Dem liegt auch die Annahme zugrunde, dass Mütter die besten Betreuungspersonen für das Kind sind und eine anderweitige Betreuung für das Kind schädlich sein könnte. Obwohl sich das Leitbild des „intensive mothering“ anfangs v.a. auf den amerikanischen Raum bezog, kann dieses auch auf Deutschland übertragen werden. Denn auch in Deutschland und insbesondere in Westdeutschland werden hohe Anforderungen an die Rolle der "guten Mutter" gestellt, indem „Mutterschaft (...) als äußerst anspruchsvolle und stark auf das Kind fokussierte Lebensweise konstruiert" wird (Diabaté 2015, S. 208). Dechant und Rinklake (2016) konnten in ihrer qualitativen Studie, in der schwangere Frauen befragt wurden, ebenfalls eine Vorherrschaft des Ideals des „intensive mothering" in Deutschland beobachten. So sind viele der befragten Frauen der Ansicht, dass sie selbst die bestmögliche Betreuungsperson für ihr Kind sind und intuitiv wissen, was inr Kind braucht. Des Weiteren betrachten sie eine Betreuung durch die Väter oder durch eine externe Betreuungsperson als nicht gleichwertig mit einer Betreuung durch die Mutter (Dechant und Rinklake 2016). Eine weitere Studie für Deutschland von Diabaté und 
Beringer (2018) belegt, dass etwa ein Viertel der Mütter das Konzept des „intensive mothering" vertritt und sich selbst für die beste Betreuungsperson hält. Viele Mütter stehen deshalb sowohl einer Betreuung ihrer Kleinkinder durch Betreuungseinrichtungen als auch durch die Großeltern der Kinder skeptisch gegenüber (Diabaté und Beringer 2018).

Eine Erweiterung von „intensive mothering“ ist das Leitbild des „intensive parenting“, bei dem Väter explizit in die Verantwortung für das Wohlergehen des Kindes mit einbezogen werden (Wall 2010; Liss et al. 2013; Craig et al. 2014; Faircloth 2014). Viele Studien bestätigen, dass Väter heutzutage mehr Zeit mit den Kindern verbringen als dies noch vor einigen Jahrzehnten der Fall war (Sayer et al. 2004a; Sullivan und Coltrane 2008; Sullivan et al. 2014). Eine andere Terminologie für „intensive parenting“ ist die „verantwortete Elternschaft" (Ruckdeschel 2015), die ebenso zum Ausdruck bringt, dass Eltern gemeinsam verantwortlich sind und möglichst viel Zeit, Energie und Geld in die Erziehung investiert werden soll, um die Kinder bestmöglich und intensiv zu begleiten (Ruckdeschel 2015). Das Leitbild des „intensive parenting“ bzw. der „verantworteten Elternschaft" ist in Deutschland stark präsent und prägt die Vorstellungen von Elternschaft (Ruckdeschel 2015). Eine Studie von Grunow et al. (2018) zeigt, dass etwa 15\% der westdeutschen Frauen und 18\% der westdeutschen Männer das Ideal des „intensive parenting" vertreten (Grunow et al. 2018, S. 52).

\section{Familienpolitische Maßnahmen als Indikator für Leitbilder}

Welche Leitbilder in einer Gesellschaft vorherrschend sind, lässt sich auch indirekt daran bemessen, welche Freiräume Eltern etwa durch Geldpolitiken, Zeitpolitiken und infrastrukturelle Rahmenbedingungen gegeben werden - oder eben nicht gegeben werden. Für die vorliegende Arbeit ist es von besonderem Interesse, welche Leitbilder für die einzelnen Altersphasen des Kindes in der Gesellschaft vorherrschend sind. Deshalb wird im Folgenden näher darauf eingegangen, welche altersspezifischen Leitbilder aus den jeweiligen, familienpolitischen Maßnahmen abgeleitet werden können. ${ }^{6}$

Als Geld- und Zeitpolitiken für Eltern von Kleinkindern sind der Mutterschutz, das Elterngeld und die Elternzeit zu nennen. Mit Ausnahme des Mutterschutzes sind diese Politiken geschlechtsneutral. Durch die Einführung der zwei Partnermonate hat die deutsche

\footnotetext{
${ }^{6}$ Auf die Zeitpolitiken, die Geldpolitiken und die Infrastruktur wird in Kapitel 5.4 zur Trias der Familienpolitik vertiefend eingegangen. Die Trias der Familienpolitik wird jedoch auch schon hier aufgegriffen, da sie Familienleitbilder prägen und beeinflussen kann. Inhaltlich kann es in den nächsten Absätzen zu leichten Überschneidungen mit den Kapiteln 5.4 und 5.5 kommen, aber die Perspektiven sind dennoch verschieden: Hier werden familienpolitische Maßnahme behandelt, weil sie ein Indikator für Familienleitbilder sein können; in Kapitel 5.4 werden die familienpolitische Maßnahmen als (Teil-)Repräsentant der allgemeinen, gesellschaftlichen Rahmenbedingungen behandelt; und in Kapitel 5.5 werden die aus den theoretischen Ansätzen abgeleiteten Bedeutungen der relativen Ressourcen und der (individuellen) Geschlechterrolleneinstellungen explizit mit den institutionellen Rahmenbedingungen und damit einhergehend auch mit den familienpolitischen Maßnahmen verknüpft.
} 
Familienpolitik darüber hinaus explizite Anreize für Väter gesetzt, Elterngeld zu nutzen. Dies zeigt, dass die Familienpolitik bei den Geld- und Zeitpolitiken für Familien mit Kleinkindern egalitäre Einstellungen vertritt. Die Vätermonate wurden gut angenommen und 2015 bereits von 35\% der Väter genutzt, allerding liegt die Bezugsdauer der Väter zu $79 \%$ bei höchstens zwei Monaten, sodass die Mehrheit der Väter lediglich die Vätermonate ausschöpfte und nicht darüber hinausging (BMFSFJ 2016c, S. 18). Dieses Nutzungsverhalten deutet darauf hin, dass Leitbilder vorherrschend sind, die Müttern eine gewichtigere Rolle beimessen als Vätern.

Während sich das Elterngeld auf die ersten 12 Monate (bzw. 14 Monate) des Kindes beschränkt, kann die Elternzeit bis zu 36 Monate genommen werden. Damit zählt die deutsche Elternzeit zu den großzügigsten Elternzeitansprüchen weltweit (Ray et al. 2010; Korpi et al. 2013; Gangl und Ziefle 2015). Dies zeigt, dass die Familienpolitik in Deutschland die traditionell-familiäre Einstellung vertritt, dass Kinder in den ersten drei Lebensjahren zu Hause betreut werden sollen. Neuere Regelungen ermöglichen Eltern eine flexiblere Nutzung der Elternzeit: Seit dem 1. Juli 2015 können Eltern 24 Monate der Elternzeit auch zwischen dem dritten und achten Geburtstag des Kindes nehmen (Deutscher Bundestag 2014). Damit signalisiert die Familienpolitik für Kinder ab der Vollendung des ersten Lebensjahres etwas modernere Einstellungen, aber durch das Festhalten an bis zu 36 Monaten Elternzeit, vertritt sie bzgl. der Elternzeit dennoch weiterhin einen familiären Kurs.

Ein weiteres, wichtiges Element ist der Ausbau von Kinderbetreuungsmöglichkeiten sowie die Betreuungsquote in den jeweiligen Altersstufen des Kindes. Denn die Verfügbarkeit externer Kinderbetreuungsmöglichkeiten spielt eine entscheidende Rolle dafür, ob Mütter und Väter mit egalitären Einstellungen eine egalitäre Arbeitsteilung realisieren können. Ohne Möglichkeiten der externen Kinderbetreuung ist eine egalitäre Aufteilung der Erwerbsarbeit nicht möglich und infolgedessen ist auch eine egalitäre Aufteilung der Hausarbeit und Kinderbetreuung unwahrscheinlicher. Im Folgenden wird daher kurz auf den Ausbau von Kinderbetreuungsstätten eingegangen und anschließend auf die Betreuungsquoten (ausführlichere Informationen hierzu finden sich in Kapitel 5.4).

In den letzten zwei Jahrzehnten wurde das Kinderbetreuungsangebot für Kinder unter drei Jahren stark ausgebaut. Durch das Tagesbetreuungsausbaugesetz (TAG) von 2005 sollte das Betreuungsangebot für Kinder unter drei Jahre verbessert werden, indem bis zum Jahr 2010230.000 zusätzliche Kinderbetreuungsplätze anvisiert werden (BMFSFJ 2008 b, S. 27). Damit sollte in Westdeutschland eine Betreuungsquote von $17 \%$ ermöglicht werden (BMFSFJ 2008a, S. 27). 2008 folgte das Kinderförderungsgesetz (KiföG), das ab dem 1. August 2013 für alle Eltern mit einem Kind ab Vollendung des ersten Lebensjahrs bis zur Vollendung des dritten Lebensjahres einen Rechtsanspruch auf einen Betreuungsplatz gewährt (BMFSFJ 2015). Hierfür sollten die Kinderbetreuungsplätze bis 2013 stufenweise auf insgesamt 750.000 Plätze ausgebaut werden, um einen Betreuungsbedarf von 35\% decken zu können (BMFSFJ 2015, S. 6). Schließlich trat 
2019 das Gute-Kita Gesetz in Kraft, das bis 2020 die Qualität der Kinderbetreuungsplätze verbessern soll.

Im Folgenden wird ein Blick darauf geworfen, wie viele Eltern Kinderbetreuungsplätze für Kinder unter drei Jahren in Anspruch nehmen und inwiefern dies den Bedarfen entspricht. Im Jahr 2006 wurden in Deutschland etwa 286.000 Kinder im Alter von unter drei Jahren betreut, 2018 waren es bereits 720.000 (BMFSFJ 2019, S. 7). Damit lag die Betreuungsquote der unter 3-jährigen Kinder 2006 bei 14\% und stieg bis 2018 auf 34\% (BMFSFJ 2019, S. 10). Diese Zahlen beziehen sich auf das gesamte Deutschland. Differenziert man zwischen West- und Ostdeutschland, so lagen 2006 noch massive Unterschiede vor, die sich bis 2018 deutlich verkleinert haben: 2006 lagen West- und Ostdeutschland bei der U3-Betreuungsquote noch 31 Prozentpunkte auseinander (Westdeutschland 8\%, Ostdeutschland 39\%), bis 2018 hat sich die Lücke auf 22 Prozentpunkte minimiert (Westdeutschland 29\%, Ostdeutschland 52\%) (BMFSFJ 2019, S. 10). Damit hat sich die Betreuungsquote der unter 3-jährigen Kinder in Westdeutschland in etwa einem Jahrzehnt mehr als verdreifacht. Der Betreuungsquote von 34\% im Jahr 2018 für das gesamte Deutschland steht ein Betreuungsbedarf von 48\% gegenüber, d.h. der Betreuungsbedarf übersteigt das Angebot um 14 Prozentpunkte (BMFSFJ 2019, S. 13).

Vergleicht man die Betreuungsquote der U3-Kinder mit der Betreuungsquote von Kindern im Kindergartenalter, so zeigt sich eine deutliche Kluft: Während 2018 „nur“ jedes dritte U3-Kind extern betreut wird, sind es bei den Kindern im Kindergartenalter 93\% (BMFSFJ 2019, S. 21). Dies zeigt, dass es sich bei einem Kindesalter von drei Jahre um ein Schwellenalter handelt: Wenn das Kind unter drei Jahre ist, wird die primäre Zuständigkeit für die Kinderbetreuung noch in der Familie gesehen. Auch wenn die Betreuungsquote der U3-Kinder in den letzten Jahren deutlich angestiegen ist, so wird die Mehrheit der Kleinkinder dennoch in der Familie betreut. Ab einem Alter von über drei Jahren besuchen dagegen fast alle Kinder einen Kindergarten. Dies zeigt, dass gesellschaftlich akzeptiert wird, dass bei Kindern über drei Jahre außerfamiliale Betreuung stattfindet. Mütter, deren Kinder in den Kindergarten gehen, verstoßen somit nicht gegen das Leitbild der "guten Mutter“. Bei den Kleinkindern ist die Gesellschaft diesbezüglich noch zwiegespaltener. Der Eintritt ins Schulalter stellt ein weiteres Schwellenalter dar: Während der Kindergartenbesuch freiwilliger Natur ist, ist der Schulbesuch verpflichtend. Dies zeigt, dass eine längere Trennung von Mutter und Kind nicht nur für zumutbar, sondern als zwingend erforderlich für die optimale Entwicklung des Kindes erachtet wird.

Zusammenfassend lässt sich auf Basis der Geld- und Zeitpolitiken festhalten, dass diese geschlechtsneutral sind und egalitäre Einstellungen zum Ausdruck bringen. Das Nutzungsverhalten der Eltern zeigt jedoch, dass diese geschlechtsneutralen Politiken sehr geschlechtsasymmetrisch genutzt werden und Mütter bei Kleinkindern weiterhin die primäre Betreuungsperson darstellen. Verwendet man die Betreuungsquoten als Indikator 
für Leitbilder, so lässt sich festhalten, dass auf der gesellschaftlichen Ebene die Erwartungen an die Mutterrolle noch relativ konservativ sind, wenn sich das Kind im Kleinkindalter befindet, sie bereits lockerer werden, wenn sich das Kind im Kindergartenalter befindet und sie nochmals lockerer werden, wenn das Kind ins Schulalter eintritt.

\section{Altersspezifische Einstellungen als Indikator für Leitbilder}

Für die vorliegende Arbeit ist es von Relevanz, welche Geschlechterrolleneinstellungen für die jeweiligen Altersphasen des Kindes vorliegen. Es wäre von Vorteil, wenn die in Surveys abgefragten Geschlechterrolleneinstellungen einen konkreten Bezug auf die Altersphasen des Kindes nähmen. Dabei interessieren vor allem diejenigen Einstellungen, die sich auf die Mutterschaft beziehen bzw. abfragen, inwiefern sich eine Erwerbstätigkeit der Mutter auf die Mutter-Kind-Beziehung auswirkt. Es zeigt sich jedoch, dass hierbei meistens nicht danach differenziert wird, wie alt das Kind ist. So wird nur allgemein danach gefragt, ob erwerbstätige Mütter eine genauso innige Beziehung zu ihren Kindern aufbauen können wie nicht erwerbstätige Mütter oder ob das Familienleben durch die Erwerbstätigkeit der Mütter im Allgemeinen leidet (so wurde es beispielsweise im „International Social Survey Programme" (ISSP) abgefragt).

Wenn in den Fragebögen bei den Einstellungen auf das Alter des Kindes Bezug genommen wird, dann meist nur auf ein bestimmtes Kindesalter. Ein Beispiel hierfür ist die Frage danach, ob ein Kind im Vorschulalter darunter leidet, wenn die Mutter erwerbstätig ist. ${ }^{7}$ Die Tatsache, dass sich diese Frage ausschließlich auf das Vorschulalter bezieht und nicht auch für jüngere oder ältere Altersphasen des Kindes abgefragt wird, könnte darauf hindeuten, dass das Vorschulalter ein wichtiges Schwellenalter ist, für das wenig gesellschaftlicher Konsens besteht. Möglicherweise wurde diese Frage nicht für Kleinkinder gestellt, weil gesellschaftlicher Konsens darüber besteht, dass Kleinkinder sehr wohl unter der Erwerbstätigkeit der Mütter leiden; und für ältere Kinder im Schulalter wurde sie nicht mehr gestellt, weil Konsens darüber besteht, dass Kinder dann nicht mehr unter der Erwerbstätigkeit der Mütter leiden.

Eine Ausnahme zu diesen allgemeinen Einstellungen, die das Alter des Kindes nicht oder nur sehr grob berücksichtigen, ist die Studie von Lietzmann und Wenzig (2017) auf Basis des deutschen Panels „Arbeitsmarkt und soziale Sicherung“ (PASS). In der 5. Erhebungswelle von 2011 wurden beim PASS-Survey einmalig Einstellungen erhoben, die abfragen ab welchem Kindesalter Mütter wieder erwerbstätig sein sollen und ab welchem Kindesalter Kinder eine Kinderbetreuungseinrichtung besuchen sollen. Auf die Ergebnisse dieser Studie wird im Folgenden näher eingegangen.

\footnotetext{
${ }^{7}$ Das Original-Item lautet „A preschool child is likely to suffer if his or her mother works" und wurde so z.B. in den amerikanischen Datensätzen „International Social Survey Programme“, "General Social Survey" und "National Survey of Families and Households" abgefragt (vgl. Davis und Greenstein 2009, S. 90).
} 
Die Ergebnisse zeigen, dass Frauen der Meinung sind, dass Mütter im Durchschnitt ab einem Kinderalter von 3 Jahren und 2 Monaten wieder in Teilzeit und ab einem Kindesalter von 7 Jahren wieder in Vollzeit erwerbstätig sein können (Lietzmann und Wenzig 2017, S. 4). Dabei zeigen sich deutliche Unterschiede zwischen Ost- und Westdeutschland: Eine Teilzeiterwerbstätigkeit der Mütter halten ostdeutsche Frauen bereits ab einem Kinderalter von 2 Jahren und 6 Monaten für vertretbar, westdeutsche Frauen dagegen erst ab einem Kindesalter von 3 Jahren und 7 Monaten (Lietzmann und Wenzig 2017, S. 7). Eine Vollzeiterwerbstätigkeit der Mütter finden ostdeutsche Frauen schon ab einem Kindesalter von 4 Jahren und 2 Monaten für angemessen. Bei den westdeutschen Frauen wird dies erst deutlich später mit einem Kindesalter von 7 Jahren und 8 Monaten für gut befunden (Lietzmann und Wenzig 2017, S. 7).

Des Weiteren wurde danach gefragt, ab welchem Alter Kinder für einige Stunden außerhalb der Familie betreut werden können und ab welchem Alter sie ganztätig außerhalb der Familie betreut werden können. Eine Betreuung für einige Stunden halten die befragten Frauen durchschnittlich ab einem Kindesalter von 2 Jahren und 7 Monaten für in Ordnung und eine ganztägige Betreuung im Durchschnitt ab 5 Jahren und 4 Monaten (Lietzmann und Wenzig 2017, S. 6). Auch hier zeigen sich wieder deutliche Ost-WestUnterschiede: Ostdeutsche Frauen finden eine Betreuung für einige Stunden ab 2 Jahre und westdeutsche Frauen ab 2 Jahren und 10 Monaten für akzeptabel; eine ganztätige Betreuung wird ab 3 Jahren und 4 Monaten in Ostdeutschland und erst ab 5 Jahren und 6 Monaten in Westdeutschland befürwortet (Lietzmann und Wenzig 2017, S. 6).

Zusammenfassend zeigt die Studie, dass eine Teilzeiterwerbstätigkeit der Mutter sowie eine stundenweise außerfamiliale Kinderbetreuung etwa ab einem Kindesalter von ca. 3 Jahren gesellschaftlich akzeptiert ist. Eine Vollzeiterwerbstätigkeit der Mutter wird ab einem Kindesalter von 7 Jahren und eine ganztätige externe Kinderbetreuung ab einem Kindesalter von ca. 5 Jahren als richtig empfunden.

Aus dieser Studie von Lietzmann und Wenzig (2017) gehen zwar Einstellungen zur Mutterschaft und zur externen Kinderbetreuung hervor, nicht jedoch zur Vaterschaft. Eine Norm, der Männer und Väter immer noch stark ausgesetzt sind, ist die „ideal worker norm“ (Williams 2000). Die „ideal worker norm“ hat eine kontinuierliche Vollzeiterwerbstätigkeit und größtmögliche, zeitliche Verfügbarkeit für den Arbeitgeber als Ideal. Die Familienstruktur der Väter und damit auch das Alter des Kindes bleiben bei dieser Norm unberücksichtigt, sodass sie auch für Väter von kleinen Kindern gilt. Dass diese Norm auch in Deutschland noch stark präsent ist, zeigt sich beispielsweise daran, dass beim Elterngeld zwar viele Väter die zwei Vätermonate beziehen, aber nur eine Minderheit mehr als zwei Monate Elterngeld bezieht.

Kombiniert man die Studienergebnisse von Lietzmann und Wenzig (2017) bzgl. der Einstellungen zur Erwerbstätigkeit der Mütter und der externen Kinderbetreuung mit der „ideal worker norm“ (Williams 2000) für Väter, so ergeben sich auf Paarebene folgende 
Normen zum Arbeitsarrangements: Bei Kindern unter 3 Jahren wird ein männliches Ernährermodell erwartet, in dem der Vater in Vollzeit erwerbstätig ist und die Mutter nicht erwerbstätig ist und das Kind zu Hause betreut. Ab einem Kindesalter von 3 bis unter 6 Jahren entspricht ein Vollzeit-/Teilzeitmodell der Norm, bei dem Väter in Vollzeit erwerbstätig sind und Mütter durch eine stundenweise, externe Kinderbetreuung wieder einer Teilzeitbeschäftigung nachgehen können. Ab einem Kindesalter von etwa 6 Jahren wird ein Vollzeit-/Vollzeit-Modell mit einer ganztätigen, externen Kinderbetreuung toleriert.

Wenn man neben diesen Einstellungen auch noch das weiter oben beschriebene Nutzungsverhalten von Elterngeld und externer Kinderbetreuung sowie Erkenntnisse aus Leitbildern wie „intensive mothering/intensive parenting“ berücksichtigt, so lassen sich folgende Annahmen für die Arbeitsteilung der Eltern in unterschiedlichen Phasen des Familienzyklus ableiten: Wenn die Kinder noch klein sind, übernehmen Mütter den Großteil der Kinderbetreuung und der Hausarbeit. Sie sind deshalb in diesem Altersspektrum des Kindes nicht oder nur in geringem Umfang erwerbstätig. Die Arbeitsteilung fällt somit bei kleinen Kindern besonders geschlechtsspezifisch aus, da angenommen wird, dass sich die ständige Anwesenheit der Mutter förderlich auf die Entwicklung und das Wohlbefinden des Kindes auswirkt. Mit zunehmendem Alter des Kindes ist die körperliche Anwesenheit der Mutter nicht mehr ganz so wichtig, sodass die Mutter wieder in stärkerem Umfang erwerbstätig sein kann. Zudem profitiert das Kind auch durch das zusätzliche Einkommen der Mutter, sodass die Erwerbstätigkeit der Mutter nicht mehr in Widerspruch zum kindlichen Wohlbefinden stehen muss. Es ist deshalb aus einer Perspektive der Familienleitbilder heraus anzunehmen, dass mit zunehmendem Alter des Kindes der Arbeitsteilung egalitärer wird.

\subsection{Zeit, Geld und Infrastruktur: Zur Entwicklung der ,Trias der Familienpolitik' in Deutschland}

Ob Eltern eine egalitärere oder traditionellere Arbeitsteilung praktizieren und ob diese über die verschiedenen Phasen des Familienzyklus stabil bleibt oder nicht, hängt insbesondere von zeitlichen, finanziellen und infrastrukturellen Rahmenbedingungen ab. Bei der sog. „Trias der Familienpolitik“ handelt es sich um einen Begriff, der durch den Siebten Familienbericht geprägt ist und der genau diese drei Aspekte von familienpolitischen Maßnahmen aufgreift: Zeitpolitiken, Geldpolitiken und Infrastruktur (BMFSFJ 2006b). So regelt die Familienpolitik etwa durch die Elternzeit und das Elterngeld, wieviel gemeinsame Zeit sie Familien zugesteht und wieviel Geld sie innen in dieser Zeit zur Verfügung stellt. Infrastrukturelle Maßnahmen, wie der Ausbau von Kinderbetreuungseinrichtungen im Kleinkindalter entscheiden darüber, ob es Müttern möglich ist, zeitnah nach der Geburt wieder in die Erwerbstätigkeit zurückzukehren. Die Trias der Familienpolitik nimmt dabei eine lebensverlaufsorientierte Perspektive ein, denn Familien mit kleinen Kindern stehen vor anderen Herausforderungen bzgl. der Vereinbarkeit von Familie und Beruf 
als Familien mit älteren Kindern. Daher benötigen erstere eine andere staatliche Unterstützung als letztere.

Im Folgenden wird auf diese Zeitpolitiken, Geldpolitiken und infrastrukturellen Rahmenbedingungen eingegangen und dabei zwischen den verschiedenen Altersstufen des Kindes differenziert, da auch die familienpolitischen Maßnahmen an spezifische Altersstufen des Kindes ausgerichtet sind. Darüber hinaus wird auch auf den Wandel eingegangen, den die deutsche Familienpolitik in den letzten Jahren (bzw. Jahrzehnten) durchlaufen hat.

\section{Vom Erziehungsgeld zum Elterngeld und ElterngeldPlus}

Das Erziehungsgeld wurde 1986 mit dem Bundeserziehungsgeldgesetz eingeführt. Es galt bis zum 31.12.2006 und wurde danach vom Elterngeld abgelöst (Deutscher Bundestag 2006). Beim Erziehungsgeld handelt es sich um eine einkommensabhängige Familienleistung, die sowohl Eltern mit vorheriger Erwerbstätigkeit als auch Eltern ohne vorheriger Erwerbstätigkeit gezahlt wurde (BMFSFJ 2006a, S. 7). Bei der Nutzung des Erziehungsgelds konnten Eltern zwischen zwei Optionen wählen: Entweder entschieden sie sich dafür, den Regelbetrag zu beziehen, der pro Monat und pro Kind $300 €$ umfasste und für die ersten 24 Monate des Kindes bezogen werden konnte (BMFSFJ 2006a, S. 13). Oder die Eltern wählten das Budget, das $450 €$ pro Monat und pro Kind betrug, aber dafür nur für die ersten 12 Monate des Kindes bezogen werden konnte (BMFSFJ 2006a, S. 13). Voraussetzung für den Bezug des Regelbetrags war, dass das gemeinsame jährliche Nettoeinkommen der Eltern in den ersten sechs Lebensmonaten des Kindes nicht über $30.000 €$ bzw. ab dem siebten Liebensmonat des Kindes nicht über $16.500 €$ lag (BMFSFJ 2006a, S. 19-20). Für den Bezug des Budgets lagen die Einkommenshöchstgrenzen bei $22.086 €$ in den ersten sechs Lebensmonaten und bei $16.500 €$ ab dem siebten Lebensmonat (BMFSFJ 2006a, S. 19-20).

Das Erziehungsgeld ermöglichte somit längere Erwerbsunterbrechungen, wobei die finanziellen Anreize durch die Absenkung der Einkommenshöchstgrenzen ab dem siebten Lebensmonat vor allem Familien mit sehr niedrigem Einkommen vorbehalten blieben (Menke und Klammer 2017, S. 218). Die Person, die sich um die Kinderbetreuung kümmerte (in der Regel die Mutter), wurde zwar einerseits finanziell entschädigt, allerding reichte diese Entschädigung mit einem Betrag von $300 €$ bzw. $450 €$ pro Monat nicht zur Existenzsicherung aus. Die Person blieb deshalb vom Einkommen des Partners abhängig oder war auf weitere Sozialleistungen angewiesen, wodurch eine traditionelle Aufteilung der Erwerbsarbeit und der Kinderbetreuung gefördert wurde (Menke und Klammer 2017, S. 218-219).

2007 kam es zu einem familienpolitischen Wandel der häufig als ein „Paradigmenwechsel“ beschrieben wird (Schutter und Zerle-Elsäßer 2012, S. 219; Bujard 2013, S. 133; Menke und Klammer 2017, S. 213): Für alle Geburten ab dem 01.01.2007 gilt nicht mehr das Erziehungs-, sondern das Elterngeld. Dies wurde im Bundeselterngeld- und Eltern- 
zeitgesetz (BEEG) beschlossen. Beim Elterngeld handelt es sich im Gegensatz zum Erziehungsgeld um eine Lohnersatzleistung. Die Lohnersatzleistung orientiert sich am Einkommen in den 12 Monaten vor der Geburt des Kindes und kompensiert $67 \%$ bis $100 \%$ des durchschnittlichen monatlichen Nettoeinkommens. Dabei gibt es eine Deckelung nach unten und oben: Es beträgt mindestens $300 €$ und höchstens $1.800 €$. Eltern, die in den 12 Monaten vor der Geburt nicht erwerbstätig waren, erhalten den Mindestbetrag von $300 €$. Das Elterngeld leistet somit einen Beitrag zur Einkommenssicherung in der Phase der Familiengründung (Deutscher Bundestag 2006, S. 1-2; Bujard 2013, S. 140). Die maximale Bezugsdauer beträgt 12 Monate und kann um zwei weitere Monate verlängert werden, wenn beide Elternteile das Elterngeld für mindestens zwei Monate beziehen (Deutscher Bundestag 2006, S. 2; BMFSFJ 2018a, S. 19). Diese zwei zusätzlichen Monate, durch die sich die gesamte Bezugsdauer auf 14 Monate erhöhen lässt, werden auch - geschlechtsneutral formuliert - als Partnermonate bezeichnet, da sie nur bezogen werden können, wenn auch das zweite Elternteil Elterngeld in Anspruch nimmt. Da jedoch in der Regel die Mütter den Großteil und damit bis zu 12 Monate Elterngeld beziehen und die Väter häufig nur die beiden zusätzlichen Monate nehmen, werden diese Partnermonate häufig auch als Vätermonate bezeichnet. Die Partner- bzw. Vätermonate sollen Väter dazu motivieren, ihre Erwerbsarbeit zu unterbrechen oder zumindest zu reduzieren und dadurch ihre Beteiligung an der Kinderbetreuung zu erhöhen. Dadurch soll eine egalitärere Aufteilung der Kinderbetreuung begünstigt werden.

Durch die Beschränkung der Lohnersatzleistung auf das erste Lebensjahr des Kindes werden Mütter gleichzeitig dazu angeregt, direkt im Anschluss wieder in die Erwerbstätigkeit zurückzukehren, wodurch nach dem ersten Lebensjahr des Kindes eine egalitärere Aufteilung der Erwerbsarbeit gefördert wird als dies beim Erziehungsgeld der Fall war. Da das Elterngeld eine Teilzeiterwerbstätigkeit im Umfang von bis zu 30 Wochenstunden zulässt, wird darüber hinaus bereits eine Teilzeiterwerbstätigkeit im ersten Lebensjahr des Kindes für Mütter und Väter attraktiver. Damit will das Elterngeld Paare in ihrer eigenständigen, finanziellen Existenzsicherung unterstützen und im Gegensatz zum Erziehungsgeld keine Anreize für eine Erwerbsunterbrechung von über einem Jahr mehr geben (Bujard 2013, S. 140).

Das Elterngeld stellt den Eltern einen zeitlichen und finanziellen Schonraum zur Verfügung, indem es innen ermöglicht, sich an die neue Rolle zu gewöhnen und die Fürsorgearbeit selbst übernehmen zu können (Deutscher Bundestag 2006, S. 2; Bujard 2013, S. 140). Allgemein soll den Menschen durch das Elterngeld mehr Mut zur Familiengründung gemacht werden und so eine Fertilitätssteigerung erzielt werden (Deutscher Bundestag 2006, S. 2; Bujard 2013, S. 140).

Zusammenfassend dient das Elterngeld damit den folgenden fünf Zielen (Bujard 2013, S. 140):

- Einkommenssicherung in der Phase der Familiengründung,

- Finanzieller und zeitlicher Schonraum, 
- Erwerbspartizipation von Müttern erhöhen,

- Fürsorgebeteiligung von Vätern fördern und

- Familiengründung erleichtern.

Mit der Einführung des Elterngeldes leistete die Familienpolitik einen wichtigen Beitrag für die Gleichstellung der Geschlechter und zur Förderung einer egalitären Arbeitsteilung.

Das Elterngeld fand bereits im ersten Jahr großen Anklang in der Bevölkerung und wurde von einem höheren Anteil der Eltern in Anspruch genommen als dies zuvor beim Erziehungsgeld der Fall war: „92 Prozent der Familien, deren Kind im Januar 2007 geboren wurde, erhalten Elterngeld. Im Jahr 2006 bezogen hingegen nur 77 Prozent der Familien Erziehungsgeld für ein Kind im ersten Lebensjahr" (BMFSFJ 2008a, S. 21). Betrachtet man die Aufteilung des Elterngeldbezugs zwischen den Eltern, so zeigt sich Folgendes:

„Nur in etwa drei Prozent aller Familien mit Elterngeldbezug nehmen ausschließlich die Väter die Elterngeldzahlungen in Anspruch, im Gegenzug sind es in 89,5 Prozent der Fälle ausschließlich die Mütter. Partnerlösungen wurden bei den Januaranträgen von 7,4 Prozent der Familien beantragt. Das bedeutet, dass in 10,5 Prozent der Familien der Vater Elterngeld nutzt, die Mutter in 96,9 Prozent der Familien" (BMFSFJ 2008a, S. 21).

Damit liegt der Anteil der Väter, die Elterngeld in Anspruch nehmen, bereits im Jahr 2007 deutlich über dem Anteil der Väter, die Erziehungsgeld in Anspruch genommen haben (dies waren lediglich 3\% der Väter) (BMFSFJ 2018b, S. 16). Dies zeigt, dass die Arbeitsteilung durch familienpolitische Maßnahmen stark beeinflussbar ist und Veränderungen bereits innerhalb kürzester Zeit eintreten können. Wie die Abbildung 5.2 zeigt, ist die Inanspruchnahme des Elterngeldes von Vätern in den nachfolgenden Jahren kontinuierlich angestiegen: 2008 waren es 21\%, 2013 waren es bereits $29 \%$ und 2015 schließlich 35\% (BMFSFJ 2018b, S. 16): 
Abbildung 5.2: Entwicklung der Väterbeteiligung am Erziehungs- und Elterngeldbezug, nach Geburtsjahrgängen der Kinder, von 2006 bis 2015

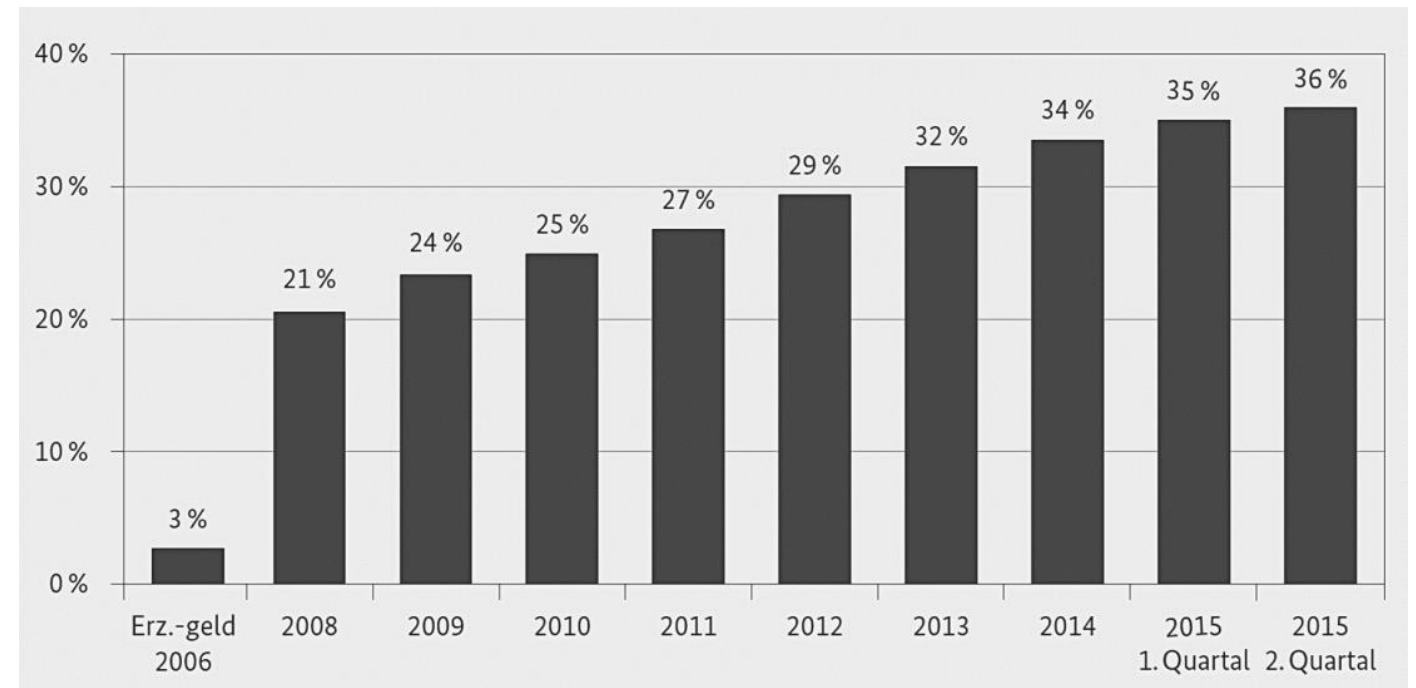

Quelle: BMFSFJ 2018b, S. 16. Datenquelle: Statistisches Bundesamt 2017.

Allerdings liegt die Bezugsdauer der Väter zu $79 \%$ bei höchstens zwei Monaten, was darauf zurückzuführen ist, dass Väter meistens die beiden Partnermonate, die zusätzlich zu den 12 Monaten gewährt werden, ausschöpfen (BMFSFJ 2016c, S. 18). Auch hier wird wieder die Beeinflussbarkeit der Arbeitsteilung durch die Politik deutlich sichtbar: Väter sind bereit, zwei Monate Elterngeld in Anspruch zu nehmen, weil diese zwei Monate zusätzlich als Bonus gewährt werden. Läge der Partnerschaftsbonus dagegen beispielsweise bei drei Monaten, so wäre anzunehmen, dass der Großteil der Väter entsprechend drei Monate Elterngeld in Anspruch nehmen würde.

Das Elterngeld leistet insofern einen wichtigen Schritt zur Gleichstellung der Geschlechter, indem es bewirkt, dass Väter Elterngeld in Anspruch nehmen. Die Tatsache, dass die Bezugsdauer der Väter in der Regel jedoch nur zwei Monate beträgt, zeigt gleichzeitig, dass die Arbeitsteilung zwischen Müttern und Vätern nach wie vor sehr geschlechtsspezifisch ist. Deshalb besteht auch nach dem Inkrafttreten des Elterngeldes immer noch eine große Diskrepanz zwischen der gewünschten und der tatsächlich praktizierten Arbeitsteilung: Etwa $60 \%$ der Eltern mit einem jüngsten Kind im Alter von ein bis drei Jahren wünschen sich, dass die Erwerbsarbeit, die Hausarbeit und die Kinderbetreuung egalitär zwischen den Eltern aufgeteilt wird; allerdings gelingt nur etwa 14\% der Eltern eine egalitäre Aufteilung der Erwerbsarbeit und von diesen 14\% handelt es sich zu 75\% um Paare, in denen beide in Vollzeit arbeiten (Müller et al. 2013, S. 3). Auf diese Diskrepanz reagierte die Bundesregierung mit dem ElterngeldPlus, das für alle Geburten ab dem 01.07.2015 gilt (BMFSFJ 2016a, S. 2).

Das ElterngeldPlus zeichnet sich dadurch das, dass es bis zu doppelt so lange bezogen werden kann wie das Elterngeld. Wenn nach der Geburt eine Erwerbspause eingelegt wird, dann ist die Höhe des ausgezahlten Lohnersatzes beim ElterngeldPlus jedoch nur halb so hoch wie beim Elterngeld. Ein Monat Elterngeld entspricht somit zwei Monaten 
ElterngeldPlus (BMFSFJ 2018a, S. 22). Wenn nach der Geburt jedoch einer Teilzeiterwerbstätigkeit nachgegangen wird, kann das ElterngeldPlus genauso hoch ausfallen wie das monatliche Elterngeld - und dennoch doppelt so lange wie das Elterngeld bezogen werden (BMFSFJ 2018a, S. 22). Darüber hinaus ist ein Partnerschaftsbonus in Form von vier zusätzlichen ElterngeldPlus-Monaten möglich. Voraussetzung für den Partnerschaftsbonus ist, dass sich beide Elternteile in vier aufeinander folgenden Monaten für eine Teilzeitbeschäftigung im Umfang von 25 bis 30 Wochenstunden entscheiden (BMFSFJ 2018a, S. 25). Damit fördert das ElterngeldPlus eine egalitärere Aufteilung der Erwerbsarbeit und Kinderbetreuung, denn eine

„frühere Rückkehr zur Erwerbstätigkeit beider Partner in Teilzeit, die gleichzeitig eine (gemeinsame) Fürsorge für das neugeborene Kind ermöglicht, wird sich stärker lohnen als bisher" (Deutscher Bundestag 2014, S. 2).

Dass ElterngeldPlus steht somit für „eine Politik, die betont, dass die Familienorientierung von Vätern genauso viel wert ist wie die Erwerbsorientierung von Müttern“ (BMFSFJ 2016c, S. 3).

Die Abbildung 5.3 zeigt die Veränderung der Erwerbstätigenquote zwischen dem Jahr 2006 und dem Jahr 2012 in Abhängigkeit vom Alter des Kindes:

Abbildung 5.3: Entwicklung der Erwerbstätigenquoten von Müttern mit minderjährigen Kindern, Deutschland, 2006-2012, Veränderung der Erwerbstätigenquoten in \%

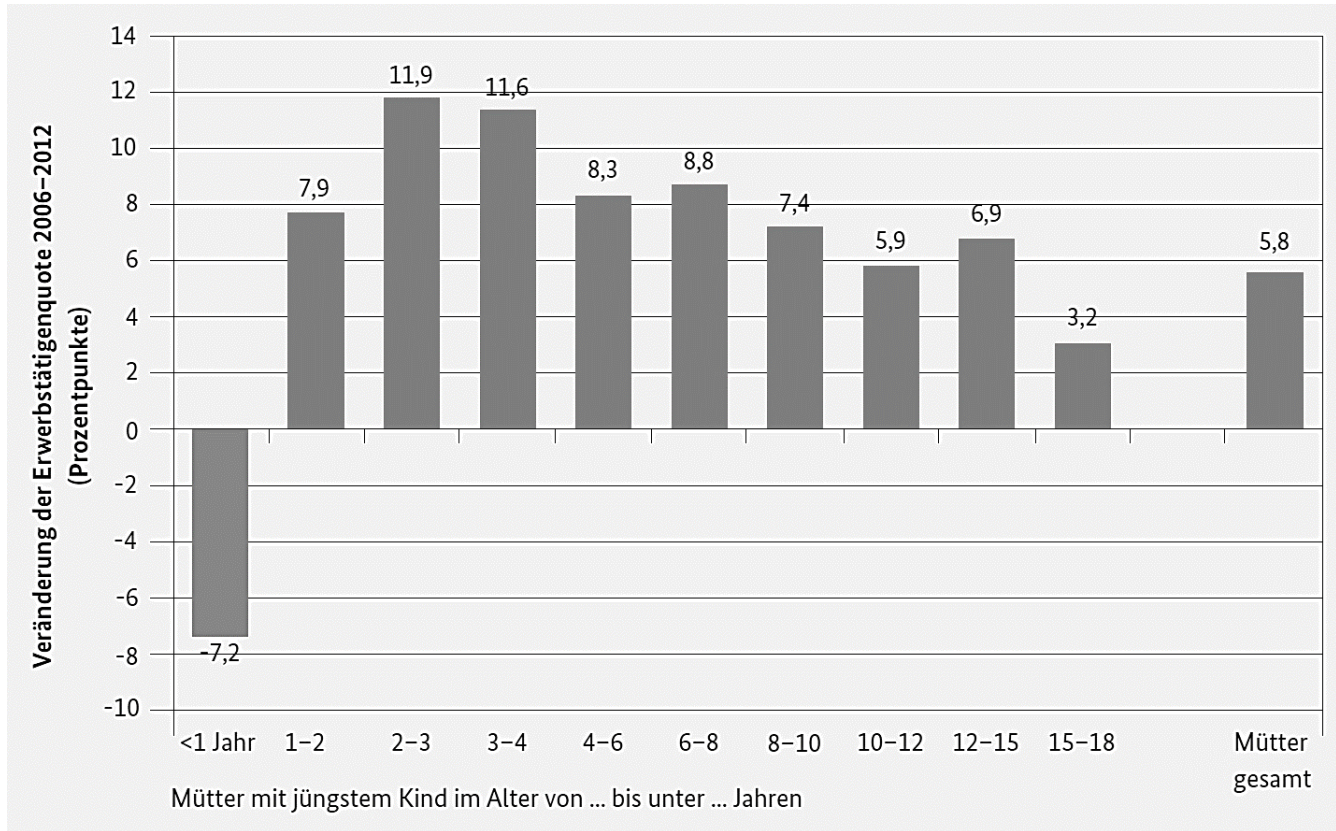

Quelle: BMFSFJ 2014: Dossier Müttererwerbstätigkeit, S. 26; Daten: Mikrozensus-Sonderauswertung s13129, Berechnung Prognos AG.

Wenn Mütter 2012 ein Kind im Alter von unter einem Jahr hatten, so war ihre Erwerbstätigenquote um 7,2\% geringer als dies 2006 der Fall war. Hier zeigt sich, dass das Elterngeld eine Erwerbsunterbrechung von 12 Monaten attraktiver gemacht hat und deshalb auch von mehr Müttern in Anspruch genommen wird. In allen weiteren Altersstufen 
des Kindes ist die Erwerbstätigenquote der Mütter 2012 jedoch höher als 2006: Sie fällt bereits bei Kindern im Alter von ein bis unter zwei Jahren um 7,9\% und bei Kindern im Alter von zwei bis unter drei Jahren sogar um 11,9\% höher aus. Das Elterngeld fördert somit die Rückkehr der Mütter in die Berufstätigkeit, wenn das Kind das erste Lebensjahr vollendet hat. Die positiven Differenzen bleiben auch bei den übrigen Altersstufen des Kindes noch bestehen, allerdings werden sie mit zunehmendem Altem des Kindes geringer.

\section{Von der Erziehungszeit zur Elternzeit}

Vor 2001 war in Deutschland zur Umschreibung der kindbedingten Erwerbspause nach der Geburt der Begriff des „Erziehungsurlaubs“ gebräuchlich. Dieser Begriff wurde ab dem 01.01.2001 durch den Begriff der „Elternzeit“ ersetzt, da die

„bisherige Bezeichnung Erziehungsurlaub (...) in der Öffentlichkeit zu steigenden Irritationen [führt], weil sie die Kinderbetreuung und die Arbeit in der Familie mit der Vorstellung über Freizeit und Muße verbindet“" (Deutscher Bundestag 2000, S. 1).

Demgegenüber erscheint der Begriff der „Elternzeit“ angemessener, weil er die „gemeinsame partnerschaftliche Verantwortung von Mutter und Vater für die Betreuung ihres kleinen Kindes“ verdeutlicht (Deutscher Bundestag 2000, S. 1).

Bei der Elternzeit handelt es sich um eine unbezahlte Freistellung vom Beruf und inkludiert einen Kündigungsschutz bzw. das Recht auf die Rückkehr zu einem äquivalenten Arbeitsplatz (BMFSFJ 2018a, 85 und 99f). Die Elternzeit beträgt drei Jahre, sie beginnt mit der Geburt des Kindes und kann bis zur Vollendung des dritten Lebensjahres des Kindes genommen werden. Ein Anteil von bis zu 12 Monaten von diesen drei Jahren kann jedoch auch bis zur Vollendung des achten Lebensjahres genommen werden (BMFSFJ 2006a, S. 49). Das Recht auf drei Jahre Elternzeit ist unabhängig von der Bezugsdauer des Elterngeldes und sie steht beiden Elternteilen zu (BMFSFJ 2006a, S. 49-50). D.h. wenn „die Eltern wollen, können sie Anteile der Elternzeit oder aber die gesamte dreijährige Elternzeit vollständig gemeinsam nutzen (also nicht etwa nur gemeinsame $11 \frac{1}{2}$ Jahre)“ (BMFSFJ 2006a, S. 50). Dass beide Elternteile die Elternzeit gemeinsam nutzen können, ist eine Neuerung zum vorherigen Erziehungsurlaub. Ebenso neu ist das Recht auf Arbeitszeitreduktion, welches im Jahr 2002 durch das Teilzeit- und Befristungsgesetz noch erweitert wurde (Leitner et al. 2004, S. 18). Im Vergleich zum vorherigen Erziehungsurlaub bietet die Elternzeit ab 2001 somit eine größere Flexibilisierung und die Möglichkeit zu einer Teilzeitbeschäftigung von bis zu 30 Wochenstunden während der Elternzeit.

An der Länge einer maximalen Elternzeit von bis zu drei Jahren hat sich auch nach 2007 mit der Einführung des Elterngeldes nichts geändert. Allerdings motiviert die durch das Elterngeld herbeigeführte Begrenzung der bezahlten Zeit auf 12 bzw. 14 Monate dazu, eine kürzere Elternzeit zu nehmen. Zudem kommt eine „unbezahlte (bis zu) zweijährige Elternzeit im Anschluss an die Elterngeldphase (...) nur für Mütter und Väter in Frage, die über den Familienkontext finanziell abgesichert sind“ (Menke und Klammer 2017, S. 
221). Das Elterngeld bedeutet somit nicht für alle Paare einen Zugewinn an Autonomie und Handlungsfreiheit, sondern „[i]m Gegenteil, ärmere Paare erleben im Vergleich zum Erziehungsgeld eine deutliche Einschränkung ihrer finanziellen Handlungsspielräume“ (Schutter und Zerle-Elsäßer 2012, S. 224).

Seit 2015 können von den bis zu drei Jahren der Elternzeit maximal 24 Monate zwischen dem dritten und dem vollendeten achten Lebensjahr genommen werden - zuvor war dies nur für bis zu 12 Monaten möglich (Menke und Klammer 2017, S. 221). Mit der Ausweitung auf bis zu 24 Monaten setzt die Familienpolitik einen weiteren Anreiz für eine kürzere Erwerbspause unmittelbar nach der Geburt und bietet dafür mehr Flexibilisierung in den nachfolgenden Jahren. Darüber hinaus „kann die Elternzeit in drei Zeitabschnitte pro Elternteil aufgeteilt und von beiden Elternteilen gleichzeitig genommen werden“ (Menke und Klammer 2017, S. 221). Insgesamt lassen diese neuen Instrumente der Familienpolitik damit eine größere Geschlechtergerechtigkeit zu, indem sie

„(1) Erwerbs- und (2) Fürsorgearbeit inzwischen für beide Geschlechter zeitgleich ermöglichen, eine eigenständige Existenzsicherung durch die Parallelität von Erwerbs- und Elternzeit prinzipiell unterstützt wird (3) und damit die Wahlmöglichkeiten zwischen Fürsorgearbeit und Erwerbsarbeit verbessert werden“ (Menke und Klammer 2017, S. 221).

An den Reformen zum Elterngeld und zur Elternzeit ist positiv hervorzuheben, dass sie für mehr Geschlechtergerechtigkeit einstehen. Negativ zu beurteilen ist, dass diese Geschlechtergerechtigkeit höchst selektiv ist (Schutter und Zerle-Elsäßer 2012; Menke und Klammer 2017). So ist das Elterngeld vor allem dann lohnend, wenn man vor der Geburt des Kindes in Vollzeit erwerbstätig war und über ein höheres (oder zumindest mittleres) Einkommen verfügt. Aber gerade in Deutschland kehren Mütter mit einem Kind und im Besonderen bei mehreren Kindern nach der Erwerbspause häufig nur in Teilzeit oder mit noch weniger als 20 Erwerbsstunden in die Erwerbsarbeit zurück (BMFSFJ 2014a, S. 47). Dadurch erfüllen Mütter jedoch vor der Geburt eines weiteren Kindes nicht die Voraussetzungen für ein Elterngeld in existenzsichernder Höhe (Schutter und Zerle-Elsäßer 2012, S. 223). Nicht nur für Mütter in bereits bestehenden Einkind- und Mehrkindfamilien hat sich die Situation verschlechtert. Eine drastische Verschlechterung erfahren langzeitarbeitslose Mütter und Väter: Da das Elterngeld mit SGB II-Leistungen verrechnet wird, erhalten sie de facto kein Elterngeld (Schutter und Zerle-Elsäßer 2012, S. 224; Menke und Klammer 2017, S. 225; Reimer et al. 2019, S. 227). Die Geschlechtergerechtigkeit geht somit zum Teil auf Kosten der sozialen Gerechtigkeit (Menke und Klammer 2017, S. 225).

Trotz diesem Paradigmenwechsel, der eine größere Geschlechtergerechtigkeit und eine partnerschaftliche Vereinbarkeit von Familie und Beruf anstrebt, sendet die Familienpolitik weiterhin widersprüchliche Signale aus. Denn institutionelle Rahmenbedingungen, die das Ernährermodell unterstützen, bleiben weiterhin bestehen. Als Beispiele hierfür seien etwa das Ehegattensplittung, das Kindergeld, die beitragsfreie Mitversicherung von Familienmitgliedern in der gesetzlichen Krankenversicherung, Teilzeitschulen und 
Teilzeitkindergärten genannt (Grunow et al. 2011, S. 398; Bertram und Bujard 2012, S. 6; Müller et al. 2013, S. 3-4; Menke und Klammer 2017, S. 226).

\section{Vom ElterngeldPlus zur Familienarbeitszeit?}

Als zukünftige Familienpolitik steht eine Familienarbeitszeit zur Debatte, die Eltern wie auch Alleinerziehenden nach dem Ablauf des Elterngeldbezugs zur Verfügung stehen soll. Das Ziel der Familienarbeitszeit ist es, der Diskrepanz zwischen der gewünschten und der tatsächlichen Arbeitsteilung entgegenzuwirken und eine egalitäre Arbeitsteilung zu ermöglichen: Beide Eltern sollen "gleich viel Zuwendungszeit für das Kind haben, ihre Chancen im Beruf ergreifen und ihre Existenzen auf Dauer sichern können - wie sie es sich wünschen" (BMFSFJ 2016b, S. 6). Eltern sollen dabei 28-36 Wochenstunden der Erwerbsarbeit nachgehen, was $80-90 \%$ einer regulären Vollzeit entspricht. Diese große Teilzeit ermöglicht beiden Elternteilen eine eigenständige finanzielle Absicherung. Eine kleine Teilzeit mit weniger als 25 Wochenstunden soll damit vermieden werden, da sie ein Armutsrisiko für Mütter mit sich bringt (BMFSFJ 2016b, S. 6). Da die meisten Väter bislang in Vollzeit und die Mütter in kleiner Teilzeit erwerbstätig waren, bedeutet ein Erwerbsumfang von 28-36 Stunden für Mütter mehr Erwerbsarbeitszeit und für Väter mehr Familienzeit. Während der Familienzeit soll ein Familiengeld in Höhe von insgesamt $300 €$ pro Monat - d.h. $150 €$ pro Elternteil - gezahlt werden. Die maximale Bezugsdauer beträgt 24 Monate und das Kind darf höchstens acht Jahre alt sein (BMFSFJ 2016b, S. 8). Die Familienarbeitszeit und das Familiengeld sollen somit die Einkommenssituation von Familien mit Kindern unter acht Jahren verbessern, das Armutsrisiko für Mütter senken, die Altersvorsorge der Mütter optimieren und den Eltern mehr gemeinsame Zeit mit der Familie ermöglichen (BMFSFJ 2017a, S. 75).

Während das Modell der Familienarbeitszeit und des Familiengeldes noch zur Diskussion steht, ist das Brückenteilzeitgesetz bereits seit dem 01.01.2019 in Kraft getreten (Deutscher Bundestag 2018). Dieses richtet sich nicht ausschließlich an Eltern, sondern gilt allgemein für Arbeitnehmer und Arbeitnehmerinnen. Es soll dafür sorgen, dass man nach einer Teilzeitbeschäftigung leichter in die Vollzeitbeschäftigung zurückkehren kann. Im Unterschied zum Teilzeit- und Befristungsgesetz, das sich für eine Reduzierung der Arbeitszeit stark gemacht hat, erleichtert das Brückenteilzeitgesetz die Rückkehr zu einer verlängerten Arbeitszeit. Die Brückenteilzeit ermöglicht somit eine zeitlich begrenzte Teilzeitarbeit (Deutscher Bundestag 2018).

\section{Ausbau der Kinderbetreuung}

In den letzten Jahren wurde die Infrastruktur für Kinderbetreuung, insbesondere für Kinder unter drei Jahren, massiv ausgebaut. Eine erste rechtliche Grundlage war 2005 das Tagesbetreuungsausbaugesetz (TAG), welches zum Ziele hatte bis 2010230.000 zusätzliche Betreuungsplätze zu schaffen und in Westdeutschland eine Betreuungsquote von $17 \%$ zu erreichen (BMFSFJ 2008b, S. 27; Menke und Klammer 2017, S. 223). Im Jahr 2008 folgte das Kinderförderungsgesetz (KiföG), das sich zur Bereitstellung von 
weiteren Betreuungsplätzen für Kinder unter drei Jahren verpflichtete (Menke und Klammer 2017, S. 223). Das Kinderförderungsgesetz wurde um einen wichtigen Rechtsanspruch erweitert: So gilt seit dem 01.08.2013 für Kinder ab dem vollendeten ersten bis zum vollendeten dritten Lebensjahr ein Rechtsanspruch auf einen Betreuungsplatz in einer Kindertageseinrichtung oder Kindertagespflege (Bertram und Bujard 2012, S. 11; Menke und Klammer 2017, S. 223). Der Ausbau der Betreuungsplätze und die gesetzlichen Änderungen zeigen sich auch empirisch durch einen deutlichen Anstieg der Betreuungsquote von Kindern unter drei Jahren: 2008 lag sie bei 17,6\%, 2010 bei 23,0\%, 2012 bei $27,6 \%$ und 2014 schließlich bei 32,2\% (BMFSFJ 2015, S. 45). Der Umfang des Kinderbetreuungsplatzes orientiert sich am Umfang der Erwerbstätigkeit beider Eltern (bzw. bei Alleinerziehenden an deren alleinigen Erwerbsumfang), sodass etwa aus einem Halbtagsjob der Rechtsanspruch auf einen halbtägigen Betreuungsplatz entsteht (Menke und Klammer 2017, S. 223). Schließlich trat 2019 das Gute-Kita Gesetz in Kraft, dass bis 2020 die Qualität der Kinderbetreuungsplätze verbessern soll.

Dieser verstärkte Ausbau der Kinderbetreuung ermöglicht Eltern eine bessere Vereinbarkeit von Beruf und Familie. Eltern haben dadurch mehr Wahlfreiheit in der Aufteilung von Erwerbsarbeit und Sorgearbeit. Eltern, die aus finanziellen Gründen bald wieder einer Erwerbstätigkeit nachgehen müssen, können dies dank dem Rechtsanspruch tun. Auch Mütter, die etwa aufgrund besserer Karrierechancen bald wieder in die Erwerbstätigkeit einsteigen wollen, werden so in ihrem Vorhaben unterstützt. Damit leistet der Rechtsanspruch auf einen Betreuungsplatz einen wichtigen Beitrag zu mehr Geschlechtergerechtigkeit (Menke und Klammer 2017, S. 223). Kritisch anzumerken ist hierbei jedoch, dass auch der Rechtsanspruch an den Kinderbetreuungsplatz für unter dreijährige Kinder - ebenso wie das weiter oben beschriebene Elterngeld - von der Erwerbstätigkeit bzw. dem Umfang der Erwerbstätigkeit der Eltern abhängig ist.

Bislang wurde auf die Kinderbetreuung für Kinder unter drei Jahren eingegangen; für Kinder ab drei Jahren bis zum Schuleintritt gilt bereits seit 1996 ein Rechtsanspruch auf einen Kindergartenplatz. Der Großteil der Kinder besucht einen Kindergarten: 2006 lag die Betreuungsquote bei $87,6 \%, 2008$ bei $91,6 \%, 2010$ bei $93,2 \%$ und 2016 bei $94,0 \%$ (BMFSFJ 2017b, S. 15). Obwohl also die Betreuungsquote für Kinder von drei bis sechs Jahren bereits 2006 relativ hoch war, so ist auch hier die Tendenz weiter steigend. 96,5\% der Eltern äußerten 2016 einen Betreuungsbedarf. Da die Betreuungsquote 2016 bei $94,0 \%$ lag, beträgt die Lücke zwischen Betreuungsbedarf und -quote somit lediglich 2,5\% (BMFSFJ 2017b, S. 16). Etwa die Hälfte der Eltern (49,0\%) buchte 2016 einen Ganztagsplatz (mehr als 35 Stunden pro Woche), 13,1\% buchten einen Halbtagsplatz (maximal 25 Stunden pro Woche) und 37,8\% einen erweiterten Halbtagsplatz (zwischen 26 und 35 Stunden pro Woche) (BMFSFJ 2017b, S. 20).

Welche Schlussfolgerungen lassen sich aus diesen familienpolitischen Rahmenbedingungen für die Arbeitsteilung der Eltern in Abhängigkeit vom Alter des Kindes ziehen? 
Sprechen diese Maßnahmen eher dafür, dass sich die Arbeitsteilung mit dem Älterwerden des Kindes verändert oder dass sich die unmittelbar nach der Geburt des Kindes gewählte Arbeitsteilung auch in den Folgejahren fortsetzt? Der ab 2007 eingeschlagene Paradigmenwechsel der Familienpolitik legt nahe, dass die traditionelle Arbeitsteilung, die im ersten Lebensjahr des Kindes besteht, nicht fortbestehen muss. Familienpolitische Maßnahmen wie die Begrenzung des Elterngeldes auf 12 bzw. 14 Monate und der Ausbau der Kinderbetreuungseinrichtungen (z.B. durch das TAG, das KiföG oder das Gute-Kita-Gesetz) unterstützen einen Wandel hin zu einer egalitäreren Arbeitsteilung. Wie aus der „policy-feedback-theory“ (Campbell 2012) hervorgeht, stehen politische Maßnahmen in Wechselwirkung zu individuelle Einstellungen, d.h. die Politik kann Einstellungen beeinflussen. Wenn die neueren familienpolitischen Maßnahmen sich für eine stärkere Geschlechtergerechtigkeit und eine bessere Vereinbarkeit von Beruf und Familie starkmachen, kann dies die Einstellungen der Bevölkerung prägen und dazu führen, dass etwa die Erwerbstätigkeit von Müttern mit kleinen Kindern gesellschaftlich akzeptierter wird als dies noch vor wenigen Jahrzehnten der Fall war. Insofern sind die Auswirkungen dieser neueren familienpolitischen Maßnahmen nicht zu unterschätzen. Gleichzeitig sind die Auswirkungen auch nicht zu überschätzen, denn ältere Regelungen, die das traditionelle männliche Ernährermodell unterstützen, wie z.B. das Ehegattensplittung, das Kindergeld oder die beitragsfreie Mitversicherung von Familienmitgliedern in der gesetzlichen Krankenversicherung (Grunow et al. 2011, S. 398; Bertram und Bujard 2012, S. 6; Müller et al. 2013, S. 3-4; Menke und Klammer 2017, S. 226), bleiben weiterhin bestehen und sprechen eher für eine stabile traditionelle Arbeitsteilung.

\subsection{Verknüpfung der theoretischen Ansätze mit den institutionel- len Rahmenbedingungen}

Aus den theoretischen Ansätzen ging hervor, dass Faktoren wie relative Ressourcen oder Geschlechterrolleneinstellungen eine zentrale Rolle für die Arbeitsteilung der Eltern spielen. Die Bedeutung dieser Faktoren vollzieht sich jedoch nicht im luftleeren Raum, sondern ist in institutionelle Rahmenbedingungen eingebettet. Um Hypothesen zur Arbeitsteilung der Eltern aufstellen zu können, müssen Einflussfaktoren wie die relativen Ressourcen oder die Geschlechterrolleneinstellungen somit unter Berücksichtigung der jeweiligen institutionellen Rahmenbedingungen betrachtet werden. Im Folgenden werden daher erneut die ökonomischen und die geschlechtsspezifischen Ansätze aufgegriffen und in Bezug zu den institutionellen Rahmenbedingungen gestellt.

Ökonomische Theorien zeichnen sich dadurch aus, dass sie auf einer Kosten-NutzenLogik basieren. Relative Ressourcen dienen dabei als Proxy für Verdienstmöglichkeiten bzw. für die innerpartnerschaftlichen Machtverhältnisse und Verhandlungspositionen. Nach der familienökonomischen Theorie ist der Haushaltsnutzen dann größer, wenn sich die Person mit den höheren, arbeitsmarktrelevanten Ressourcen auf die Erwerbsarbeit 
und die Person mit den niedrigeren Ressourcen auf die Hausarbeit spezialisiert. Bei der Verhandlungstheorie steht dagegen die individuelle Nutzenmaximierung im Vordergrund und höhere, relative Ressourcen helfen dabei, weniger von der unangenehmen Hausarbeit übernehmen zu müssen. Wie hoch der tatsächliche Nutzen ist und welche Kosten mit einer bestimmten Arbeitsteilung einhergehen, hängt jedoch entscheidend von den institutionellen Rahmenbedingungen ab.

Welche Rahmenbedingungen prägen also neben den relativen Ressourcen die Arbeitsteilung von Eltern in den jeweiligen Phasen des Familienzyklus? Für die erste Altersstufe, in der das Kind unter einem Jahr alt ist, sind als wesentliche institutionelle Rahmenbedingungen der Mutterschutz und das Elterngeld bzw. die Elternzeit zu nennen. Mit Ausnahme des Mutterschutzes handelt es sich beim Elterngeld und der Elternzeit um geschlechtsneutrale, politische Maßnahmen, da sie sowohl von Müttern als auch von Vätern bezogen werden können. Die sog. Vätermonate ermutigen Väter dazu, wenigstens zwei Monate Elterngeld zu beziehen und unterstützten dadurch eine egalitäre Arbeitsteilung. Trotz dieser modernen Familienpolitik sprechen die tatsächlichen Zahlen eine andere Sprache und deuten auf eine sehr geschlechtsasymmetrische Nutzung hin: 2007 sind $97 \%$ der Elterngeldbezieher Mütter und in 90\% ausschließlich die Mütter (in nur 3\% sind es ausschließlich die Väter) (BMFSFJ 2008a, S. 21); 2014 bezogen immerhin 34\% der Väter Elterngeld (BMFSFJ 2016c, S. 16), allerdings lag die Bezugsdauer bei 79\% nur bei höchstens zwei Monaten und geht damit nicht über die beiden Vätermonate hinaus (BMFSFJ 2016c, S. 18). Doch

„[w]enn eine augenscheinlich geschlechtsneutrale Familienpolitik vor allem von Frauen genutzt wird und wenn Männer nur wenig oder gar keine Auszeit von der Arbeit nehmen, um sich um ihre Kinder zu kümmern, bleibt die Ungleichheit der Geschlechter bei bezahlter und unbezahlter Arbeit bestehen" (Aisenbrey et al. 2009, S. 573, eigene Übersetzung vom Englischen ins Deutsche).

Wenn Väter im ersten Lebensjahr des Kindes ihre Erwerbstätigkeit nicht - oder nur für die besagten zwei Monate - unterbrechen, dann sind Mütter gezwungen, zu Hause zu bleiben, denn dass beide Elternteile im ersten Lebensjahr des Kindes wieder erwerbstätig sind, ist unwahrscheinlich und häufig ökonomisch unattraktiv. Damit Mütter nach Ablauf des Mutterschutzes wieder in die Erwerbstätigkeit einsteigen könnten und dies attraktiv und finanziell lohnenswert wäre, wären sie auf kostengünstige Betreuungseinrichtungen für Kleinkinder angewiesen, die zudem quantitativ und qualitativ gut ausgebaut sein sollten. Zwar gibt es Kinderkrippen, die Säuglinge und Kleinkinder ab einem Alter von etwa sechs Monaten betreuen, aber diese Krippenplätze sind rar und sehr kostenintensiv.

Allgemein kann angenommen werden: Je kleiner die Kinder sind, umso höher sind in der Regel die Betreuungskosten, da der Betreuungsschlüssel so ausgerichtet ist, dass eine Betreuungsperson nur eine sehr geringe Anzahl an Kleinkindern zu betreuen hat. Mit zunehmendem Alter des Kindes wird der Betreuungsschlüssel lockerer, sodass eine Be- 
treuungsperson für eine größere Anzahl an Kindern zuständig ist. Diese niedrigeren Personalkosten schlagen sich wiederum in niedrigeren Betreuungskosten nieder. Wegen der hohen Betreuungskosten wird es sich in vielen Fällen für die Mütter finanziell nicht lohnen wieder in die Erwerbstätigkeit zurückzukehren, solange sich das Kind noch im ersten Lebensjahr befindet.

Wenn das Kind im Alter von ein bis unter drei Jahren ist, ist davon auszugehen, dass Mütter mit höheren oder gleichen relativen Ressourcen wieder eher einer Erwerbstätigkeit nachgehen und die Aufteilung der Erwerbsarbeit und damit einhergehend auch die Aufteilung der Hausarbeit und Kinderbetreuung bereits etwas egalitärer ausfällt. Zwar erschweren institutionelle Rahmenbedingungen wie ein unzureichender Ausbau an Kinderbetreuungsplätzen, zu kurze Öffnungszeiten der Betreuungseinrichtungen und traditionelle Familienleitbilder den Müttern die Rückkehr in die Erwerbstätigkeit, aber durch den Wegfall des Elterngeldes erhöhen sich die Kosten, die mit einem Verbleiben in der Nicht-Erwerbstätigkeit verbunden wären. Zudem bemühen sich neuere familienpolitische Maßnahmen wie das Tagesbetreuungsausbaugesetz (TAG), das Kinderförderungsgesetz (KiföG) und das Gute-Kita-Gesetz darum, den Ausbau der U3-Kinderbetreuung sowohl quantitativ als auch qualitativ zu verbessern.

Eine deutliche Verbesserung der institutionellen Rahmenbedingungen tritt ein, wenn sich das Kind im Kindergartenalter befindet. Das Recht auf einen Kindergartenplatz besteht bereits seit 1996 und hat sich gesellschaftlich etabliert, wie sich auch an der starken Inanspruchnahme eines Kindergartenplatzes zeigt. Mütter mit höheren Ressourcen können somit leichter wieder erwerbstätig sein. Es ist deshalb davon auszugehen, dass bei Müttern mit gleichen oder höheren Ressourcen die Aufteilung der Erwerbsarbeit, der Hausarbeit und der Kinderbetreuung deutlich egalitärer ist, wenn sich das Kind bereits im Kindergartenalter befindet.

Mit dem Eintritt ins Schulalter verringert sich der Betreuungsaufwand für Eltern durch den Schulbesuch, durch externe Betreuungsangebote sowie durch die zunehmende Selbstständigkeit des Kindes. Durch den verringerten Betreuungsaufwand und die dadurch freiwerdende Zeit können Mütter wieder erwerbstätig sein bzw. ihren Erwerbsumfang erhöhen, insbesondere dann, wenn die Mütter höheren Ressourcen haben als die Väter. Bei Müttern mit höheren Ressourcen wird die Aufteilung der Erwerbsarbeit daher egalitärer sein. Mit dem Übergang ins Jugendalter verringert sich der Betreuungsaufwand weiter, sodass die Erwerbsarbeit der Mütter kaum noch durch zeitintensive Betreuung erschwert wird. Die Kinder bzw. Jugendlichen können stattdessen von dem zusätzlichen Einkommen der Mütter profitieren.

Während sich mit zunehmendem Kindesalter der benötigte Betreuungsaufwand reduziert, bleibt die Hausarbeit relativ unabhängig vom Kindesalter. Wenn davon ausgegangen wird, dass die Erwerbsarbeit mit zunehmendem Alter des Kindes egalitärer aufgeteilt wird, sie also veränderlich über die Phasen des Familienzyklus ist, so gilt gleiches nicht 
für die Hausarbeit. Dies mag damit zusammenhängen, dass sich die Trias der Familienpolitik v.a. auf die Aspekte der Erwerbsarbeit und der externen Kinderbetreuung bezieht und die Hausarbeit unberücksichtigt bleibt. Sämtliche familienpolitische Maßnahmen bewirken keine Entlastung für Mütter (und Väter) von der zu verrichtenden Hausarbeit.

Zusammenfassend lässt sich für die Verknüpfung der ökonomischen Rational-ChoiceLogik mit den institutionellen Rahmenbedingungen festhalten, dass die Bedeutung der relativen Ressourcen für die Arbeitsteilung relativ gering ist, wenn sich das jüngste Kind im Kleinkindalter befindet: Aufgrund mangelnder externer Kinderbetreuungsmöglichkeiten und hohen Betreuungskosten können Mütter von Kleinkindern ihre höheren Ressourcen (noch) nicht wirksam in Form von hohen Erwerbsumfängen umsetzen, sodass eine traditionelle Arbeitsteilung zu erwarten ist, in der Väter in Vollzeit erwerbstätig sind und Mütter den Großteil der Hausarbeit und der Kinderbetreuung übernehmen. Sobald das Kind im Kindergartenalter ist, lohnt es sich für Mütter eher wieder erwerbstätig zu sein, sodass anzunehmen ist, dass die Aufteilung der Erwerbsarbeit ab diesem Kindesalter wieder egalitärer wird - insbesondere dann, wenn die Mütter höhere Ressourcen aufweisen als die Väter. Mit dem Eintritt ins Schulalter sind Kinder längere Zeit extern betreut: Sei es etwa allein durch den Schulunterricht oder zusätzlich noch durch eine außerschulische Nachmittagsbetreuung. Die Betreuungskosten fallen dabei deutlich geringer aus als bei kleineren Kindern, da der Schulunterricht kostenlos ist und auch eine Nachmittagsbetreuung bei Schulkindern aufgrund eines ausgeweiteten Betreuungsschlüssels kostengünstiger ist als bei Kleinkindern. Somit übersteigt der Nutzen der Erwerbstätigkeit der Mütter leichter die Kosten der Kinderbetreuung, weshalb bei älteren Kindern höhere Erwerbsumfänge der Mütter und damit eine egalitärere Aufteilung der Erwerbsarbeit zu erwarten sind.

Als nächstes werden die geschlechtsspezifischen Ansätze mit den institutionellen Rahmenbedingungen verknüpft. Bei den geschlechtsspezifischen Ansätzen stehen Geschlechterrollen bzw. -stereotypen und Geschlechterrolleneinstellungen im Fokus. Aus einer Perspektive der Sozialisationstheorie heraus wird angenommen, dass sich individuelle Geschlechterrolleneinstellungen in der Kindheit, im Jungendalter und ggf. noch im frühen Erwachsenenalter herausbilden, aber im weiteren Erwachsenenalter dann relativ stabil bleiben. Die Lebenslaufforschung konnte jedoch aufzeigen, dass sich Einstellungen auch noch im Erwachsenenalter ändern können, insbesondere an bestimmten Übergängen wie etwa dem Übergang in die Elternschaft, der Einstieg in die Erwerbsarbeit oder durch die Heirat (siehe Kapitel 4.2.4 Stabilität oder Wandelbarkeit von Einstellungen).

Neben den individuellen Einstellungen spielen jedoch auch institutionelle Rahmenbedingungen eine zentrale Rolle für die tatsächlich praktizierte Arbeitsteilung. So bestimmen institutionelle Rahmenbedingungen mit darüber, inwiefern individuelle Einstellungen in Form einer einstellungskonformen Arbeitsteilung realisiert werden können. Institutionelle 
Rahmenbedingungen transportieren immer auch Ideale und Leitbilder mit. Während individuelle Einstellungen und praktizierte Arbeitsteilungsmuster durch institutionelle Rahmenbedingungen geprägt werden, können sie sich auch umgekehrt auf die institutionellen Rahmenbedingungen auswirken. Diese Zusammenhänge werden im Folgenden anhand der Geschlechterkultur und der „policy-feedback-theory“ näher erläutert.

Nach Pfau-Effinger spielt die Geschlechterkultur eine entscheidende Rolle für die individuellen Einstellungen und die Arbeitsteilung. Die Geschlechterkultur bildet zusammen mit der Geschlechterordnung das Geschlechterarrangement. Die Geschlechterkultur umfasst dabei

„dominierende Werte und Leitbilder in Bezug auf die Geschlechterbeziehungen (...), welche auch in der Form von Normen im institutionellen System verankert und deshalb relativ stabil sind" (Pfau-Effinger 2005, S. 3).

Verschiedene Geschlechterarrangements in West- und Ostdeutschland deuten auf unterschiedliche Leitbilder hin: In Westdeutschland überwiegt die Vorstellung einer privaten Kindheit, in der die Eltern möglichst viel Zeit mit den Kindern verbringen, wobei die primäre Zuständigkeit für die Kinderbetreuung bei den Müttern liegt, während die Väter die Rolle des Familienernährers innehaben. Bei sehr kleinen Kinder, die noch sehr betreuungsbedürftig sind, hat sich eine komplette Erwerbsunterbrechung der Mütter etabliert, gefolgt von einer Teilzeitbeschäftigung der Mütter bis die Kinder nicht mehr betreuungsbedürftig sind (Pfau-Effinger 2005, S. 4). Dagegen wird in Ostdeutschland von beiden Elternteilen eine Erwerbstätigkeit in Vollzeit erwartet und die Kinderbetreuung schon bei sehr kleinen Kindern ausgelagert (Pfau-Effinger 2005, S. 5).

Ein Beispiel für die Geschlechterkultur ist das Leitbild des „intensive mothering“ (Hays 1996). Aus einer Studie von Dechant und Rinklake (2016) geht hervor, dass das Ideal des „intensive mothering“ auch in Deutschland vorherrschend ist. Nach diesem Ideal sind Mütter die primäre und auch die beste Betreuungsperson (Hays 1996). Gleichzeitig werden starke Anforderungen an die Mutterrolle gestellt, denn beim Ideal des „intensive mothering" handelt es sich um einen kindzentrierten Ansatz, d.h. Mütter setzen das Wohl des Kindes an erste Stelle und damit noch vor ihre eigenen Bedürfnisse. Sie widmen sich zeitintensiv und aufopferungsvoll der Kinderbetreuung (Hays 1996). Eine „gute Mutter" ist demnach eine Mutter, bei der das Wohlergehen des Kindes oberste Priorität hat. Eine Mutter, die zu früh wieder in die Erwerbstätigkeit einsteigt, wird diesem Ideal nicht gerecht und kann leicht als „Rabenmutter" stigmatisiert werden (Diabaté 2015; Hipp 2018).

Die Geschlechterkultur im Allgemeinen sowie einzelne Leitbilder im Konkreten können in einem Spannungsverhältnis zu den individuellen Einstellungen stehen. Eine in (West-)Deutschland vorherrschende, traditionelle Geschlechterkultur mit dem Ideal der privaten Kindheit und mit Leitbildern wie dem „intensive mothering“ sprechen deshalb dafür, dass Eltern mit egalitären Einstellungen in einem Spannungsverhältnis zur traditionellen Geschlechterkultur stehen. Das Spannungsverhältnis ist besonders groß, wenn 
die Kinder noch sehr klein sind. Dies hängt damit zusammen, dass die gesellschaftlichen Erwartungen an die Mutterrolle größer sind, wenn das Kind noch sehr klein ist. Mit zunehmendem Alter des Kindes verändern sich die Erwartungen an die Mutterrolle: So ist etwa die ständige Verfügbarkeit der Mütter bei älteren Kindern nicht mehr ganz so wichtig wie noch bei kleineren Kindern. Daher muss die eigenständige Erwerbstätigkeit der Mütter bei älteren Kindern auch nicht mehr im Widerspruch zum Leitbild der "guten Mutter“ stehen. Egalitäre Einstellungen können somit bei älteren Kindern leichter in Form einer egalitären Arbeitsteilung realisiert werden, da sie dann nicht mehr in einem Spannungsverhältnis zu den gesellschaftlichen Leitbildern stehen. Es ist deshalb davon auszugehen, dass individuelle, egalitäre Einstellungen bei Eltern von älteren Kindern einen größeren Effekt auf die Arbeitsteilung haben als bei Eltern von jüngeren Kindern.

Gesellschaftliche Leitbilder lassen sich auch daran ablesen, wie gut die Kinderbetreuungsplätze in den jeweiligen Altersstufen des Kindes ausgebaut sind und inwiefern diese in Anspruch genommen werden. Der Ausbau der Kinderbetreuungsplätze bzw. der Betreuungsquote kann jedoch in Wechselwirkung zu den individuellen Einstellungen stehen. So ist etwa mit dem Ausbau der Kinderbetreuungsplätze auch die Nachfrage danach gestiegen, d.h. es besteht eine Wechselwirkung zwischen dem Ausbau und dem individuellen Nutzungsverhalten, welches sich auf die Betreuungsquote auswirkt. Die Kinderbetreuungsquote kann daher gleichzeitig auch als „ein Proxy gesehen werden, der die Wechselwirkung zwischen politischem Engagement zum Ausbau der Kinderbetreuung und der persönlichen Entscheidung zur Nutzung beschreibt“" (Diener und Berngruber 2018, S. 126). Diese Wechselwirkungen zwischen familienpolitischen Maßnahmen und individuellen Verhalten bzw. individuellen Einstellungen ähneln einem Prozess, der auch als „policy-feedback-theory“ geläufig ist (Campbell 2012).

In einer Studie von Gangl und Ziefle (2015) wird dies anhand der Folgen ersichtlich, die die Ausweitung des Bundeserziehungsurlaubs auf drei Jahre im Jahr 1992 hatte. Diese Ausweitung des Bundeserziehungsurlaubs hatte nicht nur zur Folge, dass Frauen ihr Verhalten änderten und ihre Erwerbsarbeit länger unterbrachen, sondern auch ihre Präferenzen änderten sich und der Erwerbsarbeit wurde eine geringe Bedeutung beigemessen (Gangl und Ziefle 2015). Wenn lange Elternurlaubsansprüche dazu führen, dass längere Auszeiten genommen werden und die Präferenzen familienorientierter werden, ist es naheliegend, dass umgekehrt durch eine Verkürzung der Ansprüche und einen Ausbau der Kinderbetreuungsmöglichkeiten die Präferenzen wieder berufsorientierter werden können. Familienpolitische Maßnahmen, die eine egalitäre Arbeitsteilung fördern, können somit auch dazu führen, dass die Einstellungen egalitärer werden.

Obwohl sich die Familienpolitik in den letzten Jahren intensiv darum bemüht hat, die U3Kinderbetreuung auszubauen, so sind die Möglichkeiten zur externen Kinderbetreuung bei Kindern unter drei Jahren immer noch schlechter bestellt als bei Kindern über drei Jahren. Dementsprechend repräsentiert die Familienpolitik bei Kleinkindern immer noch traditionellere Leitbilder, die eine innerfamiliale Arbeitsteilung nahelegen, als bei älteren 
Kindern. Auch am Beispiel der Kinderbetreuungssituation lässt sich daher annehmen, dass Eltern mit egalitäreren Einstellungen auf größere Widerstände stoßen, wenn die Kinder kleiner sind und die Realisierung einer egalitären Arbeitsteilung daher erschwert ist. Somit ist auch hier anzunehmen, dass individuelle Einstellungen bei kleineren Kindern - aufgrund ungünstiger institutioneller Rahmenbedingungen - einen geringeren Effekt auf die praktizierte Arbeitsteilung haben; bei älteren Kindern sind aufgrund verbesserter institutioneller Rahmenbedingungen dann stärkere Effekte auf die Arbeitsteilung zu erwarten.

Zusammenfassend lässt sich festhalten, dass sich bei einer Verknüpfung der theoretischen Ansätze mit den institutionellen Rahmenbedingungen andere Vorhersagen ergeben als bei einer ausschließlichen Betrachtung der theoretischen Ansätze: Würde man allein die theoretischen Ansätze betrachten (vgl. Kapitel 4), so wäre von einer relativ stabilen Arbeitsteilung auszugehen, die sich mit den Altersstufen des Kindes nicht oder nur sehr geringfügig ändert. Bezieht man jedoch die institutionellen Rahmenbedingungen mit ein, so ist davon auszugehen, dass sich die Arbeitsteilung mit dem Alter des Kindes verändert, da die institutionellen Rahmenbedingungen je nach Kindesalter anders ausfallen. Sowohl bei den ökonomischen als auch bei den geschlechtsspezifischen Ansätzen ist davon auszugehen, dass die Arbeitsteilung bei jüngeren Kindern traditioneller ist und bei älteren Kindern egalitärer wird, allerdings sind die dahinter stehenden Erklärungen verschieden: Gemäß den ökonomischen Theorien erscheint eine egalitäre Arbeitsteilung bei kleinen Kindern unter Berücksichtigung der institutionellen Rahmenbedingungen finanziell nicht lohnenswert, d.h. es sind Kosten-Nutzen-Überlegungen, die gegen eine egalitäre und für eine traditionelle Arbeitsteilung sprechen. Mit zunehmendem Alter des Kindes verbessern sich die Rahmenbedingungen, sodass eine egalitärere Arbeitsteilung rentabler wird. Nach den geschlechtsspezifischen Ansätzen ist eine egalitäre Arbeitsteilung bei kleinen Kindern unter Berücksichtigung der institutionellen Rahmenbedingungen unwahrscheinlicher, weil die Geschlechterkultur bzw. die gesellschaftlichen Leitbilder noch traditioneller sind. Eltern mit egalitären Einstellungen stehen daher eher im Widerspruch zu den gesellschaftlichen Leitbildern, wenn sie kleine Kinder haben, als wenn sie ältere Kinder haben. Eine Verknüpfung der theoretischen Ansätze mit den institutionellen Rahmenbedingungen ergibt daher, dass sich die Arbeitsteilung mit dem Alter des Kindes verändert, da sich die institutionellen Rahmenbedingungen mit dem Alter des Kindes verändert haben. 


\section{Hypothesengenerierung}

Bei der Ableitung der Hypothesen wird im ersten Teil zunächst eine rein theoretische Perspektive eingenommen, in der die institutionellen Rahmenbedingungen unberücksichtigt bleiben. Aus dieser theoretischen Sichtweise werden dann Hypothesen auf Basis der ökonomischen Theorien und anschließend auf Basis der geschlechtsspezifischen Ansätze abgeleitet. Im zweiten Teil werden die institutionellen Rahmenbedingungen mitberücksichtigt und darauf eingegangen, inwiefern sich unter dieser komplexeren Sichtweise dann andere Hypothesen ergeben.

Theoretische Perspektive - ohne institutionelle Rahmenbedingungen

Familienökonomische Argumentation

H1: Diejenige Person mit den höheren, relativen Ressourcen spezialisiert sich auf die Erwerbsarbeit und die andere Person auf die Hausarbeit und Kinderbetreuung.

Die eingeschlagene Spezialisierungsrichtung wird beibehalten, d.h. die Arbeitsteilung bleibt relativ stabil und ändert sich nicht oder nur geringfügig mit dem Alter des Kindes.

Verhandlungstheoretische Argumentation (nach Ott)

H2: $\quad a)^{8}$ Die Verhandlungstheorie nimmt allgemein an:

Diejenige Person mit den höheren, relativen Ressourcen hat mehr Verhandlungsmacht und muss weniger von der Hausarbeit und von der Kinderbetreuung ${ }^{9}$ übernehmen; diejenige Person mit den höheren, relativen Ressourcen kann stattdessen mehr Erwerbsarbeit übernehmen.

\footnotetext{
${ }^{8}$ Diese Hypothese wurde 2a) genannt und die nachfolgenden Hypothese 3a), weil es auf den folgenden Seiten noch weitere Varianten zu H2 und $\mathrm{H} 3$ kommen werden.

9 Die Kinderbetreuung wurde hier mitaufgenommen, da Ott, wenn sie von „Hausarbeit“ spricht, auch die innerfamiliale Kinderbetreuung miteinschließt. Dies wird z.B. an folgender Textstelle ersichtlich: „Danach ist die Nachfrage nach typischen Haushaltsgütern in der Phase, in der kleine Kinder im Haushalt leben, besonders hoch. Vor allem werden in dieser Phase die besonders zeitintensiven Güter (Kindererziehung) nachgefragt. Entsprechend kann für diese Phase von einem hohen Zeitbedarf für Haushaltsproduktion ausgegangen werden" (Ott 1993, S. 34). Ott schließt somit die Kindererziehung in die Hausarbeit mit ein und begreift Kindererziehung als typische Haushaltsgüter. An späterer Stelle erläutert sie, warum für Frauen eine Spezialisierung auf die Hausarbeit unattraktiv ist und es deswegen zu einem Geburtenrückgang kommt: „Die hohen Opportunitätskosten der Kindererziehung übersteigen deren Nutzen, was den Geburtenrückgang zumindest zum Teil erklären kann" (Ott 1993, S. 47). Aus diesem letzten Zitat wird ersichtlich, dass die Kindererziehung (auch wenn sie angenehmer sein mag als Hausarbeit im Sinne von Putzen oder Wäsche waschen) mit Opportunitätskosten einhergeht. Bei Müttern mit hohen Ressourcen (wie einem hohen Einkommenspotential) sind die Opportunitätskosten der Hausarbeit und Kindererziehung zudem besonders hoch. Die Tatsache, dass Kinderbetreuung
} 
Diejenige Person mit den niedrigeren, relativen Ressourcen hat weniger Verhandlungsmacht und muss mehr von der Hausarbeit und von der Kinderbetreuung übernehmen; diejenige Person mit den niedrigeren, relativen Ressourcen übernimmt weniger Erwerbsarbeit.

Mit dem Übergang zur Elternschaft findet bei Müttern eine Erwerbsunterbrechung und eine Spezialisierung auf die Hausarbeit und Kinderbetreuung statt. Dadurch verschlechtern sich ihre Ressourcen und ihre Verhandlungspositionen nachhal$\operatorname{tig}^{10}$, d.h. Mütter übernehmen mehr Hausarbeit und Kinderbetreuung und diese Aufteilung bleibt relativ stabil.

\section{Geschlechterrollentheoretische Argumentation}

H3: a) Wenn Mütter egalitäre (resp. traditionelle) Einstellungen haben, dann ist die Arbeitsteilung egalitärer (resp. traditioneller). ${ }^{11}$

Des Weiteren wird angenommen, dass die Einstellungen stabil sind und sich im Erwachsenenalter kaum noch ändern. Daraus lässt sich schlussfolgern:

Wenn Mütter egalitäre (resp. traditionelle) Einstellungen haben und diese Einstellungen stabil bleiben, dann ist auch die Arbeitsteilung egalitärer (resp. traditioneller) und ändert sich nicht in Abhängigkeit vom Alter des Kindes.

Die bisherigen, ausschließlich theoriegeleiteten Hypothesen nehmen an, dass die Arbeitsteilung stabil bleibt und sich mit zunehmendem Alter des Kindes nicht oder nur sehr wenig verändert. Sobald jedoch neben den Theorien auch die institutionellen Rahmenbedingungen mitberücksichtigt werden, ergibt sich ein anderes Bild, das eine größere Dynamik bei der Arbeitsteilung in Abhängigkeit vom Alter des Kindes zeigt. Im Folgenden

nutzenstiftend und angenehmer als Hausarbeit ist und deswegen keineswegs automatisch vermieden werden will, wird in den Hypothesen 4a) bis 4d) zum Leitbild des „intensive mothering“ berücksichtigt.

10 Ott schreibt, dass die Auswirkungen eines Verlustes bzw. einer Unterbrechung der Erwerbstätigkeit nahezu irreversibel sind und da es in der Regel die Frauen sind, die mit der Geburt des Kindes ihre Erwerbstätigkeit unterbrechen, geht die Geburt des Kindes für Frauen mit einer nachhaltigen Ressourcenverschlechterung einher (Ott 1992, S. 105-106): „Let us consider a situation where the wish for a child can be realized only by an interruption in the working life of one spouse, which is in most cases the wife. This is a typical situation in Germany and in many other countries because of a lack of institutions for child care. Then, the welfare gain of the household is combined with a large decrease in the bargaining position of the wife" (Ott 1992, S. 106).

11 Gleiches lässt sich auch für die Väter annehmen. Da jedoch in der hier verwendeten Datengrundlage (DJI-Survey „Aufwachsen in Deutschland: Alltagswelten“) die Fallzahlen der Väterangaben deutlich geringer ausfallen als die Fallzahlen der Mütterangaben und deshalb in der vorliegenden Arbeit auch nur die Mütterangaben empirisch überprüft werden, werden hier keine Hypothesen zu den Einstellungen der Väter aufgestellt. 
werden daher Hypothesen zu den ökonomischen Theorien und den geschlechtsspezifischen Ansätzen formuliert und die institutionellen Rahmenbedingungen miteinbezogen.

Theoretische Perspektive - mit institutionellen Rahmenbedingungen

Da die institutionellen Rahmenbedingungen stark vom Alter des Kindes abhängig sind, werden im Folgenden altersspezifische Hypothesen abgeleitet.

Verhandlungstheoretische Argumentation inkl. der institutionellen Rahmenbedingungen

Bei den ökonomischen Theorien wird im Folgenden näher auf die Verhandlungstheorie nach Ott eingegangen, da diese flexibler auf institutionelle Rahmenbedingungen reagiert als die familienökonomische Theorie nach Becker. ${ }^{12}$ Unter Berücksichtigung der institutionellen Rahmenbedingungen lassen sich aus verhandlungstheoretischer Argumentation die nachfolgenden Hypothesen aufstellen, die die Hypothese 2 a) erweitern. Hypothese 2 a) nimmt an, dass diejenige Person mit den höheren Ressourcen weniger Hausarbeit und Kinderbetreuung und mehr Erwerbsarbeit übernimmt. Wenn Mütter die höheren Ressourcen haben, dann übernehmen sie demzufolge weniger unbezahlte Arbeit und mehr Erwerbsarbeit, sodass die Arbeitsteilung egalitärer ist. Ob bei Müttern die höheren, relativen Ressourcen jedoch tatsächlich zu einer egalitäreren Arbeitsteilung führen, ist von institutionellen Rahmenbedingungen abhängig und diese Rahmenbedingungen unterscheiden sich nach dem Alter des Kindes. ${ }^{13}$

H2 altersspezifisch:

2 b) Bei einem Kind im Alter von unter einem Jahr beziehen alle Mütter zunächst 8 Wochen Mutterschutz. Anschließend nehmen die meisten Mütter unabhängig von ihren Ressourcen bis zu 12 Monate Elterngeld. Eine frühe Rückkehr in die Erwerbstätigkeit ist für viele Mütter ökonomisch nicht attraktiv, weil Krippenplätze schlecht ausgebaut und teuer sind. Die Rückkehr in die Erwerbstätigkeit wäre

\footnotetext{
12 Die familienökonomische Theorie nach Becker nimmt an, dass die eingeschlagene Spezialisierungsrichtung beibehalten wird und sich auch nicht mit dem Alter des Kindes verändert. Mit dem Beginn einer Partnerschaft werden Weichen gestellt, die die zukünftige Arbeitsteilung festlegen, wobei institutionelle Rahmenbedingungen an dieser Starrheit kaum etwas verändern können. Im Gegensatz dazu ist die Verhandlungstheorie in ihrem Ansatz dynamisch und durch institutionelle Rahmenbedingungen leichter zu beeinflussen.

${ }^{13}$ Die relativen Ressourcen werden in den empirischen Analysen anhand des relativen Berufsstatus (nach ISEI) operationalisiert. Bei den Hypothesen werden an dieser Stelle zunächst nur relative Ressourcen, d.h. der relative Berufsstatus, betrachtet. Es spielen jedoch auch absolute Ressourcen wie der absolute Berufsstatus (nach ISEI) eine wichtige Rolle: So entstehen für Mütter mit hohem Berufsstatus durch den Verzicht auf die Erwerbstätigkeit bzw. durch eine Reduktion des Erwerbsumfangs höhere Opportunitätskosten als für Mütter mit niedrigem Berufsstatus. Die Tatsache, dass auch der absolute Berufsstatus von Bedeutung ist, wird an späterer Stelle bei Hypothese 7 mitberücksichtigt.
} 
somit mit hohen Betreuungskosten verbunden, die den Nutzen der Erwerbstätigkeit leicht übersteigen, sodass eine Erwerbstätigkeit finanziell nicht lohnend ist.

Wenn das Kind unter einem Jahr alt ist, dann spielen relative Ressourcen keine oder nur eine sehr geringe Rolle.

2 c) Bei einem Kind im Alter ein bis unter drei Jahren erschweren unzureichend ausgebaute, öffentliche Kinderbetreuungsstätten und die damit verbundenen Betreuungskosten den Müttern weiterhin eine Rückkehr in die Erwerbstätigkeit. Die Möglichkeit, bis zum dritten Lebensjahr des Kindes in Elternzeit zu gehen, erscheint für viele Mütter einerseits attraktiv, weil dann eine innerfamiliale Betreuung stattfinden kann und keine Betreuungskosten anfallen. Die Tatsache, dass in der Elternzeit jedoch keine Lohnersatzzahlung mehr gegeben ist, macht die Nutzung der Elternzeit andererseits ökonomisch unattraktiver. Aufgrund des deutlich hinter dem Bedarf zurückbleibenden Ausbaus der Betreuungsplätze, beanspruchen viele Mütter weiterhin Elternzeit.

Auch wenn das Kind ein bis unter drei Jahre alt ist, dann spielen relative Ressourcen nur eine geringe Rolle; jedoch spielen sie bereits eine etwas größere Rolle als in der vorherigen Altersstufe.

2 d) Wenn das Kind drei bis unter sechs Jahre alt ist, verbessern sich durch die Möglichkeit des Kindergartenbesuchs die institutionellen Rahmenbedingungen, sodass Mütter wieder leichter erwerbstätig sein können und dies leichter rentabel ist. Eine weiter bestehende Erwerbslosigkeit wäre dagegen mit hohen Opportunitätskosten verbunden - insbesondere bei Müttern mit höheren, relativen Ressourcen. Für diese Altersgruppe sind somit deutliche Effekte zwischen den relativen Ressourcen der Mütter und der Arbeitsteilung zu erwarten.

Wenn das Kind drei bis unter sechs Jahre alt ist, dann spielen relative Ressourcen eine deutliche Rolle.

2 e) Mit dem Schuleintritt des Kindes verbessern sich die institutionellen Rahmenbedingungen weiter, da der Schulbesuch kostenlos ist und auch andere Betreuungsformen (wie z.B. der Hort) durch das fortgeschrittene Kindesalter günstiger werden. Die durch diese externe Betreuung freiwerdende Zeit können Mütter leichter in ihre Erwerbsarbeit investieren. Bei Kindern im Grundschulalter und ebenso bei Kindern im Jugendalter sind deshalb weiterhin deutliche Effekte zwischen den relativen Ressourcen der Mütter und der Arbeitsteilung zu erwarten.

Wenn das Kind sechs Jahre alt oder älter ist, dann spielen relative Ressourcen eine deutliche Rolle.

Zusammenfassend lässt sich festhalten, dass bei Kindern im Kleinkindalter ungünstige, institutionelle Rahmenbedingungen vorliegen, die Müttern selbst dann eine Rückkehr in 
die Erwerbsarbeit erschweren, wenn sie über die höheren Ressourcen verfügen. Mit zunehmendem Alter des Kindes verbessern sich die institutionellen Rahmenbedingungen, sodass bei Müttern mit höheren Ressourcen eine egalitärere Arbeitsteilung zu erwarten ist.

Geschlechterrollentheoretische Argumentation inkl. der institutionellen Rahmenbedingungen

Ausgangspunkt ist hier zunächst die Hypothese 3 a), die annimmt, dass bei Müttern mit egalitären Einstellungen die Arbeitsteilung egalitärer ist. Im Gegensatz zu Hypothese 3 a) wird jedoch nicht davon ausgegangen, dass die Arbeitsteilung über die Altersstufen des Kindes hinweg stabil bleibt, sondern dass institutionelle Rahmenbedingungen mit darüber entscheiden, ob Mütter ihre Einstellungen realisieren können:

H 3 altersspezifisch:

3 b) Bei einem Kind im Alter von unter einem Jahr stehen Mütter mit egalitären Einstellungen in einem großen Spannungsverhältnis zu gesellschaftlichen Normen zur Mutterschaft. Erwerbstätige Mütter würden im Konflikt zum Leitbild der guten Mutter stehen. Der sehr schlechte Ausbau von Betreuungsplätzen sowie die hohen Betreuungskosten verweisen darauf, dass Kinder unter einem Jahr in der Familie betreut werden sollen.

Wenn das Kind im Alter von unter einem Jahr ist, können Mütter ihre egalitären Einstellungen nicht realisieren.

3 c) Auch bei einem Kindesalter von einem bis unter drei Jahren stehen Mütter mit egalitären Einstellungen noch in großem Spannungsverhältnis zu Mutterschaftsleitbildern. So hält der Großteil der Bevölkerung eine Erwerbstätigkeit von Müttern in dieser Altersphase für verfrüht (Lietzmann und Wenzig 2017). Obwohl sich die neuere Familienpolitik intensiv darum bemüht, den Ausbau von Kleinkinderbetreuungsplätzen zu optimieren, sind diese immer noch unzureichend ausgebaut. ${ }^{14}$ Die Nutzung einer Kinderbetreuungsstätte für Kinder in dieser Altersphase hält die Mehrheit noch immer für zu früh (Lietzmann und Wenzig 2017).

Wenn das Kind im Alter von ein bis unter drei Jahren ist, können Mütter ihre egalitären Einstellungen nur schwer realisieren.

3 d) Bei einem Kind im Kindergartenalter ist die Inanspruchnahme eines Kindergartenplatzes gesellschaftlich akzeptiert und Mütter können durch die dadurch freiwerdende Zeit wieder leichter in die Erwerbstätigkeit einsteigen. Eine Teil-

\footnotetext{
14 Da die Daten, anhand denen die Hypothesen überprüft werden, von 2013/14 sind, bleiben politische Maßnahmen, die neuer sind (wie z.B. das Gute-Kita-Gesetz), an dieser Stelle unberücksichtigt.
} 
zeiterwerbstätigkeit der Mütter sowie die Nutzung einer (zumindest stundenweisen) externen Kinderbetreuung findet die Mehrheit der Deutschen für diese Altersphase angebracht (Lietzmann und Wenzig 2017).

Wenn das Kind im Alter von drei bis unter sechs Jahren ist, können Mütter ihre egalitären Einstellungen schon etwas leichter realisieren.

3 e) Bei Kindern über sechs Jahren werden die gesellschaftlichen Erwartungen an die Mutterrolle lockerer. Die Tatsache, dass der Schulbesuch verpflichtend ist, zeigt, dass eine längere Trennung von Mutter und Kind als zumutbar angesehen wird. Durch den Schulbesuch, ggf. den Besuch von Nachmittagsbetreuungen sowie die zunehmende Selbstständigkeit des Kindes, haben Mütter wieder mehr Zeit zur Verfügung, die sie für ihre Erwerbstätigkeit nutzen können. Für Kinder in dieser Altersphase wird eine Vollzeiterwerbstätigkeit der Mütter sowie eine ganztätige, außerfamiliale Kinderbetreuung vom Großteil der Bevölkerung als völlig legitim erachtet (Lietzmann und Wenzig 2017). Mütter mit egalitären Einstellungen stehen somit nicht mehr im Spannungsverhältnis zu gesellschaftlichen Normen und können ihre Einstellungen daher leicht realisieren.

Wenn das Kind im Grundschul- und Jugendalter ist, können Mütter ihre egalitären Einstellungen deutlich leichter realisieren.

Die bisherigen Hypothesen bezogen sich auf allgemeine Einstellungen zu Geschlechterrollen. Es gibt jedoch auch spezifischere Rollenerwartungen wie etwa das „intensive mothering" (Hays 1996). Wie weiter oben bereits erwähnt wurde, ist das Leitbild des „intensive mothering" auch in Deutschland präsent (Dechant und Rinklake 2016; Grunow et al. 2018). Dieses Leitbild stellt hohe Anforderungen an die Mutterrolle und richtet sich insbesondere an Mütter von kleinen Kindern. Das Leitbild des „intensive parenting“ bezieht die Väter explizit mit ein und betont die Wichtigkeit der Väter als Familienernährer und Betreuungspersonen (Wall 2010; Grunow et al. 2018). Bezugnehmend auf das Leitbild des „intensive mothering/parenting“ lässt sich daher die folgende Hypothese ableiten:

H 4 allgemein:

4 a) Mütter, die sich besonders mit dem Leitbild des „intensive mothering/parenting" identifizieren, werden sich verstärkt um die Kinderbetreuung und die Hausarbeit kümmern und wenn nötig, hierfür auch für längere Zeit ihre Erwerbstätigkeit unterbrechen oder reduzieren.

Wenn sich Mütter mit dem Leitbild des „intensive mothering/parenting“ identifizieren, dann ist die Arbeitsteilung traditioneller.

Aufgrund der institutionellen Rahmenbedingungen, die im vorherigen Abschnitt bei den allgemeinen Geschlechterrolleneinstellungen erläutert wurden, werden je nach Kindes- 
alter unterschiedlich starke Effekte zwischen dem Leitbild und der Arbeitsteilung angenommen: So wird angenommen, dass sich diese Effekte am stärksten bei noch unter 3jährigen Kindern zeigen, gefolgt von immer noch starken Effekten bei 3- bis unter 6jährigen Kindern und dass sie bei älteren Kindern nur noch in deutlich abgeschwächter Form vorliegen. Daraus ergeben sich die folgenden altersspezifischen Hypothesen ergeben:

H 4 altersspezifisch:

4 b) Wenn das Kind unter 3 Jahre alt ist, hat das Leitbild des ,intensive mothering/parenting" einen stark traditionalisierenden Effekt auf die Arbeitsteilung.

4 c) Wenn das Kind 3 bis unter 6 Jahre alt ist, hat das Leitbild des „intensive mothering/parenting" einen etwas abgeschwächten, aber immer noch traditionalisierenden Effekt auf die Arbeitsteilung.

4 d) Wenn das Kind im Grundschulalter ist, hat das Leitbild des „intensive mothering" keinen oder nur einen sehr geringen Effekt auf die Arbeitsteilung.

\section{Weitere Hypothesen}

Im Folgenden werden weitere Hypothesen aufgestellt, die Zusammenhänge zwischen Variablen und der Arbeitsteilung beschreiben. Diese Zusammenhänge mit anderen Variablen werden nur in komprimierter Form dargestellt und auf tiefergehende Ausführungen wird verzichtet.

\section{Anzahl der Kinder}

H5 allgemein:

5 a) In Familien mit mehreren Kindern ist die Arbeitsteilung traditioneller als in Familien mit einem Kind.

H5 altersspezifisch:

5 b) Wenn das jüngste Kind noch klein ist, sind die Unterschiede zwischen Einund Mehrkind-Familien besonders groß; je älter das jüngste Kind ist, umso unwichtiger wird die Anzahl der Kinder in der Familie.

\section{Ost-/West-Unterschiede}

H6 allgemein:

6 a) In Ostdeutschland ist die Arbeitsteilung egalitärer aufgeteilt als in Westdeutschland.

H6 altersspezifisch:

6 b) Wenn sich das Kind noch im Kleinkindalter befindet, dann sind die Unterschiede besonders groß; wenn sich das Kind im Kindergartenalter befindet, verringern sich die Unterschiede; bei älteren Kindern verringern sich die Unterschiede weiter, bleiben aber immer noch signifikant. 


\section{(absoluter) Berufsstatus der Mutter ${ }^{15}$}

H7 allgemein:

7 a) Bei Müttern mit hohem Berufsstatus ist die Arbeitsteilung egalitärer und bei Müttern mit niedrigem Berufsstatus ist sie traditioneller.

$\mathrm{H} 7$ altersspezifisch:

7 b) Wenn das Kind im Kleinkindalter ist, zeigen sich bereits Unterschiede zwischen Müttern mit hohem Berufsstatus und Müttern mit niedrigem Berufsstatus; wenn das Kind im Kindergarten- und Grundschulalter ist, werden die Unterschiede noch größer.

Nach dieser Darstellung der Hypothesen wird nun zum empirischen Teil der Arbeit übergegangen. In Kapitel 7 wird zunächst die Datengrundlage und die Methodik vorgestellt, während die Kapitel 8, 9 und 10 die empirischen Ergebnisse zur Aufteilung der Erwerbsarbeit, der Hausarbeit und der Kinderbetreuung beinhalten.

\footnotetext{
15 Um die Komplexität zu reduzieren, wurde der absolute Berufsstatus hier unter den weiteren Variablen positioniert und nur der relative Berufsstatus an vorderer Stelle ausführlicher behandelt. Dies hängt damit zusammen, dass der Fokus in der vorliegenden Arbeit auf der Paarebene und der relativen Arbeitsteilung zwischen den Elternteilen liegt. Die Bedeutung des absoluten Berufsstatus lässt sich jedoch auch unter Rückbezug auf die ökonomischen Theorien herleiten.
} 


\section{Daten und Methoden}

\subsection{Datengrundlage: DJI-Survey 2013/14 „AID:A“}

Als Datengrundlage zur Beantwortung der Forschungsfragen dient die zweite Welle des Surveys des Deutschen Jugendinstituts „Aufwachsen in Deutschland: Alltagswelten“ (kurz: „AID:A“). Bei diesem Survey handelt es sich um einen integrierten Survey, der frühere Einzelsurveys des Deutschen Jungendinstituts wie den Familiensurvey, den Jugendsurvey, das Kinderpanel und die Kinderbetreuungsstudie in eine große Gesamterhebung zusammenfasst (vgl. Rauschenbach 2012, S. 14). Das Ziel dieses integrierten Surveys ist es, das Aufwachsen von Kindern und Jugendlichen bis ins Erwachsenenalter hinein nachzeichnen zu können, den Alltag von Kindern, Jugendlichen, jungen Erwachsenen und deren Familien zu erfassen und Erkenntnisse über Lebenslagen, Wohlbefinden und Familienmodelle zu gewinnen. Der DJI-Survey „AID:A“ eignet sich sehr gut zur Beantwortung der Forschungsfragen, da er dezidiert nach der Aufteilung der Erwerbsarbeit, der Hausarbeit und Kinderbetreuung sowie nach Verschränkungen und Entgrenzungen der einzelnen Bereiche fragt. Auch liegen Angaben zu Geschlechterrolleneinstellungen vor. Der DJI-Survey liegt im Multi-Actor-Design vor, d.h. es wurden neben der Zielperson auch die erziehungsberechtigte Auskunftsperson (in der Regel die Mutter) und der Partner der Auskunftsperson (in der Regel der Vater) befragt.

Die zweite Welle des DJI-Surveys, auf der die folgenden Analysen basieren, wurde 2013/14 mittels telefonischer (CATI - Computer Assisted Telephone Interview) und persönlicher Interviews (CAPI - Computer Assisted Personal Interview) erfasst, die von den Instituten Infas und Infratest durchgeführt wurden (siehe Tabelle 7.1). Insgesamt wurden 22.691 Zielpersonen im Alter von 0 bis 32 Jahren erhoben. Bei Zielpersonen im Alter von 0 bis unter 9 Jahren wurden ausschließlich die Eltern, d.h. die Auskunftsperson und der Partner der Auskunftsperson befragt; bei Zielpersonen von 9 bis unter 18 Jahren wurden sowohl die Zielpersonen selbst, als auch die Eltern befragt und bei Zielpersonen von 18 bis 32 Jahren wurden ausschließlich die Zielpersonen selbst befragt (vgl. Infas Institut für angewandte Sozialwissenschaft 2015, S. 25).

Die Interviews zu den 22.691 Zielpersonen wurden in 12.903 Fällen über die Auskunftsperson von minderjährigen Zielpersonen realisiert und in 9.542 Fällen über die volljährige Zielperson selbst. Darüber hinaus gab es 4.791 Interviews mit minderjährigen Zielpersonen und 5.705 Interviews mit den Partnern von Auskunfts- und Zielpersonen (vgl. Infas Institut für angewandte Sozialwissenschaft 2015, S. 14). Die Befragung der Partner fand in einem ersten Teil ebenfalls mittels telefonischer Interviews (CATI) statt, aus ausschöpfungsrelevanten und forschungsökonomischen Gründen fand in einem zweiten Teil die Befragung mittels eines Online-Selbstausfüllerfragebogens (CAWI - Computer Assisted Web Interview) statt (vgl. Infas Institut für angewandte Sozialwissenschaft 2015, S. 25). Da Zielpersonen im Alter von 0 bis 6 Jahren im Survey überrepräsentiert 
sind, wurde von Infas ein Designgewicht erstellt, um so die Randverteilung der Stichprobe an die Randverteilung der Grundgesamtheit anzupassen. In den folgenden Analysen wird jeweils angegeben sein, ob die Daten ungewichtet oder gewichtet verwendet wurden. In Tabelle 7.1 sind diese Informationen zur Datengrundlage nochmals zusammengefasst dargestellt:

Tabelle 7.1: Datengrundlage der Studie: DJI-Survey AID:A II von 2013/14

\begin{tabular}{|c|c|}
\hline Datengewinnung & $\begin{array}{l}\text { - telefonische und persönliche Befragung (CATI und CAPI) } \\
\text { der Ziel- und Auskunftspersonen } \\
\text { - telefonische Interviews und Online-Selbstausfüllerfrage- } \\
\text { bogen (CATI und CAWI) bei den Partnern }\end{array}$ \\
\hline Auswahlverfahren & $\begin{array}{l}\text { - } \quad \text { repräsentative Zufallsauswahl von Adressen über Ein- } \\
\text { wohnermeldeamtziehungen } \\
\text { anschließend Recherche der Telefonnummern, hierbei } \\
\text { Rückgriff auf Adressen und Telefonnummern, die bereits } \\
\text { bei „AID:A I“ erhoben wurden }\end{array}$ \\
\hline Stichprobengröße & $\begin{array}{l}\text { - Gesamtstichprobengröße: } 22.691 \text { Zielpersonen im Alter } \\
\text { von } 0 \text { bis } 32 \text { Jahren } \\
\text { - davon in } 12.903 \text { Fällen über die Auskunftsperson von } \\
\text { minderjährigen Zielpersonen und in } 9.542 \text { Fällen über } \\
\text { die volljährige Zielperson selbst }\end{array}$ \\
\hline Sample & $\begin{array}{l}\text { - weibliche Auskunftspersonen von minderjährigen Ziel- } \\
\text { personen, die einen männlichen Partner haben und mit } \\
\text { diesem im selben Haushalt leben: } n=11.330\end{array}$ \\
\hline
\end{tabular}

Quelle: Infas Institut für angewandte Sozialwissenschaft 2015, eigene Darstellung.

Das Sample, auf dem die folgenden Analysen basieren, reduziert sich von $n=22.691$ auf $n=11.330$, da nur Zielkinder im Alter von 0 bis 17 Jahren $(n=13.158)$ mit weiblichen Auskunftspersonen ( $n=12.642)$ und männlichen Partnern $(n=11.590)$ ausgewählt wurden. Fälle, bei denen die Auskunftspersonen männlich und ihre Partner weiblich sind, werden nicht berücksichtig, da diese nur eine kleine Minderheit darstellen. Darüber hinaus bezieht sich das Sample auf Auskunftspersonen, die mit ihrem Partner zusammen im selben Haushalt wohnen, woraus sich eine Sample-Fallzahl von $n=11.330$ ergibt. Abbildung 7.1 verdeutlicht graphisch, wie sich aus der gesamten Fallzahl (oben in der Mitte) schrittweise das Sample (links unten) ergibt: 
Abbildung 7.1: Überblick über die Entstehung des Samples

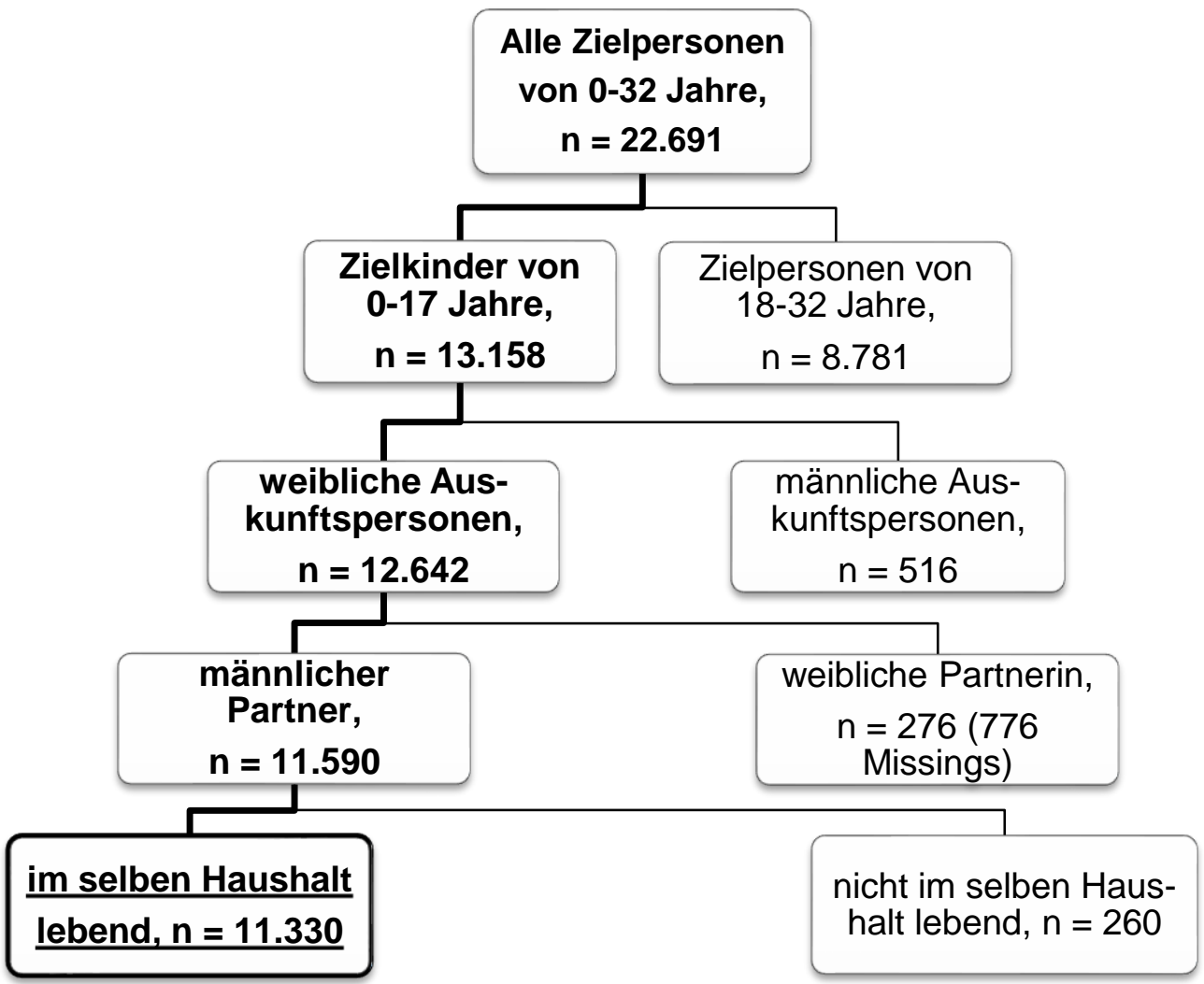

Quelle: DJI-Studie AID:A 2013/14, eigene Berechnungen.

\subsection{Operationalisierung der Variablen}

Im Folgenden wird auf die Operationalisierung der abhängigen Variablen und der zentralen unabhängigen Variablen eingegangen. Bei den abhängigen Variablen handelt es sich um die Aufteilung der Erwerbsarbeit, der Hausarbeit und der Kinderbetreuung zwischen der Auskunftsperson und ihrem Partner. Die zentralen unabhängigen Variablen sind neben dem Alter des Kindes die relativen Ressourcen sowie die Geschlechterrolleneinstellungen. Darüber hinaus spielen weitere Variablen wie die absoluten Ressourcen, die Kinderanzahl oder die Unterschiede zwischen Ost- und Westdeutschland eine wichtige Rolle für die Aufteilung der bezahlten und unbezahlten Arbeit.

\section{Abhängige Variablen}

Da in der hier vorliegenden Arbeit die Arbeitsteilung auf Paarebene im Fokus steht, wird die Aufteilung der Erwerbsarbeit als Erwerbsanteil der Mütter am gesamten Erwerbsvolumen des Paares betrachtet. Hierfür wurden zunächst die wöchentlichen Erwerbsstunden von Müttern und Vätern zum gesamten Erwerbsvolumen aufaddiert und anschließend wurden die individuelle Erwerbsstunden der Mütter in Relation zum gesamten Er- 
werbsvolumen des Paares gestellt. Der Erwerbsanteil der Mütter kann somit Werte zwischen $0 \%$ und $100 \%$ annehmen: So beträgt der Erwerbsanteil der Mütter 0\%, wenn Mütter nicht erwerbstätig sind; $50 \%$, wenn Mütter und Väter in exakt gleichem Umfang erwerbstätigt sind, und $100 \%$, wenn Mütter die Alleinverdienerinnen sind. ${ }^{16}$ Als weitere, abhängige Variable werden neben dem Erwerbsanteil der Mütter auch die individuellen Erwerbsstunden der Mütter betrachtet, denn es sind v.a. die Mütter, die ihre Erwerbsstunden an verschiedene Lebenssituationen anpassen, sodass eine Veränderung des Erwerbsanteils der Mütter in den meisten Fällen auf eine Veränderung der Erwerbsstunden der Mütter und nicht der Väter zurückzuführen ist.

Die Aufteilung der Hausarbeit und der Kinderbetreuung wurden in Form eines Hausarbeitsindexes und eines Kinderbetreuungsindexes operationalisiert. Der Hausarbeitsindex fasst die vier Items Kochen, Einkaufen, Putzen und Wäsche waschen zusammen und umfasst damit die zeitintensivsten Tätigkeiten der Hausarbeit (Coltrane 2000; Blair und Lichter 1991). Der Kinderbetreuungsindex fasst dagegen die vier Items Spielen, Bringen und Holen der Kinder, Kontakt zur Schule/Kita o.ä. sowie das Betreuen und Versorgen der Kinder zusammen. Eine explorative Faktorenanalyse bestätigt diese Vorgehensweise, da die vier Items zur Hausarbeit auf den ersten Faktor laden und die vier Items zur Kinderbetreuung auf den zweiten Faktor laden.

Bei diesen Items wurde jeweils gefragt, wer diese Aufgaben überwiegend übernimmt, wobei die Antwortkategorien „überwiegend Ihr Partner“, „überwiegend Sie selbst“, „beide gleichermaßen“, „kommt das bei Ihnen nicht vor“ oder „jemand anders“ zur Verfügung standen. Bei den letzten beiden Antwortkategorien waren die Fallzahlen jeweils so gering, dass sie aus den Analysen ausgeschlossen werden können (einzig bei dem Item „Putzen“ wurde gelegentlich die Antwort „,jemand anders“ genannt). ${ }^{17}$ Die Antwortkategorien der einzelnen Items wurden zu „-1: überwiegend der Vater“, „0: beide etwa gleich“ und „1: überwiegend die Mutter" recodiert. Indem je aus den vier Tätigkeiten zur Hausarbeit sowie zur Kinderbetreuung ein Durchschnittsscore gebildet wurde, ergeben sich auch für den Hausarbeits- und den Kinderbetreuungsindex Skalen im Wertebereich von „-1: überwiegend der Vater“ über „0: beide etwa gleich“ bis hin zu „1: überwiegend die Mutter".

\footnotetext{
${ }^{16}$ Die maximale, individuelle Erwerbsstundenanzahl eines Individuums wurde wie bei Buschner et al. (2018) auf 60 Stunden pro Woche trunkiert. In Robustheitschecks bei den späteren, multivariaten Analysen zur Aufteilung der Erwerbsarbeit wurde auch getestet, ob die Ergebnisse anders ausfallen, wenn keine Trunkierung stattfindet. Die Ergebnisse sind jedoch robust und unterscheiden sich nicht signifikant voneinander.

${ }^{17}$ Ein weiterer Punkt, der den Ausschluss dieser beiden Kategorien legitimiert, ist die Tatsache, dass in der vorliegenden Arbeit die Aufteilung der Arbeit innerhalb der Partnerschaft im Fokus steht und diese beiden Kategorien keine Relevanz für die innerpartnerschaftliche Aufteilung der Arbeit haben.
} 


\section{Unabhängige Variablen}

Relativer, sozioökonomischer Berufsstatus (ISEI): Der relative Berufsstatus wird anhand des „International Socio-EEconomic of occupational Index“ (ISEI) nach Ganzeboom et al. (1992) operationalisiert. Der ISEI basiert auf einer internationalen Standardklassifikation der Berufe (ISCO-08), die nach einem Codierschema von Ganzeboom (2010) kodiert wurden. Der ISEI beruht auf Informationen zum Einkommen, zur Bildung und zur Zugehörigkeit von verschiedenen Berufsgruppen. Der ISEI kann nach der ISCO-08-Kodierung Werte zwischen 10 und 90 annehmen, wobei sich am unteren Ende der Skala Berufe mit niedrigem Status befinden (z.B. Hilfskräfte und Reinigungspersonal) befinden, während am oberen Ende Berufe mit höherem Status stehen (etwa Richter und Ärzte) (Ganzeboom 2010). Zur Ermittlung der Differenz des Berufsstatus von Mutter und Vater wurde analog zur Studie von Hipp und Leuze (2015) vorgegangen und eine kategoriale Variable erstellt, die einen gleichen Berufsstatus ausgibt, wenn sich die ISEI-Skalenpunkte von Mutter und Vater nur um bis zu fünf Punkte unterscheiden; einen höheren Berufsstatus der Mutter, wenn die Mutter um mindestens fünf ISEI-Punkte höher liegt als der Vater („Frau > Mann“); und einen niedrigeren Berufsstatus der Mutter, wenn die Mutter um mindestens fünf ISEI-Punkte niedriger liegt als der Vater („Frau < Mann“) (Hipp und Leuze 2015, S. 671).

Geschlechterrolleneinstellungen der Mütter: Zur Abbildung der Geschlechterrolleneinstellungen wird auf einer 6-stufigen Skala (von „1: stimme voll und ganz zu“ bis „6: stimme überhaupt nicht zu“) abgefragt, inwiefern den folgenden vier Gender-Items zugestimmt wird:

1) „Wenn kleine Kinder da sind, sollte nicht nur die Frau, sondern auch der Mann Teilzeit arbeiten“;

2) „Wenn Kinder da sind, soll der Mann arbeiten gehen und die Frau zu Hause bleiben und die Kinder versorgen";

3) „Auch wenn eine Frau arbeitet, sollte der Mann der Hauptverdiener sein und die Frau die Verantwortung für den Haushalt tragen“;

4) „Ein Vater sollte genauso stark in die Erziehung und Pflege eines Kindes eingebunden sein wie die Mutter".

Die vier Items zeigen inhaltlich noch nicht in dieselbe Richtung, da bei den Items (2) und (3) niedrige Skalenwerte einer traditionellen Einstellung entsprechen, während sie bei den Items (1) und (4) für eine egalitäre bzw. aufgeschlossene Einstellung stehen. Zur Erleichterung der Interpretation wurde deshalb die Skalierung der Items (1) und (4) umgedreht, so dass nun bei allen vier Items niedrige Skalenpunkte eine traditionelle Einstellung und hohe Skalenpunkte eine egalitäre Einstellung widerspiegeln. Die 6-stufige Skala wurde entsprechend umbenannt und geht nun von „1: traditionell“ bis „6: egalitär“. In den späteren Analysen werden die Gender-Items nicht nur einzeln als unabhängige Variablen mitaufgenommen, sondern sie bilden auch die Basis für eine latente Klassenanalyse und diese latenten Klassen fließen ebenfalls als Einflussfaktoren in den Analysen mit ein. Auf die genauere Operationalisierung der latenten Klassen sowie auf die 
inhaltliche Beschreibung der Klassen wird an dieser Stelle nicht näher eingegangen, da diesem Thema in Kapitel 7.5 ein eigener Exkurs gewidmet ist.

Alter des jüngsten Kindes: Beim Alter des jüngsten noch im Haushalt lebenden Kindes wurde zwischen den folgenden Altersstufen differenziert: „unter 1 Jahr“, „1 bis unter 3 Jahre“, „3 bis unter 6 Jahre“, „6 bis unter 11 Jahre“ und „11 bis unter 18 Jahre“. Die Altersstufen repräsentieren verschiedene Lebensphasen und sind mitunter institutionell begründet: Die erste Altersstufe bezieht sich auf das erste Lebensjahr und repräsentiert das Säuglings- und frühe Krippenalter. Eltern können bis zu einem Kindesalter von 12 Monaten (bzw. 14 Monaten) Elterngeld beziehen. Als nächste Grenzziehung wurde ein Alter von einem Jahr bis zur Vollendung des dritten Lebensjahres des Kindes gewählt. Kinder befinden sich hier im Kleinkindalter und Eltern können bis zu diesem Zeitpunkt in Elternzeit gehen (neuere familienpolitische Regelungen ermöglichen jedoch auch eine flexiblere Nutzung der Elternzeit nach dem dritten Lebensjahr des Kindes). Ein Kindesalter von drei bis unter sechs Jahren markiert das Kindergartenalter und stellt die nächste Altersstufe dar. Als darauffolgende Altersstufe wurde die Grundschulzeit gewählt, die durch ein Kindesalter von sechs bis unter elf Jahren repräsentiert wird. Die letzte Altersstufe des Kindes zeichnet sich durch den Besuch einer weiterführenden Schule aus. Hier werden Kinder und Jugendliche im Alter von elf bis unter 18 Jahren zusammengefasst.

Anzahl der Kinder. Auch bei der Anzahl der Kinder wurden nur die im Haushalt lebenden Kinder berücksichtigt, da angenommen wird, dass nur diese einen Einfluss auf die Arbeitsteilung der Eltern haben. Die Ausprägungen der Kinderanzahl lauten „1 Kind“, „2 Kinder“, „3 Kinder“ und „4 und mehr Kinder“.

Ost-/Westdeutschland: Die alten Bundesländer Deutschlands werden zu West- und die neuen Bundesländer zu Ostdeutschland zusammengefasst. ${ }^{18}$

Berufsstatus der Mutter (ISEI): Die ISEI-Skala wurde für die Mütter in die Terzile mit den Ausprägungen „niedrig“, „mittel“ und „hoch“ kategorisiert.

Erwerbstätigkeit der Mutter: Als unabhängige Variable für die Aufteilung der Hausarbeit und der Kinderbetreuung wurde auch die Erwerbstätigkeit der Mutter mitaufgenommen. Dabei wird differenziert zwischen „nicht-erwerbstätig“, „Teilzeit“ (wöchentliche Stundenumfänge von 1 bis 35 Stunden), „Vollzeit“ (36-45 Wochenstunden) und "langer Vollzeit“ (mehr als 45 Wochenstunden).

\footnotetext{
${ }^{18}$ Da keine differenzierten Informationen für das frühere West- und Ostberlin vorliegen, musste Berlin geschlossen zu Ost- oder Westdeutschland zugeordnet werden. Im vorliegenden Fall wurde Berlin zu Westdeutschland gezählt.
} 
Erwerbstätigkeit des Vaters: Auch die Erwerbstätigkeit des Vaters wurde als unabhängige Variable für die Aufteilung der Hausarbeit und der Kinderbetreuung mitaufgenommen. Die Ausprägungen sind analog zur Erwerbstätigkeit der Mutter („nicht-erwerbstätig“, „Teilzeit“, „Vollzeit“ und „lange Vollzeit“).

Die genannten Variablen stellen die zentralen unabhängigen Variablen dar. Darüber hinaus werden in Robustheitschecks noch weitere Variablen miteinbezogen, auf die hier nicht gesondert eingegangen wird. Weitere Variablen sind z.B. die relative Bildung, die individuelle Bildung, das Alter der Mutter, das Alter des Vaters, der Familienstand und die Beziehungsdauer.

\subsection{Methoden}

Die Forschungsfragen beziehen sich auf die Aufteilung der Erwerbsarbeit, der Hausarbeit und der Kinderbetreuung. Für alle drei Dimensionen werden in den multivariaten Analysen Ordinary Least Square-Regressionen - kurz: OLS-Regressionen - berechnet. Damit OLS-Regressionen die bestmögliche Schätzung liefern, sollten sie gemäß dem „Gauss-Markov-Theorem“ „BLUE“ sein: Dieses Akronym setzt sich zusammen aus „B best“, „L - linear“, „U - unbiased“ und „E - estimation“ (Urban und Mayerl 2018, S. 112113). Auf die Bedeutung dieses Akronyms wird im Folgenden kurz eingegangen. Die nachfolgenden Gleichungen und Erläuterungen können z.B. bei Backhaus (2016) und Brüderl (2018) nachgelesen werden (Backhaus et al. 2016, S. 98; Brüderl 2018, S. 4950). Bei einer OLS-Regression wird ein linearer Zusammenhang zwischen einer abhängigen Variablen und einer unabhängigen Variable (= bivariate Regression) oder mehreren unabhängigen Variablen (= multiple Regression) angenommen. In der vorliegenden Arbeit werden multiple Regressionen angewandt, da die Aufteilung der Erwerbsarbeit, der Hausarbeit und der Kinderbetreuung durch sämtliche unabhängige Variablen beeinflusst werden. Daraus ergibt sich gemäß der Linearitätsannahme eine Regressionsschätzung, die sich wie folgt beschreiben lässt:

$$
Y_{i}=\beta_{0}+\beta_{1} x_{i 1}+\beta_{2} x_{i 2}+\ldots+\beta_{p} x_{i p}+\varepsilon_{i,} \quad i=1, \ldots, n .
$$

$Y_{i}$ repräsentiert dabei die abhängige, metrische Variable und $x_{i 1}, \ldots, x_{i p}$ die unabhängigen Variablen. Des Weiteren wird angenommen, dass der Erwartungswert der Störgröße $\varepsilon_{i}$ gleich null ist und die Störgrößen somit keinen systematischen Effekt haben:

$$
E\left(\varepsilon_{i}\right)=0 \quad \text { für alle } i
$$

Zwischen den Störgrößen und den Regressoren soll keine Korrelation vorliegen:

$$
\operatorname{Cov}\left(x_{i}, \varepsilon_{j}\right)=0 \quad \text { für alle } i \text { und } j
$$

Eine weitere Annahme bezieht sich darauf, dass die Varianz der Störgrößen konstant sein soll und somit eine Homoskedastizität gegeben ist:

$$
\operatorname{Var}\left(\varepsilon_{i}\right)=\sigma^{2} \quad \text { für alle } i
$$


Weiter wird angenommen, dass die Störgrößen unkorreliert sind und daher keine Autokorrelation vorliegt:

$$
\operatorname{Cov}\left(\varepsilon_{i}, \varepsilon_{j}\right)=0
$$

für alle $i \neq j, \quad i=1, \ldots, n \quad j=1, \ldots, n$

Zusätzlich wird angenommen, dass zwischen den unabhängigen Variablen $x_{i}$ keine lineare Abhängigkeit besteht und folglich

keine perfekte Multikollinearität vorliegt.

Diese Annahmen lassen sich mit folgender Gleichung

$$
\varepsilon_{i} \sim N\left(0, \sigma^{2}\right)
$$

zusammenfassen, die zum Ausdruck bringt, dass die Störgrößen normalverteilt sind, einen Erwartungswert gleich null haben und eine konstante Varianz $\sigma^{2}$ gegeben ist (Backhaus et al. 2016, S. 98). Wenn diese Voraussetzungen erfüllt sind, dann ist die „Kleinste-Quadrate-Schätzung“ „BLUE“, d.h. sie ist die beste, lineare und unverzerrte Schätzung.

Bei der Regressionsdiagnostik wird überprüft, ob bei den angewandten Regressionsmodellen diese Voraussetzungen erfüllt sind. Bei den hier verwendeten Regressionsmodellen auf Basis der Datengrundlage des DJI-Surveys „AID:A“ 2013/14 sind diese Voraussetzungen weitestgehend erfüllt. So sind bei den Analysen zur Aufteilung der Erwerbsarbeit, der Hausarbeit und der Kinderbetreuung die Residuen normalverteilt bzw. zumindest annähernd normalverteilt und haben einen Erwartungswert gleich null. Auch ist keine Multikollinearität in den multivariaten Gesamtmodellen zur Aufteilung der Erwerbsarbeit, der Hausarbeit und der Kinderbetreuung vorhanden: So beträgt der VIF-Wert (Variance Inflation Factor) bei der Aufteilung der Erwerbsarbeit 2,0 und bei der Aufteilung der Hausarbeit und der Kinderbetreuung je 2,2. Da die Werte nahe an 1 sind, spricht dies gegen eine Multikollinearität.

Des Weiteren wurde bei allen Modellen getestet, ob die Annahme der Homoskedastizität erfüllt werden kann, oder ob stattdessen Heteroskedastizität vorliegt. Dies wurde zuerst visuell anhand von sog. „Residual-versus-fitted“-Plots getestet (Stata-Befehl: „rvfplot“), woraus sich für sämtliche Modelle zur Aufteilung der Erwerbsarbeit, der Hausarbeit und der Kinderbetreuung ergibt, dass wahrscheinlich eine Heteroskedastizität vorhanden ist. Der sog. Breusch-Pagan/Cook-Weisberg-Test auf Heteroskedastizität (Stata-Befehl: „estat hettest“) ist für einige Modelle signifikant und bestätigt somit das Vorliegen einer Heteroskedastizität. Damit ist die Annahme der Homoskedastizität verletzt.

Eine Art, wie mit diesem Problem umgegangen werden kann, ist, dass anstelle von normalen Standardfehlern die robusten Standardfehler verwendet werden (in Stata durch die Option „robust“ beim regress-Befehl) (Torres-Reyna 2007). Bei allen Modellen, bei denen in dieser Arbeit das Vorliegen einer Heteroskedastizität bestätigt wurde, wurde daher getestet, wie die Standardfehler ausfallen, wenn die robuste Variante verwendet wird. Es hat sich gezeigt, dass die regulären und die robusten Standardfehler einander 
sehr ähnlich sind und sich frühestens ab der dritten oder vierten Nachkommastelle unterscheiden. Da in den nachfolgenden Analysen die Standardfehler auf die zweite Nachkommastelle gerundet werden, sind keine Unterschiede zwischen diesen beiden Varianten ersichtlich. ${ }^{19}$ Dennoch sollte das Vorliegen von Heteroskedastizität im Hinterkopf behalten werden, da dies häufig ein Indiz für nicht-lineare Zusammenhänge ist (Backhaus et al. 2016, S. 104). ${ }^{20}$

Für die Frage danach, ob die Modelle vollständig sind, lohnt sich ein Blick auf das Bestimmtheitsmaß $\mathrm{R}^{2}$, welches angibt, wieviel Varianz der abhängigen Variable durch die unabhängigen Variablen erklärt werden kann. Sehr niedrige $\mathrm{R}^{2}$-Werte sprechen gegen eine Vollständigkeit des Modells, während bei höheren Werten eine Vollständigkeit des Modells eher gegeben ist. Gleichzeitig ist bei einem Modell mit sehr vielen unabhängigen Variablen, durch die das $\mathrm{R}^{2}$ automatisch höher wird, auch die Gefahr des Overfittings gegeben. Das Finden der richtige Variablenanzahl ist gewissermaßen ein Balanceakt zwischen Underfitting und Overfitting, da das Modell zwar alle relevanten Variablen enthalten soll, aber gleichzeitig möglichst sparsam sein soll. Bei der Aufteilung der Erwerbsarbeit beträgt das korrigierte $\mathrm{R}^{2}$ im Gesamtmodell 0,22, d.h. 22\% der Varianz der Aufteilung der Erwerbsarbeit können durch die ins Modell inkludierten, unabhängigen Variablen erklärt werden. ${ }^{21}$ Bei der Aufteilung der Hausarbeit beträgt das korrigierte $R^{2}$ 0,21 und bei der Aufteilung der Kinderbetreuung 0,16. Am höchsten ist somit die Erklärungskraft bei den Modellen zur Aufteilung der Erwerbsarbeit und der Hausarbeit, während die Erklärungskraft bei den Modellen zur Aufteilung der Kinderbetreuung niedrig ist und dies darauf hindeuten könnte, dass das Modell möglicherweise noch unvollständig ist.

In Folgenden wird kurz inhaltlich darauf eingegangen, welche unabhängigen Variablen in welcher Reihenfolge in die Analysen miteinfließen. Bei allen drei Dimensionen (der

${ }^{19}$ Da die Signifikanzen der Regressionskoeffizienten auf den Standardfehlern basieren, wurde auch getestet, ob sich die Signifikanzen verändern, wenn anstelle der regulären Standardfehler die robusten Standardfehler verwendet werden. Auch hier waren keinerlei Unterschiede feststellbar.

20 Zunächst werden trotz dieser Problematik lineare Regressionen geschätzt. In Robustheitschecks wird jedoch überprüft werden, ob zwischen den abhängigen und den unabhängigen Variablen möglicherweise nicht-lineare Zusammenhänge vorliegen, die durch nicht-lineare Modelle besser beschrieben werden können. So wird beispielweise bei der abhängigen Variable zur Aufteilung der Erwerbsarbeit der „Erwerbsanteil der Mütter" verwendet, der Werte von 0\% bis $100 \%$ annehmen kann und in Robustheits-checks wird anstelle von einer OLS-Regression ein Fractional Probit Modell getestet. Die Ergebnisse erweisen sich als robust, da sie in beiden Fällen sehr ähnlich ausfallen.

${ }^{21}$ Hier sind diejenigen Modelle gemeint, in denen das Alter des Kindes noch als unabhängige Variable in die Modelle mitaufgenommen sind. Auf die Bestimmtheitsmaße von detaillierteren Modellen, die getrennt nach dem Alter des Kindes berechnet wurden, wird an dieser Stelle nicht näher eingegangen, sondern erst an späterer Stelle bei den entsprechenden, empirischen Kapiteln. 
Aufteilung der Erwerbsarbeit, der Hausarbeit und der Kinderbetreuung) werden die Modelle stufenweise aufgebaut: Hierfür wird zunächst das Alter des Kindes als unabhängige Variable mitaufgenommen, da das zentrale Forschungsinteresse in der vorliegenden Arbeit auf der Bedeutung des Kindesalters für die Arbeitsteilung der Eltern liegt. In Anlehnung an die ökonomischen Theorien werden dann die relativen Ressourcen berücksichtigt, anschließend werden in Anlehnung an die geschlechterrollen-, normen- und identitätsbasierte Ansätze die Geschlechterrolleneinstellungen mitaufgenommen. Danach werden weitere wichtige Variablen wie die Anzahl der Kinder, das Bundesgebiet, die absoluten Ressourcen oder die Erwerbstätigkeit mitaufgenommen. In nicht abgebildeten Robustheitschecks werden darüber hinaus weitere Variablen getestet, indem Variablen wie z.B. das Alter der Mutter oder die Beziehungsdauer testweise inkludiert werden.

Danach werden in den Analysen zur Aufteilung der Erwerbsarbeit, der Hausarbeit und der Kinderbetreuung die Modelle getrennt nach den Altersstufen des Kindes berechnet, um so feststellen zu können, ob sich die Effekte der anderen Einflussfaktoren in Abhängigkeit vom Alter des Kindes unterscheiden. So könnte es etwa sein, dass Einflussfaktoren wie die relativen Ressourcen oder die Geschlechterrolleneinstellungen in Familien mit sehr kleinen Kindern noch keine Rolle spielen, sondern diese Einflüsse erst ab einem bestimmten Kindesalter zum Tragen kommen (oder auch umgekehrt: dass diese Einflüsse speziell in Familien mit jungen Kindern stark ausgeprägt sind und bei älteren Kindern unwichtiger werden). Anschließend werden vertiefende Analysen zu den Geschlechterrolleneinstellungen vorgenommen, indem Einstellungsmuster, die anhand einer latenten Klassenanalyse identifiziert werden, als unabhängige Variable in die Modelle miteinfließen.

In Anlehnung an Urban und Mayerl (2018) werden bei den multivariaten Analysen unstandardisierte Koeffizienten verwendet. Zur Begründung dieser Entscheidung wird ein Zitat von Urban und Mayerl (2018) über die unterschiedlichen Einsatz- und Interpretationsmöglichkeiten von unstandardisierten und standardisierten Regressionskoeffizienten herangezogen:

\begin{abstract}
„Standardisierte Regressionskoeffizienten sind unabhängig von den empirischen Messeinheiten unabhängiger Variablen und können daher als Maß der Einflussstärke von $X$ auf $Y$ interpretiert werden. Sie können aber nur zum Vergleich der Einflussstärken mehrerer unabhängiger Variablen innerhalb einer Stichprobe benutzt werden, da sie immer dann variieren, wenn für die gleichen Variablen in verschiedenen Populationen bzw. Stichproben unterschiedliche Varianzen vorliegen“ (Urban und Mayerl 2018, S. 73, Hervorhebung im Original).
\end{abstract}

Der Vorteil von standardisierten Regressionskoeffizienten liegt somit in der Vergleichbarkeit der Effektstärken von unterschiedlichen, unabhängigen Variablen innerhalb einer Stichprobe. Zur Vergleichbarkeit der Effektstärke einer einzelnen Variable über verschiedene Stichproben sind jedoch die unstandardisierten Regressionskoeffizienten geeigneter: 
„Unstandardisierte Regressionskoeffizienten sind abhängig von den empirischen Messeinheiten unabhängiger Variablen. Sie sind aber unabhängig von deren Varianzen in verschiedenen Populationen. Sie können deshalb zum Vergleich der Einflussstärke einer einzigen Variablen in verschiedenen Stichproben benutzt werden, allerdings nur, wenn die betreffenden X-Variablen in jeder Stichprobe in gleicher Weise gemessen wurden" (Urban und Mayerl 2018, S. 73, Hervorhebung im Original).

In der vorliegenden Arbeit erscheinen die unstandardisierten Regressionskoeffizienten deshalb als geeigneter, weil im Fokus die Forschungsfrage steht, wie die Effektstärken einzelner Variablen in den verschiedenen Altersgruppen des Kindes ausfallen. D.h. es interessiert z.B. die Frage danach, ob relative Ressourcen bei kleinen Kindern eine andere Bedeutung haben als bei älteren Kindern. Wenn nun die Effektstärken der relativen Ressourcen über die verschiedenen Altersstufen verglichen werden, dann entspricht dies einem Vergleich von verschiedenen Modellen (bzw. Stichproben). Die Voraussetzung, dass die unabhängigen Variablen für eine Vergleichbarkeit in allen Stichproben gleich gemessen sein müssen, ist in der vorliegenden Arbeit erfüllt. ${ }^{22}$

\subsection{Verteilungen von zentralen Variablen}

Im Folgenden wird ein deskriptiver Überblick über die zentralen unabhängigen Variablen der Auswertung gegeben. Hierfür wird einerseits auf Variablen eingegangen, die aufgrund der ökonomischen Theorien und der geschlechterrollenbasierten Ansätze als relevant erachtet werden und andererseits auf weitere, wichtige Variablen (siehe Tabelle 7.2).

Relativer, sozioökonomischer Berufsstatus (ISEI): Die empirischen Ergebnisse zeigen, dass bei etwa einem Viertel $(25,8 \%)$ die Mütter und die Väter einen identischen Berufsstatus haben. Am häufigsten kommt es jedoch vor, dass Mütter einen niedrigeren Berufsstatus haben $(38,1 \%)$. Doch auch der gegenteilige Fall, nämlich, dass Mütter einen höheren Berufsstatus haben, kommt mit $36,1 \%$ relativ häufig vor.

22 Vgl. bei Urban und Mayerl (2018) auch das Unterkapitel „Die Problematik standardisierter Regressionskoeffizienten" sowie die folgende Tabelle aus Urban und Mayerl 2018, S. 96, die die unstandardisierten Koeffizienten (b-Koeffizienten) mit den standardisierten Koeffizienten ( $b^{*}-$ Koeffizienten) vergleicht:

\begin{tabular}{lcc}
\hline & b-Koeffizienten & b $^{*}$-Koeffizienten \\
\hline inhaltlich-substanzielle Interpretierbarkeit & ja & nein \\
\hline Prognose von $\hat{Y}$ & ja & nein \\
\hline Schätzung von $\beta$ in Population & ja & nein \\
\hline internationale/regionale (u. a.) Vergleiche & ja & (nur bedingt) \\
\hline Intergruppenvergleiche & ja & (nur bedingt) \\
\hline $\begin{array}{l}\text { Vergleichbarkeit innerhalb einer Schätzung } \\
\text { bei unterschiedlich skalierten X-Variablen }\end{array}$ & nein & ja \\
\hline
\end{tabular}

Quelle: Urban und Mayerl 2018, S. 96. 
Geschlechterrolleneinstellungen der Mütter: Zur Abbildung der Geschlechterrolleneinstellungen wurde auf einer sechsstufigen Skala abgefragt, inwiefern bei vier Items traditionelle oder egalitäre Einstellungen geäußert werden. Bei der Frage danach, ob in Familien mit kleinen Kindern nicht nur die Frauen, sondern auch die Männer in Teilzeit arbeiten sollen (Item 1), fallen die Antworten ziemlich unterschiedlich aus: $27 \%$ der Mütter lehnen dies ab (Skalenpunkte 1 und 2) und 32\% befürworten diese Aussage (Skalenpunkte 5 und 6); 41\% stehen dem neutral gegenüber (Skalenpunkte 3 und 4). Der Frage danach, ob Mütter generell zu Hause bleiben und die Kinder versorgen sollen, während die Väter arbeiten gehen (Item 2), stimmt mehr als jede zweite Mutter (56\%) nicht zu (Skalenpunkte 5 und 6). Zustimmung erfährt diese Frage lediglich von 15\% der Mütter (Skalenpunkte 1 und 2). Dass der Mann der Hauptverdiener sein soll (Item 3), wird nur von einer Minderheit der Mütter (12\%, Skalenpunkte 1 und 2 addiert) befürwortet, der Großteil (59\%, Skalenpunkte 5 und 6 addiert) lehnt dies ab und etwa 29\% (Skalenpunkte 3 und 4 addiert) der Mütter haben hierzu eine neutrale Einstellung. Eine weitere Fragestellung bezieht sich auf die Rolle des Vaters bei der Erziehung (Item 4): Sollten Väter genauso stark in die Erziehung und Pflege des Kindes eingebunden sein wie die Mütter? Die allermeisten Mütter (83\%) stimmen dem zu (Skalenpunkte 5 und 6), während dies von nur $1 \%$ abgelehnt wird (Skalenpunkte 1 und 2). Bei den berichteten Werten handelt es sich um die gewichteten Daten. Die Unterschiede zu den ungewichteten Daten (nicht abgebildet) fallen zwar nur gering aus, dennoch lässt sich die Tendenz beobachten, dass nach der Gewichtung, die egalitären Einstellungen minimal geringer und die traditionellen Einstellungen minimal häufiger auftreten. Eine mögliche Ursache hierfür könnte sein, dass durch die Gewichtung der Bildungsbias reduziert wird, so dass höher Gebildete nicht mehr oder zumindest nicht mehr so stark überrepräsentiert sind. Da eine hohe Bildung mit egalitäreren Werten assoziiert wird (van Berkel und Graaf 1999; Bolzendahl und Myers 2004), kann sich eine Reduktion des Bildungsbias in tendenziell etwas traditionelleren Geschlechterrolleneinstellungen äußern. Insgesamt fallen die Einstellungen jedoch selbst nach der Gewichtung immer noch ziemlich egalitär aus. 
Tabelle 7.2: Verteilung von zentralen unabhängigen Variablen

\begin{tabular}{|c|c|c|}
\hline & $\%$ & $\mathrm{~N}$ \\
\hline \multicolumn{3}{|c|}{ Relativer, sozioökonomischer Berufsstatus (ISEI) } \\
\hline Mutter < Vater & $38,1 \%$ & 3.633 \\
\hline Mutter = Vater & $25,8 \%$ & 2.461 \\
\hline Mutter > Vater & $36,1 \%$ & 3.445 \\
\hline Total & $100,0 \%$ & 9.539 \\
\hline \multicolumn{3}{|c|}{ Geschlechterrolleneinstellungen der Mütter } \\
\hline \multicolumn{3}{|c|}{$\begin{array}{l}\text { Item 1: „Wenn kleine Kinder da sind, sollte nicht nur die Frau, sondern auch der } \\
\text { Mann Teilzeit arbeiten.“ }\end{array}$} \\
\hline 1 stimme nicht zu (= traditionell) & $14,7 \%$ & 1.504 \\
\hline 2 & $12,3 \%$ & 1.257 \\
\hline 3 & $12,7 \%$ & 1.302 \\
\hline 4 & $28,0 \%$ & 2.875 \\
\hline 5 & $15,8 \%$ & 1.620 \\
\hline 6 stimme zu (= egalitär) & $16,5 \%$ & 1.692 \\
\hline Total & $100,0 \%$ & 10.250 \\
\hline
\end{tabular}

Item 2: „Wenn Kinder da sind, soll der Mann arbeiten gehen und die Frau zu Hause bleiben und die Kinder versorgen."

\begin{tabular}{lrr}
1 stimme zu (= traditionell) & 617 \\
2 & $6,0 \%$ & 926 \\
3 & $9,0 \%$ & 1.959 \\
4 & $19,1 \%$ & 1.143 \\
5 & $11,1 \%$ & 1.648 \\
6 stimme nicht zu (= egalitär) & $16,0 \%$ & 3.984 \\
Total & $38,8 \%$ & 10.277 \\
\cline { 2 - 3 }
\end{tabular}

Item 3: „Auch wenn eine Frau arbeitet, sollte der Mann der Hauptverdiener sein und die Frau die Verantwortung für den Haushalt tragen."

\begin{tabular}{lrr}
1 stimme zu (= traditionell) & $5,2 \%$ & 327 \\
2 & $6,8 \%$ & 424 \\
3 & $19,0 \%$ & 1.192 \\
4 & $10,2 \%$ & 642 \\
5 & $14,4 \%$ & 902 \\
6 stimme nicht zu (= egalitär) & $44,4 \%$ & 2.789 \\
\cline { 2 - 3 } Total & $100,0 \%$ & 6.277 \\
\hline
\end{tabular}

Item 4: „Ein Vater sollte genauso stark in die Erziehung und Pflege eines Kindes eingebunden sein wie die Mutter."

\begin{tabular}{lrr}
1 stimme nicht zu (= traditionell) & 23 \\
2 & $0,4 \%$ & 50 \\
3 & $0,8 \%$ & 146 \\
4 & $2,3 \%$ & 820 \\
5 & $13,1 \%$ & 1.792 \\
6 stimme zu (= egalitär) & $28,5 \%$ & 3.448 \\
\hline
\end{tabular}


Total

$100,0 \%$

6.279

Alter des jüngsten Kindes (in Jahren)

unter $1 \mathrm{~J}$.

1 bis unter $3 \mathrm{~J}$.

$8,9 \%$

914

3 bis unter $6 \mathrm{~J}$.

$19,6 \%$

2.019

6 bis unter $11 \mathrm{~J}$.

$22,4 \%$

2.314

11 bis unter $18 \mathrm{~J}$.

$26,0 \%$

2.686

Total

$23,1 \%$

2.379

$100,0 \%$

10.312

Anzahl der Kinder

1 Kind

$23,5 \%$

2.426

2 Kinder

$52,3 \%$

5.403

3 Kinder

$18,8 \%$

1.943

4 und mehr

$5,3 \%$

552

Total

$100,0 \%$

10.323

Ost-/Westdeutschland

Ostdeutschland

Westdeutschland

$14,5 \%$

1.495

Total

$85,5 \%$

8.837

$100,0 \%$

10.332

Berufsstatus der Mutter (ISEI)

niedrig

$33,8 \%$

3.323

mittel

$34,6 \%$

3.401

hoch

$31,6 \%$

3.112

Total

$100,0 \%$

9.836

Erwerbstätigkeit der Mutter

nicht-erwerbstätig (0 Std.)

Teilzeit (1-35 Std.)

Vollzeit (36-45 Std.)

mehr als Vollzeit (über 45 Std.)

Total

$34,9 \%$

3.562

$49,0 \%$

5.009

$11,9 \%$

1.218

$4,2 \%$ 428

Erwerbstätigkeit des Vaters

nicht-erwerbstätig (0 Std.)

$100,0 \%$

10.217

Teilzeit (1-35 Std.)

Vollzeit (36-45 Std.)

mehr als Vollzeit (über 45 Std.)

Total

$3,2 \%$

328

$6,6 \%$

681

Quelle: DJI-Studie AID:A 2013/14, Sample: $n=11.330$, eigene Berechnungen, gewichtet.

$57,5 \%$

5.892

$32,7 \%$

3.346

Alter des jüngsten Kindes: Im Sample sind zu 8,9\% Familien vertreten, deren jüngstes, noch im Haushalt lebendes Kind unter ein Jahr alt ist. In jeder fünften Familie ist das Kind im Alter von 1 bis unter 3 Jahre. Kinder im Alter von 3 bis unter 6 Jahren sind zu $22,4 \%$ vertreten, im Alter von 6 bis unter 11 Jahre zu 26,0\% und von 11 bis unter $18 \mathrm{zu}$ $23,1 \%$. 
Anzahl der Kinder: Auch bei der Anzahl der Kinder wurden nur jene Kinder berücksichtigt, die noch im selben Haushalt leben. In mehr als jeder zweiten Familie leben zwei Kinder im selben Haushalt, bei einem knappen Viertel nur ein Kind. Familien mit drei Kindern liegen bei 18,8\% vor und Familien mit vier oder mehr Kindern bei 5,3\%. Familien mit mehreren Kindern sind im ungewichteten Sample (nicht abgebildet) etwas überrepräsentiert, was jedoch durch die Gewichtung ausgeglichen werden kann, so dass der Anteil der Familien mit mehreren Kindern im gewichteten Sample geringfügig niedriger und der Anteil an Familien mit einem Kind geringfügig höher ist.

Ost-/Westdeutschland: Der Anteil der in Ostdeutschland lebenden Paare beträgt 14,5\% und der Anteil der in Westdeutschland lebenden Paare 85,5\%.

Berufsstatus der Mutter (ISEI): Der Berufsstatus wurde anhand von Terzilen in „niedrig“, „mittel“ und „hoch“ eingeteilt. ${ }^{23}$ Diese Kategorisierung auf Basis von Terzilen hat zur Folge, dass die drei Kategorien (annähernd) gleich groß sind.

Erwerbstätigkeit der Mutter: Bei der Erwerbstätigkeit der Mütter zeigt sich, dass etwa ein Drittel der Mütter nicht erwerbstätig ist und etwa die Hälfte der Mütter in Teilzeit erwerbstätig ist. Lediglich 11,9\% der Mutter sind in einer Vollzeit von 36-45 Stunden erwerbstätig und nur $4,2 \%$ in einer Vollzeit mit mehr als 45 Stunden (jeweils inklusive der Überstunden).

Erwerbstätigkeit des Vaters: Bei der Erwerbstätigkeit der Väter verhält es sich genau umgekehrt im Vergleich zur Erwerbstätigkeit der Mütter: Nur eine Minderheit der Väter ist nicht-erwerbstätig oder nur in Teilzeit erwerbstätig. Stattdessen sind 57,5\% der Väter in einer Vollzeit mit 36-45 Stunden erwerbstätig und etwa ein Drittel der Väter ist sogar in besonders langer Vollzeit mit mehr als 45 Wochenstunden erwerbstätig (wie bei den Erwerbsstunden der Mütter sind auch bei den Erwerbsstunden der Väter die Überstunden eingeschlossen).

Nach dieser Übersicht über die univariaten Verteilungen der zentralen Variablen, wird in den nachfolgenden Kapiteln auf die empirischen Ergebnisse zur Aufteilung der Erwerbsarbeit, der Hausarbeit und der Kinderbetreuung eingegangen. Zunächst erfolgt jedoch noch ein Exkurs zur multidimensionalen Abbildung von Geschlechterrolleneinstellungen.

\footnotetext{
${ }^{23}$ Hinter der Kategorie "niedrig“ stehen ISEI-Werte von 10 bis 44 , „mittel“ umfasst die ISEI-Werte 45 bis 62 und "hoch“ bildet ISEI-Werte von 63 bis 90 ab. Diese Grenzen wurden anhand von Terzilen gezogen, d.h. zu diesen drei Kategorien gehören jeweils etwa ein Drittel der Mütter.
} 


\subsection{Exkurs: Latente Klassenanalyse zur multidimensionalen Ab- bildung von Geschlechterrolleneinstellungen}

Die neuere Forschung plädiert für eine multidimensionale Abbildung von Geschlechterrolleneinstellungen (Knight und Brinton 2017; Grunow et al. 2018). Dieser Vorschlag wird in der vorliegenden Arbeit umgesetzt, indem anhand einer latenten Klassenanalyse multidimensionale Geschlechterrolleneinstellungen abgebildet werden. Dies kann mit den hier verwendeten Daten jedoch nur approximativ erfolgen, da nur vier Items zu Geschlechterrolleneinstellungen vorliegen und für eine präzisere und differenziertere Betrachtungsweise weitaus mehr Items zu Geschlechterrolleneinstellungen notwendig sind. Anhand der nachfolgenden Ergebnisse wird dennoch ersichtlich, dass sich auch mit den wenigen, vorhandenen Items mehrere latente Klassen ergeben.

Im Folgenden wird zunächst auf die Operationalisierung und Methodik der multidimensionalen Abbildung von Geschlechterrolleneinstellungen eingegangen. Anschließend werden in Anlehnung an Grunow et al. (2018) Strukturen vorgestellt, anhand denen die latenten Klassen eingeordnet und benannt werden können: So kann danach unterschieden werden, ob geschlechtsspezifische Sphären (wie die männliche Sphäre der Erwerbsarbeit und die weibliche Sphäre der Familienarbeit) oder gemeinsame Sphären angenommen werden und ob die (Nicht-)Zuständigkeit für eine Sphäre frei wählbar ist oder ob sie durch das Geschlecht vorgegeben ist (Grunow et al. 2018, S. 44). Anhand dieser Strukturen werden die in dieser Arbeit identifizierten, latenten Klassen eingeordnet, betitelt und anschließend genauer beschrieben. Danach wird auf bivariate Zusammenhänge und schließlich auf multivariate Ergebnisse eingegangen.

\section{Operationalisierung und Methodik}

Die latente Klassenanalyse basiert auf den folgenden vier Items, die die Einstellungen zu Geschlechterrollen messen:

- Item 1: „Wenn kleine Kinder da sind, sollte nicht nur die Frau, sondern auch der Mann Teilzeit arbeiten."

- Item 2: „Wenn Kinder da sind, soll der Mann arbeiten gehen und die Frau zu Hause bleiben und die Kinder versorgen."

- Item 3: „Auch wenn eine Frau arbeitet, sollte der Mann der ,Hauptverdiener' sein, und die Frau sollte die Verantwortung für den Haushalt tragen."

- Item 4: „Ein Vater sollte genauso stark in die Erziehung und Pflege eines Kindes einbezogen sein, wie eine Mutter.“

Als Antwortskala stand den Müttern eine Skala von „1: stimme voll und ganz zu“ bis „6: stimme überhaupt nicht zu“ zur Auswahl. Bei Item 1 und 4 wurde die Skala recodiert, damit alle Items inhaltlich in dieselbe Richtung zeigen, sodass bei allen Items niedrige Werte eine traditionellere Einstellung und höhere Werte eine modernere bzw. egalitärere 
Einstellung bedeuten: Die neue sechs-stufige Skala hat die Ausprägungen „1: traditionell“ bis „6: egalitär“. Anschließend wurde die Skala dichotomisiert, und zwar zunächst so, dass die Skalenpunkte eins bis drei zu „0: traditionell“ und die Skalenpunkte vier bis sechs zu „1: egalitär“ zugeordnet wurden. Diese Dichotomisierung stellte sich jedoch bei den Analysen als problematisch heraus, da die Items sehr schief verteilt sind und deswegen kaum Fälle in der traditionellen Gruppe enthalten sind. Deshalb erfolgte eine neue Dichotomisierung, bei der die Skalenpunkte eins bis vier zur traditionellen Gruppe und die Skalenpunkte fünf bis sechs zur egalitären Gruppe zusammengefasst wurden. Diese asymmetrische Dichotomisierung muss bei der Interpretation der Ergebnisse berücksichtigt werden. Die geringe Besetzung der traditionellen Gruppe bei einer symmetrischen Dichotomisierung zeigt deutlich, dass der Großteil der Befragten moderne Einstellungen vertritt. Hierbei ist jedoch kritisch anzumerken, dass die Antworten möglicherweise aufgrund sozialer Erwünschtheit verzerrt sein könnten.

Zur Identifizierung von Typen mit unterschiedlichen Geschlechterrolleneinstellungen wurde eine latente Klassenanalyse verwendet. Die Entscheidung darüber, wie viele Klassen angebracht sind, hängt sowohl von den statistischen Gütekriterien der Modelle als auch von theoretischen Gründen ab. Es wurden mehrere Modelle gerechnet, die alle Abstufungen zwischen einer Klasse und bis zu fünf Klassen enthielten. Anhand des Bayes-Informationskriteriums (BIC) wäre die Variante mit vier Klassen zu wählen, da das $\mathrm{BIC}$ hier am niedrigsten ist. Die vierte Klasse ist jedoch inhaltlich schwer interpretierbar und weist nur eine sehr geringe Fallzahl auf. Die geringe Besetzung der vierten Klasse macht es vertretbar, diese kleine Gruppe unberücksichtigt zu lassen. Stattdessen wurde die Variante mit drei Klassen gewählt: Diese lässt sich inhaltlich gut interpretieren, das BIC ist hier am zweitniedrigsten und alle Gruppen sind hoch besetzt. Es ist davon auszugehen, dass es noch weitere Klassen gibt, diese können aber nicht anhand von nur vier Items identifiziert werden.

Die Existenz von weiteren Klassen wird auch durch bisherige Forschung bestätigt. So konnten beispielsweise Grunow et al. (2018) anhand einer latenten Klassenanalyse basierend auf sieben Items insgesamt fünf Klassen identifizieren, die als „egalitarian“, „egalitarian essentialism“, „intensive parenting“, „moderate traditional“ und "traditional“ bezeichnet wurden (Grunow et al. 2018). Knight und Brinton (2017) konnten anhand von sieben Items die folgenden vier Klassen identifizieren: „traditionalism“, „liberal egalitarianism“, „egalitarian familism" und "flexibel egalitarianism“ (Knight und Brinton 2017, S. 1504).

Zur Interpretation der Klassen wurden die vorhergesagte Antwortwahrscheinlichkeiten für die vier Items je Klasse berechnet. Die Ergebnisse werden in Form der Mittelwerte der Items je Klasse dargestellt. Die Mittelwerte nehmen dabei Ausprägungen zwischen „0“ und „1“ an, wobei „0“ für traditionelle Einstellungen und „1“ für moderne Einstellungen steht. Mit Hilfe dieser Informationen wurden Klassennamen gefunden, die die Verteilung 
der Mittelwerte inhaltlich zusammenfassen und darüber hinaus in Einklang mit der theoretischen Rahmung und dem Forschungsstand stehen.

\section{Strukturen zur Einordnung der latenten Klassen}

Die Benennung der latenten Klassen erfolgte zudem in Anlehnung an die Studie von Grunow et al. (2018) anhand eines dreidimensionalen Gerüstes (siehe Tabelle 7.3): Die erste Dimension bezieht sich auf die Verdienstebene und differenziert danach, ob man die Einstellung vertritt, dass beide gemeinsam für den Verdienst zuständig sind oder ob es sich dabei um eine getrennte, männliche Zuständigkeit hierfür handelt; Dimension zwei beinhaltet die Ebene der Fürsorge bzw. Kinderbetreuung und unterscheidet auch hier danach, ob man der Meinung ist, dass beide gemeinsam dafür zuständig sind, oder ob es sich dabei um eine getrennte, weibliche Zuständigkeit handelt; die dritte Dimension richtet ihren Fokus auf die Wahlfreiheit, d.h. ob man die Auffassung vertritt, dass man bei den Zuständigkeiten frei wählen kann oder ob es geschlechtsspezifische Merkmale gibt, aufgrund derer man für den geschlechtstypischen Bereich besser geeignet ist als das andere Geschlecht (Grunow et al. 2018, S. 44). Die verschiedenen, latenten Klassen spiegeln Kombinationen von Einstellungen auf diesen drei Ebenen wider.

Tabelle 7.3: $\quad$ Strukturen zur Einordnung der latenten Klassen: Männliche, weibliche oder gemeinsame Sphären

\begin{tabular}{|c|c|c|c|c|c|c|}
\hline \multirow[b]{2}{*}{ Geschlechter-Ideologien } & \multicolumn{2}{|c|}{$\begin{array}{c}\text { Zuständigkeit für } \\
\text { den Verdienst }\end{array}$} & \multicolumn{2}{|c|}{$\begin{array}{l}\text { Zuständigkeit für } \\
\text { die Fürsorge }\end{array}$} & \multicolumn{2}{|c|}{ Betonung auf... } \\
\hline & $\begin{array}{l}\text { Getrennt } \\
\text { männlich }\end{array}$ & $\begin{array}{l}\text { Gemein- } \\
\text { sam }\end{array}$ & $\begin{array}{l}\text { Getrennt } \\
\text { weiblich }\end{array}$ & $\begin{array}{l}\text { Gemein- } \\
\text { sam }\end{array}$ & $\begin{array}{l}\text { Aus- } \\
\text { wahl }\end{array}$ & $\begin{array}{l}\text { Geschlechtsspezi- } \\
\text { fische Merkmale }\end{array}$ \\
\hline \multicolumn{7}{|l|}{ Eindimensional } \\
\hline Egalitär & & $x$ & & $x$ & $x$ & \\
\hline Traditionell & $x$ & & $x$ & & & $x$ \\
\hline $\begin{array}{l}\text { Mehrdimensional } \\
\text { Intensive Mutter-/ }\end{array}$ & & & & & & \\
\hline Elternschaft & $x$ & & & $x$ & & $x$ \\
\hline $\begin{array}{l}\text { Egalitärer Essentialis- } \\
\text { mus }\end{array}$ & $\mathrm{X}$ & $\mathrm{x}$ & $\mathrm{X}$ & $\mathrm{x}$ & $\mathrm{x}$ & $\mathrm{x}$ \\
\hline
\end{tabular}

Quelle: gekürzte Darstellung der Tabelle von: Grunow et al. 2018, S. 44; eigene Übersetzung ins Deutsche.

Die traditionelle Klasse ist beispielsweise der Auffassung, dass es sich bei der Verdienstebene um eine getrennte Sphäre mit männlicher Zuständigkeit handelt (Dimension eins), bei der Fürsorgeebene dagegen um eine getrennte Sphäre mit weiblicher Zuständigkeit (Dimension zwei) und dass man diesbezüglich keine Wahlfreiheit hat, sondern dass dies durch geschlechtsspezifische Merkmale vorbestimmt ist (Dimension drei). Eine traditionelle Klasse konnte in der vorliegenden Arbeit nicht identifiziert werden. Eine Ursache hierfür können methodische Mängel sein, die darin bestehen, dass nur eine geringe Anzahl an Items und folglich auch nur eine geringe Anzahl an Klassen gegeben ist. Auch 
wenn in den Studien von Grunow et al. (2018) und Knight und Brinton (2017) eine traditionelle Klasse gefunden werden konnte, steht die Tatsache, dass in der vorliegenden Arbeit keine traditionelle Klasse gefunden werden kann, nicht im Widerspruch zum Stand der Forschung. So handelt es sich in den genannten Studien bei der traditionellen Klasse um die kleinste Klasse und deren Ergebnisse zeigen, dass traditionelle Geschlechterideologien durch andere, multidimensionale Geschlechterideologien ersetzt worden sind (Grunow et al. 2018, S. 57; Knight und Brinton 2017, S. 1518).

Der entgegengesetzte Pol zur traditionellen Klasse ist die egalitäre Klasse. Hierbei wird die Einstellung vertreten, dass Männer und Frauen sowohl gemeinsam für den Verdienst als auch gemeinsam für die Fürsorgearbeit zuständig sind und dass sie diesbezüglich wählen können, d.h. dass dies nicht durch geschlechtsspezifische Eigenschaften festgelegt ist (Grunow et al. 2018, S. 44). Diese Klasse konnte auch in der vorliegenden Arbeit nachgewiesen werden (siehe unten). Neben der egalitären Klasse wurden in der vorliegenden Arbeit noch zwei weitere Klassen identifiziert, die sich nicht auf einer eindimensionalen Achse mit den Skalenenden "traditionell“ und "egalitär" einordnen lassen und am besten mit „intensive mothering/parenting“ und „egalitärer Essentialismus“ beschrieben werden können. Das Auffinden dieser beiden Klassen bestätigt, dass eine eindimensionale Betrachtung unterkomplex ist und stattdessen eine multidimensionale Herangehensweise angebracht und notwendig ist (Grunow et al. 2018; Knight und Brinton 2017). Im Folgenden werden die einzelnen Klassen inhaltlich anhand der Mittelwerte der jeweiligen Items näher beleuchtet (siehe Abbildung 7.2).

Ergebnisse: Beschreibung der latenten Klassen

Die Ergebnisse der latenten Klassen der Geschlechterrolleneinstellungen sind in Abbildung 7.2 abgetragen: 
Abbildung 7.2: Latente Klassen der Geschlechterrolleneinstellungen

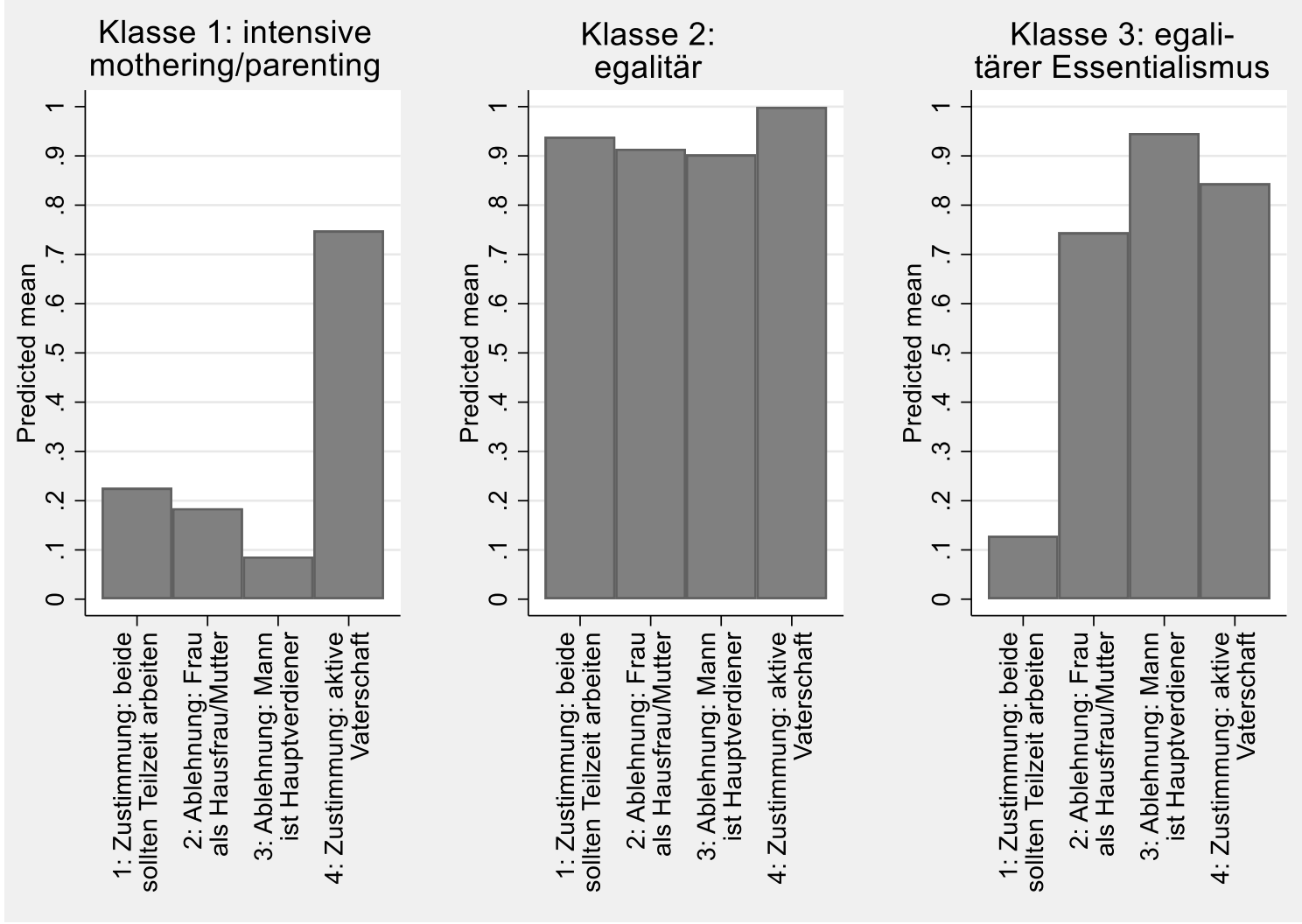

Anmerkung: Vorhergesagte Mittelwerte der Items in den jeweiligen Klassen auf einer Skala von 0 bis 1 , wobei 0 traditionellen Einstellungen und 1 egalitären Einstellungen entspricht. Quelle: DJI-Studie AID:A 2013/14, Daten gewichtet, $n$ (Gesamt) = 7.185; n (Klasse 1) = 2.801; n (Klasse 2) $=1.644 ; \mathrm{n}($ Klasse 3$)=2.741$; eigene Berechnungen.

Klasse 1: „intensive mothering/parenting“

Die erste Klasse umfasst 2.801 Fälle (39\%). Dem ersten Item, der Frage danach, ob nicht nur die Frau, sondern auch der Mann Teilzeit arbeiten sollte, wenn kleine Kinder da sind, stimmen in dieser Klasse nur 22\% zu (siehe Abbildung 7.2, linke Abbildung). D.h. die Mehrheit ist der Auffassung, dass eine Vollzeiterwerbstätigkeit des Vaters auch in Familien mit kleinen Kindern vertretbar ist. Ähnlich verhält es sich bei Item 2: Dass in Familien der Mann arbeiten geht und die Frau zu Hause bleiben und die Kinder versorgen soll, wird nur von 18\% abgelehnt, d.h. die Mehrheit stimmt dieser traditionellen Arbeitsteilung zu. Item 3, nämlich, dass der Mann der Hauptverdiener sein soll und die Frau die Hauptverantwortung für den Haushalt übernehmen soll, wird nur von $9 \%$ abgelehnt und findet somit eine starke Zustimmung. Oder anders ausgedrückt: Es wird ein Zuverdienermodell befürwortet, in dem der Mann in Vollzeit erwerbstätig ist und die Frau als Teilzeiterwerbstätige lediglich die Rolle der Zuverdienerin einnimmt. Beim vierten Item wurde danach gefragt, ob ein Vater genauso stark in die Erziehung und Pflege eines Kindes einbezogen sein sollte, wie eine Mutter. Dieser Aussage stimmen 75\% zu. Damit scheint die Zustimmung zur aktiven Vaterschaft in dieser Klasse relativ hoch zu sein, 
obwohl die Antworten zu den anderen Items in dieser Klasse im Durchschnitt relativ traditionell ausfallen. Gleichzeitig verweist die hohe Zustimmung auch darauf, dass die Erwartungen an die Vaterrolle gestiegen sind.

Diese Klasse ergibt als Ganzes betrachtet ein schlüssiges Bild: Es wird die Auffassung vertreten, dass der Mann der Haupternährer und in Vollzeit erwerbstätig ist, wohingegen die Frau primär Hausfrau und Mutter ist, weswegen sie höchstens in Teilzeit erwerbstätig ist. Da die Mutter hierbei als die primäre Betreuungsperson gilt, die sich zeitintensiv und aufopferungsvoll um die Kinder kümmert, ihre Erwerbstätigkeit gänzlich aufgibt oder zumindest stark reduziert und ihre eigenen Bedürfnisse gegenüber den kindlichen Bedürfnissen hintenanstellt, entspricht diese Gruppe dem Konzept des „intensive mothering“ (Hays 1996). Entgegen dieser traditionellen Einstellungen erfährt die aktive Vaterschaft eine hohe Zustimmung. Diese Gruppe lässt sich deswegen gleichzeitig auch als „intensive parenting" beschreiben (Wall 2010; Liss et al. 2013; Craig et al. 2014; Faircloth 2014). Beim „intensive parenting“ wird der kindzentrierte Ansatz des „intensive mothering" dahingehend erweitert, dass Väter mit in die Verantwortung für die Kinderbetreuung einbezogen werden. Eine andere Terminologie für „intensive parenting“ ist die „verantwortete Elternschaft" (Ruckdeschel 2015), die ebenso zum Ausdruck bringt, dass Eltern gemeinsam verantwortlich sind und möglichst viel Zeit und Energie in die Erziehung investiert werden soll, um die Kinder bestmöglich und intensiv zu begleiten (Ruckdeschel 2015). Gemäß Tabelle 7.3 wird beim „intensive mothering/parenting“ die Einstellung vertreten, dass es sich beim Verdienst um eine getrennte, männliche Zuständigkeit handelt und bei der Fürsorge um eine gemeinsame Zuständigkeit (vgl. Tabelle: Grunow et al. 2018, S. 44). Da diese Einstellungen auch in dieser Klasse vertreten sind, wird sie als „intensive mothering/parenting“ bezeichnet.

Klasse 2: „egalitär“

Die zweite Klasse ist mit 1.644 Fällen vertreten, was $23 \%$ entspricht. Bei allen vier Items liegen sehr egalitäre Einstellungen vor (siehe Abbildung 7.2, mittlere Abbildung). Bei Item 1 wird zu 94\% der Aussage zugestimmt, dass nicht nur die Frau, sondern auch der Mann in Teilzeit arbeiten soll, wenn kleine Kinder da sind. Umgekehrt wird zu 91\% abgelehnt, dass die Frau primär Hausfrau und Mutter sein soll (Item 2). Eine ähnliche Einstellung offenbart sich auch bei Item 3, der Frage danach, ob der Mann der Hauptverdiener sein soll, was zu $90 \%$ abgelehnt wird. Somit ist man in dieser Klasse aufgeschlossen für Konstellationen, in denen beide Partner gleich viel verdienen (oder die Frau sogar die Hauptverdienerin ist). Bei Item 4 liegt sogar eine maximal egalitäre Einstellung vor: Es wird zu 100\% befürwortet, dass der Vater genauso sehr in die Pflege und Erziehung eines Kindes einbezogen sein sollte wie die Mutter. Diese Klasse lässt sich deshalb als egalitär bezeichnen, da die Einstellungen vertreten werden, dass beide Elternteile gleichermaßen für die Erwerbsarbeit und für die Fürsorgearbeit zuständig sind und man nicht aufgrund geschlechtsspezifischer Merkmale für eine bestimmte Sphäre besser geeignet ist (vgl. Tabelle: Grunow et al. 2018, S. 44). 
Klasse 3: „egalitärer Essentialismus“

Die dritte Klasse ist mit 2.741 Fällen (38\%) vertreten. Auch hier liegen sehr egalitäre Einstellung vor (siehe Abbildung 7.2, rechte Abbildung): Es wird zu 95\% abgelehnt, dass der Mann der Hauptverdiener sein soll (Item 3), was einer noch stärkeren Ablehnung als in der vorherigen, egalitären Klasse mit $90 \%$ entspricht. Bei den weiteren Items sind die Einstellungen zwar auch sehr egalitär, aber nicht ganz so egalitär wie in der vorherigen Klasse: Es wird zu 84\% befürwortet, dass der Vater genauso stark in die Erziehung des Kindes eingebunden sein soll wie die Mutter (Item 4), in der vorherigen Klasse lag die Zustimmung bei $100 \%$. Es wird zu 74\% abgelehnt, dass die Frau primär Hausfrau und Mutter sein soll (Item 2); in der vorherigen Klasse lag die Ablehnung bei 91\%. Bei der Frage danach, ob nicht nur die Frau, sondern auch der Mann nur in Teilzeit arbeiten soll, wenn kleine Kinder da sind (=Item 1), fällt die Zustimmung mit nur 13\% sogar deutlich geringer aus als in der vorherigen Klasse, wie die Zustimmung bei 94\% lag.

Da diese Klasse bei einem Item eine egalitärere Einstellung und bei drei Items weniger egalitäre Einstellungen als die vorherige Klasse aufweist, liegt die Vermutung nahe, sie insgesamt als weniger egalitär zu bezeichnen. Gleichzeitig spricht vieles dafür, dass diese Klasse das repräsentiert, was in bisheriger Forschung als „egalitärer Essentialismus" bezeichnet wird (Charles und Grusky 2004; Cotter et al. 2011; Grunow et al. 2018). Beim „egalitären Essentialismus“ liegen sowohl Aspekte feministischer Gleichheit als auch traditioneller Mutterschaftsrollen vor (Cotter et al. 2011). D.h. traditionelle Aspekte, die es unterstützen, dass Mütter zu Hause bleiben, treffen auf egalitäre Aspekte, die das Zuhausebleiben der Mütter als ihre individuelle Wahl verstehen und die Fürsorgearbeit der Mutter als gleichwertig mit der Erwerbsarbeit betrachten (Cotter et al. 2011).

Mit nur vier Items ist es schwierig, genauere Aussagen zu dieser Klasse zu machen. Zudem lassen Zustimmungen oder Ablehnungen zu bestimmten Items verschiedene Interpretationen zu. Beispielsweise ist unklar, weshalb die Zustimmung beim ersten Item, dass beide Eltern Teilzeit arbeiten sollten, so gering ist: Wird dem nicht zugestimmt, weil Väter stattdessen in Vollzeit und Mütter in Teilzeit beschäftigt sein sollen? Oder wird dem nicht zugestimmt, weil stattdessen beide in Vollzeit erwerbstätig sein sollen? Es ist auch denkbar, dass die Wahlfreiheit, die beim „egalitären Essentialismus“ hervorgehoben wird, dazu führt, dass normative Vorgaben allgemein eher abgelehnt werden. D.h. es wird abgelehnt, dass beim Vorhandensein von kleinen Kindern auch der Vater in Teilzeit arbeiten sollte, was aber nicht bedeutet, dass der Vater nicht in Teilzeit arbeiten darf. Gleiches kann auch auf Item 2 übertragen werden: Dass es nicht die primäre Aufgabe der Mutter sein muss, Hausfrau und Mutter zu sein, heißt nicht, dass es nicht ihre primäre Aufgabe sein darf.

Die Antwortmuster dieser Klasse verleiten zu der Annahme, sie als „egalitären Essentialismus" zu betiteln. Um diese Annahme zu untermauern, wären jedoch weitere Items nötig. So geht aus einer Studie von Grunow et al. (2018) hervor, dass in der Klasse des "egalitären Essentialismus“ die Hausarbeit der Frauen befürwortet wird. Im Vergleich 
dazu erscheint die Ablehnungsquote für die Rolle der Hausfrau als primäre Rolle von Frauen in der vorliegenden Arbeit verhältnismäßig hoch. Eine Ursache für diese Diskrepanz könnte darin liegen, dass sich die Daten nur bedingt vergleichen lassen: Die Daten bei Grunow et al. (2018) stammen von 2008, die hier verwendeten Daten stammen von 2013/14 - möglicherweise sind die Einstellungen selbst innerhalb der Klasse des „egalitären Essentialismus" egalitärer geworden. Des Weiteren handelt es sich bei Grunow et al. (2018) um eine international vergleichende Studie, bei der für den deutschen Kontext Westdeutschland betrachtet wird, während hier der Fokus auf nationaler Ebene liegt und Ostdeutschland inkludiert ist. Schließlich ist denkbar, dass es sich bei der hier identifizierten Klasse des „egalitären Essentialismus“ immer noch um eine sehr heterogene Klasse handelt, die in weitere Subklassen zerlegt werden müsste, was jedoch aus methodischen Gründen nicht realisierbar ist.

Insgesamt bestätigen die hier identifizierten Klassen den Trend, dass traditionelle Einstellungen seltener werden und egalitäre Einstellungen auf dem Vormarsch sind. Dies zeigt sich etwa daran, dass in der vorliegenden Arbeit keine traditionelle Klasse identifiziert werden konnte. Selbst in der Klasse des „intensive mothering/parenting“, die im Vergleich zu den anderen beiden Klassen noch relativ traditionell ist, finden sich hohe Zustimmungen zu einer aktiven Vaterschaft, in der Väter stark in die Pflege und Erziehung des Kindes eingebunden sind. Eine aktive Vaterschaft wird somit in allen Klassen stark befürwortet.

\section{Bivariate Zusammenhänge}

Im Folgenden wird auf verschiedene bivariate Zusammenhänge eingegangen, um genauere Aussagen über die Zusammensetzung der latenten Klassen machen zu können. Begonnen wird mit dem Alter des Kindes: Die nachfolgende Abbildung 7.3 zeigt, dass bei einem unter 1-jährigen Kind etwas mehr als ein Drittel (35\%) die Einstellung des „intensive mothering/parenting“ haben. In den Altersstufen „1 bis unter 3 Jahre“ und „3 bis unter 6 Jahre" erhöht sich der Anteil auf 41\% bzw. 43\% und nimmt erst wieder in der nächsten Altersstufe („6 bis unter 9 Jahre“) ab. Da sich das Konzept des „intensive mothering" vorwiegend auf Mütter von kleineren Kindern bezieht, entspricht die Reduktion der „intensive mothering/parenting“-Klasse in der höchsten Altersstufe den Erwartungen. In der Altersstufe "unter 1 Jahr" vertritt fast die Hälfte (44\%) den egalitären Essentialismus, d.h. zum einen die egalitäre Ansicht, dass Frauen zwar erwerbstätig sein sollen und die Pflege und Erziehung von beiden Elternteilen zu gleichen Teilen übernommen werden sollen; zum anderen wird aber gleichzeitig abgelehnt, dass beide Elternteile in Teilzeit arbeiten sollten, wenn kleine Kinder da sein. Mit zunehmendem Alter des Kindes reduziert sich diese Klasse (auf 39\% bzw. 36\%) und steigt erst in der höchsten Altersstufe wieder leicht an (38\%). Die egalitäre Klasse ist in den Altersstufen „unter 1 Jahr“, „1 bis unter 3 Jahre“ und „3 bis unter 6 Jahre“ mit 21-22\% etwa gleich stark vertreten. Erst wenn das Kind im Alter von „6 bis unter 9 Jahren“ ist, steigt die egalitäre Klasse auf $27 \%$. Diese deskriptiven Befunde zeigen somit auch, dass das Schuleintrittsalter eine 
wichtige Schwelle darstellt: Bei Familien mit Kindern unter 6 Jahren sind Einstellungen wie das „intensive mothering/parenting“ stärker vertreten als in Familien mit Kindern, die im Schulalter sind; umgekehrt sind egalitäre Einstellungen erst dann häufiger vertreten, wenn das Kind bereits das Schulalter erreicht hat.

Bei der Anzahl der Kinder zeigt sich, dass die Klasse des „intensive mothering/parenting“ mit zunehmender Kinderanzahl tendenziell zunimmt. Dies mag damit zusammenhängen, dass Familien mit mehreren Kindern in der Regel familienorientiertere Einstellungen haben. Dagegen sind die Klassen des „egalitären Essentialismus“ und die egalitäre Klasse in Familien mit vielen Kindern etwas seltener vertreten als in Familien mit einem Kind.

Aus den bivariaten Zusammenhängen zwischen den latenten Klassen und dem Haushaltsnettoeinkommen geht hervor, dass die Klasse des „intensive mothering/parenting“ am stärksten vertreten ist, wenn das Haushaltsnettoeinkommen niedrig ist, während der "egalitäre Essentialismus" und die egalitäre Klasse dann am stärksten vorhanden ist, wenn das Haushaltsnettoeinkommen hoch ist. Hier gilt es zu berücksichtigen, dass das Haushaltsnettoeinkommen eine endogene Variable ist, die ihrerseits etwa durch Einstellungen beeinflusst wird: So werden Mütter, die das Leitbild des „intensive mothering/parenting" vertreten, insbesondere bei noch kleinen Kindern häufiger nicht oder nur in Teilzeit erwerbstätig sind, während Mütter mit egalitären Einstellungen häufiger und in höherem Umfang erwerbstätig sind und sie somit auch zu einem höherem Haushaltsnettoeinkommen beitragen.

Während die Unterschiede in Abhängigkeit vom relativen Status eher gering ausfallen (nicht abgebildet), zeigen sich zwischen dem absoluten Berufsstatus (ISEI) der Mütter und der Zugehörigkeit zu den latenten Klassen deutlich stärkere Zusammenhänge: Wenn Mütter einen niedrigen Berufsstatus haben, ist etwa die Hälfte von ihnen (51\%) der Klasse des „intensive mothering/parenting“ zugehörig, wohingegen es bei Müttern mit einem hohen Status nur 22\% sind. Dementsprechend sind die egalitäre Klasse und die Klasse des „egalitären Essentialismus“ stärker präsent, wenn die Mütter einen mittleren oder hohen Berufsstatus haben.

Deutliche Unterschiede in der Klassenzugehörigkeit gibt es auch zwischen Ost- und Westdeutschland. In Westdeutschland gehören mehr der Klasse des „intensive mothering/parenting" an in Ostdeutschland (Westdeutschland: 40\%; Ostdeutschland 27\%). Der egalitäre Essentialismus ist dagegen in Ostdeutschland stärker vertreten, wohingegen es bei egalitären Klassen kaum einen Unterschied zwischen Ost- und Westdeutschland gibt. 


\section{Abbildung 7.3: Bivariate Zusammenhänge der latenten Klassen}
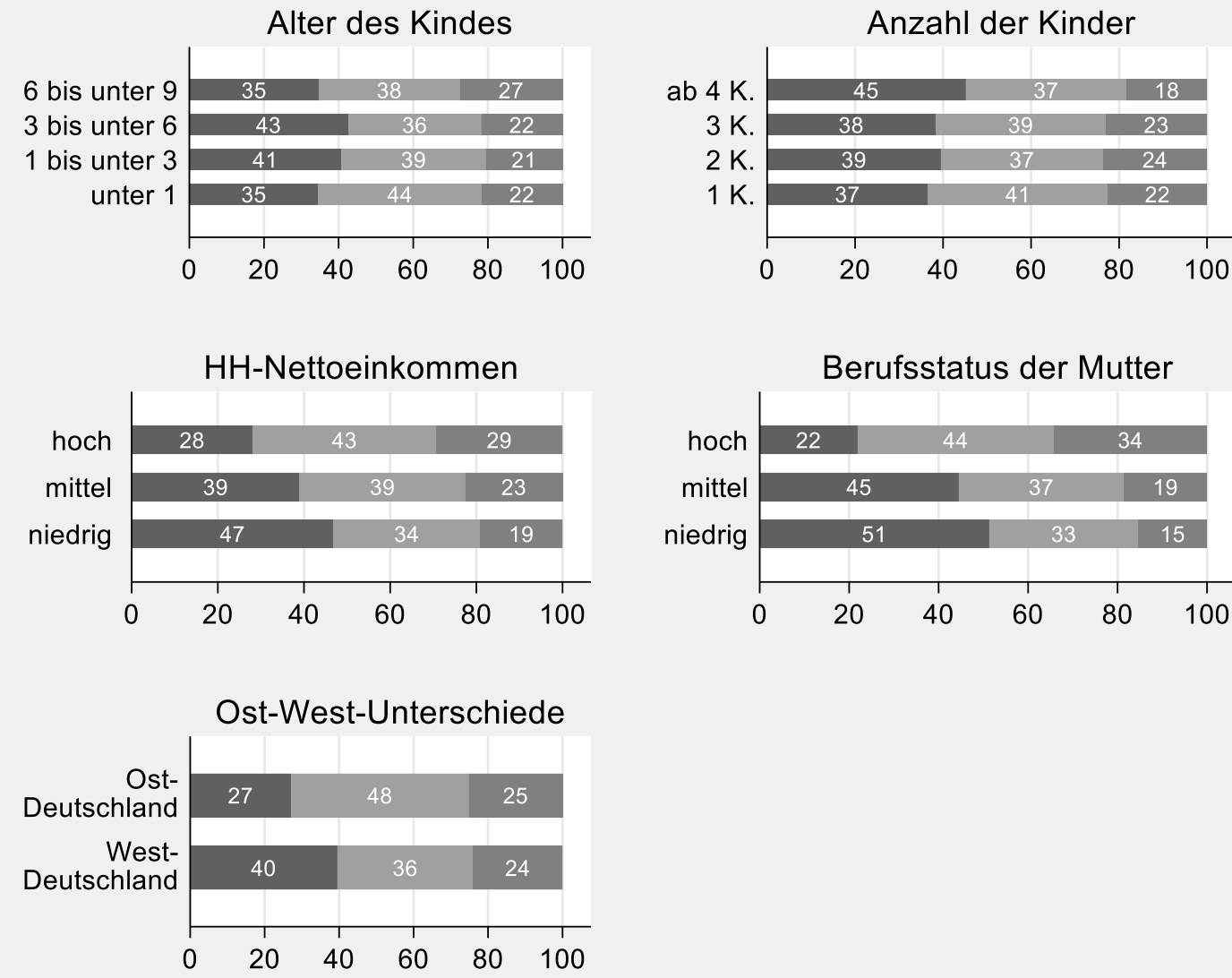

\section{intensive mothering/ parenting}

Quelle: DJI-Studie AID:A 2013/14, Daten gewichtet, $n=7.185$, eigene Berechnungen.

\section{Multivariate Ergebnisse}

Nach diesen bivariaten Zusammenhängen wird zu den multivariaten Ergebnissen übergegangen. Ausgangsbasis für die nachfolgenden Abbildungen ist ein multinomiales Logitmodell, welches als abhängige Variable die latenten Klassen mit den Ausprägungen „intensive mothering/ parenting“, „egalitärer Essentialismus“ und "egalitär" enthält (siehe Tabelle A.1 im Anhang). Für ein leichteres Verständnis der Ergebnisse werden jedoch die Predictive Margins herangezogen, die auf den Average Marginal Effects aufbauen und in Abbildung 7.4 dargestellt sind. 
Abbildung 7.4: Predictive Margins zu den latenten Klassen in Abhängigkeit von verschiedenen Einflussfaktoren
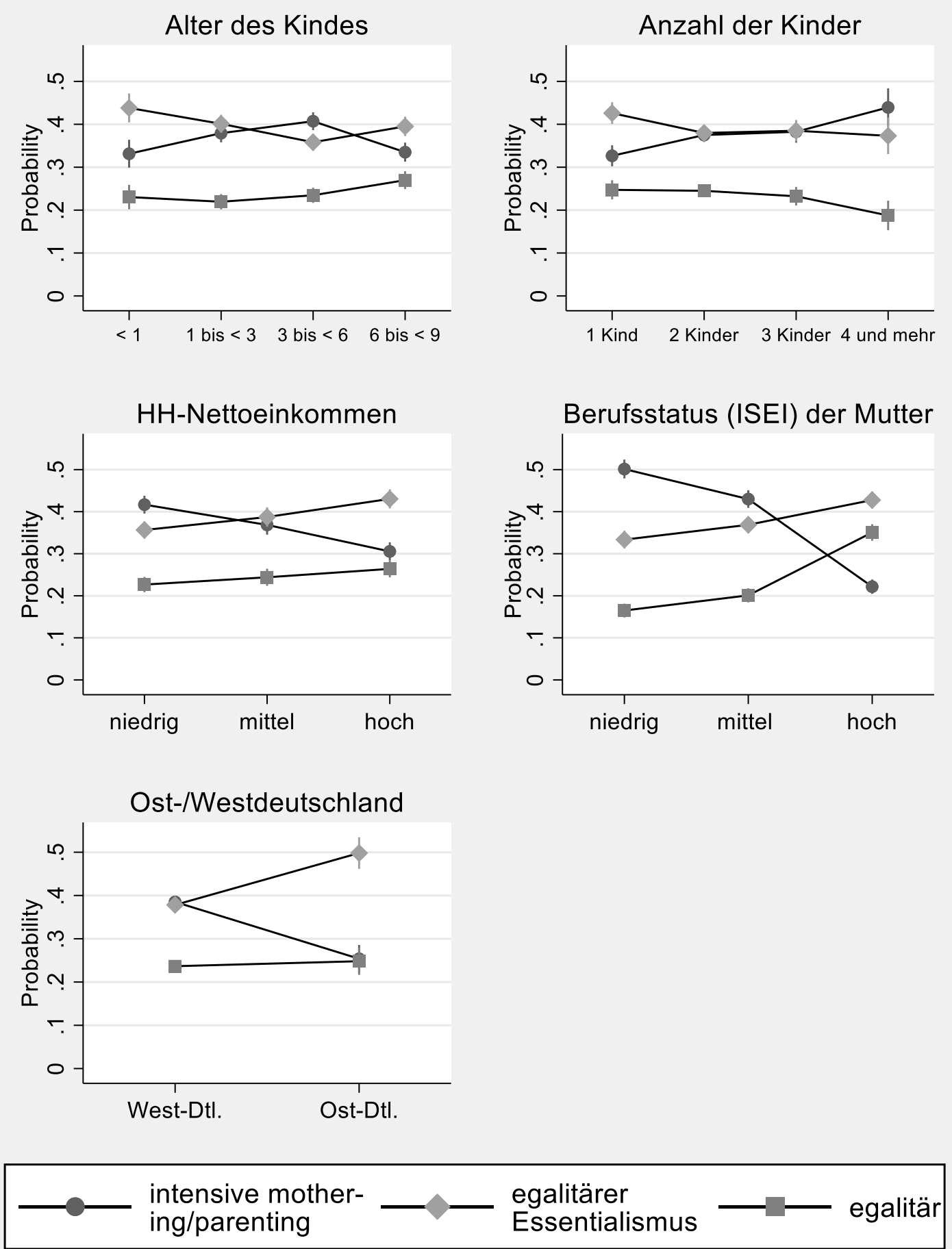

Quelle: DJI-Studie AID:A 2013/14, die Predictive Margins basieren auf dem multinomialen Regressionsmodell in Tabelle A.1 im Anhang; die weiteren Variablen des Modells wurden auf ihren Mittelwerten festgehalten; eigene Berechnungen.

Betrachtet man die multivariaten Ergebnisse zum Zusammenhang zwischen dem Kindesalter und der Zugehörigkeitswahrscheinlichkeit zu den einzelnen Klassen, so zeigt 
sich, dass die vorhergesagte Wahrscheinlichkeit für die Klasse des „intensive mothering/parenting" bis zu einem Kindesalter von unter 6 Jahren zunimmt und ab einem Kindesalter von 6 Jahren abnimmt (siehe Abbildung 7.4, oben, links). Die Klasse des „egalitären Essentialismus“ ist bei Kindern „unter 1 Jahr“ am stärksten vertreten, nimmt in den darauffolgenden Altersstufen ab und steigt erst wieder ab einem Kindesalter von 6 Jahren. Die Wahrscheinlichkeit für die egalitäre Klasse beträgt in den ersten 6 Lebensjahren des Kindes 22-23\% und steigt erst ab einem Kindesalter von 6 Jahren auf 27\%.

Bei der Kinderanzahl (siehe Abbildung 7.4, oben, rechts) steigt die Wahrscheinlichkeit für die Klasse „intensive mothering/parenting“ mit zunehmender Kinderanzahl von 33\% in Familien mit einem Kind auf $44 \%$ in Familien mit vier und mehr Kindern. Dies ist naheliegend, da Familien mit mehreren Kindern oftmals familienorientiertere Einstellungen haben. Die Wahrscheinlichkeit für die Klasse des „egalitären Essentialismus“ ist in Familien mit einem Kind am höchsten (42\%) und reduziert sich auf 37\% in Familien mit zwei oder mehr Kindern. Die Wahrscheinlichkeit für die egalitäre Klasse beträgt in Familien mit einem Kind 25\% und reduziert sich mit zunehmender Kinderanzahl auf 19\%. Eine höhere Kinderanzahl macht eine egalitäre Einstellung daher unwahrscheinlicher.

Vergleicht man die Wahrscheinlichkeiten für die latenten Klassen in Abhängigkeit vom Haushaltsnettoeinkommen (siehe Abbildung 7.4, Reihe 2, links), so zeigt sich, dass die Wahrscheinlichkeiten für die Klassen „egalitärer Essentialismus“ und „egalitär“ zum zunehmendem Haushaltsnettoeinkommen zunehmen, während die Wahrscheinlichkeit für die Klasse „intensive mothering/parenting“ mit zunehmendem Haushaltsnettoeinkommen abnimmt. ${ }^{24}$ Ein ähnlicher Zusammenhang zeigt sich auch beim absoluten Berufsstatus der Mütter (siehe Abbildung 7.4, Reihe 2, rechts): Die Klassen „egalitärer Essentialismus“ und „egalitär“ werden wahrscheinlicher, wenn Mütter anstelle eines niedrigen Status einen mittleren oder hohen Status haben, während umgekehrt die Klasse „intensive mothering/parenting" unwahrscheinlicher wird, wenn Mütter einen mittleren oder hohen Status haben. Allerdings fallen die Effekte des Berufsstatus der Mütter deutlich stärker aus als beim Haushaltsnettoeinkommen: So beträgt die Wahrscheinlichkeit für die Klasse „intensive mothering/parenting“ $50 \%$, wenn Mütter einen niedrigen Status haben und sinkt auf 43\%, wenn Mütter einen mittleren Status haben bzw. auf 22\%, wenn Mütter einen hohen Status haben.

Des Weiteren kann danach differenziert werden, ob sich der Wohnort in West- oder Ostdeutschland befindet: Bzgl. der egalitären Klasse gibt es kaum Unterschiede zwischen

\footnotetext{
${ }^{24}$ Das Einkommen ist eine höchst endogene Variable und es muss berücksichtigt werden, dass die Kausalitätsrichtung hier auch andersrum sein kann, d.h. dass nicht nur das Einkommen die Einstellungen beeinflussen kann, sondern die Einstellungen auch das Einkommen beeinflussen können. Es wurden auch Modelle gerechnet, die das Haushaltseinkommen nicht als unabhängige Variable enthalten. Die Ergebnisse der übrigen Variablen fallen jedoch auch dann sehr ähnlich aus.
} 
West- und Ostdeutschland, jedoch ist die Klasse des „intensive mothering/parenting“ Westdeutschland wahrscheinlicher als in Ostdeutschland, während die Klassen des "egalitären Essentialismus" in Westdeutschland unwahrscheinlicher ist als in Ostdeutschland (siehe Abbildung 7.4, unten).

Insgesamt lässt sich festhalten, dass sich deutliche Unterschiede zwischen den verschiedenen Profilen an Geschlechterrolleneinstellungen zeigen. Nach diesem Exkurs zur multidimensionalen Abbildung von Geschlechterrolleneinstellungen werden im Folgenden die empirischen Ergebnisse zur Aufteilung der Erwerbsarbeit, der Hausarbeit und der Kinderbetreuung vorgestellt. Hierbei werden vertiefende Analysen zu den Geschlechterrolleneinstellungen angestellt, in denen die soeben vorgestellten, latenten Klassen in die Analysen miteinbezogen werden. 


\section{Aufteilung der Erwerbsarbeit}

\subsection{Deskriptive Befunde}

Bevor die abgeleiteten Hypothesen empirisch getestet werden, wird zunächst auf die Frage eingegangen, wie viele Stunden Mütter und Väter tatsächlich erwerbstätig sind und welchen Erwerbsumfang sie sich wünschen (siehe Abbildung 8.1 für die Mütter und Abbildung 8.2 für die Väter). Ein Blick auf die gewünschten und tatsächlichen Erwerbsumfänge der Mütter zeigt, dass es große Diskrepanzen zwischen Wunsch und Wirklichkeit gibt. So wünschen sich nur 5\% der Mütter eine Erwerbslosigkeit, aber 35\% der Mütter sind ohne Erwerbstätigkeit; während eine kleine Teilzeit (16-25 Std.) von 44\% gewünscht wird, wird sie de facto nur von $24 \%$ praktiziert, so dass sich auch hier große Diskrepanzen zeigen. Von 22\% der Mütter wird eine geringfügige Beschäftigung (1-15 Std.) und von $24 \%$ eine große Teilzeit (26-35 Std.) gewünscht, aber nur von 9\% bzw. $17 \%$ tatsächlich ausgeführt. In seltenen Fällen arbeiten die Mütter auch mehr als gewünscht (siehe die Diskrepanzen bei den hohen Erwerbsumfängen in Abbildung 8.1), aber insgesamt betrachtet ist bei den Müttern in den meisten Fällen der gewünschte Erwerbsumfang höher als der tatsächliche Erwerbsumfang.

Abbildung 8.1: Vergleich der gewünschten und tatsächlichen Erwerbsumfänge der Mütter

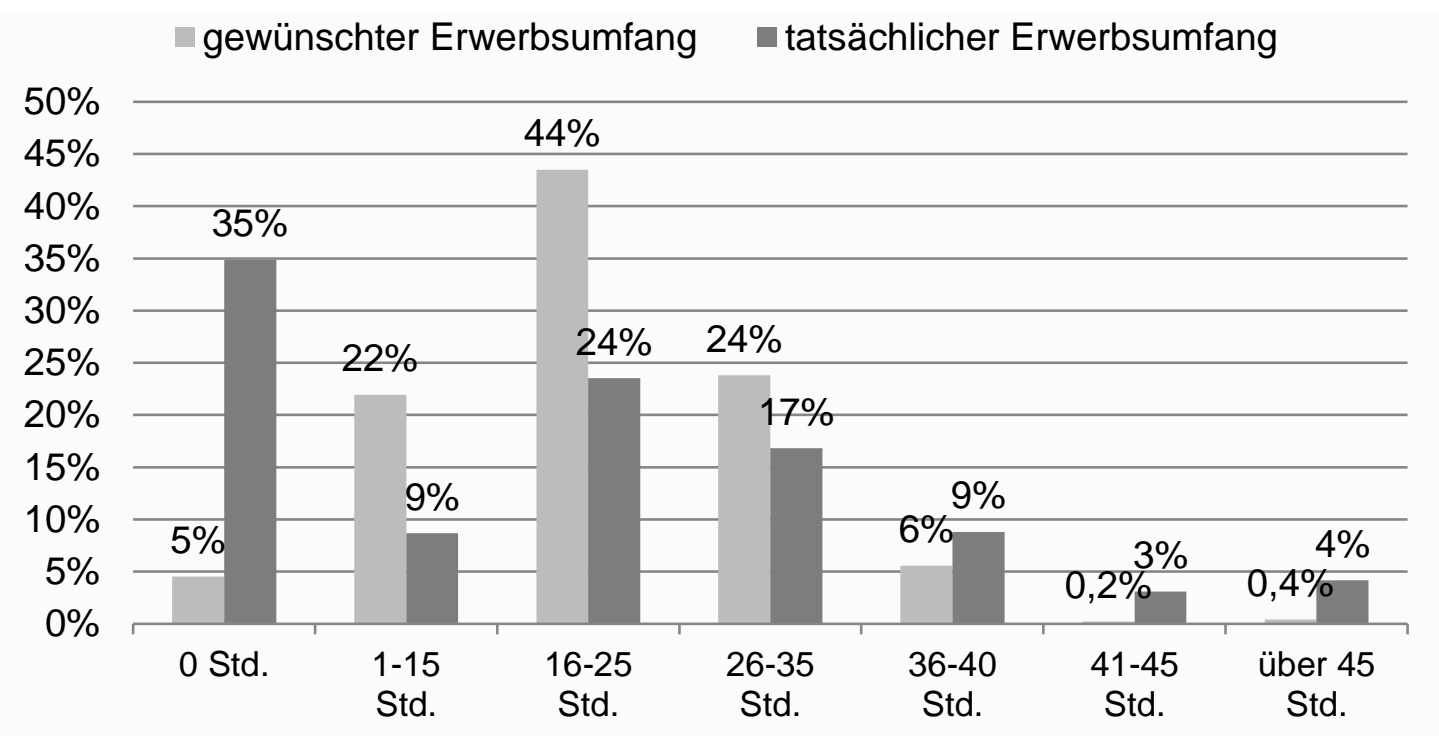

Quelle: DJI-Studie AID:A 2013/14, Sample gewichtet, N (gewünschter Erwerbsumfang) = 10.241, $N$ (tatsächlicher Erwerbsumfang) $=10.218$, eigene Berechnungen .

Gegensätzlich verhält es sich bei den Vätern: Hier wird in der Regel ein niedrigerer Umfang als der tatsächliche Erwerbsumfang gewünscht. Während der Großteil der Väter (38\%) 36-40 Std. erwerbstätig ist und dieser Umfang von etwa demselben Anteil (41\%) gewünscht wird, sind nur 5\% der Väter in großer Teilzeit (26-35 Std.) beschäftigt, obwohl sich dies $38 \%$ der Väter wünschen. Viele Väter sind über 40 Std. oder sogar über 45 
Std. erwerbstätig (19\% bzw. 33\%), obwohl sich dies nur ein Bruchteil der Väter wünscht (3\% bzw. 6\%) (siehe Abbildung 8.2). Somit liegen auch bei den Vätern große Diskrepanzen zwischen dem gewünschten und dem tatsächlichen Erwerbsumfang vor, da sie in der Regel mehr arbeiten als sie sich wünschen.

Abbildung 8.2: Vergleich der gewünschten und tatsächlichen Erwerbsumfänge der Väter

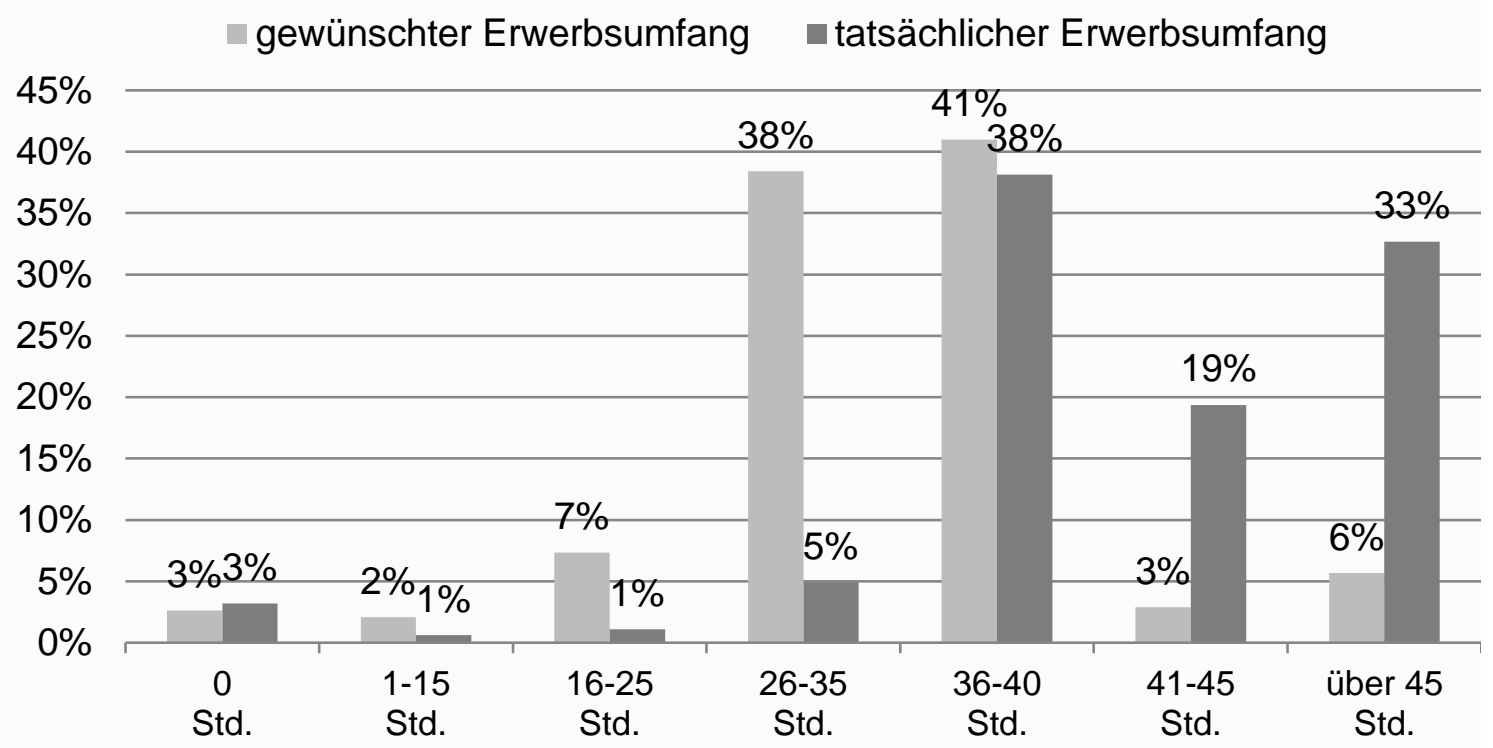

Quelle: DJI-Studie AID:A 2013/14, Sample gewichtet, N (gewünschter Erwerbsumfang) = 4.366, $N$ (tatsächlicher Erwerbsumfang) $=10.247$, eigene Berechnungen .

Da die Erwerbsumfänge der Eltern stark durch das Alter des Kindes beeinflusst werden, werden im Folgenden die Erwerbskonstellationen differenziert nach dem Alter des Kindes betrachtet. In Abbildung 8.3 ist zu sehen, dass bei einem Kind im Alter von „unter 1 Jahr" bei $85 \%$ der Paare die Väter in Vollzeit und die Mütter nicht erwerbstätig sind. Wenn das Kind „1 bis unter 3 Jahre" alt ist, liegt diese Konstellation nur noch bei etwa der Hälfte der Paare vor und 37\% praktizieren noch eine Vollzeit-/Teilzeit-Konstellation. Dass beide in Vollzeit oder beide in Teilzeit arbeiten, kommt bei $12 \%$ vor. ${ }^{25}$ Bei einem Kind im Alter von „3 bis unter 6 Jahren“ hat der Anteil des männlichen AlleinverdienerModells („Mann Vollzeit/Frau nicht erwerbstätig) weiter abgenommen und der Anteil der Vollzeit-/Teilzeit-Konstellation weiter zugenommen. Diese Vollzeit-/Teilzeit-Konstellation wird in mehr als der Hälfte aller Paare praktiziert. Damit haben sich in den ersten sechs Lebensjahren des Kindes bei den Erwerbskonstellationen der Eltern starke Veränderungen gezeigt. In den nachfolgenden Altersstufen („6 bis unter 11 Jahre“ und „11 bis unter

25 Die Kategorien „beide Vollzeit“ und „beide Teilzeit“ wurden zusammengefasst, da in beiden Fällen eine egalitäre Aufteilung der Erwerbsarbeit praktiziert wird. Inhaltlich wäre eine Unterscheidung dieser beiden Gruppen zwar interessant, aber die Gruppe „beide Teilzeit“ ist zu klein, um sie gesondert zu betrachten, da sie lediglich $2 \%$ ausmacht. 
18 Jahre") gibt es nur noch geringe Veränderungen, wobei die Vollzeit-/Teilzeit-Konstellation dennoch die gängige Erwerbskonstellation bleibt.

\section{Abbildung 8.3: Erwerbskonstellation in Abhängigkeit vom Alter des Kindes}

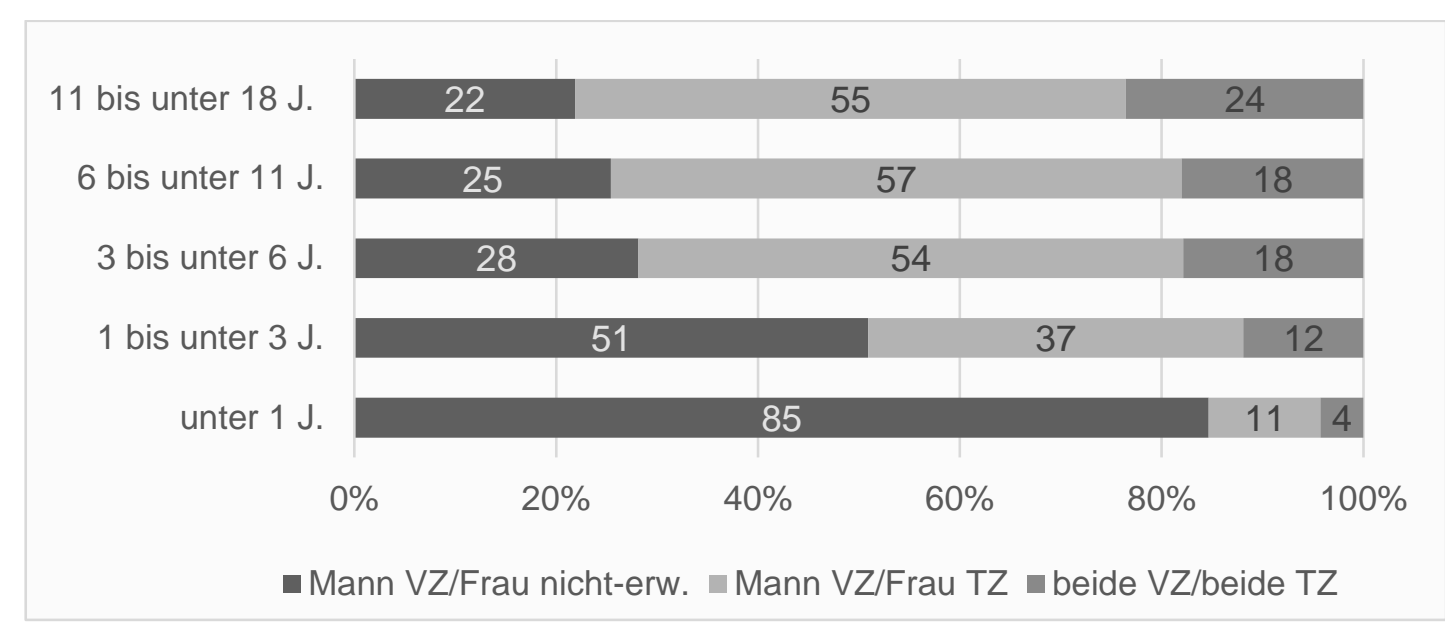

Quelle: DJI-Studie AID:A 2013/14, Sample gewichtet, $n=10.130$, eigene Berechnungen.

Um genauer herauszufinden, wie egalitär sich Eltern die Erwerbsarbeit aufteilen, werden im Folgenden anstelle der groben Erwerbskonstellationen wieder die genauen Erwerbsstunden verwendet. Um dennoch Aussagen auf Paarebene machen zu können, werden hierzu die Erwerbsstunden der Mütter am gesamten Erwerbsvolumen des Paares relativiert (siehe Abbildung 8.4). Der Erwerbsanteil der Mutter beträgt 0\%, wenn sie erwerbslos und der Vater der Alleinverdiener ist; umgekehrt beträgt ihr Erwerbsanteil 100\%, wenn der Vater erwerbslos und sie die Alleinverdienerin ist. Bei einem Erwerbsanteil von $50 \%$ (siehe eingezeichnete Linie in Abbildung 8.4) liegt eine (exakt) egalitäre Aufteilung der Erwerbsarbeit vor, d.h. beide Partner sind im gleichen Umfang erwerbstätig.

Aus Abbildung 8.4 geht hervor, dass bei 33\% der Paare der Erwerbsanteil der Mütter 0\% beträgt, d.h. bei einem Drittel aller Paare sind ausschließlich die Väter erwerbstätig. Insgesamt liegt bei vielen Müttern der Erwerbsanteil zwischen 25\% und 45\%, was daran liegt, dass viele Mütter in (kleiner) Teilzeit erwerbstätig sind, während der Großteil der Väter in Vollzeit erwerbstätig ist. Das folgende Beispiel soll die Berechnung des Erwerbsanteils veranschaulichen: Wenn die Mutter etwa 20 Std. pro Woche erwerbstätig ist und der Vater 40 Std. pro Woche, so entspricht dies einem Erwerbsanteil der Mutter von $20 /(20+40)=20 / 60=1 / 3=33,3 \%$. Dass Mütter in höherem Umfang erwerbstätig sind als die Väter, kommt nur sehr selten vor (siehe rechts von der $50 \%$-Linie in Abbildung 8.4 ) und in nur $2 \%$ sind Mütter die Alleinverdienerinnen. 


\section{Abbildung 8.4: Erwerbsanteil der Mütter am gesamten Erwerbsvolumen des Paares}

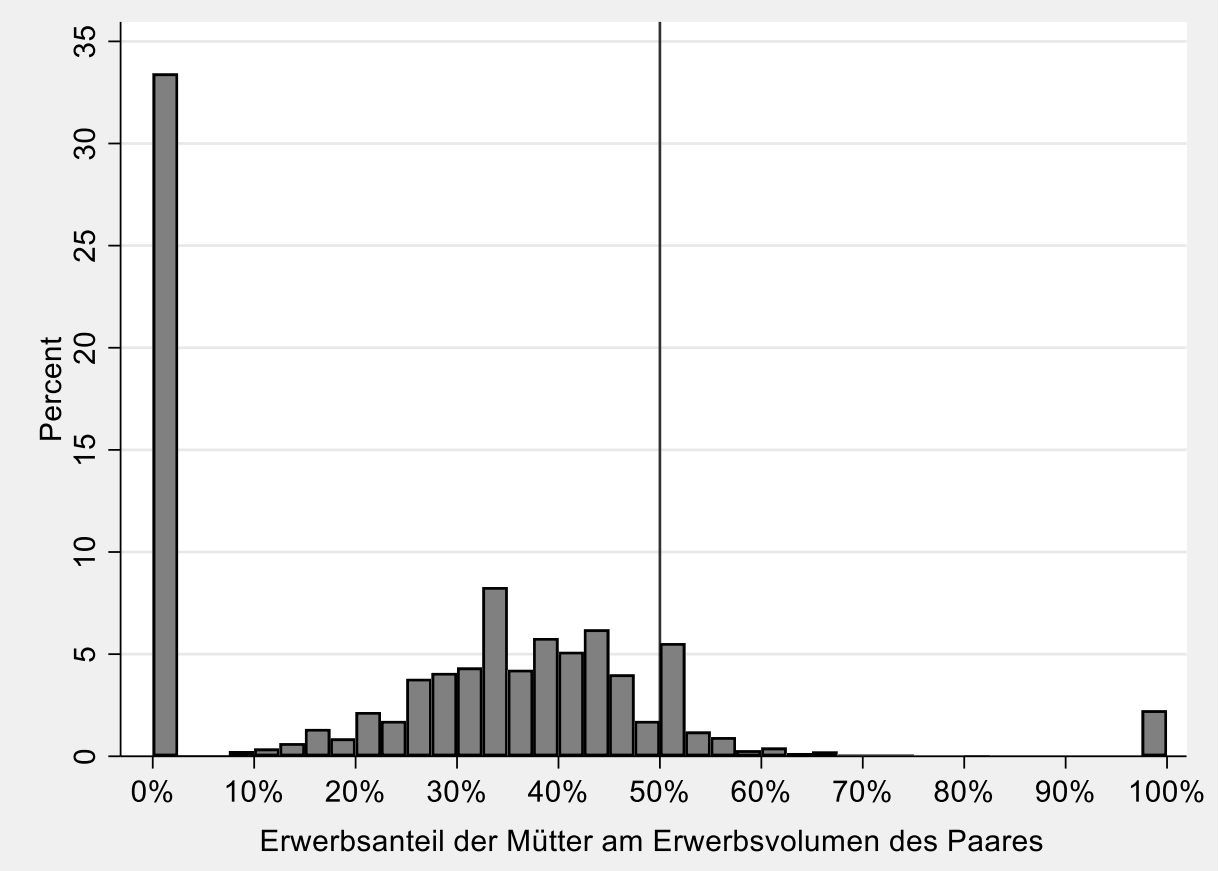

Quelle: DJI-Studie AID:A 2013/14, Sample gewichtet, $n=10.429$, eigene Berechnungen.

Anhand dieser Verteilung der Erwerbsanteile der Mütter lässt sich somit festhalten, dass die Erwerbsarbeit sehr ungleich verteilt ist. Im Folgenden wird der Frage nachgegangen, warum dem so ist, welche Rolle dabei das Alter des Kindes sowie weitere Einflussfaktoren spielen. Dieser Erwerbsanteil der Mütter stellt daher sowohl in den nachfolgenden, bivariaten Zusammenhängen als auch bei den späteren, multivariaten Analysen die abhängige Variable dar.

Nachdem die bisherigen Übersichten die univariate Verteilung der Erwerbsarbeit abgebildet haben, interessieren in einem nächsten Schritt bivariate Zusammenhänge. Die nachfolgenden Abbildungen basieren auf bivariaten Regressionsmodellen und zeigen die vorgesagten Erwerbsanteile der Mütter an. Laut den ökonomischen Theorien spielen Ressourcen eine wichtige Rolle für die Arbeitsteilung. Daher wird in Abbildung 8.5 zunächst darauf eingegangen, welche Zusammenhänge sich zeigen, wenn lediglich der relative Berufsstatus (links) oder der absolute Berufsstatus der Mutter (rechts) betrachtet wird. Die Abbildungen zeigen, dass die relativen und absoluten Ressourcen im ersten Lebensjahr des Kindes bzgl. der Erwerbsanteile der Mütter keinen signifikanten Unterschied machen. In den nachfolgenden Altersstufen werden bei den relativen Ressourcen jedoch signifikante Unterschiede ersichtlich (siehe Abbildung 8.5, links): Wenn das Kind im Alter von „1 bis unter 3 Jahre“ ist, so beträgt der Erwerbsanteil von Müttern mit einem gleichen oder höheren Berufsstatus bereits über 20\%, während er von Müttern mit niedrigerem Berufsstatus lediglich $15 \%$ beträgt. In den darauffolgenden Altersstufen steigt der Erwerbsanteil von allen Müttern, wobei Mütter mit niedrigeren Ressourcen im Vergleich zu den Müttern mit gleichen oder höheren Ressourcen weiterhin einen niedrigeren 
Erwerbsanteil haben. Zwischen den Müttern mit einem gleichen und höheren Status gibt es keine bedeutenden Unterschiede. Beim absoluten Berufsstatus der Mütter (siehe Abbildung 8.5, rechts) gibt es auch ab einem Kindesalter von einem Jahr deutliche Unterschiede: Am höchsten ist der Erwerbsanteil von Müttern mit einem hohen Status und am niedrigsten von Müttern mit einem niedrigen Status. Während Mütter mit einem mittleren Status bei einem Kind im Alter von „1 bis unter 3 Jahre“ einen signifikant höheren Erwerbsanteil haben als Mütter mit einem niedrigen Status, gibt es bei einem höheren Kindesalter keine signifikanten Unterschiede mehr zwischen diesen beiden Kategorien.

Abbildung 8.5: Bivariate Zusammenhänge des relativen Berufsstatus (ISEI) und des absoluten Berufsstatus der Mutter (ISEI) mit der Aufteilung der Erwerbsarbeit
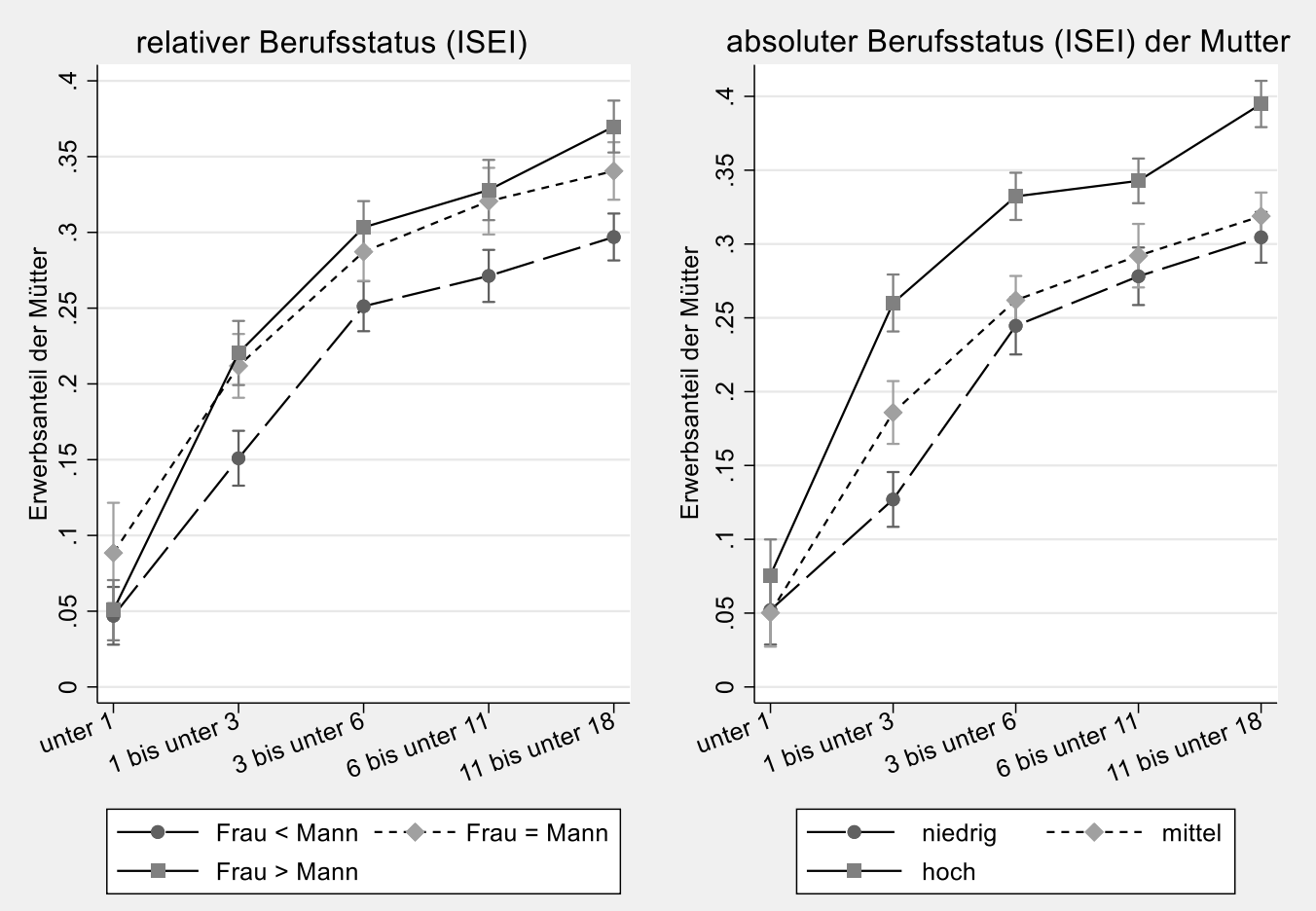

Anmerkung: Vorhergesagter Erwerbsanteil der Mütter (am Erwerbsvolumen des Paares) auf Basis von bivariaten Regressionsmodellen mit 95\%-Konfidenzintervallen. Quelle: DJI-Studie AID:A 2013/14, gewichtet, $n$ (relativer Berufsstatus) $=10.043$, n (absoluter Berufsstatus) $=10.043$; eigene Berechnungen.

Als nächstes wird auf die Bedeutung der Geschlechterrolleneinstellungen eingegangen (siehe Abbildung 8.6). Allgemein zeigt sich, dass bei beiden Items egalitäre Einstellungen mit einem höherem Erwerbsanteil der Mütter assoziiert sind. Bei Item 1 (siehe Abbildung 8.6, links) wird danach gefragt, ob nicht nur die Frau, sondern auch der Mann in Teilzeit arbeiten soll, wenn kleine Kinder vorhanden sind. In den ersten drei Lebensjahren fallen die Unterschiede zwischen egalitären, neutralen und traditionellen Einstellungen noch relativ gering aus. Mit zunehmendem Alter des Kindes und insbesondere dann, wenn das Kind „6 bis unter 11 Jahre“ alt ist, tragen egalitäre Einstellungen bei diesem Item besonders zu einem höherem Erwerbsanteil der Mütter bei. Sobald das Kind das 
Grundschulalter erreicht hat, können hier die egalitären Einstellungen leichter in eine egalitärere Aufteilung der Erwerbsarbeit umgesetzt werden. Gleichzeitig zeigt sich jedoch auch, dass die Einstellungen davor nur schwer umgesetzt werden können. Die Befürwortung einer Teilzeit-/Teilzeit-Konstellation bei kleinen Kindern kann beim Vorhandensein von kleinen Kindern somit nur schwer umgesetzt werden.

Beim zweiten Item (siehe Abbildung 8.6, rechts) wird danach gefragt, ob man der Meinung ist, dass in Familien der Vater erwerbstätig sein soll und die Mutter zu Hause bleiben und die Kinder versorgen soll. Auch hier ist die Aufteilung der Erwerbsarbeit egalitärer, wenn hierzu egalitäre Einstellungen vertreten werden. Am kleinsten sind die Zusammenhänge bei einem unter 1-jährigen Kind (die Zusammenhänge sind aber dennoch signifikant) und sie nehmen sprunghaft zu, wenn das Kind „1 bis 3 Jahre“ alt ist: Für Mütter mit einer traditionellen (resp. neutralen) Einstellung, wird für diese Altersstufe des Kindes ein Erwerbsanteil von 9\% (resp. 13\%) vorhergesagt, während für Mütter mit egalitären Einstellungen ein Erwerbsanteil von 26\% vorhergesagt wird. Insgesamt lässt sich für beide Items festhalten, dass sich deutliche Zusammenhänge zwischen den Einstellungen und der Arbeitsteilung zeigen.

\section{Abbildung 8.6: Bivariate Zusammenhänge der Gender-Items mit der Aufteilung der Er- werbsarbeit}

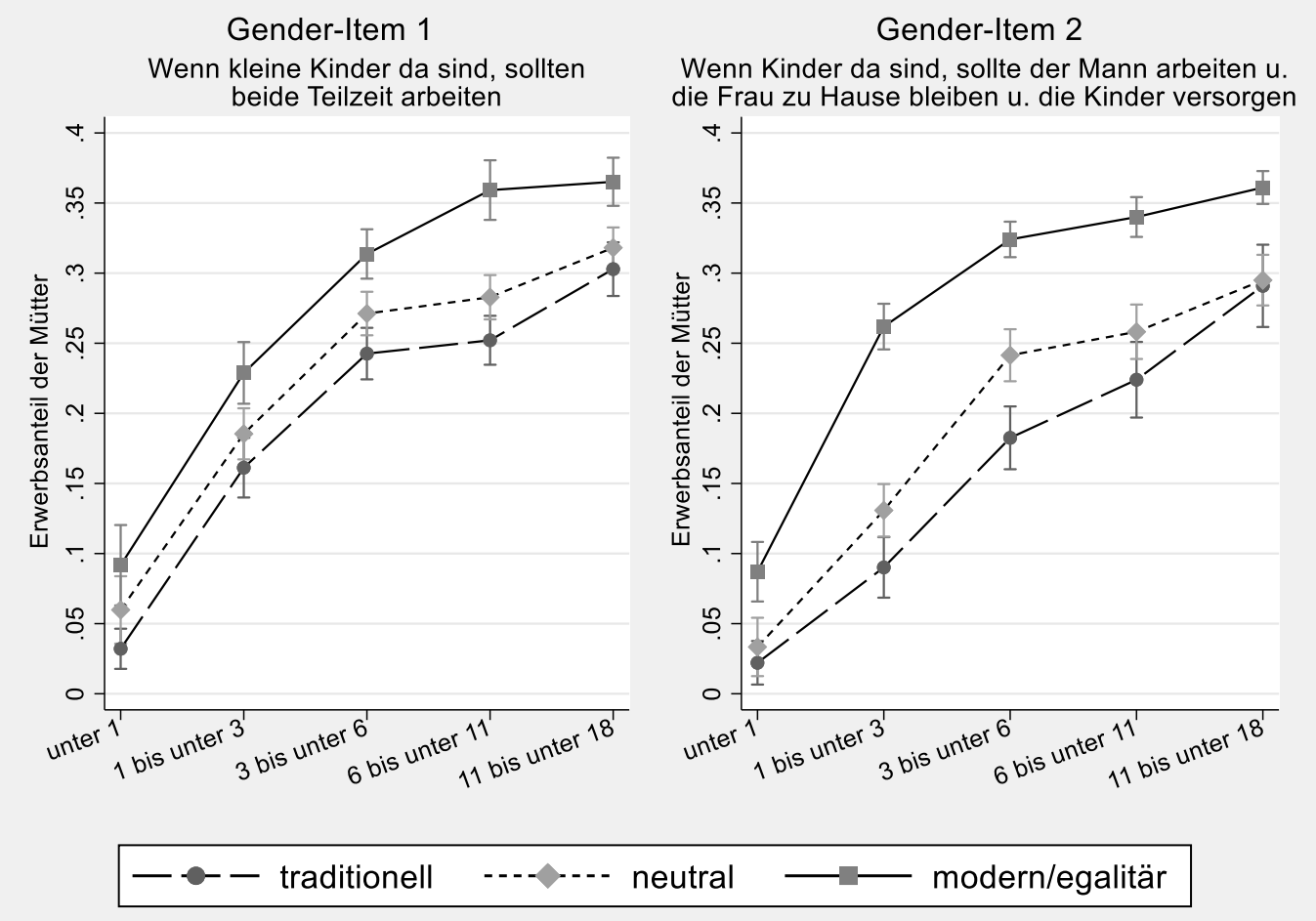

Anmerkung: Vorhergesagter Erwerbsanteil der Mütter (am Erwerbsvolumen des Paares) auf Basis von bivariaten Regressionsmodellen mit 95\%-Konfidenzintervallen. Quelle: DJI-Studie AID:A 2013/14, gewichtet, $n($ Gender-Item 1) = 9.971, n (Gender-Item 2) = 9.987; eigene Berechnungen.

Neben diesen einzelnen Items wurden latente Klassen zu Geschlechterrolleneinstellungen aufgedeckt (siehe Kapitel 7.5). Die Zusammenhänge der latenten Klassen mit der 
Aufteilung der Erwerbsarbeit sind in Abbildung 8.7 abgebildet: Bei der egalitären Klasse ist der Erwerbsanteil der Mütter in allen Altersstufen des Kindes am höchsten und die Erwerbsarbeit damit egalitärer aufgeteilt als in den anderen beiden Klassen. Am traditionellsten ist die Aufteilung der Erwerbsarbeit in der Klasse des „intensive mothering/parenting“, während die Aufteilung der Erwerbsarbeit in der Klasse des „egalitären Essentialismus" zwischen diesen beiden Extremen liegt. In der jüngsten Altersstufe des Kindes sind die Unterschiede zwischen den Klassen am geringsten und sie vergrößern sich mit zunehmendem Alter des Kindes: Bei einem Kind im Alter von „unter 1 Jahr“ gibt es kaum Unterschiede, aber im Alter von „1 bis unter 3 Jahren“ ist der Erwerbsanteil der Mütter in der Klasse des „egalitären Essentialismus" schon deutlich egalitärer als in der Klasse des „intensive mothering/parenting“. Es gibt keine Veränderung der Rangfolge der Klassen in Abhängigkeit vom Alter des Kindes: So ist auch bei einem Kind im Grundschulalter die Aufteilung der Erwerbsarbeit in der egalitären Klasse am egalitärsten und in der Klasse des „intensive mothering/parenting“ am traditionellsten.

Abbildung 8.7: Bivariate Zusammenhänge der Gender-Klassen mit der Aufteilung der Erwerbsarbeit

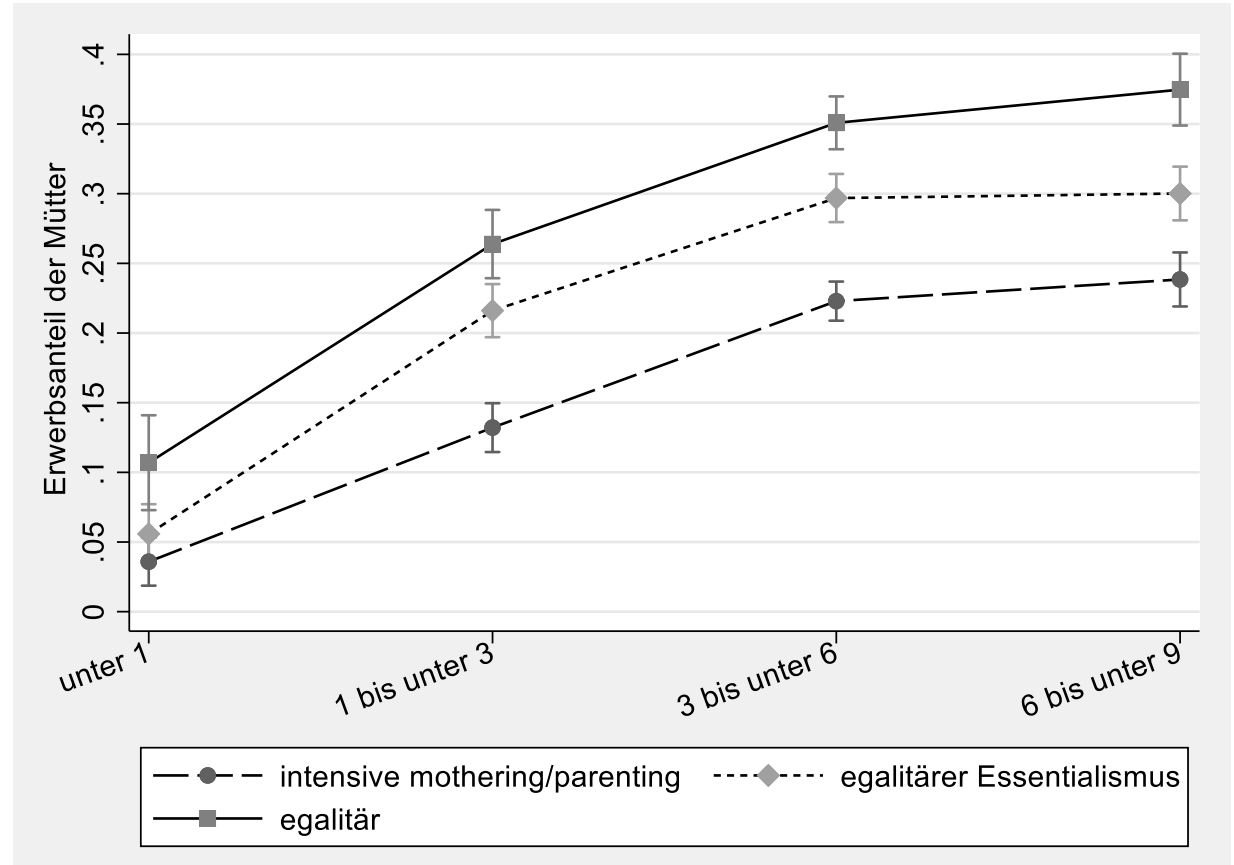

Anmerkung: Vorhergesagter Erwerbsanteil der Mütter (am Erwerbsvolumen des Paares) auf Basis von bivariaten Regressionsmodellen mit 95\%-Konfidenzintervallen. Quelle: DJI-Studie AID:A 2013/14, gewichtet, n (Gender-Klassen) = 6.633; eigene Berechnungen.

Bevor zu den multivariaten Ergebnissen übergegangen wird, wird bei den bivariaten Zusammenhängen als letztes noch auf die Bedeutung der Kinderanzahl und der Unterschiede zwischen Ost- und Westdeutschland eingegangen (siehe Abbildung 8.8). Bei der Anzahl der Kinder zeigt sich, dass der Erwerbsanteil der Mütter bei einem Kind am höchsten ist und mit steigender Kinderanzahl abnimmt. Bei einem Kind im Alter von „unter 1 Jahr" ist der Erwerbsanteil der Mütter jedoch unabhängig von der Kinderanzahl sehr 
niedrig. Aus den Unterschieden zwischen Ost- und Westdeutschland wird ersichtlich, dass der Erwerbsanteil der Mütter in Ostdeutschland höher und die Aufteilung der Erwerbsarbeit damit egalitärer ist. Bei einem Kind im Alter von „unter $1 \mathrm{Jahr"} \mathrm{fallen} \mathrm{die} \mathrm{Ost-}$ West-Unterschiede noch gering aus, sind bei einem Kind im Alter von „1 bis unter 3 Jahren" schon etwas größer und verschärfen sich mit steigendem Kindesalter weiter: So beträgt der Erwerbsanteil von Müttern mit einem Kind im Alter von „3 bis unter 6 Jahren“ in Westdeutschland lediglich $25 \%$ und in Ostdeutschland bereits $39 \%$. Somit liegen ab dem Kindergartenalter gravierende Unterschiede zwischen ost- und westdeutschen Müttern vor.

Abbildung 8.8: Bivariate Zusammenhänge der Anzahl der Kinder und Ost-West-Unterschiede mit der Aufteilung der Erwerbsarbeit
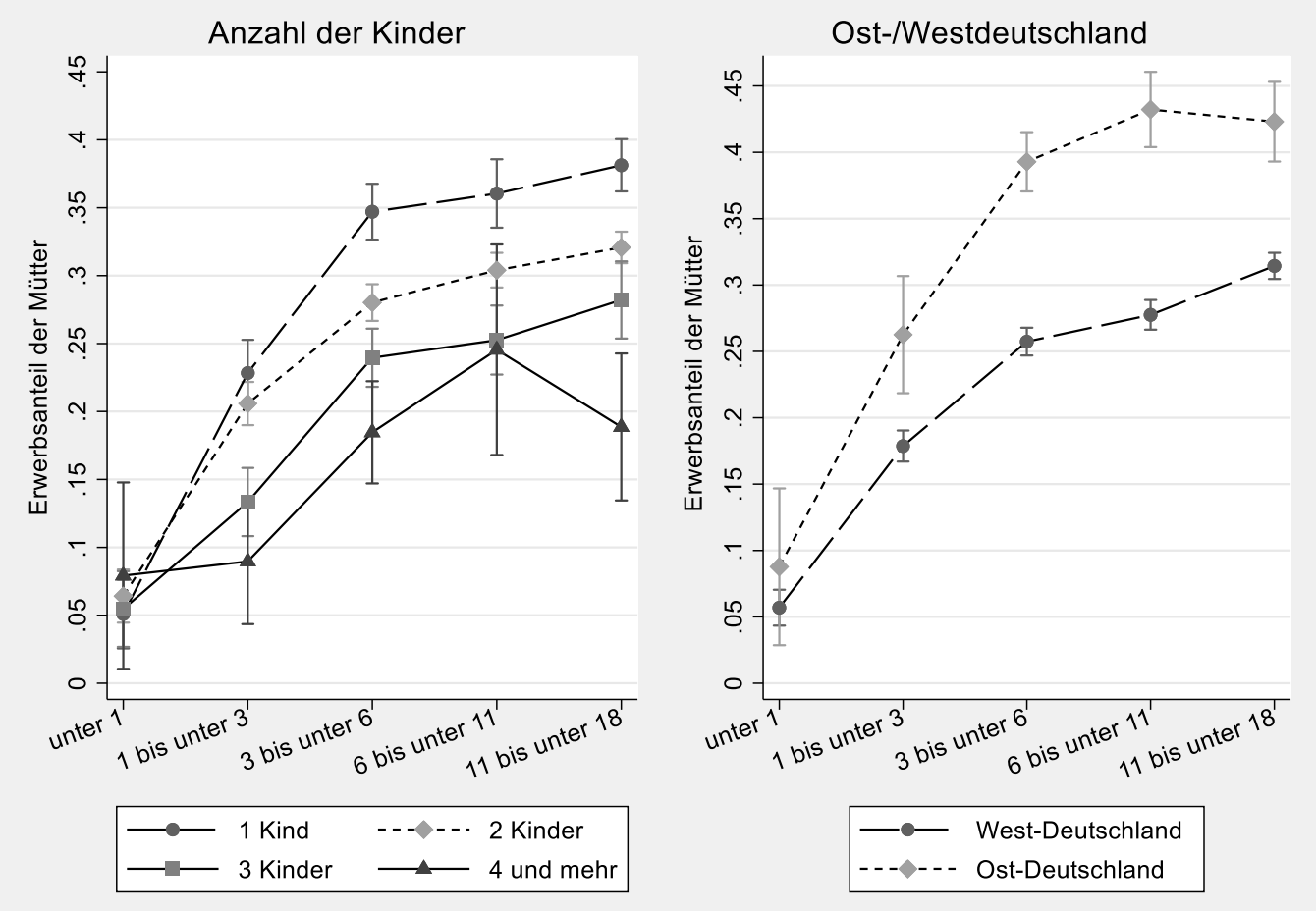

Anmerkung: Vorhergesagter Erwerbsanteil der Mütter (am Erwerbsvolumen des Paares) auf Basis von bivariaten Regressionsmodellen mit 95\%-Konfidenzintervallen. Quelle: DJI-Studie AID:A 2013/14, gewichtet, $n$ (Anzahl der Kinder) = 10.043, n (Ost-/Westdeutschland) = 10.043; eigene Berechnungen.

Aus diesen bivariaten Zusammenhängen geht insgesamt zunächst hervor, dass das Alter des Kindes einen sehr großen Einfluss auf die Aufteilung der Erwerbsarbeit hat. Andere unabhängige Variablen haben signifikante Effekte, die z.T. auch relativ groß ausfallen, aber sie spielen dennoch eine geringere Rolle als das Alter des Kindes. Bzgl. der Frage danach, inwiefern sich die Effekte der unabhängigen Variablen in Abhängigkeit vom Alter des Kindes verändern, lässt sich folgendes festhalten: Am geringsten fallen die Effekte bei einem unter 1-jährigen Kind aus, denn hier macht es nur einen kleinen Unterschied, ob Mütter etwa niedrigere oder höhere Ressourcen haben oder ob sie etwa 
traditionelle oder egalitäre Einstellungen vertreten. In den nachfolgenden Altersstufen werden die Zusammenhänge zwischen den unabhängigen Variablen und der Aufteilung der Erwerbsarbeit stärker. Wie robust diese Effekte unter der Kontrolle weiterer Variablen sind, wird im nachfolgenden Kapitel eruiert.

\subsection{Multivariate Analysen}

Da das Alter des Kindes in der vorliegenden Arbeit von zentraler Bedeutung ist, wird zunächst der Zusammenhang zwischen dem Kindesalter und der Aufteilung der Erwerbsarbeit isoliert, d.h. ohne weitere (Kontroll-)Variablen, betrachtet. In einem nächsten Schritt werden Modelle gezeigt, die neben dem Kindesalter auch relative Ressourcen, Geschlechterrolleneinstellungen und weitere Variablen berücksichtigen. Danach werden getrennte Modelle für die jeweiligen Altersstufen des Kindes berechnet, um feststellen zu können, inwiefern sich die Bedeutung der Einflussfaktoren in den verschiedenen Altersstufen unterscheidet (der stufenweise Aufbau dieser altersspezifischen Modelle wird im Anhang gezeigt).

Wie soeben angedeutet, wird zunächst auf die Bedeutung des Kindesalters für die Aufteilung der Erwerbsarbeit eingegangen. Abbildung 8.9 zeigt, welchen Einfluss das Alter des Kindes auf den Erwerbsanteil der Mütter hat. Bei einem Kindesalter von „unter 1 Jahr" beträgt der vorhergesagte Erwerbsanteil am gesamten Erwerbsvolumen des Paares $6 \%$. Im Vergleich dazu erhöht sich der vorhergesagte Erwerbsanteil der Mütter um $14 \%$, wenn das Kind „1 bis unter 3 Jahre“ alt ist, auf insgesamt 20\%; wenn das Kind „3 bis unter 6 Jahre" ist, beträgt der vorhergesagte Erwerbsanteil der Mutter 28\%; bei einem Alter von „6 bis unter 11 Jahre“ beträgt er $29 \%$ und bei „11 bis unter 18 Jahre“ schließlich $33 \%$. All diese Unterschiede sind groß und (höchst) signifikant, d.h. insgesamt spielt das Alter des Kindes eine sehr große Rolle für den Erwerbsanteil der Mütter. ${ }^{26}$

\footnotetext{
${ }^{26}$ Die in Abbildung 8.9 vorhergesagten Werte basieren auf der bivariaten OLS-Regression in Tabelle A. 2 im Anhang. Dabei wurden die Referenzkategorien variiert, da nicht nur die Frage danach interessiert, wie sich die jeweiligen Altersstufen von einer bestimmten Referenzkategorie unterscheiden, sondern auch von den restlichen Altersstufen. Wie aus den weiteren Modellen in Tabelle A. 2 hervorgeht, bleiben alle Unterschiede in den Altersstufen signifikant, egal welche Altersstufe als Referenzkategorie verwendet wird, d.h. der Erwerbsanteil der Mütter unterscheidet sich grundlegend zwischen sämtlichen Altersstufen. In den nachfolgenden Modellen wird zur besseren Übersichtlichkeit nur noch eine einzelne Altersstufe als Referenzkategorie ausgegeben. Es wurden jedoch auch weitere Modelle mit den alternativen Referenzkategorien gerechnet, wobei auf diese Ergebnisse nur dann verwiesen wird, wenn sich an der Signifikanz etwas verändert haben sollte.
} 
Abbildung 8.9: Vorhergesagter Erwerbsanteil der Mutter in Abhängigkeit vom Alter des Kindes

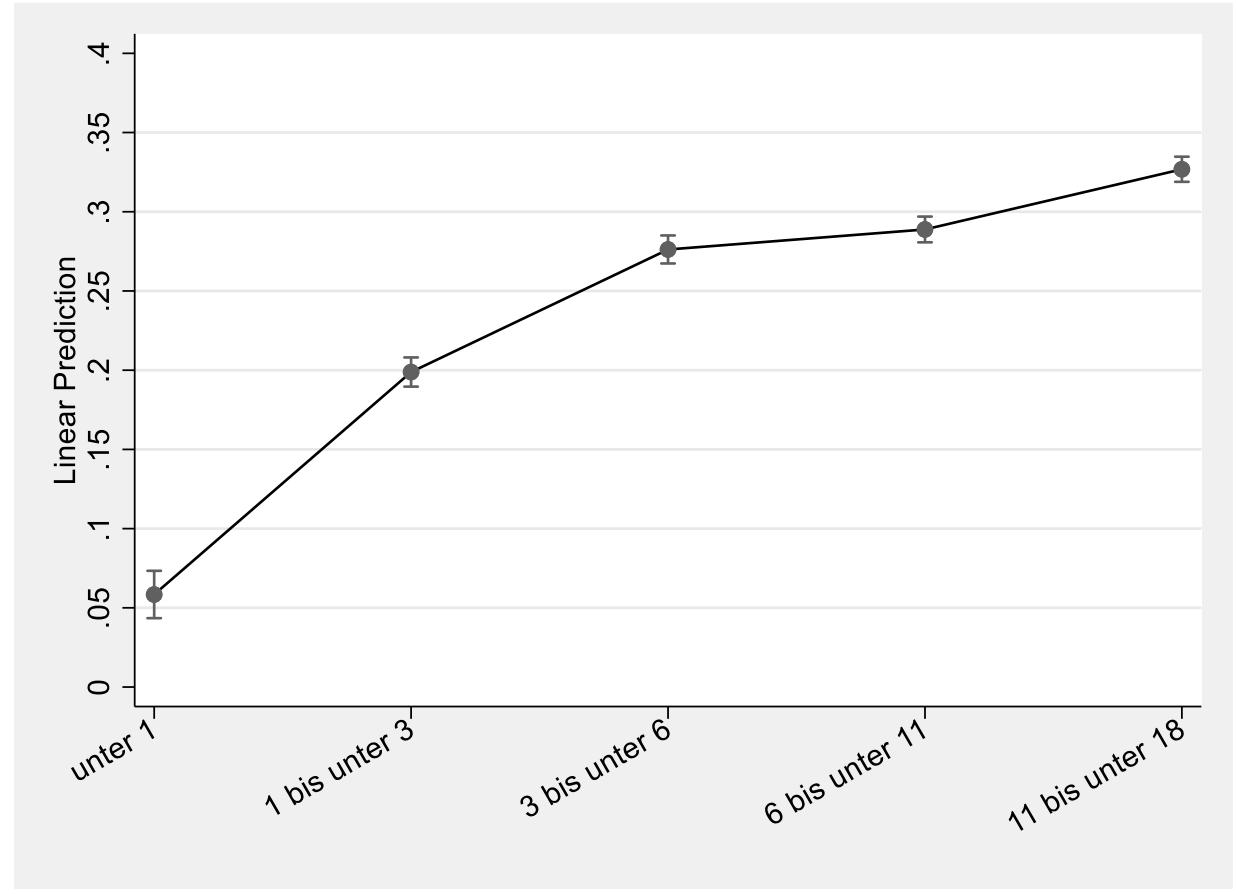

Anmerkung: Es sind die vorhergesagten Werte (Predictive Margins) mit den 95\%-Konfidenzintervallen abgetragen. Die Werte basieren auf der bivariaten OLS-Regression in Tabelle A.2 im Anhang. Quelle: DJI-Studie AID:A 2013/14, Sample: $n=10.410$, eigene Berechnungen. 
Tabelle 8.1: $\quad$ Erwerbsanteil der Mutter, stufenweiser Aufbau des Modells, OLS-Regression

\begin{tabular}{|c|c|c|c|c|c|c|c|c|c|c|}
\hline & (1) & & (2) & & (3) & & (4) & & (5) & \\
\hline VARIABLES & coef & se & coef & se & coef & se & coef & se & coef & se \\
\hline \multicolumn{11}{|l|}{$\begin{array}{l}\text { Alter des Kindes (Ref. } \\
<1 \text { ) }\end{array}$} \\
\hline 1 bis unter 3 & $0.14 * * *$ & $(0.01)$ & & & & & $0.14 * * *$ & $(0.01)$ & $0.14 * * *$ & $(0.01)$ \\
\hline 3 bis unter 6 & $0.22 * * *$ & $(0.01)$ & & & & & $0.22 * * *$ & $(0.01)$ & $0.22 * * *$ & $(0.01)$ \\
\hline 6 bis unter 11 & $0.23 * * *$ & $(0.01)$ & & & & & $0.23 * * *$ & $(0.01)$ & $0.23 * * *$ & $(0.01)$ \\
\hline 11 bis unter 18 & $0.27 * * *$ & $(0.01)$ & & & & & $0.27 * * *$ & $(0.01)$ & $0.26^{* * *}$ & $(0.01)$ \\
\hline \multicolumn{11}{|l|}{$\begin{array}{l}\text { relativer Berufsstatus } \\
\text { (Ref. Frau = Mann) }\end{array}$} \\
\hline Frau $<$ Mann & & & $-0.04 * * *$ & $(0.01)$ & & & $-0.04 * * *$ & $(0.01)$ & $-0.03 * * *$ & $(0.01)$ \\
\hline Frau $>$ Mann & & & 0.01 & $(0.01)$ & & & $0.01 *$ & $(0.01)$ & 0.01 & $(0.01)$ \\
\hline Missings & & & $-0.05 * * *$ & $(0.01)$ & & & $-0.04 * * *$ & $(0.01)$ & 0.01 & $(0.01)$ \\
\hline \multicolumn{11}{|l|}{$\begin{array}{l}\text { Geschlechterrollenein- } \\
\text { stellungen (Skala von } \\
\text {,1: traditionell“ bis } \\
\text { „6: egalitär") }\end{array}$} \\
\hline Item 1 (beide TZ) & & & & & $0.01 * * *$ & $(0.00)$ & $0.01 * * *$ & $(0.00)$ & $0.01 * * *$ & $(0.00)$ \\
\hline $\begin{array}{l}\text { Item } 2 \text { (gegen männl. } \\
\text { Alleinverdiener-M.) }\end{array}$ & & & & & $0.03 * * *$ & $(0.00)$ & $0.03 * * *$ & $(0.00)$ & $0.02 * * *$ & $(0.00)$ \\
\hline \multicolumn{11}{|l|}{$\begin{array}{l}\text { Anzahl der Kinder (Ref. } \\
\text { 2) }\end{array}$} \\
\hline $\begin{array}{l}1 \text { Kind } \\
3 \text { Kinder }\end{array}$ & & & & & & & & & $\begin{array}{l}0.04 * * * \\
-0.05 * * *\end{array}$ & $\begin{array}{l}(0.00) \\
(0.01)\end{array}$ \\
\hline
\end{tabular}




\begin{tabular}{|c|c|c|c|c|c|c|c|c|c|c|}
\hline & (1) & & (2) & & (3) & & (4) & & (5) & \\
\hline VARIABLES & coef & se & coef & se & coef & se & coef & se & coef & se \\
\hline 4 und mehr & & & & & & & & & $-0.08 * * *$ & $\overline{(0.01)}$ \\
\hline $\begin{array}{l}\text { Bundesland (Ref. West } \\
\text { dtl.) }\end{array}$ & & & & & & & & & & \\
\hline Ost-Dtl. & & & & & & & & & $0.10 * * *$ & $(0.01)$ \\
\hline $\begin{array}{l}\text { ISEI der Mutter (Ref. } \\
\text { mittel (45-62)) }\end{array}$ & & & & & & & & & & \\
\hline niedrig $(<45)$ & & & & & & & & & 0.00 & $(0.01)$ \\
\hline hoch (ab 63) & & & & & & & & & $0.04 * * *$ & $(0.01)$ \\
\hline Missings & & & & & & & & & $-0.05 * * *$ & $(0.01)$ \\
\hline Constant & $0.06 * * *$ & $(0.01)$ & $0.28 * * *$ & $(0.00)$ & $0.08 * * *$ & $(0.01)$ & $-0.09 * * *$ & $(0.01)$ & $-0.09 * * *$ & $(0.01)$ \\
\hline Observations & 10,295 & & 10,295 & & 10,295 & & 10,295 & & 10,295 & \\
\hline Adj. $R^{2}$ & 0.10 & & 0.01 & & 0.06 & & 0.17 & & 0.22 & \\
\hline
\end{tabular}

Anmerkung: Signifikanzen: ${ }^{* * *} p<0.001,{ }^{* *} p<0.01,{ }^{*} p<0.05$; Quelle: DJI-Studie AID:A 2013/14, Sample: $n=11.330$, eigene Berechnungen. 
Im Folgenden werden weitere Einflussfaktoren stufenweise mitberücksichtigt und zu einem Gesamtmodell aufgebaut. Das erste Modell in Tabelle 8.1 enthält nur das Alter des Kindes. Da die Ergebnisse redundant zur vorherigen Abbildung sind, wird auf diese nicht näher eingegangen. Modell zwei stellt einen Zusammenhang zwischen dem relativen Berufsstatus und dem Erwerbsanteil der Mütter her, wobei ein (annähernd) identischer Berufsstatus die Referenzkategorie darstellt. Entsprechend den Erwartungen wirkt sich ein im Vergleich zum Mann niedrigerer Berufsstatus der Frau negativ und ein höherer Berufsstatus der Frau positiv auf ihren Erwerbsanteil aus: Wenn die Frau einen niedrigeren Status hat, so verringert sich ihr Erwerbsanteil (im Vergleich zur Referenzkategorie) um 4 Prozentpunkte bzw. im Gesamtmodell um 3 Prozentpunkte; und wenn sie einen höheren Status hat, so erhöht sich ihr Anteil (im Vergleich zur Referenzkategorie) um 1 Prozentpunkt, allerdings ist dieser Unterschied nicht signifikant. Die Tatsache, dass sich ein niedrigerer Status der Frau negativ auf ihren Erwerbsanteil auswirkt, steht in Einklang mit den Erwartungen aus Hypothese 1 und 2. Allerdings bleibt anzumerken, dass der Einfluss der relativen Ressourcen sehr gering ausfällt und die Aufteilung der Erwerbsarbeit gemäß diesen empirischen Ergebnissen nicht wesentlich durch die relativen Ressourcen bestimmt wird. Der Teilaspekt von Hypothese 1 und 2, der annimmt, dass sich Personen mit höheren relativen Ressourcen auf die Erwerbsarbeit spezialisieren, kann für Frauen nicht bestätigt werden: Frauen mit einem höheren relativen Berufsstatus haben im Vergleich zur Referenzkategorie keinen signifikant höheren Erwerbsanteil. Insgesamt zeigen die Ergebnisse, dass die Aufteilung der Erwerbsarbeit nicht wesentlich durch die relativen Ressourcen bestimmt wird. Im Gegensatz zu diesen Ergebnissen konnten Hipp und Leuze (2015) sowohl für Frauen mit niedrigerem Status als auch mit höherem Status (im Vergleich zur Referenzkategorie, bei der kein Statusunterschied besteht) signifikante Unterschiede feststellen.

Bei den Geschlechterrolleneinstellungen in Tabelle 8.1 entsprechen die Ergebnisse dagegen den theoretischen Erwartungen: Je egalitärer die Einstellungen sind, umso höher ist der Erwerbsanteil der Mütter. Inhaltlich steckt hinter dem ersten Item die Frage danach, ob man folgendes befürwortet oder ablehnt: „Wenn kleine Kinder da sind, sollte nicht nur die Frau, sondern auch der Mann Teilzeit arbeiten“. Das zweite Gender-Item fragt die Zustimmung oder Ablehnung zu folgendem ab: „Wenn Kinder da sind, soll der Mann arbeiten gehen und die Frau zu Hause bleiben und die Kinder versorgen“. Die Skalen wurden so recodiert, dass höhere Werte eine egalitärere Einstellung repräsentieren. Wenn sich die Einstellungen auf der Skala von „1: traditionell“ bis „6: egalitär“ um einen Skalenpunkt erhöhen, so erhöht sich der Erwerbsanteil der Mütter um 1 Prozentpunkt beim ersten Gender-Item und um 3 Prozentpunkte beim zweiten Gender-Item. Die Gender-Items haben damit einen relativ großen Einfluss auf den Erwerbsanteil der Mütter, da sich die Koeffizienten nur auf die Erhöhung um einen einzigen Skalenpunkt beziehen, aber auf der 6-stufigen Skala eine Erhöhung um mehrere Skalenpunkte möglich ist. Die Effekte erweisen sich als robust und sind auch im Gesamtmodell noch höchst signifikant, sodass Hypothese $3 \mathrm{a}$ ), die eine egalitärere Arbeitsteilung bei egalitäreren 
Einstellungen annimmt, bestätigt werden kann. Bisherige Studien können dies ebenso bestätigen (Fortin 2005; Corrigall und Konrad 2007; Cunningham 2008; Davis und Greenstein 2009; Diener und Berngruber 2018).

Im Gesamtmodell in Tabelle 8.1 (Modell 5) sind als neue Variablen noch die Anzahl der Kinder, das Bundesgebiet und der absolute Berufsstatus der Mutter hinzugekommen. Bei der Anzahl der noch im Haushalt lebenden Kinder wird ersichtlich, dass der Erwerbsanteil der Mütter am höchsten ist, wenn nur ein Kind im Haushalt lebt und dass ihr Erwerbsanteil mit zunehmender Kinderanzahl abnimmt. Dies entspricht den Erwartungen von Hypothese 5 a), die somit bestätigt werden kann und steht auch im Einklang zu Ergebnissen aus bisheriger Forschung (Konietzka und Kreyenfeld 2010; Berghammer 2014; BMFSFJ 2014a; Hipp und Leuze 2015; OECD 2016).

Als nächstes wird danach differenziert, ob die Familien in Ost- oder in Westdeutschland leben: Wie angenommen, ist der Erwerbsanteil der Mütter in Ostdeutschland höher: Laut Modell 5 beträgt der Unterschied im Erwerbsanteil 10 Prozentpunkte. Dies bestätigt Hypothese 6 a), die in Ostdeutschland eine egalitärere Aufteilung der Erwerbsarbeit als in Westdeutschland annimmt und bestätigt frühere Forschungsergebnisse hierzu (PfauEffinger und Smidt 2011; Grunow und Müller 2012; BMFSFJ 2014a; Buschner et al. 2018). Beim absoluten Berufsstatus der Mutter wird gemäß Hypothese 7 a) erwartet, dass ein niedriger Status (im Vergleich zu einem mittleren Status als Referenzkategorie) zu einem reduzierten Erwerbsanteil der Mütter und ein hoher Status zu einem erhöhten Erwerbsanteil führt: Ein negativer Effekt des niedrigen Status' kann nicht bestätigt werden, ein positiver Effekt des hohen Status' dagegen schon.

Im Folgenden werden diese Modelle differenziert nach den Altersstufen des Kindes betrachtet, um feststellen zu können, welche Bedeutung die verschiedenen Einflussfaktoren in den jeweiligen Altersstufen des Kindes haben. Die folgenden Abbildungen basieren auf den Gesamtmodellen der jeweiligen Altersstufen (1c, 2c, 3c usw.) in Tabelle A.3.

Die Zusammenhänge des relativen Berufsstatus (ISEI) und des Erwerbsanteils der Mütter sind in Abbildung 8.10 veranschaulicht. Beim relativen Berufsstatus (ISEI) lässt sich für alle Altersstufen des Kindes festhalten, dass Mütter mit einem niedrigeren Status zwar einen tendenziell geringeren Erwerbsanteil haben, aber diese Effekte in den meisten Altersstufen nicht signifikant sind ${ }^{27}$ Ein negativer Zusammenhang eines niedrigeren

\footnotetext{
27 Die bivariaten Regressionsergebnisse zum relativen Berufsstatus waren über die verschiedenen Altersstufen hinweg noch signifikant (vgl. Modell 1a, 2a, 3a usw. in Tabelle A.3 im Anhang). Es wurde deshalb auch getestet, ob das Verschwinden der Signifikanzen dadurch bedingt ist, dass im Gesamtmodell neben dem relativen Berufsstatus auch der absolute Berufsstatus der Mutter enthalten ist. Es hat sich jedoch gezeigt, dass der relative Berufsstatus auch dann in den meisten Altersstufen des Kindes nicht mehr signifikant ist, wenn im Gesamtmodell der absolute Berufsstatus nicht mehr enthalten ist. Des Weiteren hat ein Test auf Multikollinearität (Varianceof-Inflation) ergeben, dass die gleichzeitige Aufnahme dieser beiden Variablen kein Problem darstellt.
} 
Status' der Mütter auf ihren Erwerbsanteil lässt sich somit im Gesamtmodell nicht mehr bestätigen. Auch zeigt sich kein signifikanter Zusammenhang zwischen einem höheren Status' der Mütter und einem höheren Erwerbsanteil. Dieses Ergebnis mag zunächst verwundern, lässt sich jedoch unter Berücksichtigung der Referenzkategorie erklären: Mütter mit höherem Status („Mutter > Vater“) haben zwar keinen signifikant höheren Erwerbsanteil als Paare mit gleichem Status („Mutter $=$ Vater“); wenn jedoch Mütter mit einem niedrigeren Status“ als Referenzkategorie verwendet werden („Mutter < Vater“), so unterscheiden sich Mütter mit einem höheren Status schon signifikant von diesen (mit Ausnahme in der jüngsten Altersstufe).

Abbildung 8.10: Koeffizienten des relativen Berufsstatus zur Vorhersage des Erwerbsanteil der Mutter, getrennt nach dem Alter des Kindes

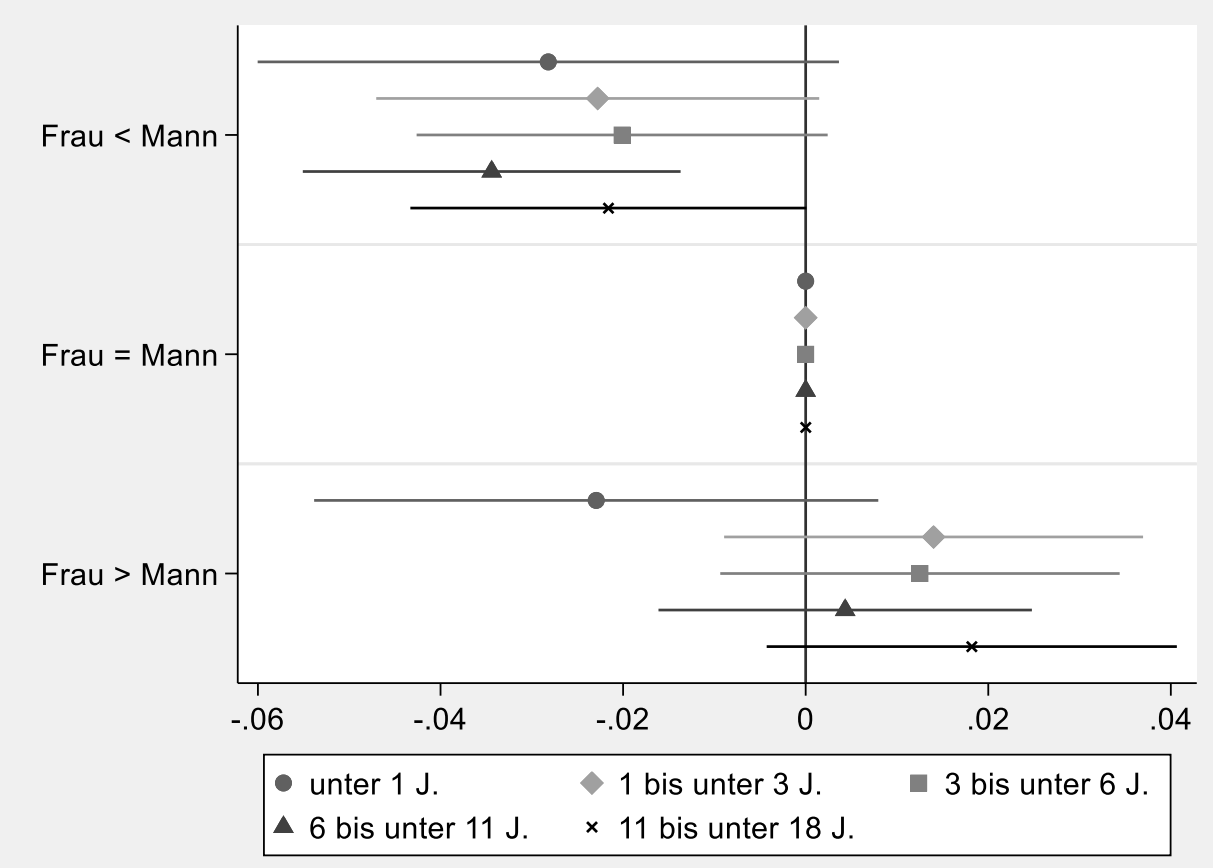

Anmerkung: Abgebildet sind die Koeffizienten mit den 95\%-Konfidenzintervallen von OLS-Regressionen; die Werte basieren auf den Gesamtmodellen (1c, 2c, 3c usw.) in Tabelle A.3, in denen weitere Variablen kontrolliert werden. Quelle: DJI-Studie AID:A 2013/14, Sample: $n$ (Gesamt) = 10.295, eigene Berechnungen.

Es wurde darüber hinaus getestet, ob sich die Effekte des relativen Status' zwischen den verschiedenen Altersstufen signifikant unterscheiden. Gemäß der Hypothese 2 b) wird angenommen, dass die relativen Ressourcen bei einem Kind im Alter von unter 1 Jahr keine oder nur eine geringe Rolle spielen. In dieser Altersgruppe zeigen sich (im Gesamtmodell) keine signifikanten Effekte, was im Einklang mit den Erwartungen steht. Die weiteren, altersspezifischen Hypothesen nehmen an, dass die Bedeutung der Ressourcen bei einem höheren Kindesalter stärker sichtbar ist als bei sehr jungen Kindern (Hypothesen 2 b) bis 2 e)). Dies kann jedoch nicht bestätigt werden, da die Effekte auch in den höheren Altersstufen nicht signifikant sind (mit Ausnahme der Altersstufe „6 bis unter 11 Jahre"). Interaktionsmodelle, die nicht abgebildet sind, belegen, dass es zwischen 
den Altersstufen keine signifikanten Unterschiede gibt. Die altersspezifischen Hypothesen 2 b) bis 2 e) können somit nicht bestätigt werden.

Als nächstes wird auf die Bedeutung von Geschlechterrolleneinstellungen für die Aufteilung der Erwerbsarbeit eingegangen. Beim ersten Gender-Item wird abgefragt, wie egalitär man zu folgender Aussage eingestellt ist: „Wenn kleine Kinder da sind, sollte nicht nur die Frau, sondern auch der Mann Teilzeit arbeiten“. Es zeigt sich, dass sich mit einer egalitäreren Einstellung bei diesem Item der Erwerbsanteil der Mütter in allen Altersstufen des Kindes erhöht (siehe Abbildung 8.11, oben, und im Anhang in Tabelle A.3). Die Effektstärke ist in beinahe allen Altersstufen gleich hoch, weshalb die altersspezifischen Hypothesen 3 b) bis 3 e) für das erste Gender-Item nicht bestätigt werden können. Eine Ausnahme bildet die Altersstufe „6 bis unter 11 Jahre": Hier ist der positive Effekt einer egalitären Einstellung höher als in den anderen Altersstufen. Allerdings sind diese Unterschiede nicht signifikant verschieden. Dies geht aus einem weiteren Regressionsmodell hervor, bei dem sämtliche Interaktionseffekte getestet wurden (nicht abgebildet).

Abbildung 8.11: Koeffizienten der Geschlechterrolleneinstellungen zur Vorhersage des Erwerbsanteils der Mutter, getrennt nach dem Alter des Kindes
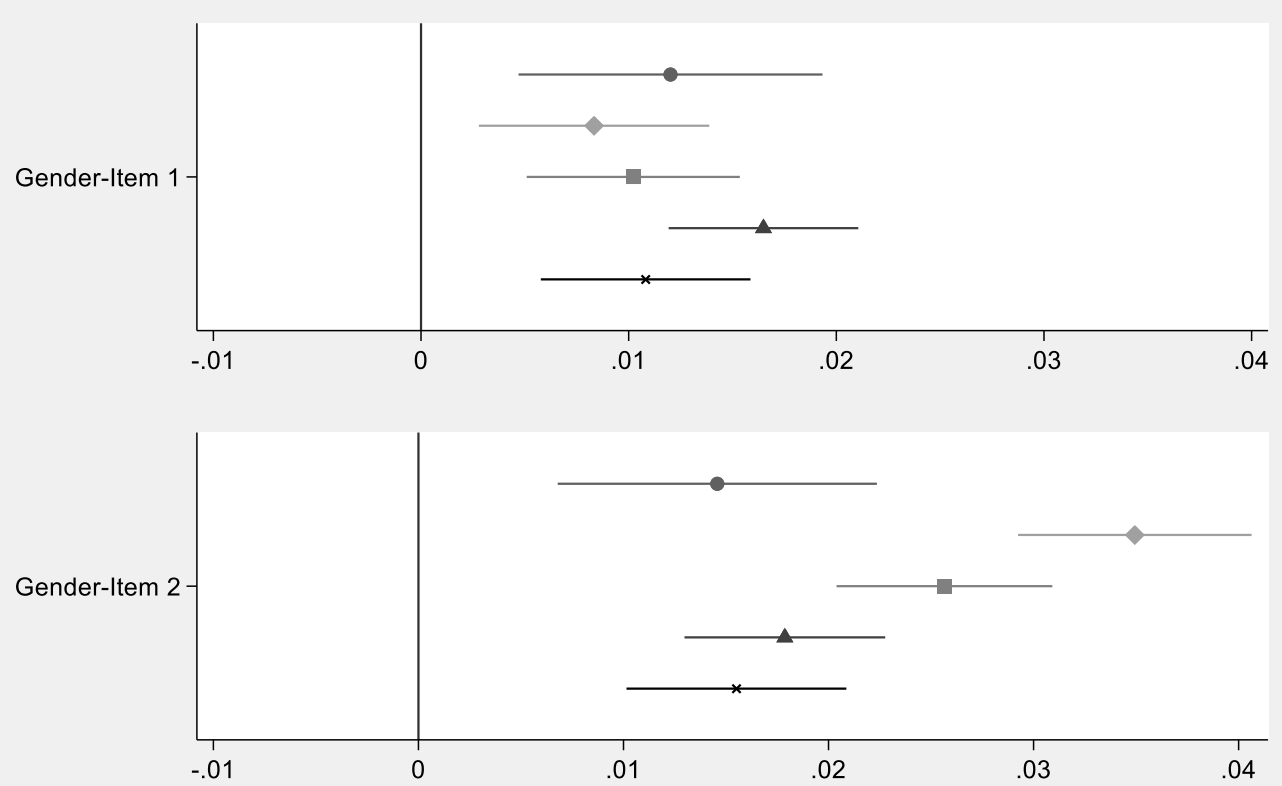

$\begin{array}{ll}\text { - unter } 1 \mathrm{~J} . & 1 \text { bis unter } 3 \mathrm{~J} .\end{array} 3$ bis unter $6 \mathrm{~J}$.

Anmerkung: Abgebildet sind die Koeffizienten mit den 95\%-Konfidenzintervallen von OLS-Regressionen; die Werte basieren auf den Gesamtmodellen (1c, 2c, 3c usw.) in Tabelle A.3, in denen weitere Variablen kontrolliert werden; Gender-Item 1 = Befürwortung, dass beide Elternteile in Teilzeit arbeiten sollen, wenn kleine Kinder da sind; Gender-Item 2 = Ablehnung eines männlichen Alleinverdiener-Modells. Quelle: DJI-Studie AID:A 2013/14, Sample: $n$ (Gesamt) $=10.295$, eigene Berechnungen.

Auch beim zweitem Gender-Item („Wenn Kinder da sind, soll der Mann arbeiten gehen und die Frau zu Hause bleiben und die Kinder versorgen“; recodiert, sodass höhere 
Werte eine egalitärere Einstellung bedeuten) lässt sich prinzipiell festhalten, dass egalitärere Einstellungen mit einem höheren Erwerbsanteil der Mütter einhergehen. Jedoch zeigen sich differenziert nach dem Alter des Kindes unterschiedlich starke Zusammenhänge (siehe Abbildung 8.11, unten, und im Anhang in Tabelle A.3): In der ersten Altersstufe („unter 1 Jahr“) sind die Effekte - wie in Hypothese 3 b) angenommen - noch relativ gering (aber dennoch höchst signifikant); in den beiden darauf folgenden Altersstufen („1 bis unter 3 Jahre“ und „3 bis unter 6 Jahre“) erhöht sich der positive Effekte der egalitäreren Einstellungen. Dies bestätigt Hypothese $3 \mathrm{c}$ ) und $3 \mathrm{~d}$ ). In den beiden höchsten Altersstufen („6 bis unter 11 Jahre“ und „11 bis unter 18 Jahre“) reduziert sich die Effektstärke jedoch wieder auf das niedrigere Niveau bei den unter 1-jährigen. Dies steht in Widerspruch zu Hypothese 3 e), die annimmt, dass sich die Effekte in dieser Altersstufe nicht reduzieren, sondern weiter vergrößern.

Als nächstes wird im Familienkontext die Anzahl der im Haushalt lebenden Kinder berücksichtigt. Hypothese 5 a) geht allgemein davon aus, dass Mütter mit einem Kind einen höheren Erwerbsanteil innehaben als Mütter mit zwei oder mehr Kindern. Die Ergebnisse bestätigen, dass in allen Altersstufen - mit Ausnahme der jüngsten Altersstufe - Mütter mit einem Kind einen höheren Erwerbsanteil haben als Mütter mit zwei Kindern; und Mütter mit drei, vier oder mehr Kindern einen geringeren Erwerbsanteil haben als Mütter mit zwei Kindern (= Referenzkategorie). Die altersspezifische Hypothese $5 \mathrm{~b}$ ) nimmt darüber hinaus an, dass die Unterschiede zwischen Ein- und Mehrkindfamilien größer sind, wenn das jüngste Kind noch klein ist und die Unterschiede geringer werden, wenn das jüngste Kind bereits älter ist. Entgegen den Erwartungen ist die Bedeutung der Kinderanzahl in der untersten Altersstufe („unter 1 Jahr“) jedoch nicht am höchsten, sondern - im Gegenteil: - am unbedeutendsten. D.h. Mütter, deren jüngstes Kind sich im ersten Lebensjahr befindet, haben nur einen sehr geringen Erwerbsanteil (etwa 6\% des gesamten Erwerbsvolumens) und dieser ist unabhängig von der Anzahl der weiteren Kinder. Diese Tatsache kann durch institutionelle Rahmenbedingungen erklärt werden, etwa dadurch, dass der Großteil der Mütter im ersten Lebensjahr des Kindes Elterngeld bezieht oder eine Auslagerung der Kinderbetreuung durch einen unzureichenden Ausbau an Betreuungsstätten erschwert ist, sodass Mütter ihre Erwerbstätigkeit im ersten Lebensjahr unterbrechen (oder zumindest drastisch reduzieren) - egal, ob sie noch weitere Kinder haben oder nicht. In den übrigen Altersstufen des Kindes spielt die Kinderanzahl schon eine Rolle: So reduziert sich der Erwerbsanteil der Mütter mit jedem weiteren Kind um ca. 3-5\%, allerdings unterscheiden sich die Effektstärken zwischen den Altersstufen nicht signifikant voneinander. ${ }^{28}$ Hypothese 5 b) kann deshalb nicht bestätigt werden.

\footnotetext{
${ }^{28}$ Dies wurde anhand von Interaktionseffekten getestet (nicht abgebildet). Dabei wurden sowohl beim Alter des Kindes als auch bei der Kinderanzahl jeweils unterschiedliche Referenzkategorien getestet.
} 
Abbildung 8.12: Vorhergesagter Erwerbsarbeitsanteil der Mutter, in Abhängigkeit von der Kinderanzahl, getrennt nach dem Alter des Kindes

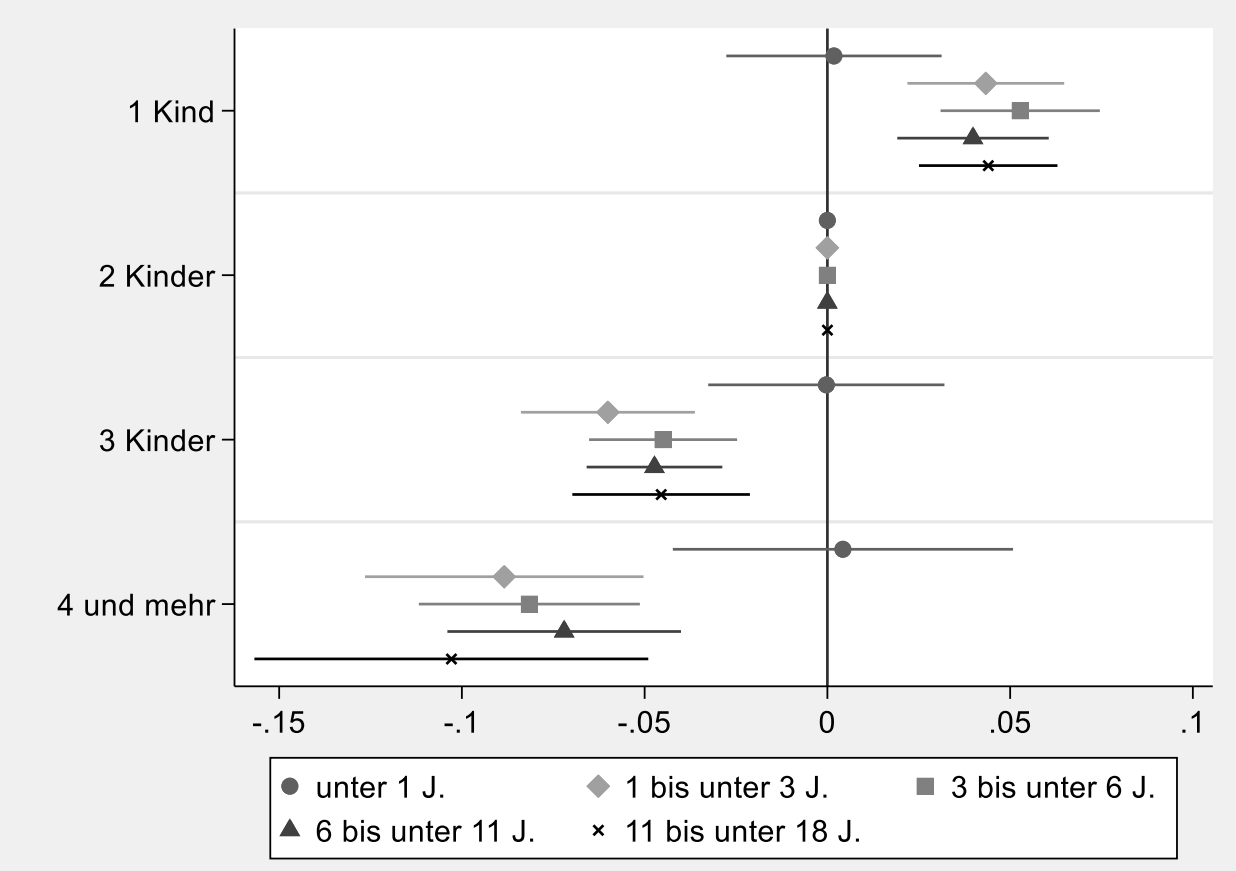

Anmerkung: Abgebildet sind die Koeffizienten mit den 95\%-Konfidenzintervallen von OLS-Regressionen; die Werte basieren auf den Gesamtmodellen (1c, 2c, 3c usw.) in Tabelle A.3, in denen weitere Variablen kontrolliert werden; Quelle: DJI-Studie AID:A 2013/14, Sample: $n$ (Gesamt) = 10.295, eigene Berechnungen.

Als nächstes werden Unterschiede zwischen Ost- und Westdeutschland betrachtet. Hypothese 6 a) nimmt allgemein an, dass die Erwerbsarbeit in Ostdeutschland egalitärer aufgeteilt ist als in Westdeutschland. Diese Annahme trifft nicht für die jüngste Altersstufe zu, da sich bei unter 1-jährigen Kindern ost- und westdeutsche Mütter nicht signifikant voneinander unterscheiden. Bei allen weiteren Altersstufen gibt es dagegen sehr große, höchst signifikante Unterschiede. Die altersspezifische Hypothese 6 b) geht davon aus, dass die Unterschiede zwischen Ost- und Westdeutschland bei kleinen Kindern besonders groß sind und mit zunehmendem Kindesalter abnehmen. In Abbildung 8.13 sind die Koeffizienten des Bundesgebietes zur Vorhersage der Erwerbsanteile der Mütter abgetragen: Bei einem Kind im Alter von unter 1 Jahr unterscheiden sich Ost- und Westdeutschland nicht signifikant voneinander. Sehr starke Unterschiede gibt es dagegen in den höheren Altersstufen. Ostdeutsche Mütter haben einen um 10 Prozentpunkte höheren Erwerbsanteil als westdeutsche Mütter; in der Altersstufe „6 bis unter 11 Jahre“ unterscheiden sie sich sogar um 13\%. Aufgrund der Tatsache, dass die Unterschiede bei Kindern unter einem Jahr nicht signifikant sind und die Effekte in den höheren Altersstufen etwa gleich stark sind (mit Ausnahme der besonders starken Unterschiede bei den 6- bis unter 11-Jährigen), kann Hypothese 6 b) daher nicht bestätigt werden. 
Abbildung 8.13: Koeffizienten des Bundesgebiets zur Vorhersage des Erwerbsarbeitsanteil der Mutter, getrennt nach dem Alter des Kindes

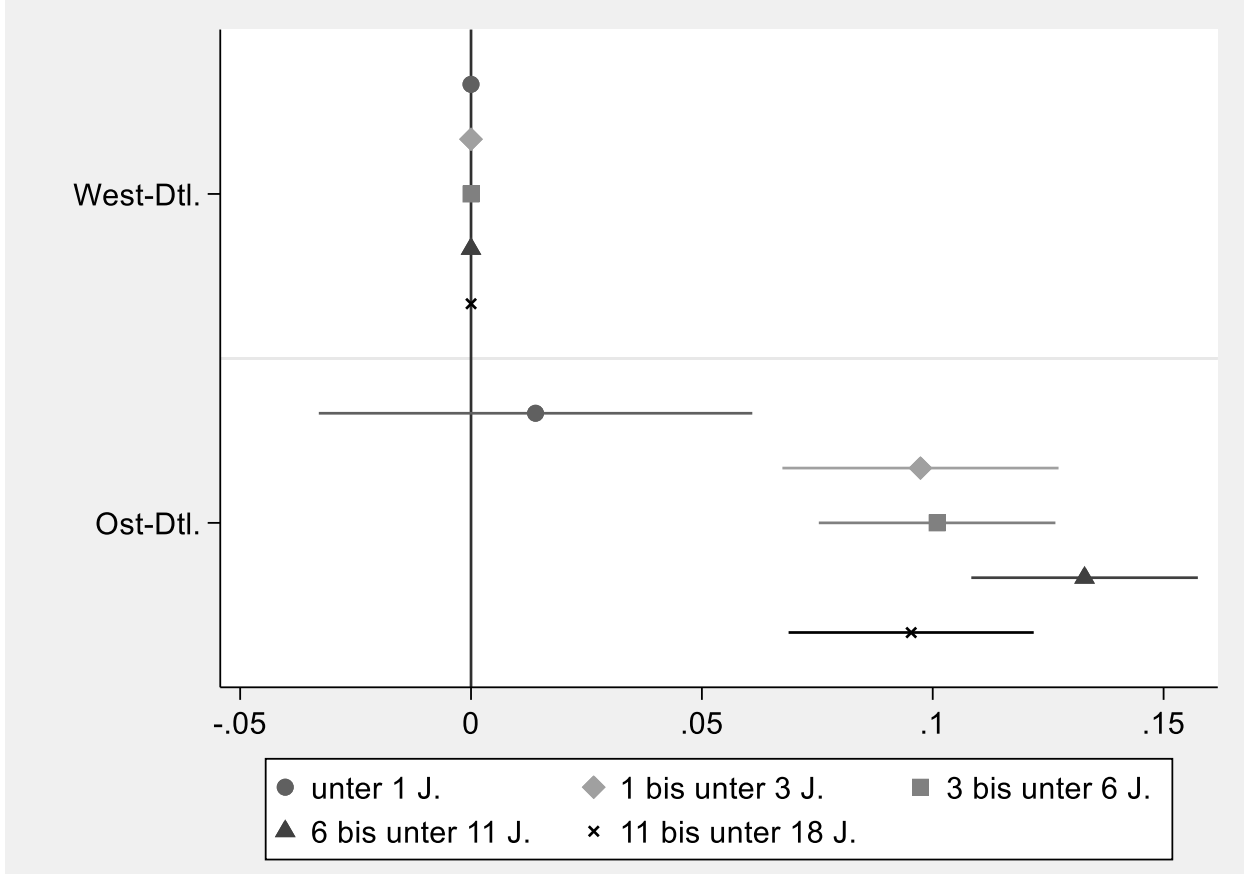

Anmerkung: Abgebildet sind die Koeffizienten mit den 95\%-Konfidenzintervallen von OLS-Regressionen; die Werte basieren auf den Gesamtmodellen (1c, 2c, 3c usw.) in Tabelle A.3, in denen weitere Variablen kontrolliert werden; Quelle: DJI-Studie AID:A 2013/14, Sample: $n$ (Gesamt) = 10.295, eigene Berechnungen.

Als letzte Variable wird auf den absoluten Berufsstatus der Mütter (ISEI) eingegangen. Nach Hypothese 7 a) kann allgemein angenommen werden, dass bei Müttern mit einem hohen, absoluten Berufsstatus der Erwerbsanteil höher ist als bei Müttern mit einem niedrigen Status. Dies kann - mit Ausnahme der untersten Altersstufe - bestätigt werden (siehe Abbildung 8.14). Bei Müttern mit einem niedrigen Berufsstatus ist der Erwerbsanteil nicht signifikant geringer im Vergleich zur Referenzkategorie der Mütter mit einem mittleren Status. Wenn als Referenzkategorie dagegen Mütter mit einem hohen Status verwendet worden wären, dann würden sich Mütter mit einem niedrigen Status signifikant von innen unterscheiden. Die altersspezifische Hypothese $7 \mathrm{~b}$ ) nimmt an, dass die Unterschiede zwischen Müttern mit hohem und Müttern mit niedrigem Status mit zunehmendem Kindesalter größer werden. Dies kann jedoch nicht bestätigt werden, da die Effektstärken nur in Referenz zur jüngsten Altersstufe signifikant größer sind, sich aber ansonsten nicht signifikant voneinander unterscheiden. 
Abbildung 8.14: Koeffizienten des absoluten Berufsstatus der Mutter zur Vorhersage des Erwerbsarbeitsanteil der Mutter, getrennt nach dem Alter des Kindes

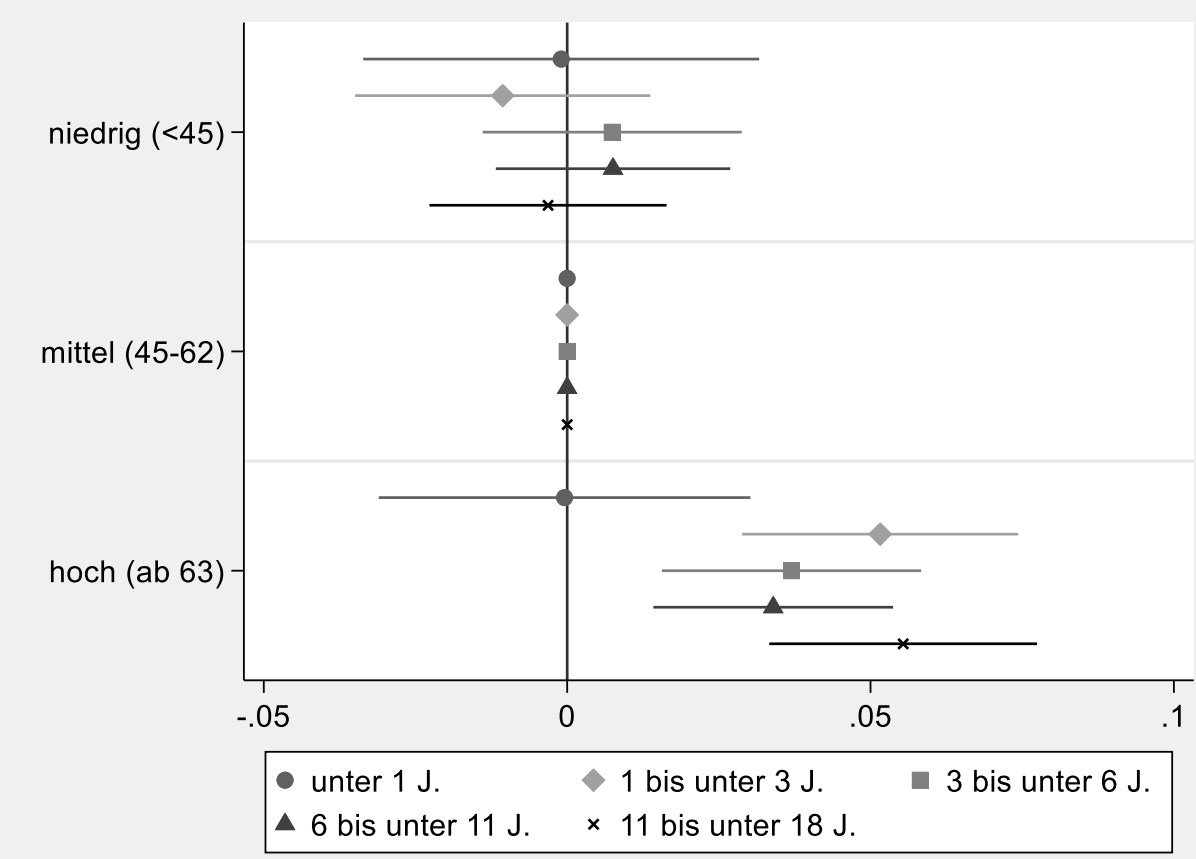

Anmerkung: Abgebildet sind die Koeffizienten mit den 95\%-Konfidenzintervallen von OLS-Regressionen; die Werte basieren auf den Gesamtmodellen (1c, 2c, 3c usw.) in Tabelle A.3, in denen weitere Variablen kontrolliert werden; Quelle: DJI-Studie AID:A 2013/14, Sample: $n$ (Gesamt) = 10.295, eigene Berechnungen.

Insgesamt fällt auf, dass sich die Effekte in allen Altersstufen sehr ähnlich sind und sich nur in der untersten Altersstufe eine andere Situation darstellt. Dies mag darin begründet liegen, dass der Großteil der Mütter von unter 1-jährigen Kindern nicht erwerbstätig ist und die Varianz der abhängigen Variable dadurch geringer ausfällt als in den höheren Altersstufen. Ein relativ großer Zusammenhang bestand zwischen den Geschlechterrolleneinstellungen und den Erwerbsanteilen der Mütter. Im Folgenden werden noch vertiefende Analysen zu den Geschlechterrolleneinstellungen behandelt, umso präzisere Aussagen darüber machen zu können, welche Einstellungsprofile mit welcher Arbeitsteilung assoziiert sind.

\section{Vertiefende Analysen zu den Geschlechterrolleneinstellungen}

Bislang wurden nur zwei verschiedene Items zu den Geschlechterrolleneinstellungen als unabhängige Variablen verwendet. Darüber hinaus existieren im DJI-Survey „Aufwachsen in Deutschland: Alltagswelten“ (2013/2014) noch zwei weitere Items. Diese wurden bis dato bei der Aufteilung der Erwerbsarbeit nicht berücksichtigt, weil sie nur bis zu einem Kindesalter von unter 9 Jahren abgefragt wurden und damit nicht für alle relevanten Altersstufen verfügbar sind. In den folgenden Analysen werden diese beiden zusätzlichen Items miteinbezogen, um so einen vertieften Einblick in Geschlechterrolleneinstellungen zu erhalten. Dementsprechend beschränken sich die nachfolgenden Analysen 
auf ein Kindesalter von unter 9 Jahren. Insgesamt werden nun die folgenden Items zu Geschlechterrolleneinstellungen betrachtet:

- Gender-Item 1 (in den bisherigen Analysen bereits enthalten): „Wenn kleine Kinder da sind, sollte nicht nur die Frau, sondern auch der Mann Teilzeit arbeiten“;

- Gender-Item 2 (in den bisherigen Analysen bereits enthalten): „Wenn Kinder da sind, soll der Mann arbeiten gehen und die Frau zu Hause bleiben und die Kinder versorgen";

- Gender-Item 3 (neu): „Auch wenn eine Frau arbeitet, sollte der Mann der ,Hauptverdiener' sein, und die Frau sollte die Verantwortung für den Haushalt tragen";

- Gender-Item 4 (neu): „Ein Vater sollte genauso stark in die Erziehung und Pflege eines Kindes einbezogen sein, wie eine Mutter". ${ }^{29}$

Zunächst wird der Frage nachgegangen, wie sehr die verschiedenen Items miteinander korrelieren. Bei sehr hohen Korrelationen erscheint es gerechtfertigt, die Items etwa in Form eines Index gemeinsam zu betrachten; niedrige Korrelationen sprechen dagegen eher dafür, die einzelnen Items differenziert zu verwenden. In Tabelle 8.2 sind die Korrelationen der Items abgetragen. Zwar zeigt sich, dass alle Korrelationen höchst signifikant sind, aber die meisten Korrelationen bewegen sich zwischen 0,18 und 0,25 und sind damit eher gering. Eine Ausnahme liegt bei den Items 2 und 3 vor, deren Korrelation 0,60 beträgt und als hoch einzustufen ist. ${ }^{30}$

Tabelle 8.2: $\quad$ Korrelationen der Gender-Items

\begin{tabular}{lllll}
\hline & Gender-Item 1 & Gender-Item 2 & Gender-Item 3 & Gender-Item 4 \\
\hline Gender-Item 1 & 1.00 & & & \\
Gender-Item 2 & $0.18^{* * *}$ & 1.00 & & \\
Gender-Item 3 & $0.18^{* * *}$ & $0.60^{* * *}$ & 1.00 & \\
Gender-Item 4 & $0.25^{* * *}$ & $0.22^{* * *}$ & $0.24 * * *$ & 1.00 \\
\hline
\end{tabular}

Quelle: DJI-Studie AID:A 2013/14, Sample: $n=6.224$, eigene Berechnung.

Des Weiteren wurde mittels einer explorativen Faktorenanalyse (nicht abgebildet) getestet, ob die Items auf einen Faktor (oder auf mehrere Faktoren) laden und eine Zusam-

${ }^{29}$ Die ursprünglichen Antwortskalen im Fragebogen gehen von „1: stimme voll und ganz zu“ bis „6: stimme überhaupt nicht zu“. Da nicht alle Items inhaltlich in dieselbe Richtung zeigen, wurden bei den Gender-Items 1 und 4 die Antwortskalen recodiert, sodass auch hier höhere Werte mit einer egalitäreren Einstellung einhergehen. Die neuen Antwortskalen gehen so bei allen vier Gender-Items von „1: traditionell“ bis „6: egalitär“.

${ }^{30}$ Es ist schwierig, allgemein anzugeben, ab wann eine Korrelation als hoch zu bewerten ist, da dies auch immer von der Forschungsfrage und dem Forschungsgegenstand abhängig ist. Cohen (1988) hat zur Einordnung von Korrelationskoeffizienten folgende Konvention vorgeschlagen: geringe Korrelationen liegen $a b r=0.10$ vor; mittlere $a b$ einem Wert von $r=0.30$ und hohe Korrelationen sind $a b r=0.50$ der Fall. 
menfassung aller (oder einiger) Items daher angebracht ist oder nicht. Aus den Ergebnissen geht hervor, dass nur ein Faktor einen Eigenwert von $>1$ hat und dass auf diesen nur Gender-Item 2 und 3 laden. Dies ist naheliegend, da bereits aus der vorherigen Korrelationstabelle hervorgeht, dass zwischen Gender-Item 2 und 3 eine relativ hohe Korrelation vorliegt. Diese beiden Items haben inhaltlich eine ähnliche Ausrichtung, da sie beide danach fragen, ob die Frau primär für die Hausarbeit und Kinderbetreuung zuständig sein soll und der Mann der Alleinverdiener oder zumindest der Hauptverdiener sein soll. Die weiteren Items (Gender-Item 1 und 4) laden weder auf diesen Faktor noch auf einen weiteren Faktor. Würde man alle Items vereinen, so wäre Cronbach's Alpha = 0,595. Dies ist als zu niedrig einzustufen, da das Cronbach's Alpha idealerweise mindestens 0,70 betragen sollte. ${ }^{31}$ Somit sprechen auch die Ergebnisse der explorativen Faktorenanalyse sowie das niedrige Cronbach's Alpha gegen einen gemeinsamen Index. Die vier Items werden daher in einem ersten Schritt als einzelne Items in die Analysen mit aufgenommen.

Dies ist in Tabelle 8.3 der Fall: Hier wurden die vier Items zur Vorhersage des Erwerbsanteils der Mütter getrennt nach den Altersstufen des Kindes abgetragen. Ebenso enthalten die Modelle die weiteren Variablen, die auch in den vorherigen Regressionsmodellen kontrolliert wurden (relativer Berufsstatus, Anzahl der Kinder, Bundesgebiet und absoluter Berufsstatus der Mutter). Die Koeffizienten dieser weiteren Variablen sind in Tabelle 8.3 nicht abgebildet, da sie (nahezu) identisch zu den vorherigen Modellen (siehe Tabelle A.3) sind und der Fokus hier lediglich auf die Gender-Items gerichtet ist.

Zunächst lässt sich festhalten, dass alle Items in die erwartete Richtung zeigen, d.h. mit egalitäreren Einstellungen erhöht sich der Erwerbsanteil der Mütter. Diese Effekte sind in der Regel signifikant. Eine Ausnahme stellt Item 3 in den beiden jüngsten Altersstufen dar. Dies hängt damit zusammen, dass Item 2 und 3 stark miteinander korrelieren und Item 3 durch die gleichzeitige Kontrolle von Item 2 nicht mehr signifikant ist. Im Folgenden wird näher auf die inhaltliche Bedeutung der Items eingegangen. Gender-Item 1 fragt danach, ob sowohl die Frau als auch der Mann Teilzeit arbeiten sollen, wenn kleine Kinder da sind. Eine egalitäre Einstellung hierzu hat einen besonders starken Effekt, wenn das Kind im Alter von 6 bis unter 9 Jahre ist. Beim zweiten und vierten Item zeigen sich die stärksten Effekte dagegen dann, wenn das Kind im Alter von 1 bis unter 3 Jahren ist. Eine ablehnende Haltung zu der Einstellung, dass die Frau zu Hause bleiben und die Kinder versorgen soll (= egalitäre Einstellung bei Item 2 ) erhöht den Erwerbsanteil bei Müttern von 1- bis 3-jährigen Kindern somit besonders; gleiches gilt für die Befürwortung, dass der Mann genauso stark in die Erziehung und Pflege eingebunden sein soll wie die

\footnotetext{
${ }^{31}$ Als Faustregel gilt, dass Cronbach's Alpha bis zu einem Wert von 0,50 inakzeptabel ist, ab 0,50 bis unter 0,60 ist es schlecht, ab 0,60 bis unter 0.70 fragwürdig, ab 0,70 bis unter 0,80 akzeptabel, ab 0,80 bis unter 0,90 gut und ab 0.90 exzellent (George und Mallery 2002, S. 231).
} 
Mutter (= egalitäre Einstellung bei Item 4). Dass die Effekte dieser beiden Items bei Kindern im Alter von 1 bis unter 3 Jahren besonders stark sind, mag u.a. damit zusammenhängen, dass im ersten Lebensjahr der Großteil der Mütter nicht erwerbstätig ist und die Varianz in der abhängigen Variable dementsprechend gering ist, während bei einem Kind im Alter von 1 bis unter 3 Jahre bereits ein größerer Anteil der Mütter erwerbstätig ist und damit auch die Varianz höher ist.

Tabelle 8.3: $\quad$ Erwerbsanteil der Mutter in Abhängigkeit vom Alter des Kindes, unter Berücksichtigung von vier Gender-Items

\begin{tabular}{|c|c|c|c|c|c|c|c|c|}
\hline & unter 1 & & 1 bis unt & & 3 bis unt & & 6 bis unt & er 9 \\
\hline & (1) & & (2) & & (3) & & (4) & \\
\hline VARIABLES & coef & se & coef & se & coef & $\mathrm{se}$ & coef & se \\
\hline \multicolumn{9}{|c|}{$\begin{array}{l}\text { Geschlechterrol- } \\
\text { leneinstellungen }\end{array}$} \\
\hline $\begin{array}{l}\text { Item } 1 \text { (beide } \\
\text { TZ) }\end{array}$ & $0.01 * *$ & $(0.00)$ & 0.00 & $(0.00)$ & $0.01 * *$ & $(0.00)$ & $0.02 * * *$ & $(0.00)$ \\
\hline $\begin{array}{l}\text { Item } 2 \text { (gegen } \\
\text { männl. Al- } \\
\text { leinverdiener- } \\
\text { M.) }\end{array}$ & $0.02 * * *$ & $(0.00)$ & $0.03 * * *$ & $(0.00)$ & $0.02 * * *$ & $(0.00)$ & $0.02 * * *$ & $(0.00)$ \\
\hline $\begin{array}{l}\text { Item } 3 \text { (gegen } \\
\text { männl. } \\
\text { Hauptverdie- } \\
\text { ner-M.) }\end{array}$ & -0.00 & $(0.00)$ & -0.00 & $(0.00)$ & $0.01^{*}$ & $(0.00)$ & 0.01 & $(0.00)$ \\
\hline $\begin{array}{l}\text { Item } 4 \text { (für } \\
\text { egali. Care- } \\
\text { Arbeit) }\end{array}$ & $0.02 *$ & $(0.01)$ & $0.03 * * *$ & $(0.01)$ & $0.02 * * *$ & $(0.01)$ & 0.01 & $(0.01)$ \\
\hline Constant & $-0.09 * *$ & $(0.04)$ & $\begin{array}{c}- \\
0.13^{* * *}\end{array}$ & $(0.03)$ & -0.01 & $(0.03)$ & 0.05 & $(0.04)$ \\
\hline Observations & 752 & & 1,923 & & 1,905 & & 1,183 & \\
\hline Adj. $R^{2}$ & 0.04 & & 0.20 & & 0.18 & & 0.17 & \\
\hline
\end{tabular}

Anmerkung: OLS-Regression. Die Skalen der jeweiligen Gender-Items gehen von „1 traditionell“ bis „,6 egalitär". Es wurden die gleichen Variablen wie in den vorherigen, multivariaten Modellen (siehe Tabelle A.3) kontrolliert, d.h. der relative Berufsstatus, die Anzahl der Kinder, das Bundesgebiet und der absolute Berufsstatus der Mutter; Quelle: DJI-Studie AID:A 2013/14, eigene Berechnungen.

Bei den vertiefenden Analysen zu den Geschlechterrolleneinstellungen wurden bis dato zwei verschiedene Wege versucht: Zum einen wurde eine Faktorenanalyse in Erwägung gezogen, um die vier Einstellungen zu einem oder mehreren Faktoren zusammenzufassen. Wie aus den Ausführungen weiter oben jedoch hervorgeht, ist diese Methoden bei den vorliegenden Items ungeeignet, da diese nur eine geringe interne Konsistenz haben. Zum anderen wurden die Items einzeln als unabhängige Variablen mitaufgenommen (siehe Tabelle 8.3).

Nachfolgend wird jedoch ein anderer Weg eingeschlagen: Mittels einer latenten Klassenanalyse wurde eruiert, ob sich latente Profile von Geschlechterrolleneinstellungen 
aufdecken lassen (siehe Kapitel 7.5). In bisherigen Studien hat sich diese Herangehensweise als fruchtbar erwiesen und es konnten multidimensionale Geschlechterrolleneinstellungen gefunden werden, die über die Bezeichnungen „traditionell“ oder „egalitär“ hinausgehen. Im Folgenden werden die drei latenten Klassen, die in Kapitel 7.5 identifiziert werden konnten (,intensive mothering/parenting“, „egalitär“ und „egalitärer Essentialismus“) als unabhängige Variablen für die Aufteilung der Erwerbsarbeit getestet.

Anhand von Tabelle A.4 im Anhang wird ersichtlich, dass der Erwerbsanteil der Mutter in Zusammenhang mit den latenten Gender-Klassen in der Klasse des „egalitären Essentialismus" höher ist als in der Klasse des "intensive mothering/parenting“. Diese Unterschiede sind in allen Altersstufen, außer in der jüngsten Altersstufe „unter 1 Jahr“, signifikant. Am höchsten ist der Erwerbsanteil der Mutter in der egalitären Klasse. Die nachfolgende Abbildung 8.15 veranschaulicht die Werte aus Tabelle A.4 im Anhang graphisch: In allen Altersstufen zeigt sich der Trend, dass der vorhergesagte Erwerbsanteil der Mütter in der Klasse des „intensive mothering/parenting“ am niedrigsten ist, gefolgt von der Klasse des "egalitären Essentialismus“ und am höchsten in der egalitären Klasse. 
Abbildung 8.15: Vorhergesagter Erwerbsarbeitsanteil der Mutter, in Abhängigkeit der drei latenten Gender-Klassen, getrennt nach dem Alter des Kindes

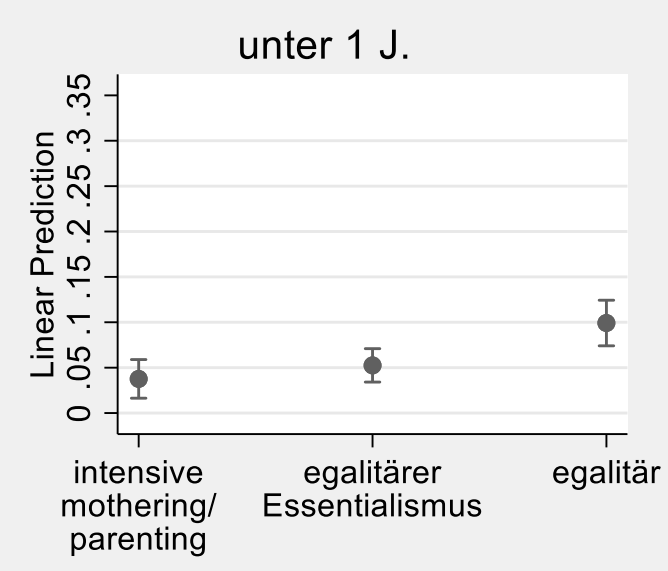

3 bis unter $6 \mathrm{~J}$.

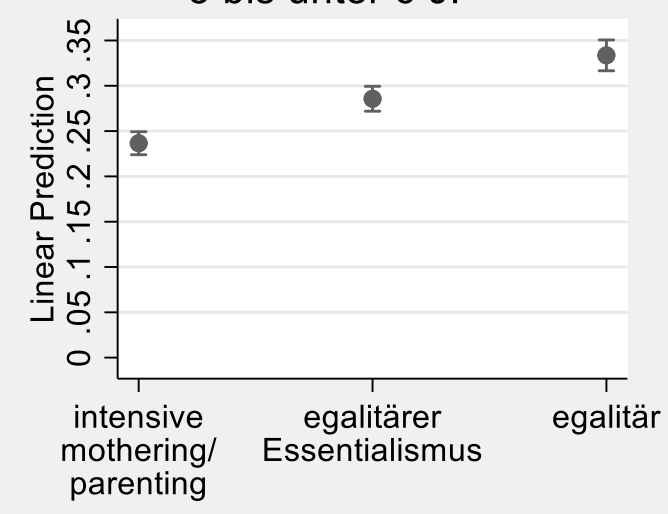

1 bis unter $3 \mathrm{~J}$.

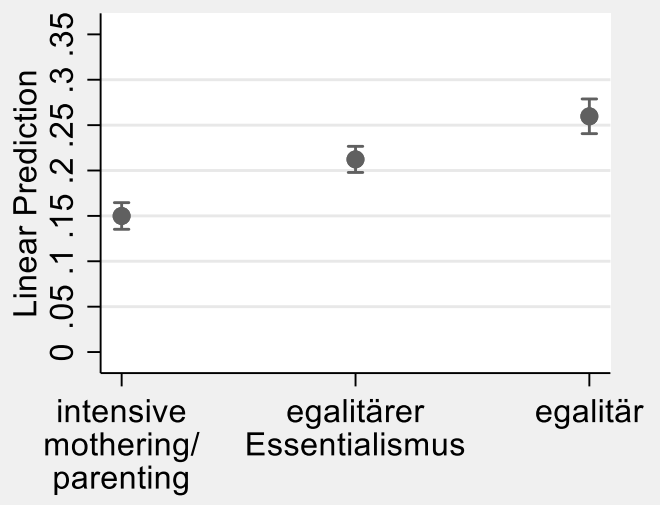

6 bis unter $9 \mathrm{~J}$.

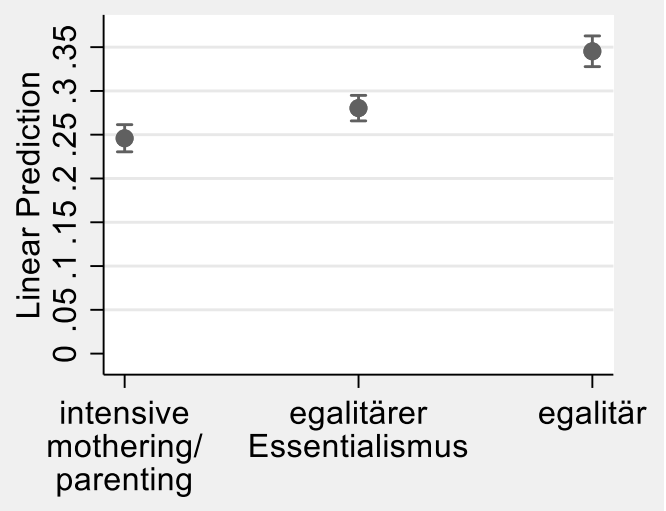

Anmerkung: Vorhergesagter Erwerbsanteil der Mutter (Predictive Margins) basierend auf Tabelle A.4; die weiteren Variablen werden auf ihrem Mittelwert festgehalten; Quelle: DJI-Studie AID:A 2013/14, Sample: $n=6.804$, eigene Berechnungen.

Ebenso zeigt sich anhand der Graphiken deutlich, wie sich mit zunehmendem Alter des Kindes der Erwerbsanteil der Mütter in allen Klassen nach oben bewegt (vgl. auch die Konstanten in Tabelle A.4). Hypothese 4 a), dass Mütter, die sich mit dem Leitbild des „intensive mothering“ identifizieren, einen geringeren Erwerbsanteil haben, kann somit bestätigt werden. Die altersspezifischen Hypothesen nehmen des Weiteren an, dass der Effekt des Leitbildes des „intensive mothering/parenting“ bei Kindern im Alter von unter 3 Jahren besonders stark ist (Hypothese 4b), bei einem Kindesalter von „3 bis unter 6 Jahre" bereits etwas abgeschwächt ist (Hypothese 4c) und bei einem Kindesalter ab 6 Jahren schließlich keine oder nur noch eine sehr geringe Rolle spielt (Hypothese 4d). Diese altersspezifischen Hypothesen können nur eingeschränkt bestätigt werden: Die Tatsache, dass der Erwerbsanteil der Mütter mit steigendem Kindesalter auch in der Klasse des „intensive mothering/parenting“ zunimmt, zeigt zwar, dass sich das Konzept des „intensive mothering/parenting“ in Abhängigkeit vom Kindesalter verändert und bei älteren Kindern ein höherer Erwerbsanteil bzw. -umfang toleriert wird. Die altersspezifischen Hypothesen 4 c) und 4 d) können jedoch insofern nicht bestätigt werden, weil die 
Unterschiede zwischen den latenten Klassen mit zunehmendem Kindesalter nicht schwächer werden: So unterscheiden sich die Klassen des „intensive mothering/parenting“ und die egalitäre Klasse in der Altersstufe „1 bis unter 3 Jahre“ als auch in den beiden höheren Altersstufen jeweils um 10-11\%.

\section{Individuelle Erwerbsstunden der Mütter}

Bislang wurde der Erwerbsanteil der Mütter auf Paarebene betrachtet, d.h. der Erwerbsumfang der Mütter wurde am gesamten Erwerbsvolumen des Paares relativiert, um so in Erfahrung bringen zu können, inwiefern die Erwerbsarbeit zwischen Paaren egalitär aufgeteilt wird bzw. welche Einflussfaktoren zu einer traditionelleren oder egalitäreren Aufteilung der Erwerbsarbeit beitragen. Da jedoch bekannt ist, dass v.a. die Erwerbsumfänge von Müttern variabel und die Erwerbsumfänge von Vätern weitgehend konstant sind, liegt die Vermutung nahe, dass eine Veränderung des Erwerbsanteils der Mütter in erster Linie auf eine Veränderung ihres eigenen Erwerbsumfangs und weniger auf eine Veränderung des Erwerbsumfangs des Partners zurückzuführen ist. Schließlich sind die meisten Männer - sowohl vor als auch nach der Familiengründung und unabhängig vom Alter des Kindes - in Vollzeit erwerbstätig. Im Folgenden wird daher getestet, ob die Ergebnisse anders ausfallen, wenn lediglich der Erwerbsumfang der Mütter betrachtet wird. Die multivariaten Modelle zur Erklärung des Erwerbsumfangs der Mütter orientieren sich dabei an den Modellen zur Erklärung des Erwerbsanteils der Mütter, werden jedoch nur in komprimierter Form dargestellt, um Redundanz zu vermeiden. Eine Form der Komprimierung besteht darin, dass direkt das Gesamtmodell vorgestellt wird, ohne gesondert auf den stufenweisen Aufbau einzugehen und ohne zusätzliche Graphiken. Die Gesamtmodelle sind getrennt nach den Altersstufen des Kindes in Tabelle 8.4 dargestellt.

Aus den Ergebnissen zum relativen Berufsstatus geht hervor, dass Mütter mit einem niedrigeren Status in allen Altersstufen des Kindes signifikant weniger Erwerbsstunden im Vergleich zur Referenzkategorie haben. Dagegen kann in keiner einzigen Altersstufe des Kindes bestätigt werden, dass Mütter mit einem höheren, relativen Berufsstatus (im Vergleich zur Referenzkategorie) signifikant mehr Erwerbsstunden verrichten. Die Effekte unterscheiden sich nicht signifikant zwischen den verschiedenen Altersstufen, was anhand von Interaktionseffekten getestet wurde, die nicht abgebildet sind. Diese Ergebnisse sind ähnlich zu den Ergebnissen, die bereits zur Erklärung des Erwerbsanteils der Mütter herausgefunden wurden. Beim Erwerbsumfang der Mütter sind die Effekte der relativen Ressourcen ebenso wie beim Erwerbsanteil der Mütter als gering einzustufen: So belaufen sich die Differenzen in den Erwerbsumfängen der Mütter in Abhängigkeit der relativen Ressourcen nur auf etwa zwei Erwerbsstunden pro Woche.

Auch bei den Geschlechterrolleneinstellungen ähneln die Zusammenhänge mit den Erwerbsstunden der Mütter den bisherigen Ergebnissen: Mit egalitäreren Einstellungen erhöhen sich die Erwerbsstunden der Mütter. Vergleicht man die Effektstärken der beiden Items, so sind die Effekte von Gender-Item 2 größer als die von Gender-Item 1. Die 
Effekte der beiden Gender-Items sind in allen Altersstufen des Kindes höchst signifikant. Während sich die Effektstärken von Gender-Item 1 nicht signifikant zwischen den Altersstufen unterscheiden sich (wie aus einem nicht abgebildeten Modell mit Interaktionseffekten hervorgeht), gibt es bei Gender-Item 2 signifikante Schwankungen zwischen den verschiedenen Altersstufen. Besonders stark sind die Effekte von Gender-Item 2 in den Altersstufen „1 bis unter 3 Jahre“ und „3 bis unter 6 Jahre“: Wenn die Einstellungen zu der Frage danach, ob beim Vorhandensein von Kindern der Mann arbeiten gehen und die Frau zu Hause bleiben und die Kinder versorgen soll (= Gender-Item 2), auf der 6stufigen Skala um eine Stufe egalitärer werden, so erhöht sich der Erwerbsumfang der Mütter um 2,43 Stunden bzw. um 1,98 Stunden.

Bei der Anzahl der Kinder konnte in der ersten Altersstufe des Kindes kein signifikanter Effekt festgestellt werden, d.h. wenn das Kind unter 1 Jahr alt ist, dann haben Mütter unabhängig von der Anzahl der weiteren Kinder nur einen sehr geringen Erwerbsumfang oder sind nicht erwerbstätig. In den höheren Altersstufen zeigen sich die erwarteten Effekte, dass Mütter mit einem Kind mehr Erwerbsstunden verrichten als Mütter mit zwei Kindern (= Referenzkategorie), während Mütter mit drei oder mehr Kindern weniger Erwerbsstunden leisten im Vergleich zu Müttern mit zwei Kindern.

Berücksichtigt man des Weiteren das Bundesgebiet, so zeigen die Ergebnisse, dass ostdeutsche Mütter in höherem Umfang erwerbstätig sind als westdeutsche Mütter, wobei die Unterschiede in allen Altersstufen, außer der jüngsten Altersstufe, signifikant sind. Die Unterschiede betragen 8,30 Stunden bei einem Kind im Alter von „1 bis unter 3 Jahre" und etwa 10 bis 12 Stunden bei einem älteren Kind. Die stärkeren Effekte in den höheren Altersstufen unterscheiden sich nicht nur signifikant von der untersten Altersstufe, sondern auch untereinander signifikant, d.h. bei einem Kind im Grundschul- und Jugendalter sind die Ost-West-Differenzen größer als bei einem Kind im Kleinkind- oder Kindergartenalter.

Beim absoluten Berufsstatus der Mütter zeigt sich, dass Mütter mit einem hohen Status in höherem Umfang erwerbstätig sind als Mütter mit einem mittleren Berufsstatus $(=\mathrm{Re}-$ ferenzkategorie). Die Effekte sind (außer bei einem Kind unter 1 Jahr) signifikant und werden mit zunehmendem Alter des Kindes größer. Wenn das Kind „11 bis unter 18 Jahre" alt ist, dann ist der Effekt eines hohen Berufsstatus' signifikant höher als dies der Fall ist, wenn das Kind „unter 1 Jahr" oder „1 bis unter 3 Jahre“ alt ist. 
Tabelle 8.4: AV: Erwerbsstunden der Mütter, getrennt nach dem Alter des Kindes

\begin{tabular}{|c|c|c|c|c|c|c|}
\hline & unter 1 & & 1 bis unter 3 & & 3 bis unter 6 & \\
\hline & (1) & & $(2)$ & & (3) & \\
\hline VARIABLES & coef & se & coef & se & coef & se \\
\hline \multicolumn{7}{|l|}{ rel. Ressourcen } \\
\hline \multicolumn{7}{|l|}{ rel. Berufsstatus (ISEI) (Ref. 2, Frau = Mann) } \\
\hline Frau $<$ Mann & $-1.93 *$ & $(0.90)$ & $-1.79 *$ & $(0.83)$ & $-1.60 *$ & $(0.78)$ \\
\hline Frau $>$ Mann & -1.12 & $(0.88)$ & 0.20 & $(0.79)$ & 0.15 & $(0.76)$ \\
\hline Missings & -1.15 & $(1.83)$ & 0.22 & $(2.25)$ & -0.80 & $(1.90)$ \\
\hline \multicolumn{7}{|l|}{ Geschlechterrolleneinstellungen (Skala von } \\
\hline \multicolumn{7}{|l|}{$, 1:$ traditionell“" bis, 6 : egalitär“") } \\
\hline Item 1 (beide TZ) & $0.40^{*}$ & $(0.20)$ & $0.43 *$ & $(0.19)$ & $0.55 * *$ & $(0.18)$ \\
\hline Item 2 (gegen männl. Alleinverdiener-M.) & $0.81 * * *$ & $(0.21)$ & $2.43 * * *$ & $(0.19)$ & $1.98 * * *$ & $(0.18)$ \\
\hline \multicolumn{7}{|l|}{ Weitere Variablen } \\
\hline \multicolumn{7}{|l|}{ Anzahl der Kinder (Ref. 2) } \\
\hline 1 Kind & -0.58 & $(0.82)$ & $3.09 * * *$ & $(0.74)$ & $4.21 * * *$ & $(0.75)$ \\
\hline 3 Kinder & -0.52 & $(0.91)$ & $-3.81 * * *$ & $(0.81)$ & $-3.74 * * *$ & $(0.70)$ \\
\hline 4 und mehr & -0.77 & $(1.30)$ & $-6.38 * * *$ & $(1.27)$ & $-6.12 * * *$ & $(1.04)$ \\
\hline \multicolumn{7}{|l|}{ Bundesland (Ref. Westdtl.) } \\
\hline Ost-Dtl. & 0.85 & $(1.25)$ & $8.30 * * *$ & $(1.02)$ & $10.10 * * *$ & $(0.89)$ \\
\hline \multicolumn{7}{|l|}{ Berufsstatus der Mutter (ISEI) (Ref. mittel) } \\
\hline niedrig $(<45)$ & 0.66 & $(0.91)$ & -0.63 & $(0.83)$ & -0.28 & $(0.73)$ \\
\hline hoch (ab 63) & -0.16 & $(0.86)$ & $3.85 * * *$ & $(0.78)$ & $3.92 * * *$ & $(0.74)$ \\
\hline Missings & -2.18 & $(2.37)$ & -2.71 & $(2.53)$ & -2.07 & $(2.05)$ \\
\hline Constant & -0.26 & $(1.44)$ & 0.35 & $(1.30)$ & $7.17 * * *$ & $(1.20)$ \\
\hline Observations & 851 & & 2,122 & & 2,274 & \\
\hline Adj. $\mathrm{R}^{2}$ & 0.02 & & 0.19 & & 0.21 & \\
\hline
\end{tabular}




\begin{tabular}{|c|c|c|c|c|}
\hline \multirow[b]{3}{*}{ VARIABLES } & \multirow{3}{*}{$\begin{array}{c}6 \text { bis unter } 11 \\
(4) \\
\text { coef }\end{array}$} & & \multicolumn{2}{|c|}{11 bis unter 18} \\
\hline & & & $(5)$ & \\
\hline & & se & coef & se \\
\hline \multicolumn{5}{|l|}{ rel. Ressourcen } \\
\hline Frau $<$ Mann & $-2.21 * *$ & $(0.71)$ & $-1.83 * *$ & $(0.70)$ \\
\hline Frau $>$ Mann & 0.46 & $(0.70)$ & 0.86 & $(0.73)$ \\
\hline Missings & -2.38 & $(1.60)$ & 0.57 & $(1.49)$ \\
\hline \multicolumn{5}{|l|}{ Geschlechterrolleneinstellungen (Skala von } \\
\hline Item 1 (beide TZ) & $0.76^{* * *}$ & $(0.16)$ & $0.62 * * *$ & $(0.16)$ \\
\hline Item 2 (gegen männl. Alleinverdiener-M.) & $1.71 * * *$ & $(0.17)$ & $1.47 * * *$ & $(0.17)$ \\
\hline \multicolumn{5}{|l|}{ Weitere Variablen } \\
\hline \multicolumn{5}{|l|}{ Anzahl der Kinder (Ref. 2) } \\
\hline 1 Kind & $3.40 * * *$ & $(0.71)$ & $2.67 * * *$ & $(0.62)$ \\
\hline 3 Kinder & $-3.40 * * *$ & $(0.63)$ & $-3.43 * * *$ & $(0.79)$ \\
\hline 4 und mehr & $-6.66 * * *$ & $(1.09)$ & $-5.62 * *$ & $(1.76)$ \\
\hline \multicolumn{5}{|l|}{ Bundesland (Ref. Westdtl.) } \\
\hline Ost-Dtl. & $11.99 * * *$ & $(0.84)$ & $10.65 * * *$ & $(0.87)$ \\
\hline \multicolumn{5}{|l|}{ Berufsstatus der Mutter (ISEI) (Ref. mittel) } \\
\hline niedrig $(<45)$ & 0.18 & $(0.66)$ & -1.06 & $(0.64)$ \\
\hline hoch (ab 63) & $3.94 * * *$ & $(0.68)$ & $5.94 * * *$ & $(0.73)$ \\
\hline Missings & 0.90 & $(1.80)$ & $-6.46^{* *}$ & $(1.98)$ \\
\hline Constant & $8.07 * * *$ & $(1.12)$ & $11.69 * * *$ & $(1.16)$ \\
\hline Observations & 2,709 & & 2,871 & \\
\hline Adj. $R^{2}$ & 0.20 & & 0.17 & \\
\hline
\end{tabular}

Anmerkung: OLS-Regression. Quelle: DJI-Studie AID:A 2013/14, Sample: $n=11.330$, eigene Berechnungen. 
Vertiefende Analysen zu den Geschlechterrolleneinstellungen

Ebenso wie beim Erwerbsanteil der Mütter werden auch beim Erwerbsumfang der Mütter vertiefende Analysen zu den Geschlechterrolleneinstellungen vorgenommen, jedoch nur in komprimierter Form vorgestellt, um Redundanz zu vermeiden. Im Folgenden wird darauf eingegangen, welcher Zusammenhang zwischen den vier Gender-Items bzw. zwischen den drei latenten Klassen und dem Erwerbsumfang der Mütter besteht und inwiefern sich diese Zusammenhänge je nach Alter des Kindes unterscheiden.

Auch hier zeigen die signifikanten Effekte in die erwartete Richtung, sodass egalitärere Einstellungen mit einem höheren Erwerbsumfang der Mütter einhergehen. Viele Effekte sind signifikant. Eine Ausnahme stellt Item 3 in den beiden jüngsten Altersstufen dar und ist damit analog zu den Ergebnissen in Tabelle 8.3, wo die vier Gender-Items bzgl. des Zusammenhangs mit dem Erwerbsanteil der Mütter analysiert wurden. Dass Item 3 in einigen Altersstufen nicht signifikant ist, kann auf die hohe Korrelation mit Item 2 zurückgeführt werden. So fragen sowohl Item 2 als auch Item 3 inhaltlich danach, ob der Mann primär für die Erwerbsarbeit zuständig ist und die Frau nicht oder nur in geringerem Umfang als der Mann arbeiten soll. D.h. inhaltlich fragen die Items nach den Einstellungen zu einem männlichen Alleinverdiener-Modell (Item 2) bzw. einem Zuverdiener-Modell (Item 3). Wenn bei Item 2 egalitäre Einstellungen vorliegen, d.h. dass ein männliches Alleinverdiener-Modell abgelehnt wird, dann schlägt sich dies in einem höheren Erwerbsumfang der Mütter nieder. Bei Gender-Item 1 sind die Effekte (außer in der höchsten Altersstufe) nicht signifikant, d.h. eine Befürwortung, dass sowohl die Frau als auch der Mann Teilzeit arbeiten sollen, wenn kleine Kinder da sind, ist nicht mit einem höherem Erwerbsumfang der Mütter assoziiert. Dagegen geht eine Befürwortung dahingehend, dass der Mann genauso stark in die Erziehung und Pflege eingebunden sein soll wie die Mutter (= egalitäre Einstellung bei Item 4) schon mit einem höheren Erwerbsumfang der Mütter einher (ausgenommen ist hierbei die jüngste Altersstufe). 
Tabelle 8.5: $\quad$ Erwerbsumfang der Mutter in Abhängigkeit vom Alter des Kindes, unter Berücksichtigung von vier Gender-Items

\begin{tabular}{|c|c|c|c|c|c|c|c|c|}
\hline & \multicolumn{2}{|c|}{ unter 1} & \multicolumn{2}{|c|}{1 bis unter 3} & \multicolumn{2}{|c|}{3 bis unter 6} & \multicolumn{2}{|c|}{6 bis unter 9} \\
\hline & (1) & & (2) & & (3) & & (4) & \\
\hline VARIABLES & coef & se & coef & se & coef & se & coef & se \\
\hline $\begin{array}{l}\text { Geschlechterrol- } \\
\text { len- } \\
\text { einstellungen }\end{array}$ & & & & & & & & \\
\hline Item 1 (beide TZ) & 0.39 & $(0.21)$ & 0.25 & $(0.20)$ & 0.34 & $(0.19)$ & $0.61 * *$ & $(0.23)$ \\
\hline $\begin{array}{l}\text { Item } 2 \text { (gegen } \\
\text { männl. Alleinver- } \\
\text { diener-M.) }\end{array}$ & $0.94 * * *$ & $(0.25)$ & $2.31 * * *$ & $(0.24)$ & $1.48 * * *$ & $(0.23)$ & $1.63 * * *$ & $(0.28)$ \\
\hline $\begin{array}{l}\text { Item } 3 \text { (gegen } \\
\text { männl. Hauptver- } \\
\text { diener-M.) }\end{array}$ & -0.33 & $(0.25)$ & -0.06 & $(0.24)$ & $0.91 * * *$ & $(0.24)$ & $0.61 *$ & $(0.29)$ \\
\hline $\begin{array}{l}\text { Item } 4 \text { (für egali. } \\
\text { Care-Arbeit) }\end{array}$ & 0.70 & $(0.41)$ & $1.84 * * *$ & $(0.36)$ & $1.43 * * *$ & $(0.35)$ & $1.02 *$ & $(0.46)$ \\
\hline Constant & -3.10 & $(2.40)$ & - & $(2.07)$ & -1.38 & (1.99) & 0.01 & $(2.58)$ \\
\hline Observations & 829 & & 2,017 & & 1,972 & & 1,230 & \\
\hline $\operatorname{Adj~} R^{2}$ & 0.03 & & 0.21 & & 0.25 & & 0.24 & \\
\hline
\end{tabular}

Anmerkung: OLS-Regression. Die Skalen der jeweiligen Gender-Items gehen von „1 traditionell“ bis „6 egalitär“. Es wurden die gleichen Variablen wie in den vorherigen, multivariaten Modellen (siehe Tabelle 8.4) kontrolliert, d.h. der relative Berufsstatus, die Anzahl der Kinder, das Bundesgebiet und der absolute Berufsstatus der Mutter; Quelle: DJI-Studie AID:A 2013/14, eigene Berechnungen.

In aller Kürze wird nun auch auf die Erwerbsumfänge der Mütter unter Berücksichtigung der latenten Klassen (,intensive mothering/parenting“, „egalitärer Essentialismus“ und „egalitär“) eingegangen (es werden darüber hinaus dieselben Variablen wie in Tabelle 8.4 kontrolliert): Aus Tabelle A.5 im Anhang geht hervor, dass laut der Konstante der Erwerbsumfang von Müttern etwa 4 Stunden beträgt, wenn das Kind unter 1 Jahr alt ist, etwa 9 Stunden bei einem 1- bis unter 3-jährigem Kind und etwa 14-15 Stunden bei einem Kind in den beiden höheren Altersstufen. Die Effekte der latenten Klassen sind in allen Altersstufen (mit Ausnahme der jüngsten Altersstufe) etwa gleich groß: Wenn Mütter der Klasse des „egalitären Essentialismus“ zugehörig sind, dann ist ihr Erwerbsumfang im Vergleich zur Referenzkategorie (,intensive mothering/parenting“) etwa um 4-5 Erwerbsstunden höher und wenn sie der egalitären Klasse angehören, dann beträgt der Unterschied zur Referenzkategorie etwa 7 Erwerbsstunden.

Diese Zusammenhänge aus Tabelle A.5 im Anhang werden in Abbildung 8.16 anhand der vorhergesagten Werten (Predictive Margins) veranschaulicht: Daraus geht hervor, dass sich das allgemeine Level des Erwerbsumfangs mit zunehmendem Alter des Kindes erhöht, aber die Unterschiede zwischen den Klassen in allen Altersstufen etwa gleich hoch sind. Eine Ausnahme bildet die Altersstufe der unter 1-Jährigen, da es hier keine 
Unterschiede zwischen der Klasse des „intensive mothering/parenting“ und des „egalitären Essentialismus“ gibt. Auch der Unterschied zwischen der Klasse des „intensive mothering/parenting" und der egalitären Klasse ist hier geringer als in den höheren Stufen, jedoch dennoch schon signifikant. Insgesamt sind auch hier die Ergebnisse zum Erwerbsumfang und den latenten Klassen sehr ähnlich zu den Ergebnissen zum Erwerbsanteil und den latenten Klassen.

Abbildung 8.16: Vorhergesagter Erwerbsumfang der Mutter, in Abhängigkeit der drei latenten Gender-Klassen, getrennt nach dem Alter des Kindes
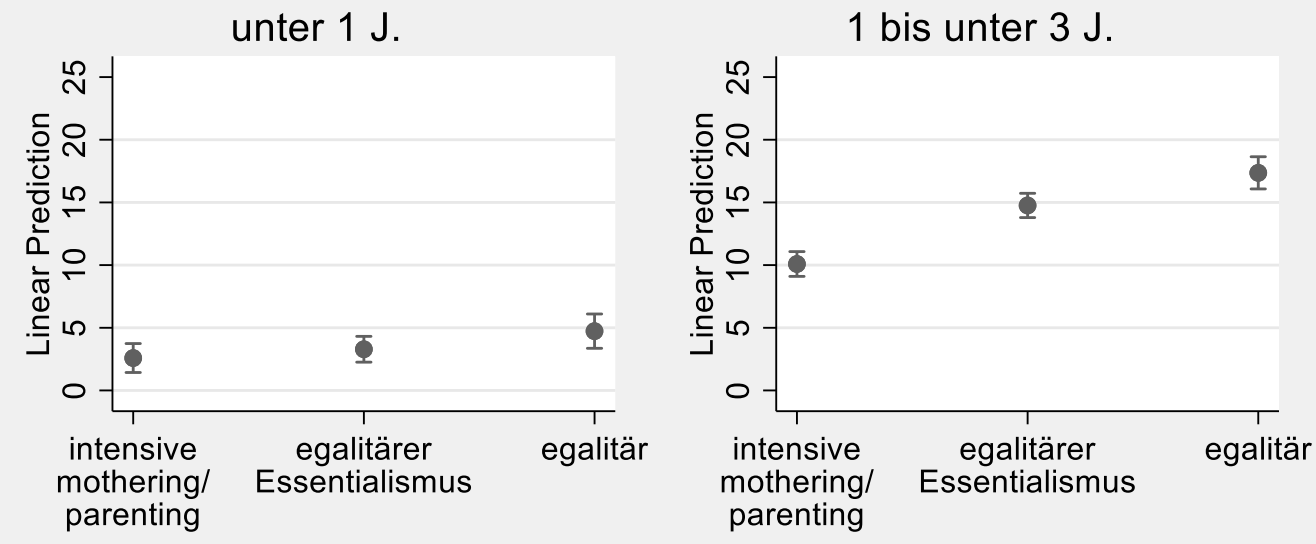

3 bis unter $6 \mathrm{~J}$.

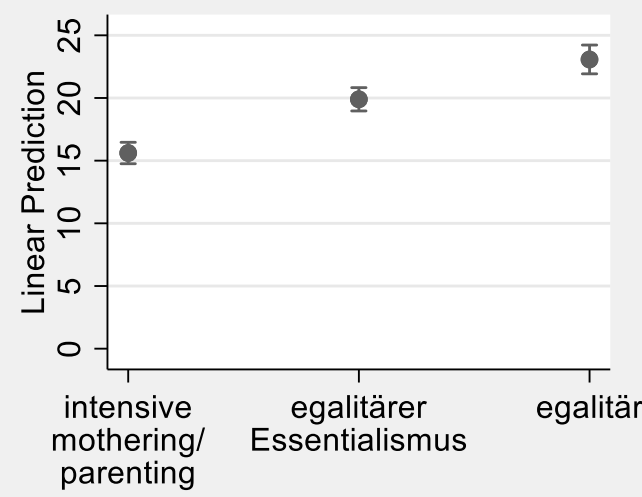

6 bis unter $11 \mathrm{~J}$.

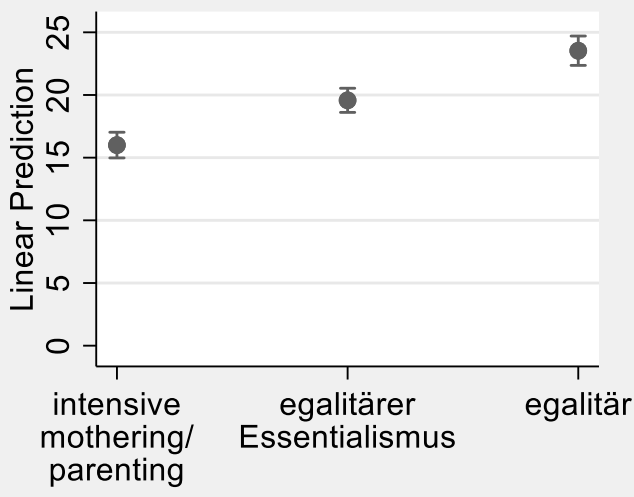

Anmerkung: Vorhergesagter Erwerbsumfang der Mutter (Predictive Margins) basierend auf Tabelle A.5; die weiteren Variablen werden auf ihrem Mittelwert festgehalten; Quelle: DJI-Studie AID:A 2013/14, eigene Berechnungen.

Zusammenfassend lässt sich festhalten, dass sich bei allen Variablen die Ergebnisse zum Erwerbsanteil der Mütter und zum Erwerbsumfang der Mütter sehr ähnlich sind. Dies ist nicht überraschend, da die meisten Väter vollzeitbeschäftigt sind und somit die Varianz in den Erwerbsumfängen der Väter relativ gering ist. Da eine Veränderung des Erwerbsanteils der Mütter v.a. auf eine Veränderung des Erwerbsumfangs der Mütter zurückzuführen ist, hat dies zur Folge, dass sich die Ergebnisse des Erwerbsanteils und des Erwerbsumfangs größtenteils entsprechen. 
Die Ergebnisse zeigen, dass relative Ressourcen wie der relative Berufsstatus nur eine geringe Rolle spielen und nur eine geringe Erklärungskraft für die Aufteilung der Erwerbsarbeit haben. Eine gewichtigere Rolle spielen dagegen Geschlechterrolleneinstellungen. Sowohl bei der Betrachtung der einzelnen Gender-Items zeigen sich starke Zusammenhänge zwischen egalitäreren Einstellungen und einer egalitäreren Aufteilung der Erwerbsarbeit; als auch bei den latenten Einstellungsprofilen zeigen sich deutliche Zusammenhänge zwischen den Klassen und der Egalität der Arbeitsteilung. Eine wichtige Rolle spielen weitere Variablen wie die Anzahl der Kinder und der absolute Berufsstatus der Mütter: Die Ergebnisse zeigen, dass sich eine zunehmende Kinderanzahl negativ auf die Erwerbstätigkeit der Mütter auswirkt und sich ein hoher Berufsstatus der Mütter positiv auf ihre Erwerbstätigkeit auswirkt. Nach wie vor stark präsent sind Unterschiede zwischen Ost- und Westdeutschland: Mütter in den neuen Bundesländern haben einen deutlich höheren Erwerbsanteil bzw. -umfang als Müttern in den alten Bundesländern. Eine Übersicht, welche Hypothesen damit genau bestätigt werden können oder nicht ist in Tabelle A.12 im Anhang enthalten.

Vergleicht man die Effektstärke der Einflussfaktoren über die Altersstufen des Kindes hinweg, so fällt auf, dass diese in den meisten Altersstufen etwa gleich stark ausfallen und nur die jüngste Altersstufe des Kindes eine Ausnahme darstellt. So sind bei einem Kind im Alter von „unter 1 Jahr“ die meisten Einflussfaktoren nicht signifikant, d.h. sie können keinen Beitrag zur Erklärung des Erwerbsanteils der Mütter leisten, da dieser scheinbar durch andere Umstände bestimmt wird. Nach diesen Ergebnissen zur Aufteilung der Erwerbsarbeit werden im Folgenden die Ergebnisse zur Aufteilung der Hausarbeit vorgestellt. 


\section{Aufteilung der Hausarbeit}

Wer übernimmt überwiegend die Hausarbeit, wovon hängt dies ab und inwiefern verändert sich dies mit dem Alter des Kindes? Diesen Fragen wird im Folgenden nachgegangen. Zunächst werden bei den deskriptiven Befunden die Verteilungen von vier, einzelnen Tätigkeiten vorgestellt: die Aufteilung der Aufgaben Kochen, Einkaufen, Putzen und Wäsche waschen. Anschließend werden diese vier Tätigkeiten zu einem Hausarbeitsindex zusammengefasst, der ein Indikator dafür ist, welcher Elternteil insgesamt die Hausarbeit überwiegend übernimmt. Dieser Hausarbeitsindex dient bei den multivariaten Analysen als abhängige Variable. Bei den multivariaten Analysen wird erforscht, welche Rolle etwa Ressourcen oder Geschlechterrolleneinstellungen für die Aufteilung der Hausarbeit spielen und welche Unterschiede es hierbei bzgl. des Alters des Kindes gibt.

\subsection{Deskriptive Befunde}

Bei den Hausarbeitstätigkeiten Kochen, Einkaufen, Putzen und Wäsche waschen handelt es sich um Aufgaben, die nahezu täglich anfallen und weitestgehend als langweilig und unangenehm empfunden werden. Coltrane fasst diese Aufgaben deshalb unter dem Begriff „routine housework“ zusammen (Coltrane 2000, S. 1210). Die regelmäßig anfallenden Aufgaben der „routine housework“ gelten als weiblich konnotiert, wohingegen nur gelegentlich anfallende Tätigkeiten („occasional housework“) wie etwa Reparaturen als männlich konnotiert gelten (Coltrane 2000, S. 1210). Darüber hinaus gibt es weitere Hausarbeitstätigkeiten wie Papierkram, worunter etwa das Begleichen von Rechnungen fällt und die als geschlechtsneutral gelten (Coltrane 2000, S. 1210).

Die Aufteilungen der einzelnen Tätigkeiten der Hausarbeit (siehe Abbildung 9.2) bestätigen, dass weiblich konnotierte Aufgaben besonders häufig überwiegend von den Müttern übernommen werden (Kochen: 75,5\%, Einkaufen: 63,4\%, Putzen: 71,9\% und Wäsche: $85,9 \%$ ) und nur sehr selten überwiegend von den Vätern (Kochen: 5,6\%, Einkaufen: $8,3 \%$, Putzen: $1,5 \%$ und Wäsche: $3,3 \%$; von beiden gleichermaßen übernommen werden am häufigsten die Einkäufe (28,4\%) und das Kochen (17,4\%). Eine mögliche Erklärung, warum Einkaufe häufig von beiden übernommen werden, kann darin liegen, dass es sich bei Einkäufen - um keine klassische innerhäusliche Tätigkeit, sondern um eine außerhäusliche Tätigkeit handelt, welche in der Regel als männlich konnotiert gelten. Einkäufe sind somit trotz ihrer Zugehörigkeit zur „routine housework“ in geringerem Maße weiblich konnotiert als etwa Putzen oder Wäsche waschen. Während die Antwortkategorien „kommt nicht vor“ bzw. ,jemand anders“ bei fast allen Items nur äußerst gering besetzt sind, ist Putzen die einzige Tätigkeit, die immerhin zu 9,9\% von jemand 
anderem übernommen wird. Insgesamt zeigen diese deskriptiven Befunde, dass die Hausarbeit immer noch sehr geschlechtsspezifisch zu Lasten der Mütter aufgeteilt ist. ${ }^{32}$

Tabelle 9.1: $\quad$ Aufteilung der einzelnen Tätigkeiten der Hausarbeit

\begin{tabular}{lllllll}
\hline \multirow{2}{*}{$\begin{array}{l}\text { Aufteilung } \\
\text { der Hausar- } \\
\text { beit }\end{array}$} & $\begin{array}{l}\text { überwie- } \\
\text { gend die }\end{array}$ & $\begin{array}{l}\text { beide } \\
\text { etwa } \\
\text { gleich }\end{array}$ & $\begin{array}{l}\text { überwie- } \\
\text { gend der } \\
\text { Vater }\end{array}$ & $\begin{array}{l}\text { kommt } \\
\text { nicht } \\
\text { vor }\end{array}$ & $\begin{array}{l}\text { jemand } \\
\text { anders }\end{array}$ & $n$ \\
\hline Kochen & 75,5 & 17,4 & 5,7 & 0,1 & 1,3 & 10.928 \\
Einkaufen & 63,4 & 28,4 & 8,1 & 0,0 & 0,2 & 10.929 \\
Putzen & 71,9 & 16,6 & 1,5 & 0,1 & 9,9 & 10.929 \\
Wäsche & 85,9 & 9,5 & 3,3 & 0,0 & 1,2 & 10.928 \\
\hline
\end{tabular}

Quelle: DJI-Studie AID:A 2013/14, gewichtet, Mütterangaben, eigene Berechnungen.

Am traditionellsten ist dabei das Wäschewaschen aufgeteilt, gefolgt vom Putzen und Kochen und am egalitärsten ist das Einkaufen aufgeteilt. Dies spiegelt sich auch in der bivariaten Zusammenhängen mit dem Alter des Kindes in Abbildung 9.1 wider, auf die im Folgenden näher eingegangen wird. Hiermit soll in einem ersten, rein deskriptiven Schritt die Forschungsfrage beantwortet werden, inwiefern sich die Aufteilung der Hausarbeit in den verschiedenen Altersstufen des Kindes unterschiedlich gestaltet oder nicht.

${ }^{32}$ Es gilt zu berücksichtigen, dass die dargestellten Befunde die Mütterangaben repräsentieren. Es wurden darüber hinaus auch Analysen mit den Väterangaben gemacht (nicht abgebildet). Vergleicht man die Verteilungen der Mütter- und Väterangaben, so sind sich diese relativ ähnlich. Die kleinen Diskrepanzen, die jedoch zwischen den Mütter- und Väterangaben vorhanden sind, deuten darauf hin, dass der eigene Anteil eher überschätzt und der Anteil des Partners eher unterschätzt wird. Dies bedeutet, dass unter Verwendung der Väterangaben eine etwas egalitärere Aufteilung der Hausarbeit hervorgeht. Ein Erklärungsansatz für die Diskrepanz zwischen den Mütter- und Väterangaben ist, dass Mütter ihren eigenen Anteil bei unangenehmen Tätigkeiten wie dem Putzen überschätzen. Dies belegt eine Studie von Schulz und Grunow 2011, die Tagebucherhebungen mit Befragungserhebungen vergleicht und aufzeigt, dass Mütter bei Befragungserhebungen einen höheren Zeitumfang für unangenehme Tätigkeiten wie dem Putzen angeben als dies bei den Tagebucherhebungen der Fall ist und somit ihren Umfang überschätzen (Schulz und Grunow 2011, S. 627-628). Des Weiteren wäre es denkbar, dass Väter ihren eigenen Anteil aufgrund sozialer Erwünschtheit überschätzen und deshalb häufiger angeben, dass Aufgaben von innen übernommen oder (annähernd) egalitär aufgeteilt werden. 
Abbildung 9.1: Aufteilung der einzelnen Hausarbeitstätigkeiten nach Alter des Kindes
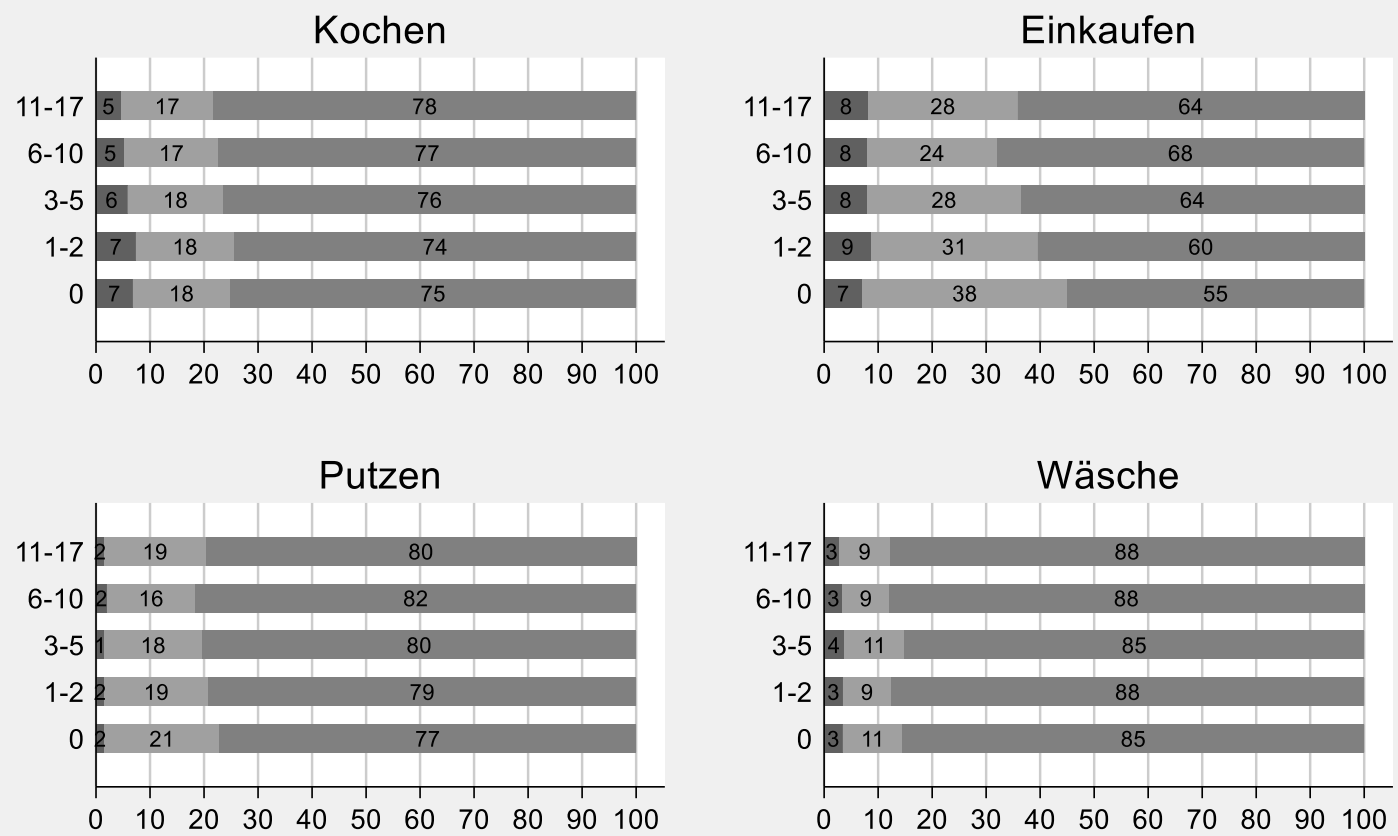

überwiegend der Vater

beide gleich

überwiegend die Mutter

Quelle: DJI-Studie AID:A 2013/14, Daten gewichtet, Mütterangaben, n (Kochen) = 10.761 , n (Einkaufen $)=10.885, \mathrm{n}($ Putzen $)=9.715, \mathrm{n}$ (Wäsche $)=10.777$, eigene Berechnungen .

Das Kochen wird bei einem jüngsten Kind im Alter von unter einem Jahr zu 75\% überwiegend von den Müttern übernommen, zu $18 \%$ von beiden gleichermaßen und zu $7 \%$ überwiegend von den Vätern. Bei einem bereits 6- bis 10-jährigen Kind wird das Kochen zu 77\% und bei einem 11- bis 17- jährigen Kind zu 78\% überwiegend von den Müttern übernommen. Damit ist das Kochen bei Müttern mit älteren Kindern etwas traditioneller aufgeteilt als bei Müttern mit jüngeren Kindern, auch wenn die Unterschiede nur sehr gering ausfallen. Beim Einkaufen zeigen sich größere Unterschiede in Abhängigkeit vom Kindesalter. Die mit Abstand egalitärste Aufteilung liegt bei unter 1-jährigen Kindern vor: Hier wird das Einkaufen nur zu 55\% überwiegend von den Müttern, zu 38\% egalitär und zu 7\% überwiegend von den Vätern übernommen. Bei Elternpaaren mit älteren Kindern erfolgt eine traditionellere Aufteilung. Besonders traditionell ist sie bei denjenigen Eltern, deren jüngstes Kind 6 bis 10 Jahre alt ist: Hier wird der Einkauf schon zu 68\% überwiegend von den Müttern und nur noch zu $24 \%$ von beiden gleichermaßen übernommen. Das Putzen weist, ebenso wie das Kochen, nur geringe Veränderungen über die Altersstufen des Kindes hinweg auf: Bei einem unter 1-jährigen Kind wird das Putzen zu 77\% überwiegend von den Müttern übernommen und zu 21\% gemeinsam übernommen. Mit zunehmendem Alter des Kindes erhöht sich der Anteil der Mütter auf $80 \%$ bei 3- bis unter 6 -jährigen Kindern bzw. auf $82 \%$ bei 6- bis 10-jährigen Kindern. Beim Wäsche waschen 
ist die Arbeitsteilung besonders geschlechtsspezifisch. Der Anteil an Müttern, die angeben, dass dies überwiegend von innen übernommen wird, variiert zwischen $85 \%$ bei einem unter 1-jährigen oder 3- bis 5-jährigen Kind und 88\% in allen anderen Altersstufen.

Diese bivariaten Zusammenhänge zeigen, dass die Hausarbeit nach wie vor weitestgehend von den Müttern selbst übernommen wird und vom Alter des Kindes nur geringfügig tangiert wird. Dennoch sind der $\mathrm{Chi}^{2}$-Test und der Rangkorrelationskoeffizient nach Spearman für alle bivariaten Zusammenhänge signifikant (nicht abgebildet). In der Tendenz liegt in Familien mit älteren Kindern eine noch geschlechtsspezifischere Aufteilung der einzelnen Tätigkeiten vor als bei Familien mit jüngeren Kindern. Dies ist am deutlichsten bei der Aufteilung des Einkaufens zu beobachten. Bei den anderen Items ist die Verschärfung der Traditionalisierung vermutlich deshalb nicht ganz so stark ausgeprägt, weil die Aufteilung bereits mit kleinen Kindern extrem traditionell ist, so dass der mögliche Spielraum dadurch geringer wird.

Zur Betrachtung der Hausarbeit als Ganzes wurden die Items Kochen, Einkaufen, Putzen und Wäsche waschen zu einem Index zusammengefasst. Eine explorative Faktoranalyse untermauert diese Vorgehensweise, indem sie belegt, dass diese Items auf denselben Faktor laden. Im Folgenden wird der Fokus auf diesen Index, der sich aus den besagten Items (Kochen, Einkaufen, Putzen und Wäsche waschen) generiert, gerichtet. Aus dem Forschungsstand geht hervor, dass diese Aufgaben die relevantesten der Hausarbeit sind, da sie regelmäßig anfallen und zeitintensiv sind, so dass sie den Alltag wesentlich mitbestimmen (Coltrane 2000, S. 1210; Lee und Waite 2005, S. 330). In Einklang mit bisherigen Studien werden diese regelmäßig anfallenden und weiblich konnotierten Aufgaben verwendet, um die Egalität der Aufteilung der Hausarbeit besser einschätzen zu können (Batalova und Cohen 2002; Cunningham 2007; Grunow et al. 2012); weitere Items wie etwa Reparaturen oder Papierkram, die nur gelegentlich anfallen und männlich konnotiert oder geschlechtsneutral sind, werden vernachlässigt. ${ }^{33}$

Nachfolgend ist die univariate Verteilung des Hausarbeitsindexes abgebildet (siehe Abbildung 9.2), der die durchschnittlichen Antworten der vier Items widerspiegelt und so von „-1: Vater übernimmt alles“, über „0: egalitär“ bis „1: Mutter übernimmt alles“ reicht. Aus Abbildung 9.2 geht hervor, dass die Hausarbeit sehr geschlechtsspezifisch zu Lasten der Mütter verteilt ist, nur sehr selten egalitär aufgeteilt ist und noch viel seltener überwiegend oder komplett von den Vätern übernommen wird. ${ }^{34}$

\footnotetext{
${ }^{33}$ Eine explorative Faktorenanalyse bestätigt diese Vorgehensweise, da aus ihr hervorgeht, dass Tätigkeiten wie Reparaturen oder Papierkram auf einen anderen Faktor laden als die Tätigkeiten Kochen, Einkaufen, Putzen und Wäsche waschen.

${ }^{34}$ Auch hier gilt es zu berücksichtigen, dass die Daten den Mütterangaben entstammen. Unter Verwendung der Väterangaben wäre die Aufteilung der Hausarbeit geringfügig egalitärer aufgeteilt. Insgesamt zeigen jedoch auch die Väterangaben, dass die Hausarbeit bzw. die einzelnen Tätigkeiten überwiegend von den Müttern übernommen werden.
} 
Abbildung 9.2: Univariate Verteilung der Aufteilung der Hausarbeit

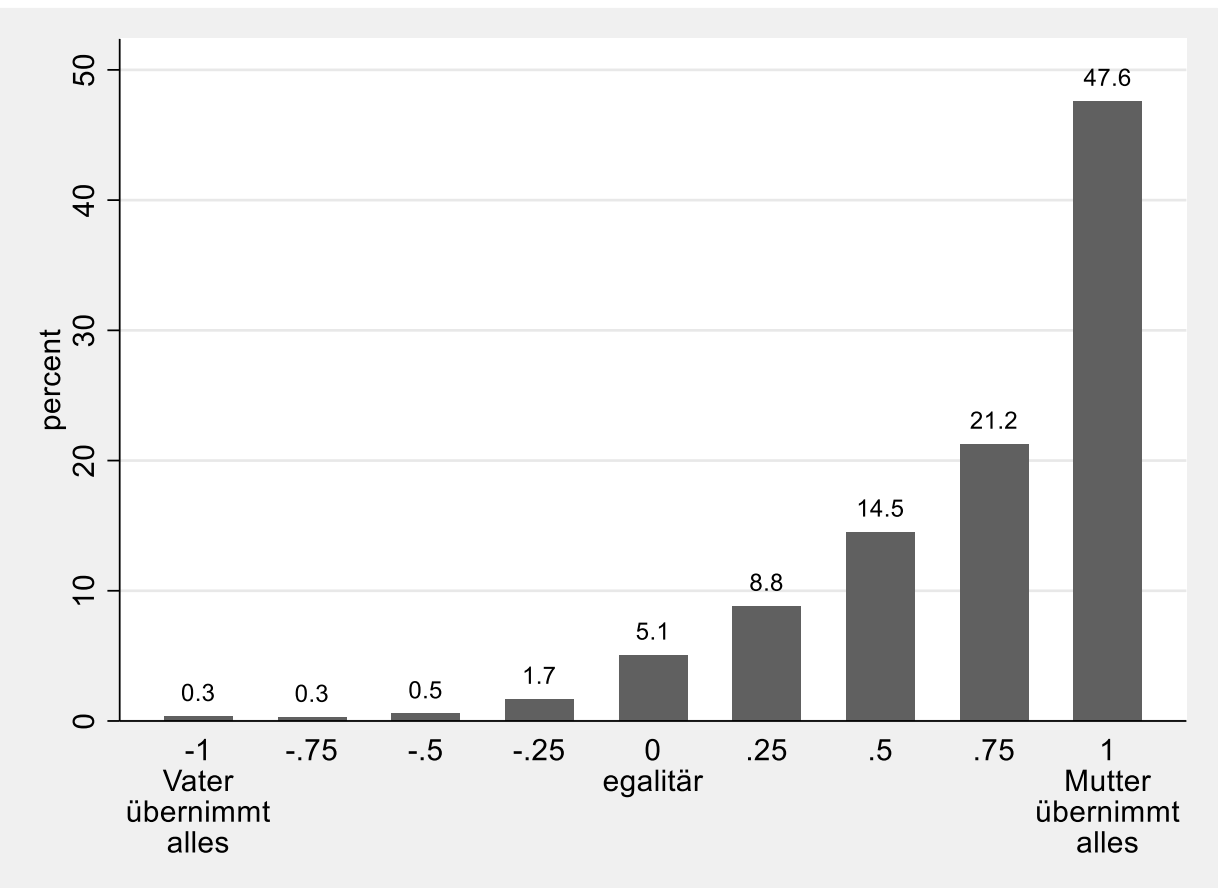

Quelle: DJI-Studie AID:A 2013/14, gewichtet, Mütterangaben, $n=10.922$, eigene Berechnungen.

Wenn man den Hausarbeitsindex zu drei Kategorien komprimiert und nach dem Kindesalter differenziert, so zeigt sich folgendes Bild (siehe Abbildung 9.3): Bei einem Kind im Alter von unter einem Jahr wird die Hausarbeit laut den Müttern zu 16\% egalitär aufgeteilt und zu $83 \%$ wird sie überwiegend von innen selbst übernommen. In Familien mit älteren Kindern ist der egalitäre Anteil etwas geringer (statt 16\% nur noch 12\%), während der Anteil der Mütter höher ist (statt 83\% jetzt 86\%). Die Unterschiede zwischen den Altersgruppen sind gering, was andeutet, dass die Aufteilung der Hausarbeit relativ stabil ist. Die geringe Veränderung, die stattfindet, deutet jedoch auf eine Traditionalisierung der Hausarbeit hin. ${ }^{35}$ Dies steht in Einklang zu bisherigen Studien, die herausfanden, dass die Aufteilung der Hausarbeit mit zunehmender Beziehungsdauer traditioneller wird (Grunow et al. 2007, 2012).

\footnotetext{
${ }^{35}$ Bei den Väterangaben (nicht abgebildet) zeigt sich ein ähnlicher Trend: Bei einem Kind im Alter von unter einem Jahr ist der egalitäre Anteil mit $29 \%$ am höchsten und reduziert sich auf einen Anteil von $15 \%$ bei einem Kind im Jugendalter, wobei der Anteil der Mütter, die die Hausarbeit überwiegend übernehmen, von $68 \%$ auf $80 \%$ ansteigt. Somit deuten auch die Väterangaben auf eine Traditionalisierung der Hausarbeit mit zunehmendem Kindesalter hin.
} 


\section{Abbildung 9.3 Aufteilung der Hausarbeit nach Alter des Kindes}

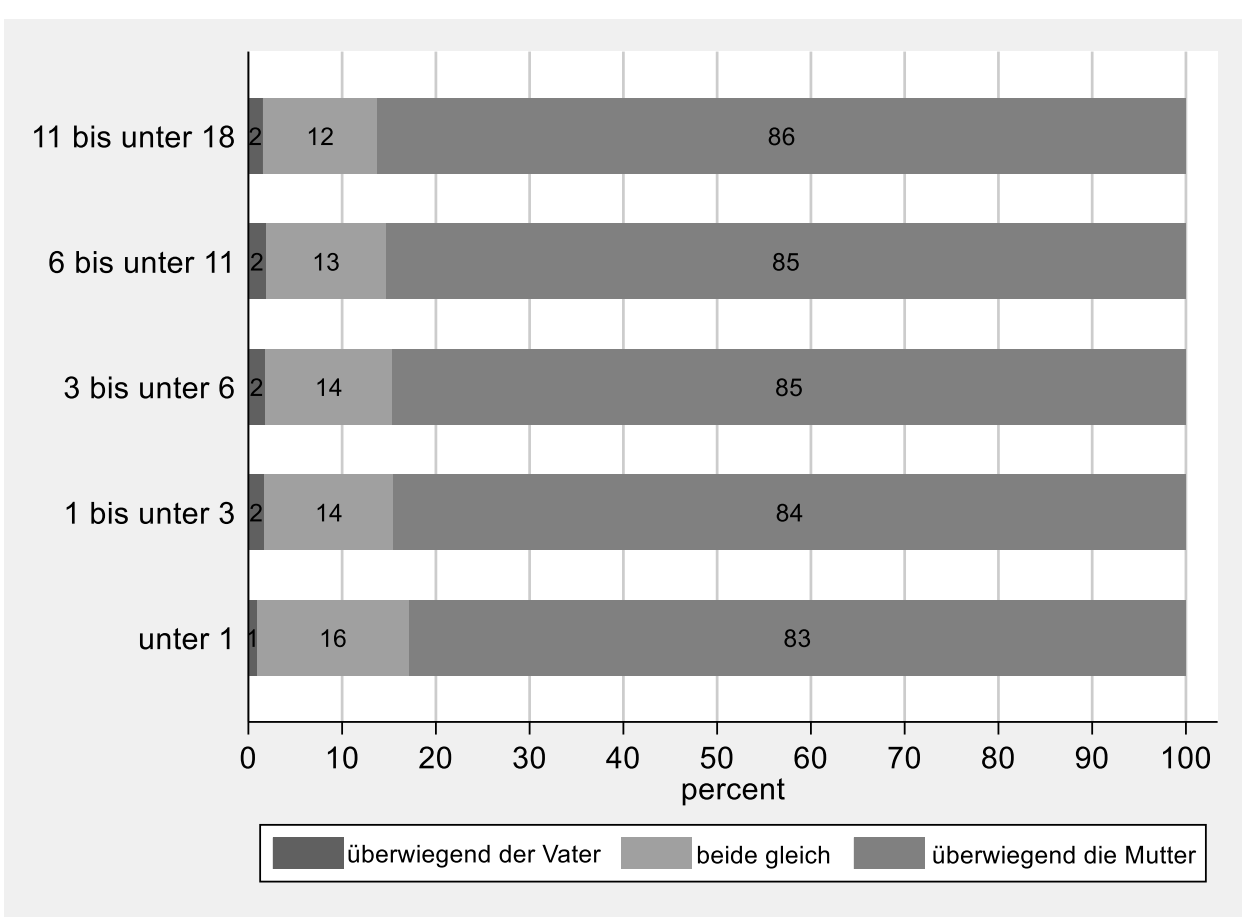

Quelle: DJI-Studie AID:A 2013/14, gewichtet, Mütterangaben, $n=10.900$, eigene Berechnungen.

Im Folgenden wird auf wichtige bivariate Zusammenhänge bzgl. der Aufteilung der Hausarbeit eingegangen. Die folgenden Abbildungen basieren auf bivariaten OLS-Regressionen und zeigen an, welche Aufteilung der Hausarbeit vorhergesagt wird (= Predictive Margins). Es ist wichtig zu berücksichtigen, dass die Werte der abhängigen Variable der Aufteilung der Hausarbeit insgesamt von „-1: Vater übernimmt alles“, über „0: egalitär/beide gleich“ bis hin zu „1: Mutter übernimmt alles“ gehen. Würde in den folgenden Abbildungen diese gesamte Skala der abhängigen Variable auf der y-Achse abgebildet werden, so wären die Zusammenhänge mit den unabhängigen Variablen kaum zu erkennen, da deren Effekte zu gering sind. Um die Zusammenhänge besser sichtbar zu machen, werden deshalb Ausschnitte der y-Achse gezeigt. Bei der Interpretation der Ergebnisse gilt es jedoch zu berücksichtigen, dass die gesamte Skala von „-1“ bis „1“ reicht.

Gemäß den theoretischen Annahmen ist zu erwarten, dass Ressourcen eine wichtige Rolle spielen. In Abbildung 9.4 sind die bivariaten Zusammenhänge zwischen dem relativen Berufsstatus und der Hausarbeit (links) bzw. dem absoluten Berufsstatus der Mutter und der Hausarbeit (rechts) abgetragen. Beim relativen Berufsstatus (ISEI) werden die höchsten Werte und damit die traditionellste Aufteilung der Hausarbeit dann vorhergesagt, wenn Mütter einen geringeren Berufsstatus haben als die Väter. Mit zunehmendem Alter des Kindes wird die Hausarbeit noch traditioneller aufgeteilt. Etwas niedrigere Werte und damit eine etwas egalitärere Aufteilung der Hausarbeit liegen dann vor, wenn beide Partner denselben Berufsstatus haben oder wenn Mütter einen höheren Berufs- 
status haben. Anhand der 95\%-Konfidenzintervalle wird jedoch ersichtlich, dass die Unterschiede in den meisten Fällen nicht signifikant sind. Eine Ausnahme stellt die Altersstufe „3 bis unter 6 Jahre“ dar: Hier ist die Hausarbeit bei Müttern mit gleichem oder höherem Status signifikant egalitärer als bei Müttern mit niedrigerem Status. Insgesamt sind die Unterschiede dennoch als gering einzuschätzen (insbesondere, wenn man berücksichtigt, dass die gesamte Skala der y-Achse von „-1“ bis „1“ geht). Etwas größere Unterschiede zeigen sich beim absoluten Berufsstatus der Mutter (ISEI): Hier ist in den meisten Altersstufen die Hausarbeitsaufteilung bei Müttern mit hohem Status signifikant egalitärer als bei Müttern mit niedrigem oder mittlerem Status.

Abbildung 9.4: Bivariate Zusammenhänge des relativen Berufsstatus (ISEI) und des absoluten Berufsstatus der Mutter (ISEI) mit der Aufteilung der Hausarbeit
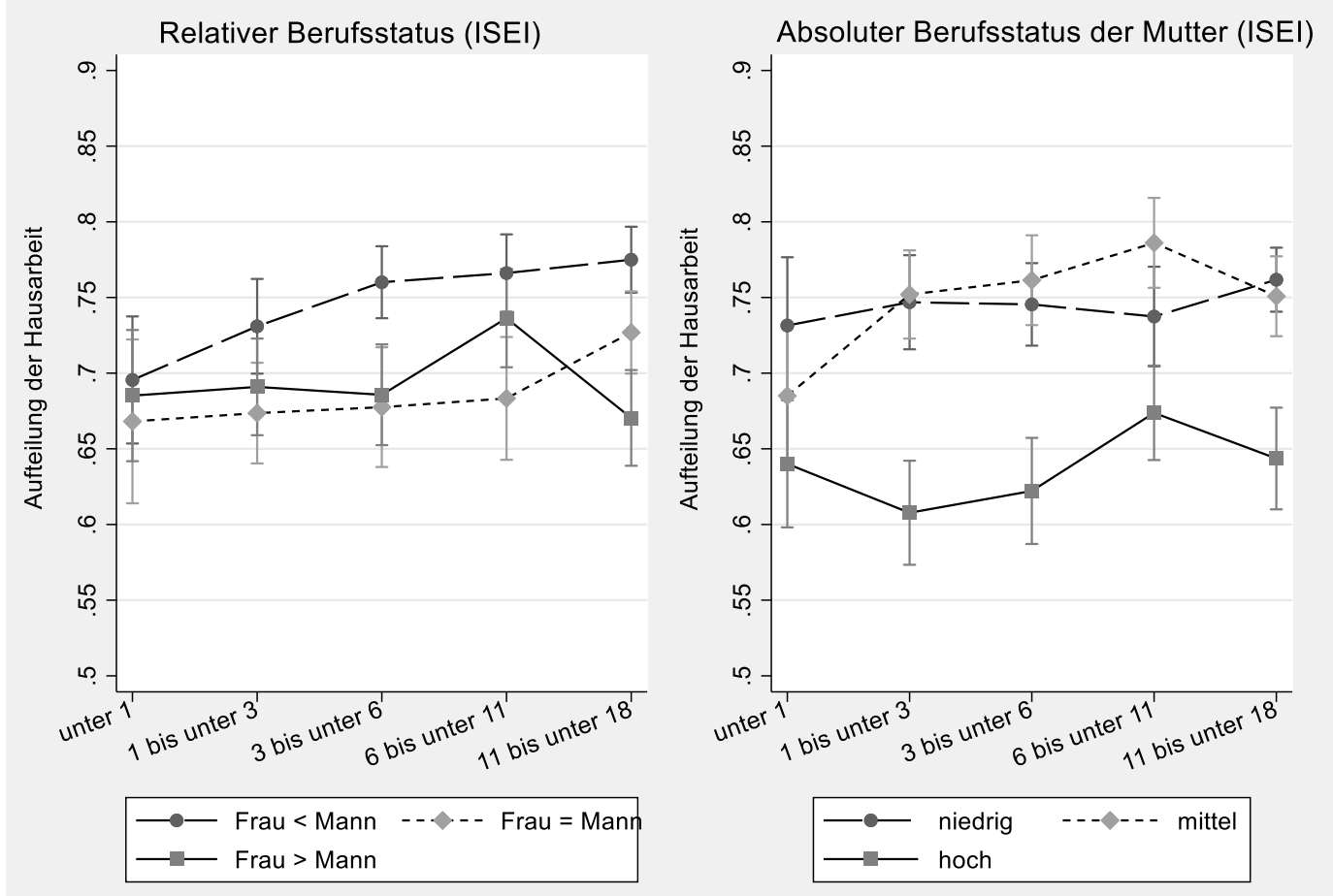

Anmerkung: Vorhergesagte Aufteilung der Hausarbeit (Predictive Margins) auf Basis von bivariaten Regressionsmodellen mit 95\%-Konfidenzintervallen; die y-Achse geht insgesamt von „-1: Vater übernimmt alles“, über „0: egalitär/beide gleich“ bis hin zu „1: Mutter übernimmt alles“. Quelle: DJI-Studie AID:A 2013/14, gewichtet, n (relativer Berufsstatus) = 10.074, n (absoluter Berufsstatus) $=10.391 ;$ eigene Berechnungen

Als nächstes wird ein Blick auf die Zusammenhänge zwischen den Gender-Items und der Aufteilung der Hausarbeit geworfen (Abbildung 9.5). ${ }^{36}$ Bei beiden Gender-Items zeigt sich, dass die Hausarbeitsaufteilung signifikant egalitärer ist, wenn egalitäre Einstellungen vertreten werden. Inhaltlich bedeutet dies folgendes: Wenn befürwortet wird, dass

${ }^{36}$ Die Skalen der Gender-Items sind ursprünglich 6-stufig, wurden hier jedoch für eine übersichtlichere Darstellung zu drei Kategorien zusammengefasst. 
beim Vorhandensein von kleinen Kindern nicht nur die Mutter, sondern auch der Vater in Teilzeit arbeitet (= egalitäre Einstellung zu Item 1), und wenn abgelehnt wird, dass in Familien nur der Vater erwerbstätig sein soll und die Mutter zu Hause bleiben soll (= egalitäre Einstellung zu Item 2), so geht dies mit einer egalitäreren Arbeitsteilung einher. Darüber hinaus lässt sich bei beiden Items und unabhängig davon, ob die Einstellung traditionell, neutral oder egalitär ausfällt, mit zunehmendem Alter des Kindes eine Traditionalisierung der Hausarbeit feststellen.

\section{Abbildung 9.5: Bivariate Zusammenhänge der Gender-Items mit der Aufteilung der Hausarbeit}

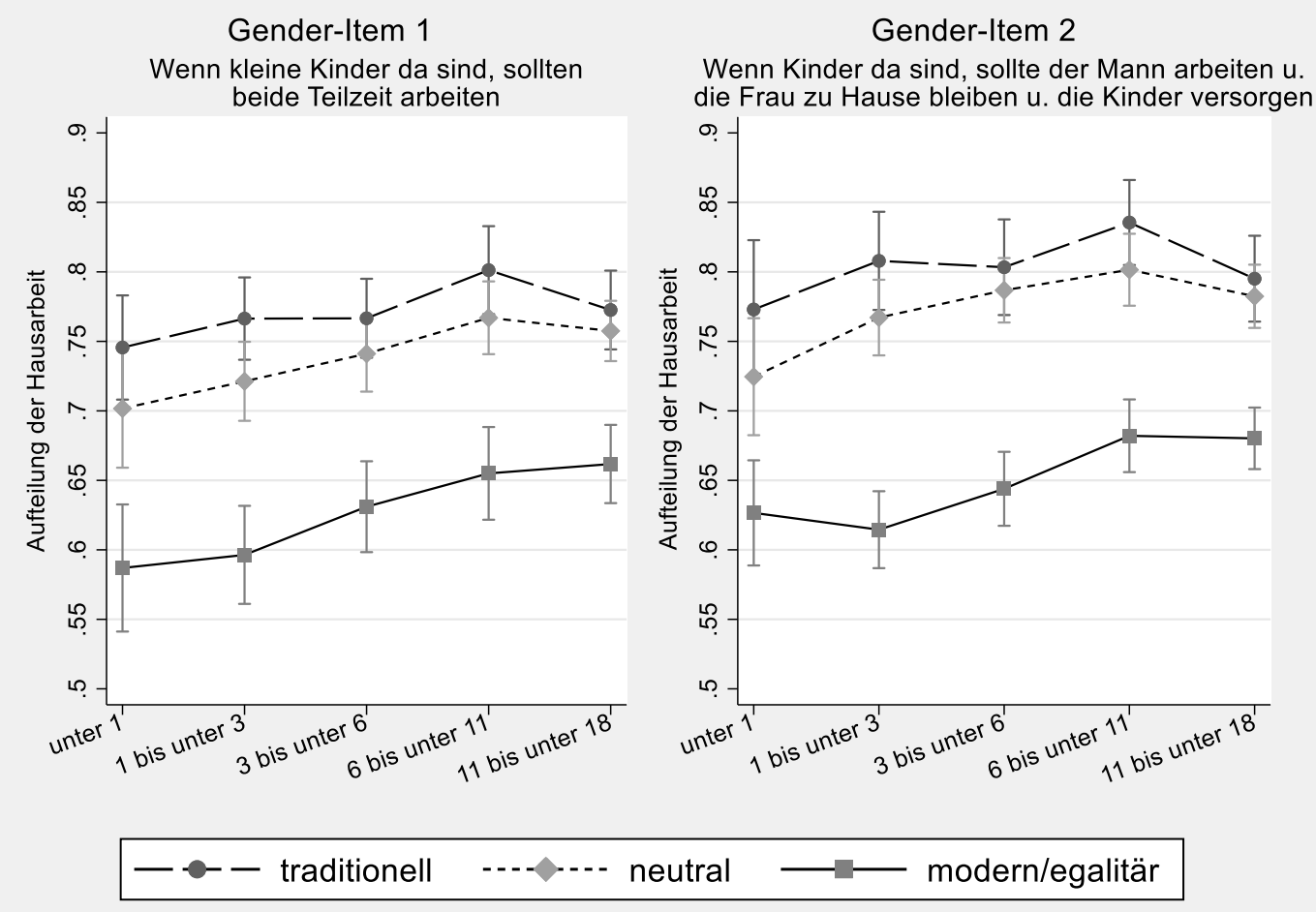

Anmerkung: Vorhergesagte Aufteilung der Hausarbeit (Predictive Margins) auf Basis von bivariaten Regressionsmodellen mit 95\%-Konfidenzintervallen; die y-Achse geht insgesamt von „-1: Vater übernimmt alles“, über „0: egalitär/beide gleich“ bis hin zu „1: Mutter übernimmt alles“. Quelle: DJI-Studie AID:A 2013/14, gewichtet, n (Gender-Item 1) = 10.807, n (Gender-Item 2) = 10.836; eigene Berechnungen.

Neben diesen beiden Items zu den Geschlechterrolleneinstellungen wurden auf Basis von insgesamt vier Einstellungsitems latente Klassen identifiziert, die als „intensiv mothering/parenting“, „egalitär" und „egalitärer Essentialismus“ beschrieben werden können (siehe Kapitel 7.5). Die vorhergesagte Aufteilung der Hausarbeit basierend auf einer bivariaten Regression mit den latenten Gender-Klassen ist in Abbildung 9.6 abgetragen. Die latenten Klassen unterscheiden sich in allen Altersstufen außer in der Altersstufe „unter 1 Jahr" signifikant voneinander: Am traditionellsten ist die Hausarbeit in der Klasse des „intensive mothering/parenting“ aufgeteilt, gefolgt von der Klasse des „egalitären Essentialismus“ und am egalitärsten ist die Aufteilung in der „egalitären“ Klasse. 
Abbildung 9.6: Bivariate Zusammenhänge der Gender-Klassen mit der Aufteilung der Hausarbeit

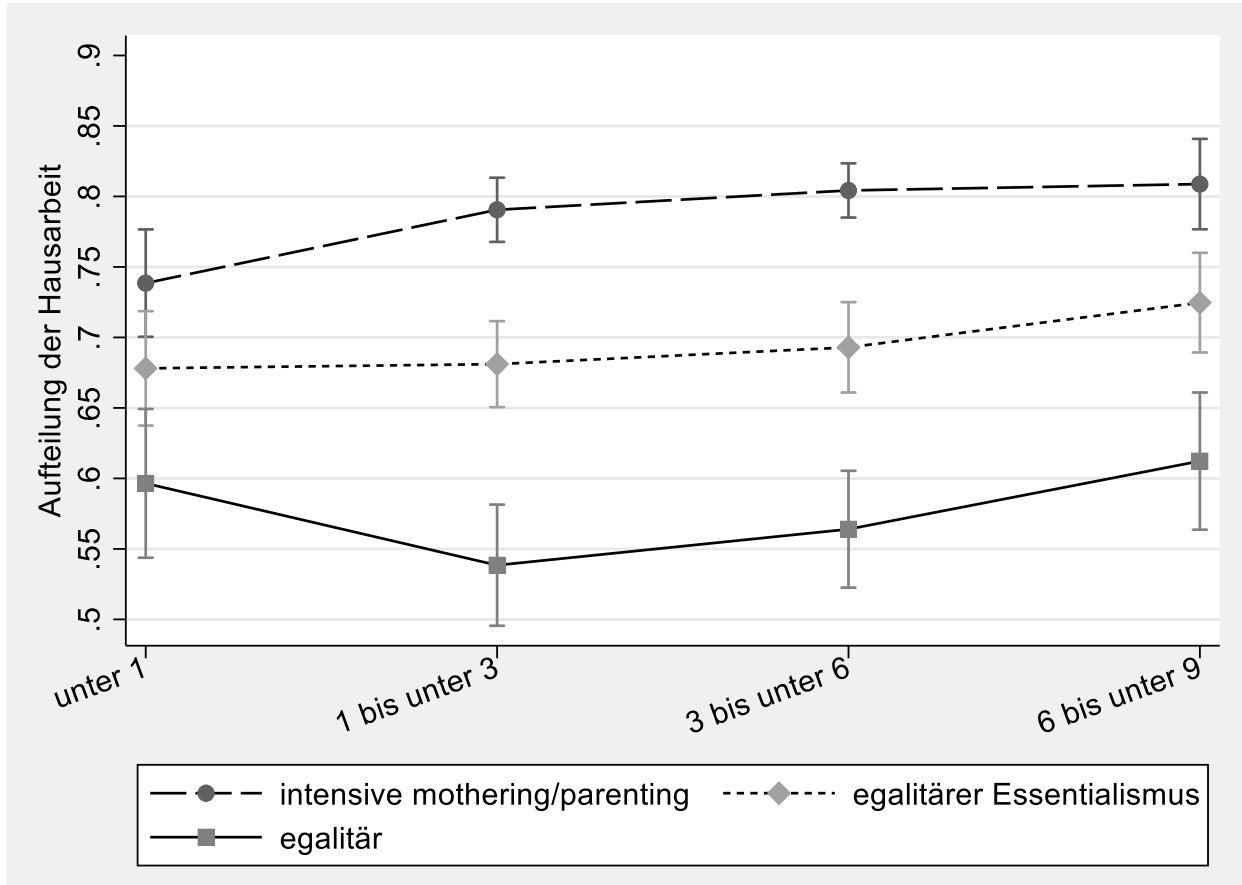

Anmerkung: Vorhergesagte Aufteilung der Hausarbeit (Predictive Margins) auf Basis von bivariaten Regressionsmodellen mit 95\%-Konfidenzintervallen; die y-Achse geht insgesamt von „-1: Vater übernimmt alles“, über „0: egalitär/beide gleich“ bis hin zu „1: Mutter übernimmt alles“. Quelle: DJI-Studie AID:A 2013/14, gewichtet, n (Gender-Klassen) = 7.181; eigene Berechnungen.

Abschließend zeigt Abbildung 9.7 je die bivariaten Zusammenhänge zwischen den OstWest-Unterschieden (links) bzw. der Erwerbskonstellation (rechts) und der Aufteilung der Hausarbeit. Bei den Unterschieden zwischen Ost- und Westdeutschland gibt es bei einem Kind im Alter von „unter 1 Jahr“ und von „1 bis 3 Jahren“ keine signifikanten Unterschiede, sondern erst in den nachfolgenden Altersstufen. Auch wenn die Hausarbeit in Ostdeutschland etwas egalitärer aufgeteilt wird, so fallen die Unterschiede dennoch ziemlich gering aus. Dagegen zeigen sich bei der Aufteilung der Hausarbeit in Abhängigkeit von der Erwerbskonstellation gravierende Unterschiede. ${ }^{37}$ Wenn beide Elternteile in Vollzeit erwerbstätig sind, dann ist die Hausarbeit deutlich egalitärer (wenn auch immer noch nicht egalitär) aufgeteilt; wenn die Mutter in Teilzeit und der Vater in Vollzeit beschäftigt ist, so ist die Hausarbeit traditioneller aufgeteilt; am traditionellsten ist die Hausarbeit dagegen dann aufgeteilt, wenn die Mutter nicht erwerbstätig und der Vater in

${ }^{37}$ Die Problematik der Erwerbsarbeit als unabhängige Variable für die Aufteilung der Hausarbeit wird bei den multivariaten Ergebnissen zur Aufteilung der Hausarbeit kritisch diskutiert. 
Vollzeit beschäftigt ist. ${ }^{38}$ Wie bereits bei einigen vorherigen, bivariaten Regressionsmodellen, zeigt sich auch hier eine Traditionalisierung der Hausarbeit mit zunehmendem Alter des Kindes. Diese Traditionalisierung ist am stärksten in der Erwerbskonstellationen zu beobachten ist, in der der Vater in Vollzeit beschäftigt und die Mutter nicht erwerbstätigt ist und am geringsten in der Konstellation, in der beide in Vollzeit arbeiten.

Abbildung 9.7: Bivariate Zusammenhänge der Ost-West-Unterschiede und der Erwerbskonstellation mit der Aufteilung der Hausarbeit
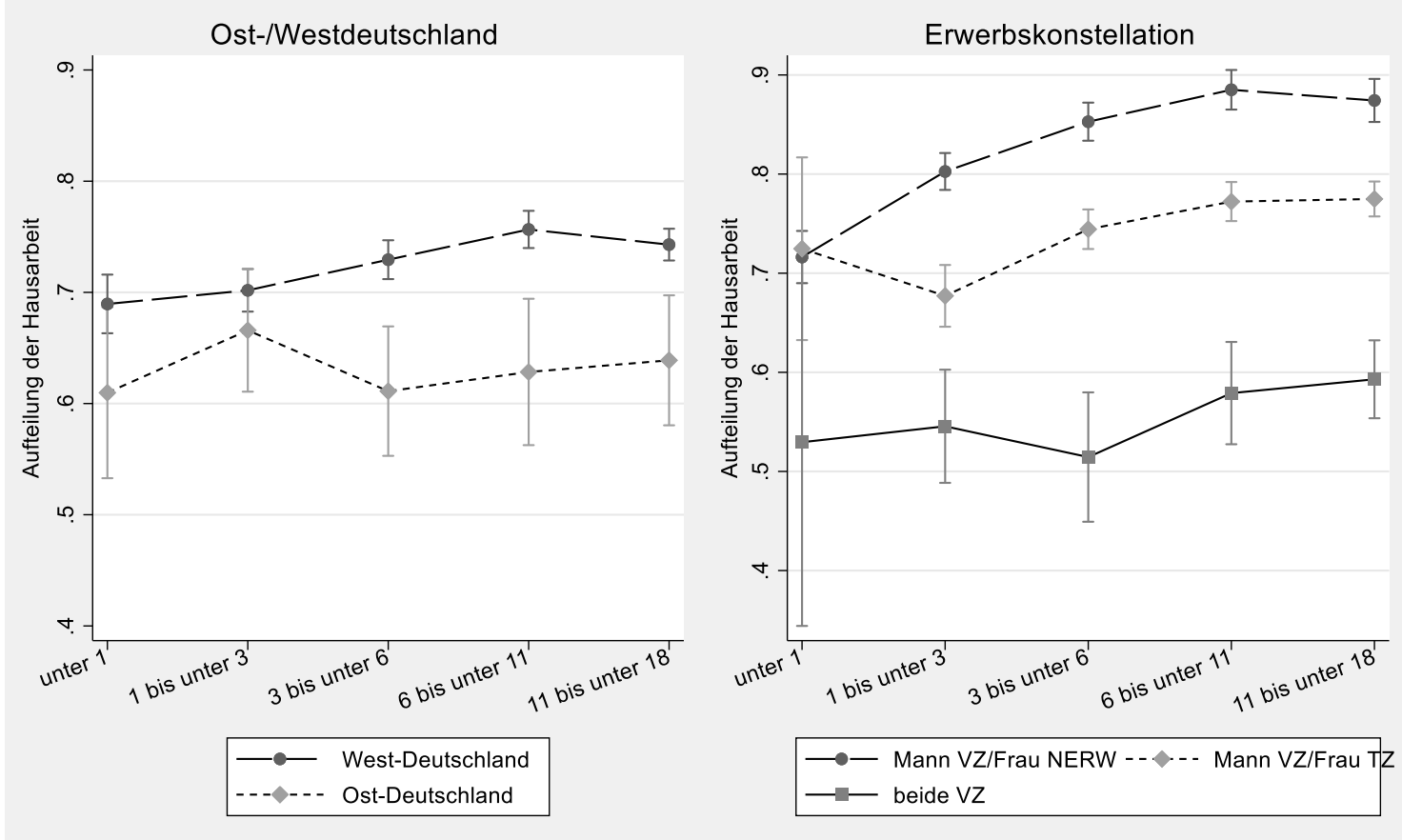

Anmerkung: Vorhergesagte Aufteilung der Hausarbeit (Predictive Margins) auf Basis von bivariaten Regressionsmodellen mit 95\%-Konfidenzintervallen; die y-Achse geht insgesamt von „-1: Vater übernimmt alles“, über „0: egalitär/beide gleich“ bis hin zu „1: Mutter übernimmt alles“. Quelle: DJI-Studie AID:A 2013/14, gewichtet, $n$ (Ost-/West-Dtl.) = 10.900, n (Erwerbskonstellation) = 9.905; eigene Berechnungen.

Insgesamt geht aus den bivariaten Ergebnissen zur Aufteilung der Hausarbeit hervor, dass der relative Berufsstatus und der absolute Berufsstatus der Mutter einen Beitrag zur Erklärung der Hausarbeit leisten, auch wenn die Effektstärken als klein einzuordnen sind. Einen etwas stärken Effekt haben die Gender-Items bzw. die latenten Gender-Klassen, aus denen hervorgeht, dass die Hausarbeit bei egalitäreren Einstellungen bzw. bei der Zugehörigkeit zur egalitären Klasse egalitärer aufgeteilt wird. Die Unterschiede zwischen den alten und den neuen Bundesländern sind zwar signifikant, fallen jedoch gering aus. Weitaus stärker sind dagegen die Zusammenhänge zwischen den Erwerbskonstellationen und der Aufteilung der Hausarbeit. Während in vielen Altersstufen

${ }^{38}$ Dass die Konfidenzintervalle in der Altersstufe „unter 1 Jahr" so groß ausfallen, ist auf die geringen Fallzahlen zurückzuführen: So gehören bei den unter 1-jährigen Kindern hier nur 3\% zur Kategorie „beide Vollzeit“ und nur 11\% zur Kategorie „Mann Vollzeit/Frau Teilzeit“. 
signifikante Effekte gegeben sind, stellt die unterste Altersstufe der unter 1-jährigen Kinder eine Ausnahme dar: Hier liegen bei den meisten Variablen noch keine signifikanten Effekte vor (ausgenommen sind etwa die beiden Gender-Items). Des Weiteren wird bei den meisten Ergebnissen ersichtlich, dass die Aufteilung der Hausarbeit mit zunehmendem Alter des Kindes traditioneller wird. Wie bereits weiter oben erwähnt, muss bei der Interpretation berücksichtigt werden, dass die gesamte Skala der Hausarbeitsaufteilung von „-1: Vater übernimmt alles“ über „0: egalitär“ bis „+1: Mutter übernimmt alles“ reicht. D.h., wenn die vorhergesagte Aufteilung der Hausarbeit anstelle von etwa 0,75 Werte von beispielsweise 0,59 annimmt (vgl. z.B. bei Gender-Item 1 den Unterschied zwischen traditionellen und egalitären Einstellungen), dann ist die Arbeitsteilung zwar egalitärer geworden, aber alles in allem immer noch traditionell aufgeteilt. Insgesamt ist die Hausarbeit somit sehr traditionell aufgeteilt und diese Ungleichheit verschärft sich mit zunehmendem Alter des Kindes. Diese bisherigen, bivariaten Ergebnisse werden in den nachfolgenden, multivariaten Analysen überprüft.

\subsection{Multivariate Analysen}

Bei den multivariaten Ergebnissen zur Aufteilung der Hausarbeit wird zunächst gesondert auf die Bedeutung des Alters des Kindes eingegangen. Um zu sehen, inwiefern sich die Altersstufen untereinander signifikant unterscheiden, werden sämtliche Altersstufen als Referenzkategorien getestet (siehe Abbildung 9.8, die auf Tabelle A.6 im Anhang beruht). Die abhängige Variable der Aufteilung der Hausarbeit kann dabei Werte zwischen „-1: Vater übernimmt alles“ über „0: beide übernehmen gleich viel“ bis hin zu „1: Mutter übernimmt alles" annehmen. Die Werte in Abbildung 9.8 bewegen sich in Abhängigkeit vom Kindesalter zwischen 0,67 und 0,74. D.h. Mütter übernehmen den Großteil der Hausarbeit, wenn sich das Kind im Alter vom „unter 1 Jahr“ befindet und mit zunehmendem Alter des Kindes verschärft sich die ungleiche Aufteilung der Hausarbeit zu Lasten der Mütter noch weiter. Auch wenn sich je die ersten beiden Altersstufen und die letzten beiden Altersstufen nicht signifikant voneinander unterscheiden (siehe die sich überlappenden Konfidenzintervalle in Abbildung 9.8; vgl. auch Tabelle A.6 im Anhang), so deuten die übrigen signifikanten Unterschiede dennoch darauf hin, dass mit zunehmendem Alter des Kindes eine Traditionalisierung der Hausarbeitsaufteilung einhergeht. Wie jedoch an dem korrigierten $\mathrm{R}^{2}$ von 0,01 ersichtlich wird, kann die Aufteilung der Hausarbeit unter Kenntnis des Kindesalters nur zu einem äußerst geringen Anteil erklärt werden. Während also die Aufteilung der Erwerbsarbeit sehr stark vom Alter des Kindes mitbestimmt wird, fallen die Zusammenhänge zwischen der Aufteilung der Hausarbeit und dem Alter des Kindes vergleichsweise gering aus. Des Weiteren unterscheiden sich die beiden Dimensionen der Arbeitsteilung darin, dass die Erwerbsarbeit mit zunehmendem Alter des Kindes egalitärer aufgeteilt wird, während bei der Hausarbeit ein höheres Kindesalter mit einer geschlechtsspezifischeren Aufteilung zusammenhängt. 
Abbildung 9.8: Vorhergesagte Aufteilung der Hausarbeit in Abhängigkeit vom Alter des Kindes

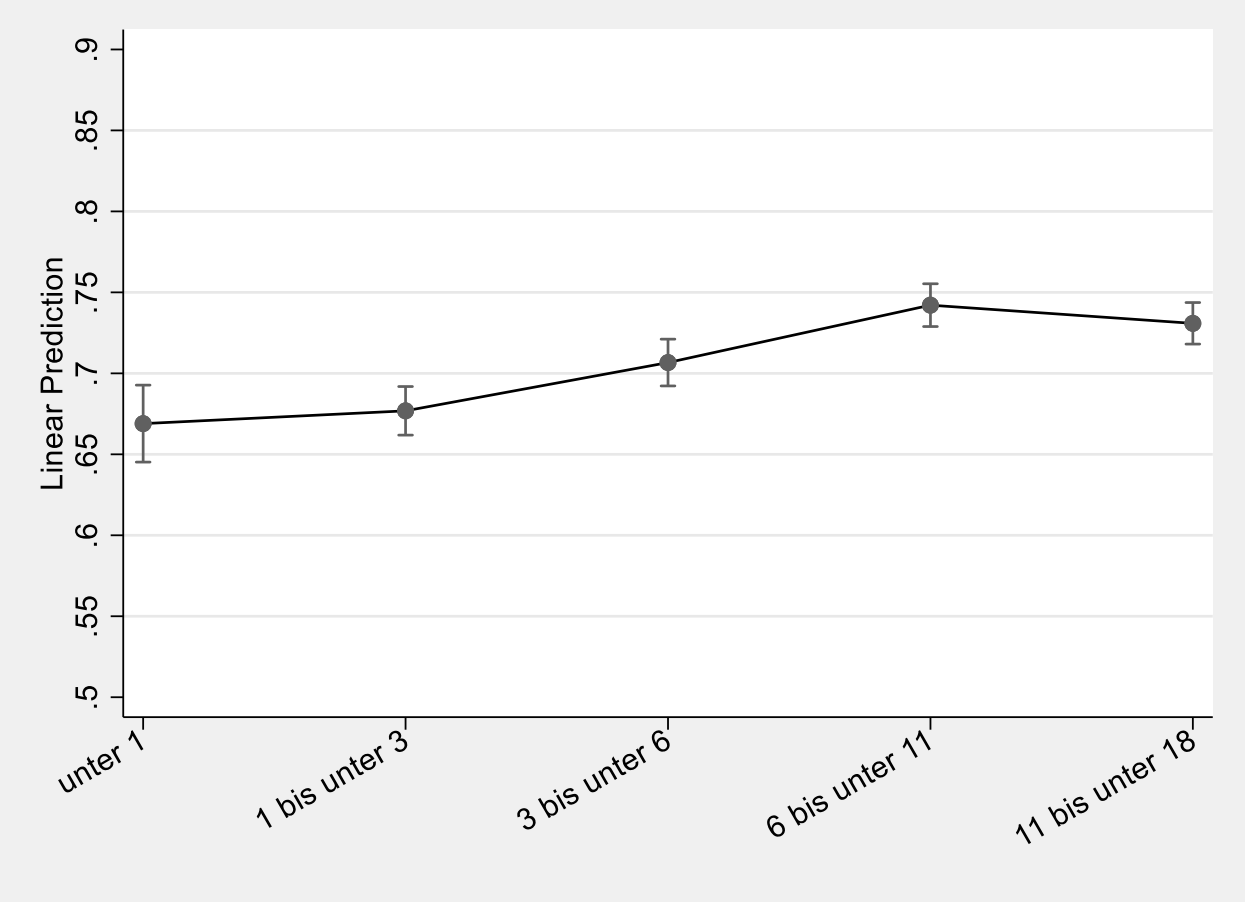

Anmerkung: Vorhergesagte Aufteilung der Hausarbeit (Predictive Margins) mit den 95\%-Konfidenzintervallen; die y-Achse geht insgesamt von „-1: Vater übernimmt alles“, über „0: egalitär/beide gleich“ bis hin zu „1: Mutter übernimmt alles“. Die Werte basieren auf der bivariaten OLS-Regression in Tabelle A.6 im Anhang. Quelle: DJI-Studie AID:A 2013/14, Sample: $n=$ 10.410, eigene Berechnungen.

Ein Blick auf Tabelle 9.2 gibt Aufschluss über weitere Einflussfaktoren, die stufenweise zum Gesamtmodell aufgebaut werden. Das erste Modell enthält nur das Alter des Kindes als unabhängige Variable und deutet, wie soeben erwähnt, daraufhin, dass die Hausarbeit mit zunehmendem Alter des Kindes traditioneller aufgeteilt wird. Das zweite Modell behandelt als unabhängige Variable den relativen Berufsstatus (ISEI). Gemäß Hypothese 1 spezialisiert sich diejenige Person mit den höheren, relativen Ressourcen auf die Erwerbsarbeit und die andere Person auf die Hausarbeit und Kinderbetreuung. Die Tatsache, dass Mütter mit einem niedrigeren relativen Berufsstatus im Vergleich zur Referenzkategorie mehr Hausarbeit übernehmen, bestätigt die theoretisch erwartete Richtung. Allerdings sind auch hier die Effektstärke und die Erklärungskraft mit einem korrigierten $\mathrm{R}^{2}$ von 0,01 sehr gering. Das Ergebnis, dass Frauen mit einem höheren, relativen Status nicht weniger Hausarbeit übernehmen als Frauen mit gleichem Status (= Referenzkategorie), widerspricht dagegen den theoretischen Erwartungen. Gleichzeitig widerspricht es auch den Annahmen von Hypothese 2 a), wonach diejenige Person mit den höheren Ressourcen mehr Verhandlungsmacht hat und sich demnach (zumindest teilweise) aus der unangenehmen Hausarbeit herausverhandeln kann. Hierbei sei noch angemerkt, dass sich die Ergebnisinterpretation bei einer anderen Wahl der Referenzkategorie anders gestaltet: So ist die Aufteilung der Hausarbeit bei Müttern mit höheren Ressourcen im Vergleich zu Müttern mit gleichen Ressourcen nicht egalitärer. Wenn 
man als Referenzkategorie jedoch Mütter mit niedrigeren Ressourcen wählen würde, so würde sich zeigen, dass Mütter mit höheren Ressourcen im Vergleich zu Müttern mit niedrigeren Ressourcen etwas weniger Hausarbeit übernehmen und die Hypothesen dann z.T. bestätigt werden können. ${ }^{39}$

Bei Modell 3 in Tabelle 9.2 sind Items zu Geschlechterrolleneinstellungen abgetragen, die nicht zu einem Index zusammengefasst werden, da sie nur eine geringe Korrelation haben (der Korrelationskoeffizient $r$ beträgt 0,18). Hinter Gender-Item 1 steckt die Frage danach, ob nicht nur die Frau, sondern auch der Mann in Teilzeit arbeiten soll, wenn kleine Kinder da sind. Wenn die Einstellungen der Mütter hierzu egalitärer werden, dann geht dies mit einer etwas egalitäreren Aufteilung der Hausarbeit einher. Auch bei Gender-Item 2, der Frage danach, ob beim Vorhandensein von Kindern der Mann arbeiten und die Frau zu Hause bleiben und die Kinder versorgen soll, zeigt sich ein ähnlicher Trend: Wenn die Einstellungen zu diesem Sachverhalt egalitärer werden, so geht auch dies mit einer egalitäreren Aufteilung der Hausarbeit einher - die aber, insgesamt betrachtet, immer noch ziemlich ungleich verteilt ist. Die beiden Gender-Items können zusammen immerhin 5\% der Varianz der Aufteilung der Hausarbeit erklären $\left(R^{2}=0,05\right)$, womit ihnen eine größere Bedeutung als dem Alter des Kindes und den relativen Ressourcen zukommt. Diese Ergebnisse bestätigen Hypothese 3 a) und stehen im Einklang mit bisherigen Studien, die gezeigt haben, dass egalitäre Einstellungen mit einer egalitäreren Aufteilung der Hausarbeit einhergehen: So übernehmen Frauen mit egalitären Einstellungen im Vergleich zu Frauen mit traditionellen Einstellungen weniger Hausarbeit und umgekehrt übernehmen Männer mit egalitären Einstellungen im Vergleich zu Männer mit traditionellen Einstellungen mehr Hausarbeit (Arrighi und Mauma 2000; Fuwa 2004; Cunningham 2005; Lewin-Epstein et al. 2006; Davis et al. 2007; Davis und Greenstein 2009; Treas und Drobnič 2010; Aassve et al. 2014; Evertsson 2014; Nitsche und Grunow 2016).

Modell 4 inkludiert alle inhaltlich relevanten Variablen und geht damit über die bivariaten Zusammenhänge in den Modellen 1 bis 3 hinaus. Durch das Zusammenführen zu einem Modell haben sich nahezu keine Veränderungen in den Koeffizienten ergeben. Modell 5 enthält neben diesen inhaltlich besonders wichtigen Variablen noch weitere Variablen wie die Anzahl der Kinder, das Bundesgebiet und den absoluten Berufsstatus (ISEI) der Mutter. In Modell 5 bleiben die Effekte des Alters des Kindes weiterhin signifikant und sind (beinahe) unverändert im Vergleich zu Modell 1. Die Effekte des relativen Status sind in Modell 5 etwas schwächer als in Modell 2, wobei die Signifikanz bei der Ausprägung „Frau < Mann“ weiterhin erhalten bleibt. Die Abschwächung des Effekts des relativen Status ist insbesondere daraufhin zurückzuführen, dass im Modell auch der absolute

\footnotetext{
39 Sobald jedoch die Erwerbstätigkeit der Mütter und Väter ins Modell mitaufgenommen wird, verschwindet der signifikante Effekt, sodass Mütter mit höheren Ressourcen nicht weniger Hausarbeit übernehmen als Mütter mit niedrigeren Ressourcen.
} 
Berufsstatus der Mutter enthalten ist. Die Effekte der beiden Gender-Items sind auch in Modell 5 noch stark vertreten: Bei Gender-Item 1 gab es keinerlei Veränderung im Vergleich zu Modell 3 und bei Gender-Item 2 hat sich die Effektstärke geringfügig verändert. Bei der Anzahl der Kinder zeigt sich, dass sich mit zunehmender Kinderanzahl der Anteil der Mütter an der Hausarbeit erhöht. Dies bestätigt Hypothese 5 a), die davon ausgeht, dass die Arbeitsteilung in Familien mit mehreren Kindern traditioneller ist als in Familien mit einem Kind und steht damit auch im Einklang zu bisheriger Forschung (Baxter et al. 2008; Kühhirt 2012; Fahlén 2016). Als weitere Variable wurde das Bundesgebiet mitaufgenommen. Die Ergebnisse zeigen, dass die Aufteilung der Hausarbeit in Ostdeutschland etwas egalitärer ist als in Westdeutschland. Dies bestätigt Hypothese $6 \mathrm{a}$ ), die von einer egalitäreren Arbeitsteilung in Ostdeutschland als in Westdeutschland ausgeht und bestätigt damit gleichzeitig die Ergebnisse bisheriger Studien (Cooke 2006; Wengler et al. 2008; Grunow 2014).

Die letzte Variable in Modell 5 beschreibt den Zusammenhang des absoluten Berufsstatus der Mutter und der Aufteilung der Hausarbeit. Die Ergebnisse zeigen, dass bei Müttern mit einem hohen und mit einem niedrigen Berufsstatus die Hausarbeit im Vergleich zur Referenzkategorie der Mütter mit einem mittleren Status egalitärer aufgeteilt ist. Dass ein hoher Berufsstatus der Mutter mit weniger Hausarbeit assoziiert ist, entspricht den theoretischen Erwartungen von Hypothese 7 a), aber dass dies auch bei einem niedrigen Status der Mutter zutreffend ist, steht konträr zu den theoretischen Erwartungen. Hypothese 7 a) kann damit nur eingeschränkt bestätigt werden.

Modell 6 enthält als weitere Variablen die Erwerbstätigkeit der Mutter und die Erwerbstätigkeit des Vaters. Bei der Interpretation der Ergebnisse ist besondere Vorsicht geboten, weil es sich bei der Erwerbstätigkeit um eine höchst endogene Variable handelt. Zunächst werden die Ergebnisse der Erwerbstätigkeit interpretiert und auch Bezug darauf genommen, inwiefern sich die anderen Variablen durch die Mitaufnahme der Erwerbstätigkeit verändern; anschließend wird die Integration der Erwerbstätigkeit als unabhängige Variable aufgrund ihrer Endogenität kritisch diskutiert.

Die Ergebnisse zeigen, dass Mütter, die in Teilzeit erwerbstätig sind, weniger Hausarbeit übernehmen als Mütter, die nicht erwerbstätig sind. Mütter, die in Vollzeit beschäftigt sind, übernehmen im Vergleich zu nicht-erwerbstätigen Müttern noch weniger Hausarbeit. Diese Ergebnisse stehen in Einklang zu bisherigen Studien (Gershuny et al. 2005; Cunningham 2007; Fuwa und Cohen 2007; Baxter et al. 2008; Hook 2010; Grunow et al. 2012; Zabel und Heintz-Martin 2013; Aassve et al. 2014; Dechant et al. 2014). Gleichzeitig zeigen die Ergebnisse jedoch auch, dass Mütter im Vergleich zu den Vätern noch immer mehr Hausarbeit übernehmen - selbst dann, wenn sie in Vollzeit beschäftigt sind. Zwischen Müttern, die in „normaler" Vollzeit (bis 45 Wochenstunden) erwerbstätig sind und Müttern, die mehr als 45 Stunden erwerbstätig sind, gibt es keine signifikanten Unterschiede in der Aufteilung der Hausarbeit. Wenn Väter in Teilzeit beschäftigt sind, anstatt nicht-erwerbstätig zu sein (= Referenzkategorie), dann erhöht sich der Anteil der 
Mütter an der Hausarbeit. Ebenso erhöht sich der Hausarbeitsanteil der Mütter, wenn Väter in „normaler“ Vollzeit erwerbstätig sind und noch mehr erhöht er sich, wenn Väter in besonders langer Vollzeit (mehr als 45 Wochenstunden) erwerbstätig sind.

Wie weiter oben bereits angedeutet, kann die Integration der Erwerbstätigkeit als unabhängige Variable problematisch sein. Im vorherigen Kapitel diente die Erwerbstätigkeit als abhängige Variable und es wurde ersichtlich, dass die Erwerbstätigkeit wesentlich durch das Alter des Kindes, durch das Bundesgebiet und durch viele weitere Variablen beeinflusst wird. Vergleicht man nun bei der Aufteilung der Hausarbeit, welche Rolle das Alter des Kindes vor und nach der Integration der Erwerbsarbeit als weitere unabhängige Variable spielt, so zeigen sich deutliche Unterschiede: Nach der Integration der Erwerbsarbeit kommt dem Alter des Kindes nicht eine geringere Rolle, sondern sogar eine höhere (!) Rolle zu, d.h. die Effektstärken sind größer geworden. Dabei handelt es sich um einen Suppressoreffekt. ${ }^{40}$ Bei allen weiteren Variablen sind die Effektstärken nach der Integration der Erwerbstätigkeit etwas geringer geworden, aber in der Regel noch weiterhin signifikant. Hinzu kommt, dass die Kausalitätsrichtung zwischen der Erwerbsarbeit und der Hausarbeit uneindeutig ist: Im hier vorliegenden Fall wird angenommen, dass die Aufteilung der Erwerbsarbeit die Aufteilung der Hausarbeit beeinflusst. Es ist jedoch ebenso denkbar, dass die Hausarbeit die Erwerbsarbeit beeinflusst. Mit den hier vorliegenden Querschnittsdaten und -analysen können keine Aussagen über die Kausalität vorgenommen werden. Ähnliche Probleme würde auch die Aufnahme des relativen Einkommens als unabhängige Variable für die Aufteilung der unbezahlten Arbeit mit sich bringen. Auch hier besteht das Problem darin, dass das Einkommen eine endogene Variable ist und beide Kausalitätsrichtungen denkbar sind (Carlson und Lynch 2017; Nitsche und Grunow 2018).

40 Üblicherweise ist die Effektstärke einer Variable in einem bivariaten Regressionsmodell am stärksten und wird durch die Aufnahme weiterer Variablen in das Regressionsmodell geringer. Bei Suppressorvariablen bzw. -effekten verhält es sich jedoch anders: „Wenn nun aus einer XVariablen gerade diejenigen Varianzanteile eliminiert werden, die zwar mit anderen X-Variablen aber nicht mit $Y$ zusammenhängen, gibt es für die bereinigte $X$-Variable einen besonders starken Zusammenhang mit $Y$, der sich auch in einer dementsprechend großen, geschätzten Einflussstärke niederschlägt. Solche unabh[ängigen] Variablen, die den ,wahren' Zusammenhang zwischen $X$ und $Y$ unterdrücken und diesen erst freigeben, wenn ihre Varianzanteile aus $X$ auspartialisiert werden, werden ,Suppressorvariablen' genannt“ (Urban und Mayerl 2018, S. 87, Hervorhebungen im Original). 
Tabelle 9.2: $\quad$ Aufteilung der Hausarbeit, stufenweiser Aufbau des Modells, OLS-Regression

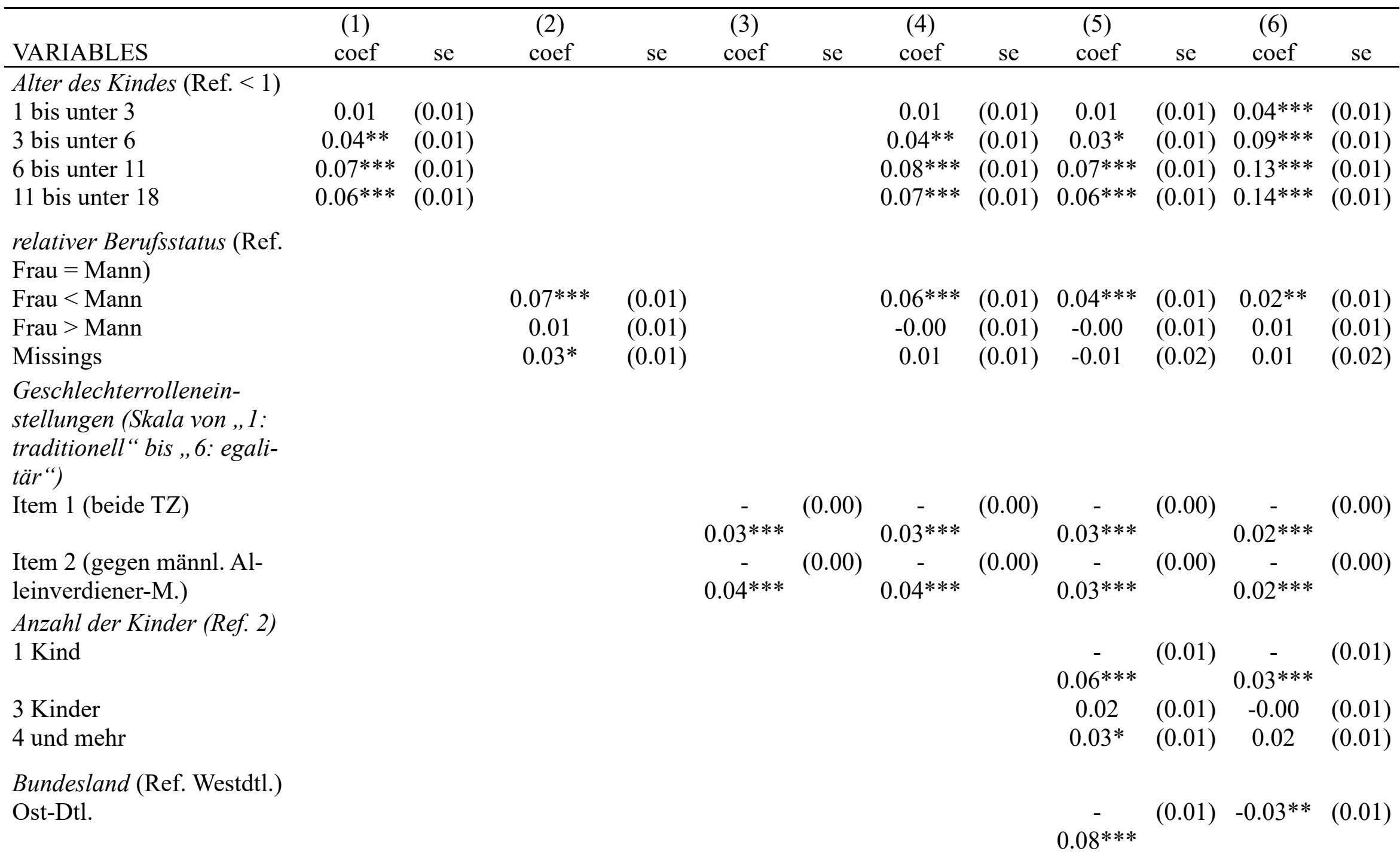




\begin{tabular}{|c|c|c|c|c|c|c|c|c|c|c|c|c|}
\hline & (1) & & (2) & & (3) & & (4) & & (5) & & (6) & \\
\hline VARIABLES & coef & $\mathrm{se}$ & coef & se & coef & se & coef & $\mathrm{se}$ & coef & se & coef & $\mathrm{se}$ \\
\hline \multicolumn{13}{|c|}{$\begin{array}{l}\text { ISEI der Mutter (Ref. mittel } \\
(45-62))\end{array}$} \\
\hline niedrig $(<45)$ & & & & & & & & & - & $(0.01)$ & - & $(0.01)$ \\
\hline & & & & & & & & & $0.04 * * *$ & & $0.03 * * *$ & \\
\hline hoch (ab 63) & & & & & & & & & $\stackrel{-}{-} 0.08^{* * *}$ & $(0.01)$ & $0.06^{-}$ & $(0.01)$ \\
\hline Missings & & & & & & & & & -0.02 & $(0.02)$ & -0.04 & $(0.02)$ \\
\hline $\begin{array}{l}\text { Erwerbstätigkeit de } \\
\text { (Ref. nicht erwerbst }\end{array}$ & & & & & & & & & & & & \\
\hline TZ (1-35 Std.) & & & & & & & & & & & $0.09^{-} * * *$ & $(0.01)$ \\
\hline VZ (36-45 Std.) & & & & & & & & & & & $\stackrel{-}{-}$ & $(0.01)$ \\
\hline mehr als VZ $(>45)$ & & & & & & & & & & & $0.27^{-} * * *$ & $(0.02)$ \\
\hline Missings & & & & & & & & & & & $\stackrel{-}{0.17^{* * *}}$ & $(0.03)$ \\
\hline $\begin{array}{l}\text { Erwerbstätigkeit de } \\
\text { (Ref. nicht erwerbst }\end{array}$ & & & & & & & & & & & & \\
\hline TZ (1-35 Std.) & & & & & & & & & & & $0.20 * * *$ & $(0.02)$ \\
\hline VZ (36-45 Std.) & & & & & & & & & & & $0.37 * * *$ & $(0.02)$ \\
\hline mehr als VZ $(>45)$ & & & & & & & & & & & $0.44 * * *$ & $(0.02)$ \\
\hline Missings & & & & & & & & & & & $0.17 * * *$ & $(0.04)$ \\
\hline Constant & $0.67 * * *$ & $(0.01)$ & $0.68 * * *$ & $(0.01)$ & $0.98 * * *$ & $(0.01)$ & $0.91 * * *$ & $(0.02)$ & $0.95 * * *$ & $(0.02)$ & $0.53 * * *$ & $(0.02)$ \\
\hline Observations & 11,157 & & 11,157 & & 11,157 & & 11,157 & & 11,157 & & 11,157 & \\
\hline Adj. $R^{2}$ & 0.01 & & 0.01 & & 0.05 & & 0.06 & & 0.08 & & 0.21 & \\
\hline
\end{tabular}

Anmerkung: OLS-Regression mit folgenden Signifikanzen: ${ }^{* *} p<0.001,{ }^{* *} p<0.01,{ }^{*} p<0.05$. Quelle: DJI-Studie AID:A 2013/14, Sample: $n=11.157$, eigene Berechnungen. 
Im Folgenden werden diese Zusammenhänge getrennt nach den Altersstufen des Kindes aufgezeigt (siehe Tabelle A.7 im Anhang). Die Koeffizienten der Gesamtmodelle (Modell 1c, 2c, 3c usw., Tabelle A.7 im Anhang) sind in den nachfolgenden Abbildungen dargestellt und liefern so eine gute Übersicht darüber, welche Koeffizienten in welchen Altersstufen signifikant sind und wie hoch ihre Effektstärken sind. ${ }^{41}$ Als erstes werden die Regressionsergebnisse des relativen Berufsstatus betrachtet: Während bei den bivariaten Regressionsmodellen (Modell 1a, 2a, 3a usw.) noch sehr viele Effekte signifikant waren, verlieren viele Effekte bei den Gesamtmodellen (Modell 1c, 2c, 3c usw.) ihre Signifikanz. Die folgende Abbildung 9.9 nimmt Bezug auf diese Gesamtmodelle und veranschaulicht die Koeffizienten des relativen Berufsstatus für die verschiedenen Altersstufen: In den Altersstufen „unter 1 Jahr" und „1 bis unter 3 Jahre“ kommt dem relativen Berufsstatus keine signifikante Bedeutung für die Aufteilung der Hausarbeit zu (siehe Modell 1c und 2c in Tabelle A.7 im Anhang sowie die entsprechenden Koeffizienten in Abbildung 9.9). In den beiden nachfolgenden Altersstufen, i.e. „3 bis unter 6 Jahre“ und „6 bis unter 11 Jahre“ übernehmen Mütter mit einem niedrigeren Berufsstatus signifikant mehr Hausarbeit im Vergleich zur Referenzkategorie, die Paare mit einem identischen Berufsstatus umfasst. Dagegen lässt sich in den besagten Altersstufen nicht nachweisen, dass Mütter mit einem höheren Status weniger Hausarbeit übernehmen, sondern dies ist erst dann der Fall, wenn das Kind im Alter von „11 bis unter 18 Jahren“ ist. (vgl. Abbildung 9.9).

\footnotetext{
${ }^{41}$ Die im folgenden vorgestellten Ergebnisse basieren auf den Modellen 1c, 2c, 3c, usw. in Tabelle A.7 im Anhang, bei denen aufgrund der soeben beschriebenen Problematik die Erwerbstätigkeit der Mütter und Väter noch nicht mitkontrolliert wird. Bei den Modellen 1d, 2d, 3d usw. werden die Erwerbstätigkeit der Mütter und Väter schon mitaufgenommen, um zu sehen, wie die Ergebnisse dann ausfallen.
} 
Abbildung 9.9: Koeffizienten des relativen Berufsstatus (ISEI) zur Vorhersage der Aufteilung der Hausarbeit, getrennt nach dem Alter des Kindes

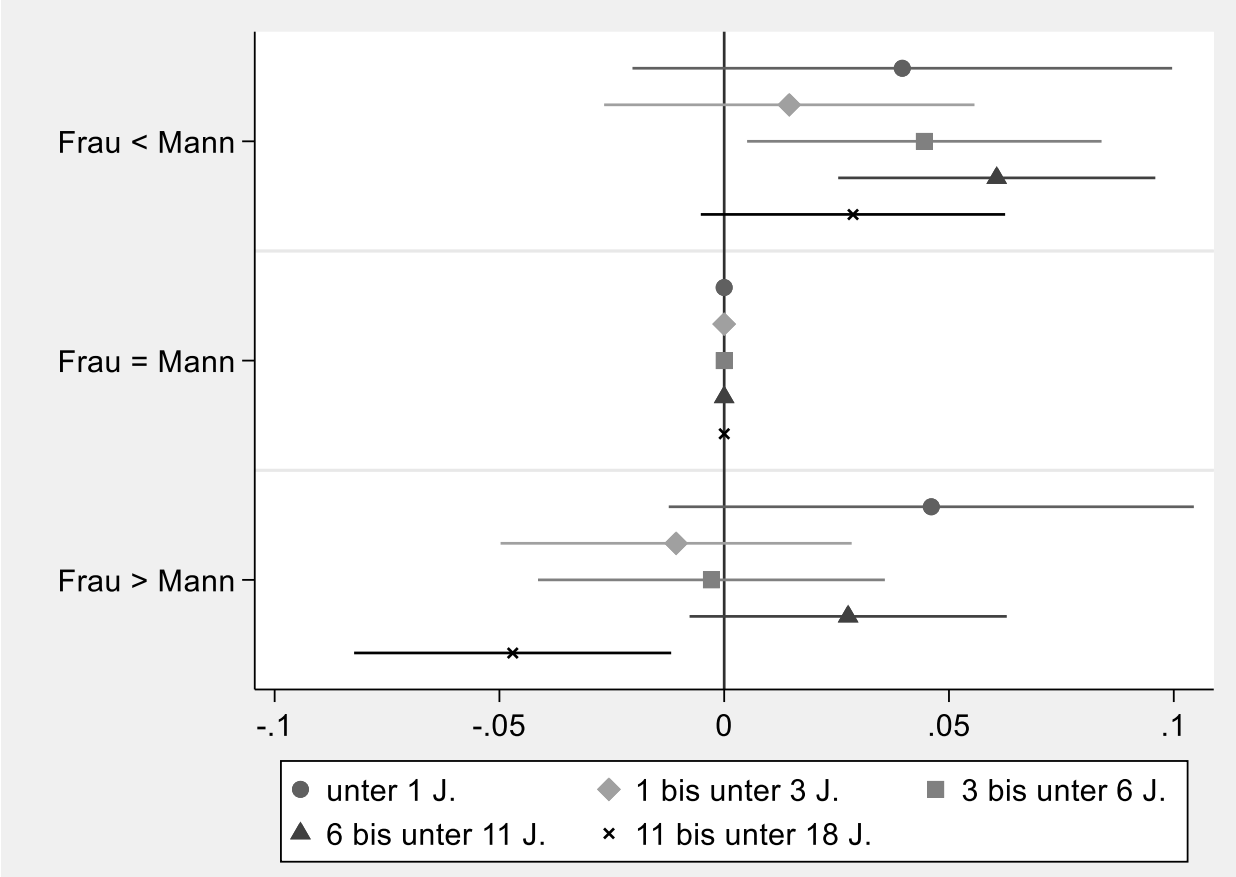

Anmerkung: OLS-Regression, dargestellt sind die Koeffizienten (Average Marginal Effects) mit 95\%-Konfidenzintervallen unter Kontrolle der weiteren Variablen aus Modell 1c, 2c, 3c usw. in Tabelle A.7 im Anhang. Quelle: DJI-Studie AID:A 2013/14, Sample: $n=11.157$, eigene Berechnungen.

Welche altersspezifischen Hypothesen zum relativen Berufsstatus (Hypothesen $2 \mathrm{~b}$ bis 2 e) lassen sich somit bestätigen? Die Hypothesen 2 b) resp. 2 c) nehmen an, dass bei einem Kindesalter von „unter 1 Jahr“ resp. von „1 bis unter 3 Jahren“ die relativen Ressourcen noch keine oder nur eine sehr geringe Rolle spielen. Da die Ergebnisse dies ebenso aufzeigen, können $\mathrm{H} 2$ b) und $\mathrm{H} 2$ c) daher bestätigt werden. Hypothese 2 d) nimmt an, dass die relativen Ressourcen bei einem Kindesalter von „3 bis unter 6 Jahren" bereits eine größere Rolle spielen als in den vorherigen Altersstufen. Auch dies lässt sich durch die empirischen Ergebnisse nachweisen, jedoch nur in die eine Richtung, dass sich bei einem niedrigeren Status der Mütter ihre Hausarbeit erhöht, und nicht, dass sich durch einen höheren Status ihre Hausarbeit reduziert. Hypothese 2 d) kann somit nur für eine Richtung bestätigt werden. Hypothese 2 e) nimmt an, dass ab einem Kindesalter von sechs Jahren, die Zusammenhänge zwischen dem relativen Berufsstatus und der Hausarbeitsaufteilung noch stärker zum Vorschein kommen. Tatsächlich sind die Koeffizienten bei der Ausprägung „Frau < Mann“ in der Altersstufe „6 bis unter 11 Jahren“ stärker ausgeprägt als in der Altersstufe „3 bis unter 6 Jahren“; da sich die Koeffizienten jedoch bei der nächsthöheren Altersstufe („11 bis unter 18 Jahre“) nicht weiter erhöhen, sondern wieder reduzieren, kann Hypothese 2 e) nur eingeschränkt bestätigt werden. Allerdings zeigt sich in dieser höchsten Altersgruppe mit einem Kind von „11 bis unter 18 Jahren" erstmalig eine signifikante Reduktion der Hausarbeit der Mütter, wenn 
sie einen höheren Berufsstatus haben. Dass dieser Effekt in den vorherigen Altersstufen noch nicht zum Vorschein gekommen ist, sondern dies erst in der höchsten Altersstufe tut, bestätigt gewissermaßen die Annahme von Hypothese 2 e). Dennoch sollten die Effektstärken des relativen Berufsstatus nicht überschätzt werden, da sie die Aufteilung der Hausarbeit nur zu einem sehr geringen Teil mitbestimmen.

Als nächstes wird zu den Bedeutungen der Geschlechterrolleneinstellungen übergegangen. Beim Gender-Item 1 (siehe Abbildung 9.10, oben), der Frage danach, ob auch der Mann in Teilzeit arbeiten soll, wenn kleine Kinder da sind, zeigen sich in allen Altersstufen signifikante Effekte, d.h. egalitärere Einstellungen führen in allen Altersstufen dazu, dass die Aufteilung der Hausarbeit egalitärer wird. Die Effektstärken von Gender-Item 1 (Abbildung 9.10) betragen in den ersten vier Altersstufen (gerundet) $-0,03$ und nur in der höchsten Altersstufe ist sie mit -0,02 etwas geringer. ${ }^{42}$ Die altersspezifischen Hypothesen 3 b) bis 3 e) nehmen an, dass egalitäre Einstellungen bei jüngeren Kindern schwerer zu realisieren sind und ihnen daher bei jüngeren Kindern eine geringere Bedeutung zukommt. Die Tatsache, dass die Effektstärken in vier der fünf Altersstufen gleich stark ausgeprägt sind, steht in Widerspruch zu diesen altersspezifischen Hypothesen. Vielmehr zeigt sich, dass die Effekte des ersten Gender-Items auf die Aufteilung der Hausarbeit weitgehend unabhängig vom Kindesalter sind.

\footnotetext{
42 In weiteren Modellen (nicht abgebildet) wurde anhand von Interaktionseffekten getestet, ob die Unterschiede der Effektstärken zwischen den Altersstufen des Kindes signifikant sind. Die Ergebnisse zeigen, dass diese Unterschiede nicht signifikant sind.
} 
Abbildung 9.10: Koeffizienten der Geschlechterrolleneinstellungen (Item 1 und 2) zur Vorhersage der Aufteilung der Hausarbeit, getrennt nach dem Alter des Kindes

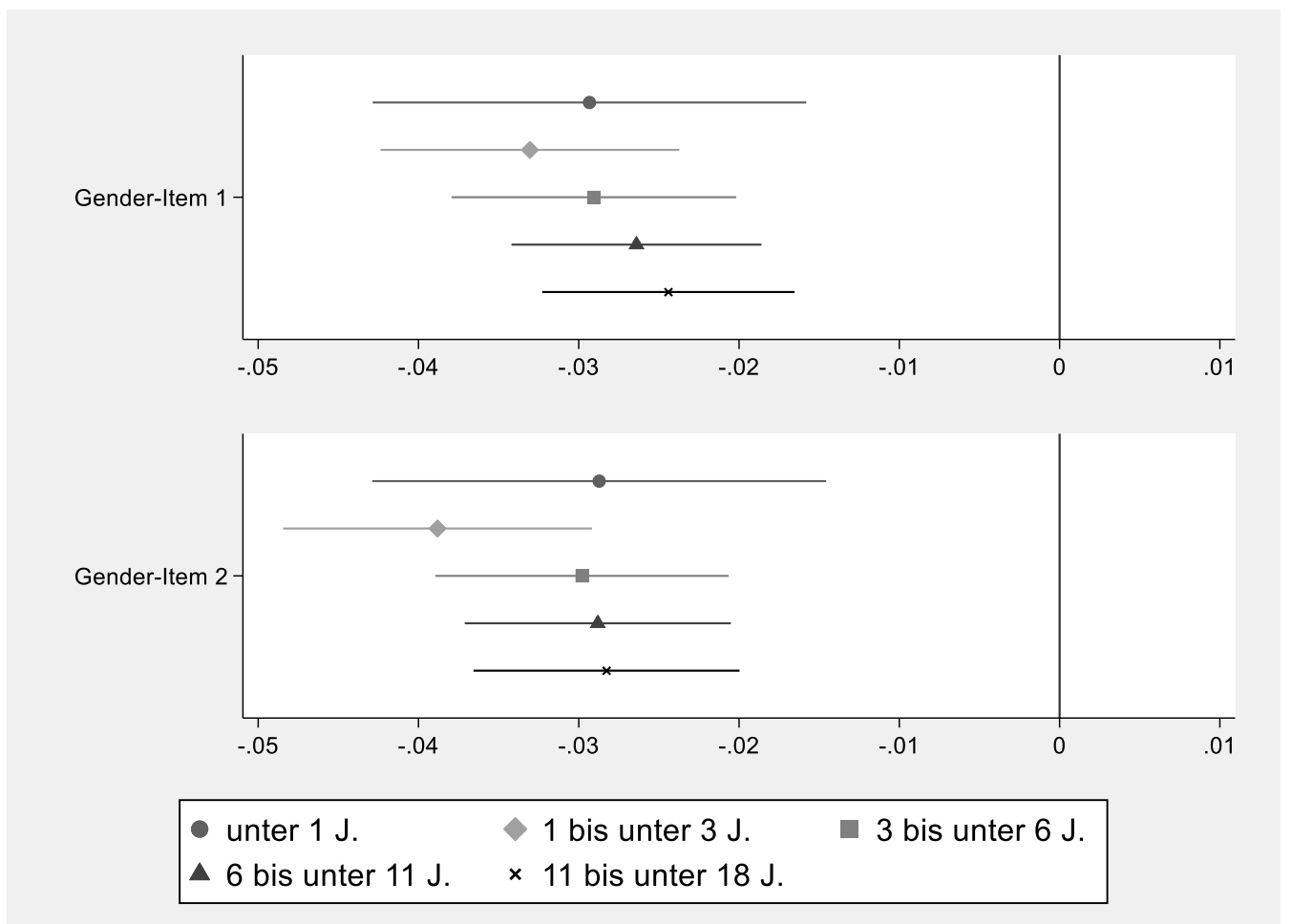

Anmerkung: OLS-Regression, dargestellt sind die Koeffizienten (Average Marginal Effects) mit 95\%-Konfidenzintervallen unter Kontrolle der weiteren Variablen aus Modell 1c, 2c, 3c usw. in Tabelle A.7 im Anhang; Gender-Item 1 = Befürwortung, dass beide Elternteile in Teilzeit arbeiten sollen, wenn kleine Kinder da sind; Gender-Item 2 = Ablehnung eines männlichen AlleinverdienerModells. Quelle: DJI-Studie AID:A 2013/14, Sample: $n=11.157$, eigene Berechnungen.

Beim nächsten Gender-Item (siehe Abbildung 9.10, unten), welches danach fragt, ob der Mann beim Vorhandensein vom Kindern arbeiten und die Frau zu Hause bleiben und die Kinder versorgen soll, zeigt sich eine sehr ähnliche Entwicklung: Die Effekte sind in allen Altersstufen signifikant und etwa gleich stark ausgeprägt. Zwar ist der Effekt in der Altersgruppe „1 bis unter 3 Jahre“ minimal stärker ausgeprägt, aber dieser ist laut weiteren Modellen, die Interaktionseffekte beinhalten und hier nicht abgebildet sind, nicht signifikant stärker. Somit lassen sich auch für Gender-Item 2 die altersspezifischen Hypothesen 3 b) bis 3 e) nicht bestätigen. Es lässt sich daher allgemein festhalten, dass sich der Anteil der Mütter an der Hausarbeit durch egalitäre Einstellungen verringert, was eine Bestätigung der Hypothese 3 a) gleichkommt; jedoch variiert der Effekt nicht in Abhängigkeit vom Alter des Kindes, sodass die altersspezifischen Hypothesen zurückgewiesen werden müssen.

Als nächstes wird der Einfluss der Kinderanzahl auf die Aufteilung der Hausarbeit untersucht und ob sich dieser Einfluss je nach Alter des jüngsten Kindes unterscheidet. In Abbildung 9.11 wird ersichtlich, dass Mütter mit einer höheren Kinderanzahl tendenziell mehr Hausarbeit übernehmen als Mütter mit einem Kind. Wenn das Kind unter einem Jahr alt ist (siehe Abbildung 9.11), dann übernehmen Mütter mit einem Kind signifikant 
weniger Hausarbeit als Mütter mit zwei Kindern; jedoch gibt es in dieser Altersgruppe keine signifikanten Unterschiede zwischen Familien mit zwei, drei und vier Kindern. In der nächsten Altersstufe, in der das Kind „1 bis unter 3 Jahre“ alt ist, zeigt sich nicht nur, dass Mütter von einem Kind signifikant weniger Hausarbeit übernehmen als Mütter von zwei Kindern, sondern dass darüber hinaus Mütter von drei oder mehr Kindern noch mehr Hausarbeit übernehmen als Mütter von zwei Kindern. In den höheren Altersstufen des Kindes bleiben zwar die Unterschiede zwischen Ein- und Zweikindfamilien signifikant, aber nicht die Unterschiede zwischen Zwei- und Mehrkindfamilien, sodass sich festhalten lässt: Bei Kindern im Alter von „1 bis unter 3 Jahren“ hängt die Aufteilung der Hausarbeit stark mit der Kinderanzahl zusammen; bei Kindern ab einem Alter von drei Jahren kommt der Kinderanzahl nur noch eine sehr geringe Bedeutung zu.

Abbildung 9.11: Koeffizienten der Kinderanzahl zur Vorhersage der Aufteilung der Hausarbeit, getrennt nach dem Alter des Kindes

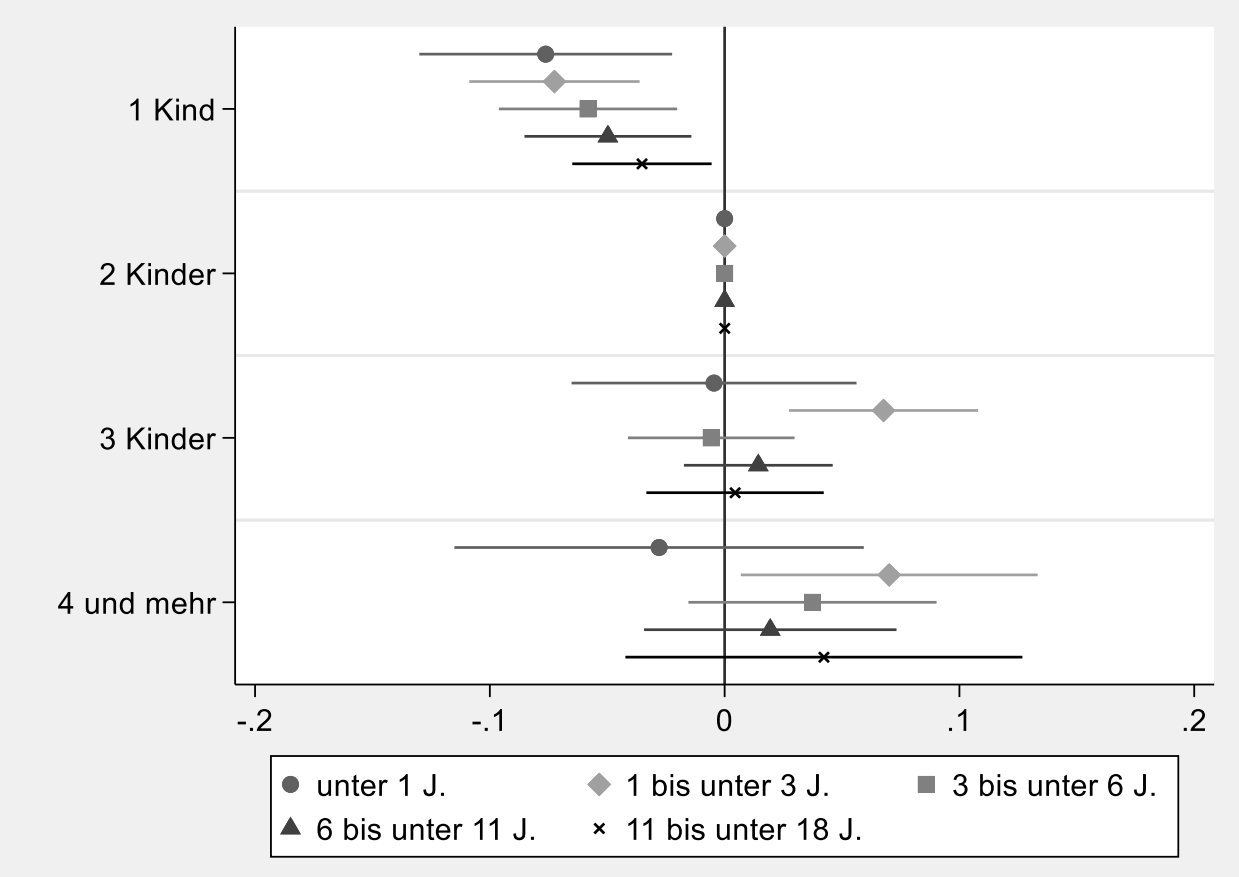

Anmerkung: OLS-Regression, dargestellt sind die Koeffizienten (Average Marginal Effects) mit 95\%-Konfidenzintervallen unter Kontrolle der weiteren Variablen aus Modell 1c, 2c, 3c usw. in Tabelle A.7 im Anhang. Quelle: DJI-Studie AID:A 2013/14, Sample: $n=11.157$, eigene Berechnungen.

Was bedeutet dies hinsichtlich der Hypothesen? Hypothese 5 a) nimmt an, dass die Arbeitsteilung in Familien mit mehreren Kindern traditioneller ist als in Familien mit einem Kind. Dies lässt sich insofern bestätigen, dass es große Unterschiede zwischen Zweiund Einkindfamilien gibt, allerdings gibt es in einigen Altersstufen keinen gravierenden Unterschied zwischen Familien mit zwei und Familien mit mehr als zwei Kindern. Die altersspezifische Hypothese 5 b) nimmt an, dass die Unterschiede zwischen Ein-, Zweiund Mehrkindfamilien dann besonders groß sind, wenn das Kind noch klein ist und dass 
die Unterschiede mit zunehmendem Kindesalter unwichtiger werden. Dies lässt sich bestätigen, da - wie soeben erwähnt - die Unterschiede bei Kindern unter drei Jahre größer sind und ab einem Alter von drei Jahren geringer werden.

Nach der Kinderanzahl wird nun auf die Bedeutung des Bundesgebietes eingegangen (siehe Abbildung 9.12). Insgesamt ist die Hausarbeit in Ostdeutschland etwas egalitärer aufgeteilt als in Westdeutschland, womit Hypothese 6 a), die dieses annimmt, bestätigt wird. Allerdings zeigen sich Unterschiede in den Altersstufen, was auf die altersspezifische Hypothese 6 b) verweist: Hypothese 6 b) geht davon aus, dass die Unterschiede in der Arbeitsteilung zwischen Ost- und Westdeutschland insbesondere dann sehr groß sind, wenn die Kinder noch klein sind und mit zunehmendem Alter des Kindes schwächer werden. Die empirischen Ergebnisse zeigen jedoch den gegenteiligen Effekt: Wenn das Kind „unter 1 Jahr" oder „1 bis unter 3 Jahre“ alt ist, so zeigen sich keine signifikanten Unterschiede zwischen Ost- und Westdeutschland, sondern diese treten erst ab einem Kindesalter von 3 Jahren auf. Hypothese 6 b) kann daher nicht bestätigt werden.

Abbildung 9.12: Koeffizienten des Bundesgebiets zur Aufteilung der Hausarbeit, getrennt nach dem Alter des Kindes

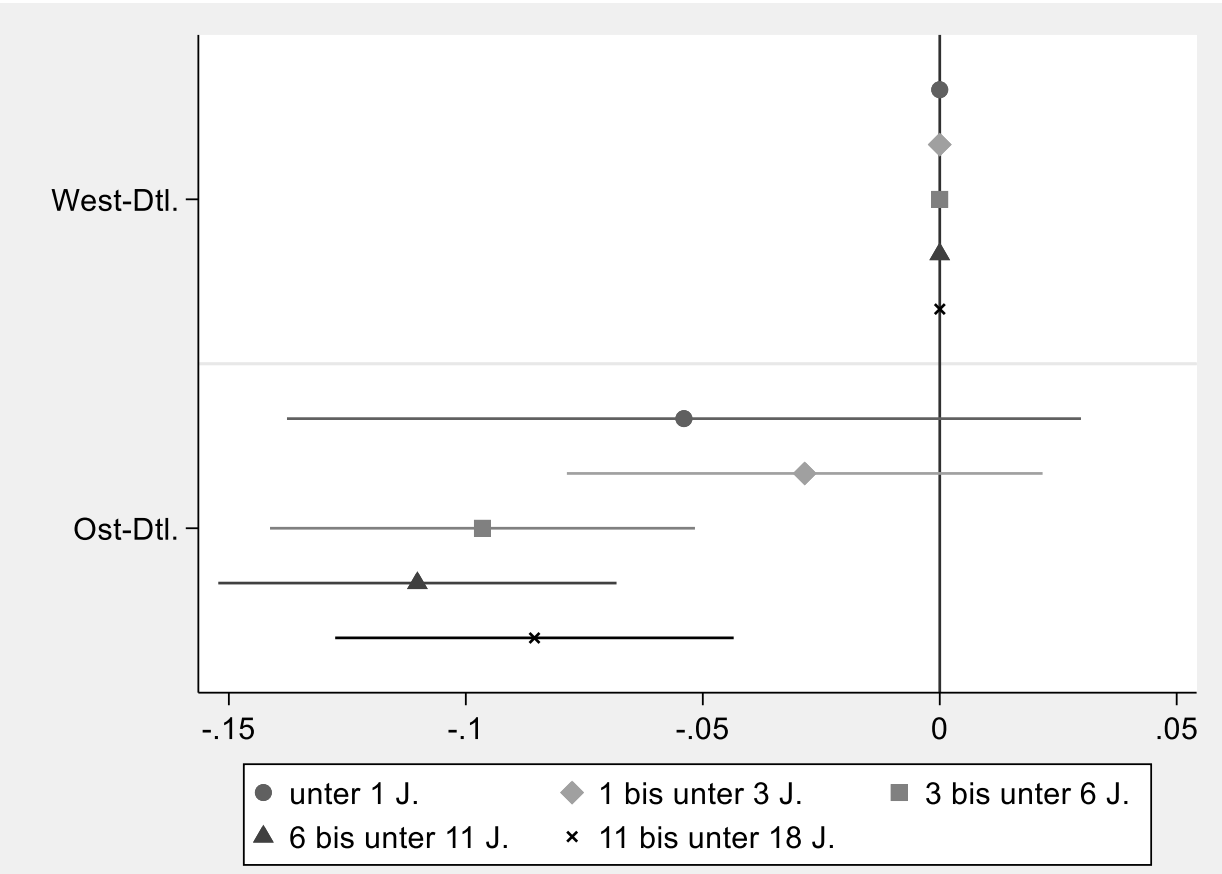

Anmerkung: OLS-Regression, dargestellt sind die Koeffizienten (Average Marginal Effects) mit 95\%-Konfidenzintervallen unter Kontrolle der weiteren Variablen aus Modell 1c, 2c, 3c usw. in Tabelle A.7 im Anhang. Quelle: DJI-Studie AID:A 2013/14, Sample: $n=11.157$, eigene Berechnungen.

Als weitere Variable wird die Bedeutung des absoluten Berufsstatus der Mutter (ISEI) für die Aufteilung der Hausarbeit betrachtet (siehe Abbildung 9.13). Es zeigt sich, dass bei einem Kindesalter von „unter 1 Jahr“ die Unterschiede noch nicht signifikant sind. In allen weiteren Altersstufen geht ein hoher absoluter Berufsstatus der Mutter jedoch mit einer egalitäreren Aufteilung der Hausarbeit einher (Referenzkategorie: mittlerer Berufsstatus 
der Mutter). Dies bestätigt einerseits Hypothese 7 a), die annimmt, dass bei einem hohem Berufsstatus die Arbeitsteilung egalitärer ist. Andererseits zeigen die Ergebnisse auch, dass bei Müttern mit einem niedrigem Berufsstatus die Hausarbeit (im Vergleich zur Referenzkategorie der Mütter mit einem mittleren Status) egalitärer ist. Dies steht jedoch im Widerspruch zu Hypothese 7 a), die des Weiteren davon ausgeht, dass die Arbeitsteilung bei Müttern mit einem niedrigen Status traditioneller sein müsste. ${ }^{43}$ Die altersspezifische Hypothese $7 \mathrm{~b}$ ) nimmt darüber hinaus an, dass die Unterschiede bei kleinen Kindern noch sehr gering sind und sich mit zunehmendem Alter des Kindes verstärken. Dies kann nicht bestätigt werden. Zwar sind die Unterschiede zwischen Müttern mit hohem Status und Müttern mit mittlerem Status bei einem Kind im Alter von „unter 1 Jahr" noch nicht signifikant, während dies bei über 1-jährigen Kindern schon der Fall ist, allerdings kommt es in den nachfolgenden Altersstufen zu keinem weiteren Anstieg der Effektstärke.

\footnotetext{
${ }^{43}$ Als mögliche Erklärung für dieses ungewöhnliche Ergebnis wurde die Tatsache in Betracht gezogen, dass in dem Modell gleichzeitig der relative und der absolute Berufsstatus enthalten sind. Es wurde deshalb auch kontrolliert, wie die Ergebnisse ausfallen, wenn nur eine der beiden Variablen im Modell enthalten ist. Es zeigt sich jedoch, dass auch dann, wenn der relative Status nicht enthalten ist, Mütter mit niedrigem, absolutem Status weniger Hausarbeit übernehmen (die Hausarbeit also egalitärer ist) im Vergleich zu Müttern mit mittlerem, absolutem Status. Das Ergebnis kann somit nicht auf Multikollinearität zurückgeführt werden. Auch anhand der VIF-Werte (Variance Inflation Factor), die deutlich kleiner als 10 sind, wird ersichtlich, dass keine Multikollinearität vorliegt und die gleichzeitige Aufnahme des relativen und absoluten Status daher kein Problem verursacht.
} 
Abbildung 9.13: Koeffizienten des Berufsstatus der Mutter zur Vorhersage der Aufteilung der Hausarbeit, getrennt nach dem Alter des Kindes

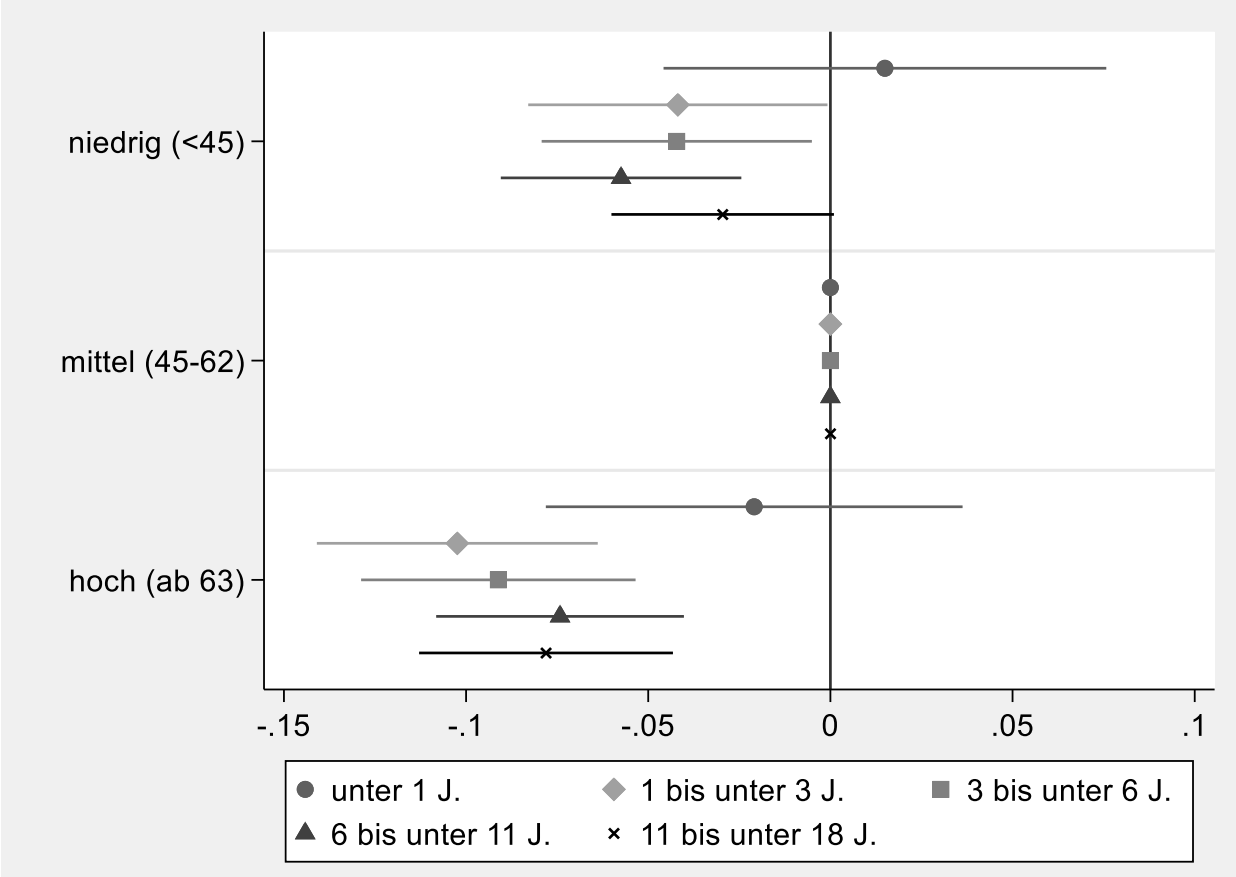

Anmerkung: OLS-Regression, dargestellt sind die Koeffizienten (Average Marginal Effects) mit 95\%-Konfidenzintervallen unter Kontrolle der weiteren Variablen aus Modell 1c, 2c, 3c usw. in Tabelle A.7 im Anhang. Quelle: DJI-Studie AID:A 2013/14, Sample: $n=11.157$, eigene Berechnungen.

Als letzte Variablen werden die Erwerbstätigkeit der Mütter und der Väter als unabhängige Variablen betrachtet. ${ }^{44}$ Bei der Erwerbstätigkeit der Mütter zeigt sich, dass ein hoher Erwerbsumfang mit negativen Koeffizienten einhergeht, d.h. erwerbstätige Mütter übernehmen im Vergleich zur Referenzkategorie der nicht-erwerbstätigen Mütter weniger Hausarbeit (siehe Abbildung 9.14). Dabei zeigen sich in allen Altersstufen des Kindes mit Ausnahme der „unter 1-Jährigen“ - signifikante Effekte. Wenn Mütter in Vollzeit erwerbstätig sind, sind die Effektstärken größer, als wenn Mütter in Teilzeit erwerbstätig sind. D.h. die Hausarbeit ist bei in Vollzeit beschäftigen Müttern egalitärer aufgeteilt als bei in Teilzeit beschäftigten Müttern. Wenn Mütter mehr als 45 Erwerbsstunden pro Woche verrichten, so sind die Effektstärken lediglich gleich hoch wie bei der „normalen“ Vollzeit. Insgesamt zeigen die Ergebnisse dennoch, dass ein höherer Erwerbsumfang der Mütter mit einer egalitäreren Aufteilung der Hausarbeit einhergeht. Berücksichtigt man zusätzlich Interaktionseffekte zwischen der Erwerbstätigkeit der Mütter und dem Alter des Kindes (Interaktionsmodell nicht abgebildet), so zeigt sich, dass bei einer in Vollzeit beschäftigten Mutter die Effekte bei einem unter 1-jährigen Kind signifikant

\footnotetext{
${ }^{44}$ Auf die Probleme der Endogenität wurde bereits an früherer Stelle bei den Erläuterungen zu Tabelle 9.2 eingegangen, sodass hierauf an dieser Stelle verzichtet wird.
} 
schwächer sind als bei Kindern, die zwischen drei und unter 18 Jahre alt sind; des Weiteren sind die Effekte einer Vollzeit erwerbstätigen Mutter bei einem Kind im Alter von „6 bis unter 11 Jahren“ signifikant stärker als bei einem Alter von „3 bis unter 6 Jahren“ ${ }^{45}$

Abbildung 9.14: Koeffizienten der Erwerbstätigkeit der Mutter zur Vorhersage der Aufteilung der Hausarbeit, getrennt nach dem Alter des Kindes

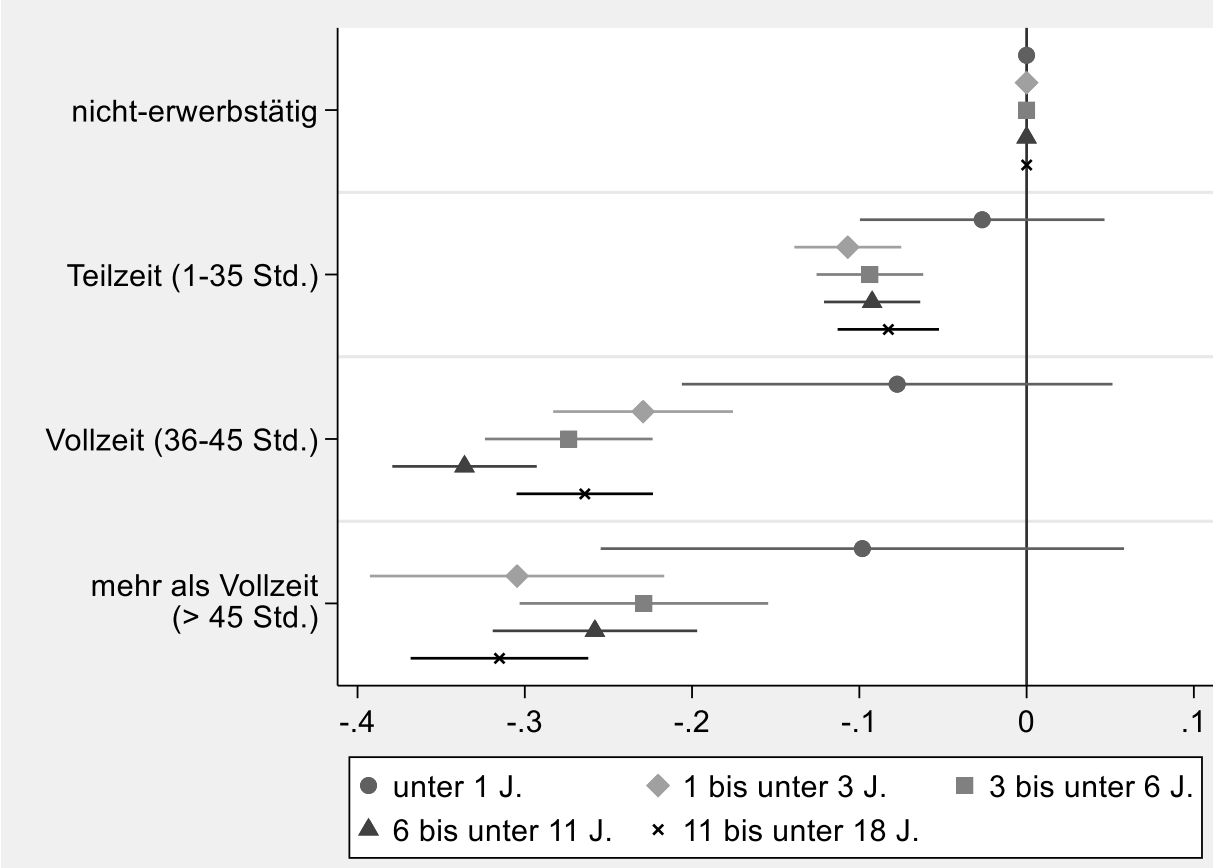

Anmerkung: OLS-Regression, dargestellt sind die Koeffizienten (Average Marginal Effects) mit 95\%-Konfidenzintervallen unter Kontrolle der weiteren Variablen aus Modell 1d, 2d, 3d usw. in Tabelle A.7 im Anhang. Quelle: DJI-Studie AID:A 2013/14, Sample: $n=11.157$, eigene Berechnungen.

Bei der Erwerbstätigkeit der Väter (siehe Abbildung 9.15) sind die Koeffizienten positiv, d.h. mit einem höheren Erwerbsumfang der Väter übernehmen sie weniger Hausarbeit und die Mütter entsprechend mehr Hausarbeit. Die Effektstärken der Erwerbstätigkeit fallen bei den Vätern insgesamt stärker aus als bei den Müttern (vgl. die unterschiedlichen Skalen in Abbildung 9.14 und Abbildung 9.15). Betrachtet man Interaktionseffekte zwischen der Erwerbstätigkeit des Vaters und dem Alter des Kindes (nicht abgebildet) ${ }^{46}$, so zeigen sich besonders große und signifikante Unterschiede zwischen den Altersstufen „1 bis unter 3 Jahre“ und „11 bis unter 18 Jahre“: Wenn das Kind „1 bis unter 3 Jahre“ alt ist, so ist die Aufteilung der Hausarbeit besonders ungleich verteilt, d.h. eine Erwerbstätigkeit des Vaters geht mit einer traditionelleren Aufteilung der Hausarbeit einher als dies in den anderen Altersstufen der Fall ist. Dagegen hat die Erwerbstätigkeit des Vaters

\footnotetext{
${ }^{45}$ Auch wenn das dahinterstehende Interaktionsmodell nicht abgebildet ist, so wird die Signifikanz der Interaktionseffekte auch daran ersichtlich, ob sich die Konfidenzintervalle der Koeffizienten in der Abbildung überlappen oder nicht.

${ }^{46} \mathrm{Vgl}$. vorherige Fußnote.
} 
in der Altersstufe „11 bis unter 18 Jahre“ eine geringere Bedeutung als in den meisten anderen Altersstufen (zur Altersstufe „unter 1 Jahr“ besteht kein signifikanter Unterschied).

Abbildung 9.15: Koeffizienten der Erwerbstätigkeit des Vaters zur Vorhersage der Aufteilung der Hausarbeit, getrennt nach dem Alter des Kindes

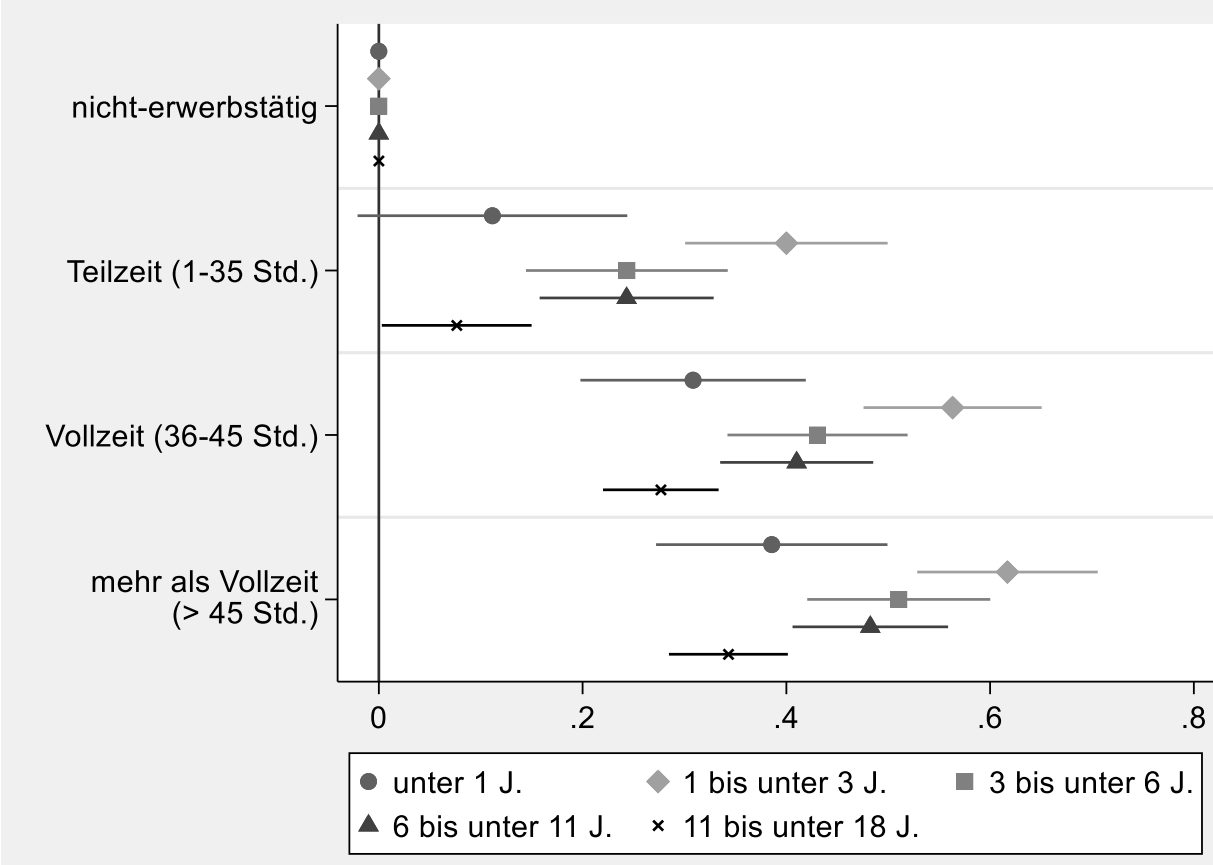

Anmerkung: OLS-Regression, dargestellt sind die Koeffizienten (Average Marginal Effects) mit 95\%-Konfidenzintervallen unter Kontrolle der weiteren Variablen aus Modell 1d, 2d, 3d usw. in Tabelle A.7 im Anhang. Quelle: DJI-Studie AID:A 2013/14, Sample: $n=11.157$, eigene Berechnungen.

\section{Vertiefende Analysen zu den Geschlechterrolleneinstellungen}

Auch bei der Aufteilung der Hausarbeit werden vertiefende Analysen zu den Geschlechterrolleneinstellungen aufgestellt. Neben den beiden bislang dargestellten Gender-Items sind im DJI-Survey „Aufwachsen in Deutschland: Alltagswelten“ (2013/2014) noch zwei weitere Items enthalten. Da diese beiden weiteren Items nur dann abgefragt wurden, wenn das Kind unter 9 Jahre alt ist, wurden diese Items in den vorherigen Analysen zur Aufteilung der Hausarbeit nicht berücksichtigt. Dies wird an dieser Stelle nachgeholt, wobei in Kauf genommen wird, dass dann nur Aussagen zur Arbeitsteilung von Eltern möglich sind, deren jüngstes Kind unter 9 Jahre alt ist. Diese vier Gender-Items wurden 
nicht zu einem Index zusammengefasst, da sie nur in schwacher Korrelation zueinanderstehen ${ }^{47}$ Alle Items haben negative Koeffizienten, d.h. wenn die Einstellungen egalitärer werden, dann geht dies mit einer egalitäreren Aufteilung der Hausarbeit einher (siehe Tabelle 9.3).

Tabelle 9.3: Aufteilung der Hausarbeit, unter Berücksichtigung von vier GenderItems, getrennt nach dem Alter des Kindes

\begin{tabular}{|c|c|c|c|c|c|c|c|c|}
\hline & \multicolumn{2}{|c|}{ unter 1} & \multicolumn{2}{|c|}{1 bis unter 3} & \multicolumn{2}{|c|}{3 bis unter 6} & \multicolumn{2}{|c|}{6 bis unter 9} \\
\hline & (1) & & (2) & & (3) & & (4) & \\
\hline VARIABLES & coef & se & coef & se & coef & se & coef & se \\
\hline $\begin{array}{l}\text { Geschlechter- } \\
\text { rolleneinstel- } \\
\text { lungen }\end{array}$ & & & & & & & & \\
\hline $\begin{array}{l}\text { Item } 1 \text { (beide } \\
\text { TZ) }\end{array}$ & $-0.03 * * *$ & $(0.01)$ & $-0.03 * * *$ & $(0.01)$ & $-0.02 * * *$ & $(0.01)$ & $-0.02 * *$ & $(0.01)$ \\
\hline $\begin{array}{l}\text { Item } 2 \text { (gegen } \\
\text { männl. Allein- } \\
\text { verdiener-M.) }\end{array}$ & $-0.02 * *$ & $(0.01)$ & $-0.02 * * *$ & $(0.01)$ & $-0.02 *$ & $(0.01)$ & -0.01 & $(0.01)$ \\
\hline $\begin{array}{l}\text { Item } 3 \text { (gegen } \\
\text { männl. Haupt- } \\
\text { verdiener-M.) }\end{array}$ & -0.01 & $(0.01)$ & $-0.02 * *$ & $(0.01)$ & $-0.02 * * *$ & $(0.01)$ & $-0.02 * *$ & $(0.01)$ \\
\hline $\begin{array}{l}\text { Item } 4 \text { (für } \\
\text { egali. Care-Ar- } \\
\text { beit) }\end{array}$ & -0.03 & $(0.01)$ & $-0.05 * * *$ & $(0.01)$ & $-0.05 * * *$ & $(0.01)$ & $-0.03 *$ & $(0.01)$ \\
\hline Constant & $1.04 * * *$ & $(0.08)$ & $1.28 * * *$ & $(0.05)$ & $1.24 * * *$ & $(0.05)$ & $1.17 * * *$ & $(0.07)$ \\
\hline Observations & 848 & & 2,070 & & 2,034 & & 1,268 & \\
\hline Adj. R-squared & 0.06 & & 0.13 & & 0.10 & & 0.09 & \\
\hline
\end{tabular}

Quelle: DJI-Studie AID:A 2013/14, eigene Berechnungen. OLS-Regression mit folgenden Signifikanzen: ${ }^{* * *} p<0.001,{ }^{* *} p<0.01,{ }^{*} p<0.05$. Anmerkung: Die Skalen der jeweiligen Gender-Items gehen von „1 traditionell“ bis „6 egalitär". Es wurden die gleichen Variablen wie in Modell 1c, 2c, $3 \mathrm{c}$ usw. in Tabelle A.7 im Anhang kontrolliert, d.h. der relative Berufsstatus, die Anzahl der Kinder, das Bundesgebiet und der absolute Berufsstatus der Mutter.

Darüber hinaus lassen sich nur wenige Gemeinsamkeiten bei den Items finden: Zwar stimmen Item 1 und 2 dahingehend überein, dass bei innen die Effekte bei einem niedrigeren Kindesalter etwas stärker ausfallen als bei einem höheren Kindesalter - dagegen

${ }^{47}$ Die inhaltliche Bedeutung der einzelnen Items wurde an früheren Stellen mehrfach erwähnt. Zum leichteren Verständnis sind sie hier jedoch erneut aufgelistet:

- Gender-Item 1 (in den bisherigen Analysen zur Hausarbeit bereits enthalten): „Wenn kleine Kinder da sind, sollte nicht nur die Frau, sondern auch der Mann Teilzeit arbeiten";

- Gender-Item 2 (in den bisherigen Analysen zur Hausarbeit bereits enthalten): „Wenn Kinder da sind, soll der Mann arbeiten gehen und die Frau zu Hause bleiben und die Kinder versorgen“;

- Gender-Item 3 (neu): „Auch wenn eine Frau arbeitet, sollte der Mann der ,Hauptverdiener' sein, und die Frau sollte die Verantwortung für den Haushalt tragen“;

- Gender-Item 4 (neu): „Ein Vater sollte genauso stark in die Erziehung und Pflege eines Kindes einbezogen sein, wie eine Mutter".

Die Items wurden so recodiert, dass alle Items von „1: traditionell“ bis „6: egalitär“ gehen. 
verhält es sich bei Item 3 umgekehrt, da der Effekt hier im jüngsten Kindesalter noch nicht signifikant ist, sondern erst ab einem Kindesalter von einem Jahr signifikant wird und sich in den weiteren Altersstufen dann nicht mehr verändert. Bei Item 4 wird der Effekt mit zunehmendem Alter des Kindes zunächst stärker, dann jedoch wieder schwächer. Diese uneinheitliche Befundlage zur Entwicklung der Gender-Items mit zunehmendem Alter des Kindes verweist darauf, dass Geschlechterrolleneinstellungen nicht eindimensional in "traditionell“ und „egalitär" eingeordnet werden können, sondern eine multidimensionale Betrachtungsweise angebracht ist, die komplexere Einstellungsmuster umfasst. ${ }^{48}$

In Folgenden werden daher anstelle der einzelnen Gender-Items die latenten Klassen an Geschlechterrolleneinstellungen (siehe Kapitel 7.5) herangezogen. Die in Abbildung 9.16 vorhergesagten Werte basieren auf der OLS-Regression in Tabelle A.6 im Anhang. Aus Abbildung 9.16 geht hervor, dass die Hausarbeit in allen Altersstufen am traditionellsten in der Klasse des „intensive mothering/parenting“ und am egalitärsten in der egalitären Klasse aufgeteilt ist, während sich die Klasse des „egalitären Essentialismus“ zwischen den beiden Extremen befindet. Damit kann Hypothese 4 a) bestätigt werden, wonach Mütter, die sich mit dem Leitbild des "intensive mothering/parenting“ identifizieren, mehr Hausarbeit übernehmen. Besonders groß sind die Unterschiede zwischen den Klassen in den Altersstufen „1 bis unter 3 Jahre“ und „3 bis unter 6 Jahre“. Was bedeutet dies für die altersspezifischen Hypothesen 4 b) bis 4 d)? Hypothese 4 b) nimmt an, dass das Leitbild des „intensive mothering/parenting“ bei einem Kindesalter von unter 3 Jahren einen besonders traditionalisierenden Effekt auf die Arbeitsteilung hat. Dies lässt sich besonders für die Altersstufe „1 bis unter 3 Jahre“ bestätigen und nur eingeschränkt für Kinder im Alter von „unter 1 Jahr“.

\footnotetext{
${ }^{48}$ Die Koeffizienten der weiteren Variablen sind in Tabelle 9.3 nicht abgebildet, da der Fokus hier auf der Bedeutung der Gender-Items liegt. Eine Erkenntnis aus den Koeffizienten der weiteren Variablen soll jedoch nicht vorenthalten bleiben: Unter der Kontrolle der vier Gender-Items verliert der relativen Berufsstatus in nahezu allen Altersstufen (mit Ausnahme der Altersstufe „3 bis unter 6 Jahre") seine Signifikanz. Dies zeigt, wie gering die Bedeutung des relativen Berufsstatus im Vergleich zur Bedeutung der Geschlechterrolleneinstellungen ist.
} 
Abbildung 9.16: Vorhergesagte Aufteilung der Hausarbeit, unter Berücksichtigung der drei Gender-Klassen, getrennt nach dem Alter des Kindes

unter $1 \mathrm{~J}$.

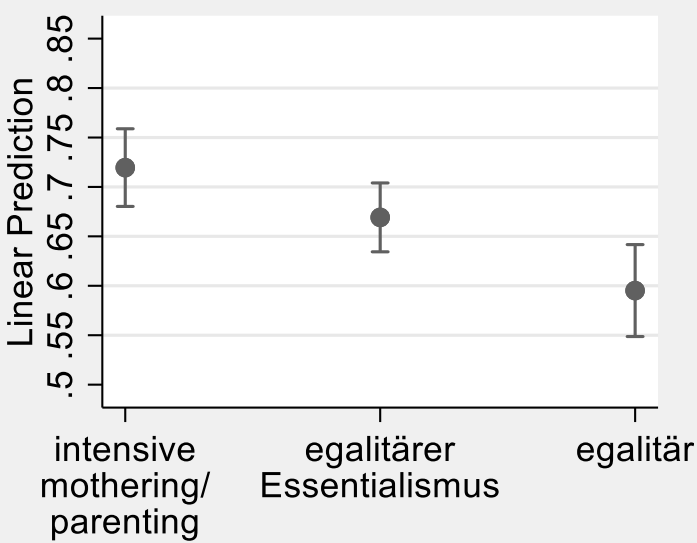

3 bis unter $6 \mathrm{~J}$.

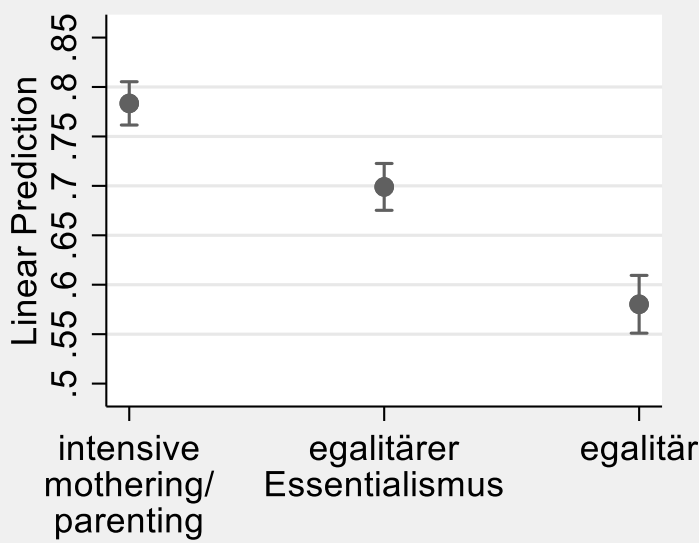

1 bis unter $3 \mathrm{~J}$.

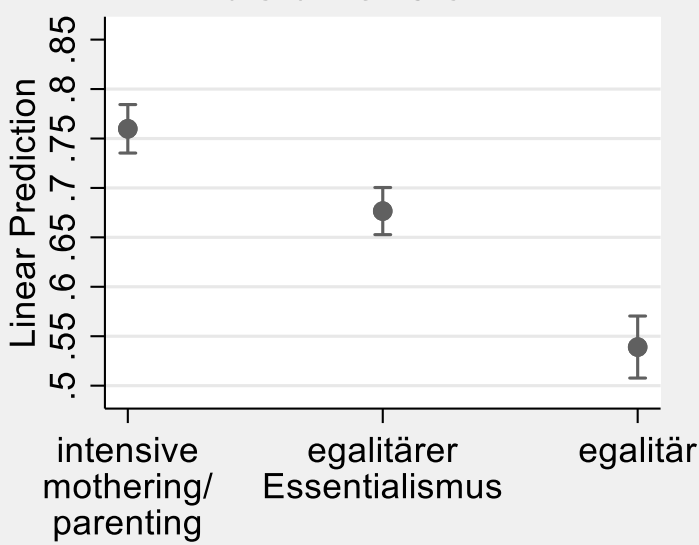

6 bis unter $9 \mathrm{~J}$.

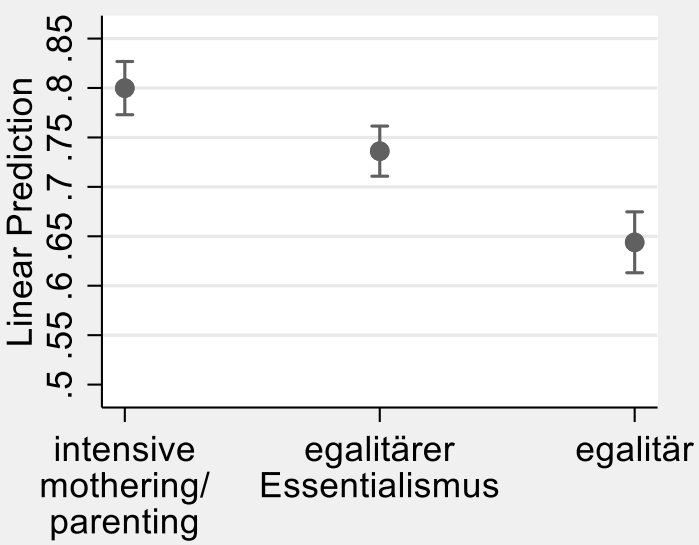

Anmerkung: Vorhergesagte Aufteilung der Hausarbeit (Predictive Margins) mit 95\%-Konfidenzintervallen basierend auf Tabelle A.8 im Anhang; die weiteren Variablen werden auf ihrem Mittelwert festgehalten Quelle: DJI-Studie AID:A 2013/14, eigene Berechnungen.

Hypothese 4 c) nimmt an, dass der Effekt des Leitbildes des „intensive mothering/parenting“ auf die Arbeitsteilung bei einem Kindesalter von „3 bis unter 6 Jahren“ bereits etwas abgeschwächt ist. Dies kann jedoch nicht bestätigt werden, da aus den Ergebnissen hervorgeht, dass die Effekte für die Aufteilung der Hausarbeit bei der Altersstufe „3 bis unter 6 Jahre“ noch gleich stark wie in der Altersgruppe „1 bis unter 3 Jahre sind“. ${ }^{49}$ Hypothese $4 \mathrm{~d}$ ) bezieht sich auf die höchste Altersstufe und nimmt an, dass das Leitbild „intensive mothering/parenting“ keinen oder nur noch einen sehr geringen Einfluss hat, wenn sich das Kind um Grundschulalter befindet. Dies kann insofern bestätigt werden,

49 Zudem geht aus Interaktionseffekten, die hier nicht abgebildet sind, hervor, dass die Effekte in der Altersgruppe „3 bis unter 6 Jahre" sogar signifikant stärker sind als in der Altersgruppe von „unter 1 Jahr". Auch dies spricht dafür, dass Hypothese 4 c) falsifiziert werden muss. 
da aus hier nicht abgebildeten Modellen mit Interaktionseffekten hervorgeht, dass der Effekt signifikant schwächer ist als in den Altersstufen „1 bis unter 3 Jahren“ und „3 bis unter 6 Jahren“. Andererseits kann nicht davon die Rede sein, dass das Leitbild des „intensive mothering/ parenting" nur noch einen geringen Effekt auf die Arbeitsteilung hat. Vielmehr zeigen die Ergebnisse, dass der Effekt zwar geringfügig schwächer geworden ist, aber dennoch sehr stark ist. Bzgl. Hypothese $4 \mathrm{~d}$ ) bedeutet dies, dass der Teilaspekt, der einen schwächer werdenden Zusammenhang annimmt, bestätigt werden kann; gleichzeitig muss der andere Teilaspekt, der annimmt, dass der Effekt in dieser Altersgruppe nicht mehr vorhanden oder nur noch sehr gering ist, abgelehnt werden.

Abschließend werden die zentralen Ergebnisse zur Aufteilung der Hausarbeit kurz zusammengefasst. Im Anhang ist darüber hinaus in Tabelle A.12 aufgelistet, welche Hypothesen damit konkret bestätigt werden können und welche nicht. Bzgl. des Alters des Kindes lässt sich festhalten, dass die Aufteilung der Hausarbeit bereits im ersten Lebensjahr des Kindes sehr traditionell ist. Dies steht im Einklang mit zahlreichen Studien, die insbesondere den Übergang zur Elternschaft als Ursache für eine traditionellere Aufteilung der Hausarbeit aufdecken (Evertsson und Nermo 2007; Baxter et al. 2008; Grunow et al. 2012; Kühhirt 2012; Schober 2013b; Dechant et al. 2014; Baxter et al. 2015). Die Ergebnisse zeigen des Weiteren, dass die Aufteilung der Hausarbeit in Familien mit älteren Kindern nicht egalitärer, sondern noch traditioneller aufgeteilt wird als in Familien mit jüngeren Kindern. Auch diese Entwicklung steht in Einklang mit bisherigen Studien, die belegen, dass die Aufteilung der Hausarbeit mit zunehmendem Alter des Kindes bzw. mit zunehmender Beziehungsdauer traditioneller wird (Schulz und Blossfeld 2006; Grunow et al. 2007, 2012; Zabel und Heintz-Martin 2013).

Als nächstes wird auf die Bedeutung der relativen Ressourcen eingegangen, die nach den ökonomischen Theorien eine wichtige Rolle spielen und in der hier vorliegenden Arbeit durch den relativen Berufsstatus (ISEI) operationalisiert wurden. Es hat sich zwar gezeigt, dass Mütter mit niedrigeren, relativen Ressourcen etwas mehr Hausarbeit übernehmen (als Mütter mit gleichen Ressourcen), aber es konnte umgekehrt nicht gezeigt werden, dass Mütter mit höheren, relativen Ressourcen weniger Hausarbeit übernehmen). Die relativen Ressourcen haben somit keine geschlechtssymmetrischen Effekte und insgesamt spielen sie nur eine sehr kleine Rolle für die Aufteilung der Hausarbeit. Betrachtet man die Ergebnisse differenziert nach dem Alter des Kindes, so zeigt sich, dass die relativen Ressourcen in den ersten Lebensjahren des Kindes noch keine signifikante Bedeutung haben. Hier scheinen die Effekte der relativen Ressourcen durch institutionelle Rahmenbedingungen überlagert zu werden.

Sobald sich das Kind im Kindergarten- und Grundschulalter befindet, übernehmen Mütter mit niedrigeren Ressourcen mehr Hausarbeit, aber Mütter mit höheren Ressourcen übernehmen in dieser Altersstufe noch nicht weniger Hausarbeit. Dass sich bei Müttern mit höheren Ressourcen ihr Hausarbeitsanteil etwas reduziert, trifft erst dann zu, wenn 
sich das Kind im Jugendalter befindet. Anscheinend tragen die höheren Ressourcen der Mütter erst ab diesem fortgeschrittenen Kindesalter zu einem niedrigerem Hausarbeitsanteil bei.

Die Geschlechterrolleneinstellungen spielen eine größere Rolle als die relativen Ressourcen. Entsprechend den Erwartungen gehen egalitäre Einstellungen mit einer egalitäreren Aufteilung der Hausarbeit einher. Aus den Analysen anhand der einzelnen Gender-Items geht hervor, dass die Effekte über das Kindesalter hinweg stabil bleiben. D.h. die Effekte der Gender-Items unterscheiden sich nicht in Abhängigkeit vom Alter des Kindes. Bei den Ergebnissen anhand der latenten Gender-Klassen hat sich gezeigt, dass die Aufteilung der Hausarbeit bei Müttern, die sich mit dem Leitbild des „intensive mothering/parenting“ identifizieren am traditionellsten ist, gefolgt von der Klasse des „egalitären Essentialismus“ und dass die Aufteilung in der „egalitären“ Klasse am egalitärsten ist. Auch wird sind die Effekte über die Altersstufen des Kindes hinweg relativ stabil: Zwar wird die Bedeutung des Leitbildes des „intensive mothering/parenting“ ab dem Grundschulalter des Kindes etwas geringer, aber die Aufteilung der Hausarbeit ist selbst dann noch deutlich traditioneller als bei den anderen beiden Gender-Klassen.

Aus den Ergebnissen zur Bedeutung der Kinderanzahl für die Aufteilung der Hausarbeit geht hervor, dass die Hausarbeit in Familien mit zwei Kindern traditioneller aufgeteilt ist als in Familien mit einem Kind, jedoch zeigen sich keine Unterschiede in Abhängigkeit vom Alter des Kindes. Bzgl. der weiteren Einflussfaktoren lässt sich festhalten, dass sich die Hausarbeit in Ostdeutschland egalitärer aufgeteilt ist als in Westdeutschland, auch wenn die Unterschiede deutlich geringer ausfallen als bei der Aufteilung der Erwerbsarbeit. Hierbei ist hervorzuheben, dass die Ost-West-Unterschiede in den ersten drei Lebensjahren des Kindes noch nicht signifikant sind und somit erst bei einem über 3-jährigen Kind zum Vorschein treten. Bei den Ergebnissen zum absoluten Berufsstatus der Mutter zeigt sich, dass ein hoher absoluter Berufsstatus der Mutter (im Vergleich zu einem mittleren Berufsstatus der Mutter) mit einer egalitäreren Aufteilung der Hausarbeit einhergeht und dieser Zusammenhang in allen Altersstufen außer bei den unter 1-jährigen Kindern signifikant ist. Die Ergebnisse zeigen weiter, dass zwischen den Erwerbstätigkeiten der Mütter und Väter starke Zusammenhänge mit der Aufteilung der Hausarbeit vorliegen. Ein höherer Erwerbsumfang der Mütter (im Vergleich zu nicht-erwerbstätigen Müttern) geht mit einer egalitäreren Aufteilung der Hausarbeit einher. Die Zusammenhänge sind in allen Altersstufen des Kindes etwa gleich groß außer bei einem Kind im ersten Lebensjahr: Hier spielt der Erwerbsumfang der Mütter noch keine signifikante Rolle für die Aufteilung der Hausarbeit. Beim Zusammenhang der Erwerbstätigkeit der Väter und der Aufteilung der Hausarbeit lässt sich festhalten, dass ein höherer Erwerbsumfang der Väter (im Vergleich zu nicht-erwerbstätigen Vätern) mit einer traditionelleren Aufteilung der Hausarbeit einhergeht. Die Zusammenhänge fallen bei 1- bis unter 3-jährigen Kindern besonders stark aus und sind bei älteren Kindern etwas schwächer. Nach diesen Ergebnissen zur Aufteilung der Hausarbeit wird nun zu den Ergebnissen der Aufteilung der Kinderbetreuung übergegangen. 


\section{Aufteilung der Kinderbetreuung}

Neben der Aufteilung der Erwerbsarbeit und der Hausarbeit interessiert als weitere Forschungsfrage die Frage danach, wer überwiegend die Kinderbetreuung übernimmt, inwiefern sich diese Aufteilung mit dem Alter des Kindes verändert und anhand welcher Einflussfaktoren die Aufteilung der Kinderbetreuung erklärt werden kann. Wie im vorherigen Kapitel zur Aufteilung der Hausarbeit wird auch bei der Aufteilung der Kinderbetreuung zunächst auf vier einzelne Tätigkeiten eingegangen. Dabei handelt es sich um die folgenden Tätigkeiten: das Spielen mit den Kindern, das Bringen und Holen der Kinder, der Kontakt zur Schule/Kita o.ä. und das Betreuen und Versorgen der Kinder. Danach werden diese vier Tätigkeiten zu einem Kinderbetreuungsindex zusammengefasst, der ein Indikator dafür ist, wie die Kinderbetreuung insgesamt zwischen den Eltern aufgeteilt ist. Nach deskriptiven Befunden hierzu dient der Kinderbetreuungsindex in den anschließenden, multivariaten Analysen als abhängige Variable, um in Erfahrung bringen zu können, wodurch die Aufteilung der Kinderbetreuung beeinflusst wird und inwiefern sie sich in Abhängigkeit vom Alter des Kindes verändert.

\subsection{Deskriptive Befunde}

Analog zur Aufteilung der Hausarbeit wird auch bei der Aufteilung der Kinderbetreuung zunächst auf die univariate Verteilung der einzelnen Tätigkeiten eingegangen, die dann an späterer Stelle zu einem Index zusammengefasst werden. In Tabelle 10.1 sind die Tätigkeiten Spielen, Bringen und Holen der Kinder, Kontakte zur Kita, Schule o.ä. sowie das Betreuen und Versorgen der Kinder abgetragen. Insgesamt fällt auf, dass die Kinderbetreuung deutlich egalitärer aufgeteilt ist als die Hausarbeit; so wird das Bringen und Holen zu 40\% von beiden gleichermaßen übernommen, der Kontakt zu Einrichtungen wie der Kita zu 29\%, das Betreuen zu 42\% und das Spielen sogar zu 74\% von beiden gleichermaßen. ${ }^{50}$ Trotz dieser egalitäreren Aufteilung der Kinderbetreuung (im Vergleich zur Aufteilung der Hausarbeit) ist der Großteil der Kinderbetreuung dennoch überwiegend die Aufgabe der Mütter.

\footnotetext{
50 Wie bei der Aufteilung der Hausarbeit werden auch bei der Aufteilung der Kinderbetreuung die Mütterangaben verwendet. Aus weiteren, nicht abgebildeten Analysen auf Basis der Väterangaben geht hervor, dass Väter die Aufteilung ähnlich einschätzen wie die Mütter, aber dass kleine Abweichungen dahingehend vorliegen, dass sie ihren eigenen Anteil etwas höher und den Anteil der Mütter etwas niedriger einschätzen.
} 
Tabelle 10.1: $\quad$ Aufteilung der einzelnen Tätigkeiten der Kinderbetreuung

\begin{tabular}{lcccccc}
\hline Aufteilung & \multicolumn{5}{c}{ in \% } \\
der Kinder- & \multicolumn{7}{c}{ betreuung } & $\begin{array}{c}\text { überwie- } \\
\text { gend die } \\
\text { Mutter }\end{array}$ & $\begin{array}{c}\text { beide } \\
\text { etwa } \\
\text { gleich }\end{array}$ & $\begin{array}{c}\text { überwie- } \\
\text { gend der } \\
\text { Vater }\end{array}$ & $\begin{array}{c}\text { kommt } \\
\text { nicht } \\
\text { vor }\end{array}$ & $\begin{array}{c}\text { jemand } \\
\text { anders }\end{array}$ & $n$ \\
\hline Spielen & 23,5 & 74,4 & 1,6 & 0,5 & 0,1 & 10.925 \\
$\begin{array}{l}\text { Bringen/Ho- } \\
\text { len }\end{array}$ & 52,6 & 39,7 & 3,9 & 3,3 & 0,5 & 10.901 \\
$\begin{array}{l}\text { Kontaktmit } \\
\text { Kita o.ä. }\end{array}$ & 66,4 & 29,4 & 1,8 & 2,3 & 0,1 & 10.886 \\
$\begin{array}{l}\text { Betreuen/ } \\
\text { Versorgen }\end{array}$ & 56,0 & 42,3 & 1,2 & 0,4 & 0,2 & 10.925 \\
\hline
\end{tabular}

Quelle: DJI-Studie AID:A 2013/14, gewichtet, eigene Berechnungen.

Um der Forschungsfrage nachzugehen, wie sich die Aufteilung der Kinderbetreuung in Abhängigkeit vom Alter des Kindes verhält, wird die Aufteilung der Kinderbetreuung in Abbildung 10.1 differenziert nach den Altersstufen des Kindes dargestellt:

Abbildung 10.1: Aufteilung der Kinderbetreuungstätigkeiten in Abhängigkeit vom Alter des Kindes
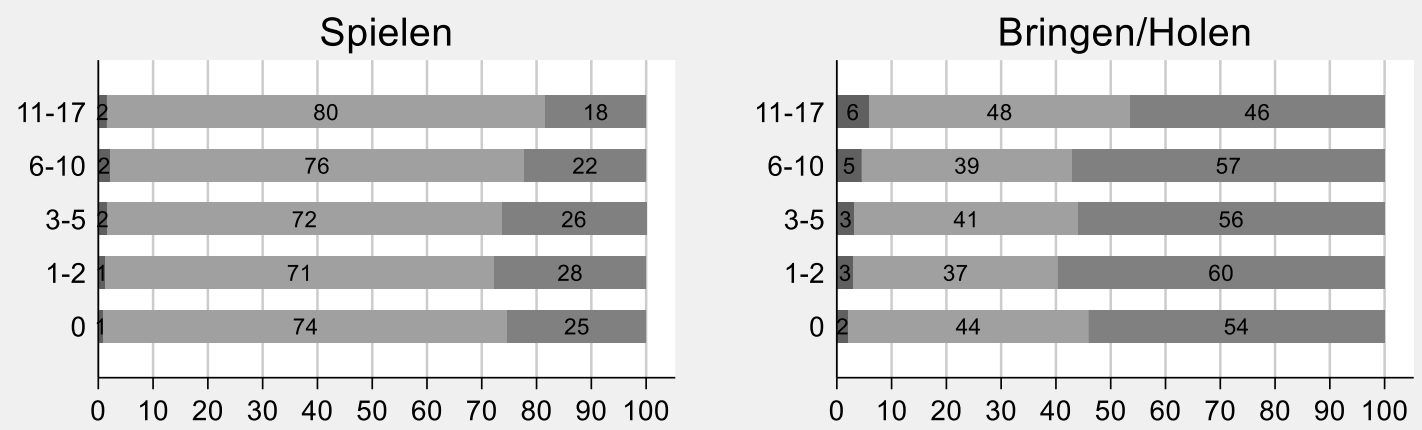

Kontakt zu Kita, Schule o.ä.
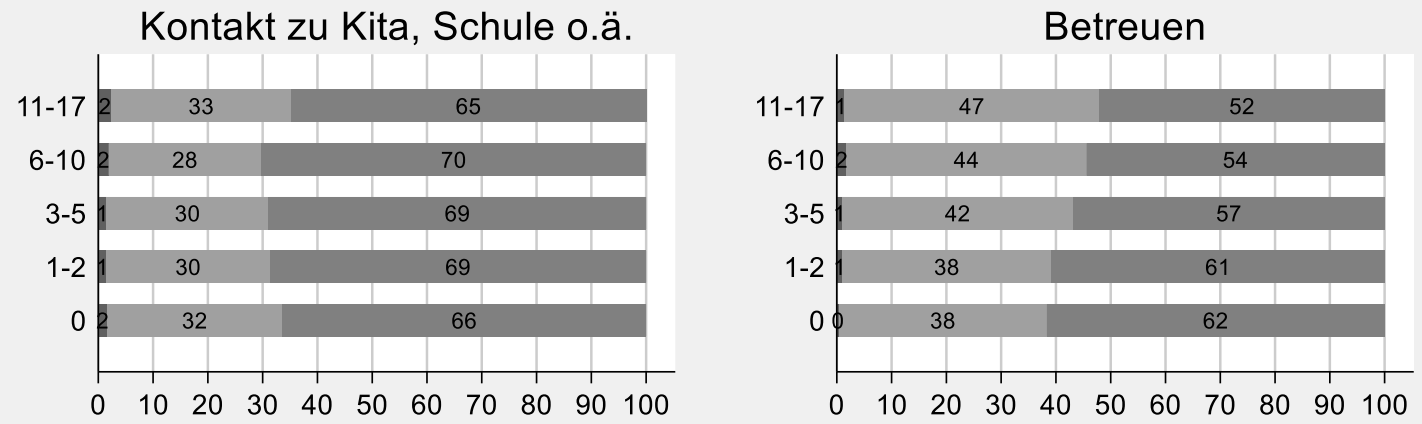

überwiegend der Vater beider gleichermaßen

überwiegend dier Mutter

Quelle: DJI-Studie AID:A 2013/14, Daten gewichtet, $\mathrm{n}$ (Spielen) $=10.845, \mathrm{n}($ Bringen/Holen $)=$ $10.444, \mathrm{n}($ Kontakt zu Kita, Schule o.ä. $)=10.636, \mathrm{n}($ Betreuen/Versorgen $)=10.835$, eigene Berechnungen.

Wie soeben aus der univariaten Verteilung hervorgegangen ist, ist das Spielen besonders egalitär aufgeteilt. Dies geschieht zu etwa 71-74\%, wenn das Kind im Kleinkind- 
und Vorschulalter ist und steigt auf 76-80\%, wenn das Kind im Grundschul- bzw. Jugendalter ist. Obwohl das Spielen somit sehr häufig von beiden Elternteilen gleichermaßen übernommen wird, kommt es selten vor, dass das Spielen überwiegend vom Vater übernommen wird: Dies ist nur bei $1 \%$ der Kinder im Kleinkindalter und bei $2 \%$ bei Kindern ab dem Vorschulalter vor. Das Bringen und Holen des Kindes wird zu 44\% egalitär aufgeteilt, wenn das Kind unter ein Jahr alt ist und zu 48\%, wenn das Kind 11- bis 17-jährig ist. In den übrigen Alterskategorien fällt der Anteil der egalitären Aufteilung mit 37-41 Prozentpunkten geringer aus. Der Anteil der Väter, die diese Aufgabe überwiegend übernehmen, steigt mit zunehmendem Alter des Kindes von 2\% auf 6\%. Der Kontakt zur Kita, Schule oder ähnlichen wird bei etwa einem knappen Drittel von beiden gleichermaßen übernommen, wobei Schwankungen über die Altersstufen des Kindes vorliegen. Der Kontakt zur Schule wird bei einem Kind im Grundschulalter nur zu 28\% von beiden gleichermaßen hergestellt, sobald das Kind jedoch im Alter einer weiterführenden Schule ist, wird der Kontakt zur Schule bereits zu 33\% von beiden hergestellt. Bei den Aufgaben Betreuen und Versorgen ist erkennbar, dass die Aufteilung bei größeren Kindern egalitärer und weniger geschlechtsspezifisch ausfällt als bei kleineren Kindern: Nur zu 38\% wird das Betreuen von beiden gleichermaßen übernommen, wenn das Kind im Kleinkindalter ist, während dies zu 39-41\% bei Kindern im Vorschul- und Grundschulalter stattfindet und sogar zu 48\% bei Kindern im Jugendalter. Die Kategorie „übernimmt überwiegend der Vater" steigt über die Altersstufen des Kindes kaum an.

Diese einzelnen Tätigkeiten wurden zu einen Kinderbetreuungsindex zusammengefasst, der in den weiteren Analysen die abhängige Variable darstellt. Die Skala geht (analog zum Hausarbeitsindex) von „-1: Vater übernimmt alles“ über „0: egalitär“ bis hin zu „+1: Mutter übernimmt alles“. Die Verteilung ist in Abbildung 10.2 dargestellt und zeigt, dass die Kinderbetreuung zwar ungleich zu Lasten der Mütter verteilt ist, aber immerhin in $16,5 \%$ egalitär aufgeteilt ist. Dass Väter mehr Kinderbetreuung übernehmen als die Mütter, ist nur sehr selten der Fall. 
Abbildung 10.2: Aufteilung der Kinderbetreuung insgesamt

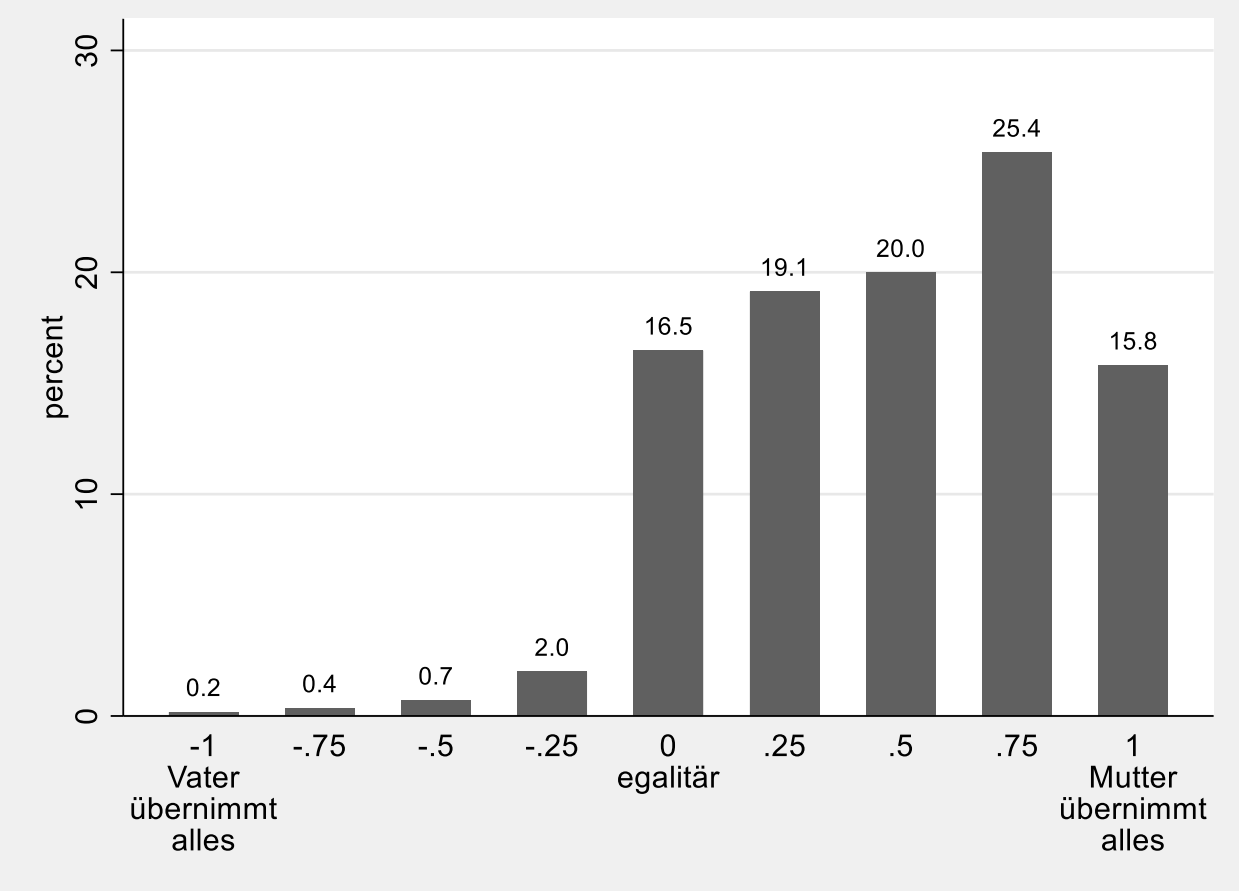

Quelle: DJI-Studie AID:A 2013/14, gewichtet, n = 10.925, eigene Berechnungen.

Im Folgenden werden bivariate Zusammenhänge zwischen diesem Kinderbetreuungsindex und weiteren Variablen dargestellt, die auf bivariaten Regressionsmodellen basieren. In den nachfolgenden Abbildungen sind die vorhergesagten Werte des Kinderbetreuungsindex abgetragen. Da sich diese vorhergesagten Werte nur zwischen 0,4 und 0,8 bewegen, ist nur dieser Ausschnitt der Skala der Aufteilung der Kinderbetreuung auf der y-Achse abgetragen. Würde die gesamte Skala abgetragen sein, die von „-1“ bis „ +1 “ geht (siehe die soeben gezeigte Abbildung 10.2), dann wären die einzelnen Zusammenhänge nur sehr schlecht sichtbar.

Um zu untersuchen, welche Rolle ökonomische Ressourcen für die Aufteilung der Kinderbetreuung spielen, werden der relative Berufsstatus (ISEI) und der absolute Berufsstatus der Mutter (ISEI) betrachtet (siehe Abbildung 10.3). Beim relativen Berufsstatus wird ersichtlich, dass die Aufteilung der Kinderbetreuung am traditionellsten ist, wenn die Mütter einen niedrigeren Status haben und am egalitärsten ist, wenn beide den gleichen Status haben. Eine Ausnahme stellt die höchste Altersstufe des Kindes dar („11 bis unter 18 Jahre"): Hier ist die Aufteilung dann am egalitärsten, wenn Mütter einen höheren Status haben. Aus der Abbildung wird jedoch auch ersichtlich, dass sich die 95\%-Konfidenzintervalle überlappen und die Unterschiede somit nicht signifikant sind. Insgesamt wird die Aufteilung der Kinderbetreuung mit zunehmendem Alter des Kindes etwas egalitärer. Nach dem absoluten Berufsstatus (ISEI) der Mutter ist die Aufteilung der Kinderbetreuung tendenziell dann am egalitärsten, wenn die Mutter einen hohen Status hat (ausgenommen bei einem unter 1-jährigen Kind). Jedoch gibt es auch hier (insbesondere in der 
jüngsten und ältesten Altersstufe des Kindes) starke Überlappungen der Konfidenzintervalle. Diese ersten bivariaten Analysen verleiten zu der Annahme, dass die Aufteilung der Kinderbetreuung nur zu einem geringen Ausmaß durch ökonomische Ressourcen mitbestimmt wird. Bevor dies anhand multivariater Analysen überprüft wird, wird zunächst auf weitere bivariate Zusammenhänge eingegangen.

Abbildung 10.3: Bivariate Zusammenhänge des relativen Berufsstatus und des absoluten Berufsstatus der Frau mit der Aufteilung der Kinderbetreuung
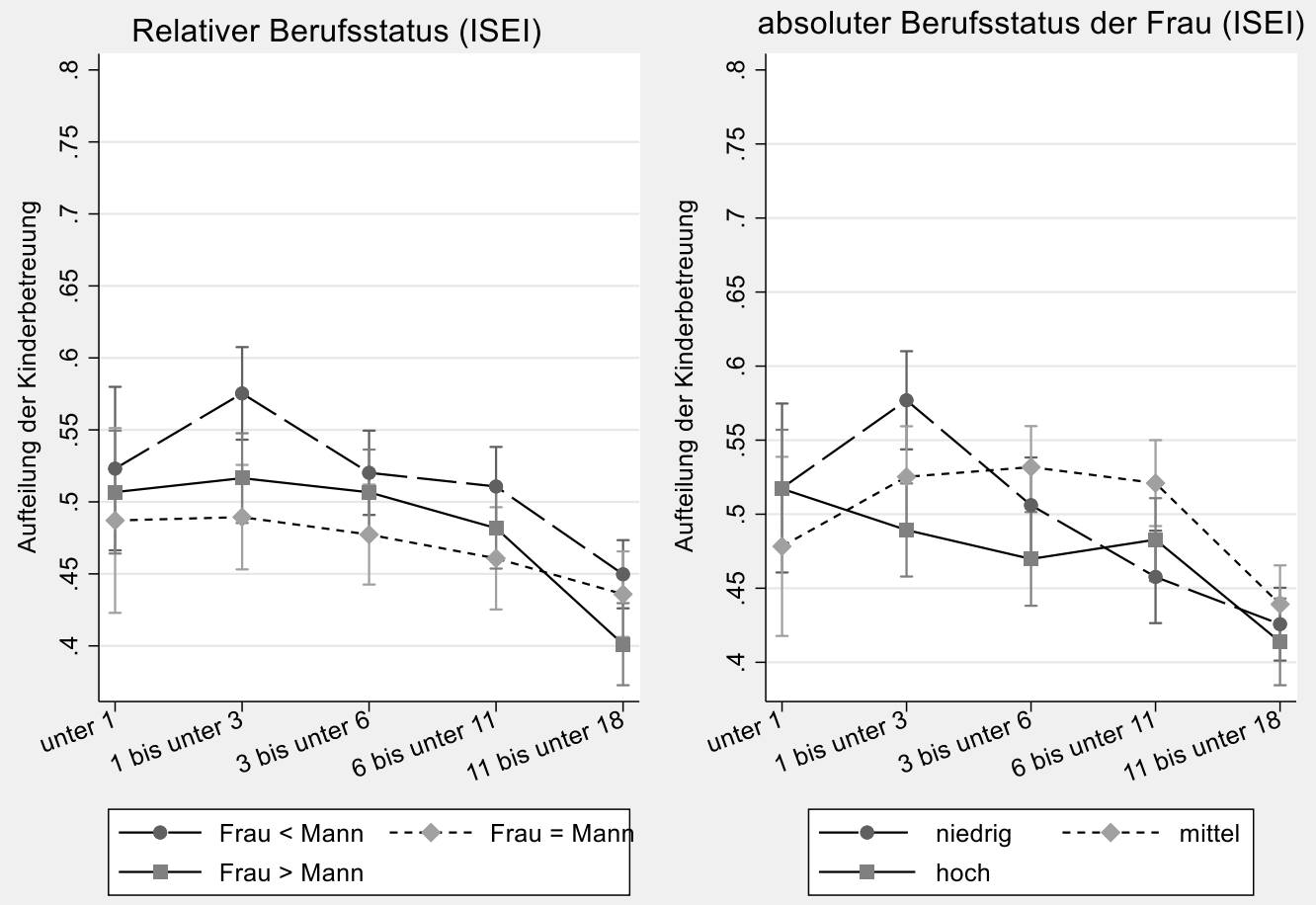

Anmerkung: Vorhergesagte Aufteilung der Kinderbetreuung auf Basis von bivariaten Regressionsmodellen mit 95\%-Konfidenzintervallen. Quelle: DJI-Studie AID:A 2013/14, gewichtet, n (relativer Berufsstatus) $=10.075, \mathrm{n}$ (absoluter Berufsstatus) $=10.392$; eigene Berechnungen

In Abbildung 10.4 ist die Aufteilung der Kinderbetreuung in Abhängigkeit von zwei verschiedenen Gender-Items abgetragen. Erwartungsgemäß ist die Aufteilung bei traditionellen Einstellungen traditioneller und bei egalitären Einstellungen egalitärer. Jedoch sind die Unterschiede zwischen einer traditionellen und einer neutralen/mittleren Einstellung bei beiden Items nur marginal. Wenn man beim Vorhandensein kleiner Kinder eine Teilzeit-/Teilzeit-Konstellation befürwortet (= egalitäre Einstellung bei Gender-Item 1 ), dann geht dies v.a. bei unter 1-jährigen Kindern und bei Kindern ab einem Alter von 6 Jahren mit einer signifikant egalitäreren Aufteilung der Kinderbetreuung einher. Wenn man ein männliches Alleinverdienermodell ablehnt (= egalitäre Einstellung bei GenderItem 2), so spiegelt sich das in einer egalitäreren Aufteilung der Kinderbetreuung wider, die besonders stark in den Altersstufen „1 bis unter 3 Jahre" und „3 bis unter 6 Jahre“ vorliegt. Während eine egalitäre Einstellung bei Item 1 gerade in der jüngsten und der 
ältesten Altersstufe signifikante Effekte aufweist, verhält es sich bei Item 2 genau andersrum. Dies zeigt, dass die beiden Items inhaltlich unterschiedliche Dimensionen abbilden und deswegen nicht zu einem Index zusammengefasst werden sollten.

Stattdessen bietet es sich an, die Einstellungsitems einer latenten Klassenanalyse zu unterziehen und so verschiedene Einstellungsprofile aufzudecken. Unter Verwendung von vier Einstellungsitems ließen sich drei latente Klassen ermitteln, die als „intensive mothering/parenting“, „egalitärer Essentialismus“ und „egalitär“ beschrieben wurden (siehe Kapitel 7.5).

Abbildung 10.4: Bivariate Zusammenhänge der Gender-Items mit der Aufteilung der Kinderbetreuung

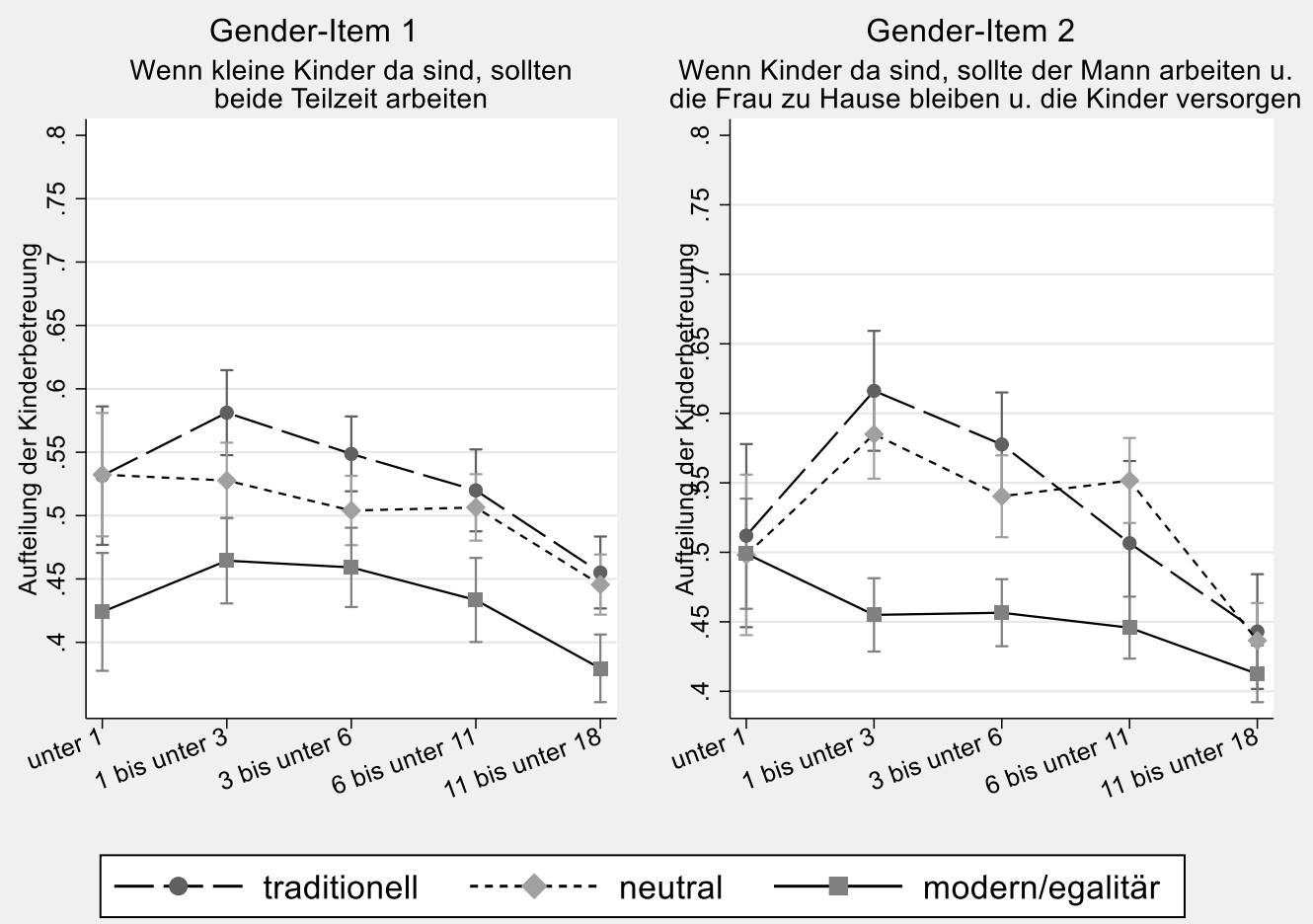

Anmerkung: Vorhergesagte Aufteilung der Kinderbetreuung auf Basis von bivariaten Regressionsmodellen mit 95\%-Konfidenzintervallen. Quelle: DJI-Studie AID:A 2013/14, gewichtet, $n($ Gender-Item 1$)=10.809, n($ Gender-Item 2$)=10.838$; eigene Berechnungen .

In Abbildung 10.5 werden Ergebnisse gezeigt, die die Aufteilung der Kinderbetreuung unter Berücksichtigung von drei latenten Klassen an Einstellungsprofilen wiedergeben. ${ }^{51}$ In allen Altersstufen außer in der jüngsten Altersstufe entsprechen die Ergebnisse den

51 Wie bereits aus vorherigen Kapiteln hervorgeht, basiert die latente Klassenanalyse auf insgesamt vier Items, wobei bei zwei Items die Angaben nur von Auskunftspersonen mit Zielkindern im Alter von unter 9 Jahren vorliegen. Daher beschränken sich die Analysen zu den latenten Klassen auf ein Kindesalter von 0 bis unter 9 Jahre. 
Erwartungen, wonach die Kinderbetreuung in der Klasse des „intensive mothering/parenting“ am traditionellsten aufgeteilt ist und in der „egalitären“ Klasse am egalitärsten, während sich die Klasse des „egalitären Essentialismus“ in der Mitte davon befindet. Lediglich in der Altersstufe „unter 1 Jahr“ ist die Kinderbetreuung in der Klasse des „intensive mothering/parenting“ egalitärer aufgeteilt als in der Klasse des „egalitären Essentialismus".

Abbildung 10.5: Bivariate Zusammenhänge der Gender-Klassen mit der Aufteilung der Kinderbetreuung

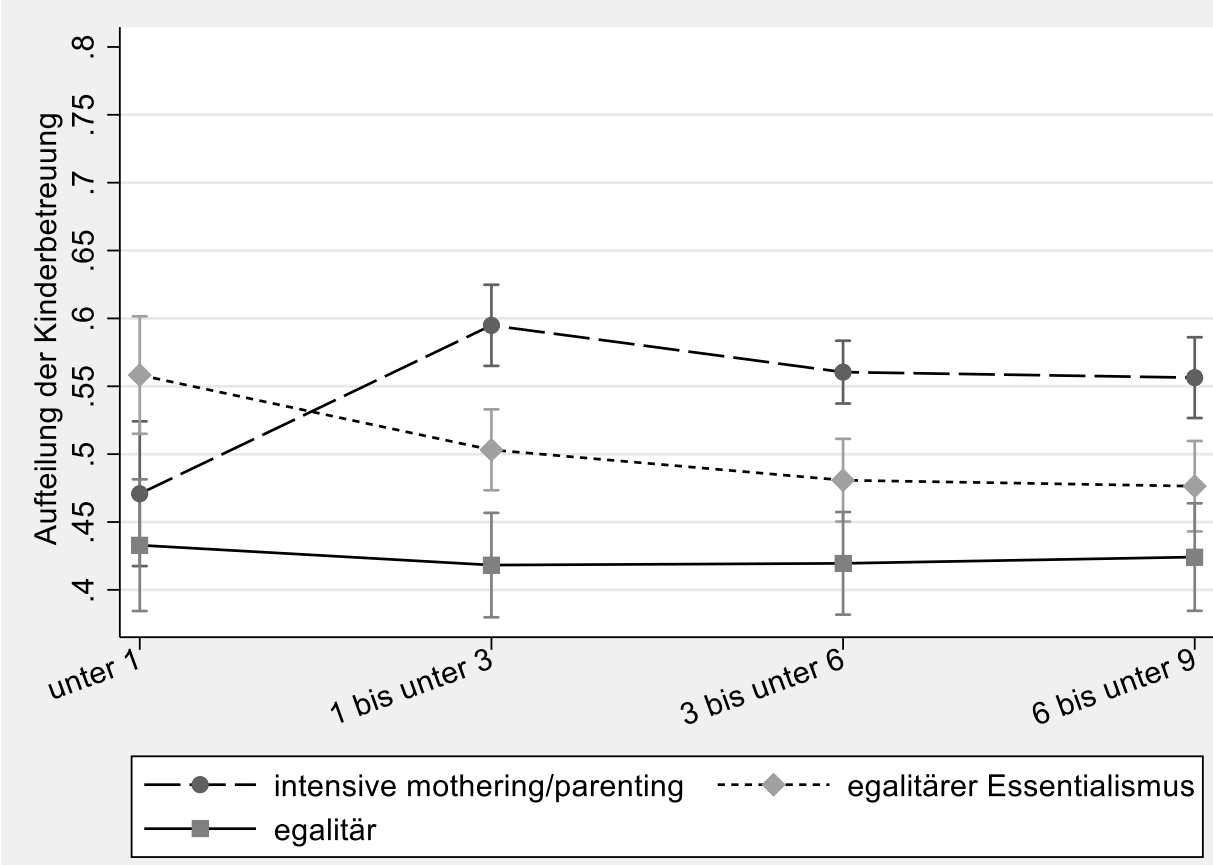

Anmerkung: Vorhergesagte Aufteilung der Kinderbetreuung auf Basis von bivariaten Regressionsmodellen mit 95\%-Konfidenzintervallen. Quelle: DJI-Studie AID:A 2013/14, gewichtet, $\mathrm{n}$ (Gender-Klassen) = 7.184; eigene Berechnungen .

Besonders große Unterschiede zeigen sich bei der Aufteilung der Kinderbetreuung in Abhängigkeit vom Bundesgebiet und von der Erwerbskonstellation (siehe Abbildung 10.6). In Ostdeutschland ist die Aufteilung der Kinderbetreuung egalitärer als in Westdeutschland und die Unterschiede reduzieren sich erst dann, wenn sich das Kind im Jugendalter befindet. Die Erwerbskonstellation spielt bei einem unter 1-jährigen Kind noch keine große Rolle, aber in den nachfolgenden Altersstufen zeigen sich gravierende Unterschiede: Am egalitärsten ist die Kinderbetreuung dann aufgeteilt, wenn beide Eltern in Vollzeit erwerbstätig sind und am traditionellsten dann, wenn die Väter in Vollzeit und die Mütter nicht erwerbstätig sind. Dieser starke Zusammenhang zwischen der Erwerbskonstellation und der Aufteilung der Kinderbetreuung. wird erst ab einem Kindesalter von 11 Jahren etwas schwächer, bleibt aber dennoch höchst signifikant. 
Abbildung 10.6: Bivariate Zusammenhänge der Ost-West-Unterschiede und der Erwerbskonstellation mit der Aufteilung der Kinderbetreuung
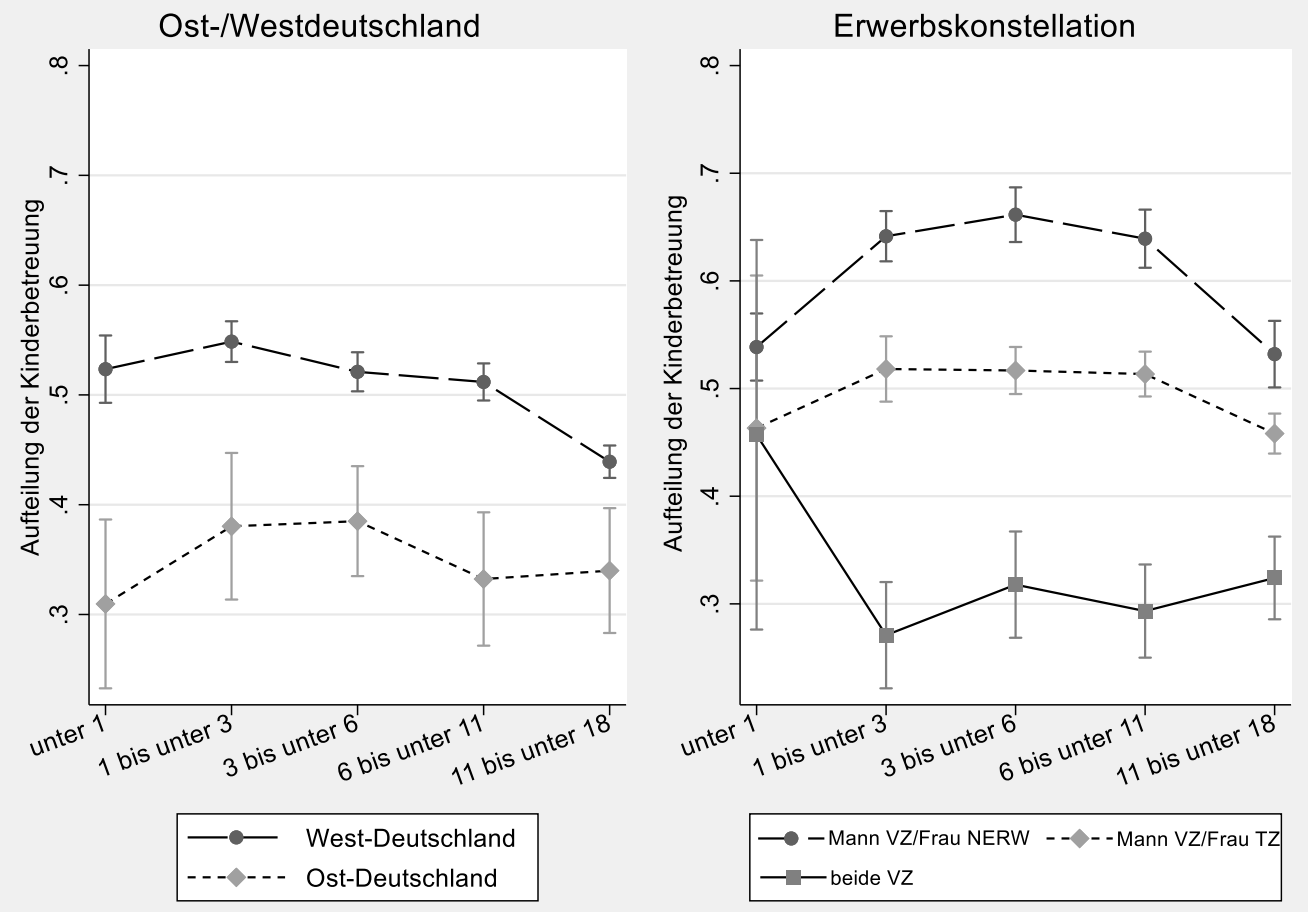

Anmerkung: Vorhergesagte Aufteilung der Kinderbetreuung auf Basis von bivariaten Regressionsmodellen mit 95\%-Konfidenzintervallen. Quelle: DJI-Studie AID:A 2013/14, gewichtet, $\mathrm{n}$ (Ost-/Westdeutschland) $=10.902 ; \mathrm{n}$ (Erwerbskonstellation) $=9.908$; eigene Berechnungen.

Aus den bivariaten Ergebnissen zur Aufteilung der Kinderbetreuung geht insgesamt hervor, dass der relative Berufsstatus und der absolute Berufsstatus der Mutter - analog zur Aufteilung der Hausarbeit - nur eine geringe Rolle spielen, während bei den Geschlechterrolleneinstellungen stärkere Zusammenhänge mit der Aufteilung der Kinderbetreuung vorliegen. Dies wird sowohl aus den Ergebnissen mit den einzelnen GenderItems als auch aus den Ergebnissen mit den latenten Gender-Klassen ersichtlich. Wenn egalitäre Einstellungen vertreten werden, so ist die Aufteilung der Kinderbetreuung in allen Altersstufen egalitärer. Bzgl. der Ost-West-Unterschiede lässt sich festhalten, dass die Kinderbetreuung in Ostdeutschland egalitärer aufgeteilt ist als in Westdeutschland und diese Unterschiede bei einem unter 1-jährigem Kind besonders deutlich zum Vorschein kommen. Deutliche Zusammenhänge zeigen sich auch zwischen den Erwerbskonstellationen und der Aufteilung der Kinderbetreuung. So ist die Kinderbetreuung bei Vollzeit-/Vollzeit-Konstellationen am egalitärsten aufgeteilt und am traditionellsten in den Konstellationen, in denen der Vater in Vollzeit und die Mutter nicht erwerbstätig ist. Wie eingangs erwähnt, ist die Kinderbetreuung insgesamt egalitärer aufgeteilt als die Hausarbeit. Dies mag damit zusammenhängen, dass die Kinderbetreuung im Gegensatz zur Hausarbeit als angenehm empfunden wird und deshalb nicht automatisch angenommen werden kann, dass sich Eltern aus der Kinderbetreuung ebenso herausverhandeln 
möchten wie aus der Hausarbeit (Raley et al. 2012; Sullivan 2013). Im Folgenden werden die deskriptiven Befunde anhand multivariater Analysen überprüft.

\subsection{Multivariate Analysen}

Bevor das Gesamtmodell präsentiert wird, wird zunächst gesondert auf die Bedeutung des Alters des Kindes eingegangen, da dieses in der vorliegenden Arbeit von zentraler Bedeutung ist. In Abbildung 10.7 (basierend auf

Tabelle A.9 im Anhang) sind diese Ergebnisse dargestellt. Die vorhergesagte Aufteilung der Kinderbetreuung ist in den ersten zehn Lebensjahren des Kindes relativ konstant und beträgt etwa 0,5. Da die gesamte Skala der Aufteilung der Kinderbetreuung (analog zur Aufteilung der Hausarbeit) von „-1: Vater übernimmt alles“ über „0: beide übernehmen gleich viel“ bis hin zu „1: Mutter übernimmt alles“ geht, bedeutet ein Wert von 0,5 inhaltlich, dass die Kinderbetreuung überwiegend von der Mutter übernommen wird. Erst ab einem Kindesalter von 11 Jahren wird die Kinderbetreuung signifikant egalitärer aufgeteilt als in den bisherigen Altersstufen. Eine mögliche Erklärung hierfür könnte sein, dass mit steigendem Alter des Kindes weniger Routinebetreuung (wie z.B. Körperpflege) anfällt und stattdessen mehr interaktive Betreuung (wie z.B. Spielen) möglich ist und Väter bevorzugt interaktive Betreuung übernehmen (Walper und Lien 2018).

Insgesamt spielt das Alter des Kindes jedoch eine erstaunlich geringe Rolle für die Aufteilung der Kinderbetreuung. Dies wird etwa daran ersichtlich, dass das korrigierte $\mathrm{R}^{2}$ nur 0,01 beträgt (vgl.

Tabelle A.9 im Anhang), d.h. nur 1\% der Streuung der Aufteilung der Kinderbetreuung kann anhand des Kindesalters erklärt werden. Während die Ergebnisse zur Aufteilung der Erwerbsarbeit einen sehr starken Zusammenhang mit dem Alter des Kindes aufzeigen, fallen die Zusammenhänge zwischen der Aufteilung der Kinderbetreuung (ebenso wie bei der Aufteilung der Hausarbeit) und dem Alter des Kindes somit gering aus.

Des Weiteren unterscheiden sich die Dimensionen der Arbeitsteilung hinsichtlich ihrer Entwicklungen mit zunehmendem Alter des Kindes: Während die Erwerbsarbeit mit zunehmendem Alter des Kindes egalitärer aufgeteilt wird, wird die Hausarbeit mit zunehmendem Alter des Kindes geschlechtsspezifischer aufgeteilt, wohingegen sich die Aufteilung der Kinderbetreuung in den ersten zehn Lebensjahren des Kindes kaum verändert und erst ab dem elften Lebensjahr des Kindes egalitärer wird. 
Abbildung 10.7: Vorhergesagte Aufteilung der Kinderbetreuung in Abhängigkeit vom Alter des Kindes

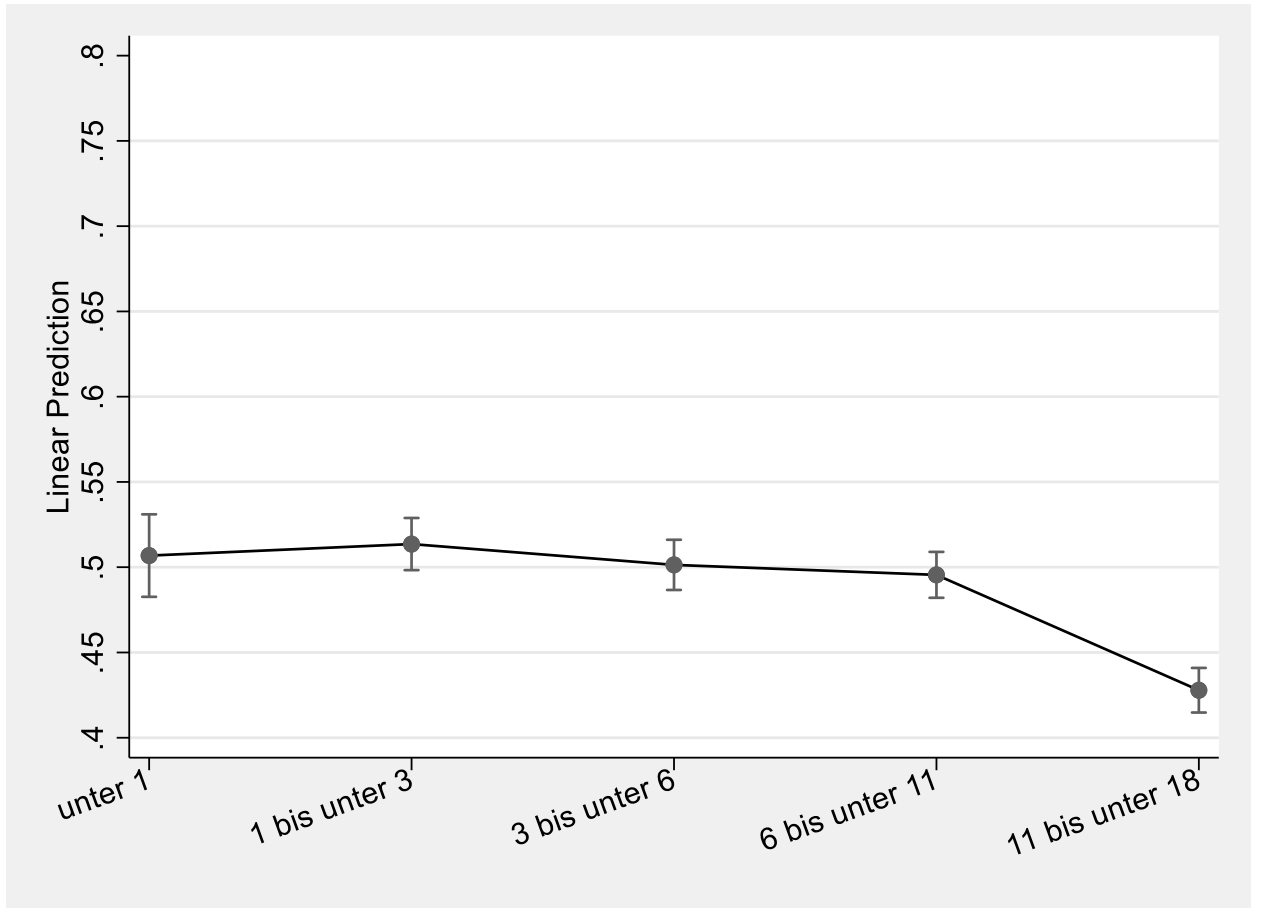

Anmerkung: Es sind die vorhergesagten Werte (Predictive Margins) mit den 95\%-Konfidenzintervallen abgetragen. Die Werte basieren auf der bivariaten OLS-Regression in Tabelle A.9 im Anhang. Quelle: DJI-Studie AID:A 2013/14, Sample: $n=10.410$, eigene Berechnungen.

Im Folgenden wird überprüft, wie robust diese Zusammenhänge unter Kontrolle von weiteren Variablen sind. In Tabelle 10.2 wird das Gesamtmodell stufenweise aufgebaut. Modell 1 enthält lediglich das Alter des Kindes als unabhängige Variable und zeigt, dass die Kinderbetreuung erst ab dem elften Lebensjahr des Kindes egalitärer wird. Der Effekt des Kindesalters bleibt in den Modellen 4 und 5 unter der Kontrolle weiterer Variablen nahezu unverändert. Sobald jedoch in Modell 6 auf die Erwerbsstunden der Mütter und Väter kontrolliert werden, kommt es zu einer Veränderung, wonach die Aufteilung der Kinderbetreuung bis zu einem Alter von unter 11 Jahren signifikant traditioneller aufgeteilt ist im Vergleich zur Referenzkategorie eines unter 1-jährigen Kindes. Laut Modell 6 zeigt sich erst in der höchsten Altersstufe kein signifikanter Unterschied mehr zur Referenzkategorie. Die ungewöhnliche und starke Veränderung der Koeffizienten unter Kontrolle der Erwerbstätigkeit verweist auf einen Suppressoreffekt. ${ }^{52}$

In Modell 2 repräsentiert der relative Berufsstatus die Bedeutung der relativen Ressourcen für die Aufteilung der Kinderbetreuung. Hypothese 1 nimmt an, dass sich diejenige Person mit den höheren, relativen Ressourcen auf die Erwerbsarbeit und die andere

52 Vgl. Fußnote 40 zur Erläuterung eines Suppressoreffekts. 
Person auf die Hausarbeit und Kinderbetreuung spezialisiert. Aus Modell 2 geht hervor, dass Mütter mit einem niedrigeren relativen Berufsstatus im Vergleich zur Referenzkategorie (= gleicher Berufsstatus) mehr Kinderbetreuung übernehmen. Dieses Ergebnis steht in Einklang mit der theoretischen Annahme und bleibt auch unter der Kontrolle weiterer Variablen (vgl. Modell 3 bis 6) stabil. Umgekehrt wurde jedoch angenommen, dass ein höherer Status der Mütter zu einer egalitäreren Aufteilung der Kinderbetreuung führt. Dies kann jedoch nicht bestätigt werden. Insgesamt spielen die relativen Ressourcen nur eine sehr geringe Rolle für die Aufteilung der Kinderbetreuung (vgl. das verschwindend geringe $R^{2}$ von (gerundet) 0,00 in Tabelle 10.2).

Als nächstes sind in Modell 3 in Tabelle 10.2 die Geschlechterrolleneinstellungen aufgelistet. Beide Items habe negative Koeffizienten, d.h. egalitärere Einstellungen gehen mit einer egalitäreren Aufteilung der Kinderbetreuung einher. Da die beiden Items nur in geringer Korrelation zueinanderstehen (der Korrelationskoeffizient $r$ beträgt 0,18 ), werden sie getrennt betrachtet und nicht zu einem Index zusammengefasst. Inhaltlich fragt Gender-Item 1 danach, ob sowohl die Frau als auch der Mann in Teilzeit arbeiten soll, wenn kleine Kinder vorhanden sind. Wenn solch eine Teilzeit-/Teilzeit-Konstellation befürwortet wird, geht dies mit einer egalitäreren Aufteilung der Kinderbetreuung einher. Bei GenderItem 2 wird die Einstellung abgefragt, ob beim Vorhandensein von Kindern der Mann arbeiten und die Frau zu Hause bleiben soll, d.h. ob man ein männliches Alleinverdienermodell befürwortet. Wenn man eine egalitäre Einstellung vertritt und ein männliches Alleinverdienermodell somit ablehnt, so ist dies mit einer egalitäreren Aufteilung der Kinderbetreuung assoziiert. Die Zusammenhänge bleiben in den Modellen 4 und 5 stabil, lediglich in Modell 6 unter Kontrolle der Erwerbsstunden der Mütter und Väter verringert sich die Bedeutung der Einstellungen, wobei sie dennoch höchst signifikant bleibt. Hypothese $3 \mathrm{a}$ ), die eine egalitärere Arbeitsteilung bei egalitäreren Einstellungen annimmt, kann somit bestätigt werden.

In Modell 5 sind neben diesen zentralen Variablen als weitere Variablen noch die Anzahl der Kinder, das Bundesgebiet und der absoluten Berufsstatus der Mutter enthalten. Bei der Anzahl der Kinder zeigt sich, dass in Familien mit einem Kind die Kinderbetreuung egalitärer aufgeteilt ist als in Familien mit zwei Kindern. Vergleicht man Familien mit zwei Kindern mit Familien mit drei, vier oder mehr Kindern, so gibt es keine Unterschiede bzgl. der Aufteilung der Kinderbetreuung. Dass die Kinderbetreuung in Einkind-Familien am egalitärsten aufgeteilt ist, steht in Einklang mit Hypothese 5 a).$^{53}$ Des Weiteren zeigen die Ergebnisse, dass die Kinderbetreuung in Ostdeutschland egalitärer aufgeteilt ist als

\footnotetext{
${ }^{53}$ Sobald in Modell 6 die Erwerbsstunden der Mütter und Väter mitkontrolliert werden, verschwindet der signifikante Effekt zwischen der Kinderanzahl und der Aufteilung der Kinderbetreuung. Dies mag damit zusammenhängen, dass die Kinderanzahl die Erwerbstätigkeit der Eltern entscheidend mitbestimmt und die Erwerbstätigkeit wiederum maßgeblich für die Aufteilung der Kinderbetreuung ist.
} 
in Westdeutschland und bestätigt somit Hypothese 6, die von einer egalitäreren Arbeitsteilung in Ostdeutschland als in Westdeutschland ausgeht. Als letzte Variable wurde in Modell 5 der absoluten Berufsstatus der Mutter mitaufgenommen und die Ergebnisse zeigen, dass bei Müttern mit einem niedrigem Berufsstatus (ISEI) die Kinderbetreuung egalitärer ist, während ein hoher Berufsstatus keinen signifikanten Effekt aufweist. Dies widerspricht Hypothese 7 a), wonach eine egalitärere Aufteilung dann zu erwarten wäre, wenn Mütter einen hohen Status haben und eine geschlechtsspezifischere Aufteilung dann, wenn Mütter einen niedrigen Status haben. ${ }^{54}$

Im letzten Modell, Modell 6, sind als weitere Variablen die Erwerbstätigkeit der Mütter und der Väter mitaufgenommen. Analog zur Aufteilung der Hausarbeit, wo die Erwerbstätigkeit ebenfalls als letzte Variable aufgenommen wurde, ist auch hier bei der Interpretation besondere Vorsicht geboten aufgrund der Endogenität der Erwerbstätigkeit. ${ }^{55}$ Aus den Ergebnissen geht hervor, dass bei in Teilzeit beschäftigten Müttern die Kinderbetreuung egalitärer aufgeteilt ist als bei nicht erwerbstätigen Müttern. Wenn Mütter in „normaler" Vollzeit (bis 45 Std. pro Woche) oder in besonders langer Vollzeit (mehr als 45 Std. pro Woche) erwerbstätig sind, so ist die Kinderbetreuung nochmals egalitärer aufgeteilt (im Vergleich zur Referenzkategorie der nicht-erwerbstätigen Mütter). Betrachtet man die Erwerbstätigkeit der Väter, so zeigt sich, dass die Aufteilung der Kinderbetreuung mit zunehmendem Erwerbsumfang mehr zu Lasten der Mütter verteilt wird. Hierbei ist hervorzuheben, dass die Kinderbetreuung durch eine lange Vollzeit der Väter (mehr als 45 Std.) nochmals deutlich geschlechtsspezifischer aufgeteilt ist als bei Vätern mit „normaler“ Vollzeit (bis 45 Std.). Dies trifft auf Mütter nicht zu, denn Mütter mit langer Vollzeit übernehmen nicht weniger als Mütter mit normaler Vollzeit, sondern sogar etwas mehr.

${ }^{54}$ Es wurde überprüft, ob dies evtl. darauf zurückzuführen ist, dass in diesem Modell sowohl der relative Berufsstatus als auch der absolute Berufsstatus enthalten sind und das Ergebnis durch Multikollinearität bedingt sind könnte. Dies ist jedoch nicht der Fall, da der VIF-Wert (Variance inflation factor) 1,58 beträgt und damit deutlich kleiner als 10 ausfällt. Ferner wurde der relative Berufsstatus testweise aus dem Modell ausgeschlossen (nicht abgebildet), doch auch dann zeigten sich dieselben Ergebnisse für den absoluten Berufsstatus.

55 Wie bereits weiter oben in Kapitel 9 zur Aufteilung der Hausarbeit erwähnt, führt die Aufnahme der Erwerbstätigkeit als unabhängige Variable zum Teil zu großen Veränderungen bei weiteren, unabhängigen Variablen wie etwa dem Alter des Kindes und der Anzahl der Kinder. Vgl. auch die Anmerkungen in Fußnote 40 zum Suppressoreffekt. 
Tabelle 10.2: Aufteilung der Kinderbetreuung, stufenweiser Aufbau des Modells, OLS-Regression

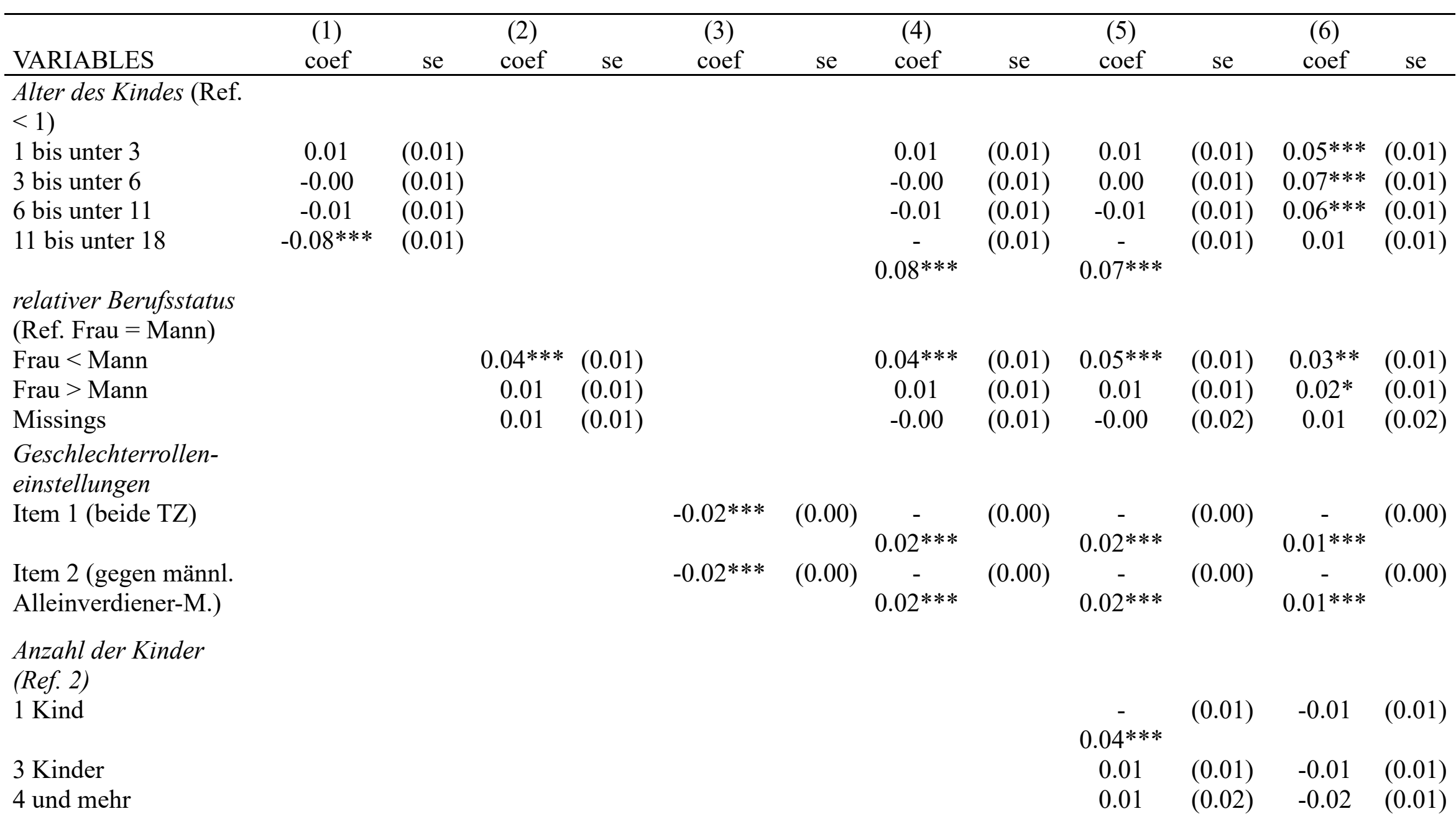

Bundesland (Ref.

Westdtl.) 
Ost-Dtl.

ISEI der Mutter (Ref.

$\begin{array}{cccc}- & (0.01) & - & (0.01) \\ 0.14 * * * & & 0.09 * * * & \\ & & & \\ & & & (0.01) \\ - & (0.01) & - & 0.03 * * * \\ 0.04 * * * & & 0.01 & (0.01) \\ -0.00 & (0.01) & 0.03 & (0.02) \\ -0.01 & (0.02) & -0.03 & \end{array}$

hoch (ab 63)

Missings

$\begin{array}{llll}-0.01 & (0.02) & -0.03 & (0.02)\end{array}$

Erwerbstätigkeit der

Mutter (Ref. nicht er-

werbstätig)

TZ (1-35 Std.)

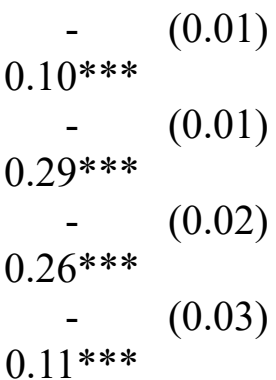

Erwerbstätigkeit des

Vaters (Ref. nicht er-

werbstätig)

TZ (1-35 Std.)

VZ (36-45 Std.)

mehr als VZ (> 45)

Missings

Constant

Observations

$0.51 * * *$

(0.01) $0.46^{* * *}(0.01)$

11,158

$0.65^{* * *}$

11,158

(0.01) $0.65 * * *$

(0.02)

0.02

0.03

$0.68^{* * * *}$

(0.02)

$0.12 * * *(0.02)$

$0.28 *(0.02)$

Adj. R-squared

0.01

0.00

0.05

$0.08 * \quad(0.04)$

Anmerkung: OLS-Regression mit folgenden Signifikanzen: ${ }^{* * *} p<0.001,{ }^{* *} p<0.01,{ }^{*} p<0.05$. Quelle: DJI-Studie AID:A 2013/14, Sample: $n=11.158$, eigene Berechnungen. 
Wie bereits bei der Aufteilung der Erwerbsarbeit und der Hausarbeit werden im Folgenden auch bei der Aufteilung der Kinderbetreuung die Analysen getrennt nach den Altersstufen des Kindes dargestellt (siehe Tabelle A.10 im Anhang). ${ }^{56}$ In der nachfolgenden Abbildung 10.8 werden die Regressionsergebnisse des relativen Berufsstatus betrachtet. Wenn Mütter einen niedrigeren Berufsstatus haben, übernehmen sie in den meisten Altersstufen mehr Kinderbetreuung (eine Ausnahme stellen die jüngste und die älteste Altersstufe dar). ${ }^{57}$ Die Hypothese 2 a), die annimmt, dass Mütter mit niedrigeren Ressourcen mehr unbezahlte Arbeit (und damit auch mehr Kinderbetreuung übernehmen) kann daher eingeschränkt bestätigt werden.

Umgekehrt zeigt sich dagegen, dass ein höherer Berufsstatus der Mutter in keiner einzigen Altersstufe einen signifikanten Effekt aufweist. Diese Tatsache, dass Mütter mit höheren Ressourcen nicht weniger Kinderbetreuung übernehmen, kann darauf hinweisen, dass Kinderbetreuung trotz der damit verbundenen Opportunitätskosten nicht vermieden werden will. So wird die Kinderbetreuung als wesentlich angenehmer empfunden als die Hausarbeit (Sullivan 2013): „The performance of child care (or at least some aspects of child care) may be a relatively desirable product of marital bargaining, but the performance of housework evidently is not" (Sullivan 2013, S. 75). Die Ergebnisse bestätigen somit, dass für die Aufteilung der Kinderbetreuung nicht dieselben Vorhersagen getroffen werden können wie für die Aufteilung der Hausarbeit, da ersteres im Gegensatz zu letzterem als angenehm und sinnstiftend empfunden wird und deswegen nicht vermieden werden will.

Bzgl. der altersspezifischen Hypothesen kann festgehalten werden, dass Hypothese 2 b), die annimmt, dass relative Ressourcen im ersten Lebensjahr des Kindes noch keine Rolle spielen, bestätigt werden kann. Hypothese $2 \mathrm{c}$ ) nimmt an, dass die relativen Ressourcen in der Altersstufe "1 bis 3 Jahre" bereits eine zumindest geringe Rolle spielen. Dies kann für die Ausprägung „Frau < Mann“ bestätigt werden, nicht jedoch für die die Ausprägung „Frau > Mann“, weshalb Hypothese 2 c) eingeschränkt bestätigt werden

\footnotetext{
${ }^{56}$ Die Koeffizienten in den nachfolgenden Abbildungen beruhen auf den Modellen 1c, 2c, $3 c$ usw. in Tabelle A.10 im Anhang. Darüber hinaus wurden in den Modellen 1d, 2d, 3d usw. (ebenfalls in Tabelle A.10 im Anhang) die Erwerbstätigkeit der Mütter und Väter mitkontrolliert. Aufgrund der Endogenitätsproblematik der Erwerbstätigkeit werden hier die Modelle zunächst ohne Kontrolle der Erwerbstätigkeit betrachtet, wobei bei den Ergebnissen darauf hingewiesen wird, inwiefern sich die Ergebnisse unter Berücksichtigung der Erwerbstätigkeit verändern würden.

57 Wenn die Erwerbstätigkeit der Mütter und Väter mitkontrolliert werden, dann bleibt der signifikante Effekt des relativen Berufsstatus bei „Frau < Mann“ lediglich in der Altersstufe „1 bis unter 3 Jahre" erhalten, in den Altersstufen „3 bis unter 6 Jahre" und „6 bis unter 11 Jahre" verlieren die Effekte ihre Signifikanz.
} 
kann. Die weiteren, altersspezifischen Hypothesen 2 d) und 2 e) müssen jedoch abgelehnt werden, da sie annehmen, dass sich die Effektstärken mit zunehmendem Alter des Kindes weiter vergrößern und dies in Widerspruch zu den Ergebnissen steht.

Abbildung 10.8: Koeffizienten des relativen Berufsstatus (ISEI) zur Vorhersage der Aufteilung der Kinderbetreuung, getrennt nach dem Alter des Kindes

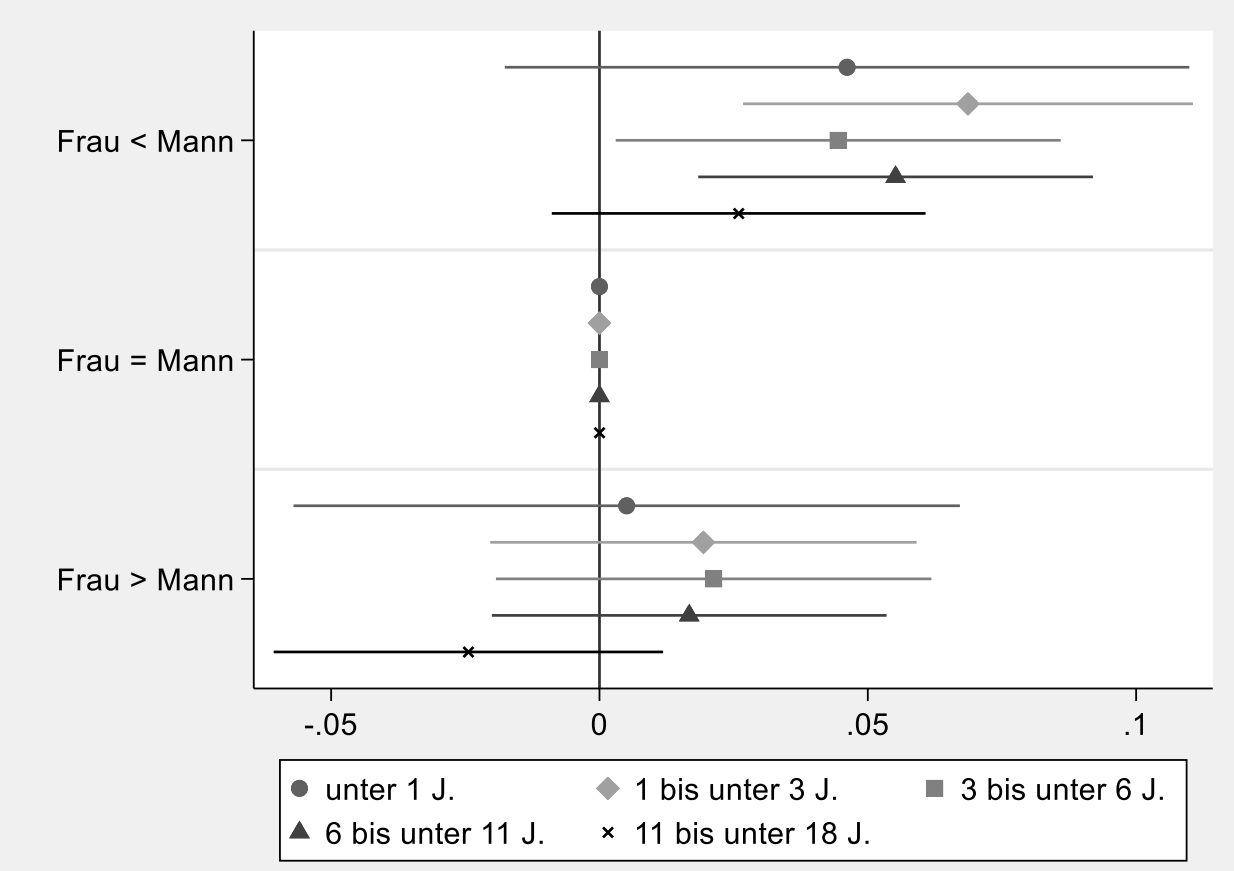

Anmerkung: OLS-Regression, dargestellt sind die Koeffizienten (Average Marginal Effects) mit 95\%-Konfidenzintervallen unter Kontrolle der weiteren Variablen aus Modell 1c, 2c, 3c usw. in Tabelle A.10 im Anhang. Quelle: DJI-Studie AID:A 2013/14, Sample: $n=11.158$, eigene Berechnungen.

Welche Zusammenhänge zeigen sich zwischen den Geschlechterrolleneinstellungen und der Aufteilung der Kinderbetreuung? Die negativen Koeffizienten in Abbildung 10.9 zeigen, dass bei beiden Gender-Items in (fast) allen Altersstufen eine egalitärere Einstellung mit einer signifikant egalitäreren Aufteilung der Kinderbetreuung einhergeht. D.h. sowohl eine Befürwortung, dass beide Elternteile bei kleinen Kindern in Teilzeit arbeiten sollen (= egalitäre Einstellung bei Gender-Item 1) als auch eine Ablehnung des männlichen Alleinverdiener-Modells (= egalitäre Einstellung bei Gender-Item 2) haben einen egalisierenden Effekt auf die Aufteilung der Kinderbetreuung. Die altersspezifischen Hypothesen 3 b) bis 3 e) gehen davon aus, dass egalitäre Einstellungen bei kleineren Kindern schwerer zu realisieren sind und innen deshalb bei kleineren Kindern eine geringere Bedeutung zukommt als bei größeren Kindern. 
Abbildung 10.9: Koeffizienten der beiden Gender-Items zur Vorhersage der Aufteilung der Kinderbetreuung, getrennt nach dem Alter des Kindes
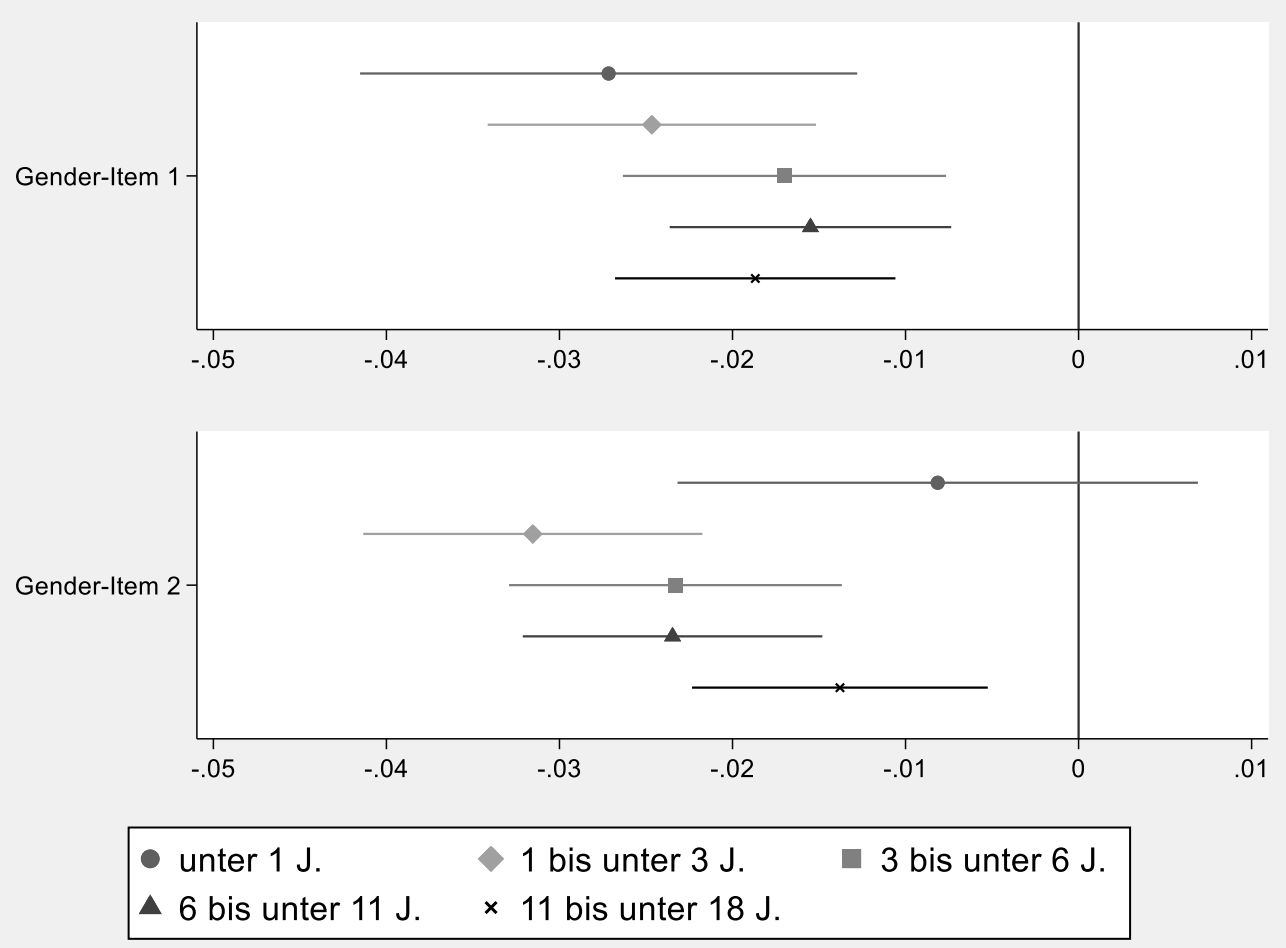

Anmerkung: OLS-Regression, dargestellt sind die Koeffizienten (Average Marginal Effects) mit 95\%-Konfidenzintervallen unter Kontrolle der weiteren Variablen aus Modell 1c, 2c, 3c usw. in Tabelle A.10 im Anhang; Gender-Item 1 = Befürwortung, dass beide Elternteile in Teilzeit arbeiten sollen, wenn kleine Kinder da sind; Gender-Item 2 = Ablehnung eines männlichen AlleinverdienerModells. Quelle: DJI-Studie AID:A 2013/14, Sample: n = 11.158, eigene Berechnungen.

Wie aus weiteren Modellen mit Interaktionseffekte hervorgeht (nicht abgebildet), unterscheiden sich die meisten Effekte zwischen den Altersstufen nicht signifikant voneinander, sodass die meisten altersspezifischen Hypothesen nicht bestätigt werden können. Eine Ausnahme liegt bei Gender-Item 2 vor: Dass der Effekt im Alter „unter 1 Jahr“ nicht signifikant ist, entspricht den Erwartungen von Hypothese $3 \mathrm{~b}$ ), wonach egalitäre Einstellungen hier besonders schwer zu realisieren sind und deswegen keinen oder nur einen sehr geringen Effekt aufweisen. Der Effekt ist in der nächsten Altersstufe, „1 bis unter 3 Jahre", signifikant höher und entspricht der Hypothese $3 \mathrm{c}$ ), die einen zunehmendem Effekt mit zunehmendem Alter des Kindes erwartet. Jedoch vergrößert sich der Effekt in den nachfolgenden Altersstufen nicht weiter (sondern wird sogar etwas geringer, wenn auch nicht signifikant geringer) und steht somit nicht im Einklang mit den Hypothesen $3 \mathrm{~d}$ ) und 3 e). ${ }^{58}$ Während für Gender-Item 1 somit alle altersspezifischen Hypothesen zu den Geschlechterrolleneinstellungen abgelehnt werden müssen, können für Gender-

\footnotetext{
${ }^{58}$ Eine weitere Ausnahme bilden bei Gender-Item 2 die Altersstufen „1 bis unter 3 Jahre“ und „11 bis unter 18 Jahre": Bei ersteren ist der Effekt signifikant größer als bei letzteren. Dies steht konträr zu den theoretischen Erwartungen.
} 
Item 2 immerhin die Hypothesen 3 b) und 3 c) bestätigt werden. Dagegen müssen die Hypothesen 3 d) und 3 e) auch bei Gender-Item 2 abgelehnt werden.

Nach den Geschlechterrolleneinstellungen wird als nächstes der Einfluss der Kinderanzahl auf die Aufteilung der Kinderbetreuung untersucht. Aus Abbildung 10.10 geht hervor, dass die Aufteilung der Kinderbetreuung in Familien mit einem Kind in den meisten Altersstufen egalitärer ist als in Familien mit zwei Kindern. Dass die Arbeitsteilung bei einem Kind egalitärer ist als bei vielen Kindern entspricht den Erwartungen von Hypothese 5 a). Dagegen zeigt sich kein signifikanter Unterschied zwischen Familien mit zwei und Familien mit drei Kindern. In Familien mit vier Kindern, bei denen das jüngste Kind unter 1 Jahr alt ist, ist die Kinderbetreuung sogar deutlich egalitärer aufgeteilt als in Familien mit zwei Kindern. Dies widerspricht Hypothese 5 b), die annimmt, dass die Unterschiede zwischen Ein- und Mehrkindfamilien besonders groß sind, wenn das Kind noch sehr klein ist, weil die Arbeitsteilung in Mehrkindfamilien dann besonders traditionell sein müsste.

Abbildung 10.10: Koeffizienten der Anzahl der Kinder zur Vorhersage der Aufteilung der Kinderbetreuung, getrennt nach dem Alter des Kindes

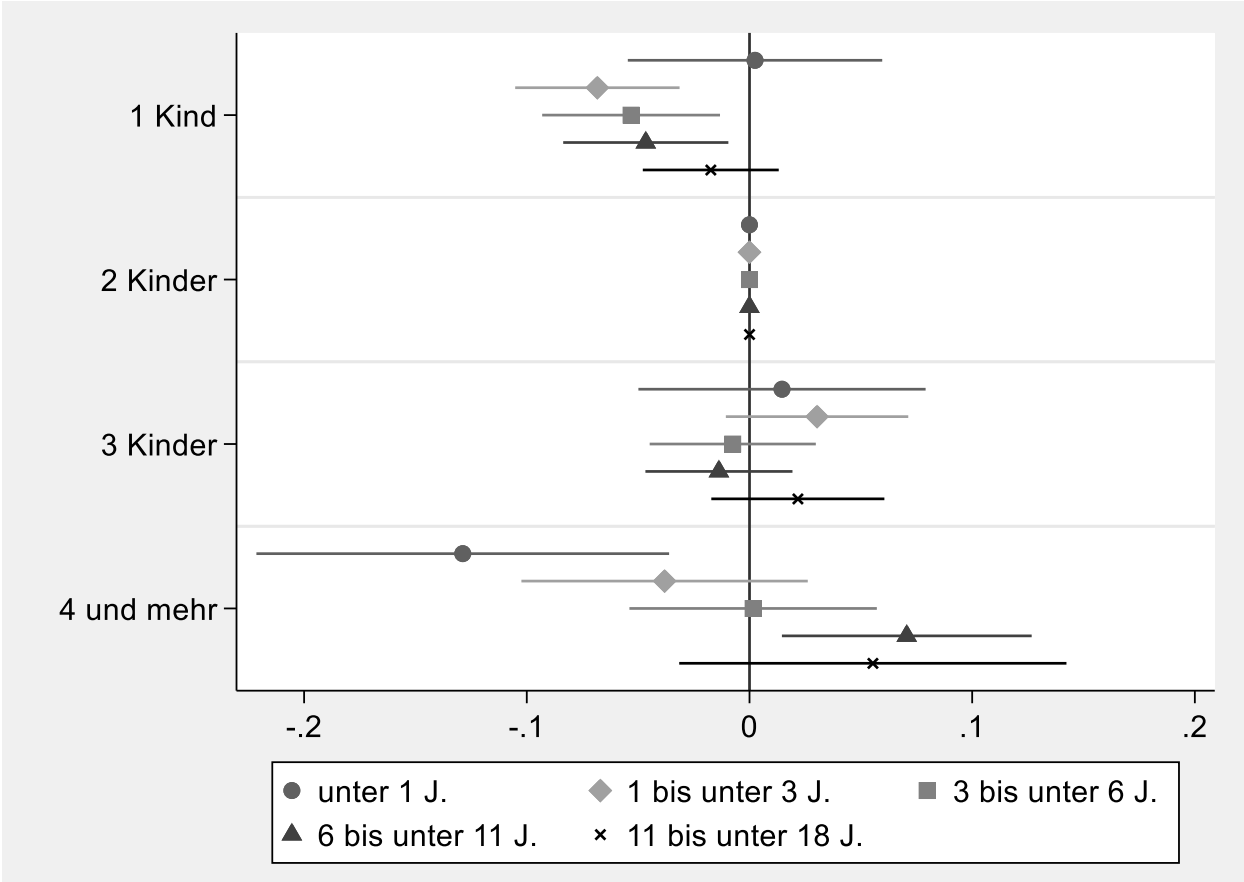

Anmerkung: OLS-Regression, dargestellt sind die Koeffizienten (Average Marginal Effects) mit 95\%-Konfidenzintervallen unter Kontrolle der weiteren Variablen aus Modell 1c, 2c, 3c usw. in Tabelle A.10 im Anhang. Quelle: DJI-Studie AID:A 2013/14, Sample: $n=11.158$, eigene Berechnungen.

Die Ergebnisse zeigen jedoch, dass die Kinderbetreuung in Familien mit vier oder mehr Kindern, bei denen das jüngste Kind unter 1 Jahr ist, nicht deutlich traditioneller, sondern deutlich egalitärer ist. Eine mögliche Erklärung hierfür könnte sein, dass eine egalitärere Aufteilung hier notwendig ist und Mütter auf die Unterstützung der Väter angewiesen 
sind, weil sie einen derart hohen Betreuungsaufwand nicht allein bewältigen können. ${ }^{59}$ Hypothese 5 b), die annimmt, dass die Unterschiede zwischen Einkind-, Zweikind- und Mehrkindfamilien in den ersten Lebensjahren des Kindes besonders groß sind und zunehmendem Alter des Kindes geringer werden, muss abgelehnt werden.

Als nächstes interessiert die Frage danach, welche Bedeutung das Bundesgebiet für die Aufteilung der Kinderbetreuung hat (siehe Abbildung 10.11). Allgemein fällt auf, dass die Kinderbetreuung in Ostdeutschland egalitärer aufgeteilt als in Westdeutschland, was in Einklang mit Hypothese 6 a) ist. Die altersspezifische Hypothese 6 b) nimmt darüber hinaus an, dass die Unterschiede in der Arbeitsteilung zwischen Ost- und Westdeutschland bei kleinen Kindern besonders groß sind und mit zunehmendem Alter des Kindes schwächer werden. Wie aus Modellen mit Interaktionseffekten (nicht abgebildet) hervorgeht, unterscheiden sich die Effekte nicht signifikant zwischen den Altersstufen, sodass Hypothese 6 b) nicht bestätigt werden kann. Wenn, wie im Anhang in Tabelle A.10 bei den Modellen 1d, 2d, 3d usw., die Erwerbstätigkeit der Mütter und Väter mitkontrolliert wird, so verringern sich die Effektstärken etwas, bleiben aber dennoch höchst signifikant. Inhaltlich kommt der Erwerbstätigkeit hierbei die Rolle eines Mediators zu: Das Bundesgebiet beeinflusst die Erwerbstätigkeit der Eltern (insbesondere der Mütter) und diese beeinflusst wiederum die Aufteilung der Kinderbetreuung. ${ }^{60}$ Unter der Kontrolle der Erwerbstätigkeit verringert sich daher der direkte Effekt des Bundesgebietes auf die Aufteilung der Kinderbetreuung.

59 Die großen Konfidenzintervalle bei der Kategorie „4 und mehr Kinder“ sind auf die geringen Fallzahlen zurückzuführen: So sind nur in 635 Familien „4 und mehr Kinder" vorhanden, was 7,6\% des Samples $(n$ (Sample) $=11.158$ ) entspricht. Von diesen $n=635$ gehören nur $n=64$ zum Modell „unter 1 Jahr“ und nur $n=68$ zum Modell „11 bis unter 18 Jahre“, deswegen sind hier die Konfidenzintervalle besonders groß.

60 Die Erwerbstätigkeit wird hier aufgrund theoretischer Überlegungen als unabhängige Variable betrachtet und die Aufteilung der Kinderbetreuung als abhängige Variable. Es ist jedoch auch die umgekehrte Kausalitätsrichtung denkbar. Die Kausalitätsrichtung kann mit den hier vorliegenden Querschnittsanalysen jedoch nicht überprüft werden. 
Abbildung 10.11: Koeffizienten des Bundesgebietes zur Vorhersage der Aufteilung der Kinderbetreuung, getrennt nach dem Alter des Kindes

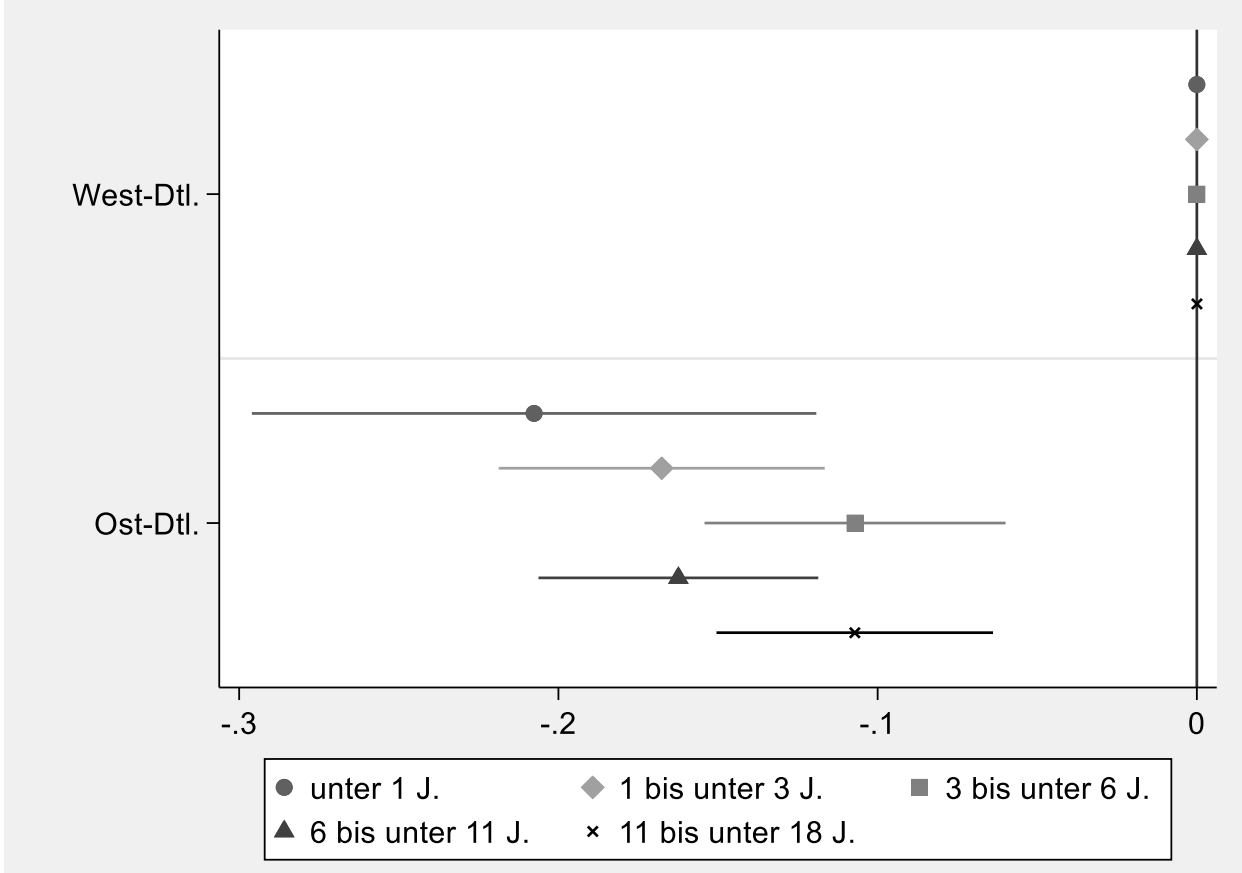

Anmerkung: OLS-Regression, dargestellt sind die Koeffizienten (Average Marginal Effects) mit 95\%-Konfidenzintervallen unter Kontrolle der weiteren Variablen aus Modell 1c, 2c, 3c usw. in Tabelle A.10 im Anhang. Quelle: DJI-Studie AID:A 2013/14, Sample: $n=11.158$, eigene Berechnungen.

Die nächste Abbildung zeigt die Bedeutung des absoluten Berufsstatus der Mutter für die Aufteilung der Kinderbetreuung (Abbildung 10.12). Es kann nicht bestätigt werden, dass Mütter mit niedrigen Berufsstatus mehr Kinderbetreuung und Mütter mit hohen Berufsstatus weniger Kinderbetreuung übernehmen - weder im Allgemeinen noch für einzelne Altersstufen. Die Hypothese 7 a), die eine egalitärere Aufteilung bei einem hohen Status der Mutter annimmt, und die Hypothese 7 b), die altersspezifische Unterschiede annimmt, müssen somit beide abgelehnt werden. 
Abbildung 10.12: Koeffizienten des absoluten Berufsstatus (ISEI) der Mutter zur Vorhersage der Aufteilung der Kinderbetreuung, getrennt nach dem Alter des Kindes

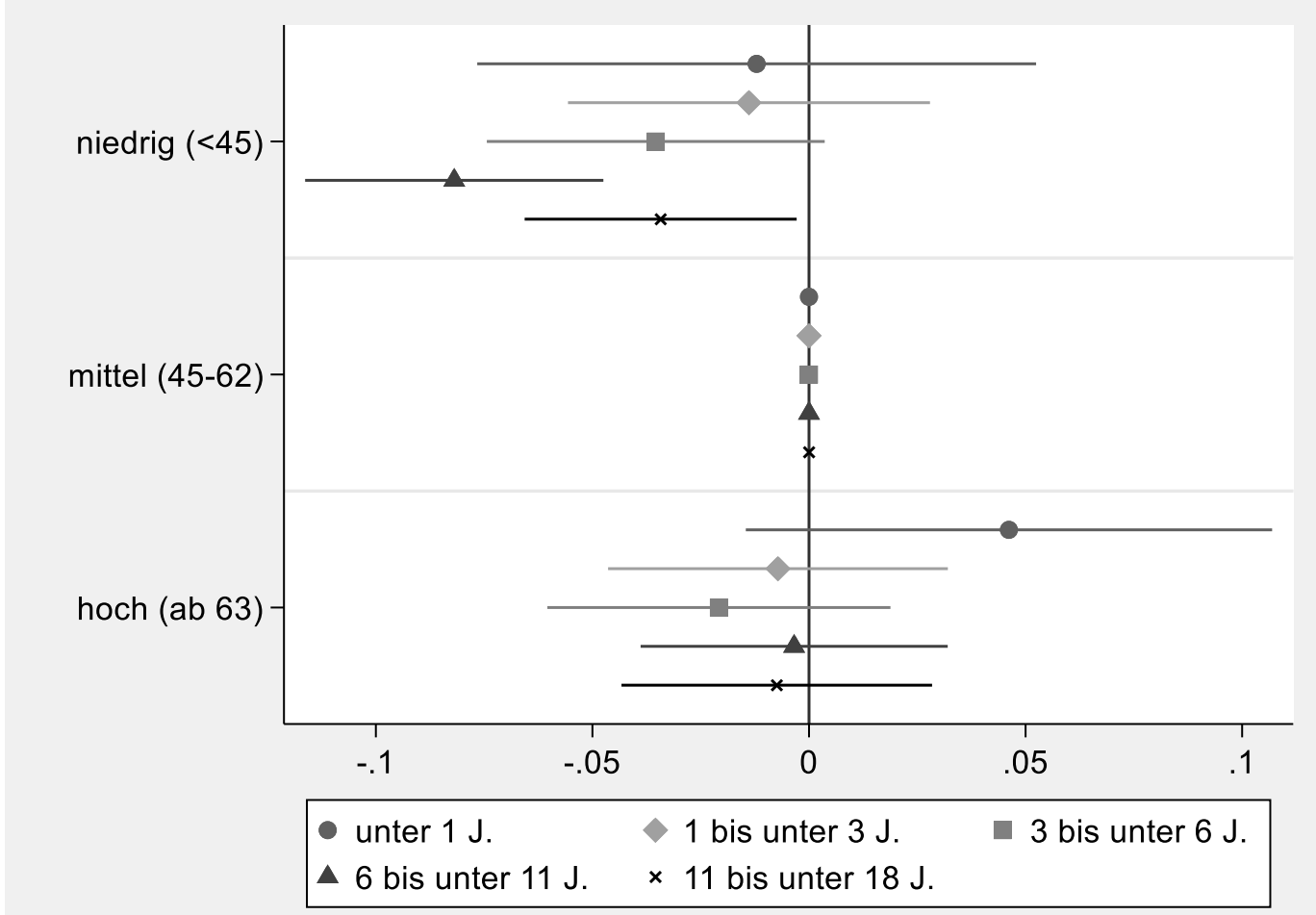

Anmerkung: OLS-Regression, dargestellt sind die Koeffizienten (Average Marginal Effects) mit 95\%-Konfidenzintervallen unter Kontrolle der weiteren Variablen aus Modell 1c, 2c, 3c usw. in Tabelle A.10 im Anhang. Quelle: DJI-Studie AID:A 2013/14, Sample: $n=11.158$, eigene Berechnungen.

In Abbildung 10.13 ist der Zusammenhang zwischen der Erwerbstätigkeit der Mütter und der Aufteilung der Kinderbetreuung abgebildet. Auffällig ist, dass die Koeffizienten weitaus größer ausfallen als bei allen bisherigen Variablen, d.h. der Erwerbsumfang der Mütter (und auch der Väter, siehe hierzu die darauffolgende Abbildung) zählt zur wichtigsten Einflussgröße für die Aufteilung der Kinderbetreuung. Wenn Mütter in Teilzeit erwerbstätig sind, so ist die Kinderbetreuung egalitärer aufgeteilt als dies bei nicht erwerbstätigen Müttern der Fall ist. Noch egalitärer ist die Kinderbetreuung dann aufgeteilt, wenn Mütter Vollzeit beschäftigt sind. Wenn Mütter statt in „normaler“ Vollzeit (bis 45 Stunden) in langer Vollzeit (über 45 Stunden) erwerbstätig sind, dann wird die Arbeitsteilung nicht noch egalitärer. Dass bei Müttern mit langer Vollzeit in der Altersstufe „unter 1 Jahr“ der Effekt nicht signifikant ist, kann damit erklärt werden, dass die Fallzahlen hier sehr gering sind und das Konfidenzintervall dementsprechend sehr groß. Auch die Fallzahlen von Müttern, die bei einem unter 1-jährigen Kind in Teilzeit oder in „normaler“ Vollzeit arbeiten, sind relativ gering, da der Großteil der Mütter nicht erwerbstätig ist und frühestens ab Vollendung des ersten Lebensjahres des Kindes wieder in die Erwerbstätigkeit einsteigt. 
Abbildung 10.13: Koeffizienten der Erwerbstätigkeit der Mutter zur Vorhersage der Aufteilung der Kinderbetreuung, getrennt nach dem Alter des Kindes

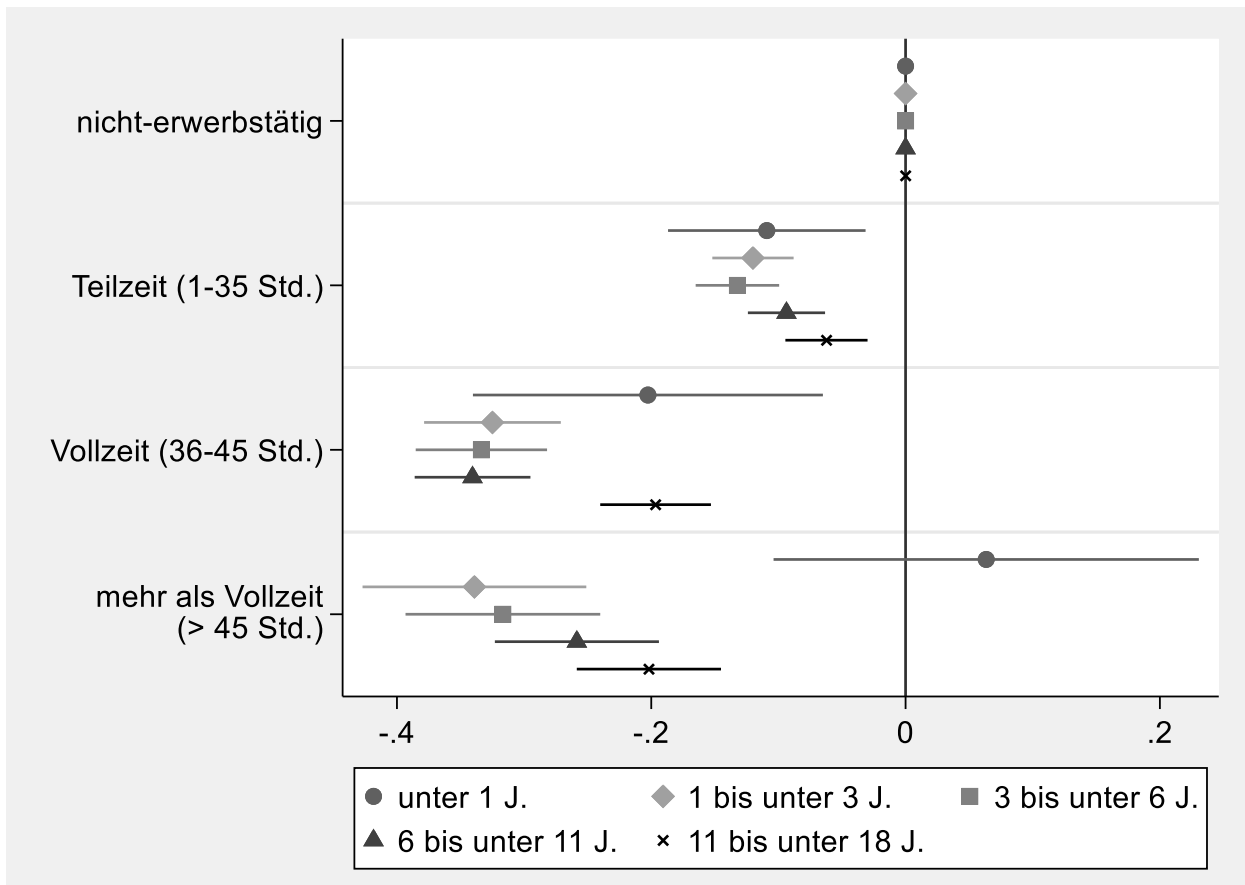

Anmerkung: OLS-Regression, dargestellt sind die Koeffizienten (Average Marginal Effects) mit 95\%-Konfidenzintervallen unter Kontrolle der weiteren Variablen aus Modell 1d, 2d, 3d usw. in Tabelle A.10 im Anhang. Quelle: DJI-Studie AID:A 2013/14, Sample: $n=11.158$, eigene Berechnungen.

Analog zum Erwerbsumfang der Mütter ist der Erwerbsumfang der Väter in Abbildung 10.14 abgebildet. Hier zeigt sich, dass die Aufteilung der Kinderbetreuung mit zunehmendem Erwerbsumfang der Väter mehr von den Müttern übernommen wird. Bei kleineren Kindern spielt die Erwerbstätigkeit der Väter eine größere Rolle für die Aufteilung der Kinderbetreuung als bei älteren Kindern. 
Abbildung 10.14: Koeffizienten der Erwerbstätigkeit des Vaters zur Vorhersage der Aufteilung der Kinderbetreuung, getrennt nach dem Alter des Kindes

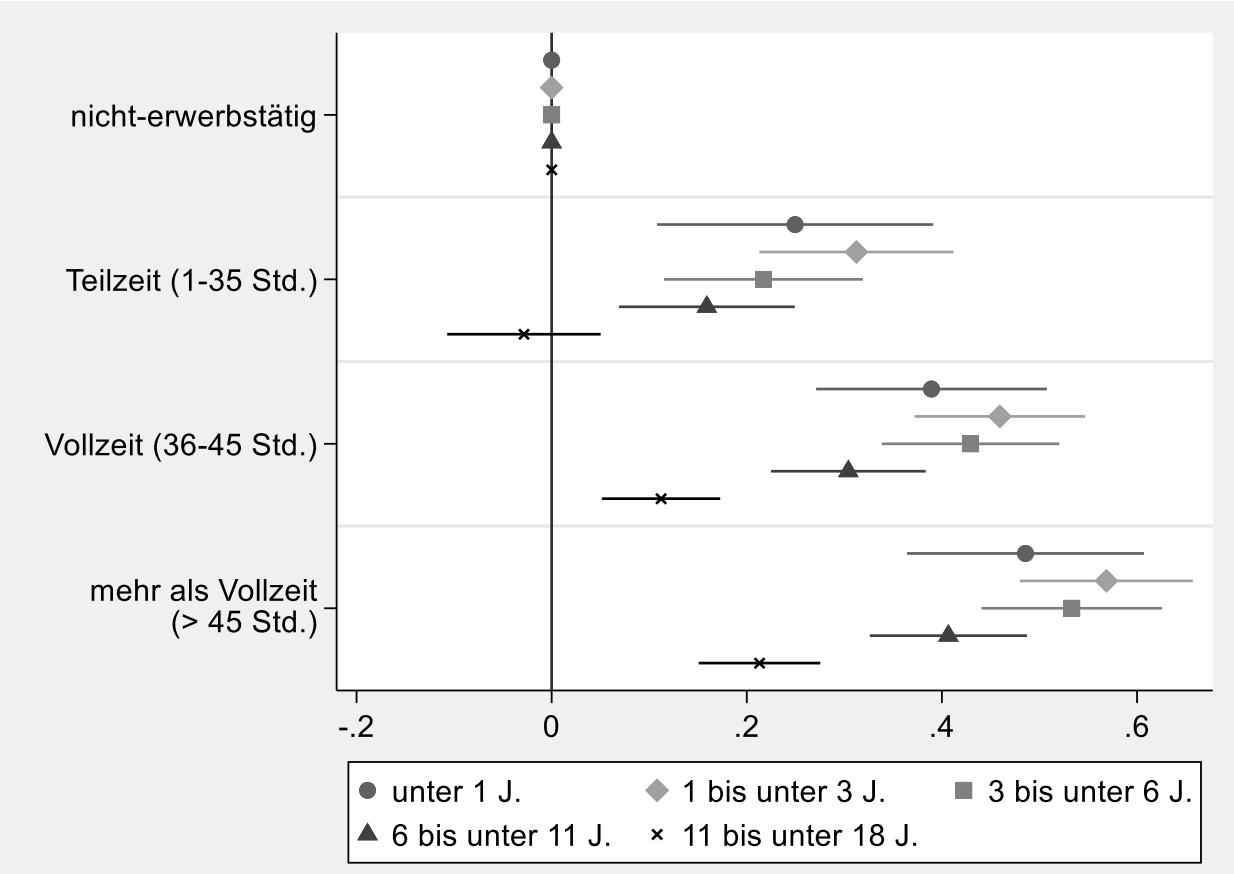

Anmerkung: OLS-Regression, dargestellt sind die Koeffizienten (Average Marginal Effects) mit 95\%-Konfidenzintervallen unter Kontrolle der weiteren Variablen aus Modell 1d, 2d, 3d usw. in Tabelle A.10 im Anhang. Quelle: DJI-Studie AID:A 2013/14, Sample: $n=11.158$, eigene Berechnungen.

\section{Vertiefende Analysen zu den Geschlechterrolleneinstellungen}

Wie auch bei den anderen beiden Dimensionen der Arbeitsteilung werden auch bei der Aufteilung der Kinderbetreuung vertiefende Analysen zu den Geschlechterrolleneinstellungen vorgenommen. Nahezu alle Gender-Items weisen negative Koeffizienten auf, was inhaltlich bedeutet, dass egalitärere Einstellungen mit einer egalitäreren Aufteilung der Kinderbetreuung einhergehen (siehe Tabelle 10.3). Am spannendsten ist Item 4 für die Aufteilung der Kinderbetreuung, da dieses Item explizit danach fragt, ob ein Vater genauso sehr in die Pflege und Erziehung eingebunden sein sollte, wie die Mutter. Wenn dies befürwortet wird, so ist die Aufteilung der Kinderbetreuung deutlich egalitärer. Die größten Effekte zeigen sich bei einem Kindesalter von „1 bis unter 3 Jahren“.

Tabelle 10.3: Aufteilung der Kinderbetreuung, unter Berücksichtigung der vier Gender-Items, getrennt nach dem Alter des Kindes

\begin{tabular}{|c|c|c|c|c|c|c|c|c|}
\hline & \multicolumn{2}{|l|}{ unter 1} & \multicolumn{2}{|c|}{1 bis unter 3} & \multicolumn{2}{|c|}{3 bis unter 6} & \multicolumn{2}{|c|}{6 bis unter 9} \\
\hline & (1) & & (2) & & (3) & & (4) & \\
\hline VARIABLES & coef & se & coef & se & coef & $\mathrm{se}$ & coef & se \\
\hline $\begin{array}{l}\text { Geschlech- } \\
\text { terrollenein- } \\
\text { stellungen }\end{array}$ & & & & & & & & \\
\hline $\begin{array}{l}\text { Item } 1 \text { (beide } \\
\text { TZ) }\end{array}$ & $-0.02 * *$ & $(0.01)$ & $-0.01 *$ & $(0.00)$ & -0.01 & $(0.01)$ & -0.00 & $(0.01)$ \\
\hline
\end{tabular}




\begin{tabular}{|c|c|c|c|c|c|c|c|c|}
\hline $\begin{array}{l}\text { Item } 2 \text { (ge- } \\
\text { gen männl. } \\
\text { Alleinverdie- } \\
\text { ner-M.) }\end{array}$ & -0.02 & $(0.01)$ & $-0.02 * *$ & $(0.01)$ & -0.01 & $(0.01)$ & $-0.02 *$ & $(0.01)$ \\
\hline $\begin{array}{l}\text { Item } 3 \text { (ge- } \\
\text { gen männl. } \\
\text { Hauptver- } \\
\text { diener-M.) }\end{array}$ & $0.02 * *$ & $(0.01)$ & -0.00 & $(0.01)$ & $-0.01 *$ & $(0.01)$ & -0.01 & $(0.01)$ \\
\hline $\begin{array}{l}\text { Item } 4 \text { (für } \\
\text { egali. Care- } \\
\text { Arbeit) }\end{array}$ & $-0.06 * * *$ & $(0.01)$ & $-0.10 * * *$ & $(0.01)$ & $-0.07 * * *$ & $(0.01)$ & $-0.08 * * *$ & $(0.01)$ \\
\hline Constant & $0.83 * * *$ & $(0.09)$ & $1.18 * * *$ & $(0.05)$ & $1.03 * * *$ & $(0.05)$ & $1.13 * * *$ & $(0.07)$ \\
\hline Observations & 850 & & 2,070 & & 2,034 & & 1,268 & \\
\hline Adj. $\mathrm{R}^{2}$ & 0.07 & & 0.12 & & 0.07 & & 0.11 & \\
\hline
\end{tabular}

Anmerkung: OLS-Regression mit folgenden Signifikanzen: ${ }^{* * *} p<0.001,{ }^{* *} p<0.01,{ }^{*} p<0.05$. Die Skalen der jeweiligen Gender-Items gehen von „1 traditionell“ bis „6 egalitär". Es wurden die gleichen Variablen wie in den Modellen 1c, 2c, 3c, usw. in Tabelle A.10 im Anhang kontrolliert, d.h. der relative Berufsstatus, die Anzahl der Kinder, das Bundesgebiet und der absolute Berufsstatus der Mutter. Quelle: DJI-Studie AID:A 2013/14, eigene Berechnungen.

Des Weiteren wurden auch die latenten Klassen an Geschlechterrolleneinstellungen als unabhängige Variablen für die Aufteilung der Kinderbetreuung betrachtet (siehe Tabelle A.11 im Anhang und Abbildung 10.15). Die Hypothese 4 a) nimmt allgemein an, dass das Leitbild des „intensive mothering/parenting“ zu einer traditionelleren Arbeitsteilung führt, was allgemein bestätigt werden kann. Die altersspezifische Hypothese 4 b) nimmt des Weiteren an, dass die Effekte bei unter 3-jährigen Kindern besonders stark ausgeprägt sind. Diese Hypothese kann nur für die Altersstufe „1 bis unter 3 Jahre“ bestätigt werden. Für die Altersstufe „unter 1 Jahr" kann dies dagegen nicht bestätigt werden, denn es zeigt sich sogar der gegenteilige Effekt, nämlich dass der traditionalisierende Effekt des ,intensive mothering/parenting“ hier besonders gering ausfällt und die Aufteilung der Kinderbetreuung hier egalitärer ausfällt als in den anderen Altersstufen. Anscheinend kommt hier das "intensive parenting“ stärker zum Tragen als in den höheren Altersstufen, d.h. die Väter engagieren sich hier vermehrt, sodass die Kinderbetreuung egalitärer aufgeteilt wird. Die weiteren, altersspezifischen Hypothesen $4 \mathrm{c}$ ) und 4 d) können nicht bestätigt werden, da sie annehmen, dass die Unterschiede zwischen den Klassen mit zunehmendem Alter des Kindes geringer werden und aus nicht abgebildeten Interaktionsmodellen hervorgeht, dass diese Interaktionseffekte nicht signifikant verschieden sind. ${ }^{61}$

\footnotetext{
61 Lediglich der Effekt der Klasse des „intensive mothering/parenting“ bei unter 1-jährigen Kindern unterscheidet sich signifikant von den Effekten derselben Klasse in den anderen Altersstufen.
} 
Abbildung 10.15: Vorhergesagte Aufteilung der Kinderbetreuung, unter Berücksichtigung der drei Gender-Klassen, getrennt nach dem Alter des Kindes

unter $1 \mathrm{~J}$.

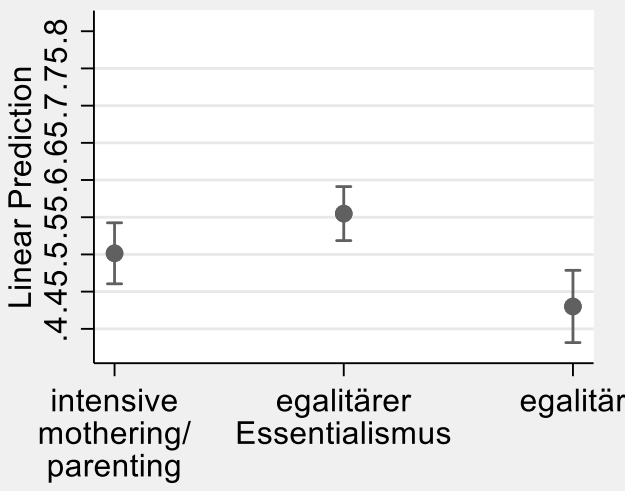

3 bis unter $6 \mathrm{~J}$.

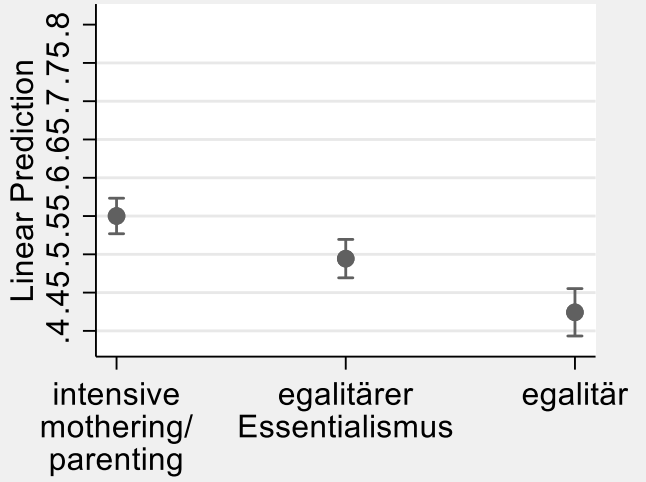

1 bis unter $3 \mathrm{~J}$.

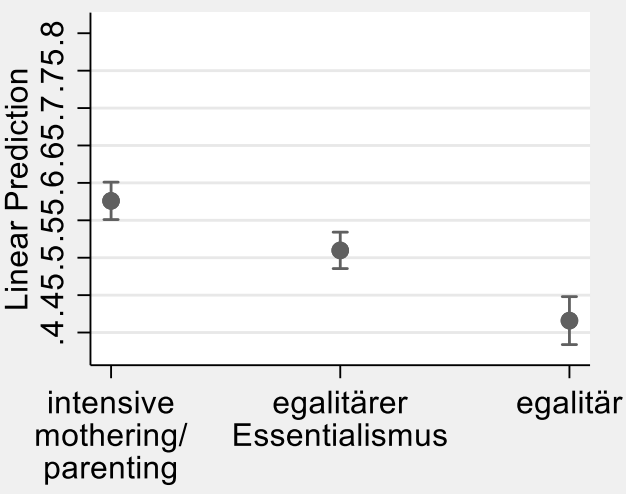

6 bis unter $9 \mathrm{~J}$.

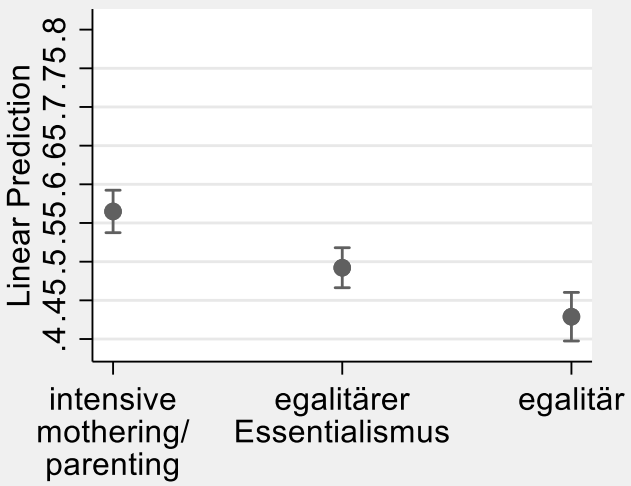

Anmerkung: Vorhergesagte Aufteilung der Kinderbetreuung (Predictive Margins) auf Basis einer OLS-Regression. Die Skalen der jeweiligen Gender-Items gehen von „1 traditionell“ bis „6 egalitär". Es wurden die gleichen Variablen wie in den Modellen 1c, 2c, 3c, usw. in Tabelle A.10 im Anhang kontrolliert, d.h. der relative Berufsstatus, die Anzahl der Kinder, das Bundesgebiet und der absolute Berufsstatus der Mutter. Quelle: DJI-Studie AID:A 2013/14, eigene Berechnungen.

Bevor zum Schlusskapitel übergegangen wird, werden die Ergebnisse des Kapitels zur Aufteilung der Kinderbetreuung zusammengefasst. In Tabelle A.12 im Anhang ist zudem eine Übersicht enthalten, welche Hypothesen damit im Einzelnen bestätigt werden können oder nicht. Die Ergebnisse zur Bedeutung des Kindesalters zeigen, dass die Kinderbetreuung in allen Altersstufen des Kindes überwiegend von den Müttern übernommen werden. Lediglich bei Kindern im Jugendalter ist die Aufteilung etwas egalitärer, was auch damit zusammenhängen mag, dass die absolute Zeit der Kinderbetreuung bei älteren Kindern geringer ist und so eine egalitärere Aufteilung leichter möglich ist.

Bzgl. der Bedeutung der relativen Ressourcen lässt sich festhalten, dass Mütter mit höheren Ressourcen nicht weniger Kinderbetreuung übernehmen, was u.a. darin begründet liegen kann, dass Kinderbetreuung im Gegensatz zur Hausarbeit nicht automatisch vermieden werden will, sondern i.d.R. als angenehm empfunden wird (Sullivan 2013). 
Entgegen den theoretischen Erwartungen zeigen sich keine signifikanten Veränderungen zwischen den unterschiedlichen Altersstufen des Kindes.

Als nächstes werden die Ergebnisse der Geschlechterrolleneinstellungen zusammengefasst. Analog zur Aufteilung der Hausarbeit spielen auch bei der Aufteilung der Kinderbetreuung die Geschlechterrolleneinstellungen eine größere Rolle als die relativen Ressourcen und egalitärere Einstellungen gehen entsprechend den Erwartungen mit einer egalitäreren Aufteilung der Kinderbetreuung einher. Bei den altersspezifischen Modellen zeigt sich, dass es meistens keine signifikanten Unterschiede zwischen den Altersstufen gibt, sodass die meisten altersspezifischen Hypothesen nicht bestätigt werden können (für detailliertere Informationen siehe auch Tabelle A.12 im Anhang). Bei den vertiefenden Ergebnissen zu den Geschlechterrolleneinstellungen anhand der vier einzelnen Items zeigt sich, dass bei Item 4 ein besonders starker Zusammenhang mit der Aufteilung der Kinderbetreuung besteht. Dies ist naheliegend, da sich dieses Item inhaltlich auf die Aufteilung der Kinderbetreuung bezieht, indem es danach fragt, ob der Vater genauso sehr in die Kinderbetreuung eingebunden sein sollte wie die Mutter. Aus den vertiefenden Ergebnissen anhand der latenten Gender-Klassen geht hervor, dass die Aufteilung der Kinderbetreuung i.d.R. in der Klasse des „intensive mothering/parenting“ am traditionellsten aufgeteilt ist (mit Ausnahme der Altersstufe „unter $1 \mathrm{Jahr}$ (), etwas egalitärer in der Klasse des „egalitären Essentialismus“ aufgeteilt ist und am egalitärsten in der „egalitären“ Klasse.

Die Ergebnisse zur Bedeutung der Anzahl der Kinder zeigen, dass Kinderbetreuung in Familien mit einem Kind i.d.R. egalitärer ist als in Familien mit zwei Kindern, jedoch besteht kein signifikanter Unterschied zwischen Familien mit zwei Kindern und Familien mit drei oder mehr Kindern. Dabei gibt es keine signifikanten Veränderungen hinsichtlich des Alters des Kindes (mit Ausnahme bei Familien mit vier oder mehr Kindern). Des Weiteren zeigen die Ergebnisse, dass die Kinderbetreuung in Ostdeutschland egalitärer aufgeteilt ist als in Westdeutschland, wobei es keine signifikanten Veränderungen in Abhängigkeit vom Kindesalter gibt. Bzgl. der Bedeutung des absoluten Berufsstatus der Mutter lässt sich festhalten, dass dieser keine signifikante Bedeutung für die Aufteilung der Kinderbetreuung hat. Bzgl. der Erwerbstätigkeit der Mütter und der Väter kann resümiert werden, dass hier starke Zusammenhänge mit der Aufteilung der Kinderbetreuung vorliegen. Ein höherer Erwerbsumfang der Mutter geht tendenziell mit einer egalitäreren Aufteilung der Kinderbetreuung einhergeht, wobei sich kaum relevante Unterschiede in Abhängigkeit vom Alter des Kindes. Umgekehrt geht ein höherer Erwerbsumfang der Väter mit einer traditionelleren Aufteilung der Kinderbetreuung einher, wobei die Zusammenhänge bei Kindern im Jugendalter geringer sind als bei jüngeren Kindern. 


\section{Schluss}

In diesem Kapitel werden abschließend die empirischen Ergebnisse zusammengefasst und diskutiert. Danach werden die Schwächen dieser Studie reflektiert sowie weitere Forschungsmöglichkeiten aufgezeigt und anschließend wird der Beitrag, den diese Arbeit leistet, resümiert.

\section{Zusammenfassung und Diskussion der Ergebnisse}

Im Folgenden werden die empirischen Ergebnisse der vorliegenden Arbeit zusammengefasst, wobei zuerst auf die Ergebnisse zur Aufteilung der Erwerbsarbeit eingegangen wird, gefolgt von den Ergebnissen zur Aufteilung der Hausarbeit und schließlich zur Aufteilung der Kinderbetreuung. In Tabelle A.12 im Anhang ist darüber hinaus eine Übersicht, aus der hervorgeht, welche allgemeinen und welche altersspezifischen Hypothesen zur Aufteilung der Erwerbsarbeit, der Hausarbeit und Kinderbetreuung damit bestätigt oder abgelehnt werden können.

\section{a) Zusammenfassung: Aufteilung der Erwerbsarbeit}

Für die Aufteilung der Erwerbsarbeit ist das Alter des Kindes der bedeutendste Einflussfaktor. Keine andere Variable bestimmt die Erwerbsumfänge der Mütter so stark wie das Kindesalter: Im ersten Lebensjahr des Kindes sind 85\% der Mütter nicht erwerbstätig, im zweiten und dritten Lebensjahr trifft dies nur noch am 51\% der Mütter zu und bei einem Kind im Alter von 3 bis 6 Jahren sind es nur noch auf 25\% der Mütter. Umgekehrt steigt mit zunehmendem Alter des Kindes der Anteil an Müttern, die in Teilzeit oder in Vollzeit erwerbstätig sind. Viele Mütter verbleiben jedoch in der Teilzeiterwerbstätigkeit, so dass der Anteil der in Vollzeit beschäftigten Mütter selbst bei einem Kind im Jugendalter nur $25 \%$ beträgt.

Aus den multivariaten Modellen zur allgemeinen Bedeutung der relativen Ressourcen operationalisiert anhand des relativen Berufsstatus (ISEI) - geht hervor, dass Mütter mit einem niedrigeren, relativen Berufsstatus im Vergleich zur Referenzkategorie (= beide haben den gleichen Berufsstatus) einen geringeren Erwerbsanteil innehaben, wohingegen umgekehrt nicht davon auszugehen ist, dass Mütter mit einem höheren, relativen Berufsstatus einen höheren Erwerbsanteil haben. Neben dieser allgemeinen Bedeutung der relativen Ressourcen wurden auch altersspezifische Hypothesen aufgestellt, die annehmen, dass die Effekte der relativen Ressourcen bei kleinen Kindern noch sehr gering ausfallen und somit keine (oder nur eine sehr geringe) Rolle spielen und dass die Effekte mit zunehmendem Alter des Kindes größer ausfallen. In den Ergebnissen findet sich jedoch keine empirische Evidenz dafür, dass die relativen Ressourcen bei kleinen Kindern eine geringe und bei älteren Kindern eine größere Rolle spielen (siehe Tabelle A.12 im Anhang). 
Bei den multivariaten Modellen zur allgemeinen Bedeutung der Geschlechterrolleneinstellungen hat sich gezeigt, dass die Erwerbsarbeit bei egalitäreren Einstellungen egalitärer aufgeteilt ist. Aus den vertiefenden Analysen zu den Geschlechterrolleneinstellungen anhand der latenten Klassen geht hervor, dass bei Müttern, die das Leitbild des „intensive mothering/parenting“ vertreten, die Erwerbsarbeit traditioneller aufgeteilt ist, während sie bei Müttern, die der Klasse des „egalitären Essentialismus“ oder der egalitären Klasse zuzuordnen sind, egalitärer ist. Bzgl. der altersspezifischen Effekte der Geschlechterrolleneinstellungen wurde ersichtlich, dass die Geschlechterrolleneinstellungen entgegen den Erwartungen bereits bei kleinen Kindern eine wichtige Rolle für die Aufteilung der Erwerbsarbeit spielen. D.h. bei Müttern mit egalitären Einstellungen ist die Erwerbsarbeit egalitärer aufgeteilt als bei Müttern mit traditionellen Einstellungen - auch bereits dann, wenn die Kinder noch sehr klein sind. Die Ergebnisse können jedoch in den meisten Fällen nicht bestätigen, dass die Bedeutung der Einstellungen bei einem höheren Kindesalter stärker ausfällt (siehe Tabelle A.12 im Anhang) ${ }^{62}$ Des Weiteren wurden die Einstellungen in vertiefenden Analysen multidimensional betrachtet. In allen Altersstufen ist die Aufteilung der Erwerbsarbeit in der Klasse des „intensive mothering/parenting" am geschlechtsspezifischsten und in der „egalitären" Klasse am egalitärsten verteilt. Die Effekte zwischen den Klassen sind in allen Altersstufen etwa gleich hoch. Die Annahme, dass die Klasse des „intensive mothering/parenting“ nur bei kleinen Kindern einen sehr starken Einfluss hat und bei älteren Kindern nur noch eine untergeordnete Rolle spielt, kann daher nicht bestätigt werden (siehe Tabelle A.12 im Anhang).

Bzgl. den Einflussfaktoren Kinderanzahl, Ost-West-Unterschiede und absolute Ressourcen lässt sich im Allgemeinen folgendes festhalten: In Familien mit mehreren Kindern ist die Erwerbsarbeit im Allgemeinen ungleicher aufgeteilt als in Familien mit einem Kind. Dieses Ergebnis steht in Einklang zu bisheriger Forschung (Berghammer 2014; Hipp und Leuze 2015; OECD 2016). Des Weiteren ist die Aufteilung der Erwerbsarbeit in Ostdeutschland egalitärer als in Westdeutschland und schließt damit an bisherige Befunde an (Pfau-Effinger und Smidt 2011; Grunow und Müller 2012; Buschner et al. 2018). Bei den absoluten Ressourcen zeigt sich, dass bei Müttern mit einem hohem Berufsstatus die Erwerbsarbeit egalitärer aufgeteilt ist (im Vergleich zu Müttern mit einem mittleren Berufsstatus). Dabei zeigen die Ergebnisse, dass die absoluten Ressourcen der Mütter für die Aufteilung der Erwerbsarbeit mindestens genauso wichtig sind wie die relativen Ressourcen. Auch diese Ergebnisse stehen in Einklang mit bisheriger Forschung, die zeigt, dass Frauen mit hoher, absoluter Bildung häufiger und in höherem Umfang erwerbstätig sind (England et al. 2012).

\footnotetext{
62 Zwar hat sich bei einem Item gezeigt, dass der Effekt bei einem Kind im Alter von 1 bis unter 3 Jahren größer ist als bei einem unter 1-jährigen Kind, aber dieser Effekt setzte sich in den höheren Altersstufen nicht fort, sodass in der Tendenz nicht angenommen werden kann, dass der Effekt der Einstellungen umso höher ausfällt, je älter das Kind ist.
} 
Neben dieser allgemeinen Bedeutung der weiteren Einflussfaktoren interessierte in der vorliegenden Arbeit insbesondere die Frage danach, ob die Bedeutung der Einflussfaktoren in den verschiedenen Altersstufen des Kindes unterschiedlich ausfällt. Welche weiteren altersspezifischen Hypothesen können bei der Aufteilung der Erwerbsarbeit bestätigt werden? Bei den Variablen der Kinderanzahl und der Ost-West-Unterschiede zeigte sich zwar, dass im Allgemeinen eine höhere Kinderanzahl mit einer traditionelleren Aufteilung der Erwerbsarbeit einhergeht und dass die Aufteilung in Westdeutschland traditioneller ist als in Ostdeutschland, jedoch konnten die altersspezifischen Hypothesen nicht bestätigt werden: D.h. die altersspezifischen Annahmen, dass die Unterschiede zwischen Familien mit einem Kind und Familien mit mehreren Kindern bei kleinen Kindern besonders groß sind und dass ebenso die Unterschiede zwischen Ost- und Westdeutschland bei kleinen Kindern besonders groß sind, können nicht bestätigt werden (siehe Tabelle A.12 im Anhang).

Bei den altersspezifischen Hypothesen zur Bedeutung des absoluten Berufsstatus der Mutter wurde angenommen, dass der absolute Berufsstatus der Mutter eine geringe Rolle spielt, wenn das Kind klein ist, und dass er eine größere Rolle spielt, wenn das Kind älter ist. Die Ergebnisse fallen hier jedoch unterschiedlich danach aus, ob der Erwerbsanteil der Mütter am gesamten Erwerbsvolumen des Paares (AV1) betrachtet wird oder ob die individuellen Erwerbsstunden der Mütter (AV2) verwendet werden. Bislang wurde bei der Zusammenfassung der Ergebnisse nicht zwischen diesen beiden Varianten differenziert, da die sie in der Regel sehr robust sind und daher sehr ähnlich ausfallen. Bei den altersspezifischen Hypothesen zur Bedeutung des absoluten Berufsstatus verhält es sich jedoch anders: Die Ergebnisse bestätigen einerseits, dass der absolute Berufsstatus der Mutter bei älteren Kindern einen größeren Effekt auf die individuellen Erwerbsstunden der Mütter hat als bei jüngeren Kindern, sodass die altersspezifischen Hypothesen bestätigt werden können. Andererseits lässt sich gleiches nicht für den Erwerbsanteil der Mütter bestätigen: Hier hat der absolute Berufsstatus der Mutter in Abhängigkeit vom Alter des Kindes keinen unterschiedlichen Effekt. Eine mögliche Erklärung hierfür kann darin liegen, dass der absolute Berufsstatus der Mutter in Abhängigkeit vom Alter des Kindes zwar einen unterschiedlichen Effekt auf die Erwerbsstunden der Mütter hat, aber sobald diese am gesamten Erwerbsvolumen des Paaren relativiert werden, werden diese altersspezifischen Unterschiede verschwindend gering und sind daher statistisch nicht mehr signifikant.

\section{b) Zusammenfassung: Aufteilung der Hausarbeit}

Während das Alter des Kindes die Aufteilung der Erwerbsarbeit entscheidend beeinflusst, variiert die Aufteilung der Hausarbeit nur geringfügig mit dem Alter des Kindes. Die Aufteilung der Hausarbeit ist bereits in den ersten Lebensjahren des Kindes sehr geschlechtsspezifisch aufgeteilt. Dies steht im Einklang zu bisheriger Forschung, die eine Traditionalisierung der Aufteilung der Hausarbeit mit dem Übergang zur Elternschaft feststellt (Evertsson und Nermo 2007; Baxter et al. 2008; Grunow et al. 2012; Kühhirt 
2012; Schober 2013b; Dechant et al. 2014; Baxter et al. 2015). Die geringe Variation, die bei der Aufteilung der Hausarbeit in Abhängigkeit vom Alter des Kindes stattfindet, deutet darauf hin, dass die Hausarbeit in Familien mit älteren Kindern noch traditioneller aufgeteilt ist als in Familien mit jüngeren Kindern. Auch dies stimmt mit früherer Forschung überein, aus der hervorgeht, dass die Aufteilung der Hausarbeit mit zunehmendem Alter des Kindes bzw. mit zunehmender Beziehungsdauer traditioneller wird (Schulz und Blossfeld 2006; Grunow et al. 2007, 2012; Zabel und Heintz-Martin 2013).

Welche Bedeutung haben die relativen Ressourcen für die Aufteilung der Hausarbeit? Hier zeigen die Ergebnisse, dass Mütter mit niedrigeren, relativen Ressourcen etwas mehr Hausarbeit übernehmen als Mütter, deren Ressourcen identisch zu denen der Väter sind; jedoch konnte umgekehrt nicht gezeigt werden, dass Mütter mit höheren, relativen Ressourcen weniger Hausarbeit übernehmen. D.h. dass die relativen Ressourcen entgegen der theoretischen Erwartungen keine geschlechtssymmetrischen Effekte haben, sondern nur einseitig wirken. Wie verhält es sich jedoch mit den relativen Ressourcen, wenn man sie in Abhängigkeit vom Alter des Kindes betrachtet? In den ersten Lebensjahren des Kindes spielen die relativen Ressourcen noch keine signifikante Rolle, was darauf zurückgeführt werden kann, dass hier die institutionellen und kulturellen Rahmenbedingungen die Oberhand haben. Erst bei Familien, deren Kind mindestens das Kindergartenalter erreicht hat, haben die relativen Ressourcen einen signifikanten Einfluss auf die Aufteilung der Hausarbeit, der jedoch - wie soeben bei der allgemeinen Bedeutung der relativen Ressourcen erwähnt - nicht geschlechtssymmetrisch ist. Der Teilaspekt, dass die Bedeutung der relativen Ressourcen bei einem höheren Kindesalter stärker zum Vorschein kommt als bei jüngeren Kindern, entspricht den theoretischen Erwartungen. Der andere Teilaspekt, dass die Effekte nicht geschlechtssymmetrisch sind, widerspricht dagegen den theoretischen Erwartungen, sodass die entsprechenden Hypothesen zu den relativen Ressourcen und der Aufteilung der Hausarbeit nur zum Teil bestätigt werden können (siehe Tabelle A.12 im Anhang). Die Tatsache, dass die relativen Ressourcen insgesamt nur eine sehr geringe Rolle für die Aufteilung der Hausarbeit spielen, ist ein weiterer Widerspruch zu den Erwartungen der ökonomischen Theorien.

Im Gegensatz zu den relativen Ressourcen spielen die Geschlechterrolleneinstellungen eine größere Rolle für die Aufteilung der Hausarbeit. Bei Müttern mit egalitären Einstellungen ist die Hausarbeit egalitärer aufgeteilt als bei Müttern mit traditionellen Einstellungen. Aus den altersspezifischen Analysen zur Bedeutung der einzelnen Gender-Items geht hervor, dass sich die Zusammenhänge nicht in Abhängigkeit vom Alter des Kindes unterscheiden. Neben den einzelnen Gender-Items wurden auch vertiefende Analysen anhand latenter Klassen vorgenommen. Hier zeigen die Ergebnisse, dass die Aufteilung der Hausarbeit bei Müttern dann am traditionellsten ist, wenn sie sich mit dem Leitbild des „intensive mothering/parenting“ identifizieren, gefolgt von der Klasse des „egalitären Essentialismus“ und dass die Aufteilung der Hausarbeit am egalitärsten in der „egalitären" Klasse ist. Auch hier wurden altersspezifische Modelle gerechnet. Aus diesen Mo- 
dellen geht hervorgeht, dass die Bedeutung des Leitbildes des „intensive mothering/parenting" ab dem Grundschulalter des Kindes zwar etwas geringer wird, aber die Aufteilung der Hausarbeit ist dabei immer noch deutlich traditioneller als bei den anderen beiden Klassen. Da die Veränderungen zwischen den Altersstufen sehr gering ausfallen, kann auch hier geschlussfolgert werden, dass die Zusammenhänge über die Altersstufen des Kindes hinweg relativ stabil (die altersspezifischen Hypothesen können daher nicht bzw. nur zum Teil bestätigt werden; für mehr Details siehe Tabelle A.12 im Anhang).

Ein weiterer Einflussfaktor für die Aufteilung der Hausarbeit ist die Kinderanzahl. Hier kann zunächst die allgemeine Hypothese bestätigt werden, dass die Aufteilung der Hausarbeit in Familien mit mehreren Kindern traditioneller aufgeteilt ist als in Familien mit einem Kind und schließt damit an bisherige Ergebnisse an (Baxter et al. 2008; Kühhirt 2012; Fahlén 2016). Darüber hinaus kann auch die altersspezifische Hypothese bestätigt werden, denn die Ergebnisse zeigen, dass die Kinderanzahl für die Aufteilung der Hausarbeit umso unwichtiger wird, je älter das jüngste Kind ist (siehe Tabelle A.12 im Anhang).

Des Weiteren wurden Unterschiede zwischen Ost- und Westdeutschland getestet, wobei angenommen wurde, dass die Hausarbeit in Ostdeutschland egalitärer aufgeteilt ist als in Westdeutschland. Dies konnte bestätigt werden und bestätigt auch gleichzeitig bisherige Befunde (Cooke 2006; Wengler et al. 2008; Grunow 2014). Jedoch konnten keine altersspezifischen Unterschiede festgestellt werden, d.h. die Ost-West-Unterschiede variieren nicht in Abhängigkeit vom Alter des Kindes (siehe Tabelle A.12 im Anhang).

Darüber hinaus wurden auch noch die absoluten Ressourcen der Mutter als Einflussfaktor für die Aufteilung der Hausarbeit untersucht. Es hat sich gezeigt, dass die Annahmen hierzu nur zum Teil bestätigt werden können: Zwar übernehmen Mütter mit hohen, absoluten Ressourcen weniger Hausarbeit, aber entgegen den Erwartungen übernehmen nicht Mütter mit niedrigen Ressourcen am meisten Hausarbeit, sondern Mütter mit mittleren Ressourcen. Die altersspezifische Hypothese, die annimmt, dass die Bedeutung der absoluten Ressourcen der Mutter bei älteren Kindern eine größere Rolle spielt als bei jüngeren Kindern kann nicht bestätigt werden (siehe Tabelle A.12 im Anhang).

Schließlich wurde auch die Erwerbstätigkeit der Mütter und Väter kontrolliert. Hier gilt es jedoch zu berücksichtigen, dass es sich bei der Erwerbsarbeit um eine höchst endogene Variable handelt (mehr kritische Anmerkungen hierzu in Kapitel 9 zur Aufteilung der Hausarbeit). Deshalb wurden diese Variablen beim stufenweisen Aufbau der Modelle auch erst im letzten Schritt testweise mitaufgenommen. Die Ergebnisse zeigen, dass bei Müttern, die in Teilzeit erwerbstätig sind, die Hausarbeit etwas egalitärer aufgeteilt ist als bei nicht-erwerbstätigen Müttern. Wenn Mütter in Vollzeit erwerbstätig sind, ist die Aufteilung nochmals etwas egalitärer. Allerdings macht es keinen Unterschied, ob Mütter in „normaler" Vollzeit (bis 45 Stunden) oder in besonders langer Vollzeit (über 45 Stunden) erwerbstätig sind, d.h. Mütter mit langer Vollzeit werden nicht stärker von der Hausarbeit 
entlastet als Mütter mit normaler Vollzeit. Es zeigen sich kaum Unterschiede in Abhängigkeit vom Alter des Kindes. Eine Ausnahme stellt hierbei das erste Lebensjahr des Kindes dar, denn hier spielt die Erwerbstätigkeit der Mütter noch keine Rolle für die Aufteilung der Hausarbeit. In allen weiteren Altersstufen spielt die Erwerbstätigkeit dagegen schon eine wichtige Rolle, wobei die Effektstärken etwa gleich groß ausfallen.

Bei der Erwerbstätigkeit der Väter zeigt sich, dass ein zunehmender Erwerbsumfang der Väter sich traditionalisierend auf die Aufteilung der Hausarbeit auswirkt. Hierbei zeigt sich auch, dass die Effekte bei 1- bis unter 3-jährigen Kindern besonders stark ausfallen und die Effekte bei älteren Kindern geringer ausfallen, d.h. bei älteren Kindern ist die Erwerbstätigkeit der Väter von geringerer Bedeutung für die Aufteilung der Hausarbeit als bei 1- bis unter 3-jährigen Kindern.

\section{c) Zusammenfassung: Aufteilung der Kinderbetreuung}

Nach den Zusammenfassungen zur Aufteilung der Erwerbsarbeit und der Hausarbeit folgt nun die Zusammenfassung zur Aufteilung der Kinderbetreuung.

Auch hier wurde zuerst die Bedeutung des Kindesalters für die Aufteilung der Kinderbetreuung betrachtet. Hier zeigt sich, dass die Aufteilung der Kinderbetreuung relativ robust gegenüber dem Alter des Kindes ist und Mütter in allen Altersstufen den Großteil der Kinderbetreuung übernehmen. Bei Kindern, die elf Jahre alt oder älter sind, ist die Aufteilung der Kinderbetreuung zwar etwas egalitärer, jedoch muss hierbei auch berücksichtigt werden, dass sich die Art und der Umfang der Kinderbetreuung stark in Abhängigkeit vom Alter des Kindes verändert. Da bei älteren Kindern der Betreuungsumfang geringer ist, kann eine egalitäre Aufteilung der Kinderbetreuung hier leichter realisiert werden.

Bei der Bedeutung der relativen Ressourcen für die Aufteilung der Kinderbetreuung konnte die Annahme, dass höhere (resp. niedrigere) relative Ressourcen mit einer geringeren (resp. höheren) Beteiligung an der Kinderbetreuung einhergehen nur zum Teil bestätigt werden: Die Ergebnisse zeigen, dass Mütter mit niedrigeren relativen Ressourcen zwar mehr Kinderbetreuung übernehmen (im Vergleich zu Müttern, deren Ressourcen identisch zu denen der Väter sind); aber es konnte umgekehrt nicht bestätigt werden, dass Mütter mit höheren relativen Ressourcen weniger Kinderbetreuung übernehmen. Hier gibt es mehrere Möglichkeiten der Interpretation: Es könnte sein, dass Mütter ihre höheren Ressourcen nicht wirksam für eine Verringerung ihres Anteils an der Kinderbetreuung einsetzen können. Dies könnte z.B. durch kulturelle Leitbilder bedingt sein, die die Mütter als die primäre Betreuungsperson ansehen. Eine andere Interpretationsweise könnte sein, dass Mütter mit höheren Ressourcen ihren Anteil an der Kinderbetreuung gar nicht verringern wollen. Denn im Gegensatz zur Hausarbeit wird die Kinderbetreuung (zumindest größtenteils) auch als angenehm und sinnstiftend empfunden (Sullivan 2013). Wenn nun die Bedeutung der relativen Ressourcen differenziert nach den verschiedenen Altersstufen des Kindes betrachtet werden, so zeigt sich, dass die meisten altersspezifischen Hypothesen abgelehnt werden müssen: Zwar kann bestätigt werden, 
dass die relativen Ressourcen bei einem unter 1-jährigen Kind noch keine Rolle spielen und bei einem Kind im Alter von 1 bis unter 3 Jahren schon, aber entgegen der Annahmen findet in den höheren Altersstufen keine weitere Vergrößerung des Zusammenhangs statt. Zudem ist der Zusammenhang (wie weiter oben erwähnt) nur einseitig, d.h. er liegt nur dann vor, wenn Mütter geringere Ressourcen haben als die Väter, und er liegt nicht vor, wenn Mütter höhere Ressourcen haben.

Des Weiteren wurde der Bedeutung von Geschlechterrolleneinstellungen für die Aufteilung der Kinderbetreuung nachgegangen. Hier zeigen die Ergebnisse, dass die Aufteilung der Kinderbetreuung egalitärer ausfällt, wenn die Einstellungen egalitärer sind. Dies steht in Einklang zu bisherigen Befunden (Bulanda 2004; Evertsson 2014; Nitsche und Grunow 2018). Bei den altersspezifischen Hypothesen wurde angenommen, dass egalitäre Einstellungen bei kleineren Kindern eine geringere Bedeutung für die Aufteilung der Kinderbetreuung haben als bei älteren Kindern. Dem liegt die Annahme zugrunde, dass egalitäre Einstellungen bei kleineren Kindern eher im Widerspruch zu kulturellen Leitbildern stehen, die insbesondere an Mütter von kleinen Kindern besonders hohe Erwartungen stellen. Diese altersspezifischen Annahmen müssen größtenteils zurückgewiesen werden. Beim ersten Gender-Item (der Frage danach, ob beide Elternteile in Teilzeit arbeiten sollen, wenn kleine Kinder da sind) zeigt sich ein Zusammenhang zwischen den Einstellungen und der Aufteilung der Kinderbetreuung, der in allen Altersstufen des Kindes etwa gleich stark ist. Zwar zeigt sich bei dem zweiten Gender-Item (der Frage danach, ob ein männliches Alleinverdienermodell befürwortet wird), dass die Bedeutung dieses Items bei unter 1-jährigen Kindern nicht signifikant ist und bei 1- bis unter 3-jährigen Kindern schon signifikant ist, allerdings wird die Bedeutung mit zunehmendem Alter des Kindes entgegen den Annahmen nicht größer. Die altersspezifischen Hypothesen müssen daher größtenteils abgelehnt werden (für detailliertere Informationen siehe Tabelle A.12 im Anhang). Neben diesen einzelnen Gender-Items wurden auch hier die Geschlechterrolleneinstellungen anhand von latenten Klassen multidimensional betrachtet. Diese Analysen zeigen, dass die Aufteilung der Kinderbetreuung in der Regel in der Klasse des „intensive mothering/parenting“ am traditionellsten und in der „egalitären“ Klassen am egalitärsten ist, während sich die Klasse des „egalitären Essentialismus" zwischen diesen beiden Extremen befindet. Dabei spielt es kaum eine Rolle, wie alt das Kind ist, denn diese Rangfolge der Klassen bleibt in Abhängigkeit vom Alter des Kindes unverändert (eine Ausnahme liegt bei unter 1-jährigen Kindern vor, da hier die Aufteilung in der Klasse des „egalitären Essentialismus“ am egalitärsten ist).

Bzgl. der Anzahl der Kinder und der Aufteilung der Kinderbetreuung lässt sich folgender Zusammenhang feststellen: Die Aufteilung der Kinderbetreuung ist in Familien mit einem Kind egalitärer als in Familien mit mehreren Kindern. Jedoch lässt sich mit den vorliegenden Daten nicht nachweisen, dass die Anzahl der Kinder in Abhängigkeit vom Alter des Kindes eine unterschiedlich starke Rolle spielt. Des Weiteren lässt sich bzgl. der Bedeutung von Ost-West-Unterschieden festhalten, dass die Aufteilung der Kinder in Ostdeutschland egalitärer ist als in Westdeutschland. Allerdings gibt es entgegen den 
Erwartungen keine Variation in Abhängigkeit vom Alter des Kindes (siehe Tabelle A.12 im Anhang).

Des Weiteren wurden die absoluten Ressourcen der Mütter anhand ihres Berufsstatus (ISEI) getestet. Hier zeigen die Ergebnisse, dass die absoluten Ressourcen der Mütter keine signifikante Rolle für die Aufteilung der Kinderbetreuung spielen, geschweige denn, dass sich deren Bedeutung in Abhängigkeit vom Alter des Kindes verändert. Ähnlich wie bei der Bedeutung der relativen Ressourcen für die Aufteilung der Kinderbetreuung könnte auch dies einerseits darauf zurückgeführt werden, dass Mütter mit hohen Ressourcen ihren Anteil an der Kinderbetreuung aufgrund weiterer Faktoren (wie etwa institutionellen und kulturellen Rahmenbedingungen) nicht reduzieren können oder dass sie ihn gar nicht reduzieren wollen, da die Kinderbetreuung trotz der damit einhergehenden Opportunitätskosten als angenehm und sinnstiftend empfunden wird (Sullivan 2013).

Schließlich wurde noch die Bedeutung der Erwerbsarbeitstätigkeit der Mütter und Väter für die Aufteilung der Kinderbetreuung untersucht. Hier zeigen die Ergebnisse, dass die Erwerbstätigkeit von entscheidender Bedeutung für die Aufteilung der Kinderbetreuung ist: Wenn die Mütter in Vollzeit erwerbstätig sind, so ist die Kinderbetreuung deutlich egalitärer aufgeteilt als dies bei nicht-erwerbstätigen Müttern der Fall ist. Hierbei macht es in der Regel kaum einen Unterschied, wie alt das Kind ist. Bzgl. der Erwerbstätigkeit der Väter zeigt sich ein umgekehrter Zusammenhang: Je höher die Erwerbsumfänge der Väter sind, umso traditioneller ist die Kinderbetreuung aufgeteilt. Dieser Zusammenhang zwischen der Erwerbstätigkeit der Väter und der Aufteilung der Kinderbetreuung wird erst dann geringer, wenn sich das Kind im Jugendalter befindet. Hierbei ist jedoch kritisch anzumerken, dass es sich bei den Erwerbstätigkeiten der Mütter und Väter um höchst endogene Variablen handelt und auch eine umgekehrte Kausalitätsrichtung nicht ausgeschlossen werden kann, d.h. die Aufteilung der Kinderbetreuung (und der Hausarbeit) kann auch die Erwerbsumfänge der Eltern beeinflussen.

\section{Rückbezug zu den Theorien und Rahmenbedingungen}

Nachdem nun die empirischen Ergebnisse zur Aufteilung der Erwerbsarbeit, der Hausarbeit und der Kinderbetreuung zusammengefasst wurden, wird abschließend noch beleuchtet, welche Rückschlüsse sich für die Theorien ergeben. Hierfür wird zunächst auf die ökonomischen Theorien eingegangen. Entgegen den theoretischen Erwartungen konnte kein geschlechtssymmetrischer Zusammenhang zwischen den relativen Ressourcen und der Arbeitsteilung gefunden werden: So zeigte sich etwa bei der Aufteilung der Hausarbeit und der Kinderbetreuung, dass Mütter mit niedrigeren, relativen Ressourcen mehr Hausarbeit und mehr Kinderbetreuung übernehmen (als Mütter mit identischen Ressourcen im Vergleich zum Partner), aber Mütter mit höheren, relativen Ressourcen umgekehrt nicht weniger Hausarbeit und nicht weniger Kinderbetreuung übernehmen. Dieser geschlechtsasymmetrische Zusammenhang widerspricht den geschlechtssymmetrischen Erwartungen der ökonomischen Theorien. Ferner scheinen die relativen 
Ressourcen nur eine geringe Rolle für die Arbeitsteilung zu spielen, obwohl es sich nach den ökonomischen Theorien dabei um die zentrale Einflussgröße handeln müsste, die die Arbeitsteilung entscheidend beeinflusst.

Welche Rückschlüsse lassen die empirischen Ergebnisse bzgl. der Geschlechterrollen-, normen- und identitätsbasieren Ansätze zu? Zunächst zeigt sich, dass die Aufteilung in den Familien nach wie vor sehr geschlechtsspezifisch und traditionell aufgeteilt ist: Die Hausarbeit und die Kinderbetreuung wird überwiegend von den Müttern übernommen, wobei die meisten Mütter in den ersten Lebensjahren des Kindes nicht erwerbstätig und anschließend in Teilzeit erwerbstätig sind, während bei den Vätern eine kontinuierliche Vollzeitbeschäftigung vorliegt. Somit wird an traditionellen Rollenbildern, in denen die Väter die Familienernährer und die Mütter die primären Betreuungspersonen des Kindes sind, festgehalten. Zwar zeigt sich, dass die Arbeitsteilung etwas egalitärer ist, wenn individuelle egalitäre Einstellungen vorhanden sind, aber alles in allem ist die Arbeitsteilung dennoch geschlechtsspezifisch. D.h. die individuellen Einstellungen (= Mikroebene) können zu einer egalitäreren Arbeitsteilung beitragen, aber der Handlungsspielraum, der durch diese individuellen Einstellungen gegeben ist, scheint durch die übergeordneten, gesamtgesellschaftlichen Rollenbilder oder durch institutionelle Anreize für traditionelle Modelle (= Makroebene) begrenzt zu sein.

Die Ergebnisse deuten auch daraufhin, dass sich die kulturellen Rahmenbedingungen in Ost- und Westdeutschland weiterhin unterscheiden, denn alle drei Dimensionen der Arbeitsteilung sind in Ostdeutschland egalitärer aufgeteilt als in Westdeutschland. Dies kann damit zusammenhängen, dass in Ostdeutschland das Leitbild der "guten Mutter" durch eine erwerbstätige Mutter nicht, oder zumindest nicht so leicht wie in Westdeutschland, verletzt wird. Mit dem Worten von Pfau-Effinger könnte man auch sagen, dass sich die Geschlechterkulturen zwischen Ost- und Westdeutschland weiterhin unterscheiden, da die beiden Bundesgebiete unterschiedliche Ansichten zur Arbeitsteilung von Müttern und Vätern haben. Es ist überraschend, dass sich diese Ost-West-Unterschiede auch drei Jahrzehnte nach der Wiedervereinigung noch so deutlich zeigen und so hartnäckig halten. Denn schließlich gibt es bei institutionellen Rahmenbedinungen wie z.B. den Zeitpolitiken und den Geldpolitiken keine Unterschiede zwischen den beiden Bundesgebieten (eine Ausnahme liegt bei den U3-Kinderbetreuungseinrichtungen vor, die in Ostdeutschland immer noch besser ausgebaut als in Westdeutschland).

\section{Schwächen der Arbeit und weitere Forschungsmöglichkeiten}

Eine Schwäche der Arbeit ist es, dass sie nur auf Querschnittsdaten und -analysen basiert. Zwar wurde ein „synthetischer Lebenslauf“ (Panova et al. 2017, S. 49) verwendet, der verschiedene Lebensphasen abbildet und einen Längsschnitt somit annäherungsweise repräsentiert. Aber um statistisch von Kausalitätszusammenhängen sprechen zu können, sind Längsschnittdaten erforderlich. Im vorliegenden Fall wäre es inhaltlich von Interesse, wie sich die Arbeitsteilung innerhalb einer Person im Zeitverlauf in Abhängigkeit vom Alter des Kindes verändert. Eine weitere Forschungsmöglichkeit könnte daher 
sein, die hier aufgeworfenen Fragestellungen anhand längsschnittlicher Daten zu überprüfen.

Die Tatsache, dass es sich bei Querschnittsanalysen um Momentaufnahmen handelt und aus methodischer Sicht keine Aussagen über die Kausalitätsrichtung zulässig sind, wird dann besonders problematisch, wenn beide Kausalitätsrichtungen denkbar sind. Dies wird etwa beim Zusammenhang zwischen den Einstellungen und der Arbeitsteilung gut ersichtlich. In dieser Arbeit wurden die Einstellungen in den multivariaten Analysen zur Aufteilung der Erwerbsarbeit, der Hausarbeit und der Kinderbetreuung als unabhängige Variablen betrachtet. Es wurde daher angenommen, dass aufgrund von egalitären Einstellungen die Arbeitsteilung egalitärer wird. Es könnte jedoch auch andersrum sein. So kann etwa ein Widerspruch zwischen Einstellungen und Verhalten - wie aus der sog. „kognitiven Dissonanz“ nach Festinger (1957) hervorgeht - auf zweierlei Arten aufgelöst werden: Entweder wird das Verhalten verändert, damit es in Einklang mit den Einstellungen steht; oder es werden die Einstellungen an das Verhalten angepasst werden. In letzterem Fall wären die Einstellungen somit die abhängige Variable und das Verhalten (wie z.B. die ausgeübte Arbeitsteilung) die unabhängige Variable.

Da die verwendeten Daten von 2013/14 sind, können neueste familienpolitische Regelungen - wie etwa das ElterngeldPlus oder das Gute-KiTa-Gesetz - nicht berücksichtigt werden. Da diese neueren Regelungen eine egalitäre Arbeitsteilung erleichtern, wäre zu erwarten, dass die Arbeitsteilung inzwischen egalitärer geworden ist als mit den vorliegenden Daten herausgefunden wurde. Diese Frage danach, ob und inwiefern die Arbeitsteilung durch familienpolitische Regelungen ab 2014 egalitärer geworden ist, bietet Anschlussmöglichkeiten für weitere Forschung.

Ein weiterer, kritisch anzumerkender Punkt ist, dass zur Operationalisierung der Aufteilung der Hausarbeit und der Kinderbetreuung jeweils ein Beteiligungsindex verwendet wurde. D.h. es wurden Items zusammengefasst, die danach fragen, welcher Elternteil welche Tätigkeiten überwiegend übernimmt. Hierbei ist jedoch problematisch, dass die einzelnen Tätigkeiten unterschiedlich zeitintensiv sein können. Des Weiteren ist anzumerken, dass die Angaben zur Aufteilung der Hausarbeit und der Kinderbetreuung retrospektiv erfasst wurden, d.h. die Befragten sollten sich nachträglich daran erinnern, wer welche Aufgaben überwiegend übernommen hat. Diese Art der Erhebung ist ungenauer und anfälliger für Verzerrungen als dies bei Zeittagebucheinträgen der Fall ist. Da das Anlegen von Tagebucheinträgen jedoch sehr zeit- und kostenintensiv ist, wird in der Forschung aus pragmatischen Gründen häufig ein retrospektives Erhebungsdesign verwendet. Eine Studie von Schulz und Grunow (2011) untersuchte die Zeitverwendung für die Hausarbeit anhand von retrospektiven Umfragedaten und anhand von Zeittagebucheinträgen, um vergleichen zu können, wie sehr die Ergebnisse einander entsprechen. Die Ergebnisse zeigen, dass die Angaben der retrospektiven Umfragedaten und der Zeittagebucheinträgen dann relativ ähnlich waren, wenn einzelnen Tätigkeiten der Hausarbeit abgefragt wurden und nicht nach der Hausarbeit im Allgemeinen gefragt wurde. Dies ist 
beim DJI-Survey „AID:A“ der Fall, da nach einzelnen Items wie z.B. dem Kochen, dem Einkaufen usw. gefragt wurde (wenn auch anhand von Beteiligungs- und nicht anhand von Zeitangaben). Diese Items wurden erst im Rahmen der Analysen in dieser Arbeit zu einem Hausarbeits- bzw. Kinderbetreuungsindex zusammengefasst. Insbesondere in Anbetracht der Ergebnisse der vergleichenden Studie von Schulz und Grunow (2011) erscheint die Verwendung der retrospektiven Erhebungsdaten vertretbar. Nichtsdestotrotz könnten Analysen auf Basis von Zeittagebucheinträgen noch präzisere Erkenntnisse über die Aufteilung der Hausarbeit und der Kinderbetreuung liefern.

Des Weiteren soll erwähnt werden, dass in der vorliegenden Arbeit die allgemeine Aufteilung der Hausarbeit und Kinderbetreuung untersucht wurde. Aus bisheriger Forschung geht jedoch hervor, dass es große Unterschiede zwischen der Aufteilung an Werktagen und an Wochenenden gibt (Walper und Lien 2018; Grunow 2007): So beteiligen sich Väter am Wochenende deutlich stärker an der Kinderbetreuung als dies an Werktagen der Fall ist. Eine Schwäche der Arbeit besteht somit darin, dass sie diese Unterschiede nicht berücksichtigen kann, da sie nicht zwischen Werktagen und Wochenenden differenziert. Zukünftige Forschung könnte daran anschließen, indem sie erforscht, wie die Hausarbeit und die Kinderbetreuung an Werktagen und an Wochenenden aufgeteilt sind - insbesondere dann, wenn die Kinder älter sind, da in bisherigen Studien häufig nur die frühe und mittlere Kindheit berücksichtigt werden (Walper und Lien 2018).

Bei den empirischen Ergebnissen wurde in den vertiefenden Analysen eine multidimensionale Abbildungsweise der Geschlechterrolleneinstellungen verwendet, die auf einer latenten Klassenanalyse basiert. Eine Schwäche in der vorliegenden Arbeit besteht darin, dass diese multidimensionale Abbildung hier nur auf vier Einstellungsitems beruht und deswegen nur drei Klassen gefunden werden konnten. Es ist anzunehmen, dass es deutlich mehr Dimensionen gibt, als in der vorliegenden Arbeit identifiziert werden konnten. So konnten etwa Knight und Brinton (2017) anhand von sieben Einstellungsitems vier Klassen finden, während Grunow et al. (2018) anhand von sieben Einstellungsitems fünf Klassen aufdecken konnten. Es ist sehr wahrscheinlich, dass mit noch mehr Items noch mehr Klassen an Geschlechterrolleneinstellungen aufgedeckt werden können. Aufgrund der begrenzten Anzahl an Einstellungsitems konnten in der vorliegenden Arbeit jedoch nur drei Klassen betrachtet werden. Dabei heißt eine Klasse „intensive mothering/parenting“ - anhand von mehr Items könnte z.B. auch besser zwischen „intensive mothering" und „intensive parenting“ unterschieden werden und sie müssten nicht mehr als eine gemeinsame Klasse betrachtet wurden.

Eine weitere kritische Anmerkung zur vorliegenden Arbeit bezieht sich auf die verwendeten Geschlechterrollenitems. Walter (2018a) kritisiert, dass in vielen Surveys die Einstellungsitems veraltet und nicht mehr zeitgemäß sind. Sie wirft einen kritischen Blick auf die Operationalisierung der Geschlechterrolleneinstellungen und stellt sie exemplarisch anhand der „ALLBUS“-Daten dar. Einige ihrer Kritikpunkte lassen sich auch auf die Items der in dieser Arbeit verwendeten „AID:A“-Daten von 2013/14 übertragen. So bemängelt 
Walter, dass sich die Items der „ALLBUS“-Daten zu sehr am männlichen Ernährermodell ausrichten und dass soziale Entwicklungen wie die gestiegene Erwerbstätigkeit der Frauen, das gestiegene Bildungsniveau oder veränderte Familienformen nicht berücksichtigt werden (Walter 2018a). Dies trifft auch auf die Items von „AID:A“-Daten von 2013/14 zu, da sich auch diese Items stark am männlichen Ernährermodell orientieren. Walter (2018a) schlägt vor, dass neue Items mehr Bezug auf eine egalitäre Arbeitsteilung und auf die Rolle von Vätern in der Familie nehmen sollten. Diese Punkte werden bei den Items der „AID:A“-Daten immerhin erfüllt, da zum einen abgefragt wird, ob beide Elternteile beim Vorhandensein von kleinen Kindern in Teilzeit arbeiten sollen und zum anderen auch danach gefragt wird, ob Väter genauso sehr in die Pflege und Erziehung eines Kindes eingebunden sein sollen wie die Mütter. Ein weiterer Hinweis von Walter (2018a) für die Gestaltung neuer Einstellungsitems bezieht sich darauf, dass es eine genauere Spezifizierung bzgl. der Erwerbsumfänge und dem Alter des Kinder geben sollte. So wird in einigen Surveys z.B. danach gefragt, ob kleine Kinder leiden, wenn die Mütter erwerbstätig sind. Stattdessen wäre es angebrachter, differenzierter zu fragen, bei welchem genauem Erwerbsumfang und bei welchem genauen Kindesalter man welche Einstellungen vertritt. Eine differenziertere Abfrage der Einstellungen in Hinblick auf das Kindesalter wäre auch für die vorliegende Arbeit von Vorteil gewesen. Hier bestehen Anschlussmöglichkeiten für weitere Forschung, indem in neuen Surveys mehr und differenziertere Items zu Geschlechterrolleneinstellungen erhoben werden, anhand denen noch mehr latente Klassen identifiziert werden können und so ein noch detaillierterer Einblick in die Bedeutung unterschiedlichster Einstellungsprofile für die Arbeitsteilung gegeben werden kann.

Die vorliegende Arbeit hat sich bei den Einflussfaktoren für die Arbeitsteilung auf die individuellen Merkmale der Eltern fokussiert (z.B. der individuelle sozio-ökonomische Status oder die individuellen Geschlechterrolleneinstellungen). Gleichzeitig spielen jedoch noch weitere Einflussfaktoren, die sich nicht auf der individuellen Ebene befinden, eine wichtige Rolle. So sind etwa bzgl. der Aufteilung der Erwerbsarbeit auch betriebliche Rahmenbedingungen wie die Betriebsgröße und das Dienstleistungsgewerbe relevant (Kümmerling und Postels 2020); ebenso spielen Kontextfaktoren wie die (außerpartnerschaftliche) Kinderbetreuung eine Rolle (Kümmerling und Postels 2020). Zukünftige Forschung könnte etwa durch die Verwendung von Mehrebenenanalysen die Bedeutung von Kontextfaktoren besser beleuchten.

\section{Beitrag der Arbeit und Ausblick}

Trotz dieser Schwächen leistet diese Arbeit in vielerlei Hinsicht einen wichtigen Beitrag. Sie erarbeitet die Bedeutung des Kindesalters für die Aufteilung der Erwerbsarbeit, der Hausarbeit und der Kinderbetreuung zunächst anhand theoretischer Ansätze und verknüpft diese dann mit konkreten, in Deutschland vorliegenden, institutionellen Rahmenbedingungen. Daraus ergeben sich Hypothesen, die das Alter des Kindes und die institutionellen Rahmenbedingungen explizit berücksichtigen. 
Anschließend liefert diese Arbeit differenzierte, empirische Ergebnisse hierzu, die zeigen, dass das Kindesalter einerseits für die Aufteilung der Erwerbsarbeit eine Schlüsselrolle spielt, während es andererseits für die Aufteilung der Hausarbeit und der Kinderbetreuung nur von geringer Bedeutung ist. Während die Aufteilung der Erwerbsarbeit somit stark vom Alter des Kindes abhängig ist und bei älteren Kindern deutlich egalitärer ausfällt als bei kleineren Kindern, erscheint die Aufteilung der Hausarbeit und der Kinderbetreuung kaum durch das Kindesalter beeinflusst zu werden. Insgesamt zeigen die Analysen, dass die Geschlechterrolleneinstellungen eine größere Bedeutung haben im Vergleich zu den relativen Ressourcen.

Ein Vorteil dieser Arbeit ist, dass sie alle Altersstufen des Kindes bis zur Volljährigkeit betrachtet und somit einen breiteren Einblick gibt als dies in Studien der Fall ist, die nur die frühe oder mittlere Kindheit kontrollieren. Ein weiterer Vorteil besteht darin, dass die Fallzahlen im gesamten Sample sehr hoch sind (Sample-Fallzahl: $n=11.330$ ) ebenso wie in den einzelnen Altersstufen des Kindes, was robuste Ergebnisse ermöglicht.

Viele Studien zur Arbeitsteilung betrachten ausschließlich die Aufteilung der Erwerbsarbeit oder der Hausarbeit oder der Kinderbetreuung. Indem diese Arbeit alle drei Dimensionen behandelt, kann sie zeigen, ob das Kindesalter sowie weitere wichtige Einflussfaktoren für alle drei Dimensionen der Arbeitsteilung bedeutsam sind oder nicht. In früheren Studien wurde häufig nicht zwischen der Aufteilung der Hausarbeit und der Kinderbetreuung differenziert. Dies ist jedoch angebracht, da sie in ihrer Art verschieden sind und unterschiedliche Präferenzen hervorrufen können (Sullivan 2013).

Bzgl. der Bedeutung von Geschlechterrolleneinstellungen leistet diese Arbeit einen wichtigen Beitrag, indem sie in vertiefenden Analysen einen neuen Ansatz in Form einer multidimensionalen Abbildung wählt. Sie kann daher aufzeigen, welcher Zusammenhang zwischen drei verschiedenen Einstellungsprofilen und der Aufteilung der Erwerbsarbeit, der Hausarbeit und der Kinderbetreuung besteht.

Die vorliegende Arbeit konnte einen detaillierten Einblick geben, wie die Erwerbsarbeit, die Hausarbeit und die Kinderbetreuung in Deutschland im Jahr 2014 aufgeteilt waren. Es bleibt spannend zu erforschen, wie sich die Arbeitsteilung in den nächsten Jahren entwickeln wird: Ist zu erwarten, dass die Arbeitsteilung egalitärer wird, indem Mütter in höherem Umfang erwerbstätig sein werden und sich Väter mehr an der Hausarbeit und an der Kinderbetreuung beteiligen werden? Oder ist von einer Stagnation oder sogar von einer (Re-)Traditionalisierung der Arbeitsteilung auszugehen? Auch gilt es zu berücksichtigen, dass sich die Arbeitsbedingungen verändern werden und beispielsweise mehr Erwerbsarbeit im Home-Office stattfinden wird, wodurch das Potential für eine bessere Vereinbarkeit von Beruf und Familie gegeben ist. Diese Entwicklung wurde durch die Covid-19-Pandemie stark beschleunigt.

Neueste Studien geben Aufschluss darüber, wie es im Covid-19-Pandemiejahr 2020 um die Arbeitsteilung der Eltern bestellt war. So geht aus einer Studie von Kohlrausch und 
Zucco (2020) hervor, dass Frauen und Männer bedingt durch die Pandemie 2020 wesentlich häufiger im Home-Office waren als in den Jahren zuvor und dass sich der Anteil der Väter an der Kinderbetreuung erhöht hat, aber dass der Großteil der Kinderbetreuung trotzdem überwiegend von den Müttern übernommen wird (Kohlrausch und Zucco 2020). Ähnliches geht auch einer Studie von Zinn et al. (2020) hervor, die die durchschnittlichen Betreuungszeiten von Müttern und Vätern vor und während dem Pandemiejahr 2020 vergleicht. Die Ergebnisse zeigen, dass in Vollzeit erwerbstätige Mütter 2019 pro Tag etwa 4,8 Stunden mit Kinderbetreuung verbracht haben und 2020 7,6 Stunden (Zinn et al. 2020, S. 3). Auch bei Vätern ist ein deutlicher Anstieg zu verzeichnen: So beteiligten sich in Vollzeit erwerbstätige Väter 2019 etwa 2,5 Stunden pro Tag an der Kinderbetreuung und 2020 waren es 4,8 Stunden (Zinn et al. 2020, S. 3). Der relative Anteil, den Väter mit der Kinderbetreuung verbringen, hat sich somit deutlich erhöht, aber insgesamt - unter der Betrachtung der absoluten Kinderbetreuungszeit - stemmen Mütter weiterhin den Großteil der Kinderbetreuung und kompensieren damit den größeren Teil der Kita- und Schulschließungen. Ähnliche Ergebnisse liefert auch eine Studie von Bujard et al. (2020), wonach sich die Zeit für Familien- und Hausarbeit für Mütter von 6,6 Stunden im Jahr 2018 auf 7,9 Stunden im Jahr 2020 erhöht hat, während sie sich für Väter von 3,3 Stunden im Jahr 2018 auf 5,6 Stunden im Jahr 2020 erhöht hat (Bujard et al. 2020, S. 37).

Auf Basis dieser Befunde ist einerseits davon auszugehen, dass Väter ihren Anteil an der Kinderbetreuung weiter erhöhen werden, insbesondere dann, wenn sie in Elternzeit gegangen sind (Tamm 2019). Ein Trend für die zukünftige Arbeitsteilung der Eltern könnte daher lauten, dass die Arbeitsteilung der Eltern etwas egalitärer wird. Andererseits ist die Arbeitsteilung dennoch noch weit von einer egalitären Arbeitsteilung entfernt, sodass davon auszugehen ist, dass geschlechtsspezifische Unterschiede weiterhin bestehen bleiben, wenn auch in geringerem Ausmaß. Gleichzeitig ist davon auszugehen, dass es nicht die eine egalitärere Arbeitsteilung geben wird, sondern aufgrund der „Pluralisierung der Lebensformen“ (Brüderl 2004; Peuckert 2012) eine Vielfalt an Arbeitsteilungsmustern, die in ihrer Tendenz wahrscheinlich egalitärer sein werden als frühere Arbeitsteilungsmuster. 


\section{A.Anhang}

Tabelle A.1: $\quad$ Multinomiales Logitmodell zu den latenten Gender-Klassen

\begin{tabular}{|c|c|c|c|c|c|c|}
\hline \multirow[t]{2}{*}{ VARIABLES } & \multicolumn{2}{|c|}{$\begin{array}{l}\text { (1) intensive mot- } \\
\text { hering/parenting }\end{array}$} & \multicolumn{2}{|c|}{$\begin{array}{l}\text { (2) egalitärer Es- } \\
\text { sentialismus }\end{array}$} & \multicolumn{2}{|c|}{ (3) egalitär } \\
\hline & AME & se & AME & se & AME & se \\
\hline Alter des Kindes $($ Ref. $<1)$ & & & & & & \\
\hline 1 bis unter 3 & $0.048 * *$ & $(0.02)$ & $-0.037 *$ & $(0.02)$ & -0.011 & $(0.02)$ \\
\hline 3 bis unter 6 & $0.076 * * *$ & $(0.02)$ & $-0.080 * * *$ & $(0.02)$ & 0.004 & $(0.02)$ \\
\hline 6 bis unter 9 & 0.004 & $(0.02)$ & $-0.043 * *$ & $(0.02)$ & $0.039 * *$ & $(0.02)$ \\
\hline $\begin{array}{l}\text { Anzahl der Kinder (Ref. } 2 \\
\text { Kinder) }\end{array}$ & & & & & & \\
\hline 1 Kind & $-0.048 * * *$ & $(0.01)$ & $0.046 * * *$ & $(0.02)$ & 0.002 & $(0.01)$ \\
\hline 3 Kinder & 0.008 & $(0.02)$ & 0.005 & $(0.01)$ & -0.013 & $(0.01)$ \\
\hline 4 und mehr & $0.064 * * *$ & $(0.02)$ & -0.007 & $(0.02)$ & $\begin{array}{c}- \\
0.058 * * *\end{array}$ & $(0.02)$ \\
\hline $\begin{array}{l}\text { HH-Nettoeinkommen } \\
\text { (Ref. mittel) }\end{array}$ & & & & & & \\
\hline niedrig & $0.048 * * *$ & $(0.02)$ & $-0.031 * *$ & $(0.02)$ & -0.017 & $(0.01)$ \\
\hline hoch & $-0.063 * * *$ & $(0.02)$ & $0.043 * * *$ & $(0.02)$ & 0.020 & $(0.01)$ \\
\hline Missings & 0.023 & $(0.02)$ & 0.014 & $(0.02)$ & $-0.037 * *$ & $(0.02)$ \\
\hline $\begin{array}{l}\text { ISEI der Mutter (Ref. mit- } \\
\text { tel }(45-62) \text { ) }\end{array}$ & & & & & & \\
\hline niedrig $(<45)$ & $0.071 * * *$ & $(0.02)$ & $-0.035 * *$ & $(0.01)$ & $\stackrel{-}{-}+036^{* * *}$ & $(0.01)$ \\
\hline hoch (ab 63) & $-0.209 * * *$ & $(0.01)$ & $0.059 * * *$ & $(0.01)$ & $0.150 * * *$ & $(0.01)$ \\
\hline Missings & -0.032 & $(0.02)$ & 0.013 & $(0.02)$ & 0.019 & $(0.02)$ \\
\hline $\begin{array}{l}\text { Bundesland (Ref. West- } \\
\text { dtl.) }\end{array}$ & & & & & & \\
\hline Ost-Dtl. & $-0.131 * * *$ & $(0.02)$ & $0.120 * * *$ & $(0.02)$ & 0.012 & $(0.02)$ \\
\hline
\end{tabular}

Quelle: DJI-Studie AID:A 2013/14, n (Gesamt) = 7.366; eigene Berechnungen. Signifikanzen: *** $p<0.001,{ }^{* *} p<0.01,{ }^{*} p<0.05$. 


\section{Tabelle A.2: $\quad$ Bivariate Regression: Erwerbsanteil der Mutter in Abhängigkeit vom Alter des Kindes}

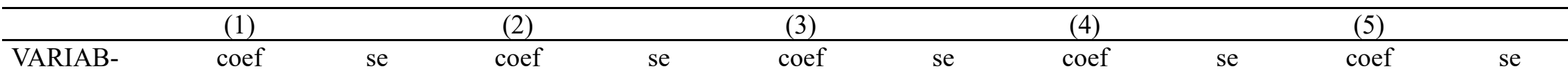

LES

Alter des Kindes (mit diversen Re-

ferenzen)

unter 1

1 bis unter 3

3 bis unter 6

6 bis unter 11

11 bis unter 18

Constant

Observations

$0.14 * * *$
$0.22 * * *$
$0.23 * * *$
$0.27 * * *$
$0.06 * * *$

(.) $-0.14 * * * \quad(0.01)$

$(0.01)$

$-0.14 * * *$

(0.01)

$-0.22 * * *$

(0.01)

$-0.23 * * *$

(0.01)

$0.08 * * *$

(.)

$-0.08 * * *$

$(0.01)$

$-0.09 * * *$

$(0.01)$

$-0.27 * * *$

$(0.01) \quad-0.13 * * *$

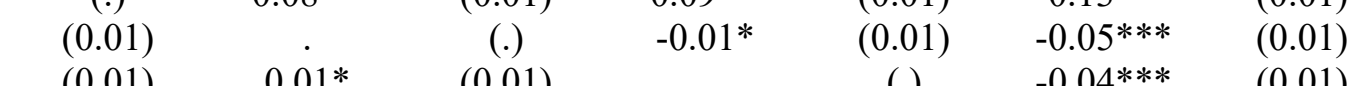

$(0.01) \quad 0.13 * * * \quad(0.01)$

$\begin{array}{lll}(0.01) & 0.13 * * * & (0.01)\end{array}$

$0.01^{*}$

$(0.01)$

R-squared

0.10

10,410

$(0.00)$

$0.05 * * *$

$0.28 * * *$

$(0.01)$

$0.04 * * * \quad(0.01)$

$(0.01)$

$-0.04 * * *$

$(0.01)$

Quelle: DJI-Studie AID:A 2013/14, Sample: $n=11.330$, eigene Berechnungen. Signifikanzen: ${ }^{* * *} p<0.001,{ }^{* *} p<0.01,{ }^{*} p<0.05$.

Tabelle A.3: $\quad$ Erwerbsanteil der Mutter, getrennt nach Alter des Kindes, OLS-Regression, stufenweiser Aufbau der Modelle

\begin{tabular}{|c|c|c|c|c|c|c|c|c|c|c|c|c|}
\hline & unter 1 & & & & & & 1 bis unt & & & & & \\
\hline & (1a) & & (1b) & & $(1 \mathrm{c})$ & & $(2 \mathrm{a})$ & & $(2 b)$ & & $(2 \mathrm{c})$ & \\
\hline VARIABLES & coef & se & coef & se & coef & se & coef & se & coef & se & coef & se \\
\hline \multicolumn{13}{|c|}{ rel. Ressourcen } \\
\hline \multicolumn{13}{|c|}{ rel. Berufsstatus } \\
\hline \multicolumn{13}{|c|}{$(I S E I)$ (Ref. 2, Frau $=$} \\
\hline \multicolumn{13}{|c|}{ Mann) } \\
\hline Frau $<$ Mann & $-0.04 *$ & $(0.02)$ & & & -0.03 & $(0.02)$ & $-0.06 * * *$ & $(0.01)$ & & & -0.02 & $(0.01)$ \\
\hline Frau $>$ Mann & -0.03 & $(0.02)$ & & & -0.02 & $(0.02)$ & 0.00 & $(0.01)$ & & & 0.01 & $(0.01)$ \\
\hline Missings & -0.04 & $(0.03)$ & & & -0.01 & $(0.03)$ & $-0.07 * *$ & $(0.02)$ & & & 0.04 & $(0.04)$ \\
\hline
\end{tabular}

Geschlechterrollen-

einstellungen 


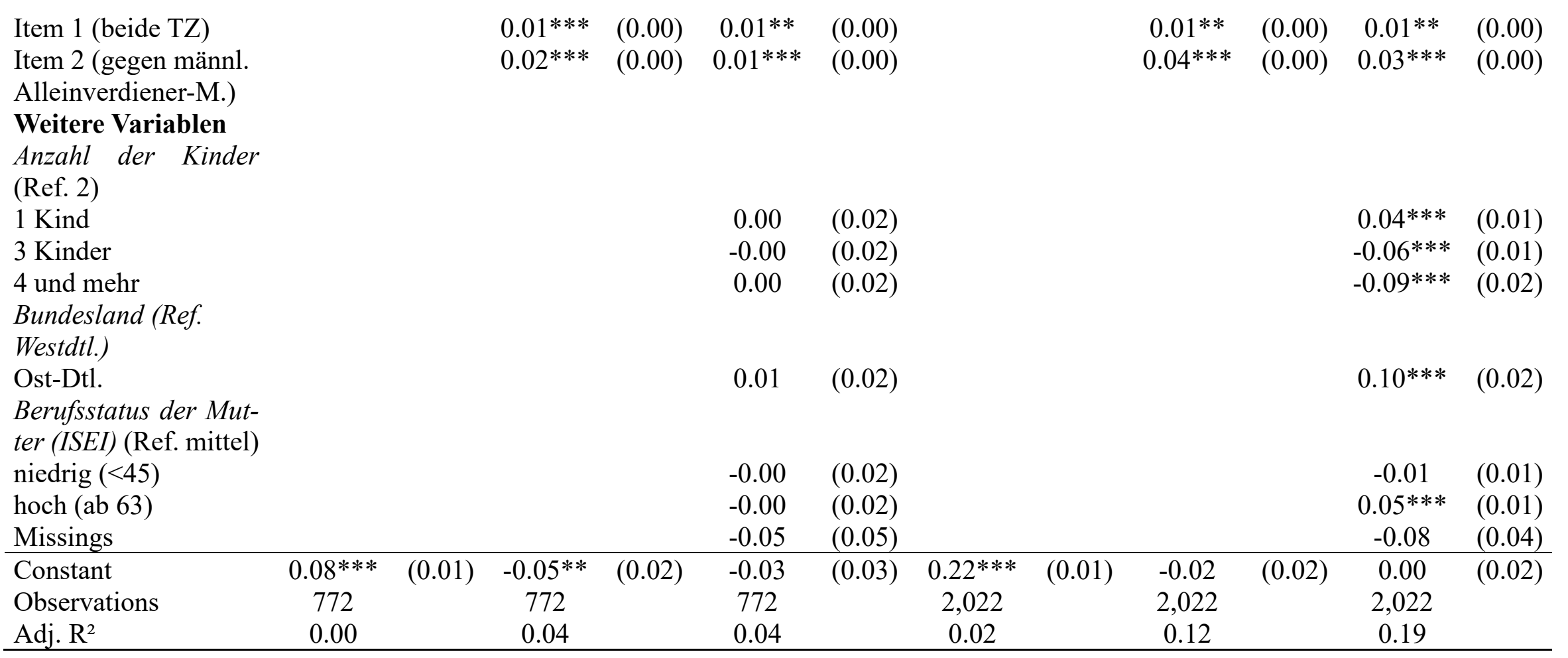

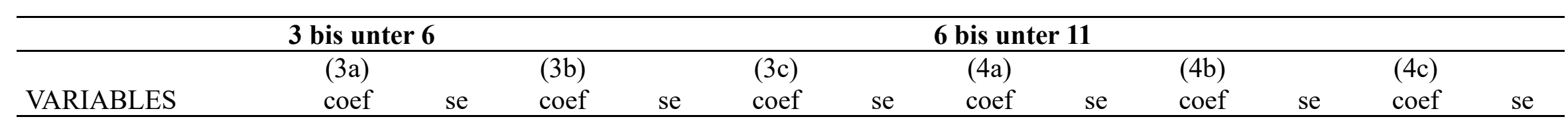

rel. Ressourcen

rel. Berufsstatus

(ISEI) (Ref. 2, Frau =

Mann)

$\begin{array}{lcc}\text { Frau }<\text { Mann } & -0.04 * * & (0.01) \\ \text { Frau }>\text { Mann } & 0.01 & (0.01)\end{array}$

$\begin{array}{cccc}-0.02 & (0.01) & -0.05 * * * & (0.01) \\ 0.01 & (0.01) & 0.00 & (0.01)\end{array}$

$-0.03 * * \quad(0.01)$ 


\begin{tabular}{|c|c|c|c|c|c|c|c|c|c|c|c|c|}
\hline Missings & $-0.05 * *$ & $(0.02)$ & & & 0.03 & $(0.03)$ & $-0.06 * * *$ & $(0.02)$ & & & -0.03 & $(0.02)$ \\
\hline \multicolumn{13}{|l|}{$\begin{array}{l}\text { Geschlechterrollen- } \\
\text { einstellungen }\end{array}$} \\
\hline Item 1 (beide TZ) & & & $0.01 * * *$ & $(0.00)$ & $0.01 * * *$ & $(0.00)$ & & & $0.02 * * *$ & $(0.00)$ & $0.02 * * *$ & $(0.00)$ \\
\hline Item 2 (gegen männl. & & & $0.03 * * *$ & $(0.00)$ & $0.03 * * *$ & $(0.00)$ & & & $0.02 * * *$ & $(0.00)$ & $0.02 * * *$ & $(0.00)$ \\
\hline \multicolumn{13}{|l|}{ Alleinverdiener-M.) } \\
\hline \multicolumn{13}{|l|}{ Weitere Variablen } \\
\hline \multicolumn{13}{|l|}{ Anzahl der Kinder } \\
\hline \multicolumn{13}{|l|}{ (Ref. 2) } \\
\hline 1 Kind & & & & & $0.05 * * *$ & $(0.01)$ & & & & & $0.04 * * *$ & $(0.01)$ \\
\hline 3 Kinder & & & & & $-0.04 * * *$ & $(0.01)$ & & & & & $-0.05 * * *$ & $(0.01)$ \\
\hline 4 und mehr & & & & & $-0.08 * * *$ & $(0.02)$ & & & & & $-0.07 * * *$ & $(0.02)$ \\
\hline $\begin{array}{l}\text { Bundesland } \quad \text { (Ref. } \\
\text { Westdtl.) }\end{array}$ & & & & & & & & & & & & \\
\hline Ost-Dtl. & & & & & $0.10 * * *$ & $(0.01)$ & & & & & $0.13 * * *$ & $(0.01)$ \\
\hline \multicolumn{13}{|l|}{ Berufsstatus der Mut- } \\
\hline & & & & & & & & & & & & \\
\hline niedrig $(<45)$ & & & & & 0.01 & $(0.01)$ & & & & & 0.01 & $(0.01)$ \\
\hline hoch (ab 63) & & & & & $0.04 * * *$ & $(0.01)$ & & & & & $0.03 * * *$ & $(0.01)$ \\
\hline Missings & & & & & $-0.06 *$ & $(0.03)$ & & & & & -0.01 & $(0.03)$ \\
\hline Constant & $0.29 * * *$ & $(0.01)$ & $0.09 * * *$ & $(0.01)$ & $0.11 * * *$ & $(0.02)$ & $0.31 * * *$ & $(0.01)$ & $0.12 * * *$ & $(0.01)$ & $0.14 * * *$ & $(0.02)$ \\
\hline Observations & 2,192 & & 2,192 & & 2,192 & & 2,590 & & 2,590 & & 2,590 & \\
\hline Adj. $R^{2}$ & 0.01 & & 0.08 & & 0.15 & & 0.02 & & 0.06 & & 0.14 & \\
\hline
\end{tabular}

\begin{tabular}{|c|c|c|c|c|c|c|}
\hline \multicolumn{7}{|c|}{11 bis unter 18} \\
\hline & $(5 a)$ & & $(5 b)$ & & $(5 c)$ & \\
\hline VARIABLES & coef & se & coef & se & coef & se \\
\hline
\end{tabular}

rel. Ressourcen

rel. Berufsstatus (ISEI)

(Ref. 2, Frau = Mann) 


\begin{tabular}{|c|c|c|c|c|c|c|}
\hline Frau $<$ Mann & $-0.04 * * *$ & $(0.01)$ & & & -0.02 & $(0.01)$ \\
\hline Frau $>$ Mann & $0.03 * *$ & $(0.01)$ & & & 0.02 & $(0.01)$ \\
\hline Missings & $-0.04 *$ & $(0.02)$ & & & 0.02 & $(0.02)$ \\
\hline \multicolumn{7}{|l|}{$\begin{array}{l}\text { Geschlechterrollen- } \\
\text { einstellungen }\end{array}$} \\
\hline Item 1 (beide TZ) & & & $0.01 * * *$ & $(0.00)$ & $0.01 * * *$ & $(0.00)$ \\
\hline Item 2 (gegen männl. & & & $0.02 * * *$ & $(0.00)$ & $0.02 * * *$ & $(0.00)$ \\
\hline \multicolumn{7}{|l|}{ Alleinverdiener-M.) } \\
\hline \multicolumn{7}{|l|}{ Weitere Variablen } \\
\hline \multicolumn{7}{|l|}{ Anzahl der Kinder } \\
\hline \multicolumn{7}{|l|}{ (Ref. 2) } \\
\hline 1 Kind & & & & & $0.04 * * *$ & $(0.01)$ \\
\hline 3 Kinder & & & & & $-0.05 * * *$ & $(0.01)$ \\
\hline 4 und mehr & & & & & $-0.10 * * *$ & $(0.03)$ \\
\hline \multicolumn{7}{|l|}{ Bundesland (Ref. West- } \\
\hline \multicolumn{7}{|l|}{$d t l)}$. \\
\hline \multicolumn{7}{|l|}{ Berufsstatus der Mut- } \\
\hline \multicolumn{7}{|l|}{ ter (ISEI) (Ref. mittel) } \\
\hline niedrig $(<45)$ & & & & & -0.00 & $(0.01)$ \\
\hline hoch (ab 63) & & & & & $0.06 * * *$ & $(0.01)$ \\
\hline Missings & & & & & $-0.08 * *$ & $(0.03)$ \\
\hline Constant & $0.34 * * *$ & $(0.01)$ & $0.19 * * *$ & $(0.02)$ & $0.20 * * *$ & $(0.02)$ \\
\hline Observations & 2,719 & & 2,719 & & 2,719 & \\
\hline Adj. $R^{2}$ & 0.02 & & 0.03 & & 0.10 & \\
\hline
\end{tabular}

Quelle: DJI-Studie AID:A 2013/14, Sample: $n=11.330$, eigene Berechnungen. Signifikanzen: ${ }^{* *} p<0.001,{ }^{* *} p<0.01,{ }^{*} p<0.05$. 
Tabelle A.4: $\quad$ Erwerbsanteil der Mutter in Abhängigkeit vom Alter des Kindes, unter Berücksichtigung der drei latenten Gender-Klassen

\begin{tabular}{|c|c|c|c|c|c|c|c|c|}
\hline \multirow[b]{3}{*}{ VARIABLES } & \multicolumn{2}{|l|}{ unter 1} & \multicolumn{2}{|c|}{1 bis unter 3} & \multicolumn{2}{|c|}{3 bis unter 6} & \multicolumn{2}{|c|}{6 bis unter 9} \\
\hline & (1) & & (3) & & (5) & & (7) & \\
\hline & coef & $\mathrm{se}$ & coef & se & coef & se & coef & se \\
\hline $\begin{array}{l}\text { Gender-Klassen } \\
\text { (Ref. ,intensive } \\
\text { mothering/paren- } \\
\text { ting) }\end{array}$ & & & & & & & & \\
\hline $\begin{array}{l}\text { egalitärer Essen- } \\
\text { tialismus }\end{array}$ & 0.01 & $(0.01)$ & $0.06 * * *$ & $(0.01)$ & $0.05 * * *$ & $(0.01)$ & $0.03 * *$ & $(0.01)$ \\
\hline egalitär & $0.06 * * *$ & $(0.02)$ & $0.11 * * *$ & $(0.01)$ & $0.10 * * *$ & $(0.01)$ & $0.10 * * *$ & $(0.01)$ \\
\hline Constant & $0.05 * *$ & $(0.02)$ & $0.13 * * *$ & $(0.01)$ & $0.22 * * *$ & $(0.01)$ & $0.24 * * *$ & $(0.01)$ \\
\hline Observations & 777 & & 2,042 & & 2,216 & & 1,767 & \\
\hline Adj. $R^{2}$ & 0.02 & & 0.15 & & 0.14 & & 0.14 & \\
\hline
\end{tabular}

Anmerkung: OLS-Regression. Es wurden die gleichen Variablen wie in Tabelle A.3 kontrolliert (d.h. der relative Berufsstatus, die Anzahl der Kinder, das Bundesgebiet und der absolute Berufsstatus der Mutter). Quelle: DJI-Studie AID:A 2013/14, eigene Berechnungen. Signifikanzen: *** $p<0.001,{ }^{* *} p<0.01,{ }^{*} p<0.05$.

Tabelle A.5: Erwerbsumfang der Mutter in Abhängigkeit vom Alter des Kindes, unter Berücksichtigung der drei latenten Gender-Klassen

\begin{tabular}{|c|c|c|c|c|c|c|c|c|}
\hline & \multicolumn{2}{|c|}{ unter 1} & \multicolumn{2}{|c|}{1 bis unter 3} & \multicolumn{2}{|c|}{3 bis unter 6} & \multicolumn{2}{|c|}{6 bis unter 9} \\
\hline & (1) & & (2) & & (3) & & (4) & \\
\hline VARIABLES & coef & se & coef & se & coef & se & coef & se \\
\hline $\begin{array}{l}\text { Gender-Klas- } \\
\text { sen (Ref. „,in- } \\
\text { tensive mothe- } \\
\text { ring/ paren- } \\
\text { ting“") }\end{array}$ & & & & & & & & \\
\hline $\begin{array}{l}\text { egalitärer Es- } \\
\text { sentialismus }\end{array}$ & 0.70 & $(0.80)$ & $4.67 * * *$ & $(0.71)$ & $4.29 * * *$ & $(0.65)$ & $3.57 * * *$ & $(0.73)$ \\
\hline egalitär & $2.14 *$ & $(0.93)$ & $7.27 * * *$ & $(0.84)$ & $7.46 * * *$ & $(0.74)$ & $7.53 * * *$ & $(0.81)$ \\
\hline Constant & $3.80 * * *$ & $(1.08)$ & $8.98 * * *$ & $(0.95)$ & $14.50 * * *$ & $(0.84)$ & $14.69 * * *$ & $(0.93)$ \\
\hline Observations & 857 & & 2,143 & & 2,301 & & 1,855 & \\
\hline Adj. $R^{2}$ & 0.00 & & 0.16 & & 0.20 & & 0.21 & \\
\hline
\end{tabular}

Anmerkung: OLS-Regression. Es wurden die gleichen Variablen wie in den vorherigen, multivariaten Modellen (siehe Tabelle 8.4) kontrolliert, d.h. der relative Berufsstatus, die Anzahl der Kinder, das Bundesgebiet und der absolute Berufsstatus der Mutter; Quelle: DJI-Studie AID:A 2013/14, eigene Berechnungen. Signifikanzen: ${ }^{* * *} p<0.001,{ }^{* *} p<0.01,{ }^{*} p<0.05$. 
Tabelle A.6: Bivariate Regression: Aufteilung der Hausarbeit in Abhängigkeit vom Alter des Kindes

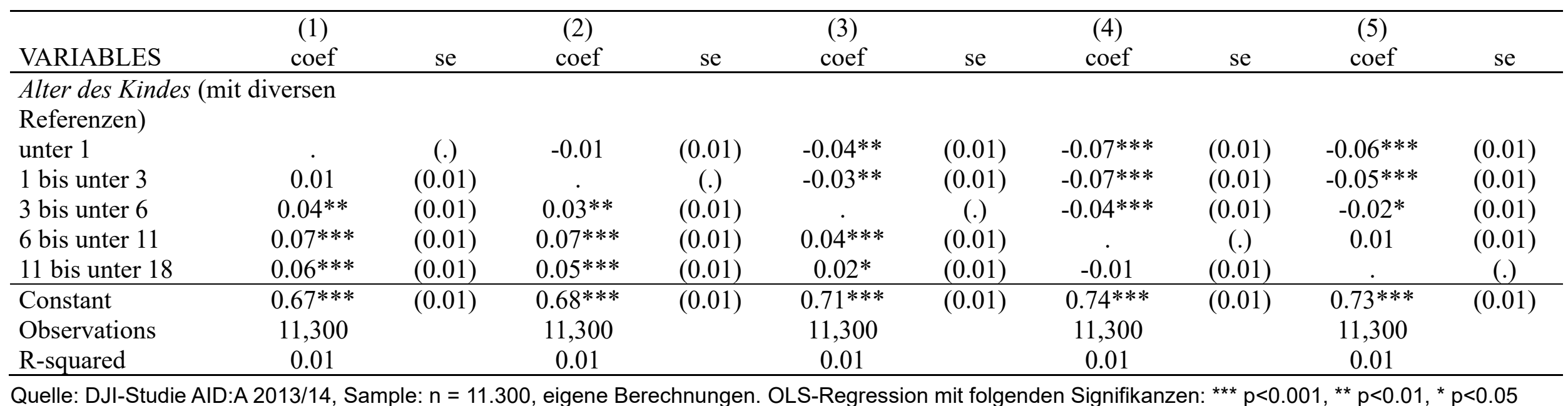

Tabelle A.7: $\quad$ Aufteilung der Hausarbeit, getrennt nach Alter des Kindes, stufenweiser Aufbau der Modelle

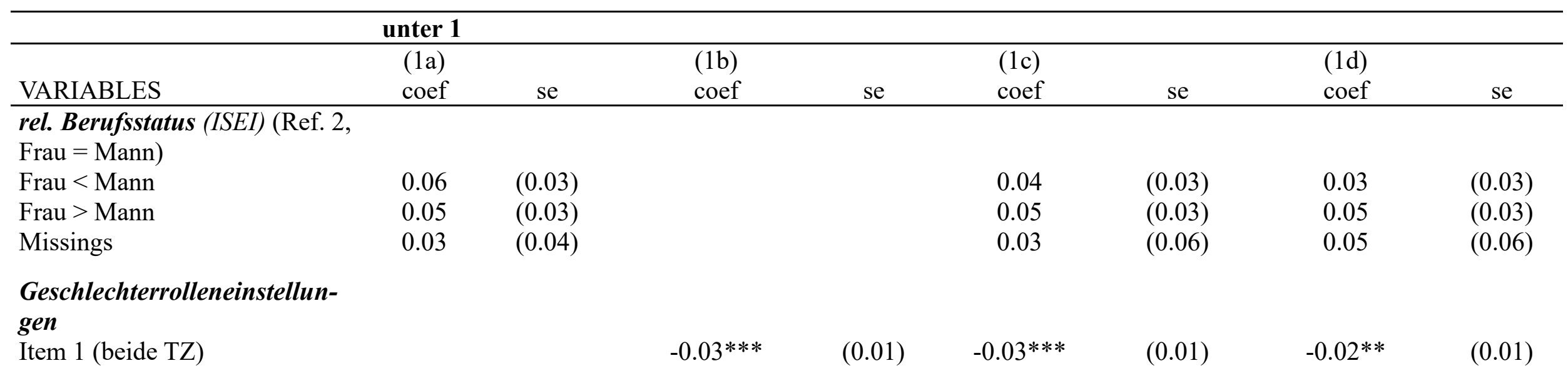




\begin{tabular}{|c|c|c|c|c|c|c|c|c|}
\hline & unter 1 & & & & & & & \\
\hline & (1a) & & (1b) & & (1c) & & (1d) & \\
\hline $\begin{array}{l}\text { Item } 2 \text { (gegen männl. Alleinver- } \\
\text { diener-M.) }\end{array}$ & & & $-0.03 * * *$ & $(0.01)$ & $-0.03 * * *$ & $(0.01)$ & $-0.03 * * *$ & $(0.01)$ \\
\hline \multicolumn{9}{|l|}{ Anzahl der Kinder (Ref. 2) } \\
\hline $1 \mathrm{Kind}$ & & & & & $-0.08 * *$ & $(0.03)$ & $-0.06^{*}$ & $(0.03)$ \\
\hline 3 Kinder & & & & & -0.00 & $(0.03)$ & -0.01 & $(0.03)$ \\
\hline Ost-Dtl. & & & & & -0.05 & $(0.04)$ & -0.04 & $(0.04)$ \\
\hline \multicolumn{9}{|l|}{$\begin{array}{l}\text { ISEI der Mutter (Ref. mittel } \\
(45-62))\end{array}$} \\
\hline niedrig $(<45)$ & & & & & 0.01 & $(0.03)$ & 0.01 & $(0.03)$ \\
\hline hoch (ab 63) & & & & & -0.02 & $(0.03)$ & -0.02 & $(0.03)$ \\
\hline Missings & & & & & -0.02 & $(0.08)$ & 0.07 & $(0.07)$ \\
\hline \multicolumn{9}{|l|}{$\begin{array}{l}\text { Erwerbstätigkeit der Mutter } \\
\text { (Ref. nicht erwerbstätig) }\end{array}$} \\
\hline mehr als VZ (> 45) & & & & & & & -0.10 & $(0.08)$ \\
\hline Missings & & & & & & & $-0.37 * * *$ & $(0.11)$ \\
\hline \multicolumn{9}{|l|}{$\begin{array}{l}\text { Erwerbstätigkeit des Vaters } \\
\text { (Ref. nicht erwerbstätig) }\end{array}$} \\
\hline TZ (1-35 Std.) & & & & & & & 0.11 & $(0.07)$ \\
\hline VZ (36-45 Std.) & & & & & & & $0.31 * * *$ & $(0.06)$ \\
\hline mehr als VZ (> 45) & & & & & & & $0.39 * * *$ & $(0.06)$ \\
\hline Missings & & & & & & & 0.02 & $(0.08)$ \\
\hline Constant & $0.63 * * *$ & $(0.02)$ & $0.91 * * *$ & $(0.04)$ & $0.90 * * *$ & $(0.05)$ & $0.57 * * *$ & $(0.07)$ \\
\hline
\end{tabular}




\begin{tabular}{|c|c|c|c|c|c|c|c|c|}
\hline & unter & & & & & & & \\
\hline & (1a) & & (1b) & & $(1 \mathrm{c})$ & & (1d) & \\
\hline VARIABLES & coef & se & coef & se & coef & se & coef & se \\
\hline Observations & 871 & & 871 & & 871 & & 871 & \\
\hline
\end{tabular}

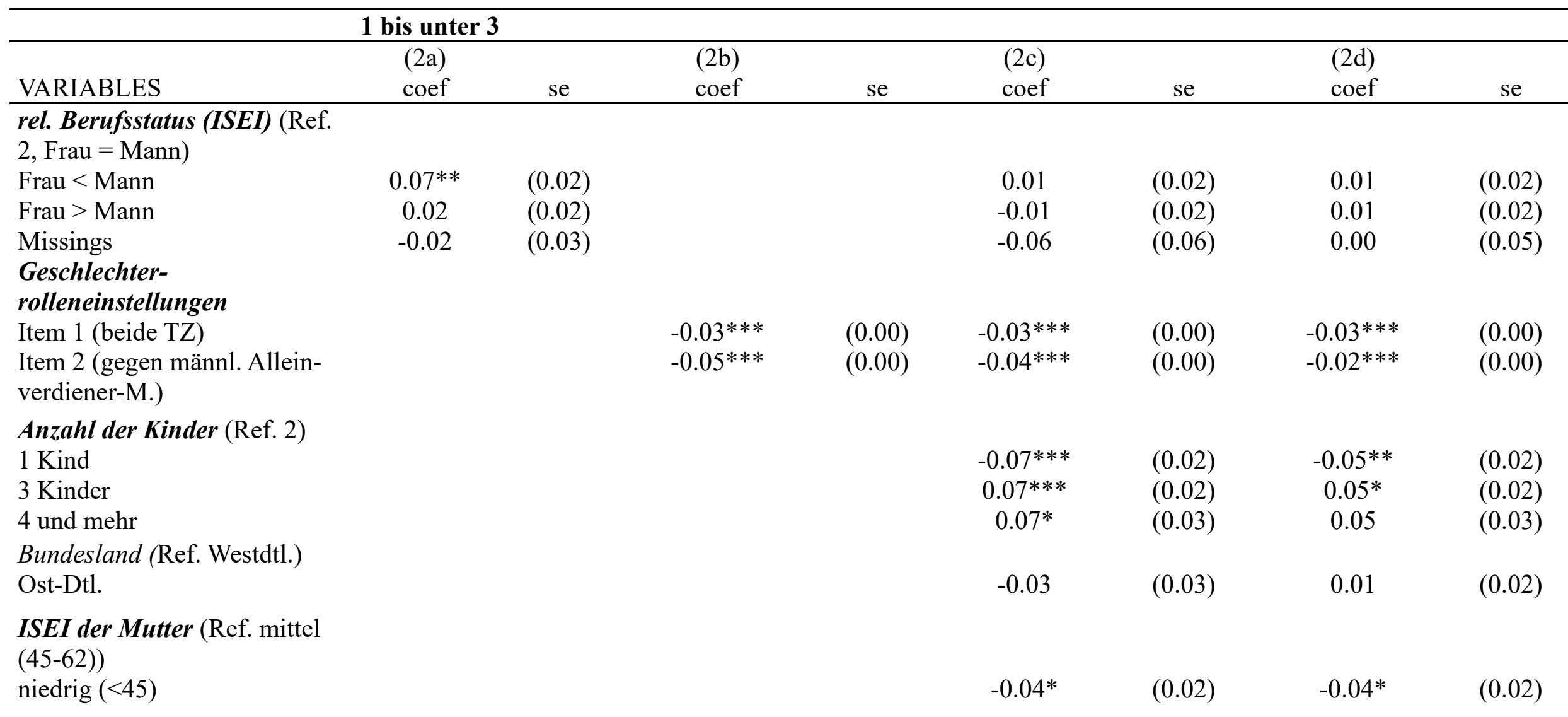




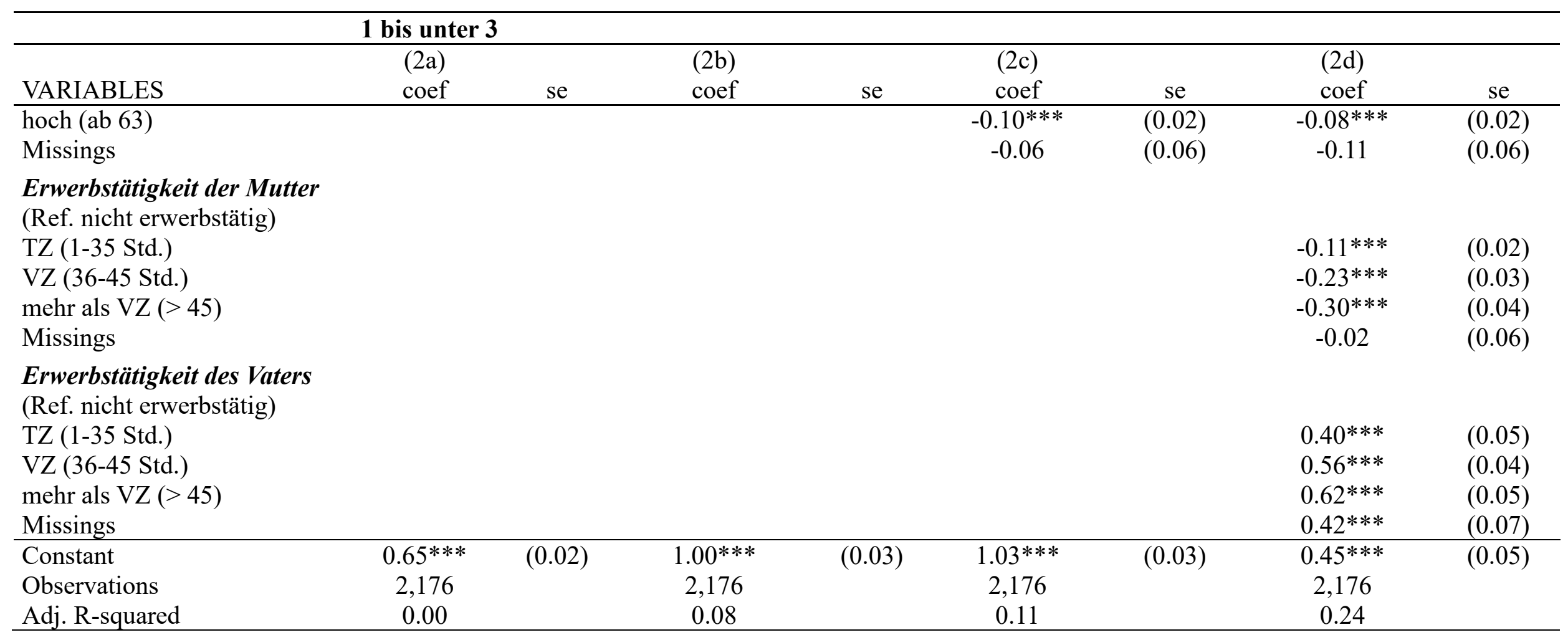

\begin{tabular}{|c|c|c|c|c|c|c|c|c|}
\hline \multicolumn{9}{|c|}{3 bis unter 6} \\
\hline & (3a) & & $(3 b)$ & & $(3 c)$ & & (3d) & \\
\hline \multicolumn{9}{|c|}{$\begin{array}{l}\text { rel. Berufsstatus (ISEI) (Ref. 2, } \\
\text { Frau = Mann) }\end{array}$} \\
\hline Frau $<$ Mann & $0.08 * * *$ & $(0.02)$ & & & $0.04 *$ & $(0.02)$ & 0.03 & $(0.02)$ \\
\hline Frau $>$ Mann & 0.01 & $(0.02)$ & & & -0.00 & $(0.02)$ & 0.01 & $(0.02)$ \\
\hline
\end{tabular}




\begin{tabular}{|c|c|c|c|c|c|c|c|c|}
\hline \multicolumn{9}{|c|}{3 bis unter 6} \\
\hline & (3a) & & (3b) & & (3c) & & (3d) & \\
\hline Missings & 0.05 & $(0.03)$ & & & -0.05 & $(0.05)$ & -0.04 & $(0.05)$ \\
\hline \multicolumn{9}{|l|}{$\begin{array}{l}\text { Geschlechterrolleneinstellun- } \\
\text { gen }\end{array}$} \\
\hline Item 1 (beide TZ) & & & $-0.03 * * *$ & $(0.00)$ & $-0.03 * * *$ & $(0.00)$ & $-0.02 * * *$ & $(0.00)$ \\
\hline $\begin{array}{l}\text { Item } 2 \text { (gegen männl. Alleinver- } \\
\text { diener-M.) }\end{array}$ & & & $-0.04 * * *$ & $(0.00)$ & $-0.03 * * *$ & $(0.00)$ & $-0.02 * * *$ & $(0.00)$ \\
\hline \multicolumn{9}{|l|}{ Anzahl der Kinder (Ref. 2) } \\
\hline 1 Kind & & & & & $-0.06 * *$ & $(0.02)$ & -0.03 & $(0.02)$ \\
\hline 3 Kinder & & & & & -0.01 & $(0.02)$ & -0.01 & $(0.02)$ \\
\hline 4 und mehr & & & & & 0.04 & $(0.03)$ & 0.02 & $(0.03)$ \\
\hline \multicolumn{9}{|l|}{ Bundesland (Ref. Westdtl.) } \\
\hline Ost-Dtl. & & & & & $-0.10 * * *$ & $(0.02)$ & $-0.05^{*}$ & $(0.02)$ \\
\hline \multicolumn{9}{|l|}{$\begin{array}{l}\text { ISEI der Mutter (Ref. mittel } \\
(45-62))\end{array}$} \\
\hline Missings & & & & & 0.06 & $(0.05)$ & 0.03 & $(0.05)$ \\
\hline \multicolumn{9}{|l|}{$\begin{array}{l}\text { Erwerbstätigkeit der Mutter } \\
\text { (Ref. nicht erwerbstätig) }\end{array}$} \\
\hline TZ (1-35 Std.) & & & & & & & $-0.09 * * *$ & $(0.02)$ \\
\hline VZ (36-45 Std.) & & & & & & & $-0.27 * * *$ & $(0.03)$ \\
\hline mehr als VZ $(>45)$ & & & & & & & $-0.23 * * *$ & $(0.04)$ \\
\hline Missings & & & & & & & $-0.18 * * *$ & $(0.05)$ \\
\hline Erwerbstätigkeit des Vaters & & & & & & & $0.24 * * *$ & $(0.05)$ \\
\hline
\end{tabular}




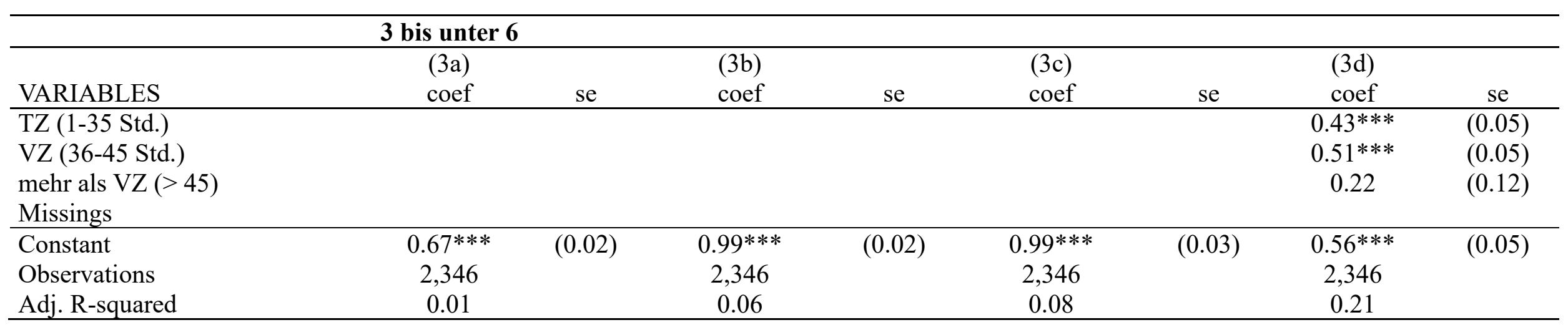

\begin{tabular}{|c|c|c|c|c|c|c|c|c|}
\hline \multirow[b]{3}{*}{ VARIABLES } & \multicolumn{4}{|c|}{6 bis unter 11} & \multirow{3}{*}{$\begin{array}{l}\text { (4c) } \\
\text { coef }\end{array}$} & \multirow{2}{*}{\multicolumn{3}{|c|}{$(4 d)$}} \\
\hline & (4a) & & (4b) & & & & & \\
\hline & coef & se & coef & se & & se & coef & se \\
\hline \multicolumn{9}{|l|}{$\begin{array}{l}\text { rel. Berufsstatus (ISEI) (Ref. 2, } \\
\text { Frau = Mann) }\end{array}$} \\
\hline Frau $<$ Mann & $0.08 * * *$ & $(0.02)$ & & & $0.06^{* * *}$ & $(0.02)$ & 0.03 & $(0.02)$ \\
\hline Frau $>$ Mann & $0.04 *$ & $(0.02)$ & & & 0.03 & $(0.02)$ & $0.03 *$ & $(0.02)$ \\
\hline Missings & $0.06^{*}$ & $(0.02)$ & & & 0.04 & $(0.04)$ & 0.04 & $(0.04)$ \\
\hline $\begin{array}{l}\text { Item } 2 \text { (gegen männl. Alleinver- } \\
\text { diener-M.) }\end{array}$ & & & $-0.03 * * *$ & $(0.00)$ & $-0.03 * * *$ & $(0.00)$ & $-0.02 * * *$ & $(0.00)$ \\
\hline \multicolumn{9}{|l|}{ Anzahl der Kinder (Ref. 2) } \\
\hline 1 Kind & & & & & $-0.05 * *$ & $(0.02)$ & -0.03 & $(0.02)$ \\
\hline 3 Kinder & & & & & 0.01 & $(0.02)$ & -0.01 & $(0.01)$ \\
\hline 4 und mehr & & & & & 0.02 & $(0.03)$ & 0.00 & $(0.03)$ \\
\hline
\end{tabular}




\begin{tabular}{|c|c|c|c|c|c|c|c|c|}
\hline \multicolumn{9}{|c|}{6 bis unter 11} \\
\hline & $(4 a)$ & & (4b) & & (4c) & & (4d) & \\
\hline Ost-Dtl. & & & & & $-0.11 * * *$ & $(0.02)$ & $-0.04^{*}$ & $(0.02)$ \\
\hline \multicolumn{9}{|c|}{$\begin{array}{l}\text { ISEI der Mutter (Ref. mittel (45- } \\
62) \text { ) }\end{array}$} \\
\hline niedrig $(<45)$ & & & & & $-0.06 * * *$ & $(0.02)$ & $-0.04 * *$ & $(0.02)$ \\
\hline hoch (ab 63) & & & & & $-0.07 * * *$ & $(0.02)$ & $-0.06 * * *$ & $(0.02)$ \\
\hline \multicolumn{9}{|c|}{$\begin{array}{l}\text { Erwerbstätigkeit der Mutter (Ref. } \\
\text { nicht erwerbstätig) }\end{array}$} \\
\hline TZ (1-35 Std.) & & & & & & & $-0.09 * * *$ & $(0.01)$ \\
\hline VZ (36-45 Std.) & & & & & & & $-0.34 * * *$ & $(0.02)$ \\
\hline mehr als VZ $(>45)$ & & & & & & & $-0.26 * * *$ & $(0.03)$ \\
\hline Missings & & & & & & & $-0.14^{*}$ & $(0.06)$ \\
\hline \multicolumn{9}{|c|}{$\begin{array}{l}\text { Erwerbstätigkeit des Vaters (Ref. } \\
\text { nicht erwerbstätig) }\end{array}$} \\
\hline Missings & & & & & & & 0.14 & $(0.12)$ \\
\hline Constant & $0.69 * * *$ & $(0.01)$ & $0.99 * * *$ & $(0.02)$ & $0.99 * * *$ & $(0.03)$ & $0.61 * * *$ & $(0.05)$ \\
\hline Observations & 2,796 & & 2,796 & & 2,796 & & 2,796 & \\
\hline Adj. R-squared & 0.01 & & 0.05 & & 0.07 & & 0.23 & \\
\hline
\end{tabular}




\begin{tabular}{|c|c|c|c|c|c|c|c|c|}
\hline \multicolumn{9}{|c|}{11 bis unter 18} \\
\hline & (5a) & & $(5 b)$ & & $(5 c)$ & & $(5 d)$ & \\
\hline \multicolumn{9}{|l|}{$\begin{array}{l}\text { rel. Berufsstatus (ISEI) (Ref. 2, } \\
\text { Frau = Mann) }\end{array}$} \\
\hline Frau $<$ Mann & $0.05^{* *}$ & $(0.02)$ & & & 0.03 & $(0.02)$ & 0.01 & $(0.02)$ \\
\hline Frau $>$ Mann & $-0.05 * *$ & $(0.02)$ & & & $-0.05^{* *}$ & $(0.02)$ & $-0.04 *$ & $(0.02)$ \\
\hline Missings & -0.00 & $(0.03)$ & & & -0.02 & $(0.04)$ & 0.01 & $(0.03)$ \\
\hline Item 1 (beide TZ) & & & $-0.03 * * *$ & $(0.00)$ & $-0.02 * * *$ & $(0.00)$ & $-0.02 * * *$ & $(0.00)$ \\
\hline $\begin{array}{l}\text { Item } 2 \text { (gegen männl. Alleinver- } \\
\text { diener-M.) }\end{array}$ & & & $-0.03 * * *$ & $(0.00)$ & $-0.03 * * *$ & $(0.00)$ & $-0.02 * * *$ & $(0.00)$ \\
\hline \multicolumn{9}{|l|}{ Anzahl der Kinder (Ref. 2) } \\
\hline 1 Kind & & & & & $-0.04 *$ & $(0.02)$ & -0.00 & $(0.01)$ \\
\hline 3 Kinder & & & & & 0.00 & $(0.02)$ & -0.01 & $(0.02)$ \\
\hline niedrig $(<45)$ & & & & & -0.03 & $(0.02)$ & -0.02 & $(0.01)$ \\
\hline hoch (ab 63) & & & & & $-0.08 * * *$ & $(0.02)$ & $-0.05 * *$ & $(0.02)$ \\
\hline Missings & & & & & -0.03 & $(0.05)$ & -0.05 & $(0.04)$ \\
\hline \multicolumn{9}{|l|}{$\begin{array}{l}\text { Erwerbstätigkeit der Mutter } \\
\text { (Ref. nicht erwerbstätig) }\end{array}$} \\
\hline TZ (1-35 Std.) & & & & & & & $-0.08 * * *$ & $(0.02)$ \\
\hline VZ (36-45 Std.) & & & & & & & $-0.26 * * *$ & $(0.02)$ \\
\hline mehr als VZ $(>45)$ & & & & & & & $-0.32 * * *$ & $(0.03)$ \\
\hline
\end{tabular}




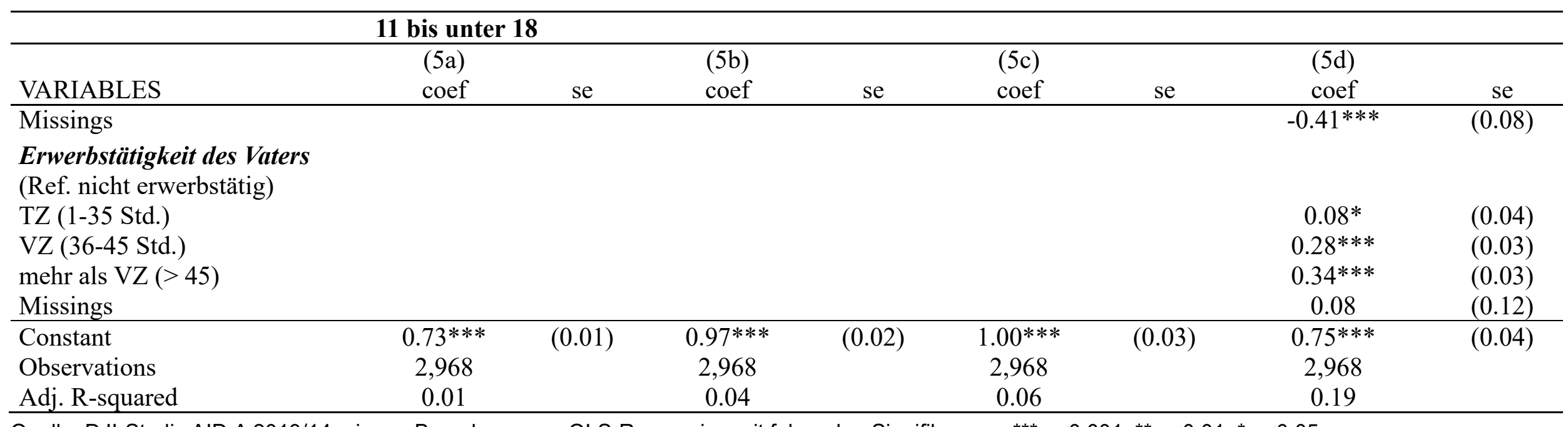

Quelle: DJI-Studie AID:A 2013/14, eigene Berechnungen. OLS-Regression mit folgenden Signifikanzen: ${ }^{* * *} p<0.001,{ }^{* *} p<0.01,{ }^{*} p<0.05$. 
Tabelle A.8: $\quad$ Aufteilung der Hausarbeit, unter Berücksichtigung der drei Gender-Klassen, getrennt nach dem Alter des Kindes

\begin{tabular}{|c|c|c|c|c|c|c|c|c|}
\hline \multirow[b]{3}{*}{ VARIABLES } & \multicolumn{2}{|c|}{ unter 1} & \multicolumn{2}{|c|}{1 bis unter 3} & \multicolumn{2}{|c|}{3 bis unter 6} & \multicolumn{2}{|c|}{6 bis unter 9} \\
\hline & $(1)$ & & (2) & & $(3)$ & & (4) & \\
\hline & coef & se & coef & se & coef & se & coef & se \\
\hline $\begin{array}{l}\text { Gender-Klassen (Ref. „,int } \\
\text { sive mothering/parenting) } \\
\text { egalitärer Essentialismus } \\
\text { egalitär }\end{array}$ & $\begin{array}{c}-0.05 \\
-0.12 * * *\end{array}$ & $\begin{array}{l}(0.03) \\
(0.03)\end{array}$ & $\begin{array}{l}-0.08 * * * \\
-0.22 * * *\end{array}$ & $\begin{array}{l}(0.02) \\
(0.02)\end{array}$ & $\begin{array}{l}-0.08 * * * \\
-0.20 * * *\end{array}$ & $\begin{array}{l}(0.02) \\
(0.02)\end{array}$ & $\begin{array}{l}-0.06 * * * \\
-0.16^{* * *}\end{array}$ & $\begin{array}{l}(0.02) \\
(0.02)\end{array}$ \\
\hline Constant & $0.71 * * *$ & $(0.04)$ & $0.83 * * *$ & $(0.02)$ & $0.83 * * *$ & $(0.02)$ & $0.85 * * *$ & $(0.02)$ \\
\hline Observations & 877 & & 2,200 & & 2,373 & & 1,913 & \\
\hline R-squared & 0.03 & & 0.10 & & 0.09 & & 0.06 & \\
\hline
\end{tabular}

Quelle: DJI-Studie AID:A 2013/14, eigene Berechnungen. Anmerkung: Es wurden die gleichen Variablen wie in Modell 1c, 2c, 3c usw. in Tabelle A.7 im Anhang

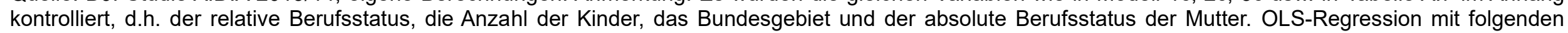

Signifikanzen: ${ }^{* * *} p<0.001,{ }^{* *} p<0.01,{ }^{*} p<0.05$.

\section{Tabelle A.9: Bivariate Regression: Aufteilung der Kinderbetreuung in Abhängigkeit vom Alter des Kindes}

\begin{tabular}{|c|c|c|c|c|c|c|c|c|c|c|}
\hline \multirow[b]{2}{*}{ VARIABLES } & \multirow{2}{*}{$\begin{array}{l}\text { (1) } \\
\text { coef }\end{array}$} & \multirow[b]{2}{*}{ se } & \multirow{2}{*}{$\begin{array}{l}(2) \\
\text { coef }\end{array}$} & \multicolumn{3}{|c|}{ (3) } & \multirow{2}{*}{$\begin{array}{l}(4) \\
\text { coef }\end{array}$} & \multicolumn{3}{|c|}{ (5) } \\
\hline & & & & se & coef & se & & se & coef & se \\
\hline $\begin{array}{l}\text { unter } 1 \\
1 \text { bis unter } 3\end{array}$ & 0.01 & $\begin{array}{c}(.) \\
(0.01)\end{array}$ & $\begin{array}{c}-0.01 \\
.\end{array}$ & $\begin{array}{c}(0.01) \\
(.)\end{array}$ & $\begin{array}{l}0.01 \\
0.01\end{array}$ & $\begin{array}{l}(0.01) \\
(0.01)\end{array}$ & $\begin{array}{l}0.01 \\
0.02\end{array}$ & $\begin{array}{l}(0.01) \\
(0.01)\end{array}$ & $\begin{array}{l}0.08^{* * *} \\
0.09 * * *\end{array}$ & $\begin{array}{l}(0.01) \\
(0.01)\end{array}$ \\
\hline 3 bis unter 6 & -0.01 & $(0.01)$ & -0.01 & $(0.01)$ & . & (.) & 0.01 & $(0.01)$ & $0.07 * * *$ & $(0.01)$ \\
\hline $\begin{array}{l}11 \text { bis unter } \\
18\end{array}$ & $-0.08 * * *$ & $(0.01)$ & $-0.09 * * *$ & $(0.01)$ & $-0.07 * * *$ & $(0.01)$ & $-0.07 * * *$ & $(0.01)$ & & (.) \\
\hline
\end{tabular}




\begin{tabular}{lccccccccc}
\hline Constant & $0.51 * * *$ & $(0.01)$ & $0.51 * * *$ & $(0.01)$ & $0.50^{* * *}$ & $(0.01)$ & $0.50^{* * *}$ & $(0.01)$ & $0.43 * * *$ \\
Observations & 11,301 & & 11,301 & & 11,301 & & 11,301 & $(0.01)$ \\
$\mathrm{R}^{2}$ & 0.01 & & 0.01 & & 0.01 & 0.01 & 11,301 & 0.01 \\
\hline
\end{tabular}

Quelle: DJI-Studie AID:A 2013/14, Sample: $n=11.301$, eigene Berechnungen. OLS-Regression mit folgenden Signifikanzen: ${ }^{* * *} p<0.001,{ }^{* *} p<0.01,{ }^{*} p<0.05$.

Tabelle A.10: Aufteilung der Kinderbetreuung, getrennt nach Alter des Kindes, stufenweiser Aufbau der Modelle

\begin{tabular}{|c|c|c|c|c|c|c|c|c|}
\hline \multirow[b]{3}{*}{ VARIABLES } & \multicolumn{4}{|c|}{ unter 1 Jahr } & \multirow[b]{2}{*}{$(1 \mathrm{c})$} & \\
\hline & (1a) & & (1b) & & & & (1d) & \\
\hline & coef & se & coef & se & coef & se & coef & se \\
\hline \multicolumn{9}{|l|}{ relativer Berufsstatus (Ref. Frau $=$} \\
\hline \multicolumn{9}{|l|}{ Mann) } \\
\hline Frau $<$ Mann & 0.03 & $(0.03)$ & & & 0.05 & $(0.03)$ & 0.04 & $(0.03)$ \\
\hline Frau $>$ Mann & 0.02 & $(0.03)$ & & & 0.01 & $(0.03)$ & -0.00 & $(0.03)$ \\
\hline Missings & -0.05 & $(0.05)$ & & & 0.01 & $(0.06)$ & -0.01 & $(0.06)$ \\
\hline \multicolumn{9}{|l|}{ Geschlechterrolleneinstellungen } \\
\hline Item 1 (beide TZ) & & & $-0.03 * * *$ & $(0.01)$ & $-0.03 * * *$ & $(0.01)$ & $-0.02 *$ & $(0.01)$ \\
\hline $\begin{array}{l}\text { Item } 2 \text { (gegen männl. Alleinverdie- } \\
\text { ner-M.) }\end{array}$ & & & -0.00 & $(0.01)$ & -0.01 & $(0.01)$ & -0.01 & $(0.01)$ \\
\hline \multicolumn{9}{|l|}{ Anzahl der Kinder (Ref. 2) } \\
\hline $1 \mathrm{Kind}$ & & & & & 0.00 & $(0.03)$ & 0.01 & $(0.03)$ \\
\hline 3 Kinder & & & & & 0.01 & $(0.03)$ & 0.01 & $(0.03)$ \\
\hline 4 und mehr & & & & & $-0.13 * *$ & $(0.05)$ & $-0.11 *$ & $(0.05)$ \\
\hline \multicolumn{9}{|l|}{ Bundesland (Ref. Westdtl.) } \\
\hline Ost-Dtl. & & & & & $-0.21 * * *$ & $(0.05)$ & $-0.18 * * *$ & $(0.04)$ \\
\hline \multicolumn{9}{|l|}{ ISEI der Mutter (Ref. mittel (45-62)) } \\
\hline niedrig $(<45)$ & & & & & -0.01 & $(0.03)$ & -0.01 & $(0.03)$ \\
\hline hoch (ab 63) & & & & & 0.05 & $(0.03)$ & 0.05 & $(0.03)$ \\
\hline Missings & & & & & -0.08 & $(0.08)$ & -0.03 & $(0.08)$ \\
\hline
\end{tabular}




\begin{tabular}{|c|c|c|c|c|c|c|c|c|}
\hline \multirow{2}{*}{ VARIABLES } & \multicolumn{4}{|c|}{ unter 1 Jahr } & \multirow[b]{2}{*}{ (1c) } & \multirow{2}{*}{\multicolumn{3}{|c|}{ (1d) }} \\
\hline & (1a) & & (1b) & & & & & \\
\hline \multicolumn{9}{|c|}{$\begin{array}{l}\text { Erwerbstätigkeit der Mutter (Ref. } \\
\text { nicht erwerbstätig) }\end{array}$} \\
\hline TZ (1-35 Std.) & & & & & & & $-0.11^{* *}$ & $(0.04)$ \\
\hline VZ (36-45 Std.) & & & & & & & $-0.20 * *$ & $(0.07)$ \\
\hline mehr als VZ $(>45)$ & & & & & & & 0.06 & $(0.09)$ \\
\hline Missings & & & & & & & 0.02 & $(0.11)$ \\
\hline TZ (1-35 Std.) & & & & & & & $0.25 * * *$ & $(0.07)$ \\
\hline VZ (36-45 Std.) & & & & & & & $0.39 * * *$ & $(0.06)$ \\
\hline mehr als VZ $(>45)$ & & & & & & & $0.49 * * *$ & $(0.06)$ \\
\hline Missings & & & & & & & $0.34 * * *$ & $(0.09)$ \\
\hline Constant & $0.50 * * *$ & $(0.02)$ & $0.61 * * *$ & $(0.04)$ & $0.63 * * *$ & $(0.05)$ & $0.21 * *$ & $(0.08)$ \\
\hline Observations & 873 & & 873 & & 873 & & 873 & \\
\hline
\end{tabular}

\begin{tabular}{|c|c|c|c|c|c|c|c|c|}
\hline \multirow{2}{*}{ VARIABLES } & \multicolumn{4}{|c|}{1 bis unter 3 Jahre } & \multirow[b]{2}{*}{$(2 \mathrm{c})$} & \multirow{2}{*}{\multicolumn{3}{|c|}{$(2 \mathrm{~d})$}} \\
\hline & $(2 a)$ & & (2b) & & & & & \\
\hline \multicolumn{9}{|c|}{$\begin{array}{l}\text { relativer Berufsstatus } \text { (Ref. Frau }= \\
\text { Mann) }\end{array}$} \\
\hline Frau < Mann & $0.09 * * *$ & $(0.02)$ & & & $0.07 * *$ & $(0.02)$ & $0.06^{* *}$ & $(0.02)$ \\
\hline Frau $>$ Mann & 0.03 & $(0.02)$ & & & 0.02 & $(0.02)$ & $0.04 *$ & $(0.02)$ \\
\hline Missings & 0.01 & $(0.03)$ & & & 0.03 & $(0.06)$ & $0.11 *$ & $(0.05)$ \\
\hline
\end{tabular}




\begin{tabular}{|c|c|c|c|c|c|c|c|c|}
\hline \multirow{2}{*}{ VARIABLES } & \multicolumn{4}{|c|}{1 bis unter 3 Jahre } & \multirow[b]{2}{*}{ (2c) } & \multirow{2}{*}{\multicolumn{3}{|c|}{ (2d) }} \\
\hline & $\begin{array}{l}\text { (2a) } \\
\text { coef }\end{array}$ & \multicolumn{2}{|r|}{$(2 b)$} & $\mathrm{se}$ & & & & \\
\hline $\begin{array}{l}\text { Item } 2 \text { (gegen männl. Alleinverdie- } \\
\text { ner-M.) }\end{array}$ & & & $-0.03 * * *$ & $(0.00)$ & $-0.03 * * *$ & $(0.00)$ & $-0.01 * *$ & $(0.00)$ \\
\hline \multicolumn{9}{|l|}{ Anzahl der Kinder (Ref. 2) } \\
\hline 1 Kind & & & & & $-0.07 * * *$ & $(0.02)$ & $-0.04 *$ & $(0.02)$ \\
\hline 3 Kinder & & & & & 0.03 & $(0.02)$ & 0.00 & $(0.02)$ \\
\hline Ost-Dtl. & & & & & $-0.17 * * *$ & $(0.03)$ & $-0.11 * * *$ & $(0.02)$ \\
\hline \multicolumn{9}{|l|}{ ISEI der Mutter (Ref. mittel (45-62)) } \\
\hline niedrig $(<45)$ & & & & & -0.01 & $(0.02)$ & -0.01 & $(0.02)$ \\
\hline hoch (ab 63) & & & & & -0.01 & $(0.02)$ & 0.01 & $(0.02)$ \\
\hline Missings & & & & & -0.05 & $(0.06)$ & $-0.12 *$ & $(0.06)$ \\
\hline \multicolumn{9}{|l|}{$\begin{array}{l}\text { Erwerbstätigkeit der Mutter (Ref. } \\
\text { nicht erwerbstätig) }\end{array}$} \\
\hline \multicolumn{9}{|l|}{$\begin{array}{l}\text { Erwerbstätigkeit des Vaters (Ref. } \\
\text { nicht erwerbstätig) }\end{array}$} \\
\hline TZ (1-35 Std.) & & & & & & & $0.31 * * *$ & $(0.05)$ \\
\hline VZ (36-45 Std.) & & & & & & & $0.46 * * *$ & $(0.04)$ \\
\hline mehr als VZ $(>45)$ & & & & & & & $0.57 * * *$ & $(0.05)$ \\
\hline Missings & & & & & & & $0.23 * *$ & $(0.07)$ \\
\hline Constant & $0.48 * * *$ & $(0.02)$ & $0.75 * * *$ & $(0.03)$ & $0.75 * * *$ & $(0.03)$ & $0.25 * * *$ & $(0.05)$ \\
\hline Observations & 2,176 & & 2,176 & & 2,176 & & 2,176 & \\
\hline Adjusted R-squared & 0.01 & & 0.04 & & 0.07 & & 0.23 & \\
\hline
\end{tabular}




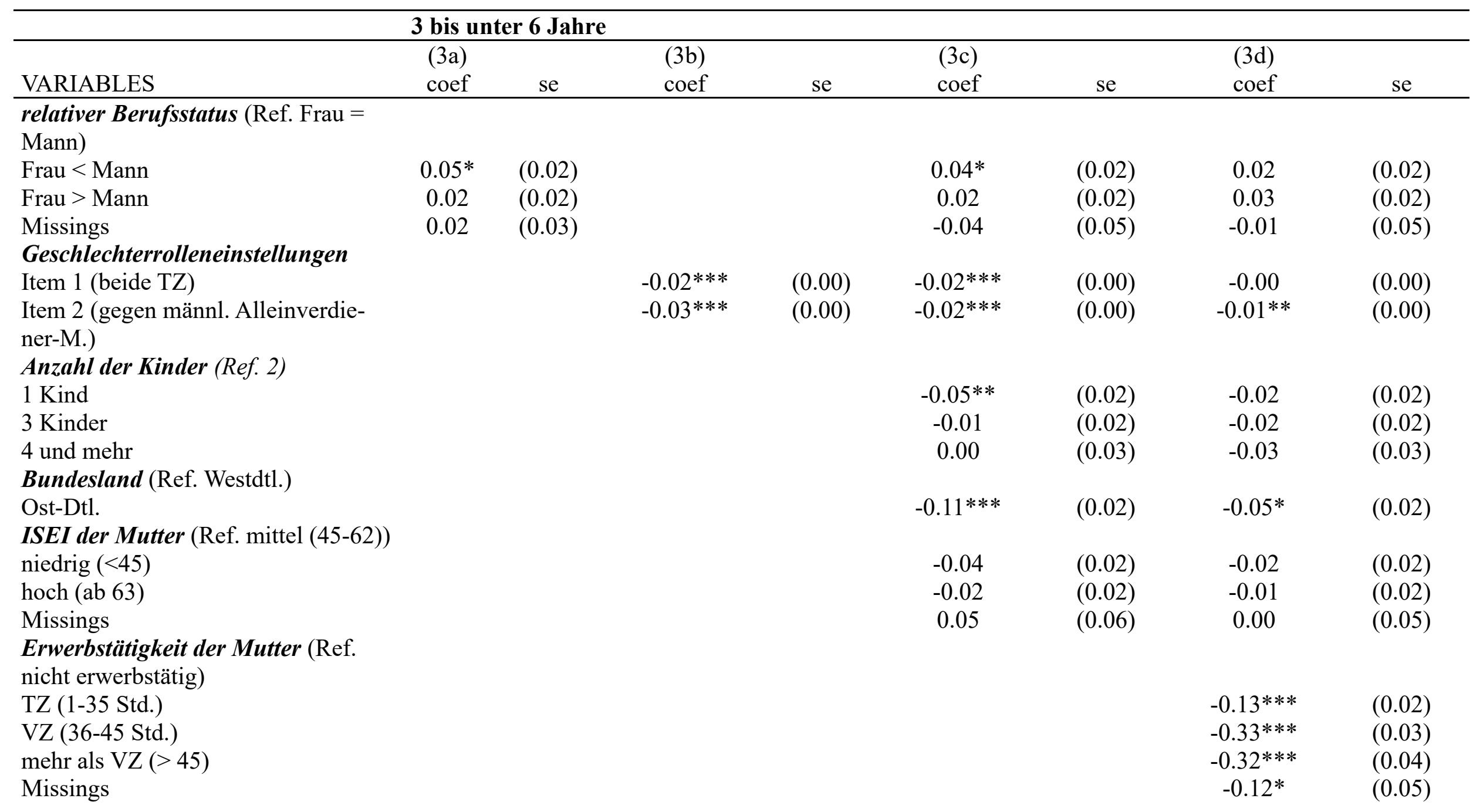




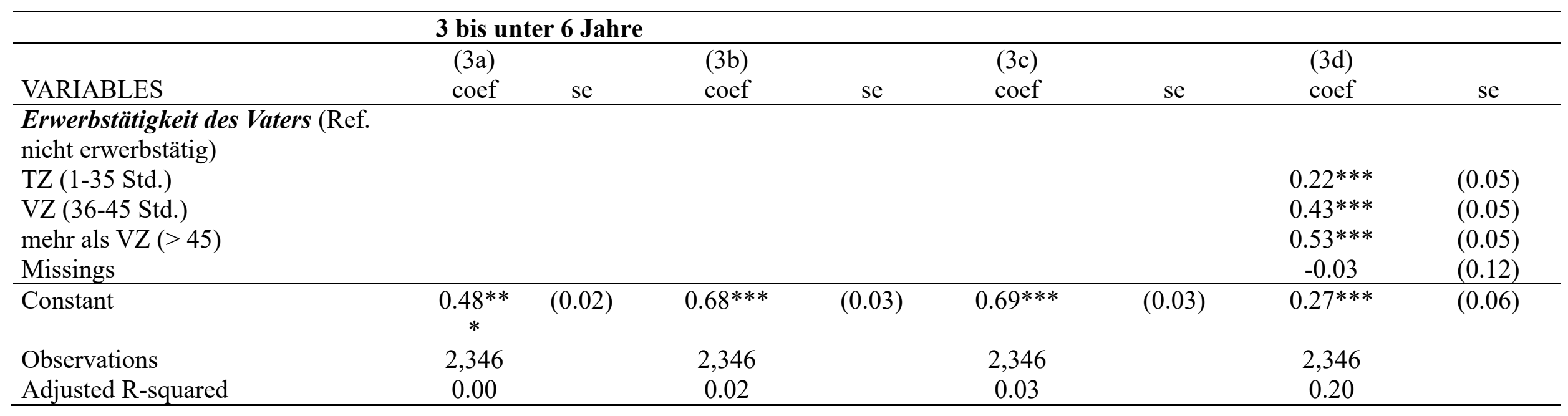

\begin{tabular}{|c|c|c|c|c|c|c|c|c|}
\hline \multirow{2}{*}{ VARIABLES } & \multicolumn{8}{|c|}{6 bis unter 11 Jahre } \\
\hline & $(4 a)$ & & $(4 \mathrm{~b})$ & & $(4 c)$ & & $(4 d)$ & \\
\hline \multicolumn{9}{|l|}{$\begin{array}{l}\text { relativer Berufsstatus (Ref. Frau }= \\
\text { Mann) }\end{array}$} \\
\hline Frau $<$ Mann & $0.05^{* *}$ & $(0.02)$ & & & $0.06^{* *}$ & $(0.02)$ & 0.02 & $(0.02)$ \\
\hline Frau $>$ Mann & 0.03 & $(0.02)$ & & & 0.02 & $(0.02)$ & 0.02 & $(0.02)$ \\
\hline Missings & 0.04 & $(0.03)$ & & & 0.02 & $(0.04)$ & 0.01 & $(0.04)$ \\
\hline $\begin{array}{l}\text { Item } 2 \text { (gegen männl. Alleinverdie- } \\
\text { ner-M.) }\end{array}$ & & & $-0.02 * * *$ & $(0.00)$ & $-0.02 * * *$ & $(0.00)$ & $-0.02 * * *$ & $(0.00)$ \\
\hline \multicolumn{9}{|l|}{ Anzahl der Kinder (Ref. 2) } \\
\hline 1 Kind & & & & & $-0.05 *$ & $(0.02)$ & -0.03 & $(0.02)$ \\
\hline 3 Kinder & & & & & -0.01 & $(0.02)$ & $-0.04 *$ & $(0.02)$ \\
\hline
\end{tabular}




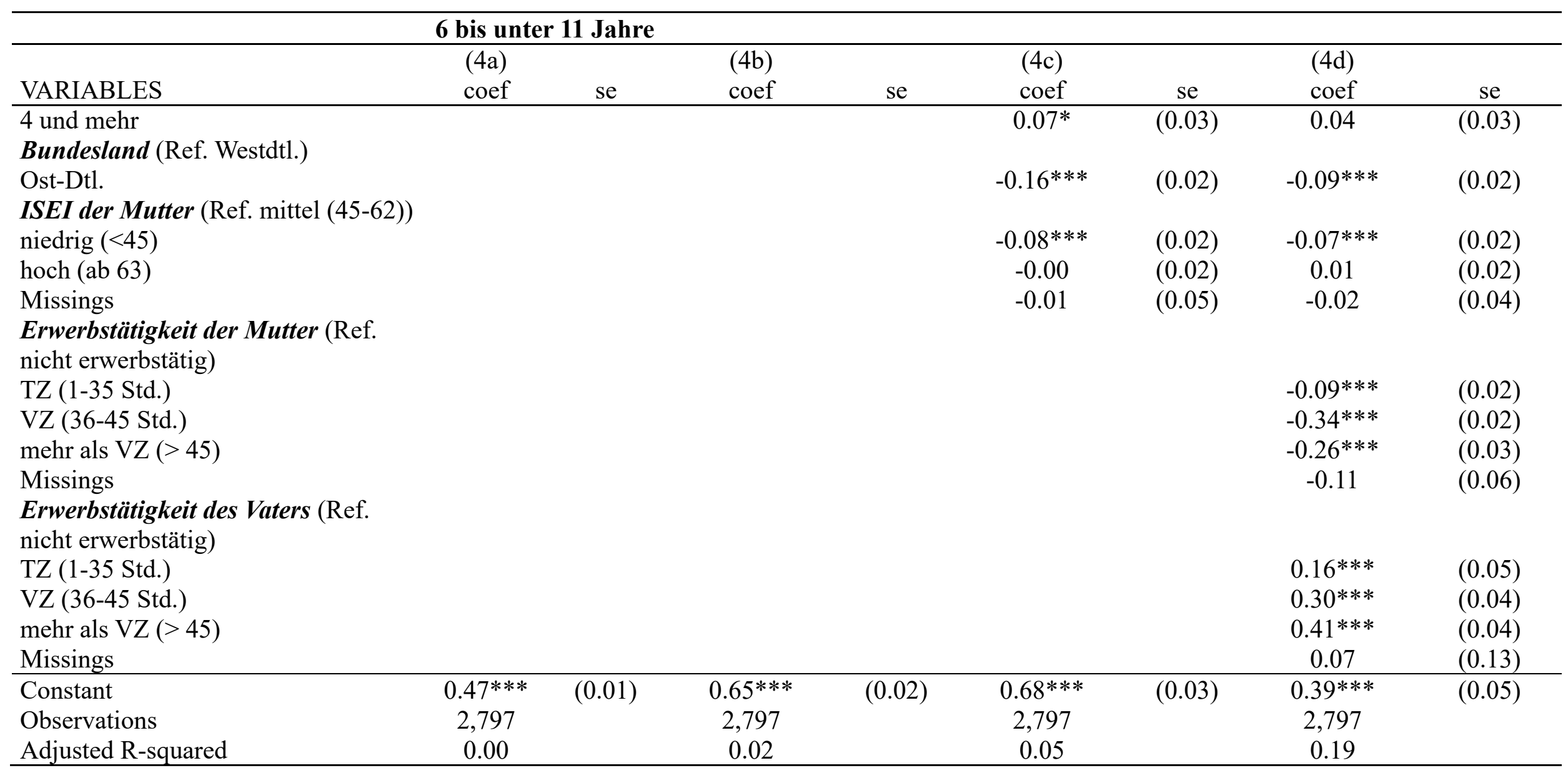




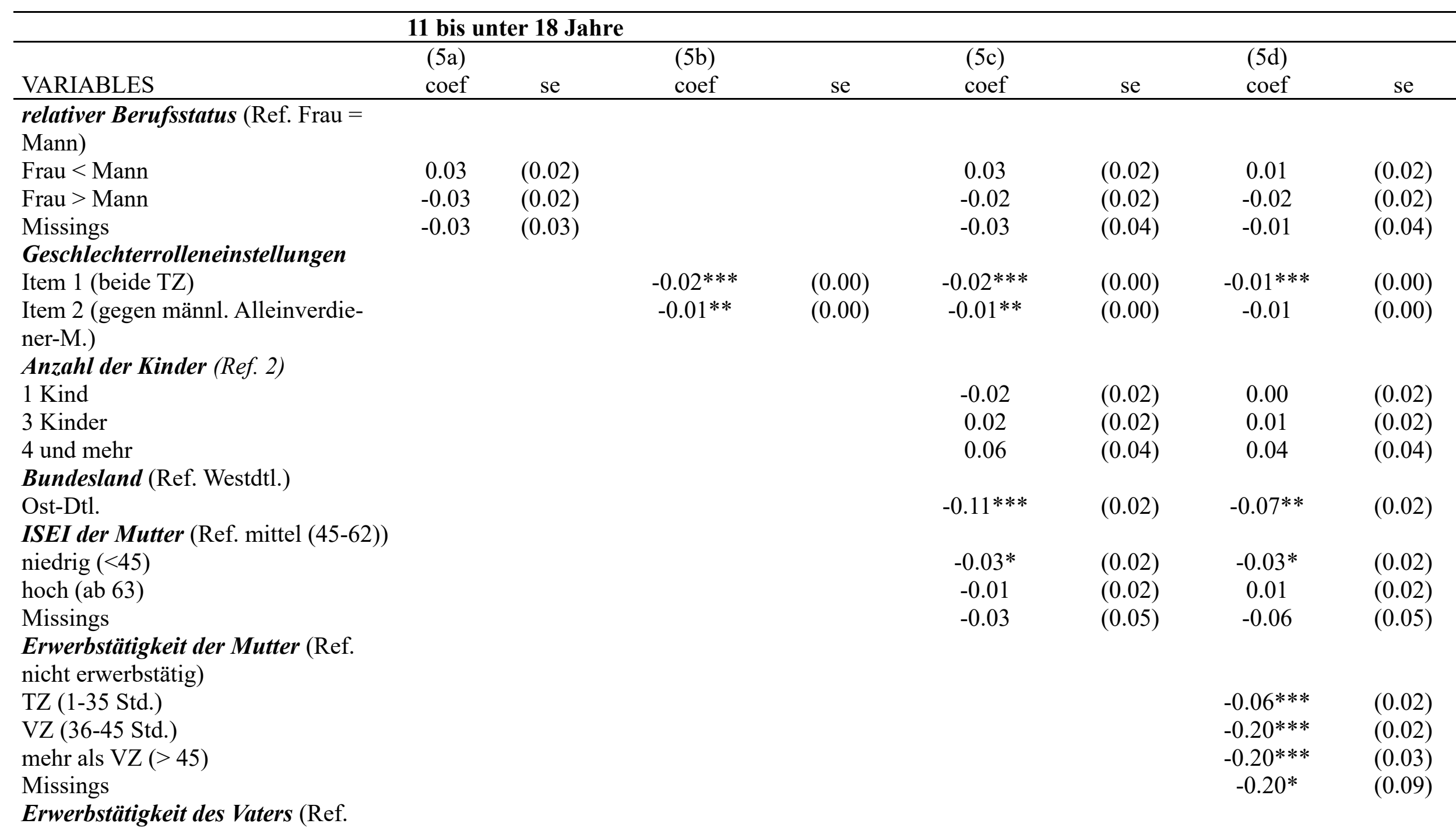




\begin{tabular}{|c|c|c|c|c|c|c|c|c|}
\hline \multicolumn{9}{|c|}{11 bis unter 18 Jahre } \\
\hline & (5a) & & $(5 b)$ & & $(5 c)$ & & (5d) & \\
\hline TZ (1-35 Std.) & & & & & & & -0.03 & $(0.04)$ \\
\hline mehr als VZ (> 45) & & & & & & & $0.21 * * *$ & $(0.03)$ \\
\hline Missings & & & & & & & -0.16 & $(0.13)$ \\
\hline Constant & $0.43 * * *$ & $(0.01)$ & $0.55 * * *$ & $(0.02)$ & $0.59 * * *$ & $(0.03)$ & $0.48 * * *$ & $(0.04)$ \\
\hline
\end{tabular}

Quelle: DJI-Studie AID:A 2013/14, eigene Berechnungen. OLS-Regression mit folgenden Signifikanzen: ${ }^{* * *} p<0.001,{ }^{* *} p<0.01,{ }^{*} p<0.05$.

Tabelle A.11: $\quad$ Aufteilung der Kinderbetreuung, unter Berücksichtigung der drei Gender-Klassen, getrennt nach dem Alter des Kindes

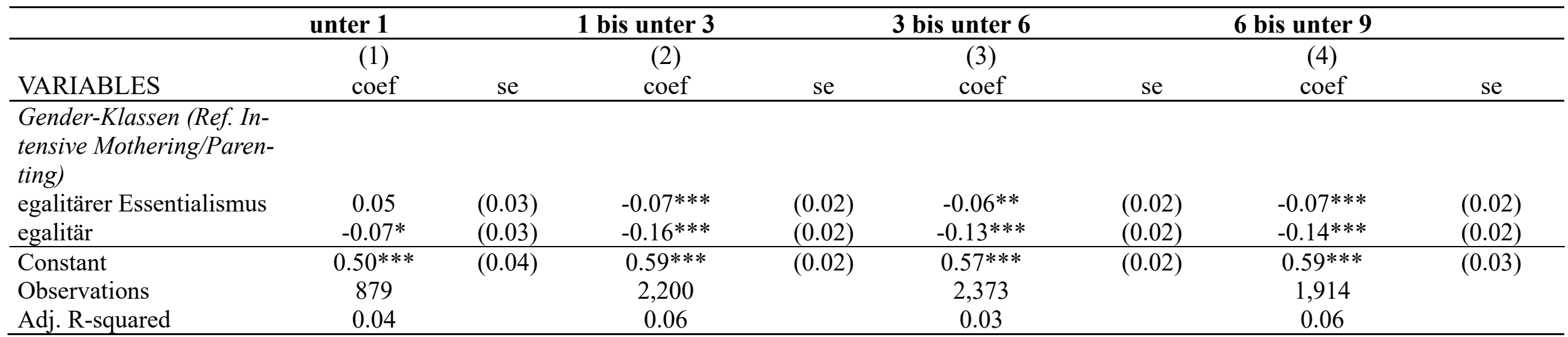

Quelle: DJI-Studie AID:A 2013/14, eigene Berechnungen. OLS-Regression mit folgenden Signifikanzen: ${ }^{* * *} p<0.001,{ }^{* *} p<0.01,{ }^{*} p<0.05$. Anmerkung: Die Skalen der jeweiligen Gender-Items gehen von "1 traditionell“ bis „6 egalitär". Es wurden die gleichen Variablen wie in den Modellen 1c, 2c, 3c, usw. in Tabelle A.10 kontrolliert, d.h. der relative Berufsstatus, die Anzahl der Kinder, das Bundesgebiet und der absolute Berufsstatus der Mutter. 
Tabelle A.12: Übersicht über die Bestätigung oder Ablehnung der Hypothesen anhand der empirischen Ergebnisse

\begin{tabular}{|c|c|c|c|c|}
\hline & Hypothesen & $\begin{array}{l}\text { Aufteilung der Er- } \\
\text { werbsarbeit }{ }^{c}\end{array}$ & $\begin{array}{l}\text { Aufteilung der } \\
\text { Hausarbeit }\end{array}$ & $\begin{array}{l}\text { Aufteilung der Kin- } \\
\text { derbetreuung }\end{array}$ \\
\hline $\begin{array}{l}\text { Hypothese 1: } \\
\text { relativer Be- } \\
\text { rufsstatus } \\
\text { (ISEI) }^{\text {a }}\end{array}$ & $\begin{array}{l}\text { Diejenige Person mit den höheren, relativen Ressour- } \\
\text { cen spezialisiert sich auf die Erwerbsarbeit und die an- } \\
\text { dere Person auf die Hausarbeit und Kinderbetreuung. }\end{array}$ & z.T. bestätigt & z.T. bestätigt & z.T. bestätigt \\
\hline \multirow{3}{*}{$\begin{array}{l}\text { Hypothese 2: } \\
\text { relativer Be- } \\
\text { rufsstatus } \\
\text { (ISEI) }{ }^{\text {b }} \\
\text { a) allgemein } \\
\text { b) - e) alters- } \\
\text { spezifisch }\end{array}$} & $\begin{array}{l}\text { b) Wenn das Kind unter einem Jahr alt ist, dann spielen } \\
\text { relative Ressourcen keine oder nur eine sehr geringe } \\
\text { Rolle. }\end{array}$ & nicht bestätigt & bestätigt & bestätigt \\
\hline & $\begin{array}{l}\text { d) Wenn das Kind drei bis unter sechs Jahre alt ist, dann } \\
\text { spielen relative Ressourcen eine deutliche Rolle. }\end{array}$ & nicht bestätigt & z.T. bestätigt & nicht bestätigt \\
\hline & $\begin{array}{l}\text { e) Wenn das Kind sechs Jahre alt oder älter ist, dann } \\
\text { spielen relative Ressourcen eine noch stärkere Rolle. }\end{array}$ & nicht bestätigt & z.T. bestätigt & nicht bestätigt \\
\hline
\end{tabular}




\begin{tabular}{|c|c|c|c|c|}
\hline & Hypothesen & $\begin{array}{l}\text { Aufteilung der Er- } \\
\text { werbsarbeit }{ }^{c}\end{array}$ & $\begin{array}{l}\text { Aufteilung der } \\
\text { Hausarbeit }\end{array}$ & $\begin{array}{l}\text { Aufteilung der Kin- } \\
\text { derbetreuung }\end{array}$ \\
\hline \multirow{4}{*}{$\begin{array}{l}\text { Hypothese 3: } \\
\text { Geschlechter- } \\
\text { rolleneinstel- } \\
\text { lungen } \\
\text { a) allgemein } \\
\text { b) - e) alters- } \\
\text { spezifisch }\end{array}$} & $\begin{array}{l}\text { a) Wenn Mütter egalitäre (resp. traditionelle) Einstellun- } \\
\text { gen haben, dann ist die Arbeitsteilung egalitärer (resp. } \\
\text { traditioneller) }\end{array}$ & $\begin{array}{l}\text { Item 1: bestätigt } \\
\text { Item 2: bestätigt }\end{array}$ & $\begin{array}{l}\text { Item 1: bestätigt } \\
\text { Item 2: bestätigt }\end{array}$ & $\begin{array}{l}\text { Item 1: bestätigt } \\
\text { Item 2: bestätigt }\end{array}$ \\
\hline & $\begin{array}{l}\text { b) Wenn das Kind im Alter von unter einem Jahr ist, kön- } \\
\text { nen Mütter ihre egalitären Einstellungen nicht realisie- } \\
\text { ren. }\end{array}$ & $\begin{array}{l}\text { Item 1: nicht bestätigt } \\
\text { Item 2: nicht bestätigt }\end{array}$ & $\begin{array}{l}\text { Item 1: nicht bestätigt } \\
\text { Item 2: nicht bestätigt }\end{array}$ & $\begin{array}{l}\text { Item 1: nicht bestätigt } \\
\text { Item 2: bestätigt }\end{array}$ \\
\hline & $\begin{array}{l}\text { c) Wenn das Kind im Alter von ein bis unter drei Jahren } \\
\text { ist, können Mütter ihre egalitären Einstellungen nur } \\
\text { schwer realisieren. }\end{array}$ & $\begin{array}{l}\text { Item 1: nicht bestätigt } \\
\text { Item 2: bestätigt }\end{array}$ & $\begin{array}{l}\text { Item 1: nicht bestätigt } \\
\text { Item 2: nicht bestätigt }\end{array}$ & $\begin{array}{l}\text { Item 1: nicht bestätigt } \\
\text { Item 2: bestätigt }\end{array}$ \\
\hline & $\begin{array}{l}\text { d) Wenn das Kind im Alter von drei bis unter sechs Jah- } \\
\text { ren ist, können Mütter ihre egalitären Einstellungen } \\
\text { schon etwas leichter realisieren. }\end{array}$ & $\begin{array}{l}\text { Item 1: nicht bestätigt } \\
\text { Item 2: bestätigt }\end{array}$ & $\begin{array}{l}\text { Item 1: nicht bestätigt } \\
\text { Item 2: nicht bestätigt }\end{array}$ & $\begin{array}{l}\text { Item 1: nicht bestätigt } \\
\text { Item 2: nicht bestätigt }\end{array}$ \\
\hline $\begin{array}{l}\text { Hypothese 4: } \\
\text { Leitbild des } \\
\text { „intensive }\end{array}$ & $\begin{array}{l}\text { a) Wenn sich Mütter mit dem Leitbild des „intensive mot- } \\
\text { hering/parenting“ identifizieren, dann ist die Arbeitstei- } \\
\text { lung traditioneller. }\end{array}$ & bestätigt & bestätigt & bestätigt \\
\hline
\end{tabular}




\begin{tabular}{|c|c|c|c|c|}
\hline & Hypothesen & $\begin{array}{l}\text { Aufteilung der Er- } \\
\text { werbsarbeit }{ }^{c}\end{array}$ & $\begin{array}{l}\text { Aufteilung der } \\
\text { Hausarbeit }\end{array}$ & $\begin{array}{l}\text { Aufteilung der Kin- } \\
\text { derbetreuung }\end{array}$ \\
\hline \multirow{3}{*}{$\begin{array}{l}\text { mothering/ } \\
\text { parenting“" } \\
\text { a) allgemein } \\
\text { b) - d) alters- } \\
\text { spezifisch }\end{array}$} & $\begin{array}{l}\text { b) Wenn das Kind unter } 3 \text { Jahre alt ist, hat das Leitbild } \\
\text { des „intensive mothering/parenting“ einen stark traditio- } \\
\text { nalisierenden Effekt auf die Arbeitsteilung. }\end{array}$ & bestätigt & z.T. bestätigt $\mathrm{g}$ & z.T. bestätigt ${ }^{j}$ \\
\hline & $\begin{array}{l}\text { c) Wenn das Kind } 3 \text { bis unter } 6 \text { Jahre alt ist, hat das } \\
\text { Leitbild des „intensive mothering/parenting“ einen etwas } \\
\text { abgeschwächten, aber immer noch traditionalisierenden } \\
\text { Effekt auf die Arbeitsteilung. }\end{array}$ & nicht bestätigt & nicht bestätigt & nicht bestätigt \\
\hline & $\begin{array}{l}\text { d) Wenn das Kind im Grundschulalter ist, hat das Leit- } \\
\text { bild des „intensive mothering" keinen oder nur einen } \\
\text { sehr geringen Effekt auf die Arbeitsteilung. }\end{array}$ & nicht bestätigt & z.T. bestätigt $\mathrm{h}$ & nicht bestätigt \\
\hline \multirow{2}{*}{$\begin{array}{l}\text { Hypothese 6: } \\
\text { Ost-/West- } \\
\text { deutschland } \\
\text { a) allgemein }\end{array}$} & $\begin{array}{l}\text { a) In Ostdeutschland ist die Arbeitsteilung egalitärer auf- } \\
\text { geteilt als in Westdeutschland. }\end{array}$ & bestätigt & bestätigt & bestätigt \\
\hline & $\begin{array}{l}\text { b) Wenn sich das Kind noch im Kleinkindalter befindet, } \\
\text { dann sind die Unterschiede besonders groß; wenn sich }\end{array}$ & nicht bestätigt & nicht bestätigt & nicht bestätigt \\
\hline
\end{tabular}




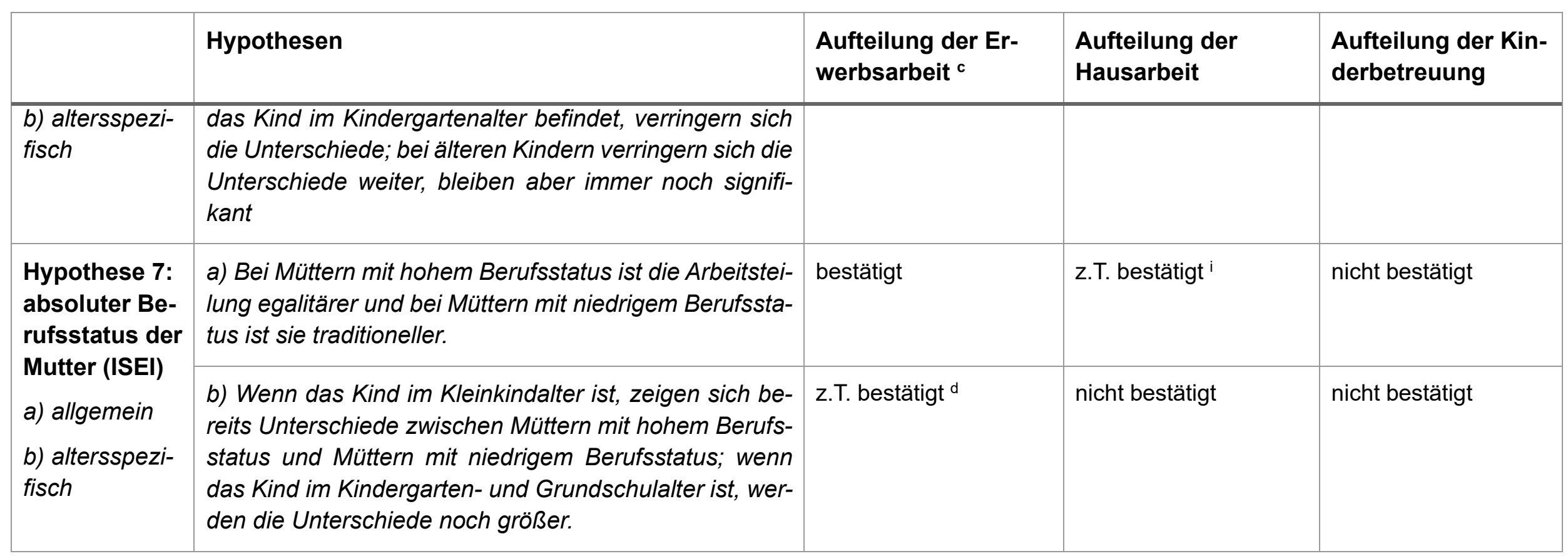

Quelle: Eigene Zusammenfassung. Anmerkungen:

${ }^{a}$ Gemäß der familienökonomischen Argumentation nach Becker.

${ }^{\mathrm{b}}$ Gemäß der verhandlungstheoretischen Argumentation nach Ott.

${ }^{\mathrm{c}}$ Bei der Aufteilung der Erwerbsarbeit wurden als abhängige Variablen der Erwerbsanteil der Mütter am gesamten Erwerbsvolumen (AV1) und die Erwerbsstunden der Mütter (AV2) verwendet. Da sich die Ergebnisse der beiden abhängigen Variablen sehr ähneln, werden sie in dieser Übersicht nicht differenziert betrachtet.

${ }^{\mathrm{d}}$ Hypothese 7 b) kann nicht für den Erwerbsanteil der Mütter am gesamten Erwerbsvolumen (AV1) bestätigt werden, aber schon für die Erwerbsstunden der Mütter (AV2). 
e Die Hypothese kann insofern bestätigt werden, weil Mütter mit niedrigeren, relativen Ressourcen etwas mehr Hausarbeit übernehmen (als Mütter, mit gleichen Ressourcen im Vergleich zu den Vätern), aber umgekehrt übernehmen Mütter mit höheren Ressourcen nicht weniger Hausarbeit (als Mütter mit gleichen Ressourcen).

${ }^{f}$ Es wird der Teilaspekt bestätigt, dass die Ressourcen bei einem Kind im Alter von „1 bis 3 Jahre“ noch keine Rolle spielen. Es kann somit nicht der Teilaspekt bestätigt werden, dass sie schon eine etwas größere Rolle als in der vorherigen Altersstufe spielen.

9 Die Hypothese 4 b) kann nur für die Altersstufe „1 bis unter 3 Jahre“ bestätigt werden: Hier ist die Aufteilung der Hausarbeit in der Klasse des „intensive mothering/parenting" signifikant traditioneller als in den anderen beiden Klassen und die Unterschiede fallen hier zudem stärker aus als in den älteren Altersstufen des Kindes.

${ }^{\mathrm{h}}$ Es kann der Teilaspekt bestätigt werden, dass das Leitbild des „intensive mothering/parenting“ bei einem über 6-jährigen Kind eine geringe Rolle spielt als bei einem jüngeren Kind, aber es muss verneint werden, dass die Effekte nicht mehr vorhanden oder nur noch verschwindend gering sind.

i Bei Mütter mit einem hohen Berufsstatus ist die Hausarbeit entsprechend den Erwartungen egalitärer aufgeteilt, aber bei Müttern mit einem niedrigen Status ist dies entgegen den Erwartungen ebenfalls der Fall. Am traditionellsten ist die Aufteilung der Hausarbeit laut den Ergebnissen bei Müttern mit einem mittleren Status.

j Für die Altersstufe „unter 1 Jahr“ kann die Hypothese, dass das Leitbild des „intensive mothering/parenting“ einen stark traditionalisierenden Effekt auf die Aufteilung der Kinderbetreuung hat, nicht bestätigt werden; für die Altersstufe „1 bis unter 3 Jahre“ kann dies dagegen schon bestätigt werden. 


\section{Literaturverzeichnis}

Aassve, Arnstein; Fuochi, Giulia; Mencarini, Letizia (2014): Desperate Housework. Relative Resources, Time Availability, Economic Dependency, and Gender Ideology Across Europe. In: Journal of Family Issues 35 (8), S. 1000-1022. DOI: 10.1177/0192513X14522248.

Aisenbrey, Silke; Evertsson, Marie; Grunow, Daniela (2009): Is There a Career Penalty for Mothers' Time Out? A Comparison of Germany, Sweden and the United States. In: Social Forces 88 (2), S. 573-605.

Aldous, Joan (1990): Family Development and the Life Course. Two Perspectives on Family Change. In: Journal of Marriage and the Family 52 (3), S. 571-583.

Alfermann, Dorothee (1996): Geschlechterrollen und geschlechtstypisches Verhalten. Stuttgart: Kohlhammer $\mathrm{GmbH}$.

Allen, Sarah M.; Hawkins, Alan J. (1999): Maternal Gatekeeping. Mothers' Beliefs and Behaviors That Inhibit Greater Father Involvement in Family Work. In: Journal of Marriage and Family 61 (1), S. 199-212. DOI: 10.2307/353894.

Allen, Tammy D.; Finkelstein, Lisa M. (2014): Work-Family Conflict Among Members of FullTime Dual-Earner Couples. An Examination of Family Life Stage, Gender, and Age. In: Journal of occupational health psychology 19 (3), S. 376-384. DOI: 10.1037/a0036941.

Almqvist, Anna-Lena; Duvander, Ann-Zofie (2014): Changes in Gender Equality? Swedish Fathers' Parental Leave, Division of Childcare and Housework. In: Journal of Family Studies 20 (1), S. 19-27. DOI: 10.5172/jfs.2014.20.1.19.

Alwin, Duane F.; Korsnick, Jon A. (1991): Aging, Cohort, and the Stability of Sociopolitical Orientations over the Life Span. In: American Journal of Sociology 97 (1), S. 169-195.

Arrighi, Barbara A.; Mauma, David J. (2000): Workplace Subordination and Men's Avoidance of Housework. In: Journal of Family Issues 21 (4), S. 464-487. DOI: 10.1177/019251300021004003.

Auspurg, Katrin; lacovou, Maria; Nicoletti, Cheti (2017): Housework Share Between Partners. Experimental Evidence on Gender-Specific Preferences. In: Social Science Research 66, S. 118-139. DOI: 10.1016/j.ssresearch.2017.01.003.

Backhaus, Klaus; Erichson, Bernd; Plinke, Wulff; Weiber, Rolf (2016): Multivariate Analysemethoden. Eine anwendungsorientierte Einführung. Berlin, Heidelberg: Springer Gabler.

Batalova, Jeanne A.; Cohen, Philip N. (2002): Premarital Cohabitation and Housework. Couples in Cross-National Perspective. In: Journal of Marriage and Family 64 (3), S. 743755.

Bauernschuster, Stefan; Schlotter, Martin (2015): Public child care and mothers' labor supply - Evidence from two quasi-experiments. In: Journal of Public Economics 123, S. 1-16. DOI: 10.1016/j.jpubeco.2014.12.013.

Baxter, Janeen (1997): Gender equality and participation in housework. A cross-national perspective. In: Journal of Comparative Family Studies 28 (3), S. 220-247.

Baxter, Janeen (2005): To Marry or Not to Marry. Marital Status and the Household Division of Labor. In: Journal of Family Issues 26 (3), S. 300-321. DOI: 10.1177/0192513X04270473.

Baxter, Janeen; Buchler, Sandra; Perales, Francisco; Western, Mark (2015): A Life-Changing Event. First Births and Men's and Women's Attitudes to Mothering and Gender Divisions of Labor. In: Social Forces 93 (3), S. 989-1014. DOI: 10.1093/sf/sou103.

Baxter, Janeen; Hewitt, Belinda; Haynes, Michele (2008): Life Course Transitions and Housework. Marriage, Parenthood, and Time on Housework. In: Journal of Marriage and Family 70 (2), S. 259-272. 
Becker, Gary S. (1960): An Economic Analysis of Fertility. In: National Bureau of Economic Research (Hg.): Demographic and Economic Change in Developed Countries: Columbia University Press, S. 209-240.

Becker, Gary S. (1965): A Theory of the Allocation of Time. In: The Economic Journal 75 (299), S. 493-517. DOI: 10.2307/2228949.

Becker, Gary S. (1975): Human Capital. A Theoretical and Empirical Analysis with Special Reference to Education. New York: National Bureau of Economic Research.

Becker, Gary S. (1976): The Economic Approach to Human Behavior. Chicago, London: University of Chicago Press (Phoenix books, 803).

Becker, Gary S. (1985): Human Capital, Effort, and the Sexual Division of Labor. In: Journal of Labor Economics 3 (1, Part 2), 33-58. DOI: 10.1086/298075.

Becker, Gary S. (1993): A treatise on the family. Enl. ed. Cambridge, Mass: Harvard University Press.

Berghammer, Caroline (2014): The Return of the Male Breadwinner Model? Educational Effects on Parents' Work Arrangements in Austria, 1980-2009. In: Work, Employment and Society 28 (4), S. 611-632. DOI: 10.1177/0950017013500115.

Berk, Richard; Berk, Sara Fenstermaker (1983): Supply-Side Sociology of the Family. The Challenge of the New Home Economics. In: Annual Review of Sociology 9, S. 375.

Bernardi, Laura; Huinink, Johannes; Settersten, Richard A. (2019): The Life Course Cube. A Tool for Studying Lives. In: Advances in Life Course Research 41, S. 1-13. DOI: 10.1016/j.alcr.2018.11.004.

Bernasco, Wim; Graaf, Paul M. de; Ulte, Wout C. (1998): Coupled Careers. Effects of Spouse's Resources on Occupational Attainment in the Netherlands. In: European Sociological Review 14 (1), S. 15-31.

Berrington, Ann; Hu, Yongjian; Smith, Peter W. F.; Sturgis, Patrick (2008): A Graphical Chain Model for Reciprocal Relationships Between Women's Gender Role Attitudes and Labour Force Participation. In: Journal of the Royal Statistical Society Series A (Statistics in Society) 17 (1), S. 89-108.

Bertram, Hans; Bujard, Martin (2012): Zur Zukunft der Familienpolitik. In: Hans Bertram und Martin Bujard (Hg.): Zeit, Geld, Infrastruktur - zur Zukunft der Familienpolitik. 1. Aufl. Baden-Baden: Nomos-Verl.-Ges (Soziale Welt Sonderband, 19).

Bianchi, Suzanne M.; Milkie, Melissa A. (2010): Work and Family Research in the First Decade of the 21st Century. In: Journal of Marriage and Family 72 (3), S. 705-725. DOI: 10.1111/j.1741-3737.2010.00726.x.

Bianchi, Suzanne M.; Milkie, Melissa A.; Sayer, Liana C.; Robinson, John P. (2000): Is Anyone Doing the Housework? Trends in the Gender Division of Household Labor. In: Social Forces 79 (1), S. 191-228. DOI: 10.2307/2675569.

Bianchi, Suzanne M.; Robinson, John P.; Milkie, Melissa A. (2006): Changing Rhythms of American Family Life. New York: Russell Sage Foundation.

Bianchi, Suzanne M.; Sayer, Liana C.; Milkie, Melissa A.; Robinson, John P. (2012): Housework. Who Did, Does or Will Do It, and How Much Does It Matter? In: Social Forces 91 (1), S. 55-63. DOI: 10.1093/sf/sos120.

Bielby, William T.; Bielby, Denise D. (1989): Family Ties. Balancing Commitments to Work and Family in Dual Earner Households. In: American Sociological Review 54 (5), S. 776 789. DOI: $10.2307 / 2117753$.

Bittman, Michael; England, Paula; Sayer, Liana; Folbre, Nancy; Matheson, George (2003): When Does Gender Trump Money? Bargaining and Time in Household Work. In: American Journal of Sociology 109 (1), S. 186-214. DOI: 10.1086/378341.

Blair, Sampson Lee; Lichter, Daniel T. (1991): Measuring the Division of Household Labor. Gender Segregation of Housework Among American Couples. In: Journal of Family Issues $12(1)$, S. 91-113. 
Blau, Peter Michael (1964): Exchange and Power in Social Life. New York/London/Sydney: Wiley.

Blau, Peter Michael (2005): Sozialer Austausch. In: Frank Adloff und Steffen Mau (Hg.): Vom Geben und Nehmen. Zur Soziologie der Reziprozität. Frankfurt am Main/New York: Campus Verlag, S. 125-138.

Blood, Robert Oscar; Wolfe, Donald MacCreery (1960): Husbands and wives. The Dynamics of Married Living. Glencoe: Greenwood Press.

Blossfeld, Hans-Peter; Timm, Andreas (2003): Who Marries Whom? Educational Systems as Marriage Markets in Modern Societiest: Dordrecht: Kluwer Academic Publishers.

BMFSFJ, Bundesministerium für Familien, Senioren, Frauen und Jugend (2006a): Eziehungsgeld, Elternzeit. Das Bundeserziehungsgesetz. Online verfügbar unter https://bit.ly/39csMZs, zuletzt geprüft am 23.11.2020.

BMFSFJ, Bundesministerium für Familien, Senioren, Frauen und Jugend (2006b): Siebter Familienbericht: Familie zwischen Flexibilität und Verlässlichkeit. Perspektiven für eine lebenslaufbezogene Familienpolitik.

BMFSFJ, Bundesministerium für Familien, Senioren, Frauen und Jugend (2008a): Das Gesetz zum Elterngeld und zur Elternzeit im internationalen, insbesondere europäischen Vergleich. Länderstudien 2008.

BMFSFJ, Bundesministerium für Familien, Senioren, Frauen und Jugend (2008b): Dossier Ausbau der Kinderbetreuung - Kosten, Nutzen, Finanzierung. Berlin. Online verfügbar unter https://bit.ly/35Xwq7F, zuletzt geprüft am 23.11.2020.

BMFSFJ, Bundesministerium für Familien, Senioren, Frauen und Jugend (2012): Ausgeübte Erwerbstätigkeit von Müttern. Erwerbstätigkeit, Erwerbsumfang und Erwerbsvolumen 2010. Berlin.

BMFSFJ, Bundesministerium für Familien, Senioren, Frauen und Jugend (2014a): Dossier Müttererwerbstätigkeit. Erwerbstätigkeit, Erwerbsumfang und Erwerbsvolumen 2012. Berlin.

BMFSFJ, Bundesministerium für Familien, Senioren, Frauen und Jugend (2014b): ElterngeIdPlus: Moderne Familienpolitik setzt auf Partnerschaftlichkeit. Online verfügbar unter https://bit.ly/2IY3rHP.

BMFSFJ, Bundesministerium für Familien, Senioren, Frauen und Jugend (2015): Fünfter Bericht zur Evaluation des Kinderförderungsgesetzes. Berlin. Online verfügbar unter https://bit.ly/370piXs, zuletzt geprüft am 23.11.2020.

BMFSFJ, Bundesministerium für Familien, Senioren, Frauen und Jugend (2016a): Bilanz 10 Jahre Elterngeld. Berlin. Online verfügbar unter https://bit.ly/3frgRIf, zuletzt geprüft am 23.11.2020.

BMFSFJ, Bundesministerium für Familien, Senioren, Frauen und Jugend (2016b): Die Familienarbeitszeit - mehr Zeit für Familie und Beruf, mehr wirtschaftliche Stabilität. Pressekonferenz zur Familienarbeitszeit. Berlin. Online verfügbar unter https://bit.ly/33b0X01, zuletzt geprüft am 23.11.2020.

BMFSFJ, Bundesministerium für Familien, Senioren, Frauen und Jugend (2016c): Väterreport 2016. Vater sein in Deutschland heute. Berlin. Online verfügbar unter https://bit.ly/3nPMNJl, zuletzt geprüft am 23.11.2020.

BMFSFJ, Bundesministerium für Familien, Senioren, Frauen und Jugend (2017a): Familienreport 2017. Leistungen, Wirkungen, Trends. Berlin. Online verfügbar unter https://bit.ly/372ufyP, zuletzt geprüft am 23.11.2020.

BMFSFJ, Bundesministerium für Familien, Senioren, Frauen und Jugend (2017b): Kindertagesbetreuung Kompakt. Ausbaustand und Bedarf 2016. Berlin. Online verfügbar unter https://bit.ly/3kZU9rQ, zuletzt geprüft am 23.11.2020.

BMFSFJ, Bundesministerium für Familien, Senioren, Frauen und Jugend (2018a): Elterngeld, ElterngeldPlus und Elternzeit. Das Bundeselterngeld- und Elternzeitgesetz. Online 
verfügbar unter https://www.bmfsfj.de/blob/jump/93614/elterngeld-elterngeldplus-und-elternzeit-data.pdf, zuletzt geprüft am 23.11.2020.

BMFSFJ, Bundesministerium für Familien, Senioren, Frauen und Jugend (2018b): Väterreport. Vater sein in Deutschland heute. Online verfügbar unter https://bit.ly/3/1n6nk, zuletzt geprüft am 23.11.2020.

BMFSFJ, Bundesministerium für Familien, Senioren, Frauen und Jugend (2019): Kindertagesbetreuung Kompakt. Ausbaustand und Bedarf 2018. Berlin. Online verfügbar unter https://bit.ly/2J5Pgk9, zuletzt geprüft am 23.11.2020.

Boeckmann, Irene; Misra, Joya; Budig, Michelle J. (2015): Cultural and Institutional Factors Shaping Mothers' Employment and Working Hours in Postindustrial Countries. In: Social Forces 93 (4), S. 1301-1333. DOI: 10.1093/sf/sou119.

Boll, Christina (2016): Die Arbeitsteilung im Paar. Theorien, Wirkungszusammenhänge, Einflussfaktoren und exemplarische, empirische Evidenz. Hamburgisches Weltwirtschaftsinstitut. Hamburg (Expertise für den Zweiten Gleichstellungsbericht der Bundesregierung).

Boll, Christina (2018): Die Familie aus der Perspektive der Wirtschaftswissenschaften. In: Astrid Wonneberger, Katja Weidtmann und Sabina Stelzig-Willutzki (Hg.): Familienwissenschaft. Grundlagen und Überblick. Wiesbaden: Springer VS (Familienforschung), S. 315-349.

Bolzendahl, Catherine I.; Myers, Daniel J. (2004): Feminist Attitudes and Support for Gender Equality. Opinion Change in Women and Men, 1974-1998. In: Social Forces 83 (2), S. 759-789. DOI: 10.1353/sof.2005.0005.

Bonke, Jens; Esping-Andersen, Gøsta (2011): Family Investments in Children. Productivities, Preferences, and Parental Child Care. In: European Sociological Review 27 (1), S. 4355. DOI: $10.1093 /$ esr/jcp054.

Brady, David (Hg.) (2011): Comparing european workers. Part A: Experiences and inequalities. Bingley United Kingdom: Emerald (Research in the Sociology of Work, v. 22).

Brewster, Karin L.; Padavic, Irene (2000): Change in Gender - Ideology, 1977 - 1996. The Contributions of Intracohort Change and Population Turnover. In: Journal of Marriage and Family 62 (2), S. 477-487.

Brines, Julie (1994): Economic Dependency, Gender, and the Division of Labor at Home. In: American Journal of Sociology 100 (3), S. 652-688.

Brines, Julie; Joyner, Kara (1999): The Ties That Bind. Principles of Cohesion in Cohabitation and Marriage. In: American Sociological Review 64 (3), S. 333-355. DOI: 10.2307/2657490.

Brooks, Clem; Bolzendahl, Catherine (2004): The Transformation of US Gender Role Attitudes. Cohort Replacement, Social-structural Change, and Ideological Learning. In: Social Science Research 33 (1), S. 106-133. DOI: 10.1016/S0049-089X(03)00041-3.

Brüderl, Josef (2004): Die Pluralisierung partnerschaftlicher Lebensformen in Westdeutschland und Europa. In: Aus Politik und Zeitgeschichte, S. 3-10.

Brüderl, Josef (2018): Querschnittsdatenanalyse: Angewandte Regressionsanalyse mit STATA. Wintersemester 2018/19. Ludwig-Maximilians-Universität München. Online verfügbar unter https://www.Is3.soziologie.uni-muenchen.de/studium-lehre/archiv/teachingmarterials/regressionsanalyse_2018.zip, zuletzt geprüft am 11.11.2020.

Buchholz, Sandra; Blossfeld, Hans-Peter (2009): Beschäftigungsflexibilisierung in Deutschland. Wen betrifft sie und wie hat sie sich auf die Veränderung sozialer Inklusion/Exklusion in Deutschland ausgewirkt? In: Rudolf Stichweh und Paul Windolf (Hg.): Inklusion und Exklusion. Analysen zur Sozialstruktur und sozialen Ungleichheit. Wiesbaden: VS Verlag für Sozialwissenschaften, S. 123-138. 
Buchler, Sandra; Perales, Francisco; Baxter, Janeen (2017): Does Parenthood Change Attitudes to Fathering? Evidence from Australia and Britain. In: Sex Roles 77 (9-10), S. 663675. DOI: 10.1007/s11199-017-0757-8.

Budig, Michelle J.; England, Paula (2001): The Wage Penalty for Motherhood. In: American Sociological Review 66 (2), S. 204-225. DOI: 10.2307/2657415.

BühImann, Felix; Elcheroth, Guy; Tettamanti, Manuel (2010): The Division of Labour Among European Couples. The Effects of Life Course and Welfare Policy on Value-Practice Configurations. In: European Sociological Review 26 (1), S. 49-66. DOI: 10.1093/esr/jcp004.

Bujard, Martin (2013): Die fünf Ziele des Elterngelds im Spannungsfeld von Politik, Medien und Wissenschaft. In: Zeitschrift für Familienforschung 25 (2), S. 132-153.

Bujard, Martin; Laß, Inga; Diabaté, Sabine; Sulak, Harun; Schneider, Norbert F. (2020): Eltern während der Corona-Krise. Zur Improvisation gezwungen. Bundesinstitut für Bevölkerungsforschung. Wiesbaden (BIB.BEVÖLKERUNGS.STUDIEN). Online verfügbar unter https://www.bib.bund.de/Publikation/2020/pdf/Eltern-waehrend-der-CoronaKrise.pdf?_blob=publicationFile\&v=8, zuletzt geprüft am 10.01.2021.

Bulanda, Ronald E. (2004): Paternal Involvement with Children: The Influence of Gender Ideologies. In: Journal of Marriage and Family 66 (1), S. 40-45.

Bünning, Mareike (2015): What Happens after the 'Daddy Months'? Fathers' Involvement in Paid Work, Childcare, and Housework after Taking Parental Leave in Germany. In: European Sociological Review 31 (6), S. 738-748.

Bünning, Mareike (2020): Paternal Part - Time Employment and Fathers' Long - Term Involvement in Child Care and Housework. In: Journal of Marriage and Family 82 (2), S. 566-586. DOI: 10.1111/jomf.12608.

Buschner, Andrea; Adam, Ursula; Schulz, Florian (2018): Relative Education, Parenthood, and Couples' Division of Paid Work. Evidence from German Census Data. In: Zeitschrift für Familienforschung 30 (1), S. 96-119.

Campbell, Andrea Louise (2012): Policy Makes Mass Politics. In: Annual Review of Political Science 15 (1), S. 333-351. DOI: 10.1146/annurev-polisci-012610-135202.

Carlson, Daniel L.; Lynch, Jamie L. (2017): Purchases, Penalties, and Power. The Relationship Between Earnings and Housework. In: Journal of Marriage and Family 79 (1), S. 199-224. DOI: 10.1111/jomf.12337.

Cesinger, Beate; König, Stefanie; Langhauser, Marc; Leicht, René (2012): Zwei Karrieren Eine Familie? Ergebnisse aus dem Projekt „Durch Selbständigkeit zur Doppelkarriere? Berufs-, Partnerschafts- und Lebenskonzepte erfolgreicher Gründerinnen“. Online verfügbar unter www.dcc-selbstaendig.de/vortrag/Broschuere_Il.pdf, zuletzt geprüft am 23.11.2020.

Charles, Maria; Grusky, David B. (2004): Occupational Ghettos. The Worldwide Segregation of Women and Men. Stanford, Calif.: Stanford University Press.

Clausen, John A. (1972): The Life Course of Individuals. In: Matilda W. Riley, Marilyn Johnson und Anne Foner (Hg.): Aging and Society, Volume 3. A Sociology of Age Stratification. New York: Russell Sage Foundation, S. 457-514.

Cohen, Jack (1988): Statistical Power Analysis for the Behavioral Sciences. 2nd ed. Hillsdale, N.J.: Lawrence Erlbaum Associates.

Coltrane, Scott (2000): Research on Household Labor. Modeling and Measuring the Social Embeddedness of Routine Family Work. In: Journal of Marriage and Family 62 (4), S. 1208-1233.

Cooke, L. P. (2006): Policy, Preferences, and Patriarchy. The Division of Domestic Labor in East Germany, West Germany, and the United States. In: Social Politics 13 (1), S. 117143. DOI: $10.1093 / \mathrm{sp} / \mathrm{jxj} 005$.

Corrigall, Elizabeth; Konrad, Alison (2007): Gender Role Attitudes and Careers. A Longitudinal Study. In: Sex Roles 56 (11), S. 847-855. 
Cotter, David; Hermsen, Joan M.; Vanneman, Reeve (2011): The End of the Gender Revolution? Gender Role Attitudes from 1977 to 2008. In: American Journal of Sociology 117 (1), S. 259-289. DOI: 10.1086/658853.

Coverdill, James; Kraft, Joan; Manley, Kelly (1996): Employment History, the Sex Typing of Occupations, Pay and Change in Gender Role Attitudes. A Longitudinal Study of Young Married Women. In: Sociological Focus 29 (1), S. 47-60.

Coverman, Shelley (1985): Explaining husbands' participation in domestic labor. In: The sociological quarterly 26 (1), S. 81-97.

Craig, Lyn (2006a): Does Father Care Mean Fathers Share? A Comparison of How Mothers and Fathers in Intact Families Spend Time with Children. In: Gender \& Society 20 (2), S. 259-281.

Craig, Lyn (2006b): Parental Education, Time in Paid Work and Time with Children. An Australian Time - diary Analysis. In: British Journal of Sociology 57 (4), S. 553-575.

Craig, Lyn; Bittman, Michael (2005): The Effect of Children on Adults' Time-use. An Analysis of the Incremental Time Costs of Children in Australia. Sydney: Social Policy Research Centre, UNSW.

Craig, Lyn; Mullan, Killian (2010): Parenthood, Gender and Work-Family Time in the United States, Australia, Italy, France, and Denmark. In: Journal of Marriage and Family 72 (5), S. 1344-1361.

Craig, Lyn; Powell, Abigail; Smyth, Ciara (2014): Towards Intensive Parenting? Changes in the Composition and Determinants of Mothers' and Fathers' Time with Children 19922006. In: British Journal of Sociology 65 (3), S. 555-579.

Craig, Lyn; Sawrikar, Pooja (2009): Work and Family: How Does the (Gender) Balance Change as Children Grow? In: Gender, Work \& Organization 16 (6), S. 684-709.

Cunningham, Mick (2005): Gender in Cohabitation and Marriage. The Influence of Gender Ideology on Housework Allocation Over the Life Course. In: Journal of Family Issues 26 (8), S. 1037-1061. DOI: 10.1177/0192513X04273592.

Cunningham, Mick (2007): Influences of Women's Employment on the Gendered Division of Household Labor Over the Life Course. Evidence From a 31-Year Panel Study. In: Journal of Family Issues 28 (3), S. 422-444. DOI: 10.1177/0192513X06295198.

Cunningham, Mick (2008): Influences of Gender Ideology and Housework Allocation on Women's Employment over the Life Course. In: Social Science Research 37 (1), S. 254267. DOI: 10.1016/j.ssresearch.2007.01.003.

Cunningham, Mick; Beutel, Ann M.; Barber, Jennifer S.; Thornton, Arland (2005): Reciprocal Relationships between Attitudes about Gender and Social Contexts during Young Adulthood. In: Social Science Research 34 (4), S. 862-892. DOI: 10.1016/j.ssresearch.2005.03.001.

Davis, Shannon N.; Greenstein, Theodore N. (2004): Cross-National Variations in the Division of Household Labor. In: Journal of Marriage and Family 66 (5), S. 1260-1271.

Davis, Shannon N.; Greenstein, Theodore N. (2009): Gender Ideology. Components, Predictors, and Consequences. In: Annual Review of Sociology 35 (1), S. 87-105. DOI: 10.1146/annurev-soc-070308-115920.

Davis, Shannon N.; Greenstein, Theodore N.; Gerteisen Marks, Jennifer P. (2007): Effects of Union Type on Division of Household Labor. Do Cohabiting Men Really Perform More Housework? In: Journal of Family Issues 28 (9), S. 1246-1272. DOI: 10.1177/0192513X07300968.

Dechant, Anna; Rinklake, Annika (2016): Anticipating Motherhood and Fatherhood in Germany. German Couples' Plans for Childcare and Paid Work. In: Daniela Grunow und Marie Evertsson (Hg.): Couples' Transitions to Parenthood: Edward Elgar Publishing, 103-124. 
Dechant, Anna; Rost, Harald; Schulz, Florian (2014): Die Veränderung der Hausarbeitsteilung in Paarbeziehungen. Ein Überblick über die Längsschnittforschung und neue empirische Befunde auf Basis der paifam-Daten. In: Zeitschrift für Familienforschung 26 (2), S. 144-168.

Dechant, Anna; Schulz, Florian (2014): Bedingungsszenarien einer partnerschaftlichen Arbeitsteilung beim Übergang zur Elternschaft in Deutschland. In: Comparative Population Studies 39 (3), S. 587-614.

Del Boca, Daniela; Pasqua, Silvia; Pronzato, Chiara (2009): Motherhood and Market Work Decisions in Institutional Context: A European Perspective. In: Oxford Economic Papers, i147-i171.

Demografie Portal (2019): Zahlen und Fakten. Je jünger die Kinder, desto seltener arbeiten Mütter. Hg. v. Bundesinstitut für Bevölkerungsforschung. Online verfügbar unter https://www.demografie-portal.de/SharedDocs/Informieren/DE/ZahlenFakten/Erwerbstaetigenquote_Kindesalter.html.

Dermott, Esther (2008): Intimate Fatherhood. A Sociological Analysis. 1. Aufl. New York [u.a.]: Routledge.

Destatis (2018): Eheschließungen, Scheidungen, Lebenspartnerschaften. Hg. v. Statistisches Bundesamt. Online verfügbar unter https://www.destatis.de/DE/ZahlenFakten/GesellschaftStaat/Bevoelkerung/Ehescheidungen/Tabellen_/lrbev06.html, zuletzt geprüft am 25.02.2018.

Destatis (2019): Kinderlosigkeit, Geburten und Familien. Ergebnisse des Mikrozensus 2018. Ausgabe 2019.

Deutscher Bundestag (2000): Gesetzentwurf zur Änderung des Begriffs „Erziehungsurlaub“. Drucksache14/4133. Berlin.

Deutscher Bundestag (2006): Gesetzentwurf der Bundesregierung zur Einführung des Elterngeldes. Drucksache 16/2454. Berlin.

Deutscher Bundestag (2014): Gesetzentwurf zur Einführung des Elterngeld Plus mit Partnerschaftsbonus und einer flexibleren Elternzeit im Bundeselterngeld- und Elternzeitgesetz. Drucksache 18/2583. Berlin.

Deutscher Bundestag (2018): Gesetzentwurf zur Weiterentwicklung des Teilzeitrechts - Einführung einer Brückenteilzeit. Berlin.

Diabaté, Sabine (2014): Mütter heute: Leitbilder, Lebensrealitäten und Wünsche. Hg. v. Bundeszentrale für politische Bildung. Online verfügbar unter https://www.bpb.de/politik/innenpolitik/familienpolitik/191689/muetter-heute?p=all, zuletzt geprüft am 23.11.2020.

Diabaté, Sabine (2015): Mutterleitbilder: Spagat zwischen Autonomie und Aufopferung. In: Norbert F. Schneider, Sabine Diabaté und Kerstin Ruckdeschel (Hg.): Familienleitbilder in Deutschland. Kulturelle Vorstellungen zu Partnerschaft, Elternschaft und Familienleben. Opladen, Berlin, Toronto: Barbara Budrich, S. 207-226.

Diabaté, Sabine; Beringer, Samira (2018): Simply the Best!? - Kulturelle Einflussfaktoren zum „intensive mothering“ bei Müttern von Kleinkindern in Deutschland. In: Zeitschrift für Familienforschung 30 (3), S. 293-315.

Diabaté, Sabine; Lück, Detlev (2014): Familienleitbilder. Identifikation und Wirkungsweise auf generatives Verhalten. In: Zeitschrift für Familienforschung 26 (1), S. 49-69.

Diener, Katharina; Berngruber, Anne (2018): Die Bedeutung öffentlicher Kinderbetreuung für die Erwerbsentscheidung und den Erwerbsumfang von Müttern beim beruflichen Wiedereinstieg. In: Zeitschrift für Familienforschung 30 (2), S. 123-150.

Domínguez-Folgueras, Marta (2012): Is Cohabitation More Egalitarian? The Division of Household Labor in Five European Countries. In: Journal of Family Issues 34 (12), S. 1623-1646. DOI: 10.1177/0192513X12464948. 
Dribe, Martin; Stanfors, Maria (2009): Does Parenthood Strengthen a Traditional Household Division of Labor? Evidence From Sweden. In: Journal of Marriage and Family 71 (1), S. 33-45.

Duvall, Evelyn Millis (1971): Family Development. 4. Aufl. New York: Lippincott.

Duvall, Evelyn Millis; Hill, Reuben (1945): When You Marry. Boston: D. C. Heath and Company.

Eckes, Thomas (2010): Geschlechterstereotype. Von Rollen, Identitäten und Vorurteilen. In: Ruth Becker und Beate Kortendiek ( $\mathrm{Hg}$.): Handbuch Frauen- und Geschlechterforschung. Theorie, Methoden, Empirie. Wiesbaden: Springer VS (Geschlecht und Gesellschaft), S. 178-189.

Elder, Glen H. (1974): Children of the Great Depression. Chicago: University of Chicago Press.

Elder, Glen H. (1994): Time, Human Agency, and Social Change: Perspectives on the Life Course. In: Social Psychology Quarterly 57 (1), S. 4-15.

Elder, Glen H. (1998): The Life Course as Developmental Theory. In: Child Development 69 (1), S. 1-12.

England, Paula (2010): The Gender Revolution. Uneven and Stalled. In: Gender \& Society 24 (2), S. 149-166. DOI: 10.1177/0891243210361475.

England, Paula; Farkas, George (1986): Households, Employment and Gender. A Social, Economic, and Demographic View. New York: Aldine De Gruyter.

England, Paula; Gornick, Janet; Shafer, Emily Fitzgibbons (2012): Women's Employment, Education, and the Gender Gap in 17 Countries. In: Monthly Labor Review 135 (4), S. 312.

England, Paula; Srivastava, Anjula (2013): Educational Differences in US Parents' Time Spent in Child Care. The Role of Culture and Cross-spouse Influence. In: Social Science Research 42 (4), S. 971-988.

Erickson, Jenet Jacob; Martinengo, Giuseppe; Hill, E. Jeffrey (2010): Putting work and family experiences in context. Differences by family life stage. In: Human Relations 63 (7), S. 955-979. DOI: 10.1177/0018726709353138.

Esping-Andersen, Gøsta (1990): The Three Worlds of Welfare Capitalism. Princeton, New Jersey: Princeton University Press.

Esping-Andersen, Gøsta (1998): Die drei Welten des Wohlfahrtskapitalismus. Zur politischen Ökonomie des Wohlfahrtsstaates. In: Stephan Lessenich und Ilona Ostner (Hg.): Welten des Wohlfahrtskapitalismus. Der Sozialstaat in vergleichender Perspektive. Frankfurt/Main: Campus Verlag (Theorie und Gesellschaft, 40).

Esping-Andersen, Gøsta (1999): Social Foundations of Postindustrial Economies. Oxford, New York: Oxford University Press.

Esping-Andersen, Gøsta; Schmitt, Christian (2020): Multi-dimensional Couple Bargaining and Housework Allocation. In: Acta Sociologica 63 (1), S. 3-22. DOI: 10.1177/0001699319859418.

Evertsson, Marie (2013): The Importance of Work: Changing Work Commitment Following the Transition to Motherhood. In: Acta Sociologica 56 (2), S. 139-153.

Evertsson, Marie (2014): Gender Ideology and the Sharing of Housework and Child Care in Sweden. In: Journal of Family Issues 35 (7), S. 927-949. DOI: 10.1177/0192513X14522239.

Evertsson, Marie; Boye, Katarina; Erman, Jeylan (2018): Fathers on call? A Study on the Sharing of Care Work Between Parents in Sweden. In: Demographic Research 39 (2), S. 33-60. DOI: 10.4054/DemRes.2018.39.2.

Evertsson, Marie; England, Paula; Hermsen, Joan; Cotter, David (2007): How Does Gender Inequality in Employment and Earnings Vary by Educational Stratum in Sweden and the United States? In: International Journal of Sociology 37 (2), S. 9-28. 
Evertsson, Marie; Nermo, Magnus (2007): Changing Resources and the Division of Housework. A Longitudinal Study of Swedish Couples. In: European Sociological Review 23 (4), S. 455-470. DOI: 10.1093/esr/jcm018.

Fahlén, Susanne (2016): Equality at Home - A Question of Career? Housework, Norms, and Policies in a European Comparative Perspective. In: Demographic Research 35, S. 1411-1440. DOI: 10.4054/DemRes.2016.35.48.

Faircloth, Charlotte (2014): Intensive Parenting and the Expansion of Parenting. In: Ellie Lee, Jennie Bristow, Charlotte Faircloth und Jan Macvarish (Hg.): Parenting Culture Studies. London: Palgrave Macmillan, S. 25-50.

Fan, Pi-Ling; Marini, Margaret Mooney (2000): Influences on Gender-Role Attitudes during the Transition to Adulthood. In: Social Science Research 29 (2), S. 258-283. DOI: 10.1006/ssre.1999.0669.

Festinger, Leon (1957): A theory of cognitive dissonance. Stanford: Stanford University Press.

Fortin, Nicole M (2005): Gender Role Attitudes and the Labour-market Outcomes of Women across OECD Countries. In: Oxford Review of Economic Policy 21 (3), S. 416-438.

Fuwa, Makiko (2004): Macro-level Gender Inequality and the Division of Household Labor in 22 Countries. In: American Sociological Review 69 (6), S. 751-767.

Fuwa, Makiko; Cohen, Philip N. (2007): Housework and Social Policy. In: Social Science Research 36 (2), S. 512-530. DOI: 10.1016/j.ssresearch.2006.04.005.

Gangl, Markus; Ziefle, Andrea (2009): Motherhood, Labor Force Behavior, and Women's Careers. An Empirical Assessment of the Wage Penalty for Motherhood in Britain, Germany, and the United States. In: Demography 46 (2), S. 341-369.

Gangl, Markus; Ziefle, Andrea (2015): The Making of a Good Woman: Extended Parental Leave Entitlements and Mothers' Work Commitment in Germany 1. In: American Journal of Sociology 121 (2), S. 511-563.

Ganzeboom, Harry B.G. (2010): International Standard Classification of Occupations. ISCO08 with ISEI-08 Scores. Online verfügbar unter http://www.harryganzeboom.nl/isco08/isco08_with_isei.pdf, zuletzt geprüft am 23.11.2020.

Ganzeboom, Harry B.G.; DeGraaf, Paul M.; Treiman, Donald J. (1992): A Standard International Socioeconomic Index of Occupational Status. In: Social Science Research (21), S. $1-56$.

Garey, Anita IIta (1995): Constructing Motherhood on the Night Shift. "Working Mothers" as "Stay-at-Home Moms". In: Qualitative Sociology 18 (4), S. 415-437. DOI: 10.1007/BF02404489.

Garfinkel, Harold (1967): Studies in Ethnomethodology. Cambridge: University Press.

Geist, Claudia (2005): The Welfare State and the Home. Regime Differences in the Domestic Division of Labour. In: European Sociological Review 21 (1), S. 23-41. DOI: 10.1093/esr/jci002.

Geist, Claudia; Ruppanner, Leah (2018): Mission Impossible? New Housework Theories for Changing Families. In: Journal of Family Theory \& Review 10 (1), S. 242-262. DOI: $10.1111 /$ jtr.12245.

George, Darren; Mallery, Paul (2002): SPSS for Windows Step by step. A Simple Guide and Reference; 11.0 Update. 4. Aufl. Boston, Mass., London: Allyn and Bacon.

Gerhards, Jürgen; Rössel, Jörg (2000): Familienkultur in den USA und in West- und Ostdeutschland. In: Jürgen Gerhards (Hg.): Die Vermessung kultureller Unterschiede. USA und Deutschland im Vergleich. Wiesbaden: VS Verlag für Sozialwissenschaften.

Gershuny, Jonathan (2000): Changing Times. Work and Leisure in Postindustrial Society. Oxford: Oxford University Press.

Gershuny, Jonathan (2013): National Utility: Measuring the Enjoyment of Activities. In: European Sociological Review 29 (5), S. 996-1009. DOI: 10.1093/esr/jcs077. 
Gershuny, Jonathan; Bittman, Michael; Brice, John (2005): Exit, Voice, and Suffering. Do Couples Adapt to Changing Employment Patterns? In: Journal of Marriage and Family 67 (3), S. 656-665.

Gildemeister, Regine (2008): Soziale Konstruktion von Geschlecht. „Doing gender“. In: Sylvia Marlene Wilz (Hg.): Geschlechterdifferenzen - Geschlechterdifferenzierungen. Ein Überblick über gesellschaftliche Entwicklungen und theoretische Positionen. Wiesbaden: VS Verlag fü Sozialwissenschaften | GWV Fachverlage GmbH Wiesbaden (Hagener Studientexte zur Soziologie), S. 167-198.

Gjerdingen, Dwenda K.; Center, Bruce A. (2005): First-Time Parents' postpartum Changes in Employment, Childcare, and Housework responsibilities. In: Social Science Research 34 (1), S. 103-116. DOI: 10.1016/j.ssresearch.2003.11.005.

Glassdoor Economic Research (2019): Progress on the Gender Pay Gap: 2019. Unter Mitarbeit von Andrew Chamberlain, Daniel Zhao und Amanda Stansell. Hg. v. Glassdoor. Glassdoor, Inc.

Glick, Paul C. (1947): The Family Cycle. In: American Sociological Review 12 (2), S. 164174.

Glick, Paul C. (1977): Updating the Life Cycle of the Family. In: Journal of Marriage and Family 39 (1), S. 5-13.

Goldscheider, Fran; Goldscheider, Calvin; Bernhardt, Eva M. (2011): Creating Egalitarian Families among the Adult Children of Turkish- and Polish-Origin Immigrants in Sweden. In: International Migration Review 45 (1), S. 68-88.

Goldscheider, Frances; Bernhardt, Eva; Lappegård, Trude (2015): The Gender Revolution. A Framework for Understanding Changing Family and Demographic Behavior. In: Population and Development Review 41 (2), S. 207-239.

Gottschall, Karin (1998): Doing Gender While Doing Work? Erkenntnispotentiale konstruktivistischer Perspektiven für eine Analyse des Zusammenhangs von Arbeitsmarkt, Beruf und Geschlecht. In: Birgit Geissler, Friederike Maier und Birgit Pfau-Effinger (Hg.): FrauenArbeitsMarkt. Der Beitrag der Frauenforschung zur sozio-ökonomischen Theorieentwicklung. Berlin: Edition Sigma, S. 63-94.

Gracia, Pablo (2014): Fathers' Child Care Involvement and Children's Age in Spain. A Time Use Study on Differences by Education and Mothers' Employment. In: European Sociological Review 30 (2), S. 137-150.

Gracia, Pablo; Esping-Andersen, Gøsta (2015): Fathers' Child Care Time and Mothers' Paid Work. A Cross-national Study of Denmark, Spain, and the United Kingdom. In: Family Science 6 (1), S. 270-281. DOI: 10.1080/19424620.2015.1082336.

Greenstein, Theodore N. (1996): Husbands' Participation in Domestic Labor. Interactive Effects of Wives' and Husbands' Gender Ideologies. In: Journal of Marriage and the Family 58 (3), S. 585-595. DOI: 10.2307/353719.

Greenstein, Theodore N. (2000): Economic Dependence, Gender, and the Division of Labor in the Home. A Replication and Extension. In: Journal of Marriage and the Family 62 (2), S. 322-335. DOI: 10.1111/j.1741-3737.2000.00322.x.

Grunow, Daniela (2007): Wandel der Geschlechterrollen und Vaterhandeln im Alltag. In: Tanja Mühling und Harald Rost (Hg.): Väter im Blickpunkt. Perspektiven der Familienforschung. Opladen: Budrich, S. 49-76.

Grunow, Daniela (2009): Geschlechtsrollen in der Familie. Perspektiven der Frauenforschung. In: Olaf Kapella (Hg.): Die Vielfalt der Familie. Tagungsband zum 3. Europäischen Fachkongress Familienforschung. Opladen: Budrich, S. 157-174.

Grunow, Daniela (2014): Die Aufteilung von Erwerbs-, Haus- und Familienarbeit in Partnerschaften im Beziehungsverlauf. Der Einfluss von Sozialpolitik in Europa. In: Detlev Lück 
und Waltraud Cornelißen (Hg.): Geschlechterunterschiede und Geschlechterunterscheidungen in Europa. Stuttgart: Lucius \& Lucius (Der Mensch als soziales und personales Wesen, v.24), S. 231-256.

Grunow, Daniela (2019): Comparative Analyses of Housework and Its Relation to Paid Work. Institutional Contexts and Individual Agency. In: Kölner Zeitschrift für Soziologie und Sozialpsychologie 71 (1), S. 247-284. DOI: 10.1007/s11577-019-00601-1.

Grunow, Daniela; Aisenbrey, Silke; Evertsson, Marie (2011): Familienpolitik, Bildung und Berufskarrieren von Müttern in Deutschland, USA und Schweden. In: Kölner Zeitschrift für Soziologie und Sozialpsychologie 63 (3), S. 395-430. DOI: 10.1007/s11577-011-0139-0.

Grunow, Daniela; Baur, Nina (2014): Die Korrespondenz von normativen Vorstellungen und Handeln. Das Beispiel männlicher Hausarbeit. In: Comparative Population Studies 39 (3), S. 479-520.

Grunow, Daniela; Begall, Katia; Buchler, Sandra (2018): Gender Ideologies in Europe. A Multidimensional Framework. In: Journal of Marriage and Family 80 (1), S. 42-60. DOI: 10.1111/jomf.12453.

Grunow, Daniela; Müller, Dana (2012): Kulturelle und strukturelle Faktoren bei der Rückkehr in den Beruf. Ostdeutsche, westdeutsche und ost-west-mobile Mütter im Vergleich. In: Johannes Huinink, Michaela Kreyenfeld und Heike Trappe (Hg.): Familie und Partnerschaft in Ost- und Westdeutschland. Ähnlich und doch immer noch anders. Zeitschrift für Familienforschung (9), S. 55-77.

Grunow, Daniela; Schulz, Florian; Blossfeld, Hans-Peter (2007): Was erklärt die Traditionalisierungsprozesse häuslicher Arbeitsteilung im Eheverlauf. Soziale Normen oder ökonomische Ressourcen? In: Zeitschrift für Soziologie 36 (3), S. 162-181.

Grunow, Daniela; Schulz, Florian; Blossfeld, Hans-Peter (2012): What determines Change in the Division of Housework over the Course of Marriage? In: International Sociology 27 (3), S. 289-307. DOI: 10.1177/0268580911423056.

Gupta, Sanjiv (2006): Her Money, her Time? Women's Earnings and their Housework Hours. In: Social Science Research 35, S. 975-999.

Gupta, Sanjiv (2007): Autonomy, Dependency or Display? The Relationship Between Married Women's Earnings and Housework. In: Journal of Marriage and Family 69, S. 399-417.

Gupta, Sanjiv; Ash, Michael (2008): Whose money, whose time? A nonparametric approach to modeling time spent on housework in the United States. In: Feminist Economics 14 (1), S. 93-120. DOI: 10.1080/13545700701716664.

Gupta, Sanjiv; Evertsson, Marie; Grunow, Daniela; Nermo, Magnus; Sayer, Liana S. (2015): The Economic Gap Among Women in Time Spent on Housework in Former West Germany and Sweden. In: Journal of Comparative Family Studies 46 (2), S. 181-201. DOI: 10.3138/jcfs.46.2.181.

Gutiérrez-Domènech, Maria (2005): Employment after Motherhood: A European Comparison. In: Labour Economics 12 (1), S. 99-123.

Hakim, Catherine (2002): Lifestyle Preferences as Determinants of Women's Differentiated Labor Market Careers. In: Work and Occupations 29 (4), S. 428-459. DOI: 10.1177/0730888402029004003.

Hausen, Karin (1976): Die Polarisierung der ,Geschlechtscharaktere'. Eine Spiegelung der Dissoziation von Erwerbs- und Familienleben. In: Werner Conze (Hg.): Sozialgeschichte der Familie in der Neuzeit Europas. Neue Forschungen. Stuttgart: Ernst Klett, S. 363393.

Hays, Sharon (1996): The Cultural Contradictions of Motherhood. New Haven, CT: Yale University Press.

Hill, Paul B.; Kopp, Johannes (2013): Familiensoziologie. Grundlagen und theoretische Perspektiven. 5. Aufl. Wiesbaden: Springer VS. 
Hipp, Lena (2018): Rabenmütter, tolle Väter. Frauen schaden kurze und lange Elternzeiten bei ihrer Karriere - Männern nicht. In: WZB Mitteilungen (161), S. 10-12.

Hipp, Lena; Leuze, Kathrin (2015): Institutionelle Determinanten einer partnerschaftlichen Aufteilung von Erwerbsarbeit in Europa und den USA. In: Kölner Zeitschrift für Soziologie und Sozialpsychologie 67 (4), S. 659-684. DOI: 10.1007/s11577-015-0343-4.

Hirschauer, Stefan (1994): Die soziale Fortpflanzung der Zweigeschlechtlichkeit. In: Kölner Zeitschrift für Soziologie und Sozialpsychologie (4), S. 668-692.

Hochschild, Arlie Russell; Machung, Anne (1989): The Second Shift. Working Parents and the Revolution at Home. New York: Viking.

Hook, Jennifer L. (2006): Care in Context. Men's Unpaid Work in 20 Countries, 1965-2003. In: American Sociological Review 71 (4), S. 639-660. DOI: 10.1177/000312240607100406.

Hook, Jennifer L. (2010): Gender Inequality in the Welfare State. Sex Segregation in Housework, 1965-20031. In: American Journal of Sociology 115, S. 1480-1523.

Hook, Jennifer L. (2017): Women's Housework: New Tests of Time and Money. In: Journal of Marriage and Family 79 (1), S. 179-198.

Hook, Jennifer L.; Wolfe, Christina M. (2012): New Fathers? Residential Fathers' Time With Children in Four Countries. In: Journal of Family Issues 33 (4), S. 415-450. DOI: 10.1177/0192513X11425779.

Huinink, Johannes; Konietzka, Dirk (2007): Familiensoziologie. Eine Einführung. Frankfurt/Main: Campus Verlag.

Hummelsheim, Dina (2009): Die Erwerbsbeteiligung von Müttern. Institutionelle Steuerung oder kulturelle Prägung?: eine empirische Untersuchung am Beispiel von Belgien, Westund Ostdeutschland. 1. Aufl. Wiesbaden: VS Verlag für Sozialwissenschaften.

Infas Institut für angewandte Sozialwissenschaft (2015): Methodenbericht. Aufwachsen in Deutschland: Alltagswelten II. Unter Mitarbeit von Folkert Aust, Julian von der Burg, Reiner Gilberg, Adam Giza und Birgit Jesske. Hg. v. Infas Institut für angewandte Sozialwissenschaft. Bonn.

Inglehart, Ronald (1977): The Silent Revolution. Changing Values and Political Styles Among Western Publics. Princeton, New Jersey: Princeton University Press.

Inglehart, Ronald (2018): Cultural Evolution. People's Motivations are Changing, and Reshaping the World. Cambridge: Cambridge University Press.

Johnston, Deirdre; Swanson, Debra (2006): Constructing the "Good Mother". The Experience of Mothering Ideologies by Work Status. In: Sex Roles 54 (7), S. 509-519.

Kan, Man Yee (2008): Does Gender Trump Money? Housework Hours of Husbands and Wives in Britain. In: Work, Employment and Society 22 (1), S. 45-66. DOI: 10.1177/0950017007087416.

Kapinus, Carolyn A.; Johnson, Michael P. (2003): The Utility of Family Life Cycle as a Theoretical and Empirical Tool. Commitment and Family Life-Cycle Stage. In: Journal of Family Issues 24 (2), S. 155-184. DOI: 10.1177/0192513X02250135.

Kaufmann, Franz-Xaver (1990): Zukunft der Familie. Stabilität, Stabilitätsrisiken und Wandel der familialen Lebensformen sowie ihre gesellschaftlichen und politischen Bedingungen. München: C.H. Beck.

Kessler, Suzanne J.; McKenna, Wendy (1978): Gender. An Ethnomethodological Approach. Chicago, London: University of Chicago Press.

Khoudja, Yassine; Fleischmann, Fenella (2018): Gender Ideology and Women's Labor Market Transitions Within Couples in the Netherlands. In: Journal of Marriage and Family 80 (5), S. 1087-1106. DOI: 10.1111/jomf.12510.

Knight, Carly R.; Brinton, Mary C. (2017): One Egalitarianism or Several? Two Decades of Gender-Role Attitude Change in Europe. In: American Journal of Sociology 122 (5), S. 1485-1532. DOI: 10.1086/689814. 
Kohli, Martin (1985): Die Institutionalisierung des Lebenslaufs. In: Kölner Zeitschrift für Soziologie und Sozialpsychologie 37 (1), S. 1-29.

Kohli, Martin (2017): Die Institutionalisierung des Lebenslaufs. In: Kölner Zeitschrift für Soziologie und Sozialpsychologie 69 (Supplement 1), S. 495-524.

Kohlrausch, Bettina; Zucco, Aline (2020): Die Corona-Krise trifft Frauen doppelt. Weniger Erwerbseinkommen und mehr Sorgearbeit. Wirtschafts- und Sozialwissenschaftliches Institut in der Hans-Böckler-Stiftung. Düsseldorf (Policy Brief, 40). Online verfügbar unter https://bit.ly/3q0WJkg, zuletzt geprüft am 10.01.2021.

Komarovsky, Mirra (1946): Cultural Contradictions and Sex Roles. In: American Journal of Sociology 52 (3), S. 184-189. DOI: 10.1086/219982.

Konietzka, Dirk; Kreyenfeld, Michaela (2010): The Growing Educational Divide in Mothers' Employment. An Investigation Based on the German Micro-censuses 1976-2004. In: Work, Employment \& Society 24 (2), S. 260-278.

Korpi, W.; Ferrarini, T.; Englund, S. (2013): Women's Opportunities under Different Family Policy Constellations. Gender, Class, and Inequality Tradeoffs in Western Countries Reexamined. In: Social Politics 20 (1), S. 1-40. DOI: 10.1093/sp/jxs028.

Kreyenfeld, Michaela; Geisler, Esther (2006): Müttererwerbstätigkeit in Ost- und Westdeutschland. In: Zeitschrift für Familienforschung 18 (3), S. 330-360. Online verfügbar unter http://nbn-resolving.de/urn:nbn:de:0168-ssoar-58022, zuletzt geprüft am 23.11.2020.

Kreyenfeld, Michaela; Konietzka, Dirk; Böhm, Sebastian (2007): Die Bildungsungleichheit des Erwerbsverhaltens mit Kindern. Westdeutschland im Vergleich zwischen 1976 und 2004. In: Zeitschrift für Soziologie 36 (6), S. 434-452.

Kroska, Amy (2004): Divisions of Domestic Work. Revising and Expanding the Theoretical Explanations. In: Journal of Family Issues 25 (7), S. 900-932. DOI: $10.1177 / 0192513 \times 04267149$.

Krüger, Helga (2010): Lebenslauf. Dynamiken zwischen Biografie und Geschlechterverhältnis. In: Ruth Becker und Beate Kortendiek (Hg.): Handbuch Frauen- und Geschlechterforschung. Theorie, Methoden, Empirie. Wiesbaden: Springer VS (Geschlecht und Gesellschaft), S. 219-227.

Krüger, Helga; Levy, René (2000): Masterstatus, Familie und Geschlecht. Vergessene Verknüpfungslogiken zwischen Institutionen des Lebenslaufs. In: Berliner Journal für Soziologie 10 (3), S. 379-401.

Kühhirt, Michael (2012): Childbirth and the Long-Term Division of Labour within Couples: How do Substitution, Bargaining Power, and Norms affect Parents' Time Allocation in West Germany? In: European Sociological Review 28 (5), S. 565-582. DOI: 10.1093/esr/jcr026.

Kulawik, Teresa (2005): Wohlfahrtsstaaten und Geschlechterregime im internationalen Vergleich. In: gender...politik...online.

Kümmerling, Angelika; Postels, Dominik (2020): Ist die Geschlechterrolleneinstellung entscheidend? Die Wirkung länderspezifischer Geschlechterkulturen auf die Erwerbsarbeitszeiten von Frauen. In: Kölner Zeitschrift für Soziologie und Sozialpsychologie 72, S. 193-224.

Künzler, Jan; Walter, Wolfgang (2001): Arbeitsteilung in Partnerschaften. Theoretische Ansätze und empirische Befunde. In: Johannes Huinink, Klaus Peter Strohmeier und Michael Wagner (Hg.): Solidarität in Partnerschaft und Familie. Zum Stand familiensoziologischer Theoriebildung. Würzburg: Ergon, S. 185-218.

Kurz, Karin (1998): Das Erwerbsverhalten von Frauen in der intensiven Familienphase. Ein Vergleich zwischen Müttern in der Bundesrepublik Deutschland und den USA. Opladen: Leske + Budrich. 
Kurz, Karin; Hillmert, Steffen; Grunow, Daniela (2006): Increasing Instability in Employment Careeres of West German Men? A Comparison of the Birth Cohorts 1940, 1955 and 1964. In: Hans-Peter Blossfeld, Melinda Mills und Fabrizio Bernardi (Hg.): Globalization, Uncertainty and Men's Careers. An International Comparison. Cheltenham: Edward Elgar, S. 75-113.

Lachance-Grzela, Mylène; Bouchard, Geneviève (2010): Why Do Women Do the Lion's Share of Housework? A Decade of Research. In: Sex Roles 63 (11-12), S. 767-780. DOI: 10.1007/s11199-010-9797-z.

Lee, Kristen S.; Alwin, Duane F.; Tufiş, Paula A. (2007): Beliefs about Women's Labour in the Reunified Germany, 1991-2004. In: European Sociological Review 23 (4), S. 487-503. DOI: $10.1093 / \mathrm{esr} / \mathrm{jcm} 015$.

Lee, YUN-SUK; Waite, Linda J. (2005): Husbands' and Wives' Time Spent on Housework. A Comparison of Measures. In: Journal of Marriage and Family 67 (2), S. 328-336.

Leitner, Sigrid (2003): Varieties of Familialism: The Caring Function of the Family in Comparative Perspective. In: European Societies 5 (4), S. 353-375.

Leitner, Sigrid; Ostner, Ilona; Schratzenstaller, Margit (2004): Was kommt nach dem Emährermodell? Sozialpolitik zwischen Re-Kommodiflzierung und Re-Familialisierung. In: Sigrid Leitner, Ilona Ostner und Margit Schratzenstaller (Hg.): Wohlfahrtsstaat und Geschlechterverhältnis im Umbruch. Was kommt nach dem Ernährermodell? 1. Aufl. Wiesbaden: VS Verlag für Sozialwissenschaften (Jahrbuch für Europa- und Nordamerika-Studien, 7), S. 9-27.

Levy, René; Ernst, Michele (2002): Lebenslauf und Regulation in Paarbeziehungen. Bestimmungsgründe der Ungleichheit familialer Arbeitsteilung. In: Zeitschrift für Familienforschung 14 (2), S. 103-132.

Lewin-Epstein, Noah; Stier, Haya; Braun, Michael (2006): The Division of Household Labor in Germany and Israel. In: Journal of Marriage and Family 68 (5), S. 1147-1164.

Lewis, Jane (1992): Gender and the Development of Welfare Regimes. In: Journal of European Social Policy 2 (3), S. 159-173.

Lewis, Jane (2001): The Decline of the Male Breadwinner Model. Implications for Work and Care. In: Social Politics: International Studies in Gender, State \& Society 8 (2), S. 152 169. DOI: $10.1093 / \mathrm{sp} / 8.2 .152$.

Lewis, Jane; Ostner, Ilona (1994): Gender and the Evolution of European Social Policy. Working Paper 4 of the Centre for Social Policy Research. Zentrum für Sozialpolitik. Universität Bremen (Arbeitspapiere des Zentrums für Sozialpolitik).

Lietzmann, Torsten; Wenzig, Claudia (2017): Arbeitszeitwünsche und Erwerbstätigkeit von Müttern. Welche Vorstellungen über die Vereinbarkeit von Beruf und Familie bestehen. Hg. v. Institut für Arbeitsmarkt- und Berufsforschung (IAB) der Bundesagentur für Arbeit (IAB-Kurzbericht, 10/2017).

Linton, Ralph (1936): The Study of Man. New York: Appleton Century Crofts, Inc.

Liss, Miriam; Schiffrin, Holly H.; Mackintosh, Virginia H.; Miles-McLean, Haley; Erchull, Mindy J. (2013): Development and Validation of a Quantitative Measure of Intensive Parenting Attitudes. In: Journal of Child and Family Studies 22 (5), S. 621-636. DOI: 10.1007/s10826-012-9616-y.

Lundberg, Shelly; Pollak, Robert A. (1993): Separate Spheres Bargaining and the Marriage Market. In: Journal of Political Economy 101 (6), S. 988-1010. DOI: 10.1086/261912.

Lundberg, Shelly; Pollak, Robert A. (1996): Bargaining and Distribution in Marriage. In: Journal of Economic Perspectives 10 (4), S. 139-158. DOI: 10.1257/jep.10.4.139.

Mattessich, Paul; Hill, Reuben (1987): Life Cycle and Family Development. In: Marvin B. Sussman und Suzanne K. Steinmetz (Hg.): Handbook of Marriage and the Family. New York: Plenum Press, S. 437-465. 
Mays, Anja (2012): Determinanten traditionell-sexistischer Einstellungen in Deutschland. Eine Analyse mit Allbus-Daten. In: Kölner Zeitschrift für Soziologie und Sozialpsychologie 64 (2), S. 277-302. DOI: 10.1007/s11577-012-0165-6.

McGill, Brittany S. (2014): Navigating New Norms of Involved Fatherhood. In: Journal of Family Issues 35 (8), S. 1089-1106. DOI: 10.1177/0192513X14522247.

Menke, Katrin; Klammer, Ute (2017): Mehr Geschlechtergerechtigkeit - weniger soziale Gerechtigkeit? Familienpolitische Reformprozesse in Deutschland aus intersektionaler Perspektive. In: Sozialer Fortschritt 66 (3-4), S. 213-228.

Mincer, Jacob (1958): Investment in Human Capital and Personal Income Distribution. In: Journal of Political Economy 66 (4), S. 281-302. DOI: 10.1086/258055.

Mincer, Jacob (1962): Labor Force Participation of Married Women. A Study of Labor Supply. In: National Bureau Committee (Hg.): Aspects of Labor Economics: Princeton University Press, S. 63-105.

Misra, Joya; Budig, Michelle; Boeckmann, Irene (2011): Work-family Policies and the Effects of Children on Women's Employment Hours and Wages. In: Community, Work \& Family 14 (2), S. 139-157.

Mückenberger, Ulrich (1985a): Die Krise des Normalarbeitsverhältnisses. Hat das Arbeitsrecht noch Zukunft? (Teil 1). In: Zeitschrift für Sozialreform 31 (7), S. 415-434.

Mückenberger, Ulrich (1985b): Die Krise des Normalarbeitsverhältnisses. Hat das Arbeitsrecht noch Zukunft? (Teil 2). In: Zeitschrift für Sozialreform 31 (7), S. 457-475.

Mückenberger, Ulrich (2010): Krise des Normalarbeitsverhältnisses - ein Umbauprogramm. In: Zeitschrift für Sozialreform 56 (4), S. 403-420. DOI: 10.1515/zsr-2010-0404.

Mühling, Tanja (2007): Wie verbringen Väter ihre Zeit? Männer zwischen „Zeitnot“ und „Qualitätszeit“. In: Tanja Mühling und Harald Rost (Hg.): Väter im Blickpunkt. Perspektiven der Familienforschung. Opladen: Budrich, S. 115-160.

Mühling, Tanja; Rost, Harald (2006): ifb-Familienreport Bayern 2006 - Zur Lage der Familie in Bayern. Schwerpunkt: Väter in der Familie. Hg. v. Staatsinstitut für Familienforschung an der Universität Bamberg (ifb). Bamberg.

Mühling, Tanja; Rost, Harald; Rupp, Marina; Schulz, Florian (2006): Kontinuität trotz Wandel. Die Bedeutung traditioneller Familienleitbilder für die Berufsverläufe von Müttern und Väter. Weinheim, München: Juventa-Verlag.

Müller, Kai-Uwe; Neumann, Michael; Wrohlich, Katharina (2013): Bessere Vereinbarkeit von Familie und Beruf durch eine neue Lohnersatzleistung bei Familienarbeitszeit. In: DIW Wochenbericht: Wirtschaft, Politik, Wissenschaft 80 (46), S. 3-11.

Nieuwenhuis, Rense; Need, Ariana; van der Kolk, Henk (2012): Institutional and Demographic Explanations of Women's Employment in 18 OECD Countries, 1975-1999. In: Journal of Marriage and Family 74 (3), S. 614-630. DOI: 10.1111/j.1741-3737.2012.00965.x.

Nitsche, Natalie; Grunow, Daniela (2016): Housework over the Course of Relationships. Gender Ideology, Resources, and the Division of Housework from a Growth Curve Perspective. In: Advances in Life Course Research 29, S. 80-94. DOI: 10.1016/j.alcr.2016.02.001.

Nitsche, Natalie; Grunow, Daniela (2018): Do Economic Resources Play a Role in Bargaining Child Care in Couples? Parental Investment in Cases of Matching and Mismatching Gender Ideologies in Germany. In: European Societies 20 (5), S. 785-815. DOI: 10.1080/14616696.2018.1473626.

Nock, Steven L. (1979): The Family Life Cycle. Empirical or Conceptual Tool? In: Journal of Marriage and the Family 41 (1), S. 15-26. DOI: 10.2307/351727.

Nomaguchi, Kei M.; Milkie, Melissa A. (2003): Costs and Rewards of Children: The Effects of Becoming a Parent on Adults' Lives. In: Journal of Marriage and Family 65 (2), S. 356374. 
Norman, Helen; Elliot, Mark; Fagan, Colette (2014): Which Fathers are the Most Involved in Taking Care of their Toddlers in the UK? An Investigation of the Predictors of Paternal Involvement. In: Community, Work \& Family 17 (2), S. 163-180. DOI: 10.1080/13668803.2013.862361.

Oberndorfer, Rotraut; Rost, Harald (2005): Neue Väter. Anspruch und Realität. In: Zeitschrift für Familienforschung 17 (1), S. 50-65.

OECD (2016): Dare to share - Deutschlands Weg zur Partnerschaftlichkeit in Familie und Beruf. Überarbeitete Ausgabe. Paris: OECD Publishing.

Orloff, Ann Shola (1993): Gender and the Social Rights of Citizenship. The Comparative Analysis of Gender Relations and Welfare States. In: American Sociological Review 58 (3), S. 303-328. DOI: 10.2307/2095903.

Ostner, llona (1995): Arm ohne Ehemann? Sozialpolitische Regulierung von Lebenschancen für Frauen im internationalen: Vergleich. In: Aus Politik und Zeitgeschichte : APuZ 45 (36), S. 3-12.

Ostner, llona (1998): Quadraturen im Wohlfahrtsdreieck. die USA, Schweden und die Bundesrepublik im Vergleich. In: Stephan Lessenich und Ilona Ostner (Hg.): Welten des Wohlfahrtskapitalismus. Der Sozialstaat in vergleichender Perspektive. Frankfurt/Main: Campus Verlag (Theorie und Gesellschaft, 40).

Ostner, llona (2004): Review Essay. Aus Anlass eines Geburtstags: "Gender and Welfare Revisited". In: Sigrid Leitner, llona Ostner und Margit Schratzenstaller (Hg.): Wohlfahrtsstaat und Geschlechterverhältnis im Umbruch. Was kommt nach dem Ernährermodell? 1. Aufl. Wiesbaden: VS Verlag für Sozialwissenschaften (Jahrbuch für Europa- und Nordamerika-Studien, 7), S. 44-61.

Ott, Notburga (1989): Familienbildung und familiale Entscheidungsfindung aus verhandlungstheoretischer Sicht. In: Gert Wagner, Notburga Ott und Hans-Joachim HoffmannNowotny (Hg.): Familienbildung und Erwerbstätigkeit im demographischen Wandel. Proceedings der 23. Arbeitstagung der Deutschen Gesellschaft für Bevölkerungswissenschaft am 28. Februar - 3. März 1989 in Bad Homburg v.d.H. Berlin, Heidelberg: Springer (Studies in Contemporary Economics), S. 97-116.

Ott, Notburga (1992): Intrafamily Bargaining and Household Decisions. Berlin, Heidelberg: Springer (Microeconomic Studies).

Ott, Notburga (1993): Zum Rationalverhalten familialer Entscheidungen. In: Claudia Born und Helga Krüger (Hg.): Erwerbsverläufe von Ehepartnern und die Modernisierung weiblicher Lebensläufe. Weinheim: Deutscher-Studien-Verlag, S. 25-51.

Ott, Notburga (1999): The Economics of Gender — Der neoklassische Erklärungsansatz zum Geschlechterverhältnis. In: Bettina Dausien, Martina Herrmann, Mechtild Oechsle, Christiane Schmerl und Marlene Stein-Hilbers (Hg.): Erkenntnisprojekt Geschlecht. Feministische Perspektiven verwandeln Wissenschaft. Opladen: Leske + Budrich (Geschlecht und Gesellschaft, 17), S. 167-196.

Panova, Ralina; Sulak, Harun; Bujard, Martin; Muth, Lisa (2017): Die Rushhour des Lebens im Familienzyklus. Zeitverwendung von Männern und Frauen. In: Statistisches Bundesamt (Hg.): Wie die Zeit vergeht. Analysen zur Zeitverwendung in Deutschland. Beiträge zur Ergebniskonferenz der Zeitverwendungserhebung 2012/2013 am 5./6. Oktober 2016 in Wiesbaden. Wiesbaden, S. 45-64.

Papastefanou, Georgios; Thies, Theresa (2018): Mütter wenden für Kinder immer mehr Zeit auf. Anstieg der täglichen Kinderbetreuungszeit seit 1991. In: Informationsdienst soziale Indikatoren 59, S. 13-17.

Parsons, Talcott (1951): The social system. New York: Free Press.

Perry-Jenkins, Maureen; Gerstel, Naomi (2020): Work and Family in the Second Decade of the 21st Century. In: Journal of Marriage and Family 82 (1), S. 420-453. 
Peuckert, Rüdiger (2012): Familienformen im sozialen Wandel. Wiesbaden: VS Verlag für Sozialwissenschaften.

Pfau-Effinger, Birgit (1997): Zum theoretischen Rahmen für die Analyse internationaler Differenzen in der gesellschaftlichen Integration von Frauen. In: Stefan Hradil (Hg.): Differenz und Integration. Die Zukunft moderner Gesellschaften. Frankfurt/Main: Campus Verlag (Verhandlungen des 28. Kongresses der Deutschen Gesellschaft für Soziologie in Dresden 1996, 28), S. 514-527.

Pfau-Effinger, Birgit (1998): Arbeitsmarkt- und Familiendynamik in Europa: theoretische Grundlagen der vergleichenden Analyse. In: Birgit Geissler, Friederike Maier und Birgit Pfau-Effinger (Hg.): FrauenArbeitsMarkt. Der Beitrag der Frauenforschung zur sozio-ökonomischen Theorieentwicklung. Berlin: Edition Sigma, S. 177-194.

Pfau-Effinger, Birgit (2000): Kultur und Frauenerwerbstätigkeit in Europa. Theorie und Empirie des internationalen Vergleichs. Opladen: Leske + Budrich.

Pfau-Effinger, Birgit (2005): Wandel der Geschlechterkultur und Geschlechterpolitiken in konservativen Wohlfahrtsstaaten. Deutschland, Österreich und Schweiz. Online verfügbar unter https://bit.ly/36RX1Cq.

Pfau-Effinger, Birgit; Smidt, Maike (2011): Differences in Women's Employment Patterns and Family Policies: Eastern and Western Germany. In: Community, Work \& Family 14 (2), S. 217-232.

Presser, Harriet B. (1994): Employment Schedules Among Dual-Earner Spouses and the Division of Household Labor by Gender. In: American Sociological Review 59 (3), S. 348364. DOI: $10.2307 / 2095938$.

Raley, Sara; Bianchi, Suzanne M.; Wang, Wendy (2012): When Do Fathers Care? Mothers' Economic Contribution and Fathers' Involvement in Child Care. In: American Journal of Sociology 117 (5), S. 1422-1459. DOI: 10.1086/663354.

Rauschenbach, Thomas (2012): Aufwachsen in Deutschland. Eine Einführung. In: Thomas Rauschenbach und Walter Bien (Hg.): Aufwachsen in Deutschland. AID:A - Der neue Survey. Weinheim, Basel: Beltz Juventa, S. 7-27.

Rauschenbach, Thomas (2015): Vorwort. In: Sabine Walper, Walter Bien und Thomas Rauschenbach $(\mathrm{Hg}$.): Aufwachsen in Deutschland heute. Erste Befunde aus dem DJI-Survey AID:A 2015. München: Deutsches Jugendinstitut, S. 4-5.

Ray, Rebecca; Gornick, Janet C.; Schmitt, John (2010): Who cares? Assessing Generosity and Gender Equality in Parental Leave Policy Designs in 21 Countries. In: Journal of European Social Policy 20 (3), S. 196-216. DOI: 10.1177/0958928710364434.

Reimer, Thordis; Erler, Daniel; Schober, Pia; Blum, Sonja (2019): Germany Country Note. In: Alison Koslowski, Sonja Blum, Ivana Dobrotic, Alexandra Macht und Peter Moss (Hg.): International Review of Leave Policies and Research 2019, S. 223-233.

Riegraf, Birgit (2010): Konstruktion von Geschlecht. In: Brigitte Aulenbacher, Michael Meuser und Birgit Riegraf $(\mathrm{Hg}$.): Soziologische Geschlechterforschung. Eine Einführung. Wiesbaden: VS Verlag für Sozialwissenschaften (Studienskripten zur Soziologie), S. 59-77.

Riley, Matilda W.; Johnson, Marilyn; Foner, Anne (1972): Elements in a Model of Age Stratification. In: Matilda W. Riley, Marilyn Johnson und Anne Foner (Hg.): Aging and Society, Volume 3. A Sociology of Age Stratification. New York: Russell Sage Foundation, S. 326.

Rodgers, Roy H. (1973): Family Interaction and Transaction. The Developmental Approach. Enlgewood Cliffs: Prentice-Hall (Prentice-Hall series in sociology).

Roeters, Anne; van der Lippe, Tanja; Kluwer, Esther (2009): Parental Work Demands and the Frequency of Child - Related Routine and Interactive Activities. In: Journal of Marriage and Family 71 (5), S. 1193-1204.

Rosenfeld, Rachel A.; Trappe, Heike; Gornick, Janet C. (2004): Gender and Work in Germany. Before and After Reunification. In: Annual Review of Sociology 30, S. 103-124. 
Rosow, Irving (1978): Status and role change through the life-span. In: Robert H. Binstock und Ethel Shanas (Hg.): Handbook of Aging and the Social Sciences. New York: Basic Books, S. 86-105.

Ruckdeschel, Kerstin (2015): Verantwortete Elternschaft. „Für die Kinder nur das Beste“. In: Norbert F. Schneider, Sabine Diabaté und Kerstin Ruckdeschel (Hg.): Familienleitbilder in Deutschland. Kulturelle Vorstellungen zu Partnerschaft, Elternschaft und Familienleben. Opladen, Berlin, Toronto: Barbara Budrich, S. 191-206.

Sanchez, Laura; Thomson, Elzabeth (1997): Becoming Mothers and Fathers. Parenthood, Gender, and the Division of Labor. In: Gender \& Society 11 (6), S. 747-772. DOI: 10.1177/089124397011006003.

Sani, Giulia M. Dotti; Treas, Judith (2016): Educational Gradients in Parents' Child-Care Time Across Countries, 1965-2012. In: Journal of Marriage and Family 78 (4), S. 1083-1096. DOI: $10.1111 /$ jomf.12305.

Sayer, Liana (2005): Gender, Time and Inequality. Trends in Women's and Men's Paid Work, Unpaid Work and Free Time. In: Social Forces 84 (1), S. 285-303.

Sayer, Liana; Bianchi, Suzanne; Robinson, John (2004a): Are Parents Investing Less in Children? Trends in Mothers' and Fathers' Time with Children. In: American Journal of Sociology 110 (1), S. 1-43.

Sayer, Liana C. (2010): Trends in Housework. In: Judith K. Treas und Sonja Drobnič (Hg.): Dividing the Domestic. Men, Women, and Household Work in Cross-National Perspective. Stanford: Stanford University Press, S. 19-38.

Sayer, Liana C.; Gauthier, Anne H.; Furstenberg, Jr., Frank F. (2004b): Educational Differences in Parents' Time with Children. Cross-National Variations. In: Journal of Marriage and Family 66 (5), S. 1152-1169.

Schleutker, Elina (2014): Fertilität, Familienpolitik und Wohlfahrtsregime. In: Comparative Population Studies 39 (1), S. 157-194.

Schmelzer, Paul; Kurz, Karin; Schulze, Kerstin (2015): Einkommensnachteile von Müttern im Vergleich zu kinderlosen Frauen in Deutschland. In: Kölner Zeitschrift für Soziologie und Sozialpsychologie 67 (4), S. 737-762. DOI: 10.1007/s11577-015-0346-1.

Schober, Pia; Scott, Jacqueline (2012): Maternal Employment and Gender Role Attitudes. Dissonance among British Men and Women in the Transition to Parenthood. In: Work, Employment \& Society 26 (3), S. 514-530.

Schober, Pia S. (2013a): The Parenthood Effect on Gender Inequality. Explaining the Change in Paid and Domestic Work When British Couples Become Parents. In: European Sociological Review 29 (1), S. 74-85.

Schober, Pia S.; Zoch, Gundula (2019): Change in the Gender Division of Domestic Work after Mothers or Fathers Took Leave: Exploring Alternative Explanations. In: European Societies 21 (1), S. 158-180. DOI: 10.1080/14616696.2018.1465989.

Schober, Pia Sophia (2013b): Maternal Labor Market Return and Domestic Work after Childbirth in Britain and Germany. In: Community, Work \& Family 16 (3), S. 307-326.

Schober, Pia Sophia; Spiess, Christa Katharina (2015): Local Day Care Quality and Maternal Employment. Evidence From East and West Germany. In: Journal of Marriage and Family 77 (3), S. 712-729. DOI: 10.1111/jomf.12180.

Schulz, Florian (2010): Verbundene Lebensläufe. Partnerwahl und Arbeitsteilung zwischen neuen Ressourcenverhältnissen und traditionellen Geschlechterrollen. Wiesbaden: VS Verlag für Sozialwissenschaften.

Schulz, Florian; Blossfeld, Hans-Peter (2006): Wie verändert sich die häusliche Arbeitsteilung im Eheverlauf? Eine Längsschnittstudie der ersten 14 Ehejahre in Westdeutschland. In: Kölner Zeitschrift für Soziologie und Sozialpsychologie 58 (1), S. 23-49.

Schulz, Florian; Grunow, Daniela (2011): Comparing Diary and Survey Estimates on Time Use. In: European Sociological Review 28 (5), S. 622-632. DOI: 10.1093/esr/jcr030. 
Schutter, Sabina; Zerle-Elsäßer, Claudia (2012): Das Elterngeld: Wahlfreiheit und Existenzsicherung für (alle) Eltern? In: WSI-Mitteilungen: Zeitschrift des Wirtschafts- und Sozialwissenschaftlichen Instituts in der Hans-Böckler-Stiftung 65 (3), S. 216-225.

Schütze, Yvonne (1986): Die gute Mutter. Zur Geschichte des normativen Musters „Mutterliebe“. Bielefeld: Kleine Verlag $\mathrm{GmbH}$.

Seel, Barbara (2001): Partnerschaftliche Arbeitsteilung und der ökonomische Erklärungsansatz. In: Zeitschrift für Familienforschung 13 (2), S. 49-68.

Shelton, Beth Anne; John, Daphne (1996): The division of household labor. In: Annual Review of Sociology 22 (1), S. 299-322.

Sørensen, Annemette (1990): Unterschiede im Lebenslauf von Frauen und Männern. In: Karl Ulrich Mayer (Hg.): Lebensverläufe und sozialer Wandel. Opladen: Westdeutscher Verlag (Kölner Zeitschrift für Soziologie und Sozialpsychologie, 31), S. 304-321.

South, Scott J.; Spitze, Glenna (1994): Housework in Marital and Nonmarital Households. In: American Sociological Review 59 (3), S. 327-347. DOI: 10.2307/2095937.

Spanier, Graham B.; Sauer, William; Larzelere, Robert (1979): An Empirical Evaluation of the Family Life Cycle. In: Journal of Marriage and the Family 41 (1), S. 27-38. DOI: $10.2307 / 351728$.

Statistisches Bundesamt (2017): Statistik zum Elterngeld. Beendete Leistungsbezüge für im 2. Vierteljahr 2015 geborene Kinder. Wiesbaden.

Steffens, Wolfgang (1988): Die Lebenszyklen. In: Manfred Cierpka (Hg.): Familiendiagnostik. Berlin, Heidelberg, New York, London, Paris, Tokyo: Springer, S. 32-47.

Steiber, Nadia; Berghammer, Caroline; Haas, Barbara (2016): Contextualizing the Education Effect on Women's Employment. A Cross-National Comparative Analysis. In: Journal of Marriage and Family 78 (1), S. 246-261. DOI: 10.1111/jomf.12256.

Steiber, Nadia; Haas, Barbara (2009): Ideals or Compromises? The Attitude-Behaviour Relationship in Mothers' Employment. In: Socio-Economic Review 7 (4), S. 639-668. DOI: 10.1093/ser/mwp015.

Sullivan, Oriel (2011): An End to Gender Display through the Performance of Housework? A review and reassessment of the quantitative literature using insights from the qualitative literature. In: Journal of Family Theory \& Review 3 (1), S. 1-13.

Sullivan, Oriel (2013): What Do We Learn About Gender by Analyzing Housework Separately From Child Care? Some Considerations From Time-Use Evidence. In: Journal of Family Theory \& Review 5 (2), S. 72-84. DOI: 10.1111/jttr.12007.

Sullivan, Oriel; Billari, Francesco C.; Altintas, Evrim (2014): Fathers' Changing Contributions to Child Care and Domestic Work in Very Low-Fertility Countries: The Effect of Education. In: Journal of Family Issues 35 (8), S. 1048-1065.

Sullivan, Oriel; Coltrane, Scott (2008): Men's Changing Contribution to Housework and Childcare. Hg. v. Council on Contemporary Families. University of Texas at Austin (Brief Reports). Online verfügbar unter https://contemporaryfamilies.org/mens-changing-contribution-to-housework-and-childcare-brief-report/, zuletzt geprüft am 23.11.2020.

Sullivan, Oriel; Gershuny, Jonathan (2016): Change in Spousal Human Capital and Housework. A Longitudinal Analysis. In: European Sociological Review 32 (6), S. 864-880. DOI: 10.1093/esr/jcw043.

Tamm, Marcus (2019): Fathers' Parental Leave-taking, Childcare Involvement and Labor Market Participation. In: Labour Economics 59, S. 184-197. DOI: 10.1016/j.labeco.2019.04.007.

Timm, Andreas (2004): Das Phänomen Bildungshomogamie und seine Entwicklung in Westdeutschland. In: Andreas Timm (Hg.): Partnerwahl- und Heiratsmuster in modernen Gesellschaften. Der Einfluss des Bildungssystems. Wiesbaden: Deutscher Universitätsverlag (Sozialwissenschaft), S. 41-47. 
Torres-Reyna, Oscar (2007): Linear Regression using Stata. Princeton University. Online verfügbar unter https://dss.princeton.edu/training/Regression101.pdf, zuletzt geprüft am 11.11.2020.

Treas, Judith K.; Drobnič, Sonja (Hg.) (2010): Dividing the Domestic. Men, Women, and Household Work in Cross-National Perspective. Stanford: Stanford University Press.

Urban, Dieter; Mayerl, Jochen (2018): Angewandte Regressionsanalyse: Theorie, Technik und Praxis. 5., überarbeitete Auflage. Wiesbaden: Springer VS (Studienskripten zur Soziologie).

Uunk, Wilfred; Kalmijn, Matthijs; Muffels, Ruud (2005): The Impact of Young Children on Women's Labour Supply: A Reassessment of Institutional Effects in Europe. In: Acta Sociologica 48 (1), S. 41-62.

van Berkel, Michel; Graaf, Nan Dirk de (1999): By virtue of pleasantness? Housework and the effects of education revisited. In: Sociology 33 (4), S. 785-808.

van der Lippe, Tanja; Ruijter, Judith de; Ruijter, Esther de; Raub, Werner (2011): Persistent Inequalities in Time Use between Men and Women. A Detailed Look at the Influence of Economic Circumstances, Policies, and Culture. In: European Sociological Review 27 (2), S. 164-179. DOI: 10.1093/esr/jcp066.

van der Lippe, Tanja; van Dijk, Liset (2002): Comparative Research on Women's Employment. In: Annual Review of Sociology 28, S. 221-241.

Verbakel, Ellen (2010): Partner's Resources and Adjusting Working Hours in the Netherlands. Differences Over Time, Between Levels of Human Capital, and Over the Family Cycle. In: Journal of Family Issues 31 (10), S. 1324-1362. DOI: 10.1177/0192513X09360188.

Verbakel, Ellen; Graaf, Paul M. de (2009): Partner Effects on Labour Market Participation and Job Level. Opposing Mechanisms. In: Work, Employment \& Society 23 (4), S. 635654.

Wall, Glenda (2010): Mothers' Experiences with Intensive Parenting and Brain Development Discourse. In: Women's Studies International Forum 33 (3), S. 253-263.

Wall, Glenda; Arnold, Stephanie (2007): How Involved Is Involved Fathering? An Exploration of the Contemporary Culture of Fatherhood. In: Gender \& Society 21 (4), S. 508-527.

Walls, Jill K.; Helms, Heather M.; Grzywacz, Joseph G. (2016): Intensive Mothering Beliefs Among Full-Time Employed Mothers of Infants. In: Journal of Family Issues 37 (2), S. 245-269.

Walper, Sabine; Lien, Shih-Cheng (2018): Routinebetreuung und interaktive "Quality Time“. Was beeinflusst, wieviel Zeit Väter wie mit ihren Kindern verbringen? In: Zeitschrift für Familienforschung 30 (1), S. 29-49.

Walter, Jessica Gabriele (2018a): Measures of Gender Role Attitudes under Revision. The Example of the German General Social Survey. In: Social Science Research 72, S. 170182. DOI: 10.1016/j.ssresearch.2018.02.009.

Walter, Jessica Gabriele (2018b): The Adequacy of Measures of Gender Roles Attitudes. A Review of Current Measures in Omnibus Surveys. In: Quality \& quantity 52 (2), S. 829848. DOI: 10.1007/s11135-017-0491-x.

Wengler, Annelene; Trappe, Heike; Schmitt, Christian (2008): Partnerschaftliche Arbeitsteilung und Elternschaft. Analysen zur Aufteilung von Hausarbeit und Elternaufgaben auf Basis des Generations and Gender Survey. Wiesbaden: Bundesinstitut für Bevölkerungsforschung.

West, Candance; Fenstermaker, Sarah (1995): Doing Difference. In: Gender \& Society 9 (1), S. 8-37.

West, Candance; Zimmerman, Don H. (1987): Doing Gender. In: Gender \& Society 1 (2), S. 125-151. DOI: $10.1177 / 0891243287001002002$. 
Wetterer, Angelika (1992a): Arbeitsteilung und Geschlechterkonstruktion. "Gender at work" in theoretischer und historischer Perspektive. Konstanz: UVK. Online verfügbar unter https://opacplus.bsb-muenchen.de/search?isbn=9783896697875\&db=100.

Wetterer, Angelika (1992b): Hierarchie und Differenz im Geschlechterverhältnis. In: Angelika Wetterer (Hg.): Profession und Geschlecht. Über die Marginalität von Frauen in hochqualifizierten Berufen. Frankfurt am Main: Campus Verlag, S. 13-40.

Williams, Joan C. (2000): Unbending Gender. New York: Oxford University Press.

Yavorsky, Jill E.; Dush, Claire M. Kamp; Schoppe-Sullivan, Sarah J. (2015): The Production of Inequality. The Gender Division of Labor Across the Transition to Parenthood. In: Journal of Marriage and the Family 77 (3), S. 662-679. DOI: 10.1111/jomf.12189.

Zabel, Cordula; Heintz-Martin, Valerie (2013): Does Children's Age Impact the Division of Housework? A Comparision of France, Eastern and Western Germany. In: European Societies 15 (5), S. 663-685.

Zerle-Elsäßer, Claudia; Li, Xuan (2017): Väter im Familienalltag - Determinanten einer aktiven Vaterschaft. In: Zeitschrift für Familienforschung 29 (1), S. 11-31. Online verfügbar unter https://opacplus.bsb-muenchen.de/search?issn=14372940\&db=255.

Ziefle, Andrea (2004): Die individuellen Kosten des Erziehungsurlaubs. In: Kölner Zeitschrift für Soziologie und Sozialpsychologie 56 (2), S. 213-231. DOI: 10.1007/s11577-0040032-1.

Zinn, Sabine; Kreyenfeld, Michaela; Bayer, Michael (2020): Kinderbetreuung in Corona-Zeiten: Mütter tragen die Hauptlast, aber Väter holen auf. Deutsches Institut für Wirtschaftsforschung DIW. Berlin (51). Online verfügbar unter https://www.diw.de/documents/publikationen/73/diw_01.c.794303.de/diw_aktuell_51.pdf, zuletzt geprüft am 10.01.2021.

Zulehner, Paul Michael; Volz, Rainer (1998): Männer im Aufbruch. Wie Deutschlands Männer sich selbst und wie Frauen sie sehen. Ostfildern: Schwabenverlag. 


\section{Tabellenverzeichnis}

Tabelle 3.1: $\quad$ Typen der Aufteilung von Erwerbs- und Familienarbeit nach Lewis ..................................................................... 26

Tabelle 4.1: Zusammenfassung der theoretischen Ansätze ..................... 83

Tabelle 5.1: $\quad$ Zusammenfassung der Regimetypen nach EspingAndersen ..................................................................... 86

Tabelle 5.2: $\quad$ Varianten von Familialismus nach Leitner............................. 89

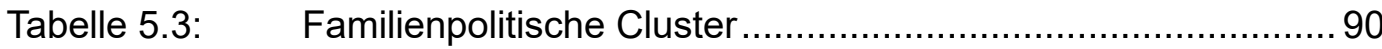

Tabelle 7.1: Datengrundlage der Studie: DJI-Survey AID:A II von

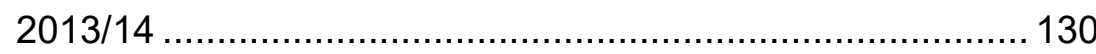

Tabelle 7.2: $\quad$ Verteilung von zentralen unabhängigen Variablen ................ 141

Tabelle 7.3: $\quad$ Strukturen zur Einordnung der latenten Klassen: Männliche, weibliche oder gemeinsame Sphären 146

Tabelle 8.1: $\quad$ Erwerbsanteil der Mutter, stufenweiser Aufbau des Modells, OLS-Regression 167

Tabelle 8.2: $\quad$ Korrelationen der Gender-Items 177

Tabelle 8.3: $\quad$ Erwerbsanteil der Mutter in Abhängigkeit vom Alter des Kindes, unter Berücksichtigung von vier Gender-Items......... 179

Tabelle 8.4: $\quad$ AV: Erwerbsstunden der Mütter, getrennt nach dem Alter des Kindes

Tabelle 8.5: $\quad$ Erwerbsumfang der Mutter in Abhängigkeit vom Alter des Kindes, unter Berücksichtigung von vier Gender-Items......... 187

Tabelle 9.1: $\quad$ Aufteilung der einzelnen Tätigkeiten der Hausarbeit 191

Tabelle 9.2: $\quad$ Aufteilung der Hausarbeit, stufenweiser Aufbau des Modells, OLS-Regression

Tabelle 9.3: $\quad$ Aufteilung der Hausarbeit, unter Berücksichtigung von vier Gender-Items, getrennt nach dem Alter des Kindes....... 217

Tabelle 10.1: $\quad$ Aufteilung der einzelnen Tätigkeiten der Kinderbetreuung .... 223

Tabelle 10.2: $\quad$ Aufteilung der Kinderbetreuung, stufenweiser Aufbau des Modells, OLS-Regression.

Tabelle 10.3: $\quad$ Aufteilung der Kinderbetreuung, unter Berücksichtigung der vier Gender-Items, getrennt nach dem Alter des Kindes 
Tabelle A.1: $\quad$ Multinomiales Logitmodell zu den latenten Gender-

Tabelle A.2: $\quad$ Bivariate Regression: Erwerbsanteil der Mutter in Abhängigkeit vom Alter des Kindes

Tabelle A.3: $\quad$ Erwerbsanteil der Mutter, getrennt nach Alter des Kindes,

OLS-Regression, stufenweiser Aufbau der Modelle.

Tabelle A.4: $\quad$ Erwerbsanteil der Mutter in Abhängigkeit vom Alter des

Kindes, unter Berücksichtigung der drei latenten Gender-

Klassen

Tabelle A.5: $\quad$ Erwerbsumfang der Mutter in Abhängigkeit vom Alter des

Kindes, unter Berücksichtigung der drei latenten Gender-

Klassen

Tabelle A.6: $\quad$ Bivariate Regression: Aufteilung der Hausarbeit in Abhängigkeit vom Alter des Kindes

Tabelle A.7: $\quad$ Aufteilung der Hausarbeit, getrennt nach Alter des Kindes, stufenweiser Aufbau der Modelle

Tabelle A.8: $\quad$ Aufteilung der Hausarbeit, unter Berücksichtigung der drei Gender-Klassen, getrennt nach dem Alter des Kindes.

Tabelle A.9: $\quad$ Bivariate Regression: Aufteilung der Kinderbetreuung in Abhängigkeit vom Alter des Kindes

Tabelle A.10: Aufteilung der Kinderbetreuung, getrennt nach Alter des Kindes, stufenweiser Aufbau der Modelle

Tabelle A.11: $\quad$ Aufteilung der Kinderbetreuung, unter Berücksichtigung der drei Gender-Klassen, getrennt nach dem Alter des Kindes

Tabelle A.12: $\quad$ Übersicht über die Bestätigung oder Ablehnung der Hypothesen anhand der empirischen Ergebnisse 


\section{Abbildungsverzeichnis}

Abbildung 2.1: Das Familienzyklus-Modell ............................................... 15

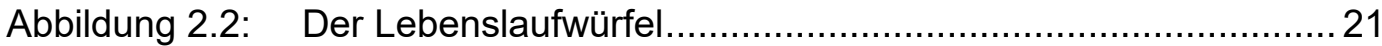

Abbildung 3.1 Erwerbstätigenquote nach Alter des jüngsten Kindes und

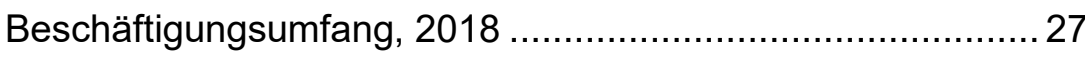

Abbildung 4.1: Wirkungsschema zur Arbeitsteilung im Paar..........................5 53

Abbildung 4.2: Teufelskreis ökonomischer Rationalität................................5 58

Abbildung 5.1: Das Geschlechterarrangement nach Pfau-Effinger .................. 93

Abbildung 5.2: Entwicklung der Väterbeteiligung am Erziehungs- und Elterngeldbezug, nach Geburtsjahrgängen der Kinder, von 2006 bis 2015

Abbildung 5.3: Entwicklung der Erwerbstätigenquoten von Müttern mit minderjährigen Kindern, Deutschland, 2006-2012, Veränderung der Erwerbstätigenquoten in \% 109

Abbildung 7.1: $\quad$ Überblick über die Entstehung des Samples ....................... 131

Abbildung 7.2: Latente Klassen der Geschlechterrolleneinstellungen .......... 148

Abbildung 7.3: Bivariate Zusammenhänge der latenten Klassen.................. 153

Abbildung 7.4: Predictive Margins zu den latenten Klassen in Abhängigkeit von verschiedenen Einflussfaktoren 154

Abbildung 8.1: Vergleich der gewünschten und tatsächlichen

Erwerbsumfänge der Mütter

Abbildung 8.2: Vergleich der gewünschten und tatsächlichen

Erwerbsumfänge der Väter 158

Abbildung 8.3: Erwerbskonstellation in Abhängigkeit vom Alter des Kindes 159

Abbildung 8.4: Erwerbsanteil der Mütter am gesamten Erwerbsvolumen des Paares 160

Abbildung 8.5: Bivariate Zusammenhänge des relativen Berufsstatus (ISEI) und des absoluten Berufsstatus der Mutter (ISEI) mit der Aufteilung der Erwerbsarbeit 161

Abbildung 8.6: Bivariate Zusammenhänge der Gender-Items mit der Aufteilung der Erwerbsarbeit 162 
Abbildung 8.7: Bivariate Zusammenhänge der Gender-Klassen mit der Aufteilung der Erwerbsarbeit 163

Abbildung 8.8: Bivariate Zusammenhänge der Anzahl der Kinder und Ost-West-Unterschiede mit der Aufteilung der Erwerbsarbeit 164

Abbildung 8.9: Vorhergesagter Erwerbsanteil der Mutter in Abhängigkeit vom Alter des Kindes 166

Abbildung 8.10: Koeffizienten des relativen Berufsstatus zur Vorhersage des Erwerbsanteil der Mutter, getrennt nach dem Alter des Kindes.

Abbildung 8.11: Koeffizienten der Geschlechterrolleneinstellungen zur

Vorhersage des Erwerbsanteils der Mutter, getrennt nach dem Alter des Kindes

Abbildung 8.12: Vorhergesagter Erwerbsarbeitsanteil der Mutter, in Abhängigkeit von der Kinderanzahl, getrennt nach dem Alter des Kindes

Abbildung 8.13: Koeffizienten des Bundesgebiets zur Vorhersage des Erwerbsarbeitsanteil der Mutter, getrennt nach dem Alter des Kindes

Abbildung 8.14: Koeffizienten des absoluten Berufsstatus der Mutter zur Vorhersage des Erwerbsarbeitsanteil der Mutter, getrennt nach dem Alter des Kindes

Abbildung 8.15: Vorhergesagter Erwerbsarbeitsanteil der Mutter, in

Abhängigkeit der drei latenten Gender-Klassen, getrennt nach dem Alter des Kindes 181

Abbildung 8.16: Vorhergesagter Erwerbsumfang der Mutter, in

Abhängigkeit der drei latenten Gender-Klassen, getrennt nach dem Alter des Kindes

Abbildung 9.1: $\quad$ Aufteilung der einzelnen Hausarbeitstätigkeiten nach Alter des Kindes. 192

Abbildung 9.2: Univariate Verteilung der Aufteilung der Hausarbeit.............. 194

Abbildung 9.3 Aufteilung der Hausarbeit nach Alter des Kindes .................. 195

Abbildung 9.4: Bivariate Zusammenhänge des relativen Berufsstatus (ISEI) und des absoluten Berufsstatus der Mutter (ISEI) mit der Aufteilung der Hausarbeit 196

Abbildung 9.5: Bivariate Zusammenhänge der Gender-Items mit der Aufteilung der Hausarbeit 197 
Abbildung 9.6: Bivariate Zusammenhänge der Gender-Klassen mit der Aufteilung der Hausarbeit 198

Abbildung 9.7: Bivariate Zusammenhänge der Ost-West-Unterschiede und der Erwerbskonstellation mit der Aufteilung der Hausarbeit 199

Abbildung 9.8: Vorhergesagte Aufteilung der Hausarbeit in Abhängigkeit vom Alter des Kindes

Abbildung 9.9: Koeffizienten des relativen Berufsstatus (ISEI) zur Vorhersage der Aufteilung der Hausarbeit, getrennt nach dem Alter des Kindes. 208

Abbildung 9.10: Koeffizienten der Geschlechterrolleneinstellungen (Item 1 und 2) zur Vorhersage der Aufteilung der Hausarbeit, getrennt nach dem Alter des Kindes.

Abbildung 9.11: Koeffizienten der Kinderanzahl zur Vorhersage der Aufteilung der Hausarbeit, getrennt nach dem Alter des Kindes

Abbildung 9.12: Koeffizienten des Bundesgebiets zur Aufteilung der Hausarbeit, getrennt nach dem Alter des Kindes

Abbildung 9.13: Koeffizienten des Berufsstatus der Mutter zur Vorhersage der Aufteilung der Hausarbeit, getrennt nach dem Alter des Kindes

Abbildung 9.14: Koeffizienten der Erwerbstätigkeit der Mutter zur

Vorhersage der Aufteilung der Hausarbeit, getrennt nach dem Alter des Kindes

Abbildung 9.15: Koeffizienten der Erwerbstätigkeit des Vaters zur

Vorhersage der Aufteilung der Hausarbeit, getrennt nach dem Alter des Kindes

Abbildung 9.16: Vorhergesagte Aufteilung der Hausarbeit, unter

Berücksichtigung der drei Gender-Klassen, getrennt nach dem Alter des Kindes

Abbildung 10.1: Aufteilung der Kinderbetreuungstätigkeiten in Abhängigkeit vom Alter des Kindes

Abbildung 10.2: Aufteilung der Kinderbetreuung insgesamt

Abbildung 10.3: Bivariate Zusammenhänge des relativen Berufsstatus und des absoluten Berufsstatus der Frau mit der Aufteilung der Kinderbetreuung

Abbildung 10.4: Bivariate Zusammenhänge der Gender-Items mit der Aufteilung der Kinderbetreuung 
Abbildung 10.5: Bivariate Zusammenhänge der Gender-Klassen mit der Aufteilung der Kinderbetreuung.....

Abbildung 10.6: Bivariate Zusammenhänge der Ost-West-Unterschiede und der Erwerbskonstellation mit der Aufteilung der Kinderbetreuung

Abbildung 10.7: Vorhergesagte Aufteilung der Kinderbetreuung in Abhängigkeit vom Alter des Kindes

Abbildung 10.8: Koeffizienten des relativen Berufsstatus (ISEI) zur

Vorhersage der Aufteilung der Kinderbetreuung, getrennt nach dem Alter des Kindes

Abbildung 10.9: Koeffizienten der beiden Gender-Items zur Vorhersage der Aufteilung der Kinderbetreuung, getrennt nach dem Alter des Kindes

Abbildung 10.10: Koeffizienten der Anzahl der Kinder zur Vorhersage der Aufteilung der Kinderbetreuung, getrennt nach dem Alter des Kindes

Abbildung 10.11: Koeffizienten des Bundesgebietes zur Vorhersage der Aufteilung der Kinderbetreuung, getrennt nach dem Alter des Kindes

Abbildung 10.12: Koeffizienten des absoluten Berufsstatus (ISEI) der Mutter zur Vorhersage der Aufteilung der Kinderbetreuung, getrennt nach dem Alter des Kindes

Abbildung 10.13: Koeffizienten der Erwerbstätigkeit der Mutter zur Vorhersage der Aufteilung der Kinderbetreuung, getrennt nach dem Alter des Kindes

Abbildung 10.14: Koeffizienten der Erwerbstätigkeit des Vaters zur Vorhersage der Aufteilung der Kinderbetreuung, getrennt nach dem Alter des Kindes

Abbildung 10.15: Vorhergesagte Aufteilung der Kinderbetreuung, unter Berücksichtigung der drei Gender-Klassen, getrennt nach dem Alter des Kindes. 


\section{Eidesstattliche Versicherung}

Eidesstattliche Versicherung gemäß § 12 der Prüfungsordnung 2013 des Promotionsstudienganges Sozialwissenschaften der Georg-August Universität Göttingen zur Erlangung des Doktorgrades für Sozialwissenschaften (Dr. disc. pol.)

1. Die Gelegenheit zum vorliegenden Promotionsvorhaben ist mir nicht kommerziell vermittelt worden. Insbesondere habe ich keine Organisation eingeschaltet, die gegen Entgelt Betreuerinnen und Betreuer für die Anfertigung von Dissertationen sucht oder die mir obliegenden Pflichten hinsichtlich der Prüfungsleistungen für mich ganz oder teilweise erledigt.

2. Ich versichere, dass ich die eingereichte Dissertation „Arbeitsteilung der Eltern: Die Aufteilung der Erwerbsarbeit, der Hausarbeit und der Kinderbetreuung in Abhängigkeit vom Alter des Kindes“ selbstständig und ohne unerlaubte Hilfsmittel verfasst habe; fremde Hilfe habe ich dazu weder unentgeltlich noch entgeltlich entgegengenommen und werde dies auch zukünftig so halten. Anderer als der von mir angegebenen Hilfsmittel und Schriften habe ich mich nicht bedient. Alle wörtlich oder sinngemäß den Schriften anderer Autoren entnommenen Stellen habe ich kenntlich gemacht.

3. Die eingereichte Dissertation habe ich nicht bereits in einem anderen Prüfungsverfahren vorgelegt.

4. Des Weiteren ist mir bekannt, dass Unwahrhaftigkeiten hinsichtlich der vorstehenden Erklärung die Zulassung zur Promotion ausschließen bzw. später zum Verfahrensabbruch oder zur Rücknahme des erlangten Titels berechtigen. 